Interpretation of equatorial current meter data as internal waves

by

Martin Benno Blumenthal

\author{
A.B., Physics \\ Princeton University \\ 1980
}

Submitted in partial fulfillment of the requirements of the degree of

Doctor of Philosophy

at the

Massachusetts Institute of Technology

and the

Woods Hole Oceanographic Institution

January 1987

Signature of the author

Joint Program in Oceanography,

Massachusetts Institute of Technology -

Woods Hole Oceanographic Institution, January 1987.

Certified by

Thesis supervisor

Accepted by

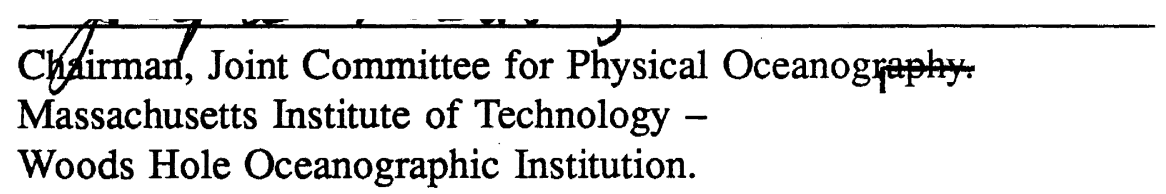




\title{
Interpretation of equatorial current meter data as internal waves
}

\author{
by
}

\author{
Martin Benno Blumenthal
}

\begin{abstract}
Submitted to the Massachusetts Institute of Technology-Woods Hole Oceanographic Institution Joint Program in January 1987 in partial fulfillment of the requirements for the degree of Doctor of Philosophy.
\end{abstract}

\begin{abstract}
Garrett and Munk use linear dynamics to synthesize frequency-wavenumber energy spectra for internal waves (GM72, GM75, GM79). The GM internal wave models are horizontally isotropic, vertically symmetric, purely propagating, and universal in both time and space. This set of properties effectively eliminates all the interesting physics, since such models do not allow localized sources and sinks of energy. Thus an important step in understanding internal wave dynamics is to make measurements of deviations from the simple GM models.

This thesis continues the search for deviations from the GM models. It has three advantages over earlier work: extensive data from an equatorial region, long time series (2 years), and relatively sophisticated linear internal wave models. Since the GM models are based on mid-latitude data, having data from an equatorial region which has a strong mean current system offers an opportunity to examine a region with a distinctly different basic state. The longer time series mean there is a larger statistical ensemble of realizations, making it possible to detect smaller internal wave signals. The internal wave models include several important extensions to the GM models: horizontal anisotropy and vertical asymmetry, resolution between standing modes and propagating waves, general vertical structure, and kinematic effects of mean shear flow. Also investigated are the effects of scattering on internal waves, effects that are especially strong on the equator because the buoyancy frequency variability is a factor of ten higher than at mid-latitudes.

In the high frequency internal wave field considered (frequencies between $.125 \mathrm{cph}$ and $.458 \mathrm{cph}$ ), several features are found that are not included in the GM models. Both the kinematic effects of a mean shear flow and the phase-locking that distinguishes standing modes from propagating waves are observed. There is a seasonal dependence in energy level of roughly $10 \%$ of the mean level. At times the wave field is zonally and vertically asymmetric, with resulting energy fluxes that are a small (4\% to 10\%) fraction of the maximum energy flux the internal wave field could support. The fluxes are, however, as big as many of the postulated sources of energy for the internal wave field.
\end{abstract}

Thesis supervisor: Dr. Charles C. Eriksen

Title:

Associate Professor of Oceanography

University of Washington 


\section{Acknowledgements}

I would first like to thank my advisor, Charlie Eriksen, whose unfailing support and patience were very important in seeing this project to completion. I would also like to thank the remainder of my committee-Terry Joyce, Jim Price, and Carl Wunsch-who waded through many drafts and made several important suggestions. Two other members of the faculty have also been very helpful. Harry Bryden has been a never-ending source of oceanographic ideas and encouragement, and Mel Briscoe has both always been ready to talk about internal waves and kindly provided the calculations on the LOTUS data presented in section 3.3.

My fellow students constitute perhaps the finest example of a scientific community that I ever hope to see, and I owe them a great deal. It is wonderful to be part of a group that is so supportive of each other, and I hope that as the students of today become the faculty of tomorrow that spirit is kept alive. It would make the world a better place.

My family has backed me no matter what I have chosen to do, even when I have spent my time plunging deeper and deeper into one particular branch of physics, an endeavor which to the outside world must resemble tilting at windmills. For their support I will always be grateful.

This work has been supported under grants from the National Science Foundation and the Office of Naval Research, grants numbered NSF-89076, ONR-88914, NSF-91002, NSF-94971, and NSF-93661. 


\section{Table of contents}

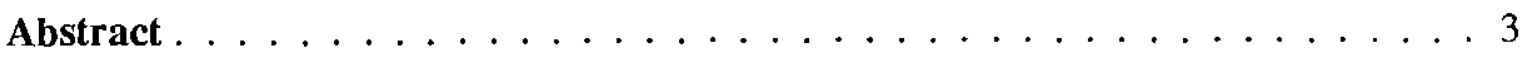

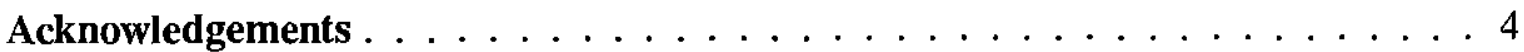

Chapter 1: Introduction and review

1.0 Introduction $\ldots \ldots \ldots \ldots \ldots \ldots \ldots \ldots \ldots \ldots \ldots \ldots$

1.1 The Garrett and Munk internal wave spectrum $\ldots \ldots \ldots \ldots . . \ldots 14$

1.2 The measurements incorporated into GM models . . . . . . . . . 15

Moored spectra . . . . . . . . . . . . . . . 15

Towed spectra $\ldots \ldots \ldots \ldots \ldots \ldots \ldots$

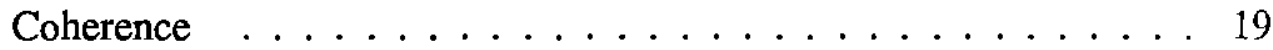

1.3 Linear internal waves $\ldots \ldots \ldots \ldots \ldots \ldots . \ldots \ldots$

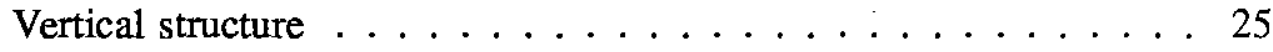

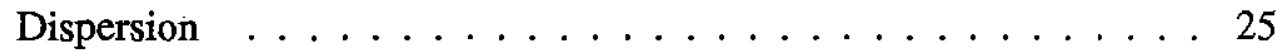

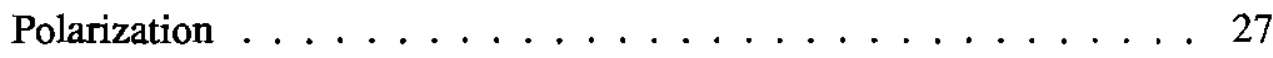

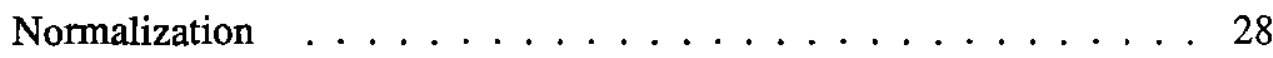

1.4 The wave continuum . . . . . . . . . . . . . . . 29

Drop boundary conditions . . . . . . . . . . . . . . . . . . . 29

Horizontal isotropy . . . . . . . . . . . . 30

1.5 Theoretical spectra and cross-spectra . . . . . . . . . . . . 31

Moored and towed spectra . . . . . . . . . . . . . 32

Moored component coherence . . . . . . . . . . . . . 32

Moored horizontal coherence $\ldots \ldots \ldots \ldots$. . . . . . . 33

Moored vertical coherence . . . . . . . . . . . . . . 33

Towed vertical coherence $\ldots \ldots \ldots \ldots \ldots$

1.6 Predictions of GM79 $\ldots \ldots \ldots \ldots \ldots \ldots \ldots \ldots$

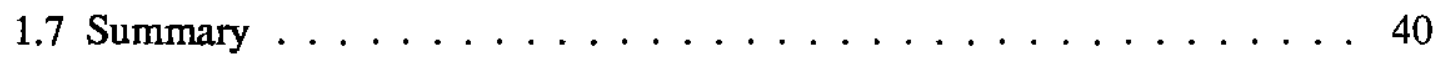

\section{Chapter 2: The PEQUOD dataset}

2.1 Data description . . . . . . . . . . . . . . 43

2.2 Rough estimates of non-GM dynamics $\ldots \ldots \ldots \ldots \ldots \ldots$ 


\section{Chapter 3: Vertical coherence calculations and interpretation}

3.1 Vertical coherence comparison of PEQUOD data with GM79 . . . . . 57

3.2 GM79 vertical coherence modifications $\ldots \ldots \ldots \ldots \ldots 75$

3.3 Coherence and phase from LOTUS $\ldots \ldots \ldots \ldots \ldots$

3.4 Summary of vertical coherence results $\ldots \ldots \ldots \ldots$

\section{Chapter 4: Single current meter data interpretation}

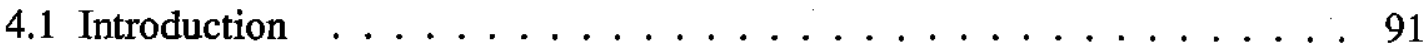

4.2 Dynamical model . . . . . . . . . . . . . . . . 92

4.3 GM79 spectral level . . . . . . . . . . . . . . . . . . 93

4.4 Covariance . . . . . . . . . . . . . . . . . . 94

Spectral estimators . . . . . . . . . . . . . . . . 94

Buoyancy frequency and vertical temperature gradient . . . . . . 95

4.5 Wave amplitude and fit estimators $\ldots \ldots \ldots \ldots$. . . . . . 97

4.6 Results and rejected models $\ldots \ldots \ldots \ldots 112$

Aliasing . . . . . . . . . . . . . . . . . 121

Mooring motion . . . . . . . . . . . . . . . . 121

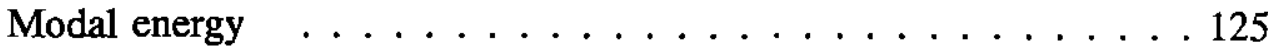

Temperature finestructure . . . . . . . . . . . . . . . 127

WKBJ shear packets . . . . . . . . . . . . . . . . . . 127

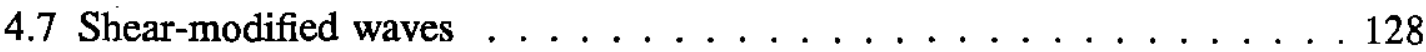

\section{Chapter 5: Internal wave models}

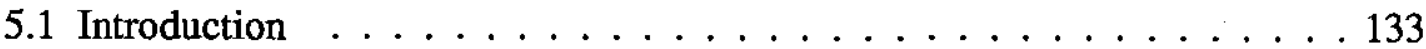

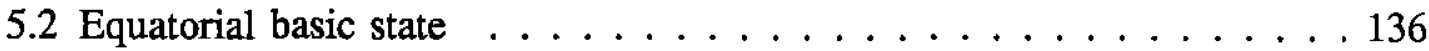

5.3 Equatorial beta plane perturbation equations . . . . . . . . . 138

5.4 Single wave solutions: motionless basic state . . . . . . . . . . . 140

5.5 Single wave solution: high frequency waves with mean flow . . . . . . 144

5.6 Vertical structure . . . . . . . . . . . . . . . . . . . . . 148

5.7 Sum of single wave solutions $\ldots \ldots \ldots \ldots \ldots$

5.8 Wave expansion of cross spectra $\ldots \ldots \ldots \ldots \ldots \ldots$

5.9 Cross spectra for a basic state at rest . . . . . . . . . . . . 156

5.10 Cross spectra for waves in a mean flow $\ldots \ldots \ldots \ldots \ldots 1$

5.11 Summary . . . . . . . . . . . . . . . . . . . . . . . 164 


\section{Chapter 6: Consistency checks}

6.1 Introduction $\ldots \ldots \ldots \ldots \ldots$

Results

6.2 Consistency check statistics and evaluation . . . . . . . . 168

6.3 Symmetries of the internal wave spectrum . . . . . . . . . 171

6.4 Model reduction results from PEQUOD . . . . . . . . . . . . 175

6.5 Internal wave symmetry results from PEQUOD . . . . . . . . . . . 182 Derivations

6.6 Consistency checks for no mean flow internal waves with general vertical struc-

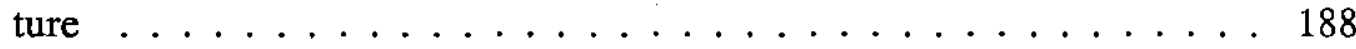

6.7 Consistency checks for no mean flow internal waves with WKBJ vertical struc-

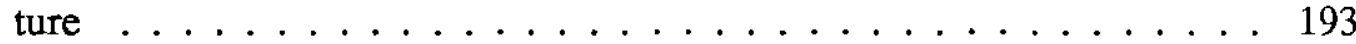

6.8 Consistency checks for internal waves in a mean shear flow . . . . . 196

\section{Chapter 7: Single internal waves in a vertically varying mean state}

7.1 Introduction . . . . . . . . . . . . . . . . 199

7.2 Vertical structure equation revisited . . . . . . . . . . . 201

7.3 Step $m(z)$ profile . . . . . . . . . . . . . . . . 205

7.4 Integrated wave solutions in the absence of mean flow . . . . . . . 206 horizontal velocity solutions ................ . . 215 comparison with WKBJ . . . . . . . . . . . . 220

7.5 Effects of buoyancy frequency profile variability . . . . . . . . . 222

Equatorial buoyancy frequency profile variability . . . . . . . 224

Analytic analysis of the effect on internal waves . . . . . . . 227

Numerical analysis of the effect on internal waves . . . . . . . 236

Summary of scattering . . . . . . . . . . . . . 247

7.6 Integrated wave solutions in a idealized mean flow . . . . . . . . . 248

7.7 Integrated wave solutions in a measured mean flow . . . . . . . . 258

7.8 Summary . . . . . . . . . . . . . . . . . . 267 


\section{Chapter 8: Spectral estimates}

8.1 Introduction . . . . . . . . . . . . . . . . . . . . . . . 269

8.2 Internal wave review and scaling $\ldots \ldots \ldots \ldots \ldots \ldots \ldots \ldots$

Boundary conditions . . . . . . . . . . . . . . 273

Polarization relations . . . . . . . . . . . . . . . . 274

8.3 Spectral model . . . . . . . . . . . . . . . . . . . . . 275

$E D P Q$ parameterization . . . . . . . . . . . 277

Direction moments . . . . . . . . . . . . . . . . . . . . . . . 279

8.4 Review of wavenumber estimation and spectral estimator statistics _ . . 281

8.5 Spectral inversion . . . . . . . . . . . . . . . 285

8.6 Spectral resolution . . . . . . . . . . . . . . . . . . . 290

Rank . . . . . . . . . . . . . . . . . . . . 290

Inversion results with a known input . . . . . . . . . . 295

Impulse response . . . . . . . . . . . . . . . 303

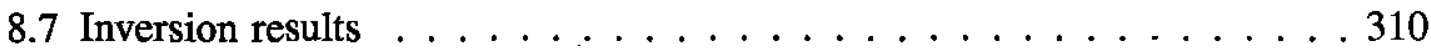

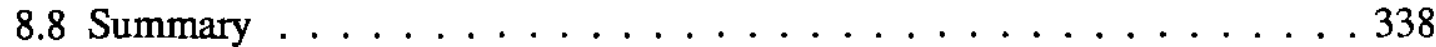

Chapter 9: Summary $\ldots \ldots . \ldots \ldots 39$

\section{Appendices}

A RVCM current meter aliasing . . . . . . . . . . . . . 345

$B$ Fourth order moments for joint-normal variables . . . . . . . . . . . 359

$C$ Covariance of cross-spectral estimators $\ldots \ldots \ldots \ldots \ldots \ldots$

$D$ Current meter spectra in terms of $E \Delta P Q$ direction moments $\ldots \ldots 363$

$E$ Model-Model covariance for the $E \Delta P Q$ direction moments $\ldots \ldots .368$

$F$ Generalized rank . . . . . . . . . . . . . . . . . 373

$G$ Beamforming and Capon estimators $\ldots \ldots \ldots \ldots \ldots \ldots$

References . . . . . . . . . . . . . . . . . . . 376 


\section{Chapter 1: Introduction and review}

\section{Introduction}

Nearly fifteen years ago, Garrett and Munk published a paper which used linear dynamics to synthesize a frequency-wavenumber energy spectrum for internal waves, a spectrum that fit all the available internal wave measurements reasonably well (Garrett and Munk 1972, henceforth referred to as GM72). This synthesis has been (and continues to be) a tremendous boon to the study of internal waves. For theoreticians it provides both a compact summary of the experimental results and a challenge to explain its universality and form. For the experimentalists it provides a standard to which new measurements can be compared, telling them the time and space scales to expect and allowing them to decide whether there is any new information in each new set of measurements.

One of the more powerful statements of GM72 is that the spectral level and scales of the internal wave field are essentially the same throughout much of the world's oceans. This universality has meant that the data from many different places has been compared to the GM72 spectrum. The additional data has led to small changes in the GM model (Garrett and Munk 1975 (GM75), Cairns and Williams 1976, Munk 1981 (GM79)). But a universal model is in some sense too simple: such a model, for example, does not allow localized sources and sinks of energy, a spatial diversity which would be expected given the diversity of processes occurring in the ocean (Wunsch 1976). If differences were found between one region and another, on the other hand, measurements of those differences would provide experimental verification that the variety of processes do in fact occur in the ocean. This has prompted many searches for anomalous regions, some of which are discussed in later sections. In spite of these efforts, the GM models have survived in large part intact.

This thesis continues the search for deviations from the GM universal models. There are three ways in which the data and methods used here increase the chances for finding differences from the GM models: the data is from a region whose basic state is different, the time series are significantly longer, and the models are more general. The data used in this thesis are from the Pacific EQUatorial Ocean Dynamics (PEQUOD) experiment, an experiment whose field work took place in the equatorial Pacific. As will be shown in section 1.2, the data incorporated into the GM models are almost all taken in midlatitude regions (though some equatorial-GM comparisons have been made, see Wunsch 
and Webb 1979). The equatorial basic state for high frequency internal waves differs significantly from its mid-latitude counterpart. These differences include enhanced scattering due to the presence of low frequency equatorially trapped motions and non-zero mean shear flow. The fact that the data are from much longer time series than were used to synthesize the GM models means that there is a larger statistical ensemble of realizations. The large ensemble allows detection of more subtle internal wave signatures than is possible with earlier measurements. The models used in this thesis are more general than those of GM in that they incorporate more dynamics and fewer arbitrary assumptions, changes which allow differentiation between inadequacies of the dynamics and inadequacies of the assumptions necessary to construct a simple result. This thesis concludes that a large number of deviations from a simple model such as GM exist in the measurements. These deviations are important because they reveal features of internal wave dynamics previously overlooked.

The first two chapters provide an introduction to internal waves and an introduction to the PEQUOD dataset. Chapter 1 reviews the GM models: the data used in forming the models, the linear internal wave theory, and the spectral analysis needed to connect the theory with the measurements. This detailed review of Garrett and Munk is included for two reasons. In some sense GM represents a widely held view of the internal wave field; it is considered to be a concise description of the internal wave field (which is fine) but it is also considered to be complete (which is not fine at all). To the extent that this thesis attempts to give a new perspective on internal waves and their observation, then, it is important to review the current perspective. But GM is also important in that it represents a good zeroth order description of the internal wave field: it includes only the simplest dynamics and wave symmetry properties and manages to describe a large fraction of the signal. In that sense, then, a review of GM is a good starting point for the more elaborate analyses that follow.

The first part of chapter 2 provides a reference description of the PEQUOD data that is used in this thesis: the two-year time series from two current meter and temperaturepressure recorder moorings $(Q$ and $U$ ) and the conductivity-temperature-depth (CTD) and horizontal velocity profiles. The second part of the chapter presents some rough measures of the non-GM dynamics present in the PEQUOD dataset. The possible importance of the mean zonal velocity shear is estimated by looking at the expected doppler shifts and Richardson numbers associated with the mean velocity profile. The scale of 
variation of the mean buoyancy frequency profile is shown to be smaller than the scales of the low modes, suggesting the non-WKBJ effects should be considered in modeling the data. Finally, a rough estimate of the expected size of energy fluxes is compared with the data set's ability to measure them; this too gives a rough idea of the results to expect from later chapters.

The zeroth order model that is given by GM79 blurs the distinction between propagating waves and vertically standing modes, modeling internal waves as a vertically symmetric field of propagating waves but writing the spectral form as a sum of modes. Chapter 3 shows that this attitude is insufficient for the PEQUOD data set: distinctions between propagating waves and modes are measurable, and the PEQUOD data set suggests that the low wavenumber energy is modal in character.

Section 3.2 discusses two measurable components of the internal wave field's modal character. The first component is the contrast between the discrete eigenvalues associated with modes and the continuous vertical wavenumber associated with purely propagating waves. The section shows that the only significant numerical effect of this discrete nature is the low wavenumber cutoff: a continuous model with a low wavenumber cutoff is indistinguishable in measurable cross-spectra from a discrete set of modes with the same low wavenumber cutoff. In practice this is a very useful result because analytically it can be quite convenient to use a model continuous in wavenumber (see Desaubies 1976), while numerically computing with a discrete sum of modes is much simpler than approximating a continuous set of modes. The model fit of the concluding chapter (8) uses a discrete set of wavenumbers, wavenumbers than correspond to the eigenvalues of the lowest modes.

The second effect of a modal internal wave field is phase-locking between upward and downward propagating waves. This effect is characterized in chapter 5 by comparing the cross-power between upward and downward waves with the power in upward and downward waves. In current meter data phase-locking is partially expressed as a difference between coherence calculated from horizontal velocity data and coherence calculated from displacement (temperature) data. Sections 3.1 and 3.3 use this property to show that significant phase-locking exists in the PEQUOD data, quite unlike the GM79 prediction. Chapter 3 thus has a mixed result as far as the comparison with GM79 is concerned. Since it is not possible to measure the difference between the sum of discrete modes and an integral of continuous waves except for the effect of the low mode cutoff, the 
smearing with cutoff of GM is partially adequate as far as measurements are concerned, but the lack of phase locking at low modes is measurably wrong as well as having no explicit theoretical justification.

The zeroth order model as given by any of the GM models presumes that the internal wave field is horizontally isotropic and vertically symmetric. Chapter 4 uses a subset of the possible current meter cross spectra to show that many of the assumptions made in formulating GM79 (the latest version of the GM models) are not consistent with the PEQUOD data. In fact the results go beyond showing that GM79 is inadequate to model the PEQUOD data; the calculations show that any vertically symmetric, horizontally isotropic model is inadequate to model the PEQUOD data. Chapter 4 concludes by showing that the subset of the data considered (cross-spectra calculated from single current meters) is consistent with two models: a zonally asymmetric modal internal wave field model and a propagating internal wave field modified by the presence of a mean zonal shear flow. It is then left to chapter 6 to use the general internal wave models of chapter 5 to differentiate between the two models.

Chapter 5 generalizes the linear internal wave theory of chapter 1 , making it possible to search for the aspects of the internal wave field that cause the misfit seen in chapter 4 . The generalizations allow vertical asymmetry, horizontal anisotropy, mixtures of vertically propagating waves and vertically standing modes, and non-WKBJ solutions to the vertical structure equation. This allows the exploration in chapter 6 of spectral features necessary to explain what is seen in the PEQUOD data. Furthermore, chapter 5 looks at the effects of a mean zonal shear flow on the internal wave field, effects shown in chapter 6 to be necessary to adequately model the PEQUOD wavefield.

Chapter 6 considers the consistency of the generalized internal wave models with the PEQUOD data. It extends the results of chapter 4 in two ways: it considers all of the spectra available from a single mooring, and it uses much more general internal wave models. All the cross-spectra can be considered at the same time because rather than checking each spectral component separately, the techniques of Müller et al. 1978 are used to combine these linear 'consistency checks' into a single statistical quantity (see section 6.2). This quantity $\left(\epsilon^{2}\right)$ can then be plotted against frequency, permitting comparison of quite general models with the complete dataset. It turns out that the most general model of an internal wave field in a resting basic state is not consistent with the PEQUOD data. This means that the data cannot be considered statistically 
consistent with any internal wave field whose basic state contains no mean flow: allowing horizontal anisotropy, vertical asymmetry, and mixtures of standing modes and vertically propagating waves improves the fit but does not reduce the misfit to the size expected of noise. An internal wave field modified by a mean shear flow, on the other hand, is possibly consistent with the dataset. This means that either the assumption that waves with different wavenumbers are uncorrelated is incorrect, or the assumption of a no mean flow basic state must be dropped. Only then are there sufficiently varied model parameters that the data can possibly be fit.

Chapter 7 considers the vertical structure of individual solutions to the internal wave perturbation equation, results necessary for the shear mode fit that is performed in chapter 8. The chapter starts by comparing integrated and WKBJ solutions to the vertical structure equation, pointing out that while the integrated solutions are 'more correct' for any given profile of buoyancy frequency, it is not clear which solution is more appropriate when the profiles contain noise. This is because only solutions which are robust in the presence of profile variability are appropriate for modeling the averaged spectra.

The results of Hayes and Powell 1980 are used to characterize the profile variability expected on the equator: the variability is enhanced by a factor of ten relative to that expected for off-equatorial latitudes. This enhancement is attributed to the low frequency waves that propagate in the equatorial waveguide and are thus closely trapped to the equator. The chapter then uses analytic and numerical results to characterize the effects of these fluctuations on the internal waves themselves. Low modes are shown to be quite robust, almost unaffected by profile variability, while the higher modes are strongly scattered. This supports the results of chapter 3 which suggest that the low wavenumbers have a modal character, the behavior expected in the absence of dissipation or strong scattering. High modes, whose structure can be quite complicated, are strongly affected by scatter, thus the complicated structures that an integration might produce are inappropriate and simpler forms can equally well be used.

Chapter 8 uses a spectral inversion to optimally fit the PEQUOD central mooring data with an internal wave model that includes the generalizations that chapters 3,4 , and 6 suggest are required. It is found that the internal wave field is both vertically and zonally asymmetric, with some evidence of both the kinematic effects of a mean shear flow and the phase-locking that distinguishes between modes and propagating waves. The chapter also finds that some features of the internal wave field change in time, changes that are 
correlated with the phase of the 1982-1983 El Niño event.

\subsection{The Garrett and Munk internal wave spectrum}

Because this thesis and much of the work in internal waves is essentially a variation on the themes of GM72, GM75, and GM79, it is appropriate to review the methods and results of the original papers. The current form of the Garrett and Munk spectrum (GM79) describes the internal wave energy $E_{I W}$ as a function of frequency $\omega$ and mode number $j$,

$$
E_{I W}(\omega, j)=N(z) E(\omega, j)=N(z) \tilde{E} B(\omega) H(j)
$$

where

$$
\begin{array}{cc}
\tilde{E}=b^{2} N_{0} E & \\
B(\omega)=\frac{2 f}{\pi \omega \sqrt{\omega^{2}-f^{2}}} & \int_{f}^{N} B(\omega) d \omega=1 \\
H(j)=\frac{\left(j^{2}+j_{*}^{2}\right)^{-1}}{\sum_{1}^{\infty}\left(j^{2}+j_{*}^{2}\right)^{-1}} & \sum_{1}^{\infty} H(j)=1
\end{array}
$$

The various constants and variables are defined as follows:

$\begin{array}{ll}E(\omega, j) & \text { Internal wave spectral level in a } 1 \mathrm{cph} \text { ocean } \\ B(\omega) & \text { Normalized frequency dependence } \\ H(j) & \text { Normalized vertical mode number dependence } \\ j_{*} & \text { Rolloff mode number }(=3) \\ E & \text { Dimensionless energy level }\left(=6.3 \times 10^{5}\right) \\ N(z) & \text { Local buoyancy frequency } \\ N_{0} & \text { Extrapolated buoyancy frequency }(3 \mathrm{cph}) \\ b & \text { Exponential depth scale for } N(z)(1.3 \mathrm{~km})\end{array}$

While it is confusing and somewhat dismaying to have such a large array of variables and constants, the basic structure of equation 1.1.1 $a$ is simple: the internal wave energy at any particular depth is factored such that it is proportional to the local buoyancy frequency $N(z)$, a function of frequency $B(\omega)$, and a function of mode number $H(j)$. The factoring into separate functions of mode number and frequency is arbitrary, justified only in that the data considered in the GM papers is consistent with such a factoring. The function of frequency is such that there is a cusp at the inertial frequency, and well away from that point the spectral level goes as $\omega^{-2}$. The function of mode number is 
such that it varies slowly when the mode number $j$ is small $\left(<j_{*}\right)$, and when $j$ is large the spectral level goes as $j^{-2}$.

This GM79 spectrum is only the third in a series of what could be many. Already changes have been suggested (Desaubies and Gregg 1981) to rid this model of certain singularities; other results suggest that the explicit cusp at the inertial frequency is unnecessary and can be found as a result of internal wave dynamics (Fu 1980 and Munk 1980). But what is of interest here is not so much how correct GM79 (or GM75, or GM72) is, but rather how such a form is fit to the data, what sorts of assumptions are necessary for the fit to be made, and in the event that one finds data that disagrees with GM79, what sorts of changes in the model would eliminate the disagreement. This process gets somewhat complicated because none of the measurements measure $E(\omega, j)$ directly: the interpretation of each measurement involves using a linear internal wave model to relate what is measured to the spectral level $E(\omega, j)$.

\subsection{The measurements incorporated into the GM models}

One of the great powers of the GM spectral fits is their ability to explain many different types of measurements. (see Jenkins and Watts 1968 for an introduction to spectra and spectral statistics). The autospectra which were included in GM72 are summarized in their figure 4, which is reproduced here as figure 1.2.1. The lower half of the figure gives measurements of the distribution of energy with frequency, while the upper half of the figure gives the distribution of energy with a component of the horizontal wavenumber. The measurements which are used to determine the distribution of energy with frequency are of two sorts: measurements from moored current meters and measurements from neutrally buoyant floats.

The horizontally stippled area labeled 'Fofonoff, Site $D$ ' represents the data from several depths at Site $D$ in the western North Atlantic (Fofonoff 1966, 1969b; Webster $1968 a, b)$. The spectra from each depth have been scaled by the local buoyancy frequency, a transformation which reduces a 10db range in energy level as a function of depth to a 3db range (Fofonoff 1969b). This scaling is consistent with WKBJ theory, a point that will be made clearer in the next section. The site $D$ data is bracketed by the data from two other locations. The stippled area marked 'Gould' represents data from a 2 week time series taken in the Bay of Biscay (Gould 1971). The area marked 'Perkins' represents two months of data taken from the Mediterranean (Perkins 1970). The other moored data used 


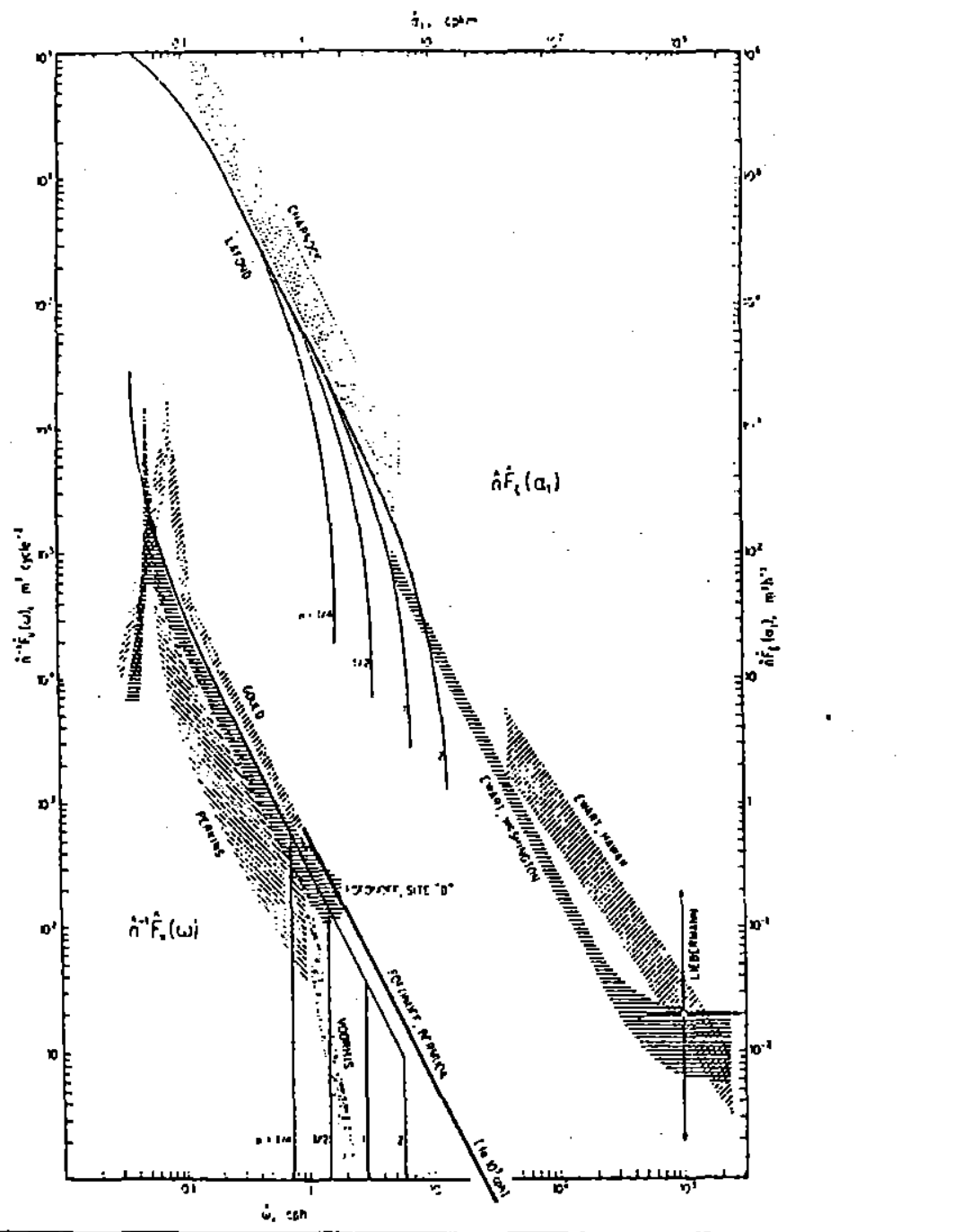

Figure 1.2.1 Autospectra from GM72

This plot is a reproduction of figure 4 from GM72. The lower part of the plot (left and bottom axes) gives the frequency $\omega$ dependence of the horizontal velocity spectra $\hat{F}_{u}$. It is scaled by the local buoyancy frequency ( $\hat{n}$ in the plot). The top half of the plot (top and right axes) gives the horizontal wavenumber component $k$ ( $\alpha_{1}$ in the plot) dependence of the displacement spectra $\hat{F}_{\eta}$. The displacement spectra are scaled by the inverse of the local buoyancy frequency. 
is from Bermuda (Fofonoff 1966, 1969b; Webster 1970): it shows a $\omega^{-2}$ dependence extending to high frequencies. Clearly the moored sensor measurements from widely separate locations are quite similar. All the moored spectra as presented in the plot are featureless except for a peak at the local inertial frequency. This result contrasts strongly with the data from neutrally buoyant floats (Voorhis 1968). The Voorhis spectra cuts off sharply at the local buoyancy frequency, unlike the moored spectra which show the same $\omega^{-2}$ slope well beyond the local buoyancy frequency. This cutoff is also found in later isotherm follower float measurements (Cairns 1975). These measurements are presented in figure 1.2.2 (which reproduces figure 2 from GM75). The models (GM72 GM75, and GM79 are indistinguishable on this plot) are given by the solid line: it cuts off sharply at the buoyancy frequency $(n)$. The data also cut off at the buoyancy frequency. GM72 suggest (and subsequent work agrees) that this discrepancy between the moored and float measurements is not a property of internal waves at all, rather it is due to finestructure contamination of the moored spectra, contamination that the float measurements are not subject to. Therefore the cusp at the inertial frequency, the general $\omega^{-2}$ frequency dependence, and the scaling as the local buoyancy frequency of energy level are the features of the frequency spectra that the GM spectra try to characterize with a combination of linear internal wave theory and empirical fit.

The measured distribution of displacement spectral energy with horizontal wavenumber is given in the top part of figure 1.2.1. The stippled areas marked Charnock and LaFond respectively are from towed thermistor chains: the depth of a particular isotherm is followed by interpolating between sensors (Charnock 1965 and LaFond and LaFond 1971). The measurements by Ewart, on the other hand, are made by a self propelled isobaric vehicle so that he obtains the horizontal spectrum of temperature $\hat{F}_{T}(K)$. That spectrum is then converted to a displacement spectrum by dividing by the mean potential temperature gradient squared. The cross marked 'Liebermann' gives the results from measurements by Liebermann 1951 with thermistors mounted on a submarine. All the results are consistent with one another (though there is little overlap in wavenumber). The Ewart measurements are analogous to the moored measurements because they too are at a fixed depth and are thus subject to the same sort of finestructure contamination. The isotherm following measurements, on the other hand, are more like Voorhis's neutrally buoyancy float measurements, and are not subject to the same contamination. So in GM72 not much attempt was made to fit the Ewart measurements, and the model 

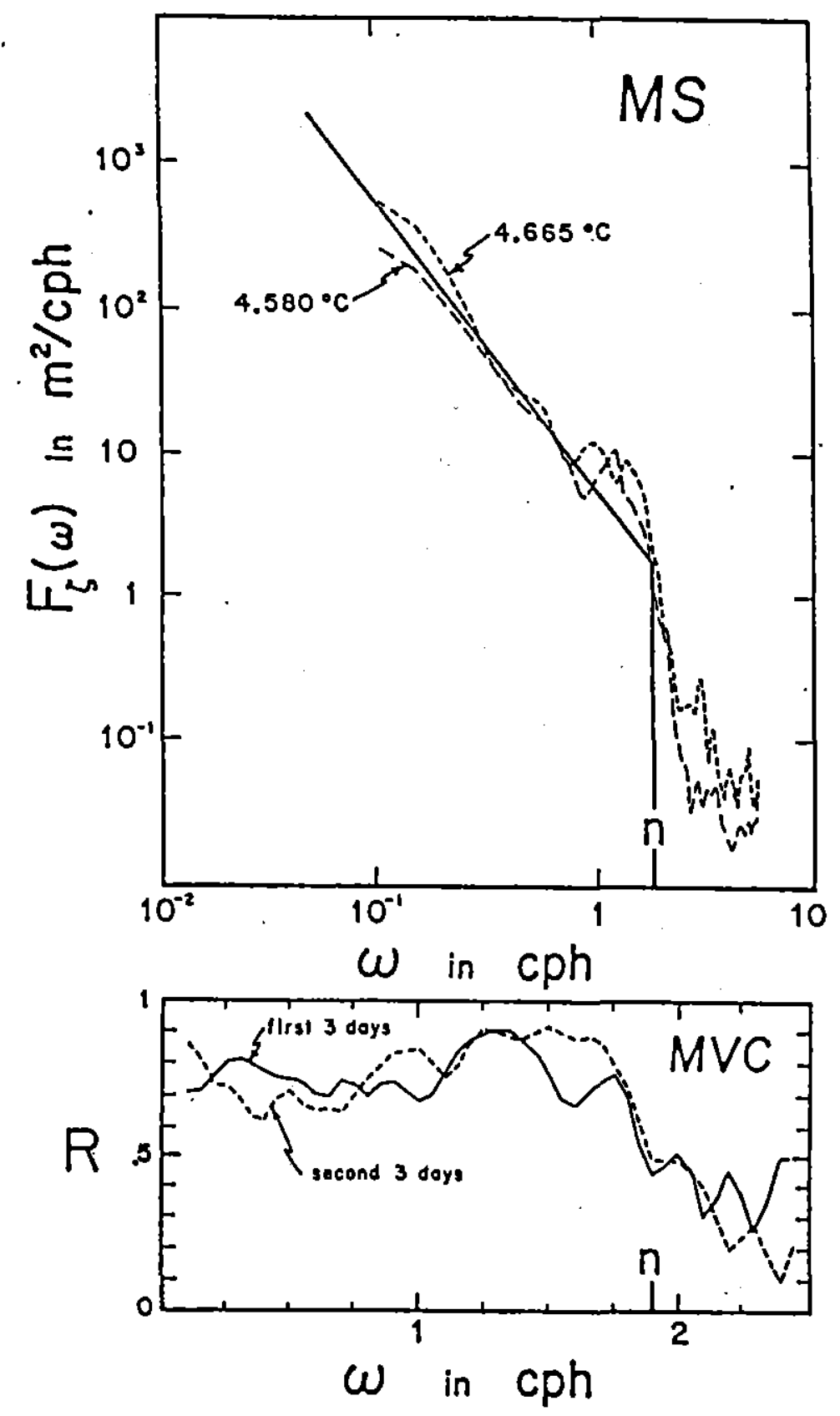

Figure 1.2.2 Moored autospectra and coherence from GM75

The spectrum of vertical displacement and the coherence for a vertical separation of $100 \mathrm{~m}$ from Cairns 1975 . The solid curve that drops sharply at $n$ could be any of the three GM internal wave models (GM72, GM75, or GM79). The figure is copied from GM75. 
(solid) curves drop off sharply. GM75, on the other hand, differs from GM72 in that it does not have such a sharp cutoff, and is a much better fit to the towed measurements. This improvement can be seen in figure 1.2.3 (taken from the correction to GM75) which compares the GM72 model spectrum, the GM75 model spectrum, and tow measurements from Katz 1974.

GM75, unlike GM72, also attempted to fit dropped spectra (DS), i.e. displacement spectra as a function of vertical wavenumber. This plot (given here as figure 1.2.4) clearly shows the difference between GM75 and GM72: GM72 simply bears no resembiance to the dropped data. This result is exactly what Garrett and Munk expected to happened new information would force an update of the model.

Coherence measures the degree of correlation between two time series at a particular frequency (see Jenkins and Watts 1968). While GM72 did look at some coherences between moored instruments that had purely horizontal separation, the data were of low quality and did not strongly influence their calculation. Measurements of coherence as a function of vertical separation were relatively useful and are presented in GM72's figure 5 (here reproduced as figure 1.2.5). Coherence at the the Site $D$ moorings was characterized by Webster 1972 leading to "Webster's Rule": At any separation $Z$, the frequency at which the coherence drops to $\frac{1}{2} \omega_{\frac{1}{2}}$ times the separation is equal to a constant,

$$
\omega_{\frac{1}{2}} Z=13 \mathrm{cph} \mathrm{m}
$$

This rule is given by the $45^{\circ}$ line in figure 1.2.5: it fits the Site $D$ data quite well at separations above $10 \mathrm{~m}$. The Siedler points are also from site $D$. Along with Webster's measurements at short separations, the Siedler points suggest that coherence drops off even faster as a function of frequency than Webster's rule would suggest. All these measurements are subject to the same finestructure contamination mentioned in the discussion of autospectra. The measurements by Pinkel, on the other hand, are not subject to that contamination, and in fact show no frequency dependence. Pinkel's result turns out to be what is expected from internal wave theory. Thus GM72 conclude that the decrease characterized by Webster's rule is due to finestructure contamination.

The lower part of figure 1.2.2 presents vertical coherence calculated by Cairns 1975 . These isotherm follower measurements are not subject to fine structure, and the plot shows that the coherence is fairly independent of frequency until a buoyancy frequency 


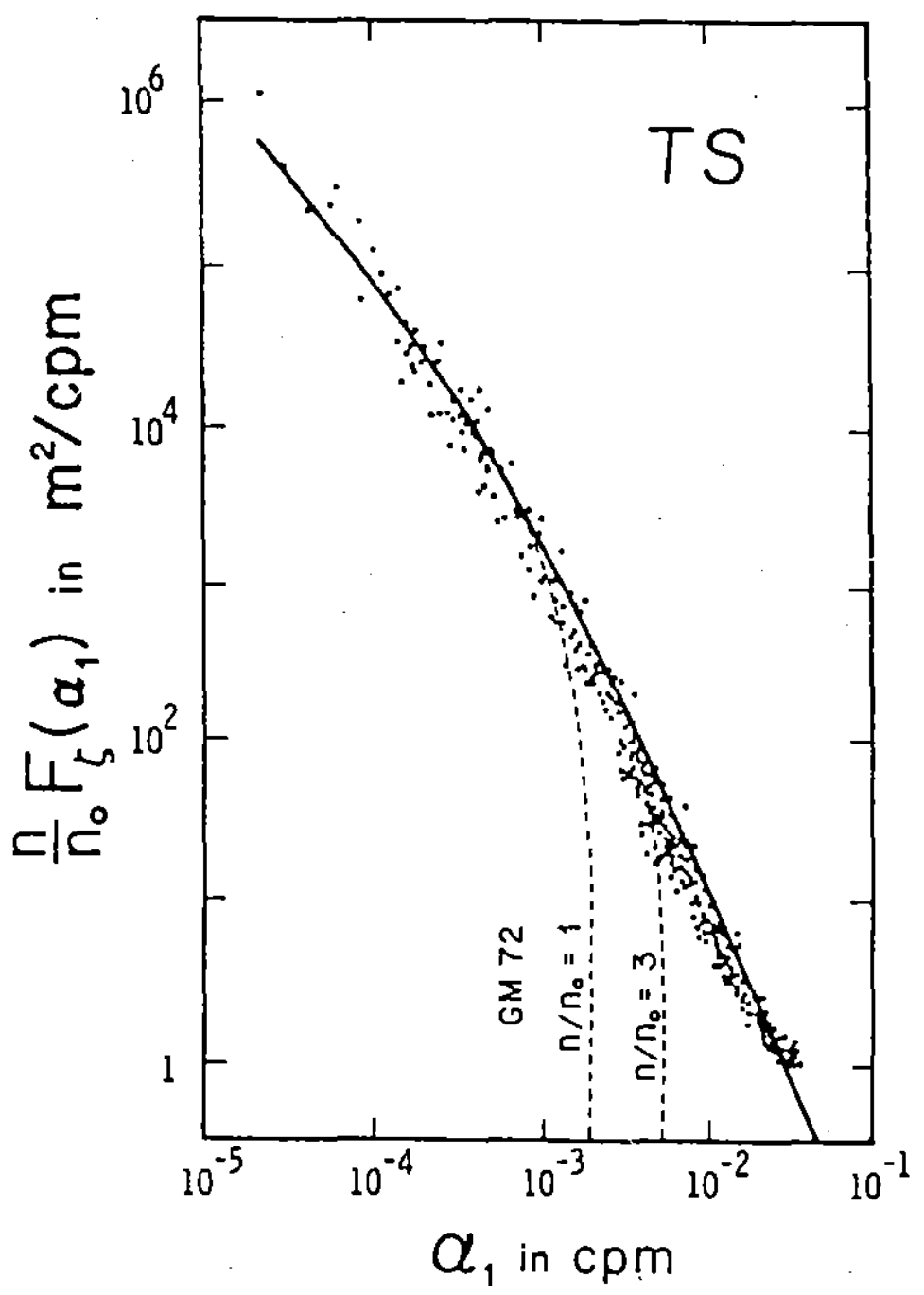

Figure 1.2.3 Towed spectra from GM75 (corrected)

The normalized towed spectrum of vertical displacement from Katz 1974. The solid line corresponds to the GM75 spectral form while the dashed curves correspond to GM72. The figure is from the correction to GM75. 


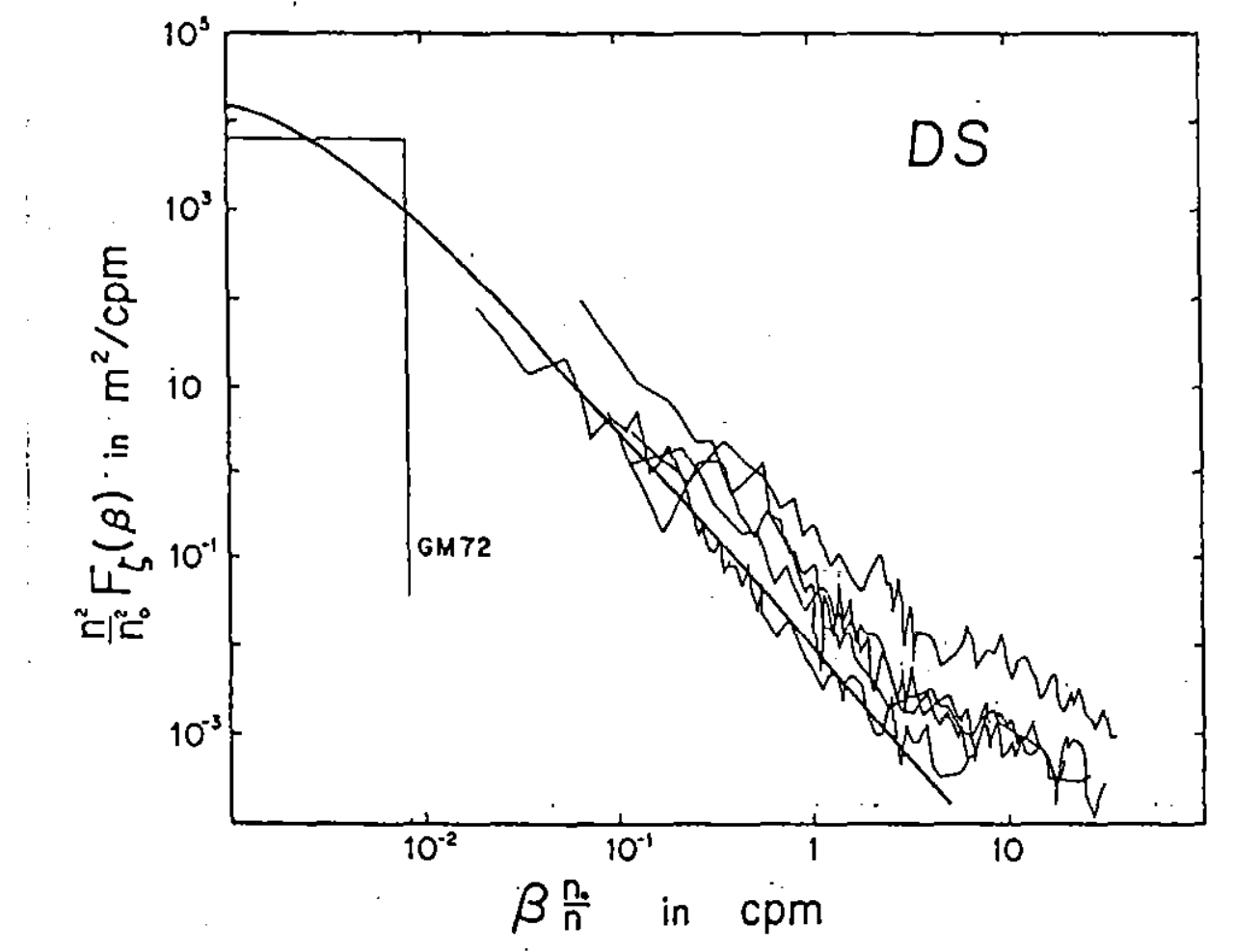

Figure 1.2.4 Dropped spectra from GM75

The normalized dropped spectrum of vertical displacement from Millard 1972. The heavy solid curve is from the GM75 model; the light line ('top hat') is from the GM72 model. The figure is from GM75. 


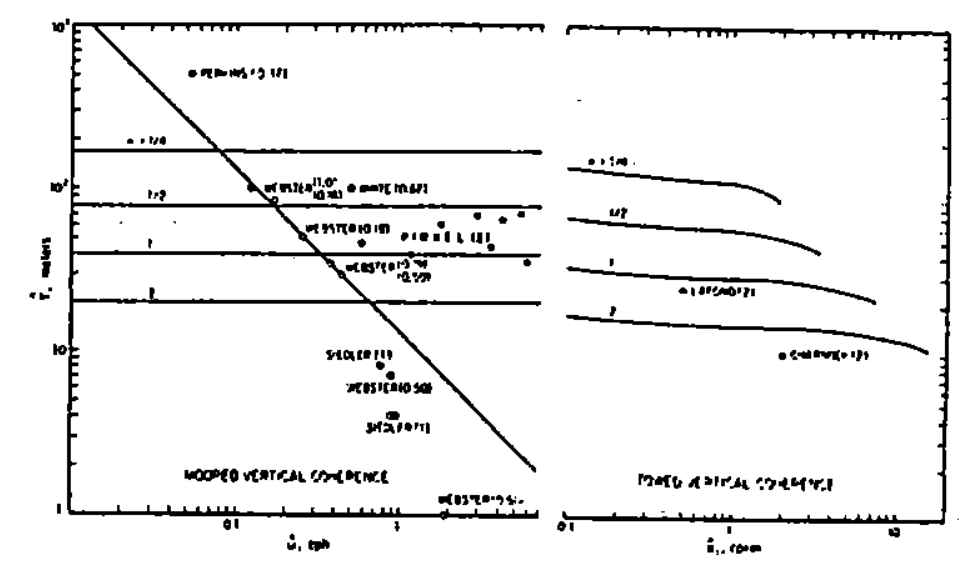

Figure 1.2.5 Coherence between vertically separated sensors

This figure is a reproduction of figure 5 in GM72. The points give for a given separation the frequency at which the coherence drops below .5. The open circles are points from measurements that are subject to finestructure contamination, while the solid circles are not. The $45^{\circ}$ line is Webster's rule (see equation 1.2.1). 


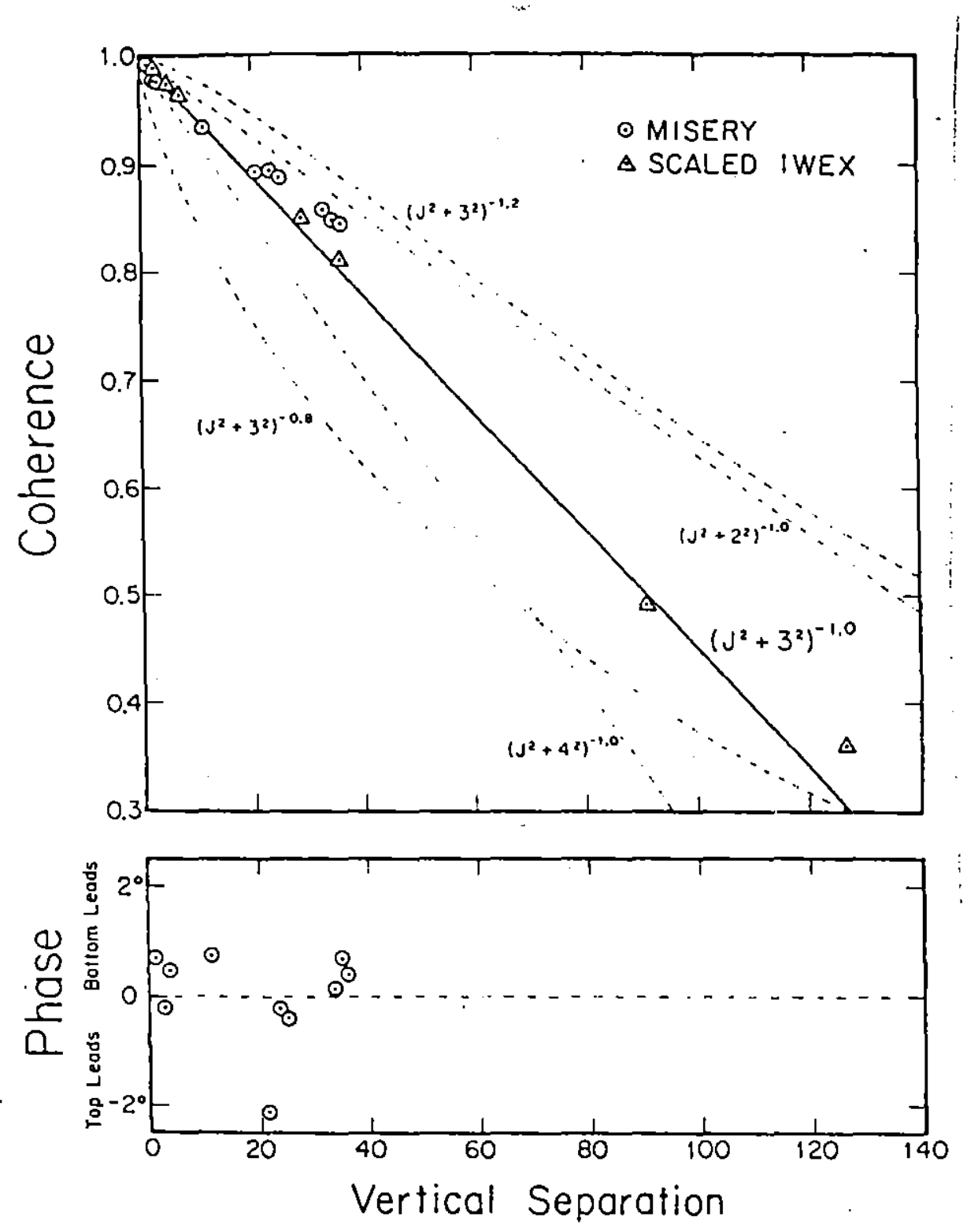

Figure 1.2.6 Vertical coherence from Cairns and Williams 1976

This figure presents vertical coherence from Caims and Williams 1976. The circles are coherences from Cairns and Williams isotherm follower measurements; the triangles are scaled data from the IWEX experiment. Each estimate is averaged over the subbuoyancy frequency range $(0 \leq \omega \leq 0.5 N(z)$ and has approximately 660 degrees of freedom. The expected variance of the coherence is .0016 and $95 \%$ confidence limits on phase are $\pm 2.6^{\circ}$. This plot is the basis for the spectral form which is called GM79. 
cutoff, much as GM72 predicted.

Cairns and Williams 1976 made more isotherm follower measurements. Coherences from the measurements are presented as circles in figure 1.2.6: IWEX data are presented as well. These results suggested changing the $H(j)$ function from what was used in GM75 to the form used in GM79; it also determined that the rolloff mode number $j_{*}$ is 3.

In some sense, then, GM79 contains the essential information presented in these six plots. The problem now is to understand how linear internal wave theory is used by the GM models to link the scaled spectral level $E(\omega, j)$ to all of the different types of measurements.

\subsection{Linear internal waves}

GM72 uses linear internal wave dynamics to devise a unified model for all the measurements in figures 1.2.1 through 1.2.6. This synthesis is done by using the dispersion relation to relate frequency, horizontal wavenumber and vertical structure, and using polarization relations to relate measurements of displacement and horizontal velocity. Both the dispersion relation and the polarization relations are results of seeking wavelike solutions to the equations of motion.

The usual $f$-plane Boussinesq equations are

$$
\begin{array}{r}
u_{t}-f v+p_{x}=0 \\
v_{t}+f u+p_{y}=0 \\
w_{t}-b+p_{z}=0 \\
b_{t}+N^{2}(z) w=0 \\
u_{x}+v_{y}+w_{z}=0
\end{array}
$$

where $b$ is the buoyancy perturbation and $p$ is the pressure perturbation divided by the mean density. Derivations of these equations can be found in several textbooks - Phillips 1980 and Gill 1982 are two possibilities — and they are also discussed in chapter 5. The textbooks do a much deeper exploration of internal wave dynamics than this section which is concerned only with the first order structure of the solutions: unlike this section the textbooks include such important topics such as group velocity and energy transfer by wave packets. 
While this section is reproducing the results of GM72, I have chosen notation to match the later chapters of this thesis (and many other papers) rather than match GM72. The table in figure 1.3.1 translates the notation of GM72 to that used in this discussion.

Since the coefficients of the equations are independent of time $t$ and horizontal position $x, y$, there are solutions wavelike in those three coordinates. That means that each dynamical variable $u, v, w, p, b$ can be written as the product of wavelike dependence, a complex constant and a function of vertical position $z$. Inspection of the equations suggests relations between those functions of vertical position. In particular, equations 1.3.1 $a, b$ suggest that $u, v$ and $p$ could all have the same vertical structure $G_{z}(z)$, and equation 1.3.1e relates that vertical structure to the vertical structure $G(z)$ of $w$. Equation 1.3.1 $d$ relates the vertical structure of $b$ to the vertical structure of $w$ and the buoyancy frequency profile $N(z)$. Thus we seek solutions of the form

$$
\left[\begin{array}{c}
u \\
v \\
w \\
p \\
b
\end{array}\right](x, y, z, t)=\left[\begin{array}{c}
\hat{u} G_{z}(z) \\
\hat{v} G_{z}(z) \\
\hat{w} G(z) \\
\hat{p} G_{z}(z) \\
\hat{b} N^{2}(z) G(z)
\end{array}\right] e^{i(k x+l y-\omega t)}
$$

where letters marked by a ${ }^{\wedge}$ denote complex constants (quantities independent of $x, y, z, t$ ). Plugging the form 1.3.2 into the equations 1.3.1a, $b, d, e$ then gives a set of algebraic equations relating the complex constants to each other, and plugging the form 1.3.2 into equation 1.3.1c gives an equation for $G(z)$. That vertical structure equation is of the form

$$
G_{z z}+m^{2}(z) G=0
$$

where

$$
m^{2}(z)=\frac{N^{2}(z)-\omega^{2}}{\omega^{2}-f^{2}} K^{2}
$$

and boundary conditions of no normal flow at a rigid top $(z=0)$ and rigid (and flat) bottom $(z=-D)$ become

$$
G(0)=G(-D)=0
$$

Equation 1.3.2 rewritten with explicit relationships between the hatted complex constants 
Notation

$\begin{array}{ccl}\text { Variable } & \text { GM72 } & \text { Description } \\ N(z) & \hat{n} & \text { buoyancy frequency } \\ u & u_{1} & \text { zonal velocity } \\ v & u_{2} & \text { meridional velocity } \\ w & v & \text { vertical velocity } \\ z & -y & \text { vertical position (height) } \\ K & \alpha & \text { horizontal wavenumber } \\ k & \alpha_{1} & \text { zonal component of wavenumber } \\ l & \alpha_{2} & \text { meridional component of wavenumber } \\ \omega & \omega & \text { frequency }\end{array}$

Figure 1.3.1 Notation and GM72 equivalents 
gives the polarization relations,

$$
\left[\begin{array}{l}
u \\
v \\
w \\
p \\
b
\end{array}\right](x, y, z, t)=\left[\begin{array}{c}
\left(i \cos \theta-\frac{f}{\omega} \sin \theta\right) \frac{1}{K} G_{z}(z) \\
\left(i \sin \theta+\frac{f}{\omega} \cos \theta\right) \frac{1}{K} G_{z}(z) \\
G(z) \\
i \frac{\left(\omega^{2}-f^{2}\right)}{\omega K^{2}} G_{z}(z) \\
-\frac{i}{\omega} N^{2}(z) G(z)
\end{array}\right] \hat{A}(\omega, k, l) e^{i(k x+i y-\omega t)}
$$

where $(k, l)=K(\cos \theta, \sin \theta)$. Using boldface to denote vectors, this becomes

$$
\mathbf{u}(x, y, z, t)=\mathbf{g}(z, \omega, k, l) \hat{A}(\omega, k, l) e^{i(k x+l y-\omega t)}
$$

The elements of $\mathbf{u}$ are written $u_{i}$, etc. Equation 1.3.4 reduces the problem of determining the structure of internal waves to solving for $G(z)$.

The vertical dependence of the buoyancy frequency $N(z)$ is as yet unspecified. After checking that the effect of a mixed layer is unimportant, GM72 consider the buoyancy frequency to have an exponential profile.

$$
N(z)=N_{0} e^{z / b}
$$

where the vertical scale $b$ is taken to be $1.3 \mathrm{~km}$ and the buoyancy frequency at the surface $N_{0}$ is $3 \mathrm{cph}$. They argue that this is a reasonable fit to the world's oceans (though the plot they present shows that it is a coarse fit at best). More importantly, they continue, most of the results depend only on the local buoyancy frequency $N(z)$ and it does not matter which model $N(z)$ profile is chosen. These local properties can be found by using a WKBJ approximation to solve equation $1.3 .3 a, b$ without the boundary conditions $1.3 .3 c$.

$$
\begin{aligned}
G_{ \pm}(z) & =\sqrt{\frac{N_{r}}{m(z)}} e^{ \pm i \int_{z_{0}}^{z} m\left(z^{\prime}\right) d z^{\prime}} \\
G_{ \pm z}(z) & = \pm i m(z) G(z)
\end{aligned}
$$

where $N_{r}(\omega, K)$ is a normalization constant that is to be determined. The difference between the two solutions $G_{+}$and $G_{-}$are that $G_{+}$corresponds to a wave which has upward phase propagation while $G_{-}$corresponds to a wave with downward phase propagation. Taking $z_{0}=-D$ and imposing the bottom boundary condition from $1.3 .3 \mathrm{c}$ results in a solution

$$
G(z)=G_{+}(z)-G_{-}(z)
$$


so that $G(z)$ is proportional to $\sin \int_{z_{0}}^{z} m\left(z^{\prime}\right) d z^{\prime}$ and thus is zero at the bottom $z=-D$. Imposing the top boundary condition from $1.3 .3 c$ as well gives a constraint that

$$
\int_{-D}^{0} m\left(z^{\prime}\right) d z^{\prime}=j \pi
$$

where the mode number $j$ is an integer. Because $j$ only takes on integer values, only a discrete set of frequencies $\omega$ are allowed for a given horizontal wavenumber $K$. This can be expressed as $\omega=\omega_{j}(K)$, where the $j$ is the mode number. In particular, were $N(z)$ constant then

$$
\omega_{j}^{2}(K)=\frac{N^{2}+\left(\frac{j \pi}{K D}\right)^{2} f^{2}}{1+\left(\frac{j \pi}{K \bar{D}}\right)^{2}}
$$

which suggests that as the index becomes large, the difference between the curves $\omega_{j}(K)$ becomes small. Essentially the results that GM72 get by using exact solutions to 1.3.3 with an exponential $N$ profile and then averaging can be obtained from the WKBJ solution as long as $f \ll \omega \ll N(z)$ (limits that GM72 explore by using the exact solutions). Using equations 1.3.3 and 1.3.6, we can now determine an approximate expression for the normalization constant $N_{r}(\omega, K)$. The mean square energy density normalized by density $(\bar{E})$ is

$$
\bar{E}=\frac{1}{2}\left(\overline{u^{2}}+\overline{v^{2}}+\overline{w^{2}}\left(1+N^{2} / \omega^{2}\right)\right)
$$

For a wave such as equation 1.3 .4 with $G(z)$ given by $G_{+}$(or $G_{-}$) from 1.3 .6 , that expression for averaged energy becomes

$$
\begin{aligned}
\bar{E} & =\frac{N^{2}-f^{2}}{\left(\omega^{2}-f^{2}\right)} \frac{|\hat{A}|^{2} N_{r}}{m(z)} \\
& \approx N(z)|\hat{A}|^{2}
\end{aligned}
$$

where the normalization $N_{r}$ has been chosen such that

$$
N_{r}=K\left(\omega^{2}-f^{2}\right)^{1 / 2}
$$

The approximation in equation $1.3 .9 b$ is to neglect the frequency $\omega$ against the buoyancy frequency $N(z)$ (a hydrostatic approximation). By considering the exact solutions to the exponential profile, GM72 conclude that this is a better approximation than retaining the 
non-hydrostatic effect in the WKBJ approximate solution. In any case, the mean square velocity $\left(\overline{U^{2}}\right)$ and mean square displacement $\left(\overline{\eta^{2}}=\overline{(w / \omega)^{2}}\right)$ are

$$
\begin{aligned}
\overline{U^{2}}=\overline{u^{2}}+\overline{v^{2}} & =N(z)\left(\frac{\omega^{2}+f^{2}}{\omega^{2}}\right)|\hat{A}|^{2} \\
\overline{\eta^{2}} & =N^{-1}(z)\left(\frac{\omega^{2}-f^{2}}{\omega^{2}}\right)|\hat{A}|^{2}
\end{aligned}
$$

which are identical to the results obtained by GM72 by averaging solutions to the exponential $N(z)$ profile.

\subsection{The wave continuum}

The ultimate goal in GM72 is to write a wave energy spectrum $E(\omega, k, l)$ that uses the internal wave structures just derived to fit the available measurements. The next step in achieving that fit is to consider what happens when there are many waves with the properties outlined in the last section. In particular, since $m(z)$ depends on frequency and wavenumber, for each value of the three component vector $(\omega, k, l)$ there are two solutions $G_{ \pm}(z)$ to the vertical structure equation. By using properties of the exact solutions to the exponential profile (which are also properties of the general problem), GM72 narrow the range of possible wavenumber values and solutions $G_{ \pm}(z)$.

One of the results obtained from GM72's exact solution to the exponential $N$ profile is that there is a discrete set of solutions $G_{i}(z)$ to the vertical structure equation. This is also seen in the WKBJ solutions when the boundary conditions are imposed. The discrete nature of the solutions is a consequence of applying top and bottom boundary conditions. This result can be summarized by saying that the effect of having an finite depth ocean is that, rather than having two solutions $G_{ \pm}(z)$ for each choice of $\omega$ and $K$, there is a single solution $G_{i}(z)$ that corresponds to a curve in $\omega-K$ space: $\omega=\omega_{i}(K)$. The questions then becomes, how close are these curves $\omega_{i}(K)$ ? If they are quite close together, then any pair of values $(\omega, K)$ can be found on or near some curve, and then the only difference between the problem with and without boundary conditions is that there are two solutions $G_{ \pm}$for each frequency-wavenumber pair when no boundary conditions are imposed, while those two solutions are forced to have equal amplitude and a fixed phase relationship (equation $1.3 .7 a$ ) when the boundary conditions are imposed. GM72 cite Munk and Phillips 1968 who show that the vertical distance $z$ beyond which the coherence is small is on the order of the reciprocal bandwidth $z=O\left(\Delta m^{-1}\right)$, i.e. more waves 
imply a shorter coherence scale. This falling off of coherence with separation is due to destructive interference of the waves with one another, a phenomena that can happen only if waves of approximately the same wavenumber have approximately the same energy. Because measurements show that the vertical coherence falls off rapidly with vertical separation, a model which uses few modes with well separated $\omega_{i}(K)$ curves cannot describe the observations. Consequently, GM72 consider the discrete modes as being an equivalent continuum, where the solutions are considered to exist as continuous functions of their index. Thus GM72 have ignored all the effects of top and bottom boundary conditions except for the imposition of a lowest mode beyond which the spectrum does not extend.

A second property of the internal wave spectrum that GM72 derive by considering the exact solution to the exponential profile solutions is that the solutions become exponentially small in regions where the frequency exceeds the local buoyancy frequency (since the profile is monotonically decreasing, there can be at most one depth where the buoyancy frequency makes the transition from being greater than to being less than the wave frequency). GM72 incorporate this property into their internal wave spectrum by not allowing any energy at frequencies which exceed the local buoyancy frequency.

In smoothing out a discrete series of modes into an equivalent continuum, GM72 force the amplitude of the upward $\left(G_{+}(z)\right)$ and the downward solution $\left(G_{-}(z)\right)$ to be the same, thus introducing the idea of vertical symmetry. There is also a great simplification if horizontal symmetry is assumed as well; i.e. the energy spectrum can be written as $E(\omega, K)$ and there is no dependence on the wavenumber direction. This simplification is justified by the measurements available to GM72, which did not show any strong indications of anisotropy. GM72 also points out that there is also some theoretical basis in that strong resonant triad interactions (Phillips 1980, Martin et al. 1969) would lead to horizontal isotropy. The explicit connection between $E(\omega, K)$ and $E(\omega, k, l)$ is

$$
\begin{aligned}
E(\omega, K) & =\int E(\omega, k, l) K d \theta \\
& =2 \pi K E(\omega, k, l)
\end{aligned}
$$

so that

$$
\int E(\omega, K) d K=\int E(\omega, k, l) d k d l
$$

By using the dispersion relation 1.3.3b, $E(\omega, K)$ can be rewritten in terms of the local 
vertical wavenumber $m(z)$,

$$
m=K \sqrt{\frac{N^{2}-\omega^{2}}{\omega^{2}-f^{2}}} \approx K N\left(\omega^{2}-f^{2}\right)^{-1 / 2}
$$

We would also like to define a vertical mode number $j$ related in a simple way to frequency and horizontal wavenumber. GM79 use the definition

$$
j \pi=b \sqrt{\frac{N_{0}^{2}-\omega^{2}}{\omega^{2}-f^{2}}} K \approx \frac{N_{0} b}{\sqrt{\omega^{2}-f^{2}}} K
$$

This relation can be justified by using the integral constraint equation 1.3.7b and an exponential profile for $N(z)=N_{0} e^{z / b}$. The local vertical wavenumber $m(z)$ can be considered approximately exponential as well as long as $\omega<N(z)$, so that equation 1.3.7b becomes

$$
j \pi=b[m(0)-m(-D)] \approx b m(0)
$$

which is the same as equation 1.4.3. This only justifies the approximate form of $j$, but since that is the only version that will be used it is unnecessary to justify the exact form.

\subsection{Theoretical spectra and cross-spectra}

Using the assumptions of the last section, we are now in a position to relate measured spectra and cross-spectra to an internal wave energy $E(\omega, k, l)$. The assumption of vertical symmetry means that the wave energy is equally split into upward and downward components. Thus the energy at a particular frequency and horizontal wavenumber can be calculated by doubling the results for a single upward component. Equation 1.3.9b gives the single component energy density, relating it to a mean-square amplitude $\left\langle|\hat{A}(\omega, k, l)|^{2}\right\rangle$. This suggests the following identification

$$
\left\langle|\hat{A}(\omega, k, l)|^{2}\right\rangle=E(\omega, k, l) d \omega d k d l
$$

so that the averaged energy becomes

$$
\begin{aligned}
\bar{E} & =N(z) \int d \omega \int d k \int d l E(\omega, k, l) \\
& =N(z) \int d \omega \int d K E(\omega, K)
\end{aligned}
$$


where the second expression is explicitly horizontally isotropic. The identification and the vector notation introduced in equation 1.3.4 allow the cross-spectrum between any dynamical variable evaluated at location one $u_{i}\left(x_{1}, y_{1}, z_{1}\right)$ and any dynamical variable evaluated at location two $u_{j}\left(x_{2}, y_{2}, z_{2}\right)$ to be written in terms of the spectral level $E(\omega, k, l)$ (This will be more carefully derived in sections 3.7 and 3.8 ).

$$
\begin{aligned}
C_{12}(\omega)+i Q_{12}(\omega)=\left\langle u_{i}^{*}\left(x_{1}, y_{1}, z_{1}\right) u_{j}\left(x_{2}, y_{2}, z_{2}\right)\right\rangle \\
\quad=\int_{-\infty}^{\infty} d k \int_{-\infty}^{\infty} d l E(\omega, k, l) g_{i}^{*}\left(z_{1}, \omega, k, l\right) g_{j}\left(z_{2}, \omega, k, l\right) e^{i k\left(x_{2}-x_{1}\right)+i l\left(y_{2}-y_{1}\right)}
\end{aligned}
$$

$C_{12}$ is the cospectrum and $Q_{12}$ is the quadrature spectrum. Since coherence is a normalized version of this cross-spectrum, and an autospectrum is the special case where the location and dynamical variables are the same for both 1 and 2, equation 1.5.2 contains all the information necessary for connecting the spectral level to the autospectra and coherence calculated from moored sensor measurements. Explicit expressions for those quantities are given in figure 1.5.1. The moored autospectra and component coherence (MCC) correspond to data from a single current meter: time series of $u, v$, and $T$ measured at a single point. The expressions predict that the zonal velocity spectra and the meridional velocity spectra will be equal, while the ratio of kinetic energy spectra to potential energy spectra is a weak function of frequency

$$
\frac{\left\langle u^{*} u\right\rangle(\omega)+\left\langle v^{*} v\right\rangle(\omega)}{N^{2}(z)\left\langle\eta^{*} \eta(\omega)\right\rangle}=\frac{\omega^{2}+f^{2}}{\omega^{2}-f^{2}}
$$

Fofonoff (1969) calculated this ratio for internal waves in a constant $N$ ocean, thus this combination of kinetic and potential energy is frequently referred to as Fofonoff's consistency check (see Wunsch 1976, Wunsch and Webb 1979). Neither equality of zonal and meridional velocity autospectra nor Fofonoff's consistency check depend on the form of $E(\omega)$, thus they provide a check on the internal wave model independent of the spectral form chosen for $E(\omega)$. The moored component coherences are also independent of the spectral form chosen for $E(\omega)$ : the velocity-displacement coherences are zero as long as the spectra are either horizontally isotropic or vertically symmetric or both, and the coherence between $u$ and $v$ holds as long as the waves are horizontally isotropic (the phase difference between $u$ and $v$ corresponds to a clockwise rotation). The one linear combination that does depend on the form of $E(\omega)$ is the total energy

$$
\frac{1}{2}\left(\left\langle u^{*} u\right\rangle+\left\langle v^{*} v\right\rangle+N^{2}(z)\left\langle\eta^{*} \eta\right\rangle\right)=E(\omega)
$$


This provides the one check of the spectral form using moored autospectra and component coherence. It does not constrain the wavenumber dependence at all.

The moored horizontal coherence (MHC) gives the coherence as a function of purely horizontal separation. The expression given in the figure is for a zonal separation, but since the system is isotropic, the result holds when rotated to an arbitrary direction as well. GM72 points out that this integral expression is invertible, so that in principle the spectrum can be determined directly from the horizontal coherence and the function of frequency $E(\omega)$.

The moored vertical coherence (MVC) give the coherence as a function of purely vertical separation. The result given here differs somewhat from what is given in GM72, since GM72 give an expression that is only valid for short separations. (Their result is equivalent to assuming that $N(z)$ is constant over the vertical interval that the coherence is being computed). Unlike the MC spectra and coherences, the moored vertical coherence (MVC) provides information about the wavenumber dependence of $E(\omega, K)$. This relation also can be inverted, so that in principle $E(\omega, K)$ can be determined from the MVC coherence to within the function of frequency $E(\omega)$.

Expressions analogous to those just derived for moored sensors can be derived for towed autospectra and coherence as well. Rather than write an exact analog to equation 1.5.1, consider the towed version of just the displacement autospectra. A fast tow will measure equal contributions from positive and negative horizontal wavenumbers. Therefore

$$
\begin{aligned}
\left\langle\eta^{*} \eta\right\rangle(k) & =N^{-1} \int_{f}^{N} d \omega \frac{\omega^{2}-f^{2}}{\omega^{2}} \int_{-\infty}^{\infty}[E(\omega, k, l)+E(\omega,-k, l)] d l \\
& =4 N^{-1} \int_{f}^{N} \int_{0}^{\infty} E(\omega, k, l) d l
\end{aligned}
$$

The second expression takes advantage of the horizontal isotropy of $E(\omega, k, l)$. Both expressions assume the $x$ axis is along the direction of the tow. Rewriting equation 1.5.5 in terms of $E(\omega, K)$ gives the expressions in figure 1.5.2. Clearly these measurements depend on both the frequency and wavenumber dependence of $E(\omega, K)$. 


\section{Autospectra and coherence from moored sensors}

in terms of $E(\omega, K)$

$$
E(\omega)=\int_{0}^{\infty} E(\omega, K) d K
$$

\section{Moored Autospectra}

Horizontal velocity autospectra

$$
\left\langle u^{*} u\right\rangle(\omega)=\left\langle v^{*} v\right\rangle(\omega)=\frac{1}{2} N(z)\left(\frac{\omega^{2}+f^{2}}{\omega^{2}}\right) E(\omega)
$$

Displacement autospectra

$$
\left\langle\eta^{*} \eta\right\rangle(\omega)=N^{-1}(z)\left(\frac{\omega^{2}-f^{2}}{\omega^{2}}\right) E(\omega)
$$

Moored component coherence (MCC)

Velocity component coherence

$$
\gamma_{u v} e^{\phi_{u v}}=\frac{\left\langle u^{*} v\right\rangle}{\sqrt{\left\langle u^{*} u\right\rangle\left\langle v^{*} v\right\rangle}}=-i \frac{2 \omega f}{\omega^{2}+f^{2}}
$$

Velocity-displacement coherence

$$
\left\langle u^{*} \eta\right\rangle=\left\langle v^{*} \eta\right\rangle=0
$$

Horizontal separation coherence (MHC)

$$
\gamma(X, \omega)=E^{-1}(\omega) \int_{0}^{\infty} E(\omega, K) J_{0}(K X) d K
$$

where $J_{0}(K X)$ is the zeroth order Bessel function

$$
\begin{gathered}
J_{0}(K X)=\int_{0}^{2 \pi} d \theta \frac{1}{2 \pi} e^{K X \cos \theta} \\
\phi=0
\end{gathered}
$$

Vertical separation coherence (MVC)

$$
\gamma\left(z_{1}, z_{2}, \omega\right) e^{i \phi}= \begin{cases}E^{-1}(\omega) \int_{0}^{\infty} E(\omega, K) \cos K \psi d K & \text { vertically symmetric } \\ E^{-1}(\omega) \int_{0}^{\infty} E(\omega, K) e^{i K \psi} d K & \text { upward phase }\end{cases}
$$

where

$$
\begin{aligned}
\psi & =\int_{z_{1}}^{z_{2}} \frac{m\left(z^{\prime}\right)}{K} d z^{\prime} \\
& \approx \int_{z_{1}}^{z_{2}} \frac{N\left(z^{\prime}\right)}{\sqrt{\omega^{2}-f^{2}}} d z^{\prime}
\end{aligned}
$$

Figure 1.5.1 Moored sensor cross-spectra 


\section{Autospectra and coherence from dropped and towed sensors} in terms of $E(\omega, K)$

Towed displacement autospectra (TS)

$$
\left\langle\eta^{*} \eta(k)\right\rangle=N^{-1}(z) \frac{2}{\pi} \int_{f}^{N(z)} d \omega \frac{\omega^{2}-f^{2}}{\omega^{2}} \int_{k}^{\infty} E(\omega, K)\left(K^{2}-k^{2}\right)^{-1 / 2} d K
$$

Towed vertical separation coherence (TVC)

$$
\gamma\left(z_{1}, z_{2}, k\right) e^{i \phi}=\left\{\begin{array}{r}
\frac{1}{\left\langle\eta^{*} \eta(k)\right\rangle} \frac{2}{\pi} \int_{f}^{N} d \omega \frac{\omega^{2}-f^{2}}{\omega^{2}} \int_{k}^{\infty} E(\omega, K)\left(K^{2}-k^{2}\right)^{-1 / 2} \cos K \phi d K \\
\quad \text { vertically symmetric } \\
\frac{1}{\left\langle\eta^{*} \eta(k)\right)} \frac{2}{\pi} \int_{f}^{N} d \omega \frac{\omega^{2}-f^{2}}{\omega^{2}} \int_{k}^{\infty} E(\omega, K)\left(K^{2}-k^{2}\right)^{-1 / 2} e^{i K \phi} d K \\
\text { vertically propagating }
\end{array}\right.
$$

Dropped autospectra (DS)

$$
\left\langle\eta^{*} \eta(m)\right\rangle=N^{-1}(z) \int_{f}^{N} \frac{\omega^{2}-f^{2}}{\omega^{2}} E(\omega, m) d \omega
$$

Figure 1.5.2 Towed and Dropped sensor cross-spectra 


\subsection{Predictions of GM79}

Figures 1.5.1 and 1.5.2 give the autospectra and correlations in terms of a general spectral density $E(\omega, K)$. In this section the particular form of the GM79 spectral level is used to make concrete predictions for the measurements, that form being

$$
E(\omega, j)=\widetilde{E} B(\omega) H(j)
$$

where $B(\omega)$ and $H(j)$ are given in parts of equation 1.1.1. Most of the points and algebra found in this section can be found in Desaubies 1976. Since some of the expressions in figures 1.5.1 and 1.5.2 are in terms of $E(\omega, K)$, it is important to write the GM79 spectrum in terms of those independent variables as well. Thus

$$
\begin{gathered}
E(\omega, K)=\widetilde{E} B(\omega) H(K) \\
H(K)=\frac{\frac{2}{\pi} K_{*}}{K^{2}+K_{*}^{2}} \quad \int_{0}^{\infty} H(K) d K=1 \\
K_{*}=\frac{\pi}{b} \frac{\sqrt{\omega^{2}-f^{2}}}{N_{0}} j_{*}
\end{gathered}
$$

While this expression is not precisely the same as using the dispersion relation 1.4.4 and the relation $E(\omega, j) \delta j=E(\omega, K) d K$ (as Munk 1981 gives as the correct procedure), it is sufficiently close for comparisons with measured spectra. Note that the smearing of modes into a continuum is implicit in the transformation of $H(j)$ to $H(K)$. It is also important that the horizontal wavenumber rolloff is a function of frequency, unlike the rolloff mode number $j_{*}=3$.

The same can be done for $E(\omega, m)$, where $m$ is local vertical wavenumber.

$$
\begin{gathered}
E(\omega, m)=\widetilde{E} B(\omega) H(m) \\
H(m)=\frac{\frac{2}{\pi} m_{*}}{m^{2}+m_{*}^{2}} \quad \int_{0}^{\infty} H(m) d m=1 \\
m_{*}=\frac{\pi}{b} \frac{N(z)}{N_{0}} j_{*}
\end{gathered}
$$

It is clear from the structure of 1.1.1 that the $E(\omega)$ appearing in figure 1.5.1 is given by

$$
E(\omega)=\widetilde{E} B(\omega)=\widetilde{E} \frac{2 f}{\pi \omega \sqrt{\omega^{2}-f^{2}}}
$$


i.e. $E(\omega)$ is only dependent on $B(\omega)$ not on $H(j)$.

Using the GM79 spectral form for $E(\omega, K)$, figure 1.6.1 presents expressions for each of the moored sensor spectra presented in figure 1.5.1. The moored autospectra only depend on $E(\omega)$, thus, since the form chosen in GM79 is separable, they only depend on $B(\omega)$. In the limit where the frequency $\omega$ is much greater than the inertial frequency $f$, both the horizontal velocity autospectra and the displacement autospectra have the same frequency dependence $\omega^{-2}$. Near the inertial frequency, however, the displacement autospectrum goes to zero while the horizontal velocity autospectra have an integrable singularity. Munk 1981 and Fu 1980 point out that this singularity is a property of linear internal waves that can be seen if the dynamics in the near inertial limit are done properly: $E(\omega)$ should be written without the explicit singularity and the corrected dynamics would introduce it at this point. The final expressions for the moored autospectra, however, would be unchanged. The velocity component coherence and velocity-displacement coherence are both independent of the form of $E(\omega, K)$; consequently they are the same as they were in figure 1.5.1.

The horizontal separation coherence (MHC) depends on frequency only through the rolloff wavenumber $K_{*}$ which is given by equation 1.6.1c. In figure 1.6.1 the coherence is given as the difference between a modified Bessel function $I_{0}\left(K_{*} X\right)$ and a modified Struve function $\mathrm{L}_{0}\left(K_{*} X\right)$, both of zeroth order (the integral is given in terms of these functions in Bateman 1954). Values of this difference are tabulated in Abramowitz and Stegun 1972 page 501, and are plotted in figure 1.6.2. Using the GM79 choices of parameters in $K_{*}$ (see equation 1.1.1) and a frequency of $.2 \mathrm{cph}$, unit value of $K_{*} X$ corresponds to horizontal separation $X$ of $2 \mathrm{~km}$ (see Desaubies 1976).

The vertical separation coherence (MVC) has only a weak dependence on frequency (as long as $\omega \ll N(z)$ there is no frequency dependence). In the vertically symmetric case (which is the prediction according to GM79), the coherence has an exponential dependence and the phase is zero. In the vertically propagating case, the real part is unchanged, but there is now an imaginary part given by the exponential integral functions $E_{1}\left(K_{*} \psi\right)$ and $E_{i}\left(K_{*} \psi\right)$ (see Abramowitz and Stegun 1972 for definitions and properties of the exponential integrals). Plots of the real part $\langle H(K) \cos K \psi\rangle$ and the imaginary part $\langle H(K) \sin K \psi\rangle$ are in figure 1.6.3. Using the GM79 choices of parameters in $K_{*}$ (see equation 1.1.1) and given the buoyancy frequency as a function of depth $N(z)$, values of $K_{*} \psi$ can be converted into physical separation. If the buoyancy frequency is a constant 


\section{Autospectra and coherence from moored sensors} in terms of GM79

Moored autospectra

Horizontal velocity autospectra

$$
\left\langle u^{*} u\right\rangle(\omega)=\left\langle v^{*} v\right\rangle(\omega)=\frac{\widetilde{E} f}{\pi} N(z) \frac{\omega^{2}+f^{2}}{\omega^{3} \sqrt{\omega^{2}-f^{2}}}
$$

Displacement autospectra

$$
\left\langle\eta^{*} \eta\right\rangle(\omega)=\frac{\widetilde{E} f}{\pi} N^{-1}(z) \frac{\sqrt{\omega^{2}-f^{2}}}{\omega^{3}}
$$

Horizontal separation coherence (MHC)

$$
\begin{gathered}
\gamma(X, \omega)=I_{0}\left(K_{*} X\right)-\mathbf{L}_{0}\left(K_{*} X\right) \\
K_{*}=\frac{\pi}{b} \frac{\sqrt{\omega^{2}-f^{2}}}{N_{0}} j_{*}
\end{gathered}
$$

Vertical separation coherence (MVC)

$$
\gamma\left(z_{1}, z_{2}, \omega\right) e^{i \phi}=\left\{\begin{array}{lr}
e^{-K_{*} \psi} & \text { vertically symmetric } \\
e^{-K_{*} \psi}+\frac{i}{\pi}\left[e^{K_{*} \psi} E_{1}\left(K_{*} \psi\right)+e^{-K_{*} \psi} E_{i}\left(K_{*} \psi\right)\right] & \text { upward phase }
\end{array}\right.
$$

where

$$
\begin{aligned}
K_{*} \psi & =\frac{\pi j_{*}}{b N_{0}} \int_{z_{1}}^{z_{2}} \sqrt{N^{2}\left(z^{\prime}\right)-\omega^{2}} d z^{\prime} \\
& \approx \frac{\pi j_{*}}{b N_{0}} \int_{z_{1}}^{z_{2}} N\left(z^{\prime}\right) d z^{\prime}
\end{aligned}
$$

$\psi$ is always taken to be positive.

Figure 1.6.1 GM79 moored sensor cross-spectra 


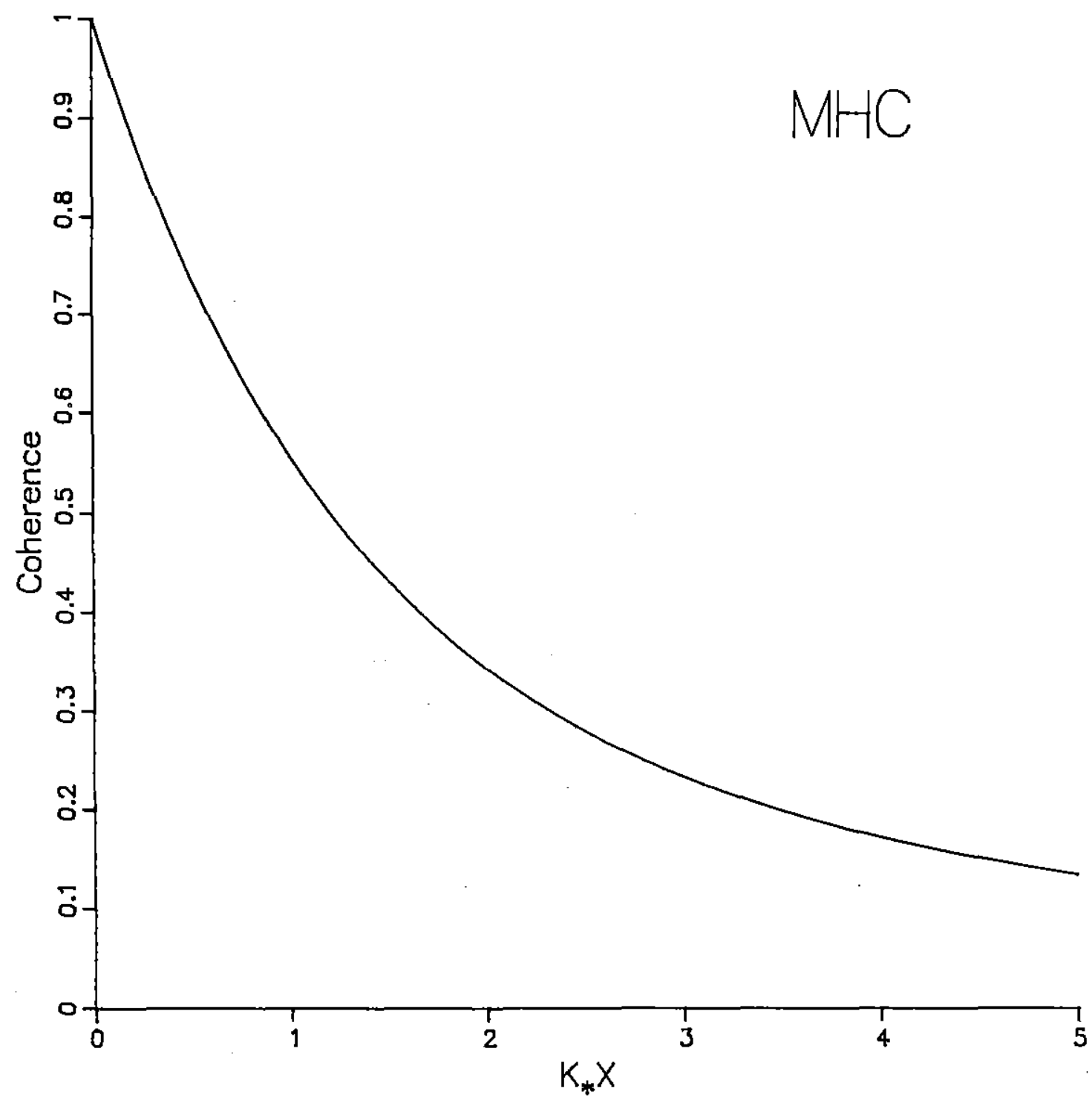

Figure 1.6.2 Moored horizontal separation coherence

This figure gives the moored horizontal separation coherence (MHC) for the GM79 model internal wave spectrum. It is the difference between a modified Bessel function and a modified Struve function (see text). 
$1 \mathrm{cph}$, then unit value of $K_{*} \psi$ corresponds to a vertical separation of $410 \mathrm{~m}$.

The same sorts of calculations can be done for the dropped and towed spectra as well. The dropped spectra is fairly simple in that the frequency dependence integrates out, leaving the mode number dependence to determine the dropped autospectra,

$$
\left\langle\eta^{*} \eta(m)\right\rangle=\widetilde{E} N^{-1}(z) H(m)
$$

While the moored and dropped measurements separate fairly cleanly in that they are either dependent on $B(\omega)$ or $H(j)$, the same is not true for the towed measurements. Therefore, while explicit expressions can be written, there are more complicated than those given in figure 1.6.1.

\subsection{Summary}

In summary, then, Garrett and Munk use linear internal wave theory, vertical symmetry, and horizontal isotropy to relate each of the various measurable spectra to a normalized internal wave spectrum $E(\omega, K)$. Linear wave theory is used by finding WKBJ solutions to the wave equations, then energy and measurable cross-spectra is calculated for each wave solution. Then the assumptions of vertical symmetry and horizontal isotropy are used to argue that the total cross-spectra is simply the sum of that due to each single wave. Once those interrelations between measured cross-spectra are found, then a fit to measurements of $E(\omega, K)$ is done. The form chosen for GM79 is separable in frequency and mode number, in part for convenience and in part because the measurements that go into GM79 tend to be integrals over either mode number or frequency, so that they are easily fit by separable functions. Comparison with the measurements that go into GM79 shows a good fit, making GM79 immensely useful: it is a succinct summary of a large fraction of the internal wave measurements. Even when more sophisticated models are available, and more sophisticated measurements (such as direct frequencywavenumber measurements) have shown aspects of the GM79 model to be wrong, its simplicity combined with its essential correctness will make it useful for a long time to come. 


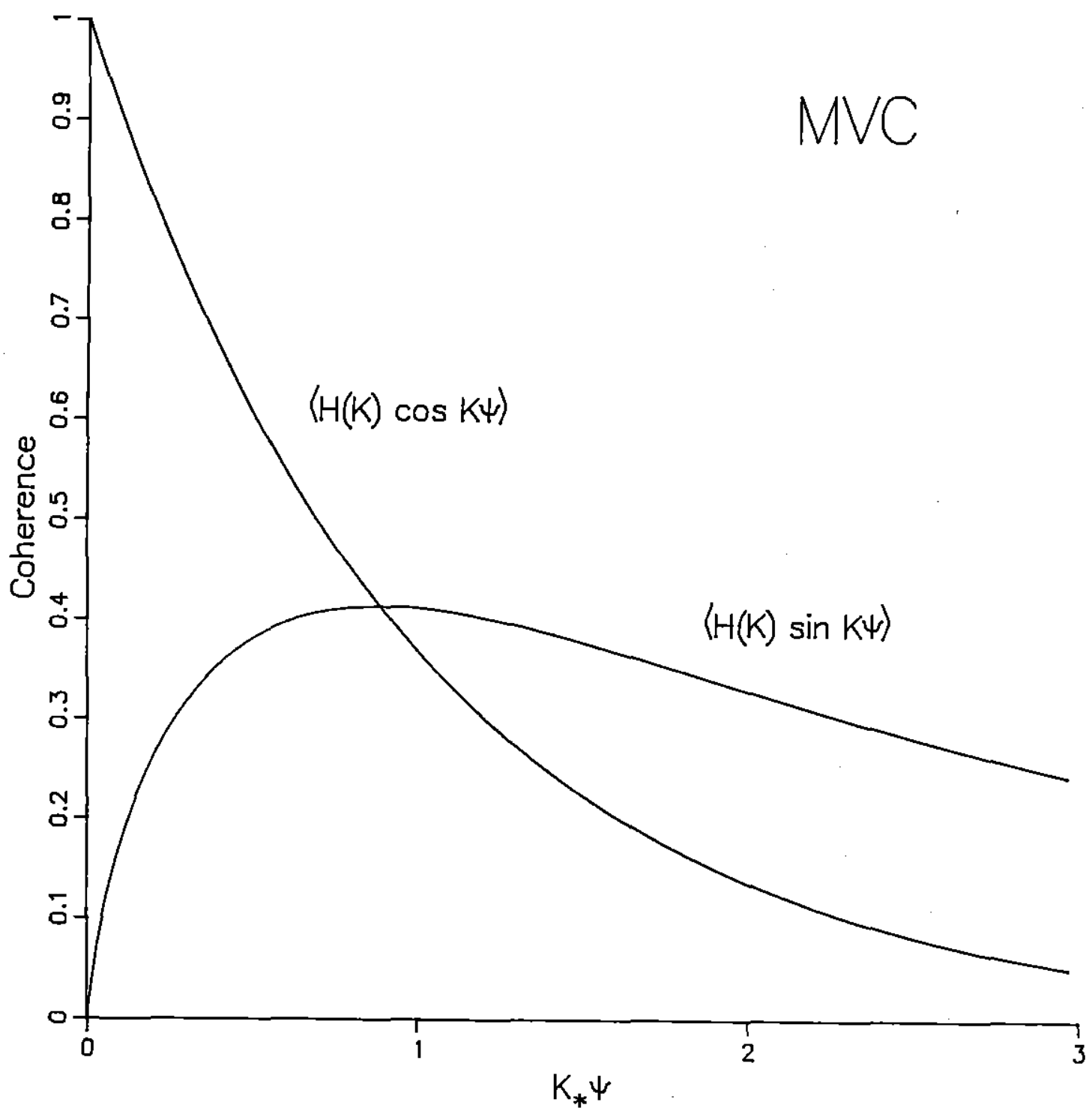

Figure 1.6.3 Moored vertical separation coherence

This figure gives the moored vertical separation coherence (MVC). The real part is given by a simple exponential while the imaginary part is the difference of two exponential integral functions (see text). 


\section{Chapter 2: The PEQUOD dataset}

\subsection{Data description}

The primary objective of the Pacific Equatorial Dynamics Experiment (PEQUOD) was to understand the dynamics of the equatorial ocean, deep equatorial dynamics in particular (PEQUOD 1980). Inspired by the discovery by Luyten and Swallow 1976 of multiple equatorially trapped high vertical wavenumber jets in the Indian Ocean and subsequent observations of similar jets in the Pacific (Eriksen 1980a), a significant portion of the PEQUOD experiment was devoted to definitive measurements of these equatorial jets: intense currents $(10-30 \mathrm{~cm} / \mathrm{s})$ found between $300 \mathrm{~m}-3000 \mathrm{~m}$ depths. These jets are small both in meridional $(O(200 \mathrm{~km}))$ and vertical $(O(200 \mathrm{~m}))$ extent (Eriksen 1980a). The PEQUOD experiment combines bimonthly velocity profiler sections (Firing 1982) with a two year current meter array, providing much more information about the temporal behavior of the jets than the earlier measurements.

Portions of the PEQUOD current meter array are used in this thesis to study high frequency internal waves. The array consists of six moorings; the moorings are lettered P-E-Q-U-O-D. The instrumentation, nominal depths, horizontal placements, and data return are given in figure 2.1.1, while a full table of data records used is given in figure 2.1.2. The six moorings were in place for two years: January 1981 to March 1983 with a redeployment in February 1982. The central moorings $(Q, U)$ have twelve instruments each (alternating Aanderaa current meters with temperature-pressure recorders) clustered at three depths. This means that there is resolution of both fairly short $(O(50 \mathrm{~m}))$ and long $(O(1000 \mathrm{~m}))$ vertical scales. The remaining moorings $(\mathrm{P}, \mathrm{E}, \mathrm{O}, \mathrm{D})$ have less vertical resolution: $\mathrm{P}$ and D have a single current meter at each cluster depth $(500 \mathrm{~m}, 1500 \mathrm{~m}, 3000 \mathrm{~m})$ while $\mathrm{E}$ and $\mathrm{O}$ have both a current meter and a TP recorder at each cluster depth. This thesis focuses on data from the two central moorings $(Q, U)$.

Aanderaa current meters are simple rotor-vane instruments which record temperature, rotor count, and direction once a sampling interval (In PEQUOD, this was once an hour), Differenced rotor counts divided by the sampling interval provide an average speed: this speed is combined with the instantaneous direction measurement to get velocity components. This scheme results in aliasing of direction, which restricts the extent to which internal wave information can be extracted from the data. How this aliasing comes about and the measures taken to circumvent it are discussed in Appendix $A$. 


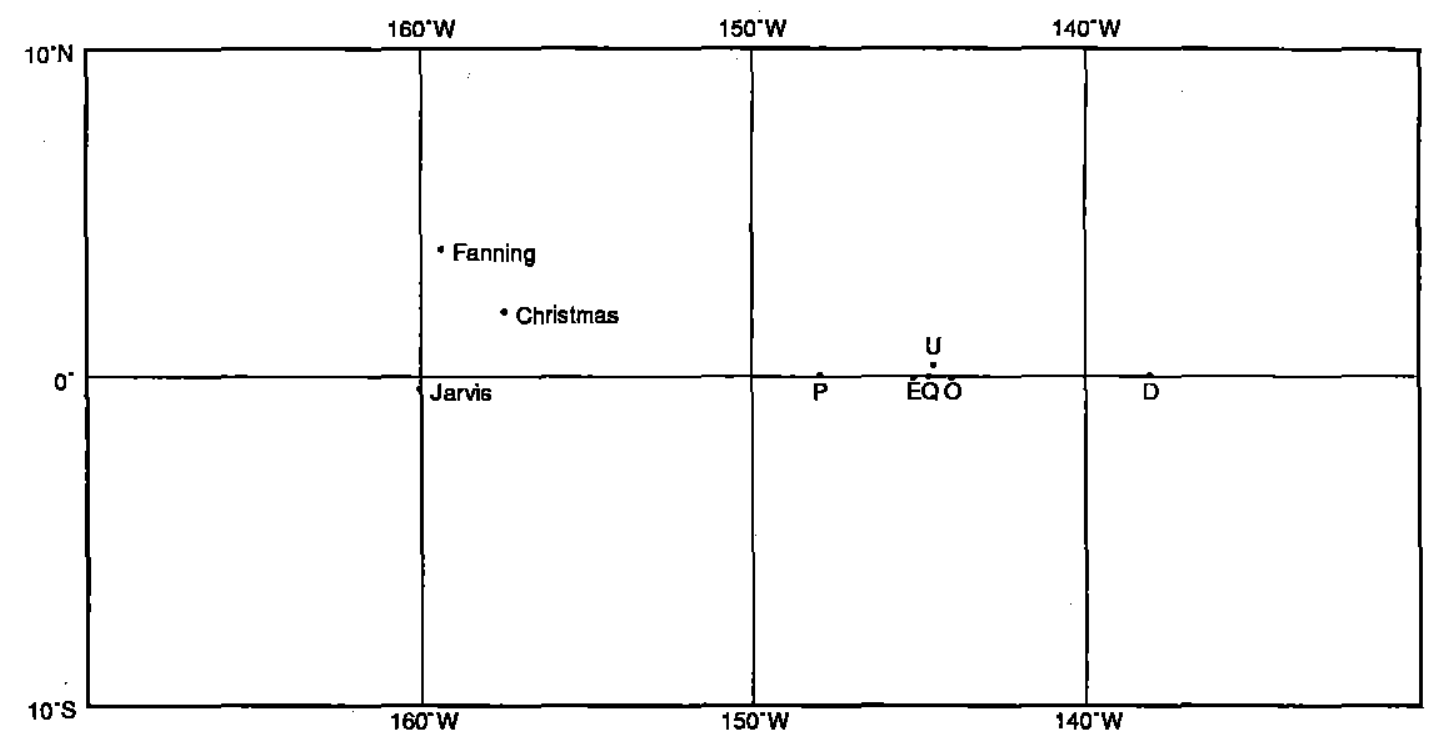

\begin{tabular}{|c|c|c|c|c|c|c|c|c|c|c|c|c|c|}
\hline \multirow{2}{*}{$\begin{array}{l}\text { Nominal } \\
\text { Depth }\end{array}$} & \multirow[t]{2}{*}{ Instrument } & \multicolumn{12}{|c|}{ Data Return } \\
\hline & & & $\ln 8$ & $1-$ & $-F$ & b 82 & & & b 8 & $2-$ & $-M$ & $\operatorname{ar} 8$ & \\
\hline 500 & current meter & $\mathbf{P}$ & $\mathrm{E}$ & 0 & - & 0 & $\mathrm{D}$ & $\mathrm{P}$ & $\mathrm{E}$ & $\mathrm{O}$ & $\mathrm{U}$ & $\mathrm{O}$ & $\mathrm{D}$ \\
\hline 575 & TP recorder & & E & $\mathrm{Q}$ & $\mathrm{U}$ & 0 & & & $\mathrm{E}$ & $\mathrm{Q}$ & $\mathrm{U}$ & $\mathrm{O}$ & \\
\hline 600 & current meter & & & $\mathrm{Q}$ & $\mathrm{U}$ & & & & & $Q$ & $\mathrm{U}$ & & \\
\hline 650 & TP recorder & & & $\mathrm{Q}$ & $\mathrm{U}$ & & & & & $\mathrm{Q}$ & $\mathrm{U}$ & & \\
\hline 1500 & current meter & $\mathbf{P}$ & $\mathrm{E}$ & & $\mathrm{U}$ & $\mathrm{O}$ & D & $P$ & $\mathrm{E}$ & $\mathrm{Q}$ & $\mathrm{U}$ & $\mathrm{O}$ & D \\
\hline 1575 & TP recorder & & E & $\mathrm{Q}$ & $\mathrm{U}$ & $\mathrm{O}$ & & & $\mathrm{E}$ & $\mathrm{Q}$ & $\mathrm{U}$ & $\mathrm{O}$ & \\
\hline 1600 & current meter & & & $\mathrm{Q}$ & $\mathrm{U}$ & & & & & $\circ$ & $\mathrm{U}$ & & \\
\hline 1650 & TP recorder & & & $\mathrm{Q}$ & & & & & & $\mathrm{Q}$ & $\mathrm{U}$ & & \\
\hline 3000 & current meter & $\mathbf{P}$ & $\mathrm{E}$ & Q & $\mathrm{U}$ & $\mathrm{O}$ & D & $\mathbf{P}$ & $\mathrm{E}$ & $\mathrm{Q}$ & $\mathrm{U}$ & $\mathrm{O}$ & $\mathrm{D}$ \\
\hline 3075 & TP recorder & & $\mathrm{E}$ & $Q$ & $\mathrm{U}$ & $\mathrm{O}$ & & & $\mathrm{E}$ & $\mathrm{Q}$ & $\mathrm{U}$ & $\mathrm{O}$ & \\
\hline 3100 & current meter & & & $\mathbf{Q}$ & $\mathrm{U}$ & & & & & Q & $\mathrm{U}$ & & \\
\hline 3150 & TP recorder & & & Q & $\mathrm{U}$ & & & & & $Q$ & $\mathrm{U}$ & & \\
\hline
\end{tabular}

- $\quad$ missing $u$ and $v$

- $\quad$ short record for $u$ and $v$

Figure 2.1.1 PEQUOD mooring summary 
Figure 2.1.2 PEQUOD time series table

Current meters and temperature-pressure (TP) recorders

\begin{tabular}{|c|c|c|c|c|c|}
\hline Label & Latitude & Longitude & Start time & $\begin{array}{c}\text { Length } \\
\text { hrs }\end{array}$ & $\begin{array}{c}\text { Depth } \\
\mathrm{m}\end{array}$ \\
\hline $\begin{array}{l}\text { Q101 } \\
\text { Q102(TP) }\end{array}$ & $0^{\circ} 00.3 \mathrm{~S}$ & $144^{\circ} 40.9 \mathrm{~W}$ & $24 \operatorname{Jan} 1981$ & $\begin{array}{l}9251 \\
9241\end{array}$ & $\begin{array}{l}514.7 \\
589.7\end{array}$ \\
\hline Q103 & & & & 9100 & 614.7 \\
\hline Q104(TP) & & & & 9100 & 664.6 \\
\hline Q106(TP) & & & & 9100 & 1590.0 \\
\hline Q107 & & & & 8834 & 1615.0 \\
\hline Q108(TP) & & & & 9100 & 1664.9 \\
\hline Q109 & & & & 8802 & 3015.0 \\
\hline Q110(TP) & & & & 9100 & 3090.0 \\
\hline Q111 & & & & 9248 & 3114.9 \\
\hline U101 & $0^{\circ} 20.7 \mathrm{~N}$ & $144^{\circ} 32.6 \mathrm{~W}$ & 29 Jan 1981 & 1582 & 491.7 \\
\hline U102(TP) & & & & 9184 & 566.6 \\
\hline U்103 & & & & 8659 & 591.6 \\
\hline U104(TP) & & & & 9184 & 641.6 \\
\hline U105 & & & & 9095 & 1492.2 \\
\hline U106(TP) & & & & 9184 & 1567.3 \\
\hline U107 & & & & 9037 & 1592.1 \\
\hline U109 & & & & 9199 & 2992.4 \\
\hline U110(TP) & & & & 9184 & 3067.4 \\
\hline U111 & & & & 9198 & 3092.4 \\
\hline U112(TP) & & & & 9198 & 3142.4 \\
\hline Q201 & $0^{\circ} 00.3 \mathrm{~S}$ & $144^{\circ} 40.9 \mathrm{~W}$ & 14 Feb 1982 & 9062 & 482.9 \\
\hline Q202(TP) & & & & 9052 & 557.7 \\
\hline Q203 & & & & 8216 & 582.7 \\
\hline Q204(TP) & & & & 6016 & 632.7 \\
\hline Q205 & & & & 6593 & 1482.3 \\
\hline Q207 & & & & 7580 & 1582.2 \\
\hline Q208(TP) & & & & 9052 & 1632.2 \\
\hline Q209 & & & & 9283 & 2981.5 \\
\hline Q210(TP) & & & & 9269 & 3056.5 \\
\hline Q211 & & & & 9277 & 3081.5 \\
\hline Q212(TP) & & & & 9269 & 3131.5 \\
\hline
\end{tabular}


Figure 2.1.2 PEQUOD time series table (cont)

Current meters and temperature-pressure (TP) recorders

$\begin{array}{lllccc}\text { Label } & \text { Latitude } & \text { Longitude } & \text { Start time } & \begin{array}{c}\text { Length } \\ \text { hrs }\end{array} & \begin{array}{c}\text { Depth } \\ \text { m }\end{array} \\ \text { U201 } & 0^{\circ} \text { 20.7'N } & 144^{\circ} 32.6^{\prime} \mathrm{W} & 18 \text { Feb } 1982 & 8978 & 495.3 \\ \text { U202(TP) } & & & & 8979 & 570.4 \\ \text { U203 } & & & & 8978 & 595.4 \\ \text { U204(TP) } & & & & 6383 & 645.4 \\ \text { U205 } & & & & 8979 & 1495.7 \\ \text { U206(TP) } & & & & 8979 & 1570.8 \\ \text { U207 } & & & & 8979 & 1595.8 \\ \text { U208 } & & & 8979 & 1645.8 \\ \text { U209 } & & & & 8979 & 2996.3 \\ \text { U210(TP) } & & & & 8979 & 3071.4 \\ \text { U211 } & & & & 8979 & 3096.4 \\ \text { U212(TP) } & & & & 8979 & 3146.4\end{array}$

Ocean depth at $Q$ mooring: $4250 \mathrm{~m}$

Ocean depth at $U$ mooring: $4400 \mathrm{~m}$ 
The temperature-pressure recorders are the same as discussed by Wunsch and Dalen (1974). The major distortion that the instruments introduce is the delayed response of the thermistors relative to the pressure sensor; a delay that is primarily due to the thermal mass of the sensor. For the instruments used in PEQUOD that delay (e-folding time) is either three minutes or twenty-three minutes. This is much greater than the thermal mass of the Aanderaa temperature sensors where the lag is on the order of seconds. Because an $e$-folding time of 23 minutes corresponds to a phase lag as large as $50^{\circ}$ at a period of two hours, the temperature records were processed to remove the effects of thermal lag. The transfer function used was

$$
T_{c}(\omega)=(1+i \omega \lambda) T_{m}(\omega)
$$

where $T_{m}(\omega)$ are the Fourier coefficients as calculated from the data, $T_{c}(\omega)$ are the corrected Fourier coefficients which are subsequently averaged to calculate spectra, $\omega$ is the frequency, and $\lambda$ is the $e$-folding time for the thermistor.

In addition to the moored array data, there are three sets of Whitehorse velocity profiles taken in January 1981, February 1982, and April 1982 respectively. The Whitehorse velocity profiler is a freely falling instrument which contains an acoustic transponder and a CTD (Luyten and Swallow 1976). To make velocity measurements with the instrument, first three acoustic transponders are dropped in a triangle, then the Whitehorse is released on the center. As it drops, it is tracked acoustically relative to the three transponders. The acoustic travel times can then be used to calculate the path of the instrument; differencing furnishes the velocities. Profiles of temperature and salinity are calculated from the CTD measurements.

There are 28 Whitehorse profiles taken near the sites of the moorings. Figure 2.1.3 gives a table of sites ('nets') and profiles that were used in calculating an average buoyancy frequency profile (figure 2.1.4) and average velocity profiles (figure 2.1.5). The velocity profiles give a detailed vertical structure which complements the detailed temporal records of the current meter data. In particular, shear estimates from internal wave models are compared with shear estimates from the direct but infrequent velocity profiles (see section 4.7). The buoyancy frequency profile is used is section 7.4 to calculate the structure of vertically propagating waves in a basic state with no mean flow, while in section 7.5 both profiles are used to calculate the vertical structure of waves in the presence of a mean flow. 
Figure 2.1.3 Whitehorse drops

\begin{tabular}{|c|c|c|c|c|c|c|}
\hline \multirow[t]{2}{*}{ Net } & \multirow[t]{2}{*}{ Cast } & \multirow[t]{2}{*}{ Latitude } & \multirow[t]{2}{*}{ Longitude } & \multirow[t]{2}{*}{ Date } & \multicolumn{2}{|c|}{ Used in profile } \\
\hline & & & & & $N(z)$ & Velocity \\
\hline \multirow[t]{8}{*}{ K } & 1 & $0^{\circ} 2^{\prime} \mathrm{N}$ & $145^{\circ} 3^{\prime} \mathrm{W}$ & $22 \operatorname{Jan} 1981$ & $\mathrm{Y}^{\prime}$ & $\mathrm{Y}$ \\
\hline & 2 & & & & $\mathbf{N}$ & $\mathrm{Y}$ \\
\hline & 3 & & & & $\mathrm{~N}$ & $\mathrm{Y}$ \\
\hline & 4 & & & $28 \operatorname{Jan} 1981$ & $\mathrm{Y}$ & $\mathrm{Y}$ \\
\hline & 5 & & & 15 Feb 1982 & $\mathrm{Y}$ & $\mathrm{Y}$ \\
\hline & 6 & & & 20 Feb 1982 & $\mathrm{Y}$ & $Y$ \\
\hline & 8 & & & 16 Apr 1982 & $\mathrm{Y}$ & $\mathrm{Y}$ \\
\hline & 9 & & & 22 Apr 1982 & $Y$ & $\mathrm{Y}$ \\
\hline \multirow[t]{7}{*}{$\mathrm{L}$} & 1 & $0^{\circ} 33^{\prime} \mathrm{S}$ & $144^{\circ} 57^{\prime} \mathrm{W}$ & 22 Jan 1981 & $\mathrm{Y}$ & $\hat{Y}$ \\
\hline & 2 & & & 23 Jan 1981 & $\mathrm{Y}$ & $\mathrm{Y}$ \\
\hline & 4 & & & & $\mathrm{~N}$ & $\mathrm{Y}$ \\
\hline & 5 & & & 14 Feb 1982 & $\mathrm{Y}$ & $\mathbf{N}$ \\
\hline & 7 & & & 20 Feb 1982 & $\mathrm{Y}$ & $\mathrm{Y}$ \\
\hline & 8 & & & 16 Apr 1982 & $\mathrm{Y}$ & $\mathrm{Y}$ \\
\hline & 9 & & & 21 Apr 1982 & $\mathrm{Y}$ & $\mathrm{Y}$ \\
\hline \multirow[t]{6}{*}{ M } & 1 & $1^{\circ} 18^{\prime} S$ & $144^{\circ} 58^{\prime} \mathrm{W}$ & $23 \mathrm{Jan} 1981$ & $\mathrm{Y}$ & $\mathrm{Y}$ \\
\hline & 2 & & & 27 Jan 1981 & $\mathrm{Y}$ & $\mathrm{Y}$ \\
\hline & 3 & & & $14 \mathrm{Feb} 1982$ & $\mathrm{Y}$ & $\hat{\mathrm{Y}}$ \\
\hline & 4 & & & 19 Feb 1982 & $\mathrm{Y}$ & $\mathrm{Y}$ \\
\hline & 5 & & & $16 \mathrm{Apr} 1982$ & $\mathrm{Y}$ & $\mathrm{Y}$ \\
\hline & 6 & & & 21 Apr 1982 & $\mathbf{Y}$ & $\mathrm{Y}$ \\
\hline \multirow[t]{5}{*}{$\mathbf{N}$} & 1 & $0^{\circ} 31^{\prime} \mathrm{N}$ & $144^{\circ} 58^{\prime} \mathrm{W}$ & $25 \operatorname{Jan} 1981$ & $\mathrm{Y}$ & $\mathrm{N}$ \\
\hline & 3 & & & $29 \mathrm{Jan} 1981$ & $\mathrm{Y}$ & $\mathrm{N}$ \\
\hline & 4 & & & $16 \mathrm{Feb} 1982$ & $\mathrm{Y}$ & $\mathrm{N}$ \\
\hline & 6 & & & 16 Apr 1982 & $Y$ & $\mathrm{~N}$ \\
\hline & 7 & & & 22 Apr 1982 & $\mathrm{Y}$ & $\mathrm{N}$ \\
\hline \multirow[t]{5}{*}{$\mathrm{O}$} & 1 & $1^{\circ} 16^{\prime} \mathrm{N}$ & $145^{\circ} 0^{\prime} \mathrm{W}$ & $25 \mathrm{Jan} 1981$ & $\mathrm{Y}$ & $\mathrm{Y}$ \\
\hline & 2 & & & $16 \mathrm{Feb} 1982$ & $\mathrm{Y}$ & $\mathrm{Y}$ \\
\hline & 3 & & & $21 \mathrm{Feb} 1982$ & $\mathrm{Y}$ & $\mathrm{Y}$ \\
\hline & 4 & & & 17 Apr 1982 & $\mathrm{Y}$ & $\mathrm{Y}$ \\
\hline & 5 & & & 22 Арг 1982 & $\mathbf{Y}$ & $\mathbf{Y}$ \\
\hline
\end{tabular}




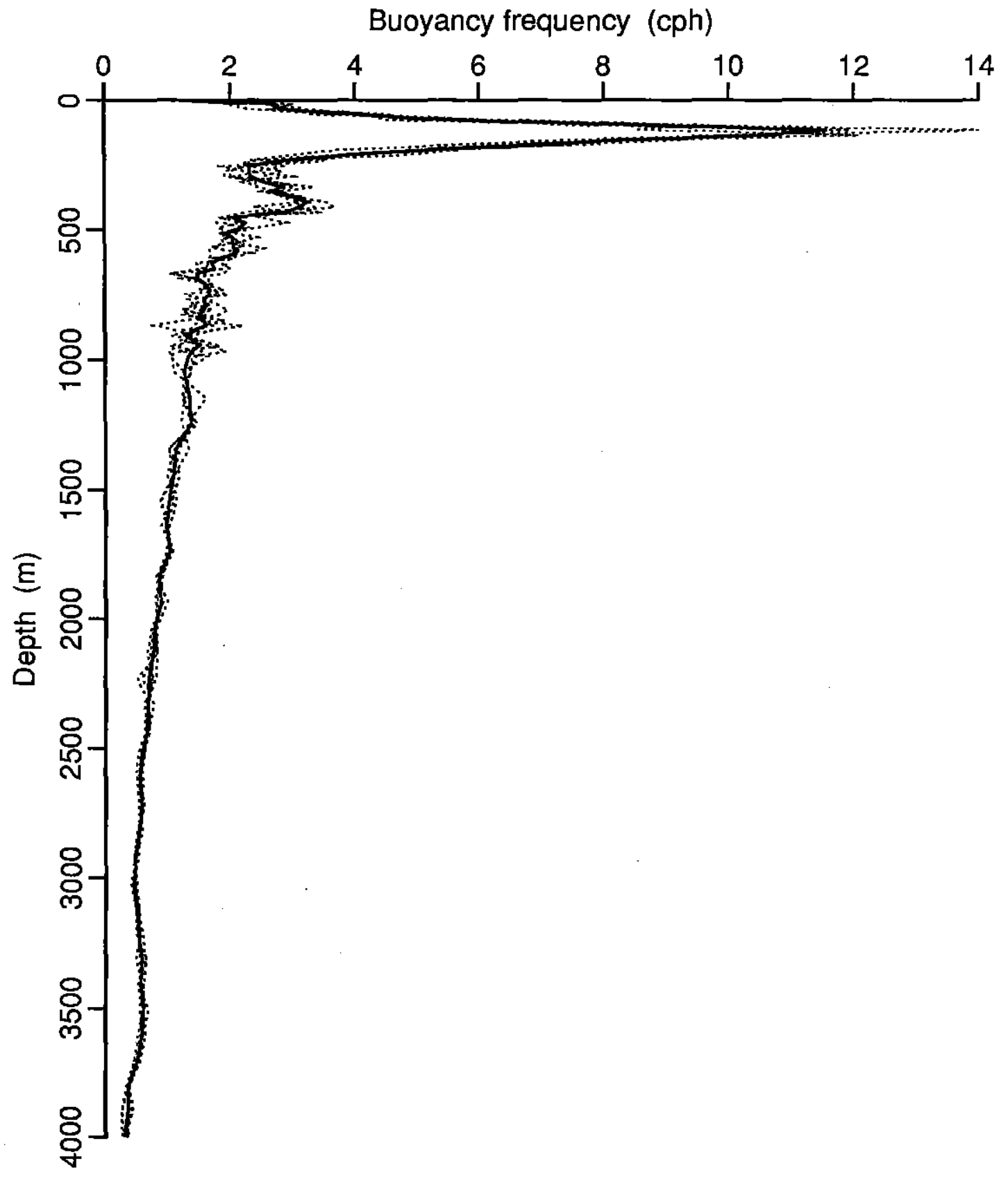

Figure 2.1.4 Averaged buoyancy frequency profile

Plotted as the solid curve is the buoyancy frequency calculated by averaging all 28 profiles, while the averages over each net separately are plotted as dotted lines. 


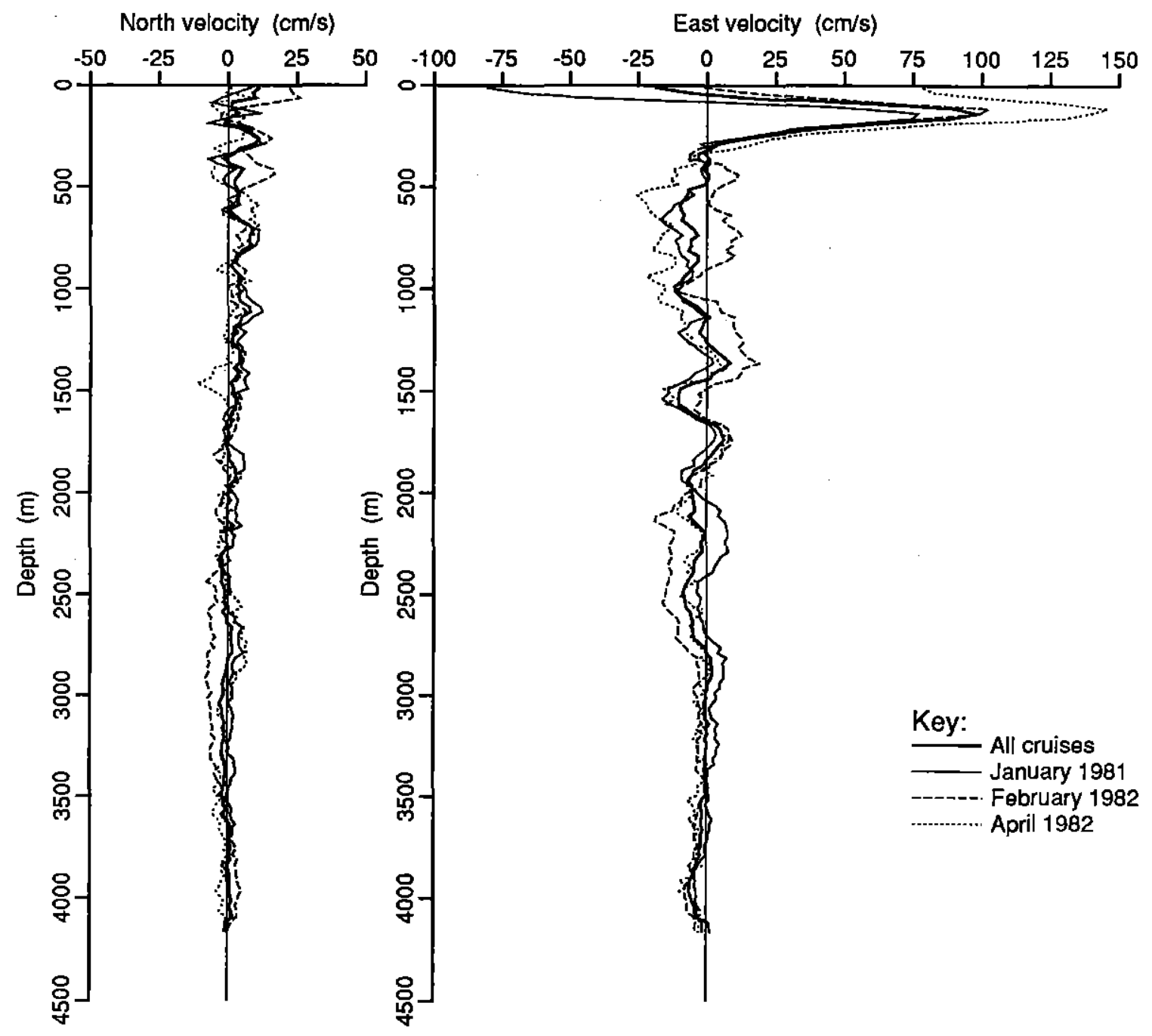

Figure 2.1.5 Whitehorse velocity profiles at $\left(0^{\circ}, 145^{\circ} \mathrm{W}\right)$.

These profiles of east and north velocity are calculated from Whitehorse drops at net $K\left(0^{\circ}, 145^{\circ} \mathrm{W}\right)$. In addition to the profile averaged over all nine drops, averages over three subgroups are also plotted: drops 1 through 4 (January 1981), drops 5 through 7 (February 1982), and drops 8 and 9 (April 1982). Comparison of the mean curve with the three curves computed from subsets shows that there is quite a bit of time variability. 


\subsection{Rough estimates of non-GM dynamics}

Without going into too much detail, it is possible to show that the presence of strong mean flows at the equator mean that the dynamics included in the GM79 model are not sufficient to model the PEQUOD dataset. Furthermore, some of the assumptions of GM79 are unwarranted and inconsistent with other aspects of the model, leading to modifications that should be made before modeling internal waves anywhere. Finally, comparison of the expected size of fluxes in the internal wave field with the PEQUOD array's ability to measure them suggest that it should be possible to see non-zero net fluxes with the PEQUOD data.

As will be shown in detail in chapters 5 and 7 , the most important effects of a mean shear flow on the internal wave field can be measured with two parameters: the Doppler shift $d$ and the inverse root Richardson number $\lambda$. The Doppler shift measures the difference between the frequency $\omega$ measured in a reference frame fixed with respect to the earth and the intrinsic frequency $\sigma$ which measures the frequency in a reference frame moving with the mean flow. The two frequencies are related by

$$
\sigma=\omega(1-d \cos \theta)=\omega(1-U / c \cos \theta)
$$

where $c$ is the phase speed for the wave being considered, $\theta$ is the direction the wave is propagating, and $U$ is the strength of the mean flow (presumed to be in the zonal direction). When the intrinsic frequency $\sigma$ reaches zero $(d= \pm 1)$ then the wave is said to be critical and linear theory breaks down. If the wave is not critical, the effects of Doppler shift are primarily local, i.e. only the local Doppler shift is important for interpreting measurements at a particular point. Thus it is important to find both the depths at which the Doppler shift reaches \pm 1 and the depths at which the Doppler shift merely approaches 1 .

It is clear from equation 2.2.1 that the strength of the Doppler shift very much depends on the speed of the internal waves as well as the strength of the mean flow. Plotted in right side of figure 2.2.1 is the Doppler shift for three selected modes. The solid curve gives the Doppler shift for mode 1 (phase speed $2.69 \mathrm{~m} / \mathrm{s}$ ) and is almost indistinguishable from zero except at the peak of the undercurrent. The dashed line corresponds to mode 10 (phase speed $.294 \mathrm{~m} / \mathrm{s}$ ): the Doppler shift is significant only at the peak of the undercurrent. Since the current meter measurements are between $500 \mathrm{~m}$ and $3200 \mathrm{~m}$, as far as local Doppler shift effects are concerned mode 10 is only slightly 


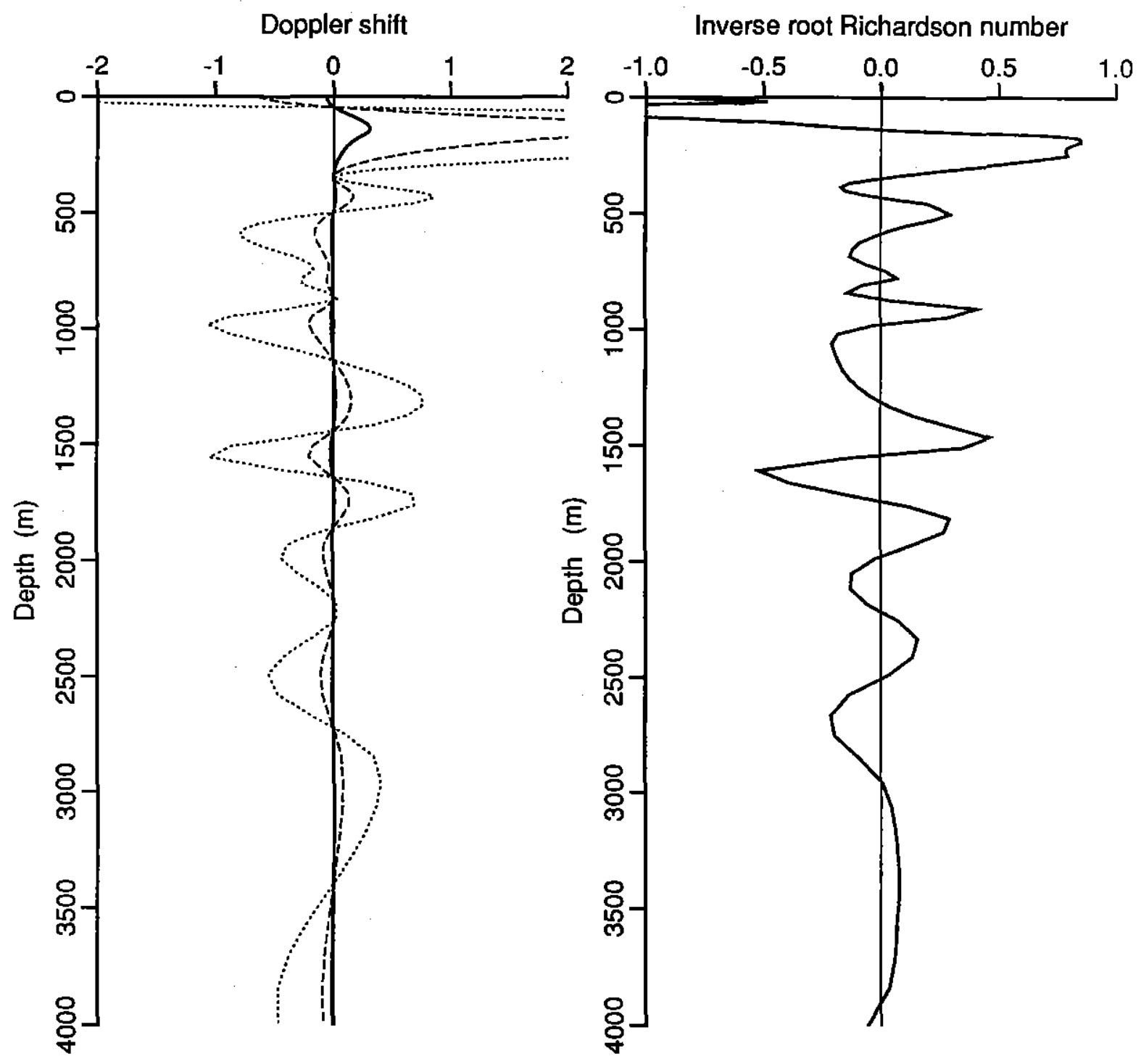

Figure 2.2.1 Doppler shifts and inverse root Richardson number

The left plot gives the Doppler shift $d$ as a function of depth for modes 1 (solid line), 10 (dashed line) and 50 (dotted line). The right plot gives the inverse root Richardson number $\lambda$ as a function of depth (the strength of the effect on internal waves does not depend on wavenumber). Both plots are computed from a spline fit to the average of the zonal (east) velocity profiles listed in figure 2.1.3: the Richardson number plot also uses a spline fit to the buoyancy frequency plotted in figure 2.1.4. The plots show that for depths below $500 \mathrm{~m}$, Doppler shifts are only important for high modes, while the shear (Richardson number) effects are important at certain depths for all wavenumbers. 
affected by Doppler shifts. Finally, the dotted line shows that mode 50 (phase speed .056 $\mathrm{m} / \mathrm{s}$ ) has critical or almost critical Doppler shifts at many different depths. Only to the extent that it is necessary to model the higher modes, then, will it be necessary to include the effects of Doppler shifts.

The right side of figure 2.2.1 gives another measure of the effect of a mean shear flow on the internal wave field, the inverse root Richardson number $\lambda=U_{z}(z) / N(z)$. As will be made clear when the internal wave solutions are scaled in section 8.2 , the addition to the meridional component of vorticity made by the mean shear is only important to the extent that it overwhelms the effect of gravity. The ratio between the two effects is given by the inverse root Richardson number. This turns out to be a purely local effect once the vertical structure equation is scaled (see chapter 7). Figure 2.2.1 shows that the ratio reaches .5 at several depths that are near the depths of PEQUOD current meters, suggesting that the mean shear can affect the measurements at those locations.

One of the assumptions made in forming the Garrett and Munk spectra is that top and boundary conditions are not important (see section 1.4). Consequently the internal wave field is viewed as a purely propagating field of waves, energy being a continuous function of wavenumber and frequency rather than being found only at the discrete wavenumbers that can satisfy both top and bottom boundary conditions. Consider Müller and Olbers 1975

“... an appreciable part of the internal wave energy is in high mode numbers, i.e. has vertical length scales which are small in comparison with the vertical length scale of the mean Brunt-Väisälä profile $(\approx 1.3 \mathrm{~km})$.

The concept of well-defined normal modes for the full water column, however, appears rather questionable for high mode numbers, since typical vertical propagation times of the wave field are comparable to typical interaction times. The phases will thus be randomized before a mode can be formed."

Desaubies 1976 goes so far as to object to the sum of modes formulation that Cairns and Williams 1976 give (equation 1.1.1d) on the grounds that interactions will destroy the modal character. The notion that interactions destroy phase structure is given some precision in McComas and Bretherton 1977 who classify wave-wave interactions into three groups: elastic scattering, induced diffusion, and parametric subharmonic instability. Elastic scattering in particular scatters vertically propagating waves into their oppositely traveling counterpart so that not only does the wave loses phase information, the wave field tends to become vertically symmetric. This tendency of the wavefield leads McComas 
and Bretherton to dismiss measurements of a net high-frequency vertical energy flux by saying

"To see any significant vertical energy propagation at the higher frequencies, one must be within a few wavelengths of the source!"

While these effects are important for high wavenumber waves, a closer look at the GM79 spectrum reveals that almost all of the energy is in low wavenumbers, wavenumbers such that the entire watercolumn is 'within a few wavelengths' of the boundaries and consequently that close to any possible source.

The continuous form of the GM79 wavenumber spectrum (Desaubies 1976) is given in equation $1.6 .1 b$, namely the wavenumber dependence $H(K)$ is given by

$$
H(K)=\frac{\frac{2}{\pi} K_{*}}{K_{*}^{2}+K^{2}}
$$

This expression is rather easily integrated to get $T(K)$, a function which gives that total amount of energy found in wavelengths shorter than wavenumber $K$,

$$
T\left(K / K_{*}\right)=\frac{2}{\pi} \tan ^{-1}\left(K / K_{*}\right)=\frac{2}{\pi} \tan ^{-1}\left(j / j_{*}\right)
$$

where the ratio of wavenumbers $K / K_{*}$ is replaced by the ratio of mode numbers $j / j_{*}$ for convenience in the following comparisons. Not surprisingly, as the mode number $j$ goes to infinity the total energy approaches 1 . Less obvious is how rapidly 1 is reached. Since the rolloff mode number $j_{*}$ is 3 , and the arctangent of of 1 is $\frac{\pi}{4}$, modes 1 through 3 contain $50 \%$ of the energy. Note that mode 3 has $1 \frac{1}{2}$ wavelengths between the top and boundaries, so it is not significantly affected by scattering. If one presses a little farther, one could argue that a wavenumber corresponding to mode 10 (5 wavelengths from top to bottom) can support a vertical flux spanning most of the water column, even if it may not be able to carry phase information from top to bottom. Evaluating $T\left(j / j_{*}\right)$ for mode 10 shows that modes 1 through 10 contain $80 \%$ of the energy. Since low wavenumbers have higher group velocities, they probably carry an even higher proportion of the total vertical energy flux. So while rapid interaction strongly affects high wavenumbers and thus can determine the overall shape of the wavenumber spectrum, the actual amount of energy at high wavenumbers is relatively small and an analysis which concentrates on the low wavenumbers will explain most of the energy density and fluxes. Waves at these low wavenumbers do not fit the definition of 'high wavenumber waves' and thus could possibly 
1) show some of the phase-locking characteristic of modes,

2) support a net vertical energy flux, and

3) have a vertical structure that is not given by the WKBJ approximation.

(Comparisons are made in chapter 7 between the exact and the WKBJ approximate solutions to understand the character of the differences). There are thus good theoretical reasons to consider extensions to the GM models.

Olbers notes in his review of internal wave models (Olbers 1983) that most of the sources of energy postulated for the internal wave field result in vertical fluxes that are roughly $1 \mathrm{erg} / \mathrm{s} / \mathrm{cm}^{2}$. Before starting an elaborate analysis of the internal wave field it would be nice to know whether such a flux is distinguishable from zero using the PEQUOD measurements.

The noise level can be roughly determined from the energy level and the degree of spectral averaging. Since low wavenumbers and frequencies have higher group velocities, consider the low wavenumber vertical energy flux estimated from a frequency band centered at $.158 \mathrm{cph}$ with width $1 / 15 \mathrm{cph}$. According to the GM spectrum this band contains somewhat less than $.7 \mathrm{erg} / \mathrm{cm}^{3}$. If yearlong time series are used in computing the spectral averages, this corresponds to $\mathbf{5 7 6}$ degrees of freedom and thus an uncertainty of roughly $.024 \mathrm{erg} / \mathrm{cm}^{3}$. This can be translated to a vertical energy flux knowing the energy weighed average vertical group velocity (roughly $20 \mathrm{~cm} / \mathrm{s}$ ). Thus the uncertainty in the vertical energy flux estimates is roughly $\frac{1}{2} \mathrm{ergs} / \mathrm{s} / \mathrm{cm}^{2}$, small enough to get significant estimates of fluxes that are the expected size.

In summary, then, there are three facets of the GM model for which extensions seem a priori necessary. Because of the presence of mean shear flows in the PEQUOD region, some inclusion of mean shear effects should be made, though the effects of local shear as measured by the Richardson number are more important for the energetic low modes than the effects of Doppler shifts. Secondly, because most of the energy is in low wavenumbers, there is a strong possibility that the phase-locking associated with modes will be measurable or that net vertical fluxes will not scattered sufficiently to make the wavefield vertically symmetric. Finally, the length of the time series available is sufficient so that fluxes of the size postulated for many internal wave sources are distinguishable from zero. 


\section{Chapter 3: Vertical coherence calculations and interpretation}

\subsection{Vertical coherence comparison of PEQUOD data with GM79}

Figures 1.5.1 and 1.6.1 list GM predictions for a set of spectral estimators that can be calculated from moored sensor time series. These include the autospectra of each component (zonal velocity, meridional velocity, and displacement), the coherence between each component at a single moored instrument (MCC), the coherence between like components measured with moored sensors that have a purely horizontal separation (MHC), and the coherence between like components measured with moored sensors that have a purely vertical separation (MVC). The coherence between the PEQUOD $Q$ and $U$ moorings is not significant at internal wave frequencies. The two moorings are roughly $40 \mathrm{~km}$ apart, thus zero coherence is consistent with the GM predictions. Thus the moored horizontal coherence MHC does not provide a very interesting comparison for the PEQUOD data and the GM model. But both the moored vertical coherence MVC and the moored component coherence MCC are more interesting. Since the vertical coherence is somewhat simpler to present than the moored component coherence, and because the GM papers tend to focus on modeling coherence as function of separation rather than the moored component coherence, we will consider the moored vertical coherence first.

The moored vertical coherences provide a check of the GM choice of wavenumber dependence $H(j)$. Comparison of the PEQUOD data with the GM79 model shows two quite distinct results. For short separations the coherences observed with the PEQUOD array are essentially the same as those predicted by GM79 (figure 3.1.1): the coherence can be modeled as being due to a field of internal waves plus some finestructure which is advected past the mooring. But for longer separations the results from PEQUOD are distinctly different from the GM79 prediction (figures 3.1.3-5). This latter result is not particularly surprising, since the GM79 model is based on relatively short separation coherences. The PEQUOD-GM79 differences show that there are aspects of the internal wave field that are measurable but not included in the GM models.

To facilitate a discussion of the short separation coherences, the moored vertical coherences for the first year $Q$ mooring data are plotted against separation in figure 3.1.1. The abscissa gives the WKBJ scaled separation $\zeta$ which is defined as

$$
\varsigma\left(z_{1}, z_{2}\right)=\frac{1}{1 \mathrm{cph}} \int_{z_{1}}^{z_{2}} N\left(z^{\prime}\right) d z^{\prime}
$$


where $z_{1}$ is the depth of the first instrument, $z_{2}$ is the depth of the second, and $N(z)$ is in cycles per hour. This definition implies that if $N_{(z)}$ is a constant $1 \mathrm{cph}$ then the WKBJ scaled separation is identical to the actual separation. Consequently the WKBJ scaled separation can be thought of as the equivalent separation in a $1 \mathrm{cph}$ ocean. The $K_{*} \psi$ that appears as the dimensionless variable in the GM79 formula for MVC in figure 1.6.1 can be written in terms of the stretched separation $\zeta$.

$$
K_{*} \psi=m_{*} \zeta\left(z_{1}, z_{2}\right)
$$

where

$$
m_{*}=\frac{\pi j_{*}(1 \mathrm{cph})}{b N_{0}}
$$

Equation 3.1.2b is equation 1.6.2c evaluated for a local buoyancy frequency of $1 \mathrm{cph}$.

The WKBJ separations available from the $Q$ and $U$ moorings fall into three distinct ranges - short $(10-300 \mathrm{~m})$, medium $(1000-1600 \mathrm{~m})$, and long $(2250-2600 \mathrm{~m})$ - because the instruments are arranged as three groups such that there are short spacings within each group and long spacings between groups. The medium range measurements can be further split into two subgroups: the $900-1100 \mathrm{~m}$ spacings, which are between the center and bottom group of instruments, and the $1200-1600 \mathrm{~m}$ spacings, which are between the center and top groups of instruments. Note the the slightly longer medium range separations are between the center and bottom group of instruments, the opposite of what would occur if actual separations rather than WKBJ separations were used: in that case the longer medium separations would be between the center and bottom instruments.

The coherences in figure 3.1 .1 are the separations shorter than $300 \mathrm{~m}$. They are distinctly lower than the GM79 reference line. The symbols at a given separation are mostly ordered such that the lower frequencies have higher coherence, suggesting that at these separations the coherence decreases with increasing frequency. This decrease is qualitatively consistent with the measurements used in GM. The difference between the GM reference curve and the measurements is usually attributed to finestructure contamination of the moored sensors. This supposition can be checked quantitatively by comparing the data with Webster's rule for the frequency and separation at which the coherence drops to $\frac{1}{2}$. The comparison is plotted in figure 1.2.5 for the data used in GM72. A comparison with the PEQUOD data can be made by considering the dotted lines in figure 3.1.1. The separations at which the dotted lines give coherences of .5 are the separations 


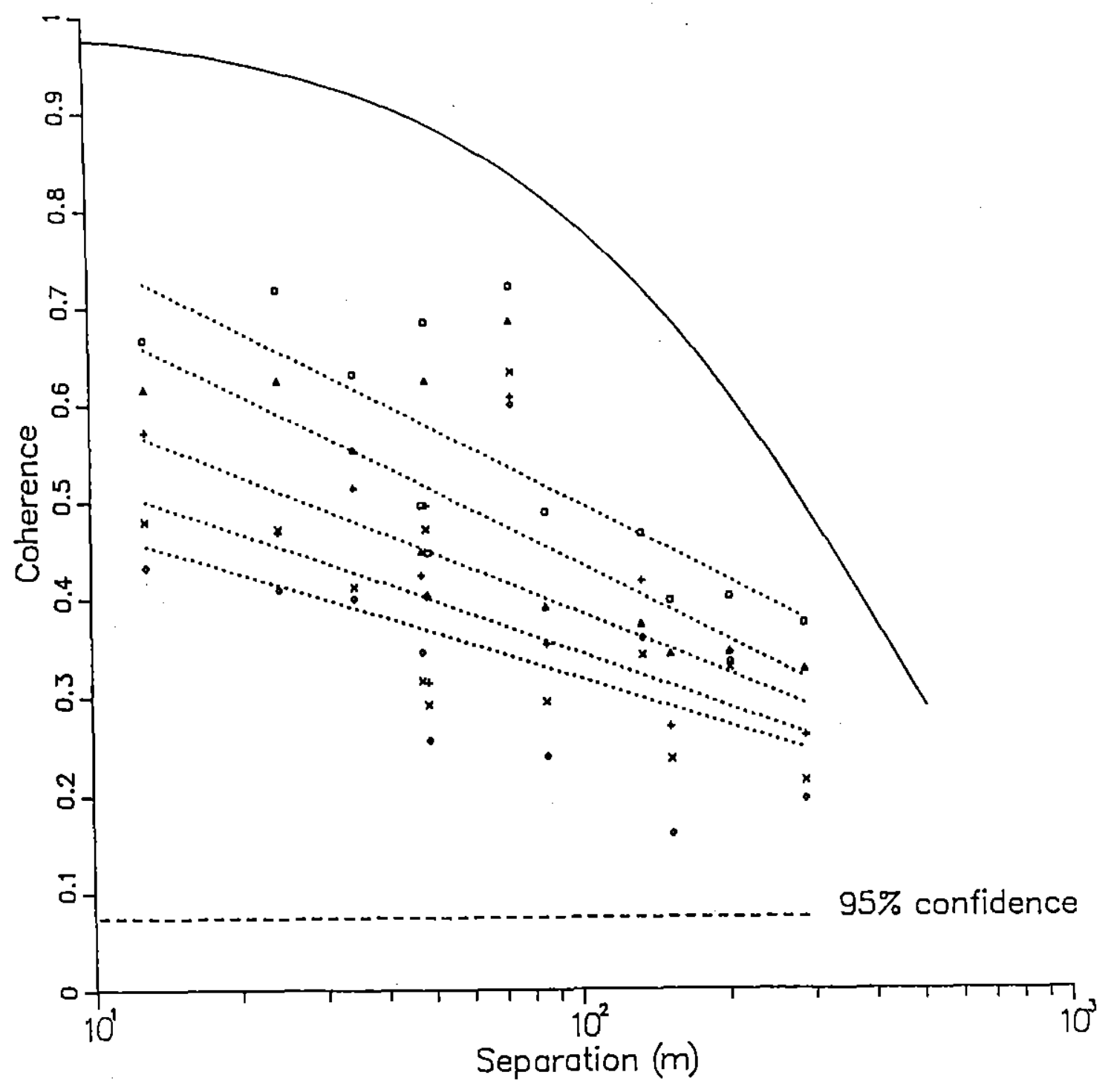

Figure 3.1.1 Vertical temperature coherence as a function of short WKBJ stretched separation

The figure contains a scatterplot of vertical temperature coherence as a function of WKBJ stretched separation for frequencies between $.1 \mathrm{cph}$ and $.5 \mathrm{cph}$. The data used are temperature measurements from the first year $Q$ mooring records. Each symbol corresponds to a different frequency bin, with the lowest frequency bin centered at .158 cph and a frequency bin width of $1 / 15 \mathrm{cph}$. The degree of averaging $N_{r}$ is 576 for each estimate, and the corresponding $95 \%$ zero significance level is given by the dashed line. The solid curve is the GM prediction based on the calculations of chapter 1 . The dotted lines are linear best fits of the coherence as a function of log separation for each frequency. Their intercepts at a coherence of .5 form the basis of the comparison of the data with Webster's rule given in figure 3.1.2. The short separation PEQUOD coherences are roughly consistent with Webster's rule. 


\section{Separations at which the coherence drops to .5}

$\begin{array}{crrr}\text { Symbol } & \text { Frequency } & \text { Webster's Rule } & \text { PEQUOD } \\ \square & .1583 \mathrm{cph} & 82 \mathrm{~m} & 100 \mathrm{~m} \\ \triangle & .2250 \mathrm{cph} & 58 \mathrm{~m} & 55 \mathrm{~m} \\ + & .2916 \mathrm{cph} & 45 \mathrm{~m} & 30 \mathrm{~m} \\ \times & .3583 \mathrm{cph} & 36 \mathrm{~m} & 15 \mathrm{~m} \\ \diamond & .4250 \mathrm{cph} & 30 \mathrm{~m} & \end{array}$

\section{Figure 3.1.2 Table of Webster's rule comparisons}

This table compares Webster's rule with PEQUOD measurements. The first column gives the symbol plotted in figure 3.1.1, while the second column gives the frequency. The last two columns give the separation at which the coherence drops to $\frac{1}{2}$ for the rule and the measurements respectively, showing that the two are about the same.

of half coherence for the five frequency bins. The intercepts are tabulated in figure 3.1.2 along with Webster's rule. These measurements suggest that there is roughly the same finestructure in the PEQUOD measurements as in the mid-latitude data that GM summarize: the coherence drops to .5 at the same separations for a given frequency as Webster's rule. On the other hand, while examining equatorial Indian ocean data (from INDEX), Wunsch and Webb 1979 saw almost no vertical coherence above .5 between sensors that had as little as $100 \mathrm{~m}$ separations. Presuming that the coherence drops are in fact due to finestructure, these results imply that the finestructure at the equatorial PEQUOD site is closer to what is found at mid-latitudes than what is found at the equatorial INDEX site. It is important to note, however, that the dotted lines are quite poor fits to the data, suggesting that the short separation coherences are not modeled well by a function that depends only on separation.

Coherences calculated from the PEQUOD measurements at $1000 \mathrm{~m}$ vertical separations are distinctly different from GM predictions. Figure 3.1.3a shows coherences calculated from the first year $Q$ mooring data plotted against linear axes. The short separation coherences are the coherences presented in figure 3.1.1, but figure 3.1.3a includes the longer separations as well. Figure 3.1.3a shows that the measured coherences at mooring $Q$ are $.2 \pm .08$ at $1000 \mathrm{~m}$; all these measurements are significantly higher than the .1 predicted by GM. The same thing is seen in figure $3.1 .3 b$, which is calculated from second year $U$ mooring data. Furthermore, unlike smaller separations, there is no strong indication of frequency dependence. At slightly longer separations (1200-1600m), many measurements are greater than the GM prediction, and most of the estimates in the bin centered at $.158 \mathrm{cph}$ are significant, but there are also many estimates indistinguishable 
from zero. There are a few significantly non-zero coherences at $2500 \mathrm{~m}$ separations as well; none of them correspond to $.158 \mathrm{cph}$.

The GM model predicts that the coherence calculated between vertically separated velocity sensors will be identical to the coherence calculated between vertically separated temperature sensors. But the velocity coherences calculated from PEQUOD are distinctly different from both the GM predictions and the PEQUOD temperature coherences. Figure 3.1.4a gives the moored vertical coherence (MVC) as calculated from the first year $Q$ mooring zonal velocity data, and figure 3.1.4b gives the same as calculated from the second year $U$ mooring data. Figures 3.1.5a and 3.1.5b give the corresponding plots for the meridional velocity. The estimates at $1000 \mathrm{~m}$ separation are centered about or below the GM prediction, quite unlike the temperature coherence. But even more striking is the fact that the coherence increases as separation increase above $1000 \mathrm{~m}$. In fact, the velocity coherences at $2500 \mathrm{~m}$ are as strong as the temperature coherences at $1000 \mathrm{~m}$ separation. This mid-depth minimum in the coherence suggests that the energy that is responsible for the coherence has some sort of modal structure that includes a node at mid-depths. This hypothesis is supported by the phase plots for velocity and temperature.

As might be expected from the coherence calculations, in the PEQUOD measurements the phase differences calculated from velocity records are distinctly difference from the phase differences calculated from temperature records. Figures 3.1.6a, $b$ give the phase differences calculated from the $Q 1$ temperature data and the $U 2$ temperature data respectively. The plots show that while all of the temperatures have phase differences indistinguishable from zero at $1000 \mathrm{~m}$ separation, the few temperature phase differences at $2500 \mathrm{~m}$ that are significant are all close to $180^{\circ}$. Note that the two frequency bins that have significant phases at $2500 \mathrm{~m}$ separation are not contiguous: one is centered at 225 $\mathrm{cph}$ and the other is centered at $.358 \mathrm{cph}$, and the intervening bin does not show any significant phase differences above $1200 \mathrm{~m}$ separation. The temperature phase differences at $1500 \mathrm{~m}$ separations are harder to characterize. The temperature phase difference for $.158 \mathrm{cph}$ is zero for both the $Q 1$ and $U 2$ data. The phase difference for $.225 \mathrm{cph}$ is slightly less than $-45^{\circ}$, which is statistically indistinguishable from zero. Finally, one of the two frequency bins which have significant phases differences at $2500 \mathrm{~m}$ separation has the same phase difference $\left(180^{\circ}\right)$ at approximately $1500 \mathrm{~m}$ separations, and the highest frequency bin has $180^{\circ}$ phase differences at $1500 \mathrm{~m}$ and no significant coherence at $2500 \mathrm{~m}$ separation. So while there are some differences in the temperature phase structure 


\section{Coherence}

WKBJ stretched depth

Q1T: frequencies between .1 cph and .5 cph

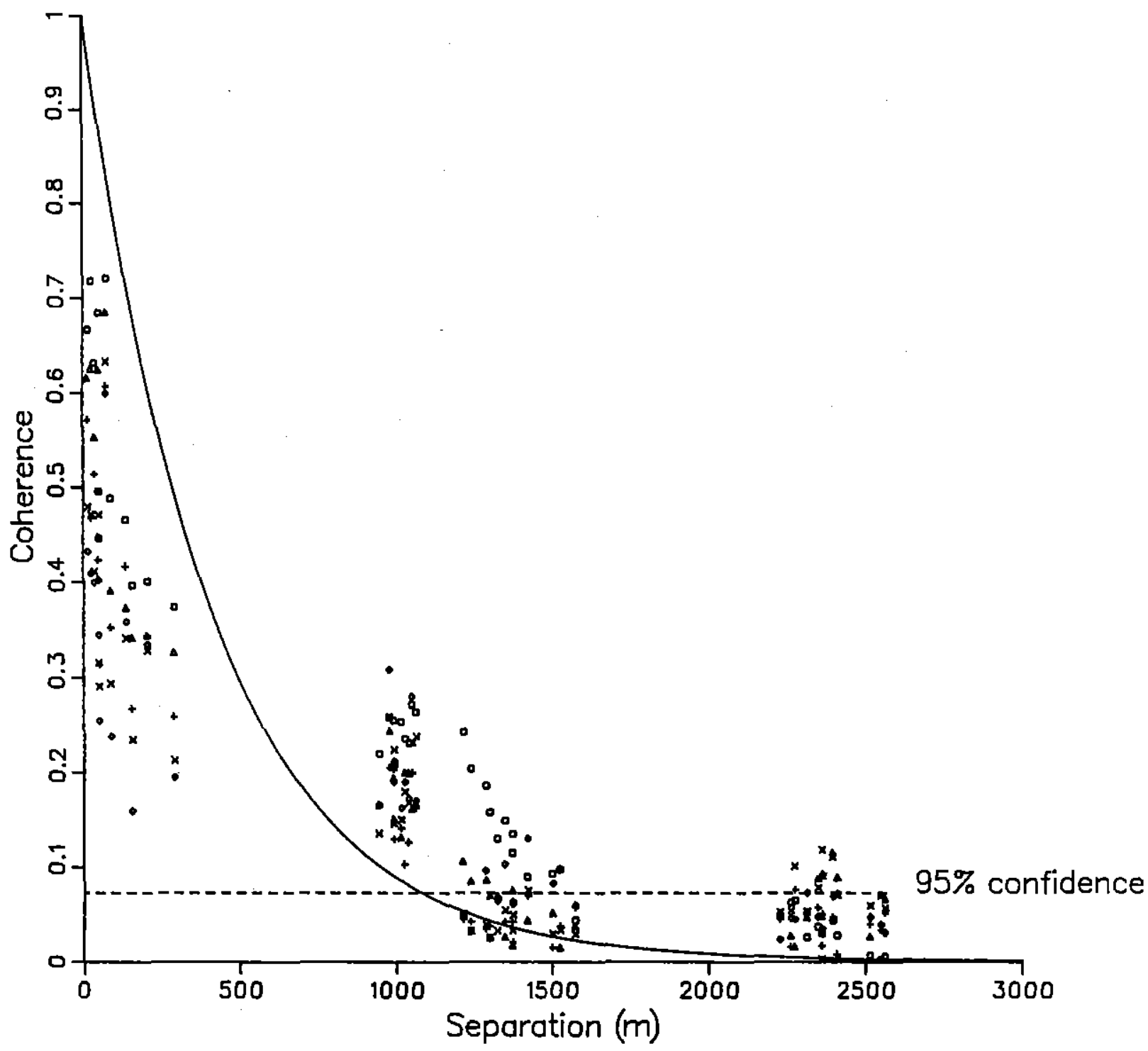

Figure 3.1.3 $a$ Vertical coherence calculated from $Q 1$ temperature data as a function of WKBJ stretched separation

The figure contains a scatterplot of vertical coherence as a function of WKBJ stretched separation calculated from temperature data for frequencies between $.1 \mathrm{cph}$ and $.5 \mathrm{cph}$. The data used are from the first year $Q$ mooring records. Each symbol corresponds to a different frequency bin, with the lowest frequency bin centered at $.158 \mathrm{cph}$ and a binwidth of $1 / 15 \mathrm{cph}$. The degree of averaging $N_{r}$ is 576 for each estimate, and the corresponding $95 \%$ zero significance level is given by the dashed line. The solid curve is the GM prediction based on the calculations of chapter 1 . The measured coherences at $1000 \mathrm{~m}$ separation are distinctly higher than the GM prediction. 


\section{Coherence}

WKBJ stretched depths

U2T: frequencies between $.1 \mathrm{cph}$ and $.5 \mathrm{cph}$

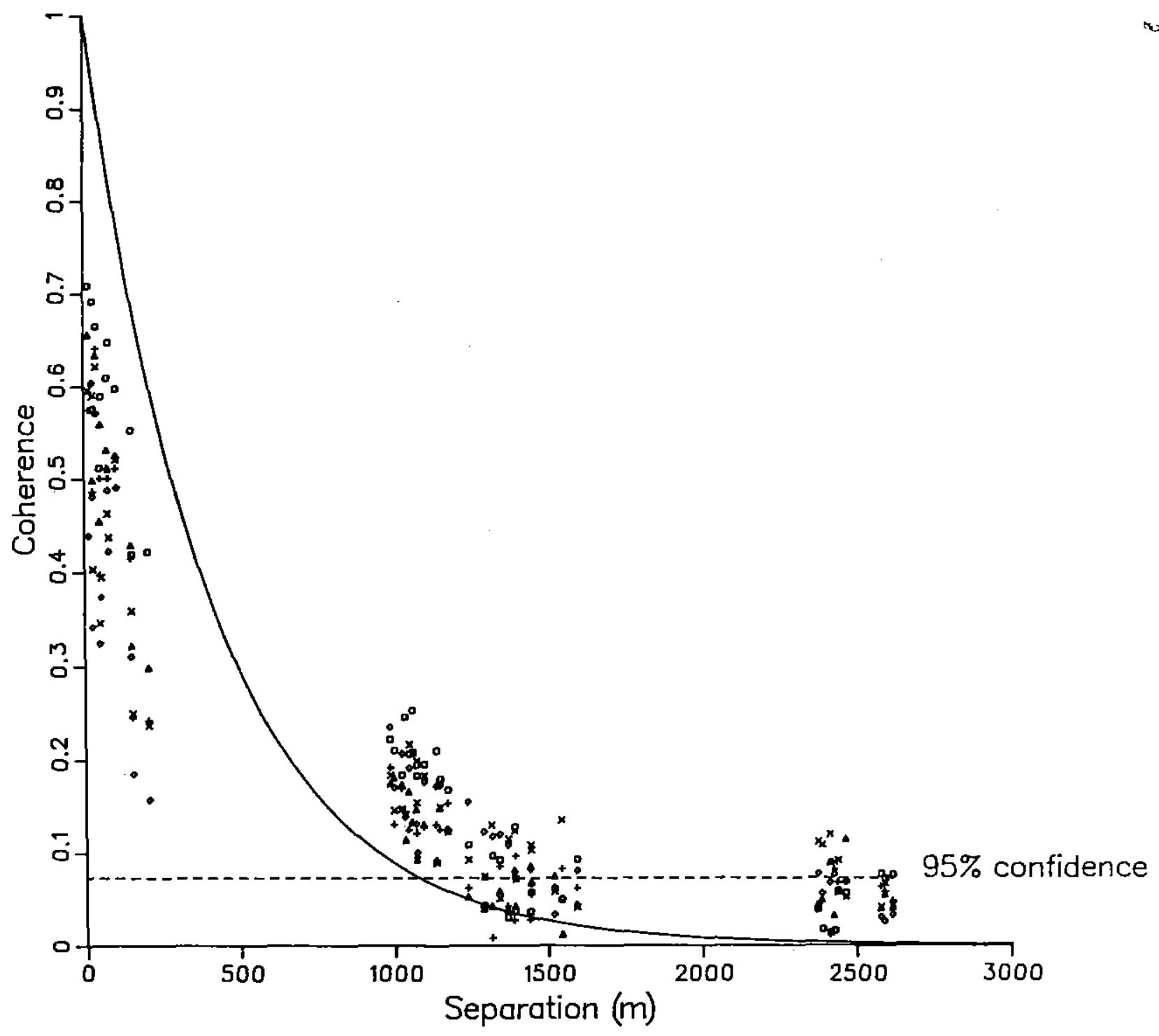

Figure 3.1.3b Vertical coherence calculated from $U 2$ temperature data as a function of WKBJ stretched separation

The figure contains a scatterplot of vertical coherence as a function of WKBJ stretched separation calculated from temperature data for frequencies between $.1 \mathrm{cph}$ and $.5 \mathrm{cph}$. The data used are from the second year $U$ mooring records. Each symbol corresponds to a different frequency bin, with the lowest frequency bin centered at .158 cph and a binwidth of $1 / 15 \mathrm{cph}$. The degree of averaging $N_{r}$ is 576 for each estimate, and the corresponding $95 \%$ zero significance level is given by the dashed line. The solid curve is the GM prediction based on the calculations of chapter 1 . The measured coherences at $1000 \mathrm{~m}$ separation are distinctly higher than the GM prediction. 


\section{Coherence}

WKBJ stretched depths

Q1U: frequencies between $.1 \mathrm{cph}$ and $.5 \mathrm{cph}$

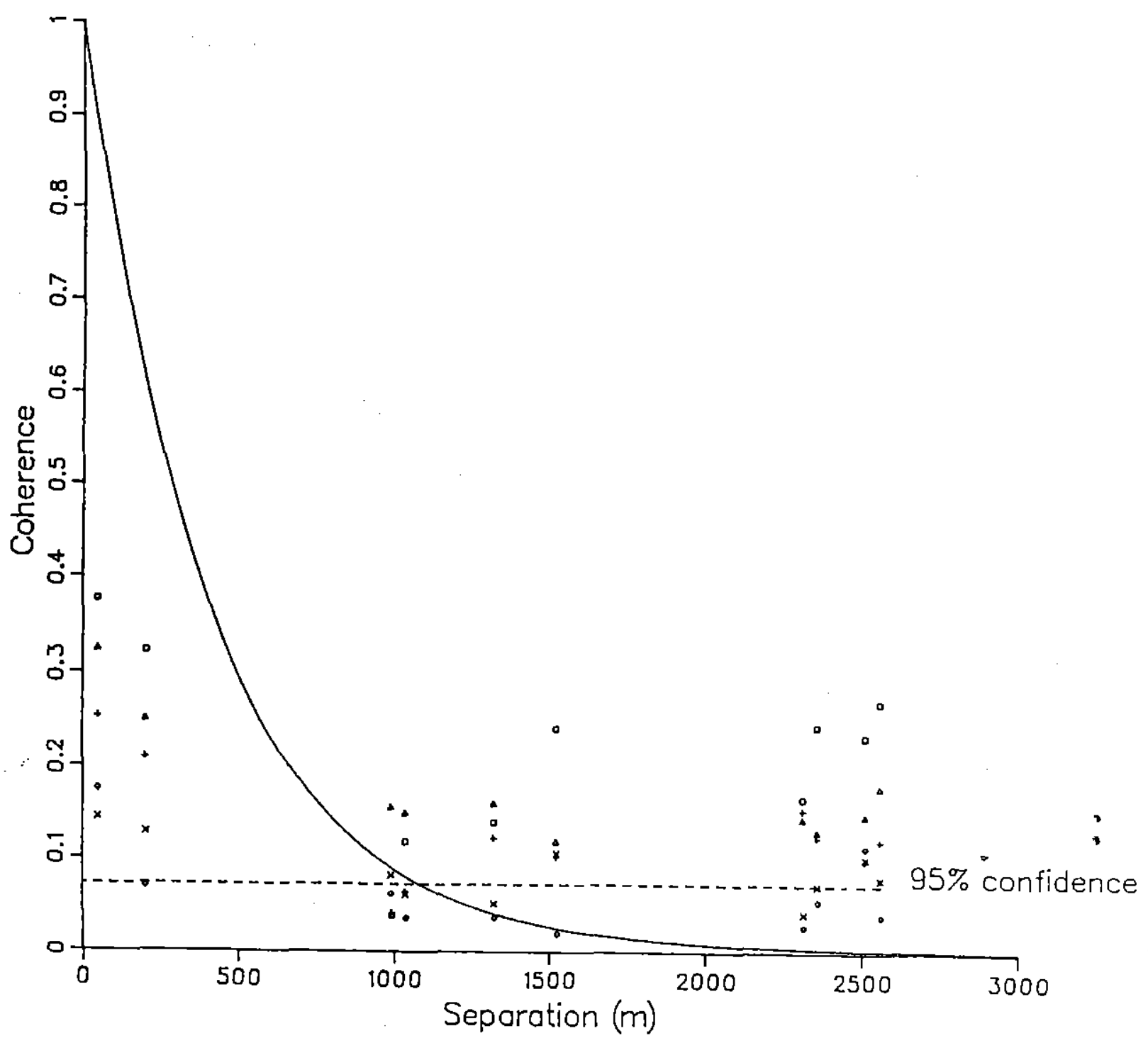

Figure 3.1.4a Vertical coherence calculated from $Q 1$ zonal velocity data as a function of WKBJ stretched separation

The figure contains a scatterplot of vertical coherence as a function of WKBJ stretched separation calculated from zonal velocity data for frequencies between .1 cph and $.5 \mathrm{cph}$. The data used are from the first year $Q$ mooring records. Each symbol corresponds to a different frequency bin, with the lowest frequency bin centered at .158 $\mathrm{cph}$ and a binwidth of $1 / 15 \mathrm{cph}$. The degree of averaging $N_{r}$ is 576 for each estimate, and the corresponding $95 \%$ zero significance level is given by the dashed line. The velocity coherences do not match either the GM predictions or the temperature coherences, especially at the longest separations. 


\section{Coherence}

WKBJ stretched depths

U2U: frequencies between $.1 \mathrm{cph}$ and $.5 \mathrm{cph}$

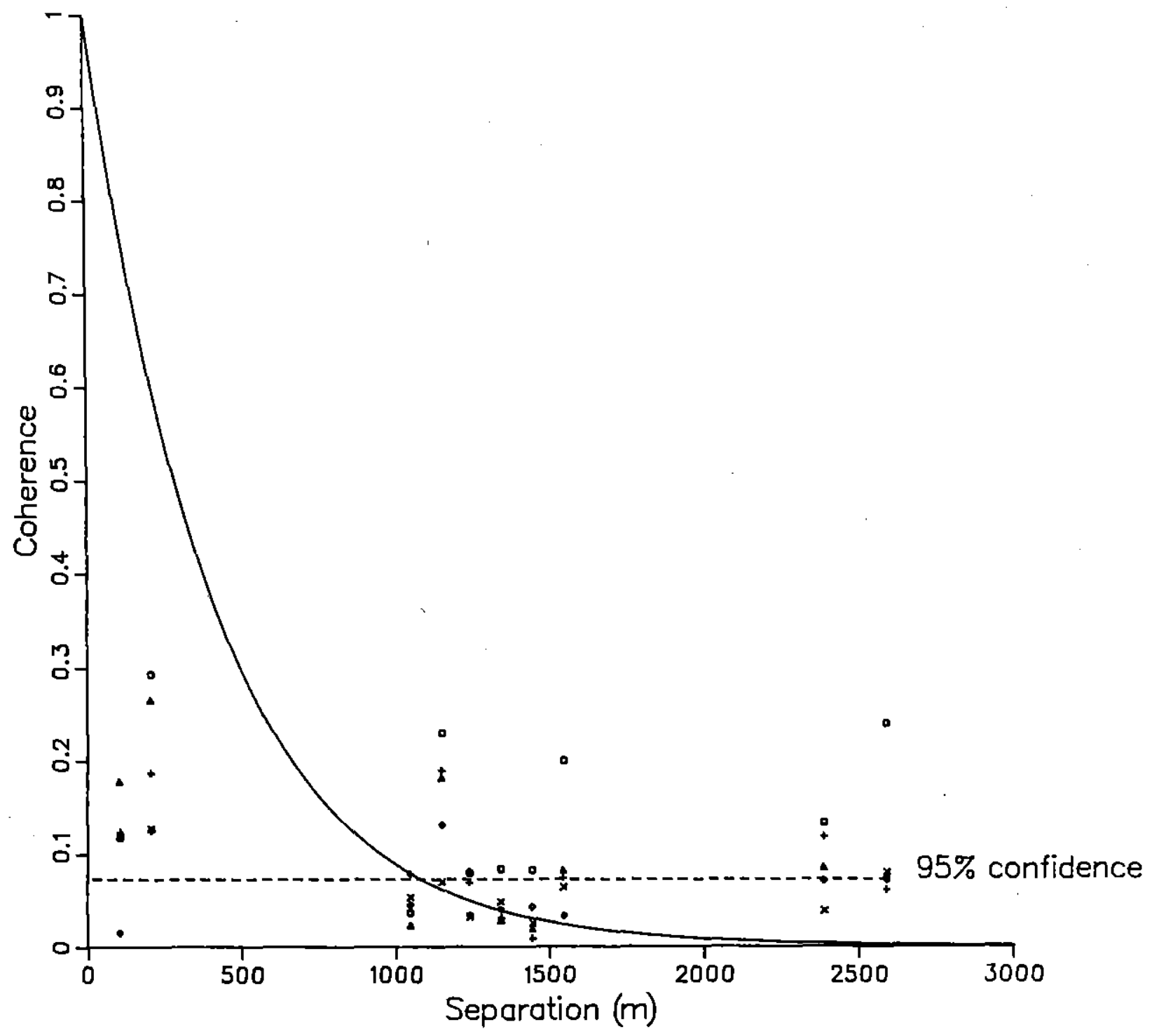

Figure 3.1.4b Vertical coherence calculated from $U 2$ zonal velocity data as a function of WKBJ stretched separation

The figure contains a scatterplot of vertical coherence as a function of WKBJ stretched separation calculated from zonal velocity data for frequencies between $.1 \mathrm{cph}$ and $.5 \mathrm{cph}$. The data used are from the second year $U$ mooring records. Each symbol corresponds to a different frequency bin, with the lowest frequency bin centered at .158 $\mathrm{cph}$ and a binwidth of $1 / 15 \mathrm{cph}$. The degree of averaging $N_{r}$ is 576 for each estimate, and the corresponding $95 \%$ zero significance level is given by the dashed line. The velocity coherences do not match either the GM predictions or the temperature coherences, especially at the longest separations. 


\section{Coherence}

WKBJ stretched depths

Q1V: frequencies between .1 $\mathrm{cph}$ and $.5 \mathrm{cph}$

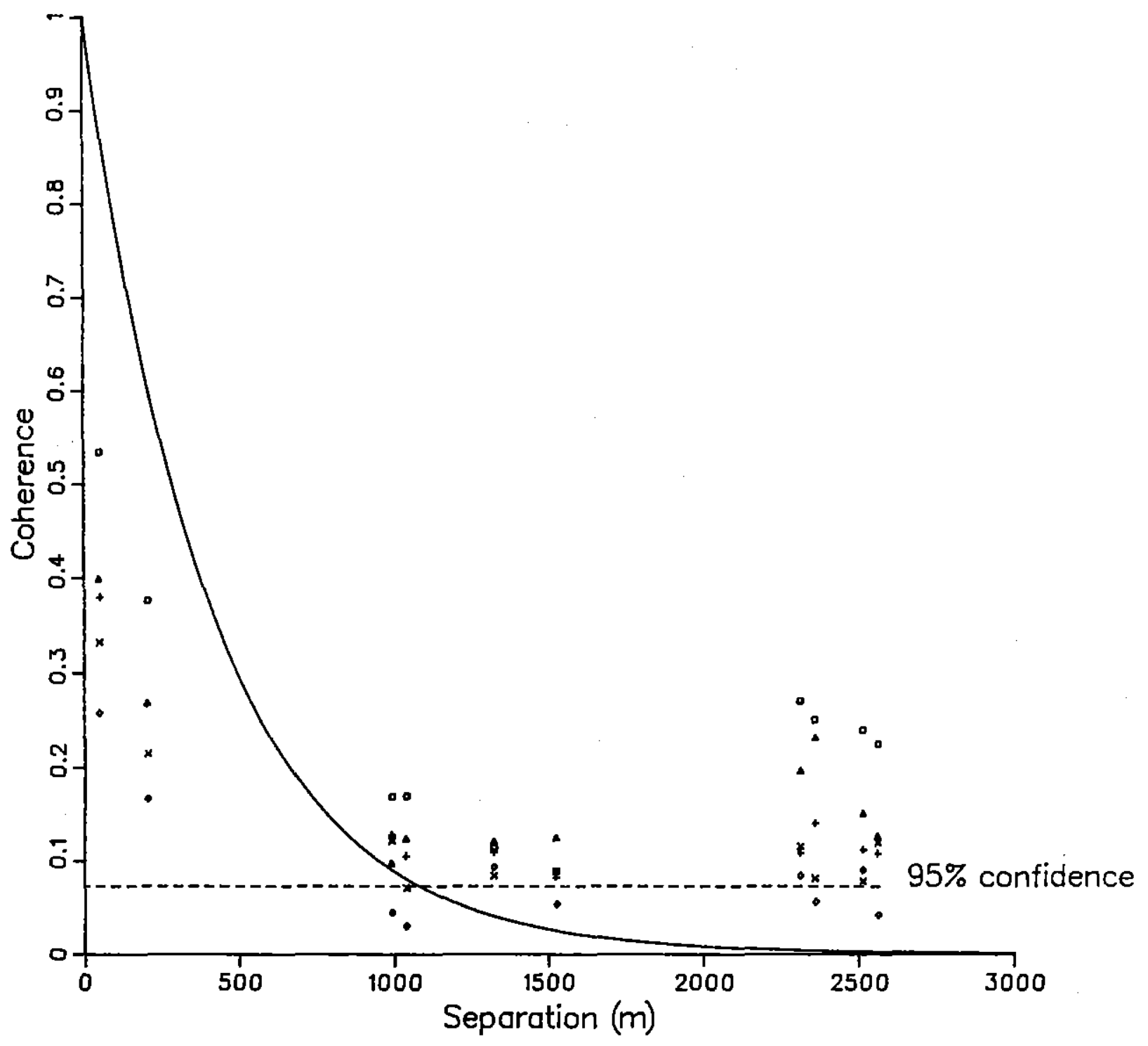

Figure 3.1.5 $a$ Vertical coherence from $Q 1$ meridional velocity data as a function of WKBJ stretched separation

The figure contains a scatterplot of vertical coherence as a function of WKBJ stretched separation calculated from meridional velocity data for frequencies between $.1 \mathrm{cph}$ and $.5 \mathrm{cph}$. The data used are from the first year $Q$ mooring records. Each symbol corresponds to a different frequency bin, with the lowest frequency bin centered at .158 $\mathrm{cph}$ and a binwidth of $1 / 15 \mathrm{cph}$. The degree of averaging $N_{r}$ is 576 for each estimate, and the corresponding $95 \%$ zero significance level is given by the dashed line. The solid curve is the GM prediction based on the calculations of chapter 1 . The velocity coherences do not match either the GM predictions or the temperature coherences, especially at the longest separations. 


\section{Coherence}

WKBJ stretched depths

U2V: frequencies between $.1 \mathrm{cph}$ and $.5 \mathrm{cph}$

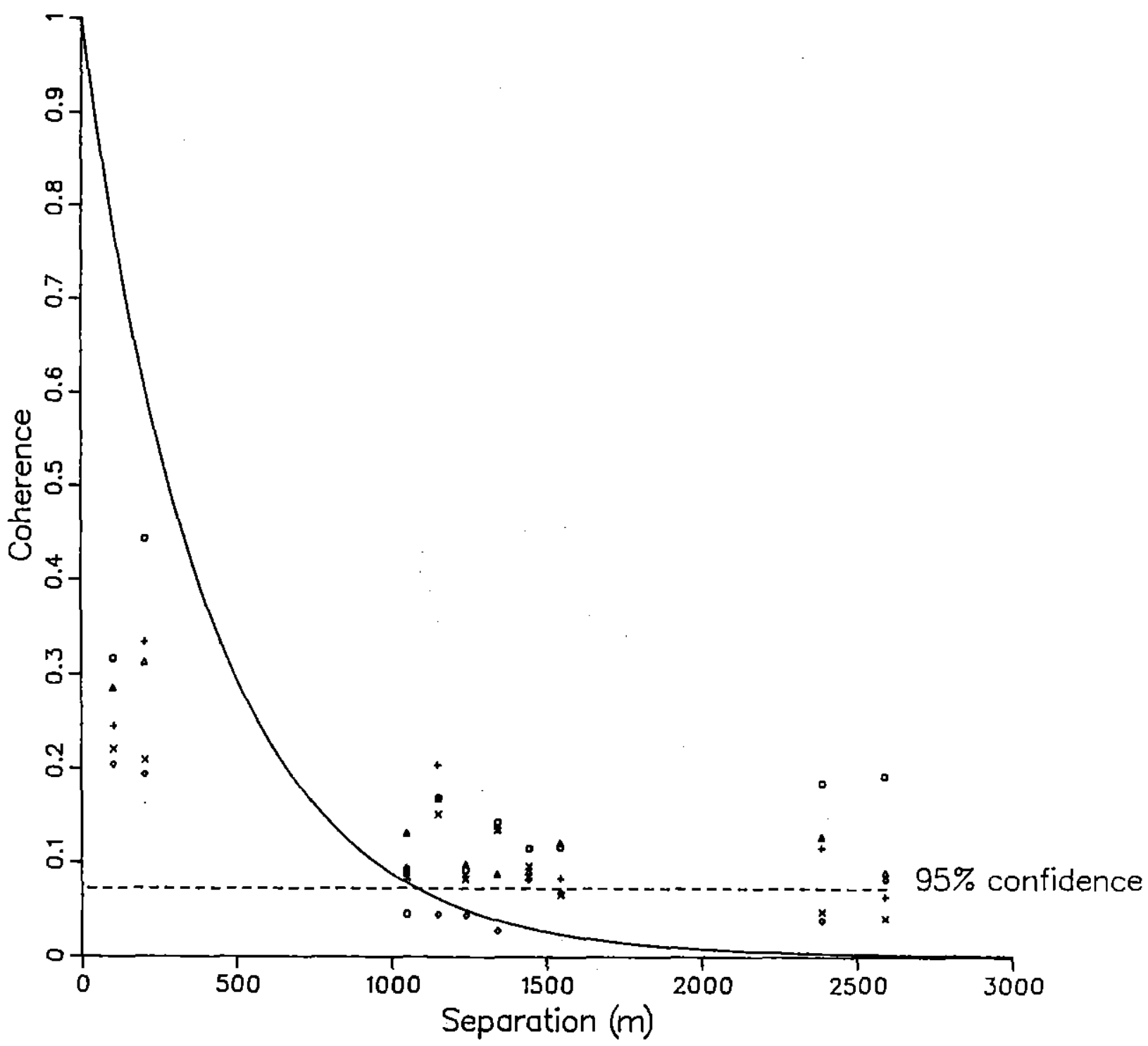

Figure 3.1.5 $b$ Vertical coherence from $U 2$ meridional velocity data as a function of WKBJ stretched separation

The figure contains a scatterplot of vertical coherence as a function of WKBJ stretched separation calculated from meridional velocity data for frequencies between $.1 \mathrm{cph}$ and $.5 \mathrm{cph}$. The data used are from the second year $U$ mooring records. Each symbol corresponds to a different frequency bin, with the lowest frequency bin centered at $.158 \mathrm{cph}$ and a binwidth of $1 / 15 \mathrm{cph}$. The degree of averaging $N_{r}$ is 576 for each estimate, and the corresponding $95 \%$ zero significance level is given by the dashed line. The solid curve is the GM prediction based on the calculations of chapter 1 . The velocity coherences do not match either the GM predictions or the temperature coherences, especially at the longest separations. 
as a function of frequency, there is no systematic trend with increasing frequency. Note that all of the complicated phase structure is at separations where the coherence is relatively small. Small coherence means that only a small fraction of the energy is involved in causing such motions, and that not modelling such a feature will only make a small contribution to the model-data misfit. So a simple characterization of the temperature data could ignore those complications and simply say that the coherence falls off from .2 to .1 between a separation of $1000 \mathrm{~m}$ and a separation of $1500 \mathrm{~m}$, and the phase difference for all separations is zero.

Like the velocity coherence plots, the velocity phase plots differ from their temperature counterparts. Figures 3.1.7 $a, b$ and 3.1.8a,b show the phase as a function of separation calculated from zonal and meridional velocity data from both years. The meridional velocity phases are quite simple to characterize: all separations greater than $1000 \mathrm{~m}$ have $180^{\circ}$ phase differences. The phase differences calculated from zonal velocities are somewhat different in that, while the significant phases corresponding to separations greater than $1200 \mathrm{~m}$ are all $180^{\circ} \pm 45^{\circ}$, the phase difference at $1000 \mathrm{~m}$ separation is close to zero. Note that the $U 2$ zonal velocity data has very few significant phases, none of which are at $1000 \mathrm{~m}$, so the phase difference at $1000 \mathrm{~m}$ is not a very robust result. Thus a simple characterization of the velocity data is that the coherence rises from approximately zero at $1000 \mathrm{~m}$ separation to .2 at $2500 \mathrm{~m}$ separation, with a phase difference of $180^{\circ}$ for all separations greater than $1000 \mathrm{~m}$. This is exactly opposite to the simple characterization of the temperature coherence and phase.

The temperature coherence for long separations can be simply characterized as decreasing from .2 at $1000 \mathrm{~m}$ separation to .1 at $1500 \mathrm{~m}$ separation, with zero phase lag for all separations, while the velocity coherence is characterized by increasing from roughly zero at $1000 \mathrm{~m}$ separation to .2 at $2500 \mathrm{~m}$ separation with a $180^{\circ}$ phase difference for all separations greater than $1000 \mathrm{~m}$. These coherence structures represents a significant deviation from GM, but it is not particularly surprising. Because the GM fit is separable in frequency and wavenumber, the moored vertical coherence (MVC) depends only on the choice of wavenumber dependence function $\mathrm{H}(\mathrm{j}$ ) (see chapter 1). The choice of $H(j)$ used in GM79 is based largely on the measurements of Caims and Williams 1976 presented in figure 1.1.6. The float measurements were only made for separations up to $40 \mathrm{~m}$ and the IWEX measurements that were also included only went up to $140 \mathrm{~m}$. Therefore the predictions for $1000 \mathrm{~m}$ and more are very great extrapolations indeed, and 
Phase

WKBJ stretched depth

Q1T: Points with expected error less than 45 deg are plotted.

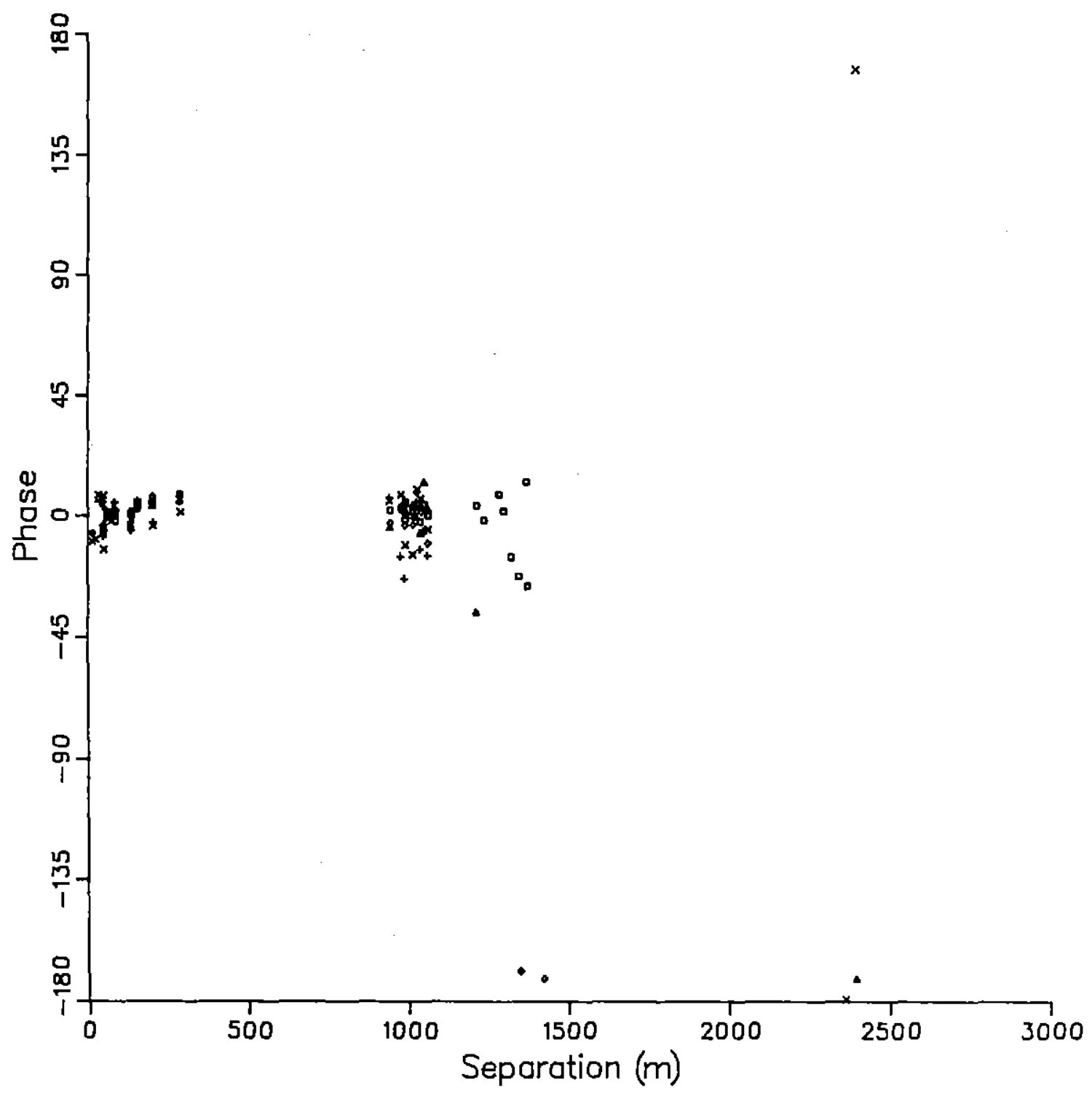

Figure 3.1.6a Phase difference from temperature as a function of WKBJ stretched vertical separation

The figure contains a scatterplot of vertical temperature phase difference as a function of WKBJ stretched separation for frequencies between $.1 \mathrm{cph}$ and $.5 \mathrm{cph}$. The data used are temperature measurements from the first year $Q$ mooring records. Each symbol corresponds to a different frequency bin, with the lowest frequency bin centered at .158 $\mathrm{cph}$ and a binwidth of $1 / 15 \mathrm{cph}$. The degree of averaging $N_{r}$ is 576 for each estimate, and only points with uncertainties less than $45^{\circ}$ are plotted. The points with phases of $\pm 180^{\circ}$ are inconsistent with the GM model. 


\section{Phase}

WKBJ stretched depths

U2T: Points with expected error less than 45 deg are plotted.

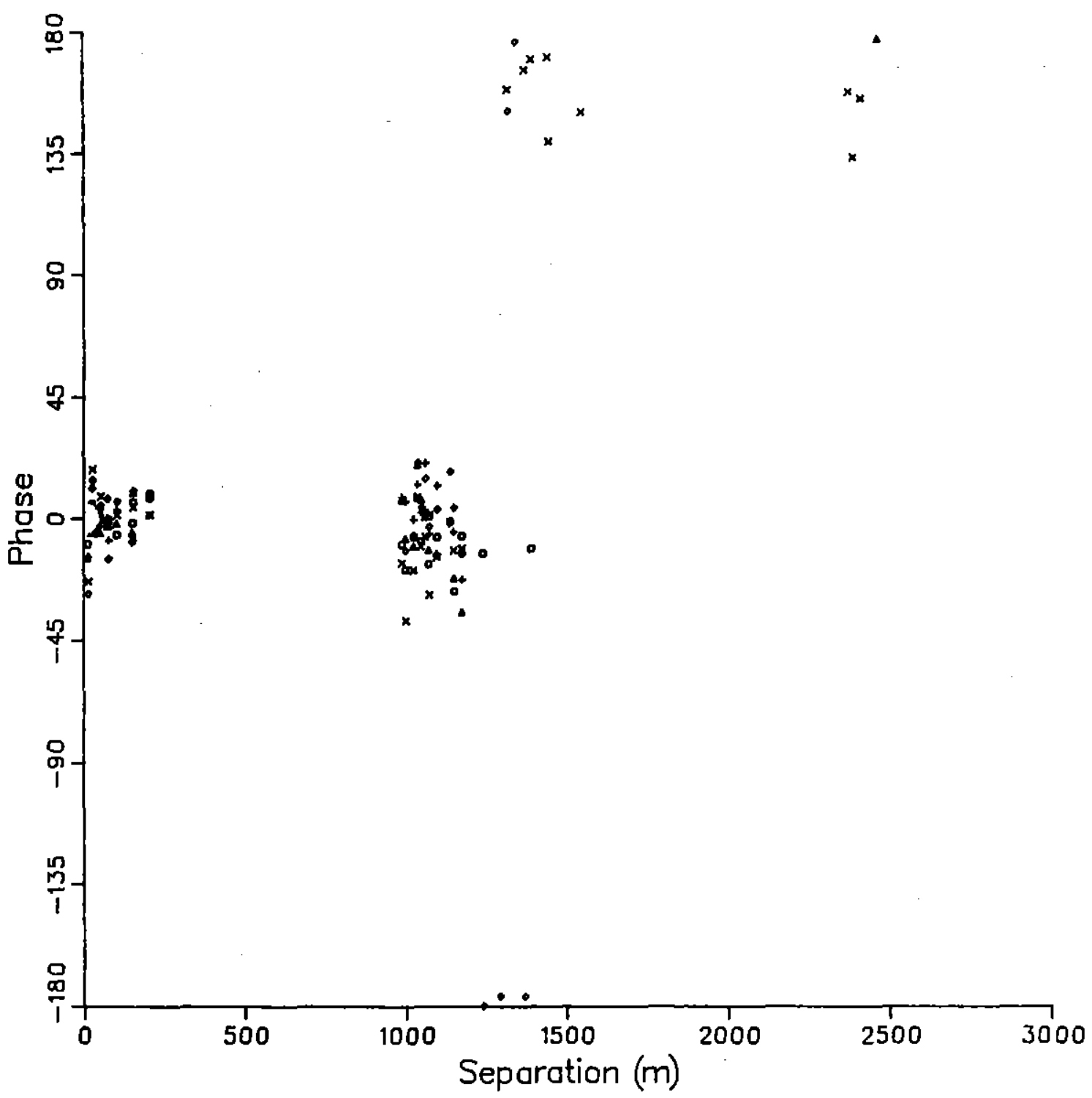

Figure 3.1.6b Phase difference from temperature as a function of WKBJ stretched vertical separation

The figure contains a scatterplot of vertical temperature phase difference as a function of WKBJ stretched separation for frequencies between $.1 \mathrm{cph}$ and $.5 \mathrm{cph}$. The data used are temperature measurements from the second year $U$ mooring records. Each symbol corresponds to a different frequency bin, with the lowest frequency bin centered at .158 cph and a binwidth of $1 / 15 \mathrm{cph}$. The degree of averaging $N_{\tau}$ is 576 for each estimate, and only points with uncertainties less than $45^{\circ}$ are plotted. The points with phases of $\pm 180^{\circ}$ are inconsistent with the $\mathrm{GM}$ model. 
Phase

WKBJ stretched depths

Q1U: Points with expected error less than 45 deg are plotted.

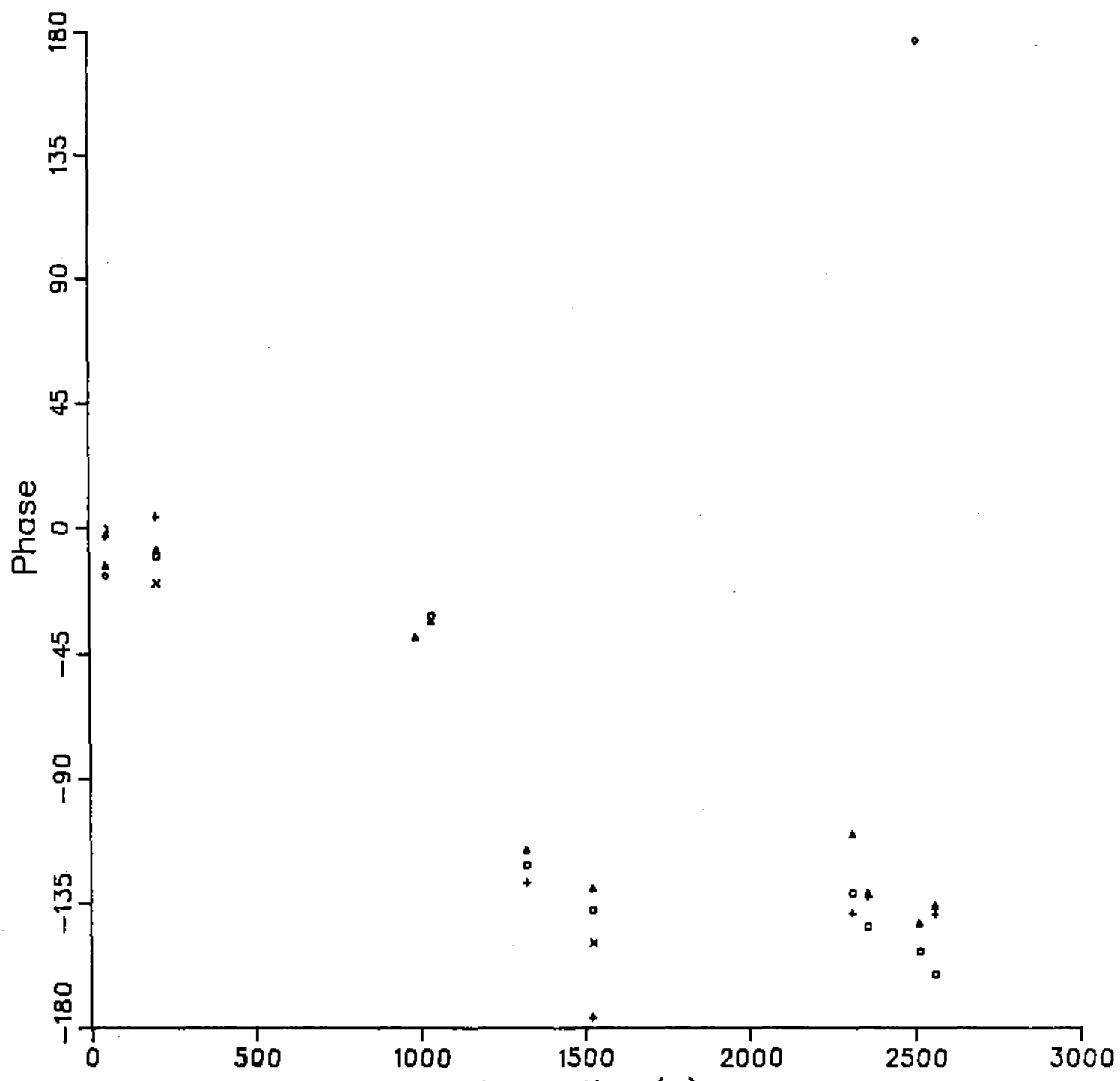

Separation $(m)$

Figure 3.1.7 $a$ Phase difference from zonal velocity as a function of WKBJ stretched vertical separation

The figure contains a scatterplot of zonal velocity phase difference as a function of WKBJ stretched vertical separation for frequencies between $.1 \mathrm{cph}$ and $.5 \mathrm{cph}$. The data used are from the first year $Q$ mooring records. Each symbol corresponds to a different frequency bin, with the lowest frequency bin centered at $.158 \mathrm{cph}$ and a binwidth of $1 / 15 \mathrm{cph}$. The degree of averaging $N_{r}$ is 576 for each estimate, and only points with uncertainties less than $45^{\circ}$ are plotted. Unlike the temperature records, even the $1000 \mathrm{~m}$ separations show $180^{\circ}$ phase differences. 


\section{Phase}

WKBJ stretched depths

U2U: Points with expected error less than 45 deg are plotted.

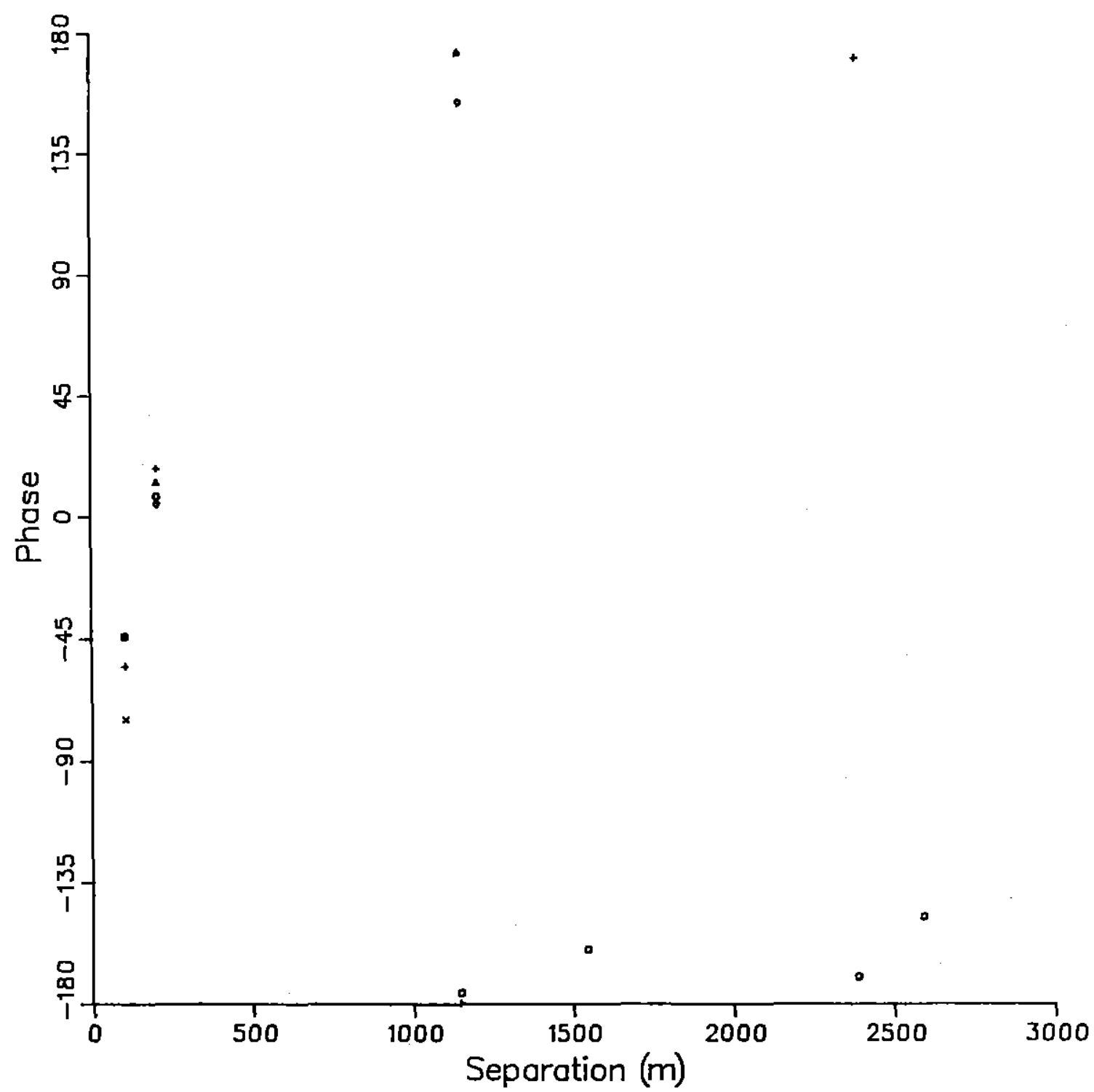

Figure 3.1.7b Phase difference from zonal velocity as a function of WKBJ stretched vertical separation

The figure contains a scatterplot of zonal velocity phase difference as a function of WKBJ stretched vertical separation for frequencies between $.1 \mathrm{cph}$ and $.5 \mathrm{cph}$. The data used are from the second year $U$ mooring records. Each symbol corresponds to a different frequency bin, with the lowest frequency bin centered at .158 cph and a binwidth of $1 / 15 \mathrm{cph}$. The degree of averaging $N_{r}$ is 576 for each estimate, and only points with uncertainties less than $45^{\circ}$ are plotted. Unlike the temperature records, even the $1000 \mathrm{~m}$ separations show $180^{\circ}$ phase differences. 
Phase

WKBJ stretched depths

Q1V: Points with expected error less than 45 deg are plotted.

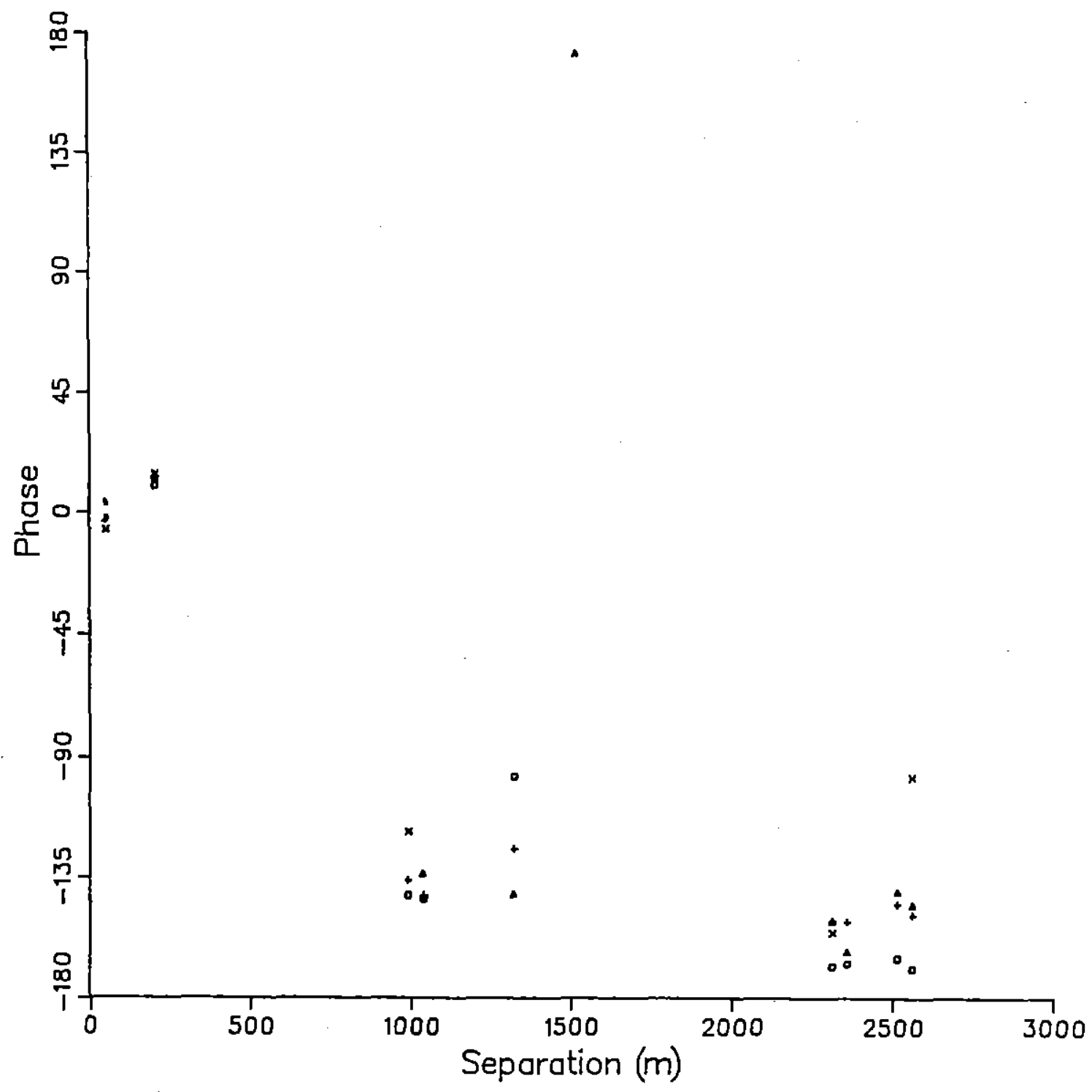

Figure 3.1.8 $a$ Phase difference from meridional velocity as a function of WKBJ stretched vertical separation

The figure contains a scatterplot of meridional velocity phase difference as a function of WKBJ stretched vertical separation for frequencies between $.1 \mathrm{cph}$ and $.5 \mathrm{cph}$. The data used are from the first year $Q$ mooring records. Each symbol corresponds to a different frequency bin, with the lowest frequency bin centered at $.158 \mathrm{cph}$ and a binwidth of $1 / 15 \mathrm{cph}$. The degree of averaging $N_{r}$ is 576 for each estimate, and only points with uncertainties less than $45^{\circ}$ are plotted. Unlike the temperature records, even the $1000 \mathrm{~m}$ separations show $180^{\circ}$ phase differences. 


\section{Phase}

WKBJ stretched depths

U2V: Points with expected error less than 45 deg are plotted.

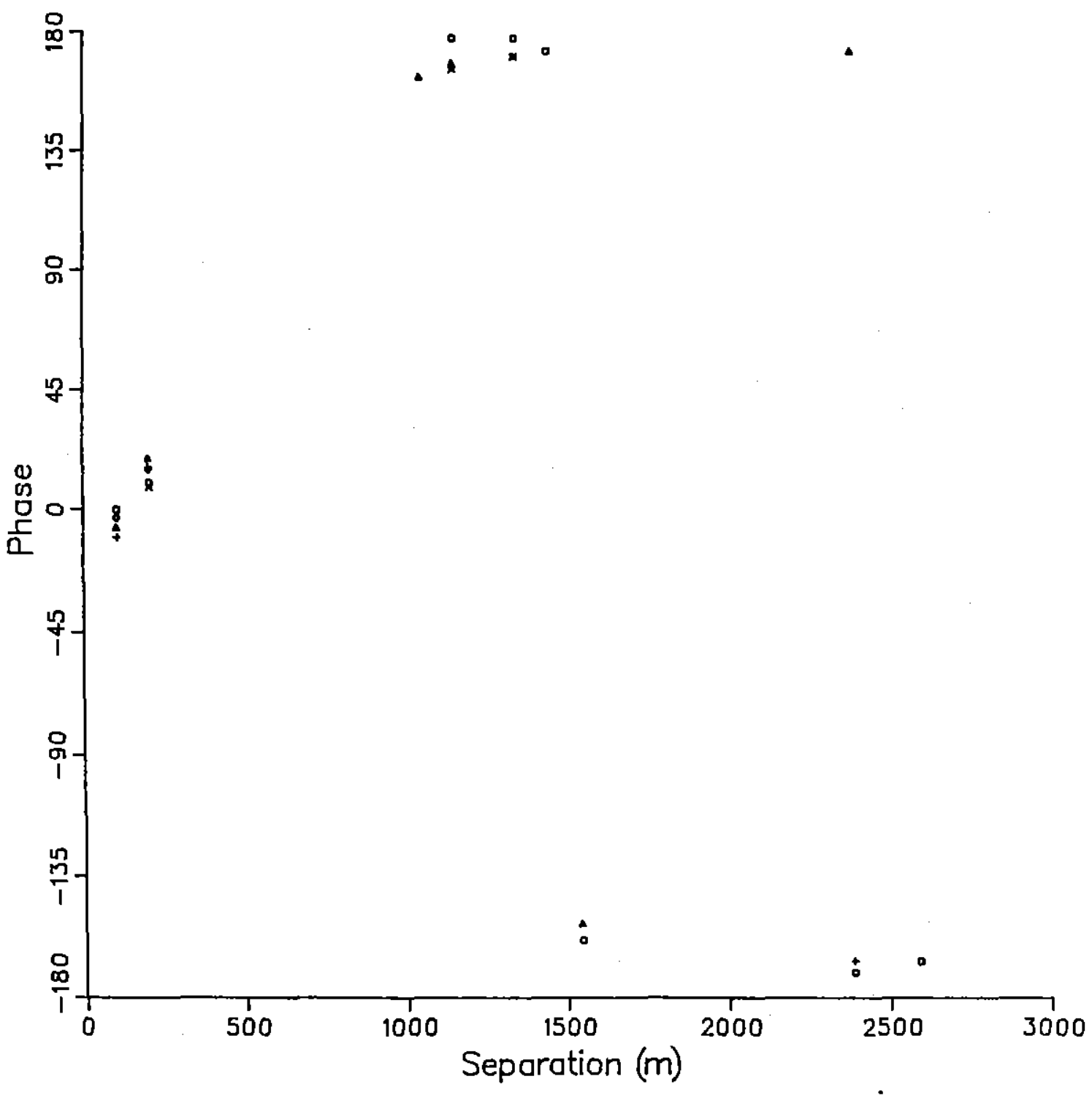

Figure 3.1.8b Phase difference from meridional velocity as a function of WKBJ stretched vertical separation

The figure contains a scatterplot of meridional velocity phase difference as a function of WKBJ stretched vertical separation for frequencies between $.1 \mathrm{cph}$ and $.5 \mathrm{cph}$. The data used are from the second year $U$ mooring records. Each symbol corresponds to a different frequency bin, with the lowest frequency bin centered at .158 cph and a binwidth of $1 / 15 \mathrm{cph}$. The degree of averaging $N_{r}$ is 576 for each estimate, and only points with uncertainties less than $45^{\circ}$ are plotted. Unlike the temperature records, even the $1000 \mathrm{~m}$ separations show $180^{\circ}$ phase differences. 
it would be quite surprising if the GM model would predict spectra accurately. The next section considers the modifications necessary to transform GM79 into a model that can adequately describe the PEQUOD data. Since the short separation behavior of PEQUOD is qualitatively the same as the data used in designing GM79, it would be better if any GM79 modifications were such that the short separation predictions of GM79 were unchanged. Then will the new model will be consistent with both the PEQUOD data and the data that GM79 summarizes.

\subsection{GM79 vertical coherence modifications}

The GM model spectra predict the MVC of velocity and temperature to be the same. Since that is not what is observed in the PEQUOD data, it is important to understand which features of the GM model lead to this prediction, and what the effect would be if these features were changed. While there are several mathematical perspectives to explain this result, all are manifestations of the claim that top and bottom boundary conditions are unimportant. This physical idea is a reasonable assumption under several circumstances. In particular, it is a good assumption when the vertical separations considered are small, and thus a good assumption for the data that Garrett and Munk consider (see Cairns and Williams 1976); it is not a good assumption for the PEQUOD array with its $1000 \mathrm{~m}$ and $2500 \mathrm{~m}$ separations.

The first step in discussing coherence is to consider the wave forms given in equation 1.3.4. The expressions for $u, v$, and $\eta$ are reproduced here,

$$
\begin{aligned}
{\left[\begin{array}{l}
u \\
v \\
\eta
\end{array}\right](x, y, z, t) } & =\left[\begin{array}{l}
\tilde{u} \\
\tilde{v} \\
\tilde{\eta}
\end{array}\right](\omega, k, l, z) e^{i(k x+l y-\omega t)} \\
& =\left[\begin{array}{c}
\left(i \cos \theta-\frac{f}{\omega} \sin \theta\right) \frac{1}{K} G_{z}(z) \\
\left(i \sin \theta+\frac{f}{\omega} \cos \theta\right) \frac{1}{K} G_{z}(z) \\
\frac{i}{\omega} G(z)
\end{array}\right] \widehat{A}(\omega, k, l) e^{i(k x+l y-\omega t)}
\end{aligned}
$$

The vertical structure function $G(z)$ depends on frequency $\omega$ and vector wavenumber $(k, l)$ as well as depth $z$. Consider cross-spectra for a purely vertical separation. At a particular frequency and horizontal wavenumber, the velocity cross-spectrum $\Phi_{12}^{u}$ and the displacement cross-spectrum $\Phi_{12}^{\eta}$ for the two depths $z_{1}$ and $z_{2}$ are given in terms of the 
spectral level $E=\left\langle A^{*} A\right\rangle$ by

$$
\begin{aligned}
\Phi_{12}^{u}(\omega, K) & =\left\langle\tilde{u}_{1}^{*} \tilde{u}_{2}+\tilde{v}_{1}^{*} \tilde{v}_{2}\right\rangle(\omega, K) \\
& =\left(1+\frac{f^{2}}{\omega^{2}}\right) \frac{1}{K^{2}} G_{z}^{*}\left(z_{1}\right) G_{z}\left(z_{2}\right) E(\omega, K)
\end{aligned}
$$

and

$$
\begin{aligned}
\Phi_{12}^{\eta}(\omega, K) & =\left\langle\tilde{\eta}_{1}^{*} \tilde{\eta}_{2}\right\rangle(\omega, K) \\
& =\frac{1}{\omega^{2}} G^{*}\left(z_{1}\right) G\left(z_{2}\right) E(\omega, K)
\end{aligned}
$$

where for simplicity the direction factors have been eliminated by summing the velocity cross-spectra. According to equation 1.5.2, the integral of these expressions over all wavenumber gives the cross-spectra $\Phi_{12}^{u}(\omega)$ and $\Phi_{12}^{\eta}(\omega)$ between two depths as measured at fixed points (i.e. moored current meters). The problem of calculating cross-spectra is thus reduced to finding an appropriate set of solutions $G(z ; \omega, K)$ and performing the integral over wavenumber.

Propagating WKBJ solutions for $G(z)$ were derived in chapter 1 . This choice is equivalent to the smearing of modes that Garrett and Munk did in their original paper (GM72). Combining the solutions given in equations $1.3 .6 a, b$ with the normalization given in equation 1.3.9c, gives expressions for the vertical structure of the upward and downward propagating waves $G_{ \pm}(z ; \omega, K)$

$$
G_{ \pm}(z)=\sqrt{\frac{\omega^{2}-f^{2}}{N(z)}} e^{ \pm i \int^{z} m\left(z^{\prime}\right) d z^{\prime}}
$$

where

$$
m(z)=\frac{N(z)}{\sqrt{\omega^{2}-f^{2}}} K
$$

and

$$
G_{z}(z)= \pm i m(z) G(z)
$$

Just as in figure 1.5.1, by defining a coordinate $\psi$ we can get quite simple expressions for the cross-spectra: $\psi$ is given by

$$
\psi_{12}=\int_{z_{1}}^{z_{2}} \frac{N\left(z^{\prime}\right)}{\sqrt{\omega^{2}-f^{2}}} d z^{\prime}
$$

and the cross-spectra for velocity and displacement are

$$
\Phi_{12}^{u}(\omega)=\left(1+\frac{f^{2}}{\omega^{2}}\right)\left(N\left(z_{1}\right) N\left(z_{2}\right)\right)^{1 / 2} \int_{0}^{\infty} e^{ \pm i K \psi_{12}} E(\omega, K) d K
$$


and

$$
\Phi_{12}^{\eta}(\omega)=\left(1-\frac{f^{2}}{\omega^{2}}\right)\left(N\left(z_{1}\right) N\left(z_{2}\right)\right)^{-1 / 2} \int_{0}^{\infty} e^{ \pm i K \psi_{12}} E(\omega, K) d K
$$

where the plus sign holds for upward propagating waves and the minus sign for downward propagating waves. These expressions for cross-spectra have identical dependence on the equivalent vertical coordinate $\psi$, thus the velocity and displacement coherences are identical,

$$
\gamma\left(z_{1}, z_{2}, \omega\right) e^{i \phi\left(z_{1}, z_{2}, \omega\right)}=E^{-1}(\omega) \int_{0}^{\infty} d K E(\omega, K) \begin{cases}e^{i K \psi_{12}} & \text { upward propagating } \\ \cos K \psi_{12} & \text { vertically symmetric }\end{cases}
$$

This is the result that is given in figure 1.5.1. In figure 1.6.1, these expressions are evaluated for the particular spectral form used in GM79: there the spectrum is vertically symmetric so that the coherence $\gamma\left(z_{1}, z_{2}, \omega\right)=C\left(K_{*} \psi_{12}\right)$ is a purely real exponential function of scaled separation, $C(X)=e^{-|X|}$.

By comparing equations $3.2 .2 a$ and 3.2.2b, one can see that the MVC calculated from velocities and the MVC calculated from displacement are the same only because the WKBJ vertically propagating solutions have the property that the ratio

$$
\frac{G_{z}^{*}\left(z_{1}\right) G_{z}\left(z_{2}\right)}{K^{2} G^{*}(z) G(z)}
$$

is independent of $K$. This would not be true if the solutions were not vertically propagating or if the solutions were not WKBJ approximations. Only if both of these conditions hold is the ratio between $G_{z}(z)$ and $K G(z)$ strictly independent of $K$.

Including top and bottom boundary conditions has two effects on the solutions $G(z)$ : the upward and downward propagating waves become phase-locked, and the horizontal wavenumber $K$ takes on discrete rather than continuous values for any particular frequency (these two effects are given explicitly in equations 1.3.7a,b). The WKBJ solution with the bottom boundary condition imposed is

$$
G(z)=\sqrt{\frac{\omega^{2}-f^{2}}{N(z)}} \sin K \psi_{D}(z)
$$

where $\psi_{D}(z)$ is given by

$$
\psi_{D}(z)=\int_{-D}^{z} \frac{N\left(z^{\prime}\right)}{\sqrt{\omega^{2}-f^{2}}} d z^{\prime}
$$


This expression insures that the $G(z)$ is zero at the bottom boundary. Imposing a upper boundary condition results in a discrete set of possible values of $K$ for a particular frequency. In particular, the rigid lid upper boundary condition is $G(0)=0$. This restricts $K \psi_{D}(0)$ to be a integral multiple of $\pi$,

$$
K \psi_{D}(0)=K \frac{D \bar{N}}{\sqrt{\omega^{2}-f^{2}}}=\pi j
$$

$\bar{N}$ is the depth averaged buoyancy frequency. This equation should be compared with equation 1.6.1c, which is the discretization that Garrett and Munk derive by considering solutions to a exponential profile. The condition that must be satisfied in order to have the two expressions identical is that $b N_{0}=D \bar{N}$, a condition that is satisfied in the PEQUOD area.

Consider the function $C(X)$ defined to be

$$
C(X)=\sum_{j=1}^{\infty} H\left(K_{j}\right) \cos \left(\frac{K_{i}}{K_{*}} X\right)
$$

where

$$
H\left(K_{j}\right)=\frac{E\left(\omega, K_{j}\right) d K_{j}}{E(\omega)}
$$

Comparing equations 3.2.12 and 3.2.8 reveals that $C\left(K_{*} \psi\right)$ differs from the coherence function for vertically propagating solutions in two ways: it is a sum rather than an integral, and the sum starts from mode 1 rather than wavenumber zero. Using the expression for $C(X)$ in place of the expression in equation 3.2.8 modifies the MVC such that it includes the discretization of wavenumber effect of imposing boundary conditions (the effects of phase locking are considered in the next section). To the extent that $H\left(K_{j}\right)$ is smooth, the sum is a close approximation to the integral, so that is not the important difference between the two equations. But the fact the the sum starts at 1 rather than zero is important, precisely because the spectral form chosen by Garrett and Munk has most of the energy in the lowest modes (over half the energy in the sum is in the first four baroclinic modes). Combining these two ideas means that as far as the discretization effect of boundary conditions is concerned, the imposition of boundary conditions could be modeled as a low wavenumber cutoff of a continuous spectrum. Figure 3.2.1 compares three versions of $C(X)$ that use the wavenumber form chosen in 
GM79: a direct evaluation of equation 3.2.12 using equation 1.6.1b (labeled 'sum'), and two continuous integral approximations for $C(X)$,

$$
C(X)= \begin{cases}e^{-|X|} & \text { 'continuous' } \\ \frac{e^{-|x|}-\epsilon}{1-\epsilon} & \text { 'cutoff' }\end{cases}
$$

where the 'continuous' version is the integral in equation 3.2.8 evaluated for $K=0$, and $\epsilon=.106$ is the contribution that the zero wavenumber mode makes to the integral. The plot shows that the sum and the cutoff integral give identical results, emphasizing the point that the only important effect of the discretization is the low wavenumber cutoff. The important distinction between the continuous and discrete cases is that while the first expression approaches 0 for large $X$, the second expression approaches -.1 . This limiting value of -.1 can be conceptualized as the signature of the missing zeroth mode. Were the zeroth mode present in the sum, the limiting value of this coherence would be zero. As discussed in section 1.4, this lack of coherence is due to the presence of many modes. On the other hand, were the zeroth mode present by itself, motions would be perfectly coherent for all depth pairs with no phase difference. Thus in the actual case, when then zeroth mode is absent but all the other modes are present, what is seen is the coherence structure that when added to the zeroth mode gives zero, i.e. a small amount of energy with $180^{\circ}$ phase differences at long separations.

There is one difference between the 'sum' and the 'cutoff' versions of $C(X)$ : since the sum is a fourier sum of cosines, it is periodic with a period that is determined by the lowest mode. In this case that period is twice the depth of the ocean $(8000 \mathrm{~m}$ for the functions plotted), so that between $4000 \mathrm{~m}$ and $8000 \mathrm{~m}$ separations, the sum version of $C(X)$ will show a dependence that is a simple reflection of its behavior between 0 and $4000 \mathrm{~m}$. While $C(X)$ is not evaluated for such large separations in this section, in the next section it will be shown that the phase locking effects can be expressed in terms of $C(X)$ evaluated at the sum of the two instrument depths. Thus the fact that the $4000 \mathrm{~m}$ to $8000 \mathrm{~m}$ behavior of $C(X)$ is a simple reflection of its 0 to $4000 \mathrm{~m}$ behavior insures that top boundary effects will be felt as well as bottom boundary effects. Therefore above separations of $4000 \mathrm{~m}$, the cutoff approximation to $C(X)$ should mirror the 0 to $4000 \mathrm{~m}$ behavior.

A modified $C(X)$ function alone, however, cannot explain why velocity and displacement show different coherence structures. In order to model that difference, the effects of phase-locking must be included. 


\section{$C\left(m_{*} z\right)$ approximations}

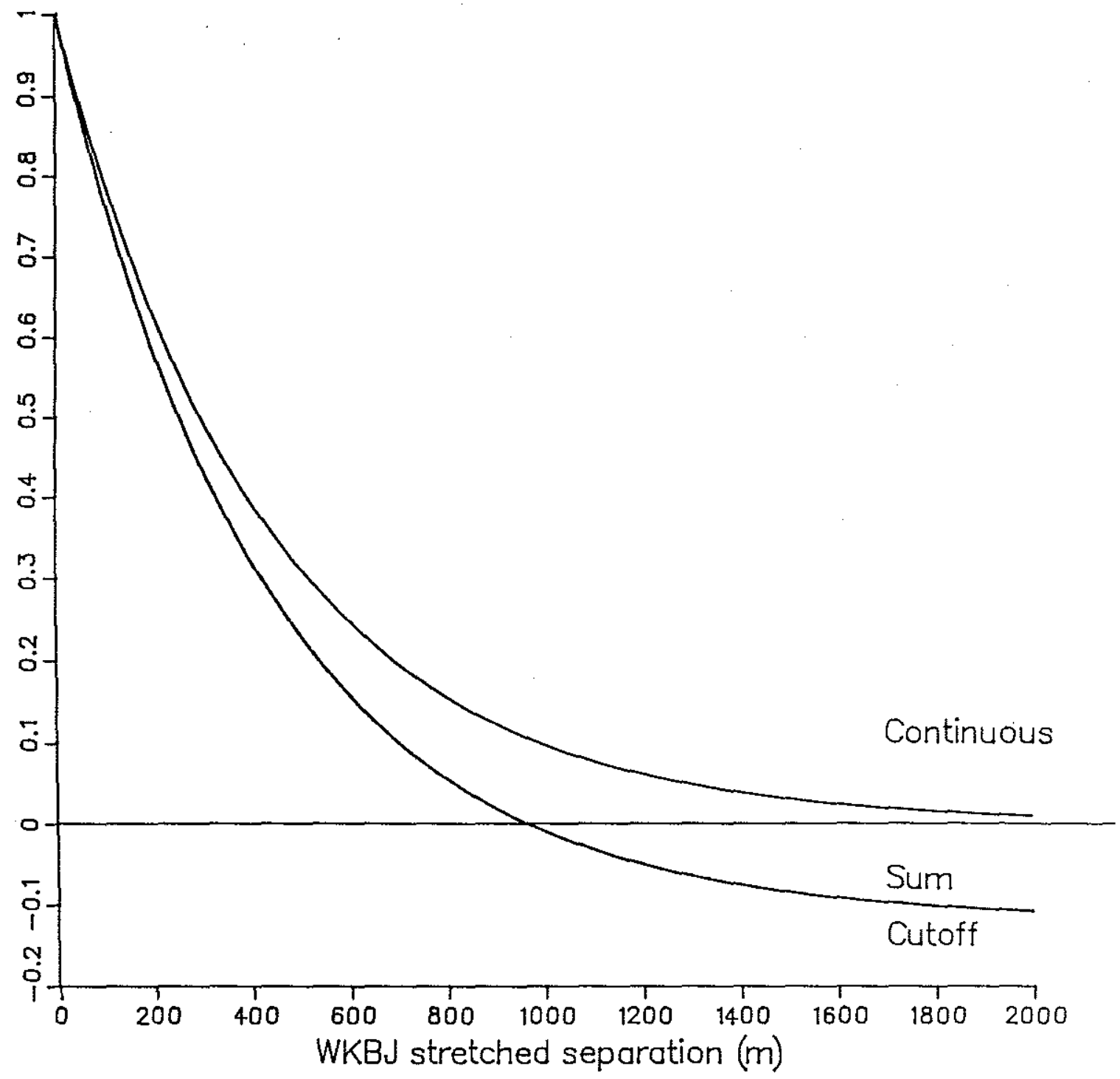

Figure 3.2.1 Vertical coherence function comparisons

This figure compares three approximations to the function $C(X)$, a function which gives the vertical coherence for an internal wave field that is purely vertically propagating. The functions are plotted against WKBJ stretched separation $\zeta$, which is proportional to the equivalent vertical coordinate $\psi$ as given in equation 3.1.2,

$$
m_{*} \zeta=K_{*} \psi \text {. }
$$

In this plot $m_{*}$ is $2.33 \times 10^{-3} \mathrm{~m}^{-1}$. The plot shows that the continuous spectrum with a low wavenumber cutoff is indistinguishable from the discrete sum of modes. 
As it turns out, the effects of phase-locking can be fairly simply expressed in terms of the function $C(X)$. This allows a fairly direct comparison of the cross-spectra from the WKBJ propagating wave model with the cross-spectra from the WKBJ modal model. The velocity and displacement cross-spectra using WKBJ modal solutions are

$$
\Phi_{12}^{u}(\omega)=\left(1+\frac{f^{2}}{\omega^{2}}\right)\left[N\left(z_{1}\right) N\left(z_{2}\right)\right]^{1 / 2} \sum_{j=1}^{\infty} \cos \left(K_{j} \psi_{D 1}\right) \cos \left(K_{j} \psi_{D 2}\right) E\left(\omega, K_{j}\right)
$$

and

$$
\Phi_{12}^{\eta}(\omega)=\left(1-\frac{f^{2}}{\omega^{2}}\right)\left[N\left(z_{1}\right) N\left(z_{2}\right)\right]^{-1 / 2} \sum_{j=1}^{\infty} \sin \left(K \psi_{D 1}\right) \sin \left(K \psi_{D 2}\right) E(\omega, K)
$$

These two equations can be rewritten in terms of the function $C(X)$ by using trigonometric identities as

$$
\Phi_{12}^{u}(\omega)=\left(1+\frac{f^{2}}{\omega^{2}}\right)\left[N\left(z_{1}\right) N\left(z_{2}\right)\right]^{1 / 2}\left(C\left[K_{*} \psi_{12}\right]+C\left[K_{*}\left(\psi_{D 1}+\psi_{D 2}\right)\right]\right) E(\omega)
$$

and

$$
\Phi_{12}^{\eta}(\omega)=\left(1-\frac{f^{2}}{\omega^{2}}\right)\left[N\left(z_{1}\right) N\left(z_{2}\right)\right]^{-1 / 2}\left(C\left[K_{*} \psi_{12}\right]-C\left[K_{*}\left(\psi_{D 1}+\psi_{D 2}\right)\right]\right) E(\omega)
$$

Note that the effect of the phase-locking is to add an extra term to the purely propagating solution, an extra term that is a function of the average scaled depth $\psi_{a v}$ of the two points being considered. This extra term means that the wave field is no longer vertically homogeneous, and the term is responsible for the differences between velocity and displacement cross-spectra. As long as the two depths $z_{1}$ and $z_{2}$ being considered are sufficiently far from the boundaries (according to figure 3.2.1, sufficiently far is such that the sum of their WKBJ stretched separations from a boundary is greater than $1500 \mathrm{~m}$ and less than $6500 \mathrm{~m}$ ), the added term will be the limiting value of $C(X)$ for large argument. That limiting value depends on the choice of function $C(X)$. Choosing the expression in equation 3.2.14 for $C(X)$ (an expression that includes the zeroth mode) means that the limiting value for large argument is zero and the expressions for velocity and displacement cross-spectra are the same. On the other hand, choosing the second expression (which excludes the zeroth mode) means that the limiting value is -.1 and there is a difference between velocity and displacement cross-spectra. Thus both the effects of phase-locking 
and of the missing zeroth mode are required before there will be differences between the velocity coherence and the temperature coherence.

GM along with the above two modifications predicts that for very long separations the velocity cross-spectra is .2 with a phase difference of $180^{\circ}$, while the displacement cross-spectra goes to zero. In terms of WKBJ stretched depth, the crossover point where the velocity phase difference goes from zero (for short separations) to $180^{\circ}$ (for long separations) occurs at $800 \mathrm{~m}$, while the temperature phase difference is always zero. This phase information matches the simple characterizations of the phase differences from temperature and velocity observed in PEQUOD. The model also predicts that the displacement cross-spectra at $800 \mathrm{~m}$ WKBJ stretched depth should be twice what the vertically propagating model predicts, which also is consistent with observations at $1000 \mathrm{~m}$ WKBJ stretched depth. Furthermore, the prediction says that the coherences at a WKBJ stretched separation of $1500 \mathrm{~m}$ are closer to the coherences at a WKBJ stretched separation of $2500 \mathrm{~m}$ than the coherences at a WKBJ stretched separation of $1000 \mathrm{~m}$, something also seen in the observations. Finally, these model predictions are such that the predicted coherences at short separations are the same as the GM79 predictions, so that all the data used in designing GM79 is also consistent with the modified model.

\subsection{Coherence and phase from LOTUS}

In order to see whether these modal effects can be seen in mid-latitude data, Dr. Briscoe has kindly provided coherences calculated from four of the second year Long Term Upper Ocean Study (LOTUS) current meter records. Details of the mooring structure are given in a LOTUS technical report (Tarbell et al. 1985). The instruments used are located at $1000 \mathrm{~m}, 1500 \mathrm{~m}, 2500 \mathrm{~m}$ and $4000 \mathrm{~m}$ depth. The mooring is in $5200 \mathrm{~m}$ of water, thus all the instruments are well away from the boundaries. The top $(1000 \mathrm{~m})$ instrument is in the bottom part of the thermocline, while the other instruments are well away from any rapid changes in the buoyancy frequency. It turns out that all the coherences between the $1000 \mathrm{~m}$ instrument and the other instruments are very low, not at all consistent with the PEQUOD observations. This lack of coherence is probably due to thermocline effects which are not included in the model of modal effects, thus the $1000 \mathrm{~m}$ record is omitted from the remaining comparisons.

Figure 3.3.1 gives the local buoyancy frequency and the WKBJ stretched separations for the LOTUS instruments used. Omitting the $1000 \mathrm{~m}$ instrument leaves estimates at 
LOTUS WKBJ stretched separations

\begin{tabular}{lrrrrrr} 
Depth & $\bar{N}$ & WKBJ depth & \multicolumn{4}{c}{ WKBJ Separations } \\
& $\mathrm{cph}$ & & $1000 \mathrm{~m}$ & $1500 \mathrm{~m}$ & $2500 \mathrm{~m}$ & $4000 \mathrm{~m}$ \\
$1000 \mathrm{~m}$ & 1.384 & 1000 & & & & \\
$1500 \mathrm{~m}$ & .674 & 1692 & 692 & & & \\
$2500 \mathrm{~m}$ & .576 & 2366 & 1366 & 674 & & \\
$4000 \mathrm{~m}$ & & 3230 & 2230 & 1538 & 864 &
\end{tabular}

\section{Frequency grid}

$\begin{array}{cl}\text { Symbol } & \text { Frequency } \\ \square & .1700 \mathrm{cph} \\ \triangle & .2367 \mathrm{cph} \\ + & .3033 \mathrm{cph} \\ \times & .3700 \mathrm{cph} \\ \diamond & .4367 \mathrm{cph}\end{array}$

Figure 3.3.1 Frequency grid and WKBJ stretched separations for LOTUS instruments

$674 \mathrm{~m}, 864 \mathrm{~m}$, and $1538 \mathrm{~m}$ WKBJ stretched separations, smaller separations than are available from the PEQUOD mooring. This points out one of the difficulties with performing long separation coherence calculations with mid-latitude records: the much deeper thermocline and the smaller deep water buoyancy frequency combine to limit the longest WKBJ stretched separation to be distinctly shorter than in the equatorial Pacific. But the LOTUS data does provide an interesting contrast to the PEQUOD data.

Figure 3.3.2 shows the temperature coherences for the three deep instruments. The temperature records are for the most part incoherent, and the significant coherences that exist are tied to the local buoyancy frequency. For example, the $2500 \mathrm{~m}$ and $4000 \mathrm{~m}$ records are coherent for most of the frequencies between .1 and $1 \mathrm{cph}$. This fairly broad peak is probably associated with frequencies approaching the local buoyancy frequency. All the nearest neighbor pairs show some sort of peak near the local buoyancy frequency, though none of the others are as broad as the peak in the $2500 \mathrm{~m}-4000 \mathrm{~m}$ case. Essentially the temperature coherences are not conclusive: they are consistent with either a modal or propagating internal wave model.

The velocity coherences, on the other hand, do show features similar to what was found in the PEQUOD data. Both zonal and meridional velocities (zonal velocity is shown) have a coherence of .2 with a $180^{\circ}$ phase difference for long separations in the .1 to $.5 \mathrm{cph}$ frequency band. There are no bumps at local buoyancy frequencies, though there are bumps of significant coherence at frequencies above the buoyancy frequency. The 


\section{Coherence}

WKBJ stretched depth

L234T: frequericies between $.11 \mathrm{cph}$ and $.5 \mathrm{cph}$

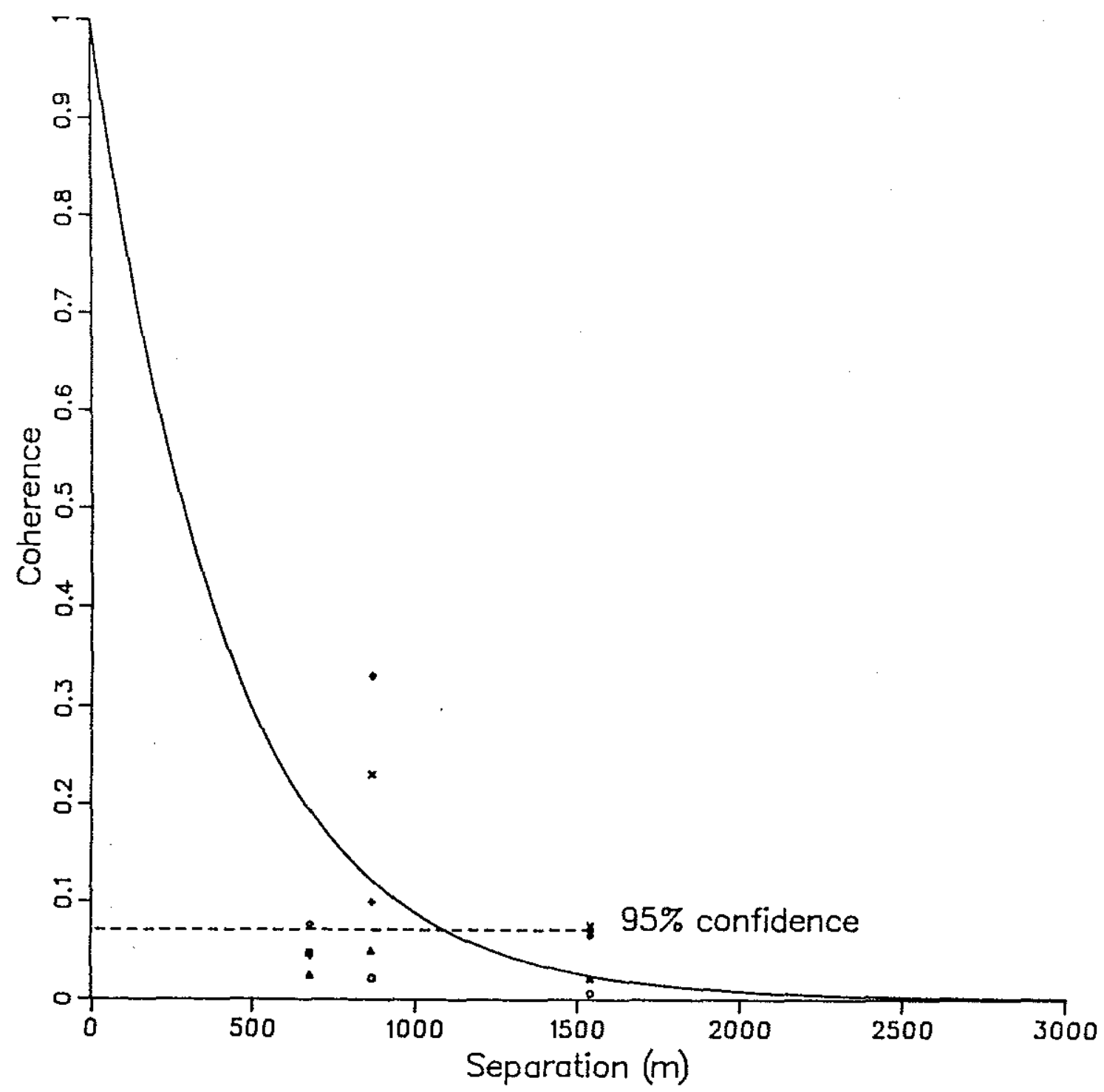

Figure 3.3.2 $a$ Vertical coherence calculated from LOTUS temperature data as a function of WKBJ stretched separation

The figure contains a scatterplot of vertical coherence as a function of WKBJ stretched separation calculated from temperature data for frequencies between $.1 \mathrm{cph}$ and $.5 \mathrm{cph}$. Each symbol corresponds to a different frequency bin, with the lowest frequency bin centered at $.170 \mathrm{cph}$ with a binwidth of $2 / 15 \mathrm{cph}$. 


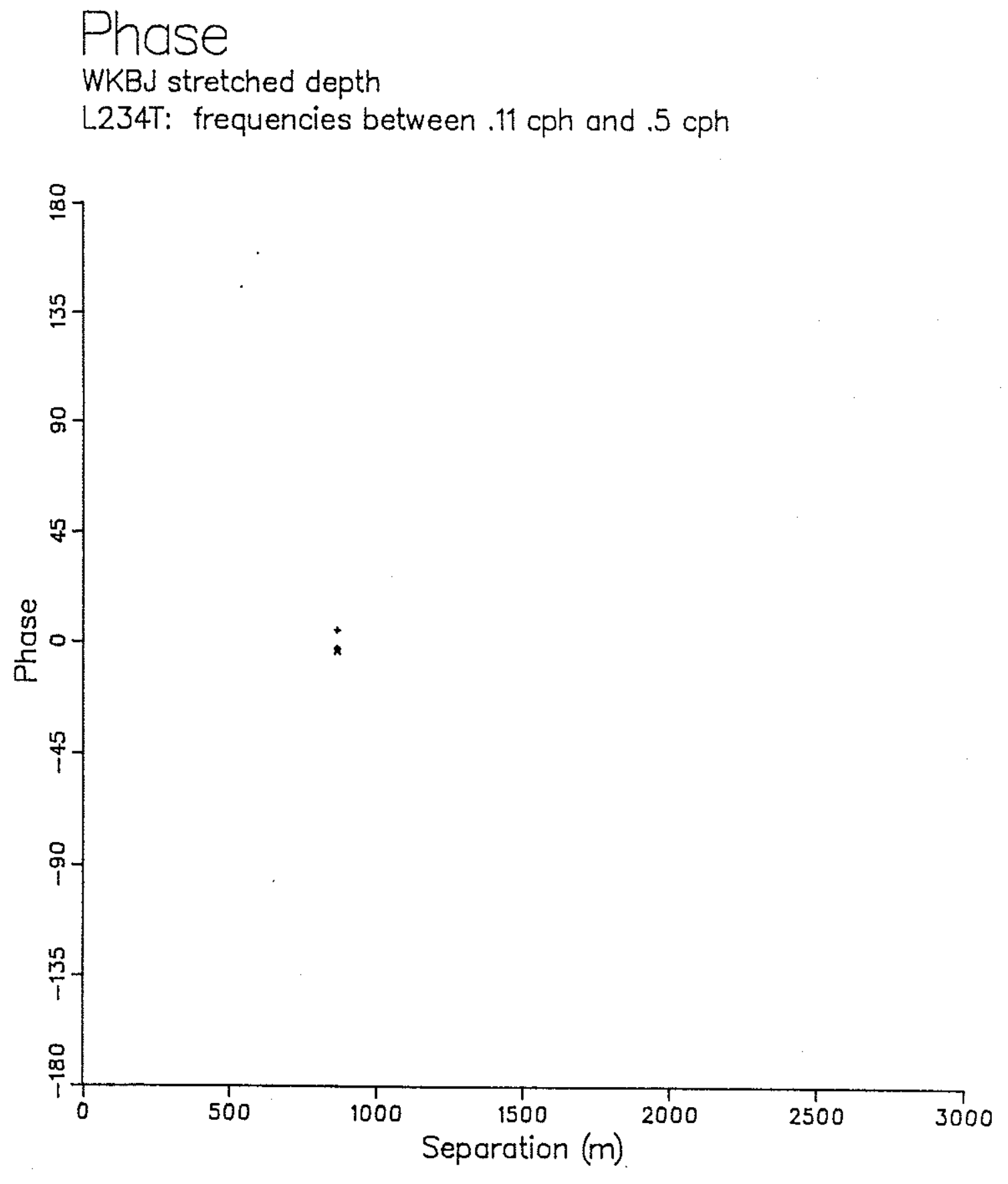

Figure 3.3.2 $b$ Vertical phase calculated from LOTUS temperature data as a function of WKBJ stretched separation

The figure contains a scatterplot of vertical phase as a function of WKBJ stretched separation calculated from temperature data for frequencies between $.1 \mathrm{cph}$ and $.5 \mathrm{cph}$. Each symbol corresponds to a different frequency bin, with the lowest frequency bin centered at $.170 \mathrm{cph}$ with a binwidth of $2 / 15 \mathrm{cph}$. 


\section{Coherence}

WKBJ stretched depth

L234U: frequencies between . $11 \mathrm{cph}$ and $.5 \mathrm{cph}$

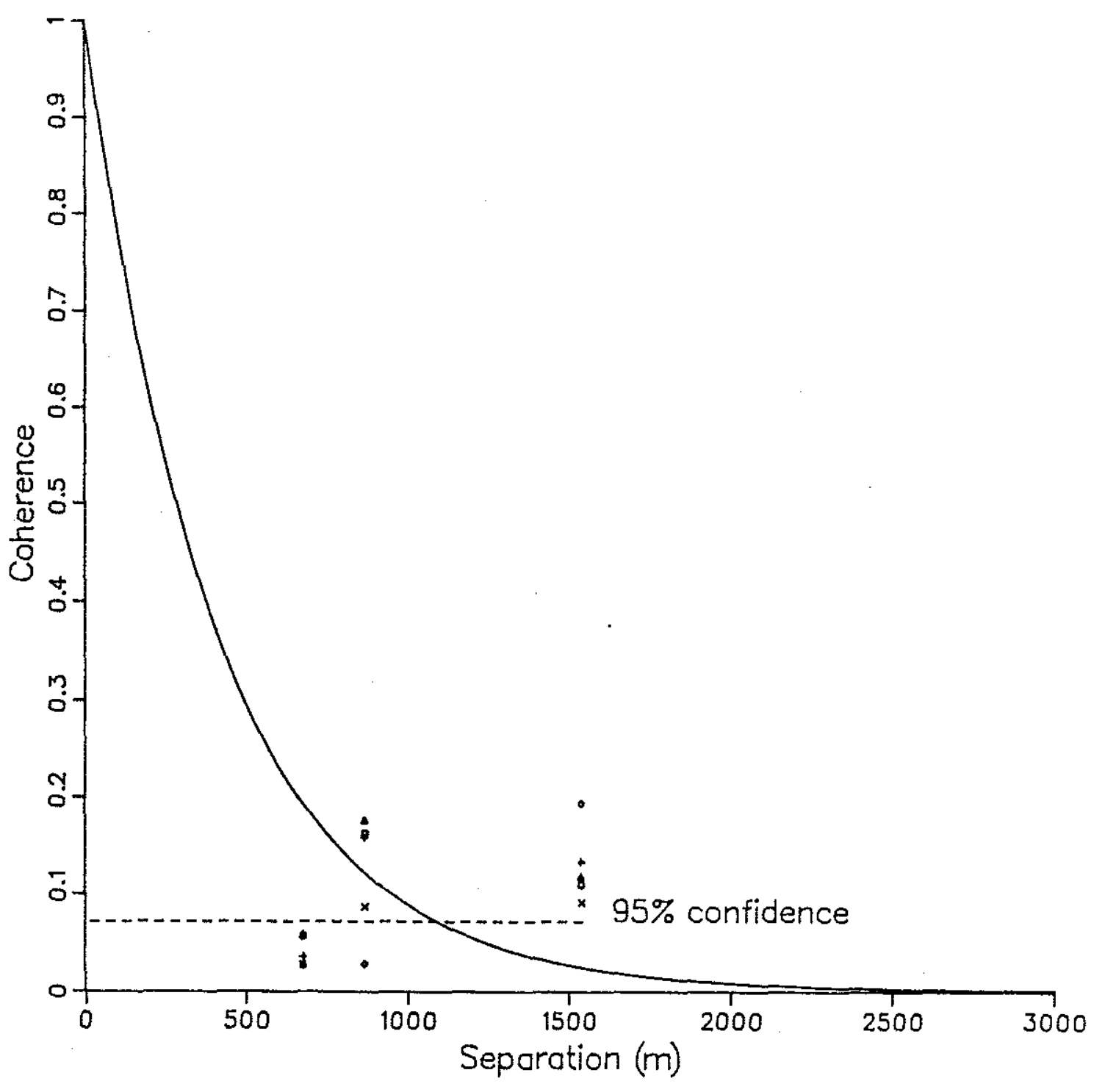

Figure 3.3.3 $a$ Vertical coherence calculated from LOTUS zonal velocity data as a function of WKBJ stretched separation

The figure contains a scatterplot of vertical coherence as a function of WKBJ stretched separation calculated from zonal velocity data for frequencies between $.1 \mathrm{cph}$ and $.5 \mathrm{cph}$. Each symbol corresponds to a different frequency bin, with the lowest frequency bin centered at $.170 \mathrm{cph}$ with a binwidth of $2 / 15 \mathrm{cph}$. 


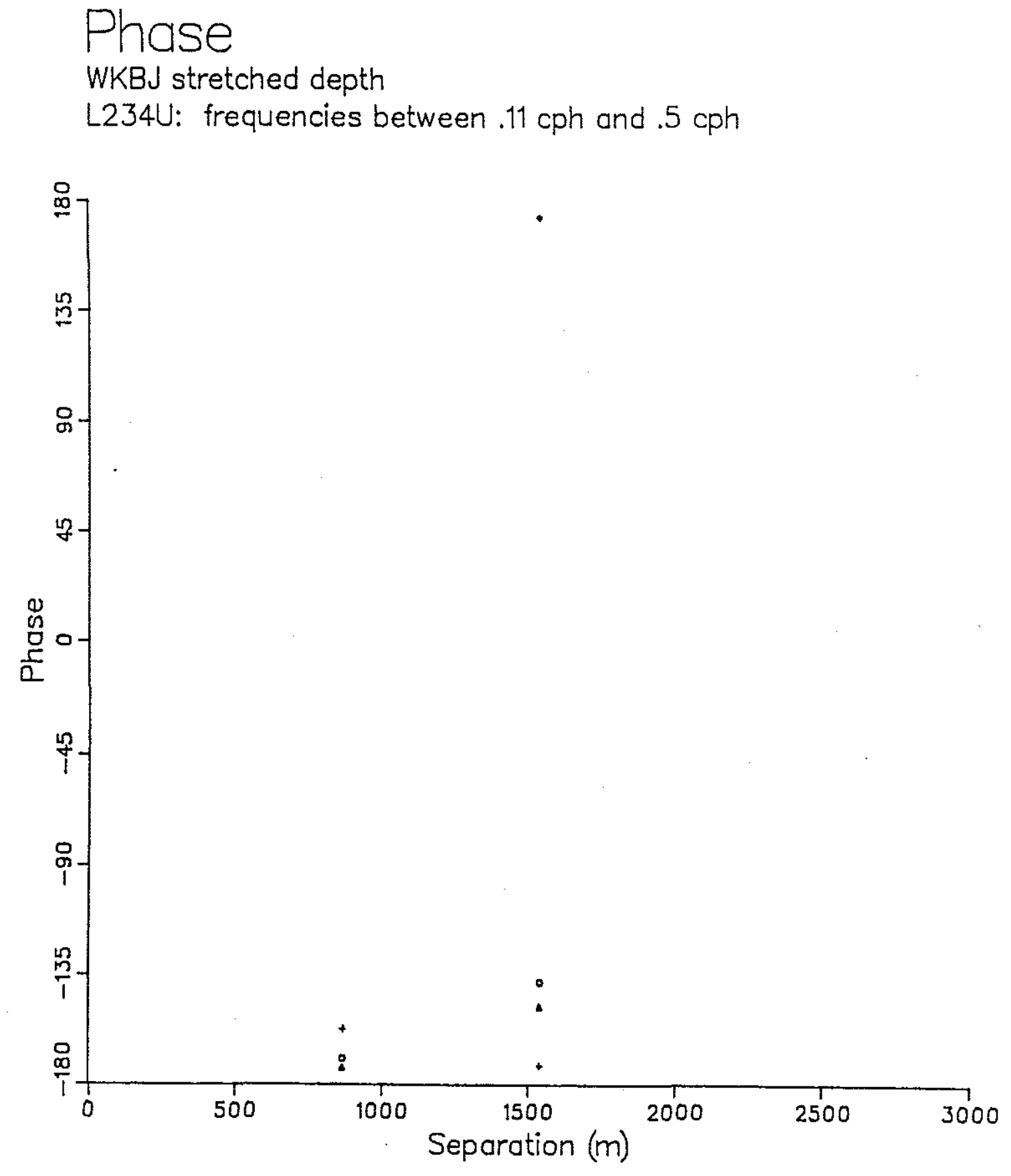

Figure 3.3.3 $b$ Vertical phase calculated from LOTUS zonal velocity data as a function of WKBJ stretched separation

The figure contains a scatterplot of vertical coherence as a function of WKBJ stretched separation calculated from zonal velocity data for frequencies between $.1 \mathrm{cph}$ and $.5 \mathrm{cph}$. Each symbol corresponds to a different frequency bin, with the lowest frequency bin centered at $.170 \mathrm{cph}$ with a binwidth of $2 / 15 \mathrm{cph}$. 
coherence vs. frequency plots show that the $4000 \mathrm{~m}$ records have significant coherences starting at frequencies below $.1 \mathrm{cph}$ and continuing throughout the internal wave band with both $1500 \mathrm{~m}$ and $2500 \mathrm{~m}$ records. These coherences correspond to phase differences of $180^{\circ}$. The $1500 \mathrm{~m}$ velocity records, on the other hand, are not coherent with the $2500 \mathrm{~m}$ records except at frequencies near the local buoyancy frequency. This is similar to the features in the PEQUOD data, except that it requires a model with a phase crossover closer to $700 \mathrm{~m}$ WKBJ stretched separation than the $900 \mathrm{~m}$ found with the PEQUOD data.

Thus the LOTUS velocity data is roughly consistent with low wavenumber energy being modal in character. The temperature data is not so clear, mainly because the deep water buoyancy frequency is so low $(.6 \mathrm{cph})$ that it is hard to separate the effects seen in the PEQUOD data from the near-buoyancy frequency effects (Desaubies 1975) discussed in the next section.

\subsection{Summary of vertical coherence results}

The coherence and phase as a function of vertical separation computed from PEQUOD data show distinct differences from the GM predictions. The GM prediction using vertically propagating waves is that coherence should be an exponential function of WKBJ stretched separation, and the phase difference should be zero for all separations. This is modified somewhat by the presence of finestructure contamination of moored sensor measurements. The short separation coherences are similar to what is expected from the GM internal wave spectrum and finestructure, but it is fairly clear that these coherences do not depend simply on separation. Coherences and phases at separations of $1000 \mathrm{~m}$ and longer are also different from the GM predictions. Temperature coherence is $.2 \pm .08$ at $1000 \mathrm{~m}$ separation, significantly higher than the GM prediction, but it drops off quickly so that by $1500 \mathrm{~m}$ separations the coherence is indistinguishable from zero. In contrast, velocity coherence is indistinguishable from zero at $1000 \mathrm{~m}$ separation, but increases for longer separations so that it is roughly .2 at $2500 \mathrm{~m}$ separations. Temperature phase can be roughly characterized as zero for all separations by ignoring some of the phases that correspond to low coherences, while velocity phase is roughly characterized as $180^{\circ}$ for all separations greater than $1000 \mathrm{~m}$. These characterizations can be understood in terms of a modified GM model which includes two effects that are a result of imposing top and bottom boundaries: phase-locking between upward and downward propagating waves to form modes, and a low wavenumber cutoff such that there is little energy below the 
first baroclinic mode. The data thus provides clear evidence of low wavenumber cutoff, phase-locked behavior at frequencies throughout the supertidal to the subbuoyancy frequency range, the frequency range in which one would expect to see primarily internal wave motions.

This is not the first experimental evidence that there is more modal character in the internal wave field than is included in the GM model. Pinkel 1975 made upper ocean observations from FLIP which indicate that there is phase-locking of upward and downward propagating waves as the frequency considered approaches the local buoyancy frequency. Peters 1980 and Peters 1983 look at the upper ocean internal wave field as seen in the JASIN and GATE experiments respectively. He finds differences from GM which are then modeled in in terms of shear modes, that is, modes where the effects of a mean velocity shear are included. In fact, there is much experimental evidence (see Olbers 1983 and Levine 1985 for reviews that include discussions) that the upper ocean internal wave field is quite variable and not well modeled by a stationary, universal model. But observations of modal effects in the deep ocean internal wave field are not so numerous. Desaubies 1975 predicts that there should be phase-locking as the frequency approaches the local buoyancy frequency, and by using Airy function solutions in the region where the two frequencies become equal, constructs predictions of enhanced spectra and coherence. He notes that the IWEX data shows features that support his results. But the PEQUOD results differ from these results in several respects. Most importantly, the measurements are from sensors in the deep ocean, and the coherences are over separations which are a sizable fraction of the ocean depth. Furthermore, the coherences show a modal character that is uniform for a large part of the internal wave band, unlike the earlier results where the modal character is confined to frequencies near the local buoyancy frequency. These considerations suggest that the model that has modal effects due to boundaries is more appropriate for the PEQUOD data.

Determining the low wavenumber cutoff and, more generally, determining the low wavenumber distribution of energy are important because the low modes contain most of the internal wave energy. Such an effect is even more pronounced when considering energy fluxes, because not only are the low modes more energetic, they are also faster, thus making proportionally an even stronger contribution to the energy flux. Thus it is important to see whether the data can be used to determine such a cutoff. The GM modifications discussed so far have only been compared to the data in a qualitative sense: 
no real attempt has been made to quantitatively allocate energy between a model spectrum and noise to get a full accounting of the energy measured in the data. Furthermore, the model is vertically symmetric and horizontally isotropic: such a model has net energy fluxes that are identically zero. In the next section, PEQUOD records are examined for evidence of vertical asymmetry and horizontal anisotropy. Later chapters do a quantitative comparison, extracting the low mode information and deriving estimates of fluxes carried by the internal wave field. 


\section{Chapter 4: Single current meter data interpretation}

\subsection{Introduction}

As a first comparison of data with an internal wave model, this chapter compares data from single current meters with the Garrett and Munk internal wave spectrum (GM79). While this comparison is superseded by the more elaborate calculations done in chapters 6 and 8 (which use all the available cross-spectra), the calculation is important in that it shows that relatively simple analyses show many of the same anomalies. Confining the information to that which can be obtained from a single current meter means that the data consists of the autospectra of and the cross-spectra between east velocity $(u)$, north velocity $(v)$ and temperature measured at a single depth. Using only a subset of the available cross-spectra greatly simplifies the comparison without destroying its utility: if there is a significant difference between the data and the model then the model needs to be amended.

The model-data comparison is done in several steps. First the dynamics of internal waves in a resting basic state are examined, specializing the results of section 1.5 to the spectra measurable from a single current meter at the equator. The next section extrapolates the GM79 data fit given in section 1.1 to the equator, thus determining the equatorial expected spectral level. As a result, the spectral covariance can be calculated: the covariance exists both because variance is a fundamental property of spectral estimators and because of nonlinearities in the equations of motion. Finally, the comparison between data and model is made. This chapter concludes that the PEQUOD data is actually significantly different from the GM model, and explores some model modifications and their respective abilities to account for the data-GM79 differences. The modifications include additional dynamical mechanisms as well as relaxing assumptions such as horizontal isotropy and (to a lesser extent) vertical symmetry, assumptions that have little theoretical basis but are consistent with the data GM79 summarizes. 


\subsection{Dynamical model}

The variables measured by a single current meter are zonal velocity $u$, meridional velocity $v$, and temperature fluctuations $T$. Thus a nine-component cross-spectral matrix can be calculated from single current meter data: three real autospectra $\left\langle u^{*} u\right\rangle(\omega),\left\langle v^{*} v\right\rangle(\omega)$, $\left\langle T^{*} T\right\rangle(\omega)$, and three complex cross-spectra $\left\langle u^{*} v\right\rangle(\omega),\left\langle u^{*} T\right\rangle(\omega),\left\langle v^{*} T\right\rangle(\omega)$. Linear internal wave theory interrelates these nine components. Specializing the results given in figure 1.5.1 to the equator by setting $f$ to zero, the single current meter spectra can be written in terms of the spectral level $E(\omega)$

$$
\left[\begin{array}{ccc}
\tilde{u}^{*} \tilde{u} & \tilde{u}^{*} \tilde{v} & \tilde{u}^{*} \tilde{T} \\
\tilde{v}^{*} \tilde{u} & \tilde{v}^{*} \tilde{v} & \tilde{v}^{*} \tilde{T} \\
\tilde{T}^{*} \tilde{u} & \tilde{T}^{*} \tilde{v} & \tilde{T}^{*} \tilde{T}
\end{array}\right]=\left[\begin{array}{ccc}
\frac{1}{2} N & 0 & 0 \\
0 & \frac{1}{2} N & 0 \\
0 & 0 & \frac{1}{N T_{z}^{2}}
\end{array}\right] E(\omega)
$$

A linear relation has been used to go from displacement $(\eta)$ to temperature fluctuation $(T)$. This result assumes that the internal wave field is horizontally isotropic, vertically symmetric, and WKBJ vertically propagating (see section 1.4 ). The result also assumes that the waves are hydrostatic. The single current meter cross-spectra can also be obtained from the general internal wave models of chapter 5. In particular, the constant $N$ results of section 5.9 can be modified to be valid for WKBJ solutions $\alpha$ to the vertical structure equation, giving the non-hydrostatic results:

where

$$
\left[\begin{array}{ccc}
\tilde{u}^{*} \tilde{u} & \tilde{u}^{*} \tilde{v} & \tilde{u}^{*} \tilde{T} \\
\tilde{v}^{*} \tilde{u} & \tilde{v}^{*} \tilde{v} & \tilde{v}^{*} \tilde{T} \\
\tilde{T}^{*} \tilde{u} & \tilde{T}^{*} \tilde{v} & \tilde{T}^{*} \tilde{T}
\end{array}\right]=\left[\begin{array}{ccc}
\frac{1}{2} \lambda N & 0 & 0 \\
0 & \frac{1}{2} \lambda N & 0 \\
0 & 0 & \frac{1}{\lambda N T_{z}^{2}}
\end{array}\right] \lambda_{0} E(\omega)
$$

$$
\lambda=\sqrt{1-\omega^{2} / N(z)^{2}}
$$

and $\lambda_{0}$ is evaluated for $N=1 \mathrm{cph}$. For the frequencies of interest there is little difference between the hydrostatic and non-hydrostatic expressions since $\lambda$ is very close to one. The calculations in this chapter are all based on the non-hydrostatic model.

To simplify algebra later in this chapter, it is useful to define a wave amplitude squared $\gamma$ which is related to the spectral level $E(\omega)$,

$$
\gamma(\omega)=\left\langle\lambda N \lambda_{0} E(\omega)\right\rangle
$$

Note that the nine elements of the cross-spectral matrix have reduced to a single quantity, $\gamma . \gamma$ can then be related to the total energy

$$
\frac{1}{2}\left(\left\langle\tilde{u}^{*} \tilde{u}\right\rangle+\left\langle\tilde{v}^{*} \tilde{v}\right\rangle+\left(N^{2}+\sigma^{2}\right)\left\langle\tilde{\eta}^{*} \tilde{\eta}\right\rangle\right)=\lambda^{-2} \gamma
$$


So in the hydrostatic, noise-free limit the wave amplitude squared $\gamma$ is equal to the energy. Equation 4.2.5 means that there are now two ways of estimating energy level: the direct calculation, which is simply the left-hand-side of equation 4.2.5, and a calculation using $\gamma$, which uses the relations in equation 4.2.2 to estimate $\gamma$ from the data and then uses equation 4.2.5 to calculate the energy from $\gamma$. The advantage of estimating energy via $\gamma$ is two-fold. Energy calculated this way is due only to waves with the proper relations between all nine spectral components: the variability with orthogonal structure is filtered out. Furthermore, unlike the energy density, there are three ways to estimate $\gamma$ (as will be detailed below), so the expected variance in $\gamma$ (and anything calculated from it) is smaller than it would be if there were only one estimate.

\subsection{Model spectral level}

While the linear theory of section 4.2 interrelates the cross-spectral components, the overall model spectral level remains to be determined. To solve this problem, Garrett and Munk did an empirical fit of the available data to a chosen spectral form, the most recent version of which is GM79 (Munk 1981). The basic form and parameter definitions are given in section 1.1. Unfortunately, the spectral form as written in section 1.1 is singular at the equator. This singularity occurs because Garrett and Munk normalized their spectrum so that the total energy (integrated from $f$ to $N$ ) is a constant. This assumption was not really tested in the data they originally used, since it was almost all from mid-latitudes. As Munk points out (Munk 1981), there has subsequently been some evidence that it is instead the energy density that is independent of latitude (Wunsch and Webb 1979, Eriksen 1980b). This requires a slight renormalization, letting

$$
f_{30^{\circ}} E=N_{0} \hat{E}
$$

and replacing $f E$ with $N_{0} \hat{E}$. After making this adjustment, $E(\sigma)$ no longer goes to zero as $f$ goes to zero. Replacing all the parameters by their numerical values, the expected spectral level at the equator is then

$$
\frac{F_{e}(\sigma)}{N}=6.4 \frac{\mathrm{cm}^{2} / \mathrm{s}^{2}}{(\mathrm{cph})^{2}}\left(\frac{.2 \mathrm{cph}}{\sigma}\right)^{2}
$$

This is an expression for the total spectral density at a particular frequency, having integrated over all wavenumbers. For example, the total spectral density in an ocean with a buoyancy frequency of one cph at a frequency of $.2 \mathrm{cph}$ is 6.4 ergs. 
The linear model and the GM79 spectral level combine to determine the model cross-spectra. The next step is to construct estimators that compare the data to that completed model. Equations 4.2.2 and 4.2.4 determine all nine components of the crossspectral matrix given the squared wave-amplitude $\gamma$. To compare the nine measured spectra with the model spectra, it would be nice to construct nine estimators that will gauge the consistency of data and model. If there were no noise in the coefficients of $\gamma$, then it would be sufficient simply to compare the measured cross-spectral matrix with the model cross-spectral matrix: all the variance would be due to the measured spectra, and the comparison would be simply checking the difference between the model and the measurements against the expected variance of the measured spectra. But two parameters (buoyancy frequency $N$ and vertical temperature gradient $T_{z}$ ) are measured quantities (and thus random variables) as well. The next section (4.4) calculates the covariances for both the cross-spectral estimators and the two parameters so that the estimators to measure fit can be constructed (section 4.5).

\subsection{Covariances}

As long as the distribution can be approximated by a joint-normal distribution, the covariance matrix elements are the only distribution parameters required. The general properties of the smoothed cross-spectral covariance matrix are discussed in Jenkins and Watts 1968 (see also appendix $C$ ). In particular, the smoothed spectral estimators have a covariance which depends on the true cross-spectrum, $X$, and the degree of averaging, $n_{r}$. In this case, the true cross-spectra are determined from the GM model: the cross-spectra from the GM model level (equation 4.3.2) are converted to wave amplitude squared $\gamma$ (equation 4.2.4), and then the cross-spectral matrix can be determined from equation 4.2.2. In order for the covariance of the cross-spectral estimators to be a matrix, the cross-spectral estimators must be rewritten into a vector. Thus equation 4.2 .2 becomes

$$
\left[\begin{array}{c}
\tilde{u}^{*} \tilde{u} \\
\tilde{u}^{*} \tilde{v} \\
\tilde{v}^{*} \tilde{v} \\
\tilde{u}^{*} \tilde{T} \\
\tilde{v}^{*} \tilde{T} \\
\tilde{T}^{*} \tilde{T}
\end{array}\right]=\left[\begin{array}{c}
\frac{1}{2} \\
0 \\
\frac{1}{2} \\
0 \\
0 \\
\frac{1}{\left(N^{2}-\sigma^{2}\right)^{2} T_{z}^{2}}
\end{array}\right] \gamma
$$

Using this vector as the true cross-spectra, it is apparent that true cross-powers are all zero. 
That means that $u, v, T$ are independent, and the covariance matrix for the cross-spectral estimates is diagonal.

$$
\frac{1}{n_{r}}\left[\begin{array}{cccccc}
P_{u u}^{2} & 0 & 0 & 0 & 0 & 0 \\
0 & P_{u u} P_{v v} & 0 & 0 & 0 & 0 \\
0 & 0 & P_{v v}^{2} & 0 & 0 & 0 \\
0 & 0 & 0 & P_{u u} P_{T T} & 0 & 0 \\
0 & 0 & 0 & 0 & P_{v v} P_{T T} & 0 \\
0 & 0 & 0 & 0 & 0 & P_{T T}^{2}
\end{array}\right]
$$

where $P_{u u}$ denotes the true value of $\left\langle u^{*} u\right\rangle$, etc. Note that the variance for the crosspowers is evenly split between the real (co-spectrum) and imaginary (quadrature spectrum) parts.

The second contribution to the variance comes from noise in buoyancy frequency $N$ and vertical temperature gradient $T_{z}$. These variables are in the coefficients of equation 4.4.1, thus under certain circumstances they can make a significant contribution to the noise in the final result. A major contribution to their variability is the internal wave field itself advecting the isopycnals and isotherms up and down. Desaubies and Smith (1982) show the connection between the variance and internal wave strain as follows: first define $\eta$ as internal wave displacement, and let $\phi$ represent either density or temperature. Then

$$
\phi=\phi_{0}-\eta \phi_{0 z}
$$

where $\phi_{0}$ denotes the basic state (where $\eta \equiv 0$ ). The approximate equation

$$
\phi_{z} \approx \phi_{0 z}\left(1-\eta_{z}\right)
$$

follows: the approximation is valid as long as $\phi_{0 z} / \phi_{0 z z} \gg \eta / \eta_{z}$ i.e. the scale of the mean is much greater than the scale of the motion. The resulting variance is simply,

$$
\frac{\left\langle\left(T_{z}-T_{0_{z}}\right)^{2}\right\rangle}{T_{0 z}^{2}}=\frac{\left\langle\left(N^{2}-N_{0}^{2}\right)^{2}\right\rangle}{N_{0}^{4}}=\left\langle\eta_{z}^{2}\right\rangle=\lambda^{2}
$$

By choosing a spectral form (similar to GM but with an additional high wavenumber cutoff), they find the mean square strain rate $\lambda^{2}=.25(\lambda=.5)$ for the data they considered. Thus there is an expected variance of 50 percent in temperature gradient and 25 percent in buoyancy frequency. They are quick to point out this is not what would 
be measured in a finite difference approximation to the gradients: differencing over 5 meters depth produces an apparent strain rate $\lambda_{e}$ of .35 , differencing over 20 meters depth produces $\lambda_{e}=.2$. In interpreting the linear internal wave model, the percentage error of $T_{z}$ and $N$ will be taken to be $50 \%$ and $25 \%$ respectively. Having such a large percentage error in some of the model coefficients alters the best choice of estimator for squared wave amplitude significantly. Having such a large error in these coefficients is not a new idea. Wunsch (1976) figures a factor of two uncertainty in $N$, and thus concentrates on making estimates with velocities. The next section will explore the effect on the optimal estimators of these errors in $N$ and $T_{z}$. Note that this model predicts the errors in $N$ and $T_{z}$ to be completely correlated. In the actual ocean there are other sources of error, so they will not be completely correlated - the effects of different choices of correlation will also be looked at in section 4.5 . Furthermore, to the extent that the physics of the internal wave model is inadequate and the true mean vector calculated from GM79 is inaccurate, the variances might have to be altered. For example, were temperature and/or velocity finestructure a significant fraction of the total variance, the true mean vector (equation 4.4.1) would have to be altered. This is not a serious problem, however, since the largest uncertainty is not in the spectral quantities. The largest uncertainties are in the buoyancy frequency and temperature gradient values, thus changing the estimated noise level in the spectral estimators will make little difference. 


\subsection{Wave amplitude and fit estimators}

Equations 4.2.2 and 4.2.4 determine all nine components of the cross-spectral matrix given the squared wave amplitude $\gamma$. If the only quantities with noise were the crossspectra, comparing the data with the model would be simply a matter of checking the difference between the model values and the measurements again the expected noise in the measurements. Unfortunately the coefficients of $\gamma$ in equation 4.2.2 depend on the noisy variables $N$ and $T_{z}$. It would be simpler if all the variables that contained random noise were on the same side. This is equivalent to solving for the model parameters such as $\gamma$.

First consider estimating $\gamma$ in a system that assumes a WKBJ vertical dependence. That implies that $\gamma$ is proportional to buoyancy frequency as evidenced by equation 4.2.2, where the wave amplitude component $E$ (which is the WKBJ solution) has to be multiplied by $N$ to get $\gamma(N \gg \sigma$ so that $\lambda$ is one). Thus there are the following three estimators for square wave amplitude $\gamma$

$$
\begin{array}{ll}
A & 2\left(N_{0} / N\right)\left\langle u^{*} u\right\rangle \\
B & 2\left(N_{0} / N\right)\left\langle v^{*} v\right\rangle \\
C & \left(N_{0} / N\right)\left(N^{2}-\sigma^{2}\right) / T_{z}^{2}\left\langle T^{*} T\right\rangle \\
& \langle A\rangle=\langle B\rangle=\langle C\rangle=\gamma
\end{array}
$$

where $N_{0}$ is the true value of $N$. Appendix $F$ describes how to optimally combine correlated estimates of some random variable. The optimal estimator is given by equation $F .4$ and requires only the covariance matrix. First define

$$
\lambda^{2}(\sigma)=\frac{N_{0}^{2}+\sigma^{2}}{N_{0}^{2}-\sigma^{2}}
$$

The covariance matrix of the estimates $A, B, C$ is then

$$
\gamma^{2}\left[\begin{array}{ccc}
\frac{1}{n_{r}}+\langle\epsilon \epsilon\rangle & \langle\epsilon \epsilon\rangle & \langle\epsilon \delta\rangle-\lambda^{2}\langle\epsilon \epsilon\rangle \\
\langle\epsilon \epsilon\rangle & \frac{1}{n_{r}}+\langle\epsilon \epsilon\rangle & \langle\epsilon \delta\rangle-\lambda^{2}\langle\epsilon \epsilon\rangle \\
\langle\epsilon \delta\rangle-\lambda^{2}\langle\epsilon \epsilon\rangle & \langle\epsilon \delta\rangle-\lambda^{2}\langle\epsilon \epsilon\rangle & \frac{1}{n_{r}}+4\langle\delta \delta\rangle+\left(\lambda^{2}\right)^{2}\langle\epsilon \epsilon\rangle-4 \lambda^{2}\langle\delta \epsilon\rangle
\end{array}\right]
$$

where $\epsilon$ is the percentage error in $N=N_{0}(1+\epsilon)$ and $\delta$ is the percentage error in $T_{z}=T_{z 0}(1+\delta)$.

Another model differs only in the presumed vertical dependence. Rather than assuming a WKBJ vertical dependence, it assumes energy is depth independent. There 
is no particular theoretical basis for this, it is merely presented by way of contrast: a simpler alternate theory. The three estimators in this case are

$$
\begin{array}{ll}
A & 2\left\langle u^{*} u\right\rangle \\
B & 2\left\langle v^{*} v\right\rangle \\
C & \left(N^{2}-\sigma^{2}\right) / T_{z}^{2}\left\langle T^{*} T\right\rangle \\
& \\
& \langle A\rangle=\langle B\rangle=\langle C\rangle=\gamma
\end{array}
$$

The covariance matrix in this case is much simpler since the three estimators are independent.

$$
\gamma^{2}\left[\begin{array}{ccc}
\frac{1}{n_{r}} & 0 & 0 \\
0 & \frac{1}{n_{r}} & 0 \\
0 & 0 & \frac{1}{n_{r}}+4\langle\delta \delta\rangle+\left(\frac{2 N_{0}^{2}}{N_{0}^{2}-\sigma^{2}}\right)^{2}\langle\epsilon \epsilon\rangle-\frac{8 N_{0}^{2}}{N_{0}^{2}-\sigma^{2}}\langle\delta \epsilon\rangle
\end{array}\right]
$$

The five lines of figure 4.5.1 illustrate the wave amplitude and energy estimators for various choices of the parameters for both models. The parameters varied are

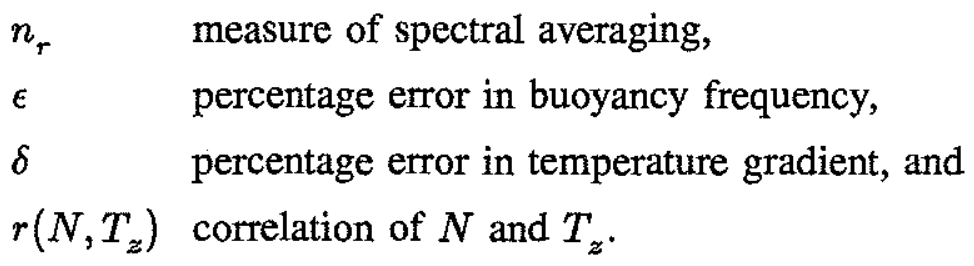

The first two lines show the estimators for the simple (not WKBJ) model. Unless there is very little spectral averaging, the best estimator is in fact using just the horizontal kinetic energy as a measure of energy level. The WKBJ model results are somewhat different. The third line shows the estimators assuming 20 percent error in both $N$ and $T_{z}$ calculations. This choice might be made as it seems reasonable in light of the 20 percent error seen in finite differenced $N$ calculations. The weighing is much more even than the simple model. The fourth line has $N$ and $T_{z}$ still uncorrelated, but the error levels correspond to Desaubies and Smith's results: 25 percent error in $N$ and 50 percent error in $T_{z}$. The $C$ estimator is downweighed relative to the less noisy case. Finally, the last line shows the case where correlation is included. While error in the best-fit estimator is the same, the relative weighing is vastly different. The $C$ estimator is now subtracted from the other two: since the three estimates are positively correlated, the optimal estimator now subtracts the noisiest estimate. This final choice of parameters is 
Figure 4.5.1 Weights for combining A, B, and C estimators

\begin{tabular}{|c|c|c|c|c|c|}
\hline $\mathrm{N}_{\mathrm{sK}}$ & $\varepsilon$ & $\delta$ & $\mathrm{r}$ & $\mathrm{W}_{\mathrm{A}+\mathrm{B}}$ & $\mathrm{w}_{\mathrm{c}}$ \\
\hline \multicolumn{6}{|c|}{ Isotropic model } \\
\hline 10 & $\square_{0.25}$ & E.50 & Elpoigo & Ellio. & Odo.10 \\
\hline Q7erag & $\bigotimes_{0.25}$ & 80.50 & Elloo.90 & Elllioo & 0.00 \\
\hline \multicolumn{6}{|c|}{ WKBJ model } \\
\hline ETriegs & $\bigotimes_{0.20}$ & 0.20 & 0.00 & DPo.75 & $Q_{0.25}$ \\
\hline V7rag & $\bigotimes_{0.25}$ & 80.50 & 0.00 & Ello.90 & 80.10 \\
\hline Elize: & $\square_{0.25}$ & Co.50 & Ello. 0.90 & QDPDPli.28 & $-0.28 \square$ \\
\hline
\end{tabular}

Figure 4.5.1 Weights for combining $A, B$, and $C$ estimators 
the most reasonable, and it results in an estimator that is considerably different from the estimator one might pick at first glance.

Plotted in figures 4.5.2 are several different estimators of the energy level. Each curve is plotted twice: once on the lower half of the page, where all the curves are correctly scaled, and once on the top of the page, where each curve is offset by a decade relative to its nearest neighbors. The solid straight line gives the level predicted by GM79 (equation 4.3.2); on either side are dashed lines which give the $95 \%$ confidence limits. The other solid line gives the level as estimated from the data using the estimator that corresponds to the third WKBJ weighing of figure 4.5.1. The dotted line gives the level as estimated from the data using the second isotropic model weighing of figure 4.5.1. Finally, the dashed curve gives the direct estimate of energy, i.e.

$$
E=\frac{1}{2}\left(\left\langle u^{*} u\right\rangle+\left\langle v^{*} v\right\rangle+\left(N^{2}+\sigma^{2}\right)\left\langle T^{*} T\right\rangle / T_{z}^{2}\right)
$$

21 such plots were made from data taken with the PEQUOD central moorings $Q$ and $U$, one plot for each yearlong current meter $(u, v, T)$ record (there were two missing records in the first year, and one in the second). Eight plots are presented here as a summary: they are figures 4.5.2a through 4.5.2h. Each figure refers to its original set of time series by instrument label, and figure 2.1.2 translates those labels into horizontal location, start times, duration, and depth. Figure 4.5.2a contains the results for instrument Q103, which is the first year data from the $615 \mathrm{~m}$ depth current meter on the $Q$ mooring. It is quite typical of the results seen in the other 20 plots. There is a strong peak in energy in the frequency bin centered at $12 \frac{1}{4}$ hour period which is tidal. This peak appears to divide the frequency range into two parts: frequencies lower than that tidal peak, where all three estimators are significantly lower than the GM79 level, and frequencies higher than that tidal peak, where all three levels are consistent with the GM79 level. It is important to realize that the GM79 error bars are due to both spectral estimator noise and noise in buoyancy frequency $N(z)$ and vertical temperature gradient $T_{z}$. For the spectral estimators, each frequency is independent, and were the noise entirely due to spectral noise the estimators would distribute evenly about the expected mean (which in this case is GM79). But part of the noise is due to the buoyancy frequency and the temperature gradient, and as far as that noise is concerned different frequencies are not independent realizations. Thus it is not surprising that the variability in figure $4.5 .2 a$ is not centered about GM79. 
What difference there is between the three estimators appears primarily at higher frequencies. The differences are due to temperature variance in excess of that expected from GM79. The plot shows the direct energy estimator is larger than the isotropic model estimator which is larger than the WKBJ model estimator. Since the isotropic model estimator essentially ignores the temperature data, the WKBJ model estimator subtracts it from the kinetic energy (since there is correlated noise between them), and the direct energy estimator adds the temperature data to the kinetic energy, the estimators' relative sizes indicate excess temperature variance. This will be more easily seen in the plots of figure 4.5.4.

By way of comparison, figure 4.5.2b presents the energy estimates from instrument U103, the first year data from the $592 \mathrm{~m}$ instrument on mooring $U$. It is almost indistinguishable from the plot from instrument Q103, indicating that there is little difference between the two moorings $Q$ and $U$, a result that is also true of the other plots. Figure 4.5.2c presents the energy estimates from instrument Q203, the second year version of Q103. Again all the differences are within the error bars, though the three estimators appear closer to each other and to GM79 then before (not a statistically significant difference, though). The same can be said of figure 4.5.2d, which presents the second year mooring $U$ data from approximately the same depth. Figures 4.5.2e through 4.5.2h present a series of plots from the second year $U$ mooring data that are successively deeper. The results are not different from figures 4.5.2a $-d$, though the plots of the data from U209 and U211 serve as a visual reminder that the internal wave model used breaks down as the buoyancy frequency is approached. There is some suggestion in figure 4.5.2h that the spectral slope calculated from the data might be less than the -2 used in the GM79 model, a result seen in some of the plots that are not shown too, but the difference is not statistically significant, and it is in a frequency range where the model is not particularly appropriate. Thus these eight plots, typifying the full set of 21 , suggest that there is no statistically significant difference in energy level from GM79 above the tidal peak, results that do not depend on year, mooring, or depth. There also appears to be little difference between the three energy estimators.

The energy levels computed in the last section are directly related to the squared wave amplitude $\gamma$. There are eight other model parameters which together with $\gamma$ span the nine-dimensional data space. They all have true value zero, though in the presence of noise the corresponding estimators will not necessarily be zero. Once a true mean has 


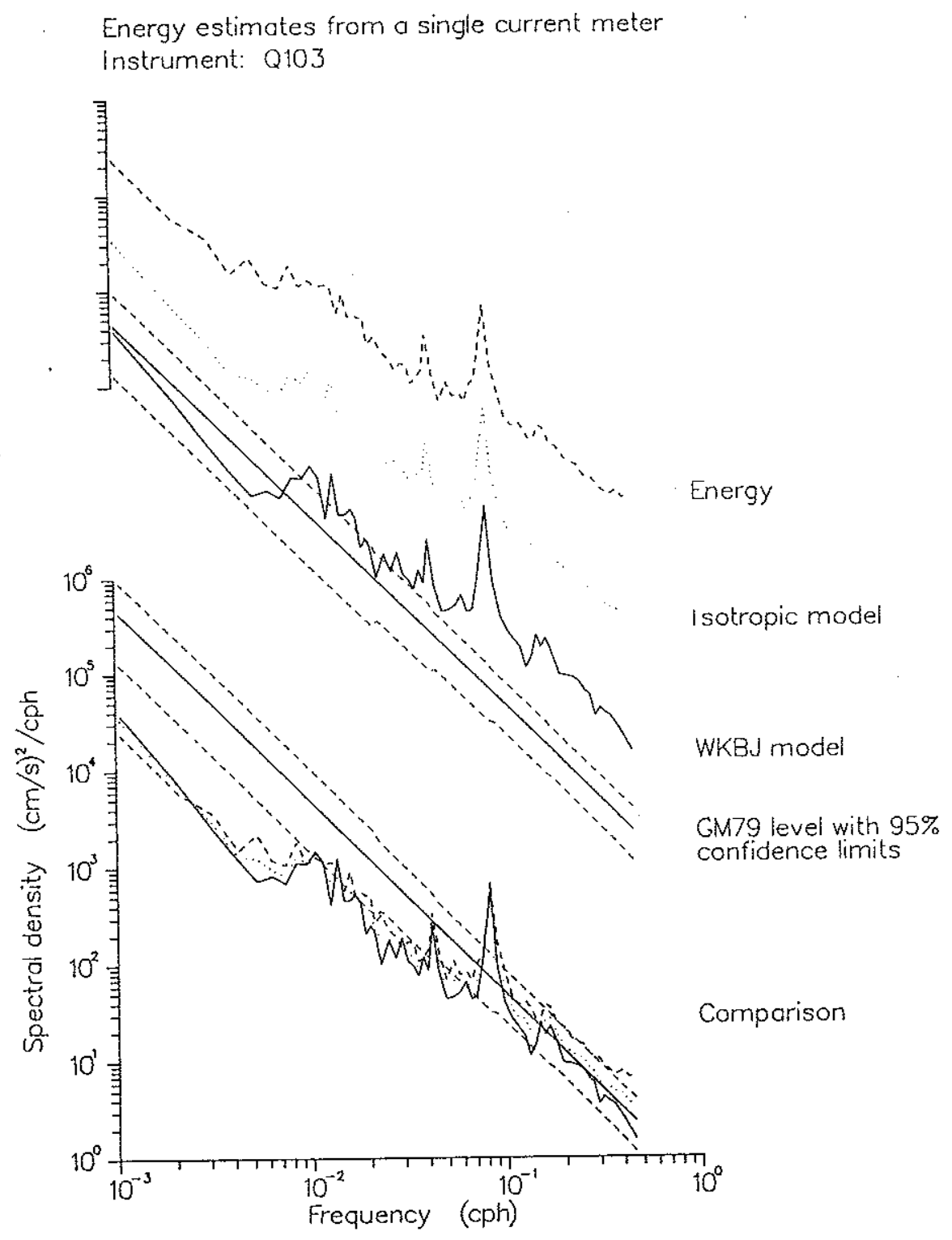

Figure 4.5.2a Energy estimates from Q103

This plot compares three different estimators of energy level with that expected from GM79. The bottom half of the plot has the three curves properly scaled; the top of the plot has each plot offset by a decade relative to its neighbors. 


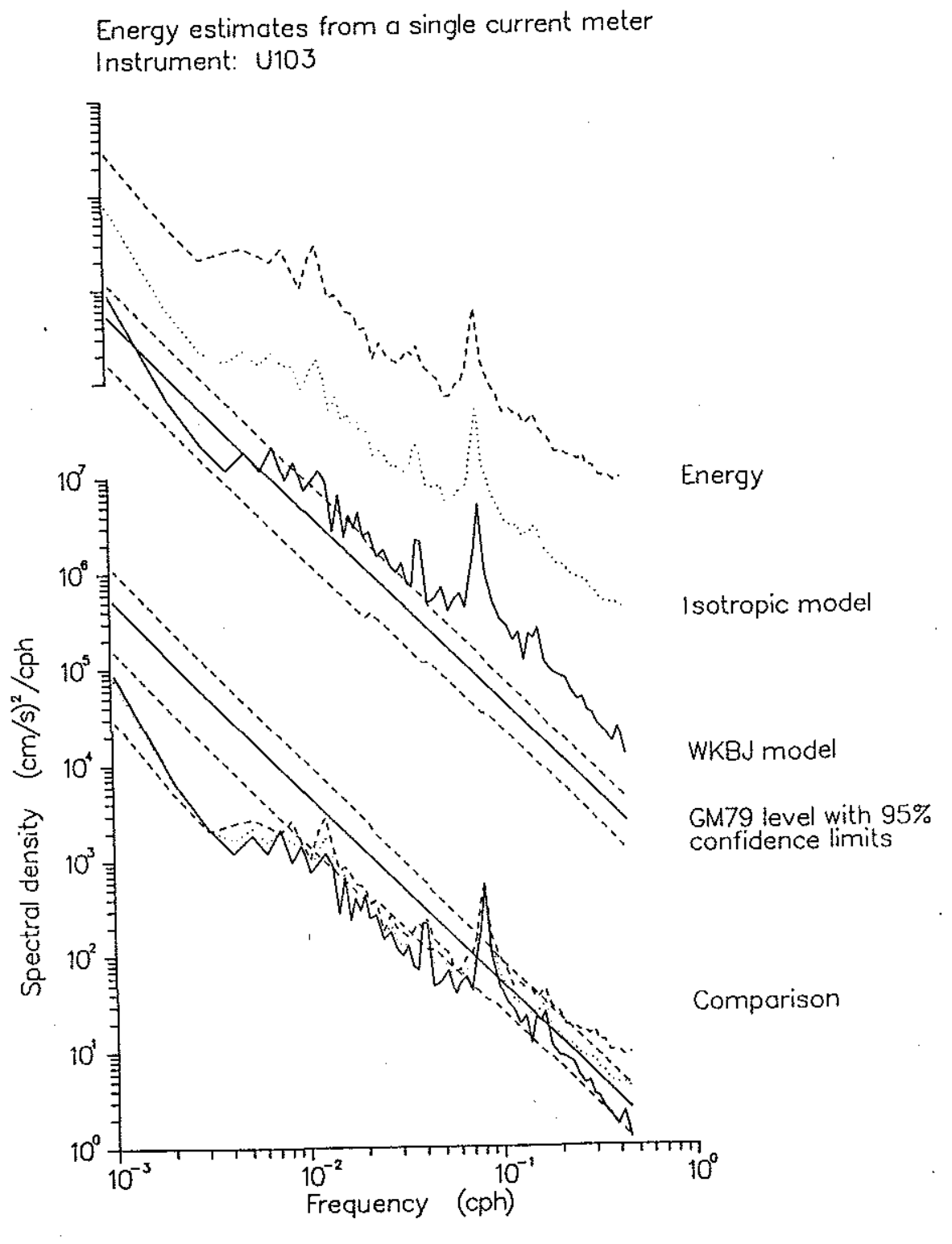

Figure 4.5.2b Energy estimates from U103

This plot compares three different estimators of energy level with that expected from GM79. The bottom half of the plot has the three curves properly scaled; the top of the plot has each plot offset by a decade relative to its neighbors. 


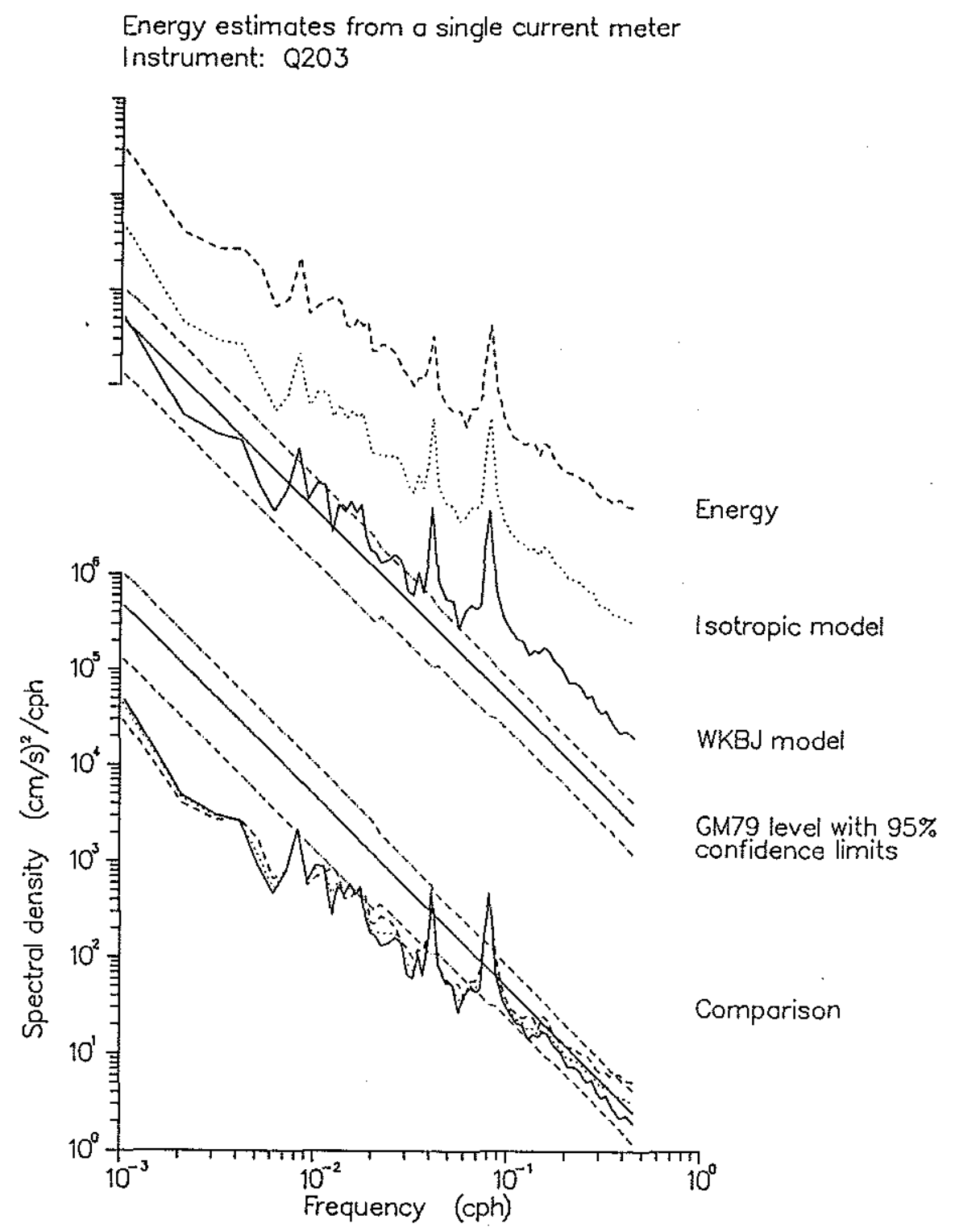

Figure 4.5.2c Energy estimates from Q203

This plot compares three different estimators of energy level with that expected from GM79. The bottom half of the plot has the three curves properly scaled; the top of the plot has each plot offset by a decade relative to its neighbors. 


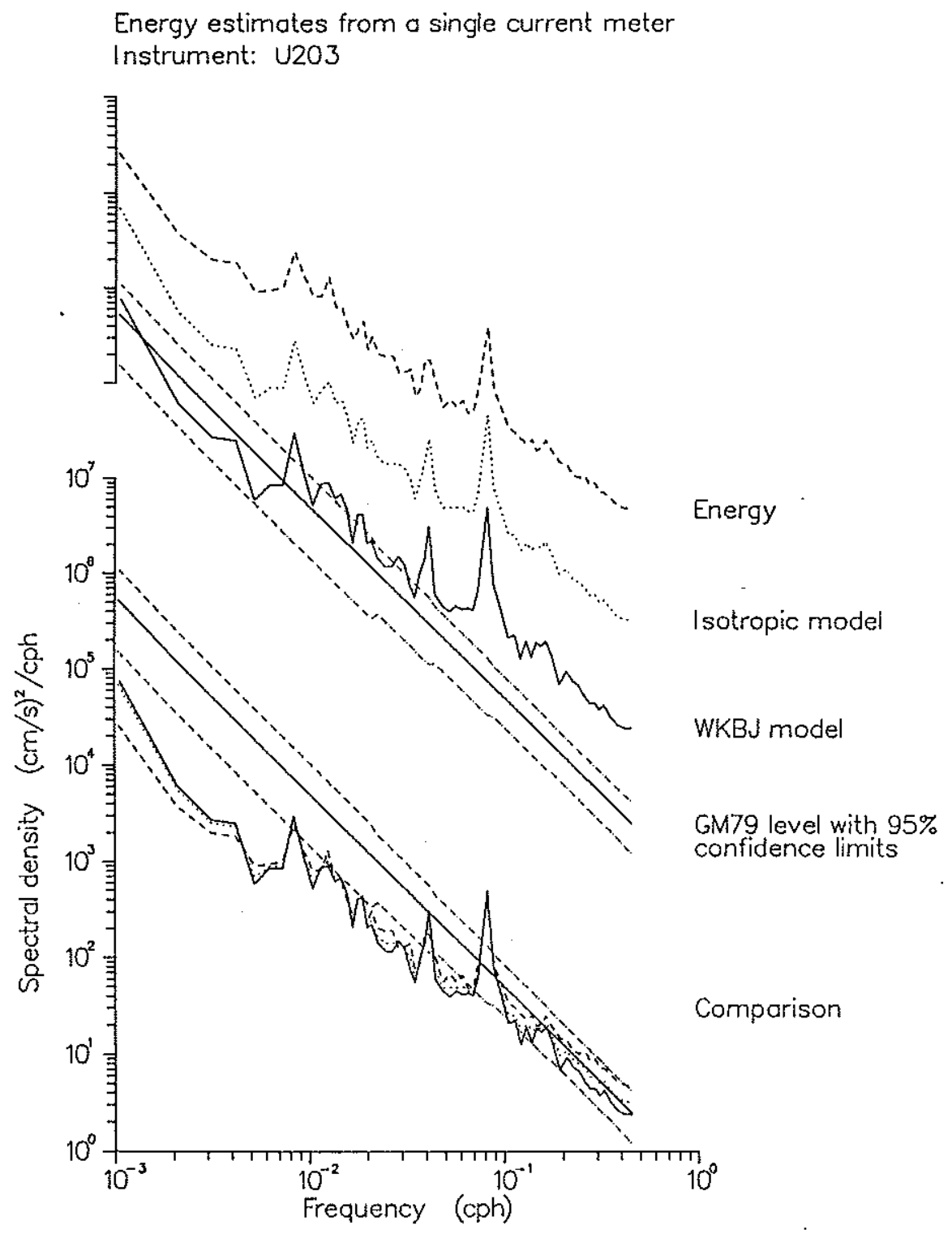

Figure 4.5.2 $d$ Energy estimates from U203

This plot compares three different estimators of energy level with that expected from GM79. The bottom half of the plot has the three curves properly scaled; the top of the plot has each plot offset by a decade relative to its neighbors. 


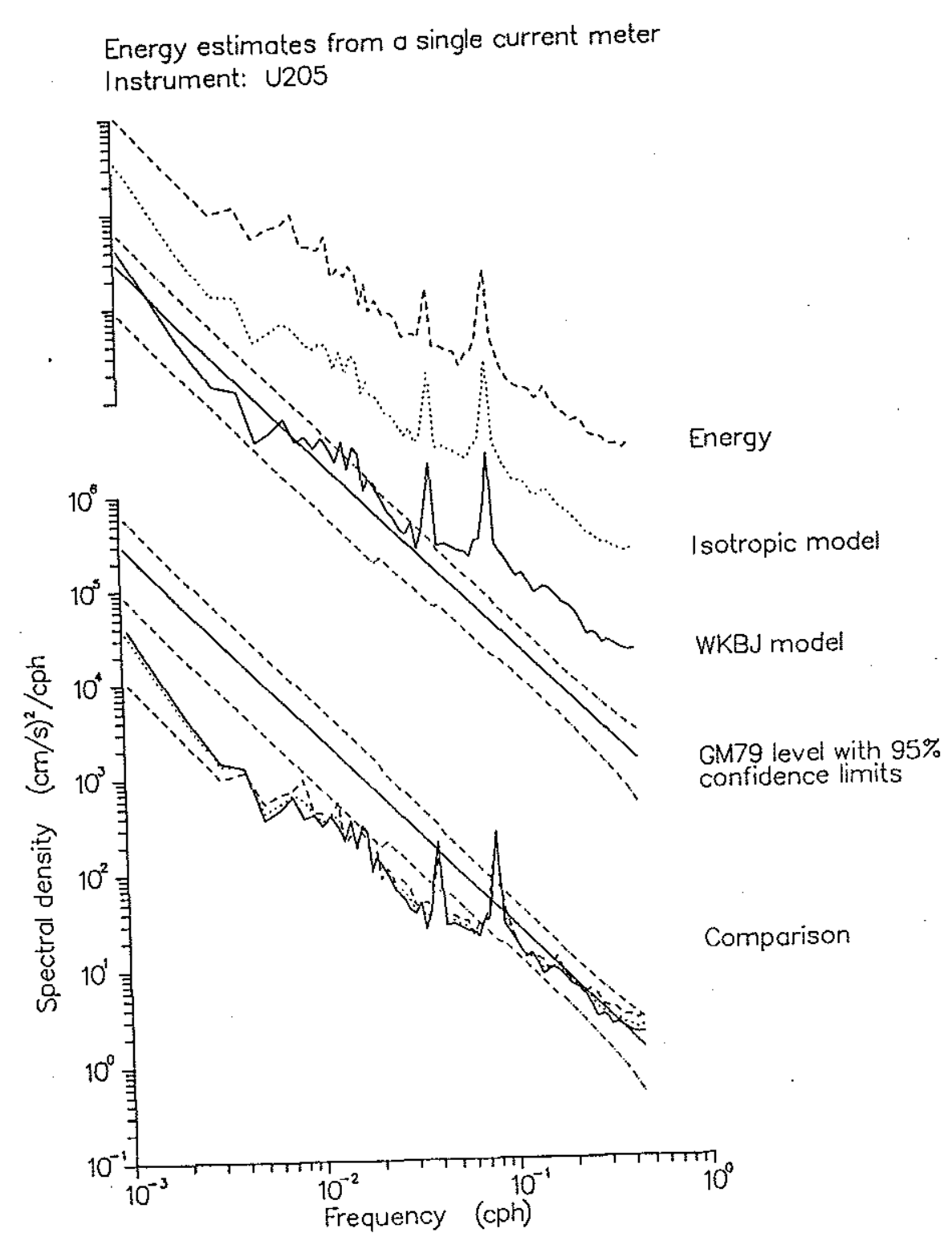

Figure 4.5.2 $e$ Energy estimates from U205

This plot compares three different estimators of energy level with that expected from GM79. The bottom half of the plot has the three curves properly scaled; the top of the plot has each plot offset by a decade relative to its neighbors. 


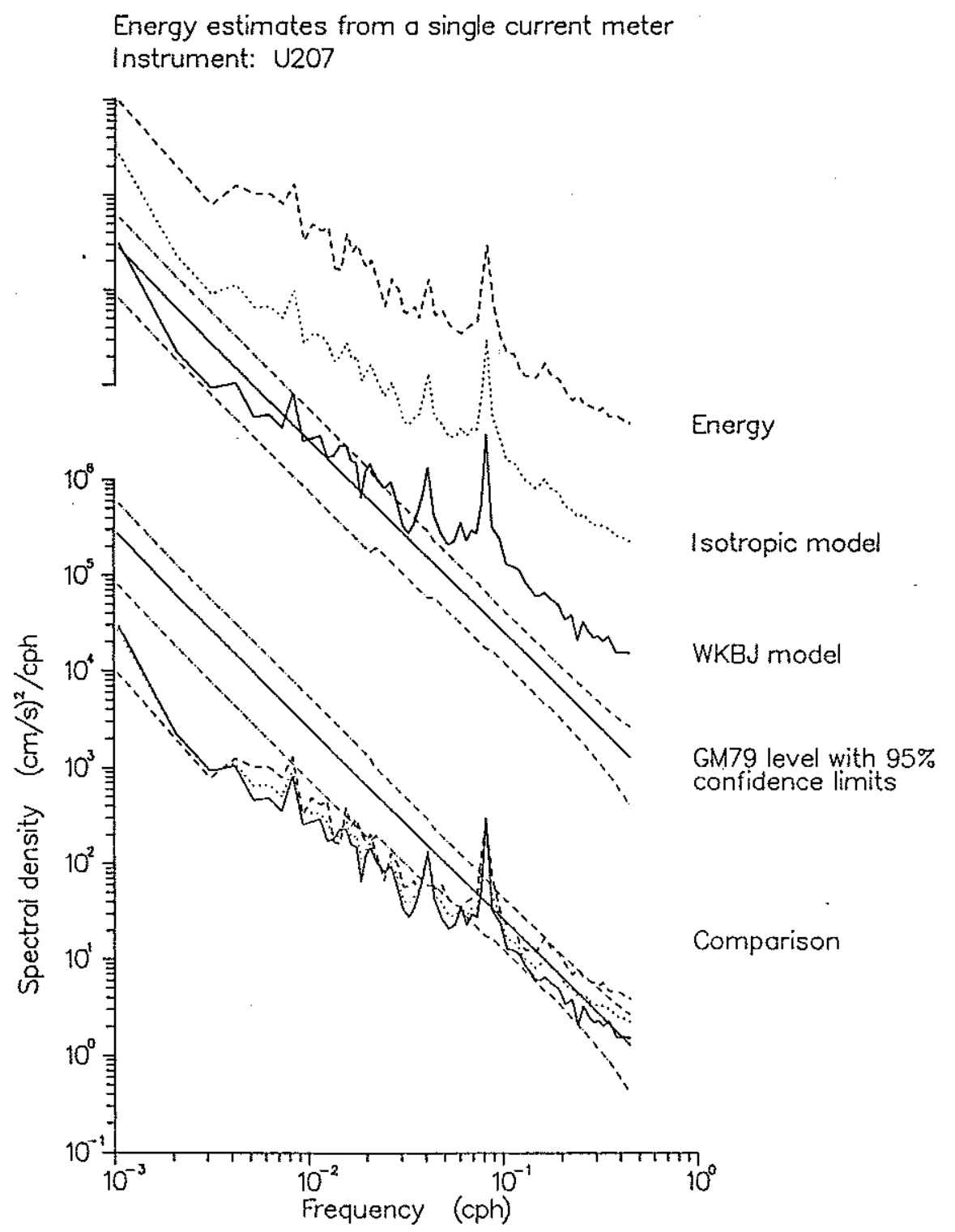

Figure 4.5.2 $f$ Energy estimates from U207

This plot compares three different estimators of energy level with that expected from GM79. The bottom half of the plot has the three curves properly scaled; the top of the plot has each plot offset by a decade relative to its neighbors. 


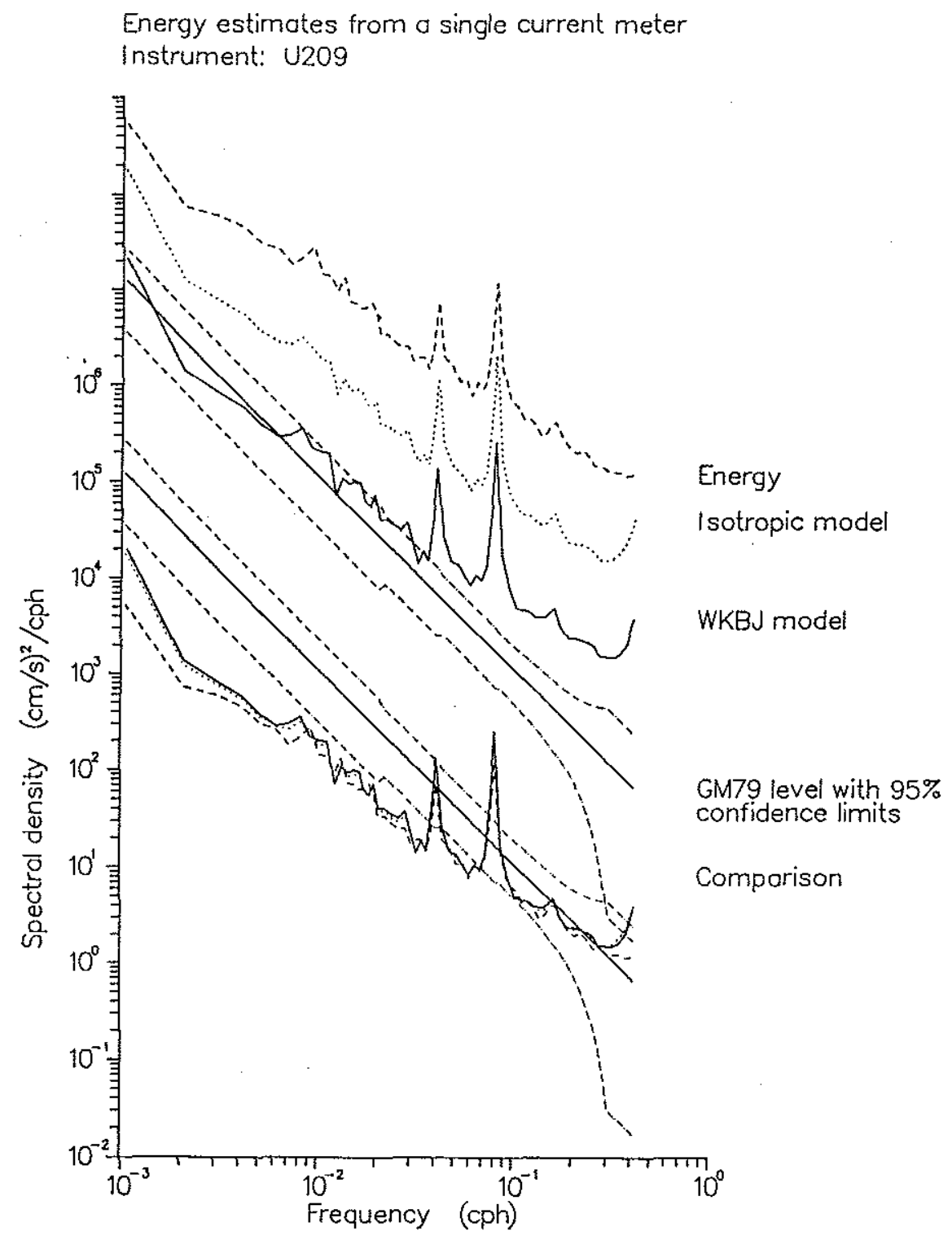

Figure 4.5.2 $g$ Energy estimates from U209

This plot compares three different estimators of energy level with that expected from GM79. The bottom half of the plot has the three curves properly scaled; the top of the plot has each plot offset by a decade relative to its neighbors. 


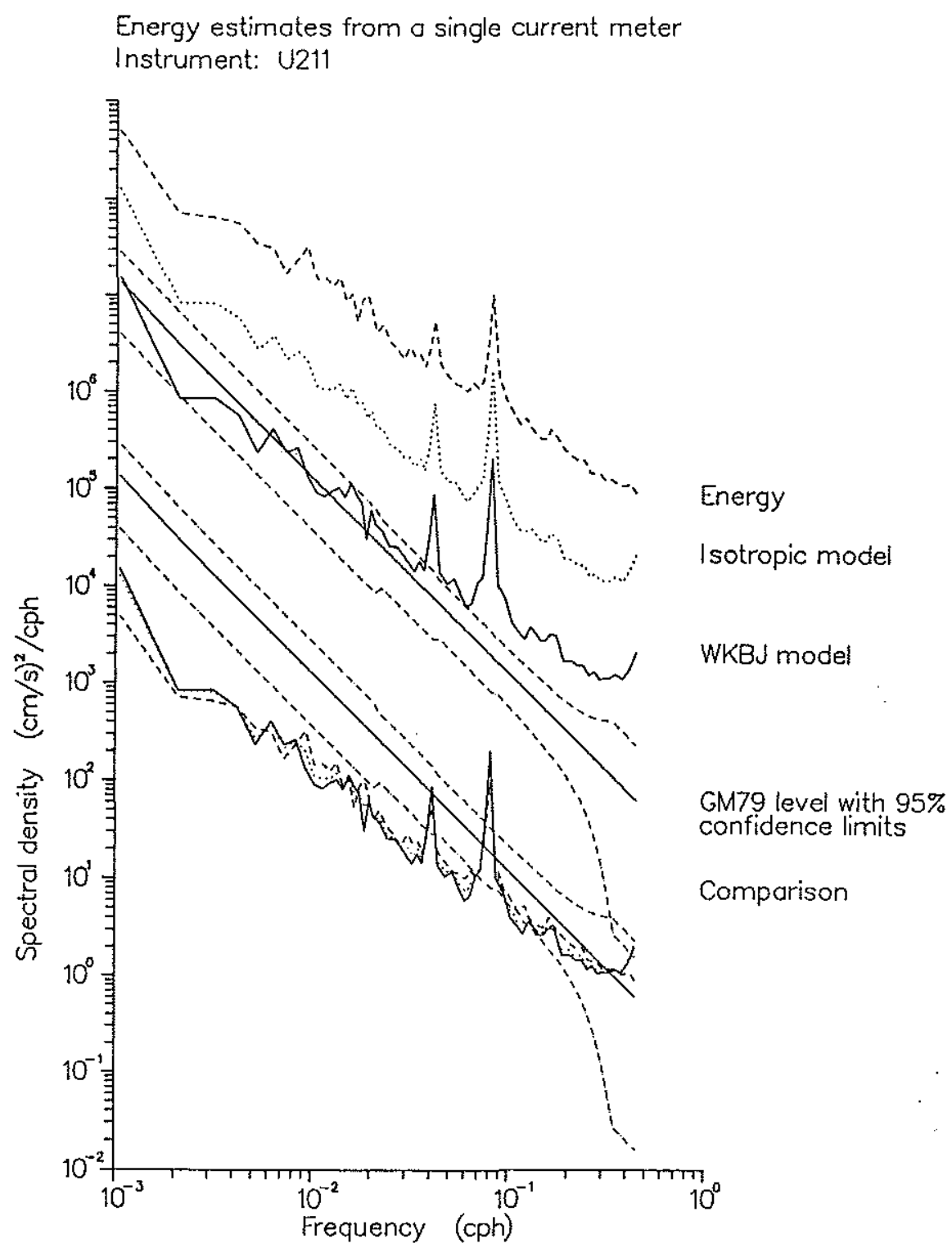

Figure 4.5.2h Energy estimates from U211

This plot compares three different estimators of energy level with that expected from GM79. The bottom half of the plot has the three curves properly scaled; the top of the plot has each plot offset by a decade relative to its neighbors. 
Figure 4.5.3 Table of zero mean estimators

$$
\begin{aligned}
& \text { Estimator } \\
& \gamma-\gamma_{0} \\
& \left(R_{K}\left\langle T^{*} T\right\rangle-\left\langle u^{*} u\right\rangle-\left\langle v^{*} v\right\rangle\right) \quad\left(\frac{3}{2} \frac{1}{n_{r}}+4\langle\delta \delta\rangle+\left(1+\lambda^{2}\right)^{2}\langle\epsilon \epsilon\rangle\right. \\
& \text { Variance } \\
& \gamma_{\mathrm{err}}^{2} \\
& \left\langle u^{*} u\right\rangle-\left\langle v^{*} v\right\rangle \\
& \left\langle u^{*} v\right\rangle \\
& \left\langle u^{*} T\right\rangle \\
& \left\langle v^{*} T\right\rangle \\
& \begin{array}{l}
\left.-\left(2+4 \lambda^{2}\right)\langle\delta \epsilon\rangle\right) \gamma_{0}^{2} \\
\frac{1}{2} \frac{1}{n_{r}} \gamma_{0}^{2} \\
\frac{1}{8} \frac{1}{n_{r}} \gamma_{0}^{2} \\
\frac{1}{4 R_{K}} \frac{1}{n_{r}} \gamma_{0}^{2} \\
\frac{1}{4 R_{K}} \frac{1}{n_{r}} \gamma_{0}^{2}
\end{array}
\end{aligned}
$$

been chosen, there is a ninth estimator that has true value zero: the difference between the estimator $\gamma$ and its true value $\gamma_{0}$. There is some freedom in choosing the eight estimators, but they are more useful statistically independent. These nine zero mean estimators (consistency checks) are given in figure 4.5.3 where

$$
R_{K}=\left(N^{2}-\sigma^{2}\right) / T_{z}^{2}
$$

and $\gamma_{\text {err }}$ is computed using equation 2.3.5.

In the plots 4.5.3 these estimators have been normalized so that they are all unit variance. Estimator 4.5.5a compares the level as measured by the WKBJ estimator to that expected by GM79. It thus duplicates the information presented in the plots of energy estimators 4.5 .2 (though there is a small difference in that here a normal distribution is used to calculate confidence limits from the expected variance, while a chisquared distribution is used in the energy estimator plot). Estimator 4.5.5b compares the relative sizes of the kinetic and potential energies to the relationship expected for internal waves. It was introduced in section 1.5 and is usually called Fofonoff's consistency check (Fofonoff 1969, see also Wunsch 1976, Wunsch and Webb 1979). Estimator 4.5.5c measures the difference between zonal and meridional kinetic energy; positive values correspond to excess zonal kinetic energy. The remaining estimators are normalized cross-spectra. Estimator 4.5.5d gives the real part of $\left\langle u^{*} v\right\rangle$ while estimator 4.5.5e gives the corresponding imaginary part. Estimator 4.5.5 $f$ gives the real part of $\left\langle u^{*} T\right\rangle$ while estimator $4.5 .5 \mathrm{~g}$ gives the corresponding imaginary part. And estimator 4.5.5 $h$ and 4.5.5i give respectively the real and imaginary parts of $\left\langle v^{*} T\right\rangle$. Note that the estimators $4.5 .5 b-i$ are the same for both the simple and the WKBJ models. That is because their expected value is zero, so that variations in the coefficient (to first order) have no effect. 
Like the energy estimator plots, there are 21 plots of these nine zero-mean estimators from the central mooring PEQUOD data. But because the results are so much more complicated, they are much harder to summarize with a selection of plots. So while figure 4.5.4 presents the results of eight particular instruments, figures 4.6.1 and 4.6.2 present two additional tabulations of the the results.

Figure 4.5.5a compares the data from instrument Q103 with GM79. The estimator 4.5.5a indicates that the measured level is less than that expected from GM79 for frequencies below the large tidal peak at roughly $12 \frac{1}{4}$ hour period, much higher than GM79 at the tidal peak, and roughly consistent at higher frequencies. This is the part of the information that was presented on the spectral level plot. The estimator 4.5.5b indicates a slight excess of temperature variance over what is expected from Fofonoff's consistency check, most of the excess being between the two vertical lines that mark the Nyquist frequency and two octaves below the Nyquist frequency. This is the range where the Aanderaa aliasing problem arises (see Appendix $A$ ), and so anomalies in that frequency range must be viewed with suspicion. The only other estimator that shows significant deviation from GM79 for Q103 is estimator 4.5.5c, which indicates an excess of meridional kinetic energy, an excess no doubt exaggerated by the effects of the Aanderaas.

Figure 4.5.4b compares the data from U103 with GM79. It shows the same deviations as Q103, suggesting that the two central moorings $U$ and $Q$ behave very much as two realizations of the same thing and are pretty much interchangeable. Figure 4.5.4c compares the data from Q203 with GM79. The deviations of estimator 4.5.5c noted in Q103 and U103 are present, plus there appears to be significant positive $\left\langle v^{*} T\right\rangle$ in-phase correlation indicated by estimator 4.5.5h. But there are no significant deviations in 4.5.5b. The data from U203 (presented in figure 4.5.4d) also has nothing in 4.5.5b. U203 also has nothing in estimator 4.5.5 h. So the only result that is found at this depth independent of time and mooring is the there is excess meridional kinetic energy, and that is only in a frequency range that is possibly badly affected by aliasing.

The results at somewhat deeper instruments are much more dramatic. Figure 4.5.4c presents the comparison of U105 data with GM79. Here estimator 4.5.5 $f$ indicates a strongly significant positive in-phase $u T$ correlation at all frequencies, while estimator 4.5.5 $\mathrm{g}$ indicates that there is a significant positive quadrature (out-of-phase) $u T$ correlation at high frequencies. To a lesser extent estimator 4.5.5 $\mathrm{h}$ indicates that there is a significant positive in-phase $v T$ correlation as well. These correlations are found in all three of the 
records taken at this depth (as can be verified by looking at the tables in figure 4.6.1 and 4.6.2).

In stark contrast, the data from instruments $100 \mathrm{~m}$ lower have a strong correlation with opposite sign. Figure 4.5.4f shows the data from U107. Here estimator 4.5.5 indicates a strong negative in-phase $u T$ correlation, while estimator $4.5 .5 \mathrm{~g}$ indicates a barely significant negative out-of-phase correlation for $u T$. Estimator $4.5 .5 h$, on the other hand, shows a tendency towards a positive $v T$ correlation, but it is not clearly significant.

Figures 4.5.4g and 4.5.4h show the comparison with GM79 using U109 and U111 data respectively. Neither plot shows much except for a slight excess of meridional kinetic energy at high frequencies. Clearly there is a great deal of depth dependence in the strength of the cross-power between horizontal velocities and temperature.

\subsection{Isotropic model results}

Two summaries of the GM79 - PEQUOD current meter data comparisons are presented in figures 4.6.1 and 4.6.2. Figure 4.6.1 is a rough visual survey of the significant deviations found for frequencies higher than $.1 \mathrm{cph}$. Deviations are given to the nearest two standard deviations, and deviations less than 2 are omitted. Figure 4.6 .2 presents the deviations averaged over the frequency band $[.1 \mathrm{cph}, .125 \mathrm{cph}]$. Here the deviations are truncated to an integer number of standard deviations. Like figure 4.6.1, deviations less than 2 are omitted. The surveys differ in that the visual survey considers data from frequencies higher than .125 in determining a value, while the numerical survey does not. Both these tables bear out what was found in the discussions of individual plots in the last section. Primarily the PEQUOD data differed from GM79 in two ways. Most records had meridional velocity spectra higher than the zonal, though the difference was not statistically significant in some of the records. The significance of this excess meridional energy is hard to judge since it occurs exclusively in the frequency range where Aanderaas have trouble (see appendix $A$ ). The other major significant deviation occurred in $\left\langle u^{*} T\right\rangle$. The in-phase component of the $u T$ correlation is very much outside the $95 \%$ confidence limits for the mid-depth $(1500 \mathrm{~m}$ and $1600 \mathrm{~m}$ ) records (labels of the form -05 and 07). It is also outside the $95 \%$ confidence limits at other depths as well, though not so spectacularly. The in-phase component of the $v T$ correlation is also significantly nonzero for the -07 records for both surveys, and for the -05 records in the visual survey but the not numerical average. To some extent the out-of-phase $u T$ correlation is significant, 


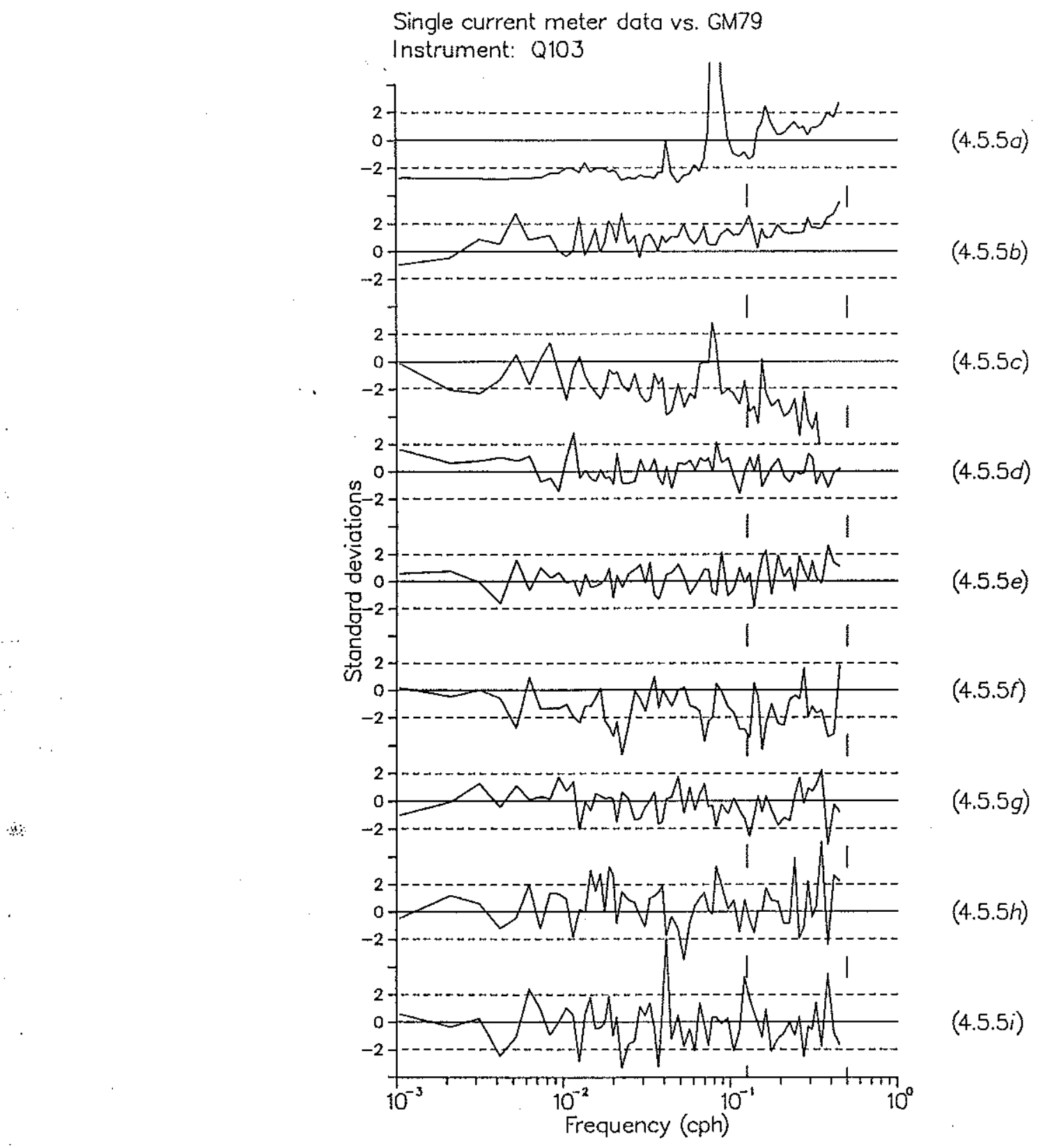

Figure 4.5.4a Q103 data compared to GM79

These nine plots compare the zero mean estimators of figure 4.5 .3 to their expected noise levels. The $95 \%$ confidence limits are given by the dashed lines. The pairs of vertical lines mark the Nyquist frequency $(.5 \mathrm{cph})$ and a point two octaves below the Nyquist frequency. This is the frequency range in which the Aanderaas are expected to have trouble (see Appendix $A$ ). Curves are omitted at the points which are further than six standard deviations from the mean. 


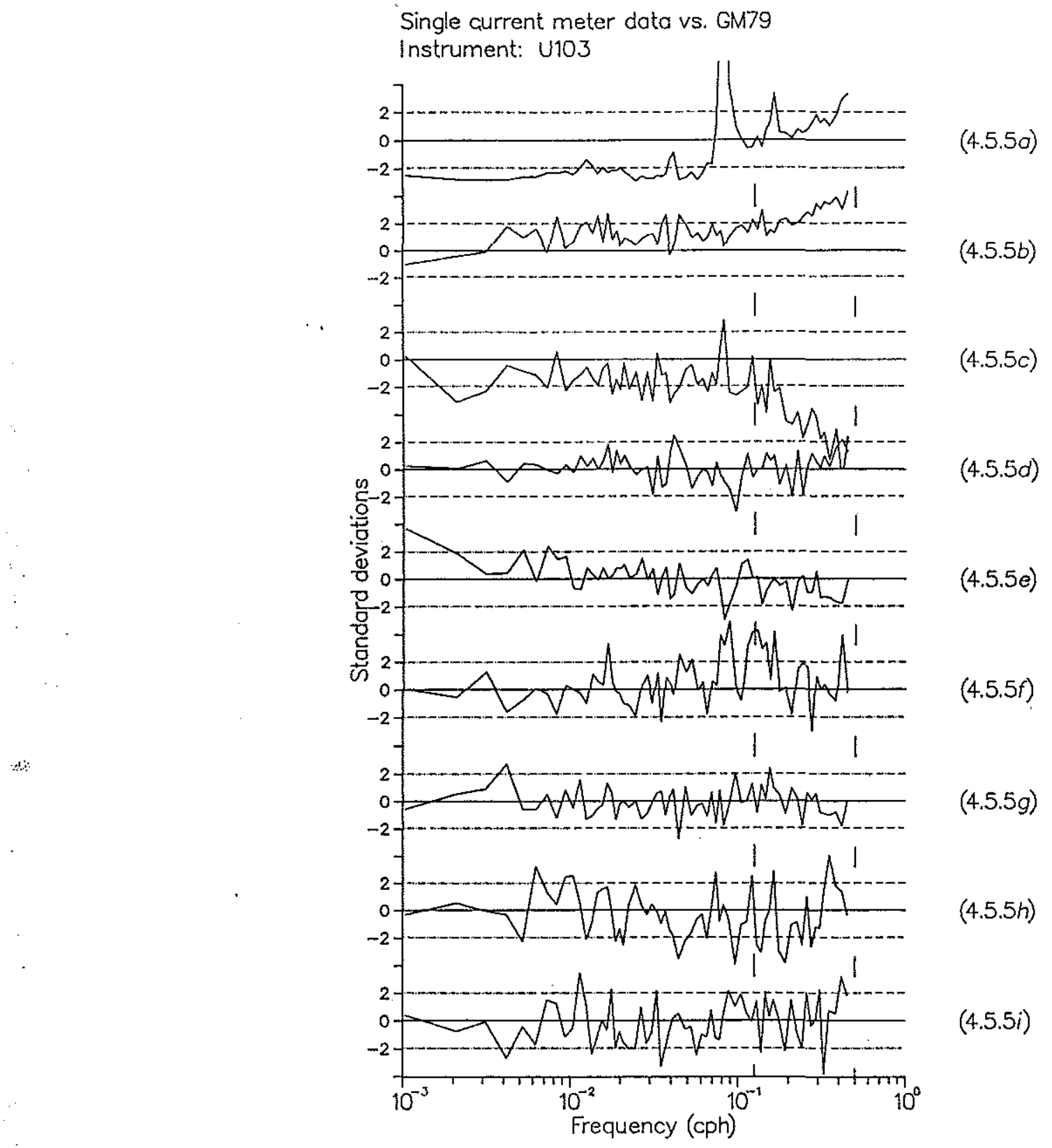

\section{Figure 4.5.4b U103 data compared to GM79}

These nine plots compare the zero mean estimators of figure 4.5.3 to their expected noise levels. The $95 \%$ confidence limits are given by the dashed lines. The pairs of vertical lines mark the Nyquist frequency $(.5 \mathrm{cph})$ and a point two octaves below the Nyquist frequency. This is the frequency range in which the Aanderaas are expected to have trouble (see Appendix $A$ ). Curves are omitted at the points which are further than six standard deviations from the mean. 
Single current meter dota vs. GM79

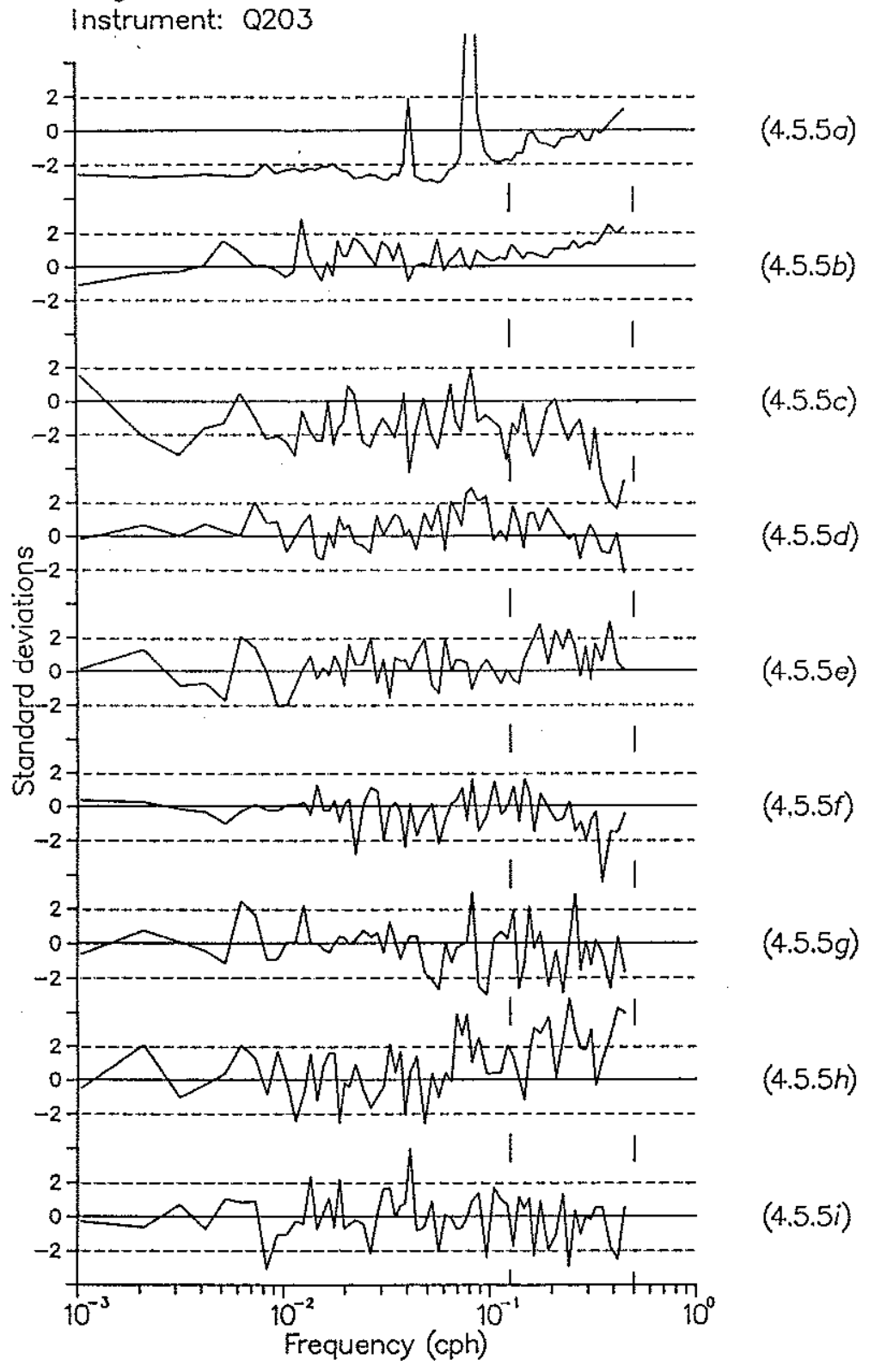

\section{Figure 4.5.4c Q203 data compared to GM79}

These nine plots compare the zero mean estimators of figure 4.5 .3 to their expected noise levels. The $95 \%$ confidence limits are given by the dashed lines. The pairs of vertical lines mark the Nyquist frequency $(.5 \mathrm{cph})$ and a point two octaves below the Nyquist frequency. This is the frequency range in which the Aanderaas are expected to have trouble (see Appendix $A$ ). Curves are omitted at the points which are further than six standard deviations from the mean. 
Single current meter dato vs. GM79

Instrument: U203

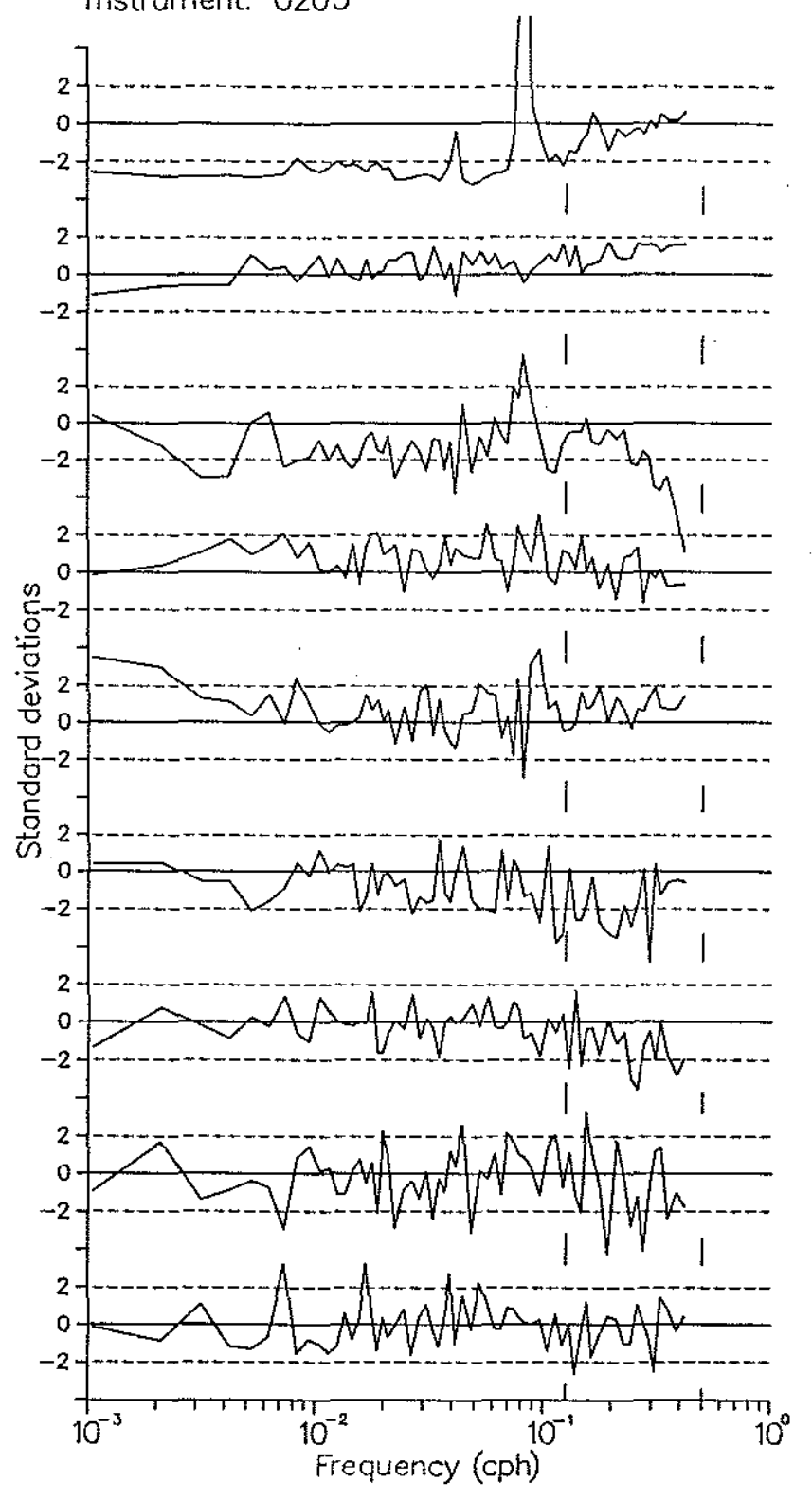

\section{Figure 4.5.4d U203 data compared to GM79}

These nine plots compare the zero mean estimators of figure 4.5 .3 to their expected noise levels. The $95 \%$ confidence limits are given by the dashed lines. The pairs of vertical lines mark the Nyquist frequency $(.5 \mathrm{cph})$ and a point two octaves below the Nyquist frequency. This is the frequency range in which the Aanderaas are expected to have trouble (see Appendix $A$ ). Curves are omitted at the points which are further than six standard deviations from the mean. 


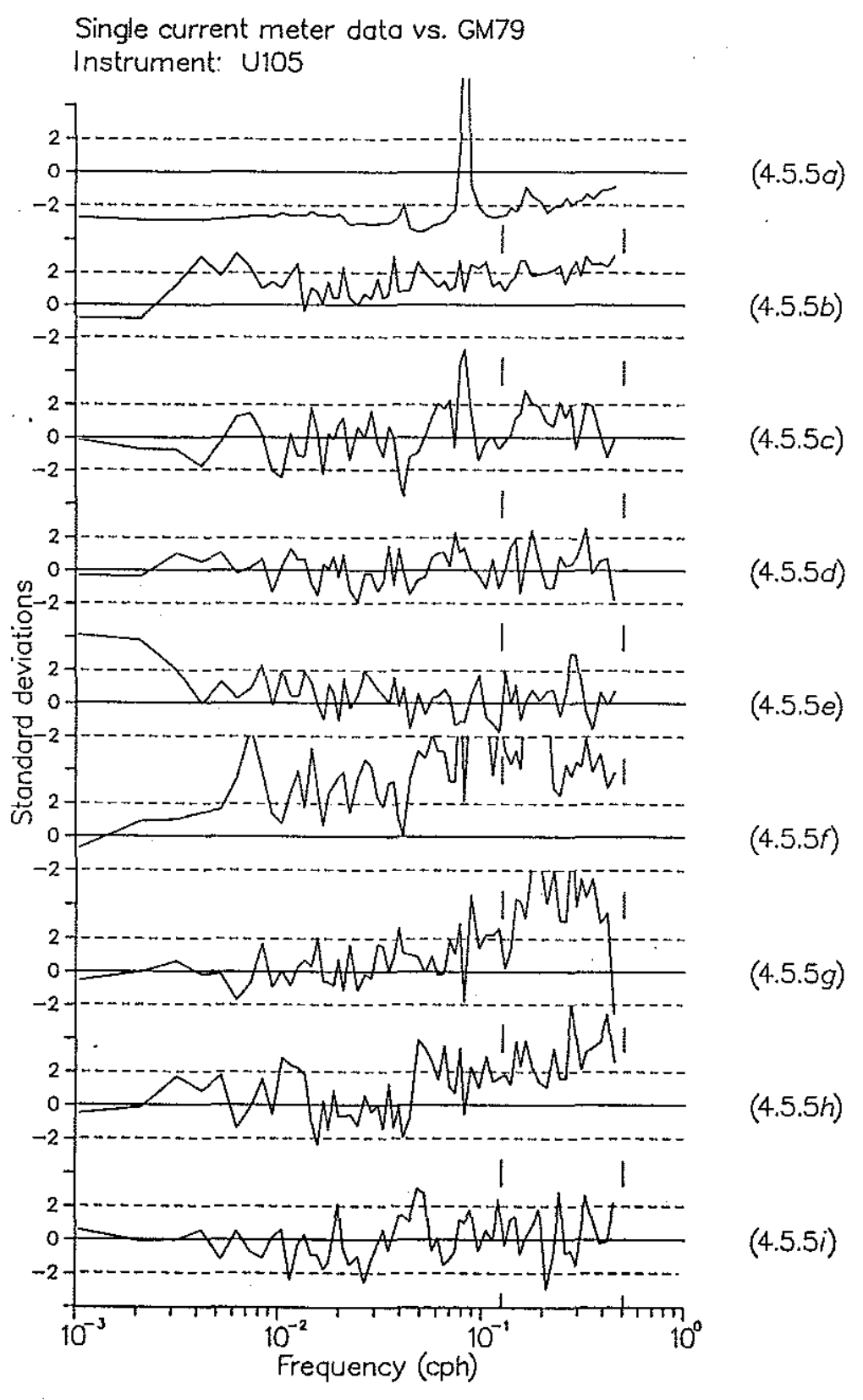

Figure 4.5.4e U105 data compared to GM79

These nine plots compare the zero mean estimators of figure 4.5.3 to their expected noise levels. The $95 \%$ confidence limits are given by the dashed lines. The pairs of vertical lines mark the Nyquist frequency $(.5 \mathrm{cph})$ and a point two octaves below the Nyquist frequency. This is the frequency range in which the Aanderaas are expected to have trouble (see Appendix $A$ ). Curves are omitted at the points which are further than six standard deviations from the mean. 


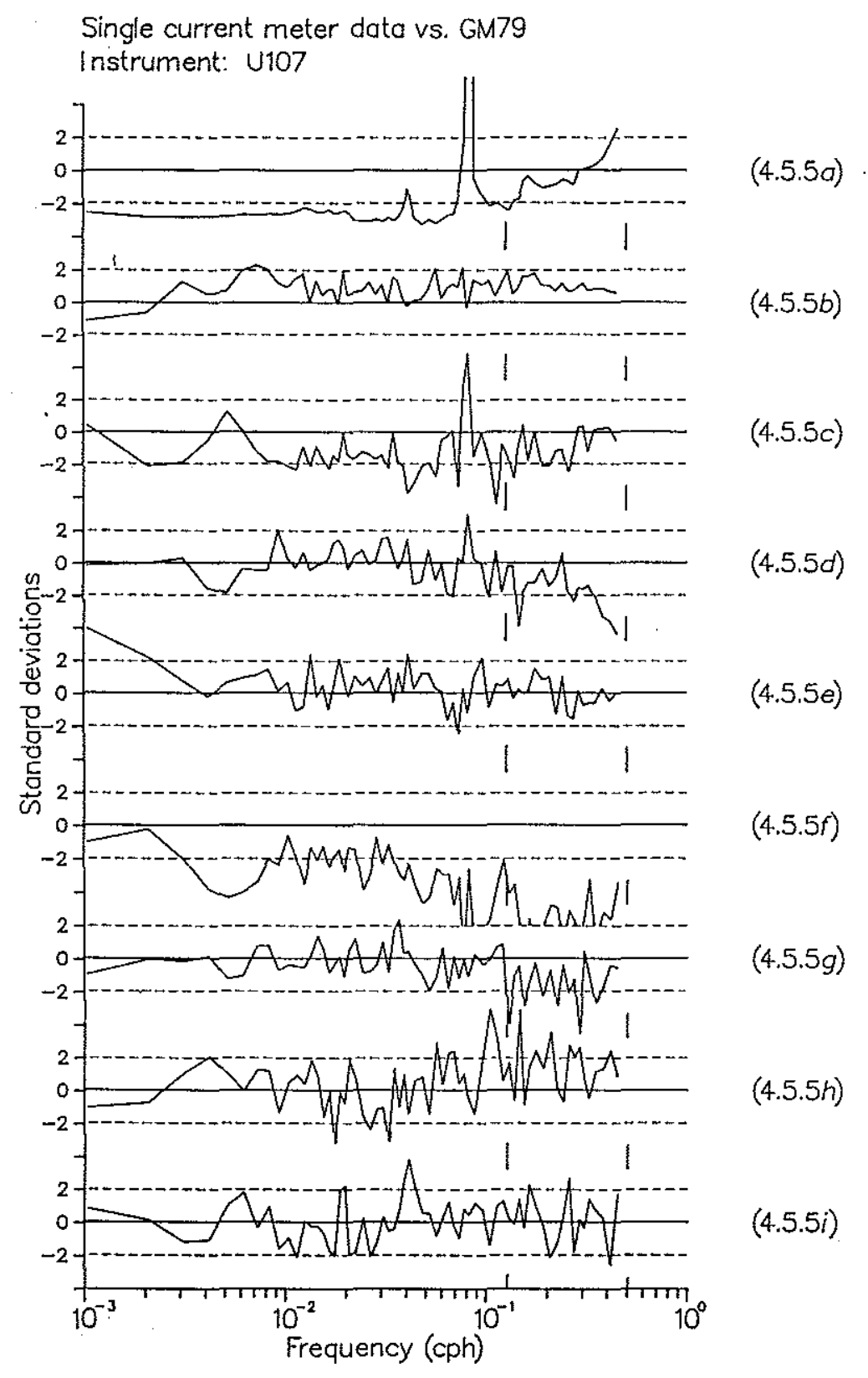

\section{Figure 4.5.4f U107 data compared to GM79}

These nine plots compare the zero mean estimators of figure 4.5.3 to their expected noise levels. The $95 \%$ confidence limits are given by the dashed lines. The pairs of vertical lines mark the Nyquist frequency $(.5 \mathrm{cph})$ and a point two octaves below the Nyquist frequency. This is the frequency range in which the Aanderaas are expected to have trouble (see Appendix $A$ ). Curves are omitted at the points which are further than six standard deviations from the mean. 
Single current meter data vs. GM79

Instrument: U109

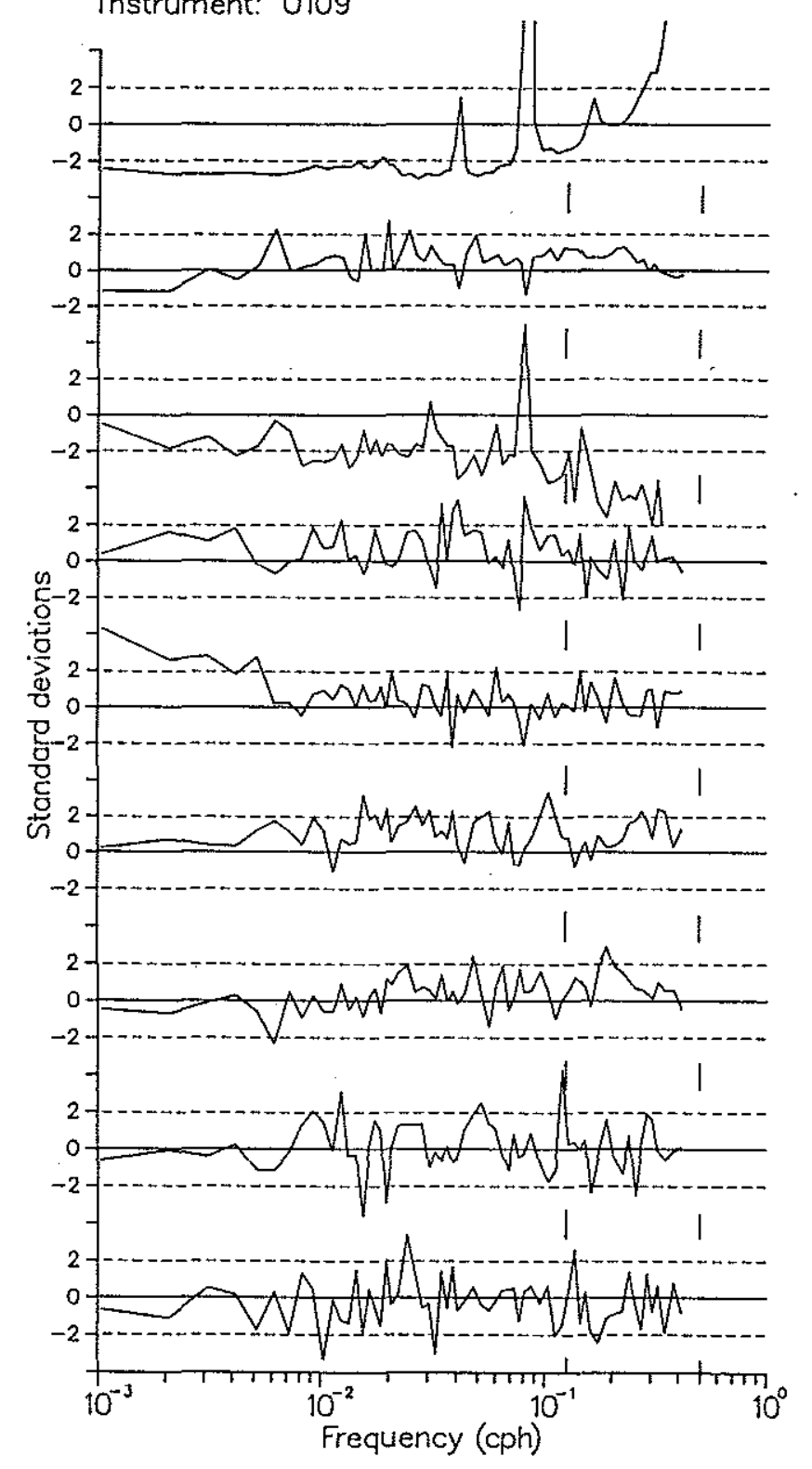

\section{Figure 4.5.4g U109 data compared to GM79}

These nine plots compare the zero mean estimators of figure 4.5 .3 to their expected noise levels. The $95 \%$ confidence limits are given by the dashed lines. The pairs of vertical lines mark the Nyquist frequency $(.5 \mathrm{cph})$ and a point two octaves below the Nyquist frequency. This is the frequency range in which the Aanderaas are expected to have trouble (see Appendix $A$ ). Curves are omitted at the points which are further than six standard deviations from the mean. 
Single current meter dato vs. GM79 Instrument: U111

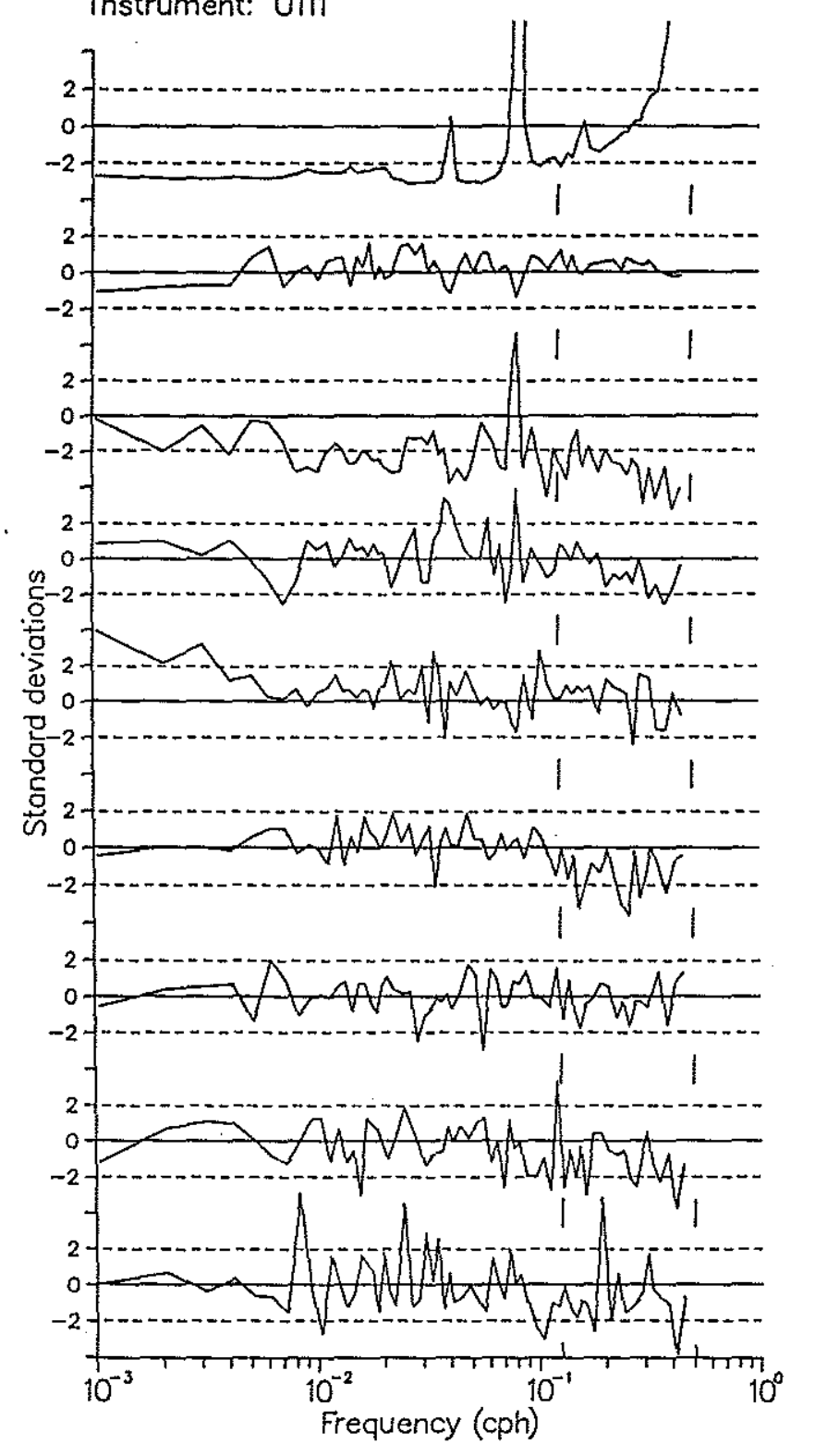

\section{Figure 4.5.4h U111 data compared to GM79}

These nine plots compare the zero mean estimators of figure 4.5 .3 to their expected noise levels. The $95 \%$ confidence limits are given by the dashed lines. The pairs of vertical lines mark the Nyquist frequency $(.5 \mathrm{cph})$ and a point two octaves below the Nyquist frequency. This is the frequency range in which the Aanderaas are expected to have trouble (see Appendix $A$ ). Curves are omitted at the points which are further than six standard deviations from the mean. 
too: the visual survey shows significant deviations for the -05 instruments. But certainly the most robust results are the positive $u T$ correlation at the -05 instruments, and the negative $u T$ correlations at the -07 instruments. It is this signal that the remainder of this section will attempt to explain.

\section{Alternate models}

The next step is to determine which of the many possible modifications to the GM79 model can possibly account for those two major differences between the model and data: the tendency to have more meridional energy than zonal energy, and the strong in-phase $\langle u T\rangle$ correlations. The possible modifications can be broadly grouped as instrumental errors, alternate assumptions, and additional dynamics.

One possible instrument problem has been referred to several times and is discussed at length in appendix $A$ : the Aanderaa current meter's sampling scheme introduces an aliasing error which forces discarding the highest two octaves of data. The essence of the the argument is simple. The Aanderaa is a standard rotor-vane (RV) current meter: it periodically stores direction and rotor count. By differencing rotor counts, an averaged speed is calculated. That speed is then combined with the instantaneous direction to get velocity components. This creates an aliasing problem in direction. To avoid that aliasing problem, a vector averaging current meter (VACM) stores vector averaged velocities in addition to the variables that the RV instruments store. Since a VACM stores both kinds of records, one can compare the two different sampling schemes for a particular environment. Comparisons using this property of VACM's have already been done for JASIN wind measurements by Weller et al. 1978. Because the aliasing process is inherently nonlinear, it is not possible to directly transfer results calculated on atmospheric data to results for oceanic data. Thus a comparison made with oceanic data is necessary, and it is presented in figure 4.6.3. It is calculated with data from the Indian Ocean Experiment (INDEX79), an equatorial experiment made in the Indian Ocean. The figure shows clear differences in both velocity components, with the Aanderaas showing excess energy, but the differences are small for frequencies less than two octaves below the Nyquist frequency. So these sorts of aliasing errors could not account for all the discrepancies between GM79 and the measurements.

Mooring motion is another possible instrumental problem, but mooring motions cannot account for the model-measurement differences. Strong temperature-pressure 
Instrument (4.5.5a) (4.5.5b) (4.5.5c) (4.5.5d) (4.5.5e) (4.5.5f) (4.5.5g) (4.5.5h) (4.5.5i)

Q101

Q201

U201

Q103

U103

Q203

U203

U105

Q205

U205

Q107

U107

U207

Q109

U109

Q209

U209

Q111

U111

Q211

U211
$-2$

$-2$

$-2$

$-2$

$-2$

$-2$

$-2$

$-2$

$-2$

$-2$

$-2$

$-2$
$-2$

$\begin{array}{lll}4 & 2 & 2 \\ 6 & 2 & \\ 6 & 2 & 2\end{array}$

$\begin{array}{lll}-6 & & 2 \\ -6 & -2 & 2 \\ -2 & & -2\end{array}$

2

2

$-2$

2

2

2

2

2

$-2$

$-2$

$-2$

$-2$

Figure 4.6.1 Table of significant deviations

This table presents the results of a qualitative survey of the single current meter vs. GM79 plots. The numbers are standard deviations exceeded, that number being truncated to the nearest multiple of 2 . 


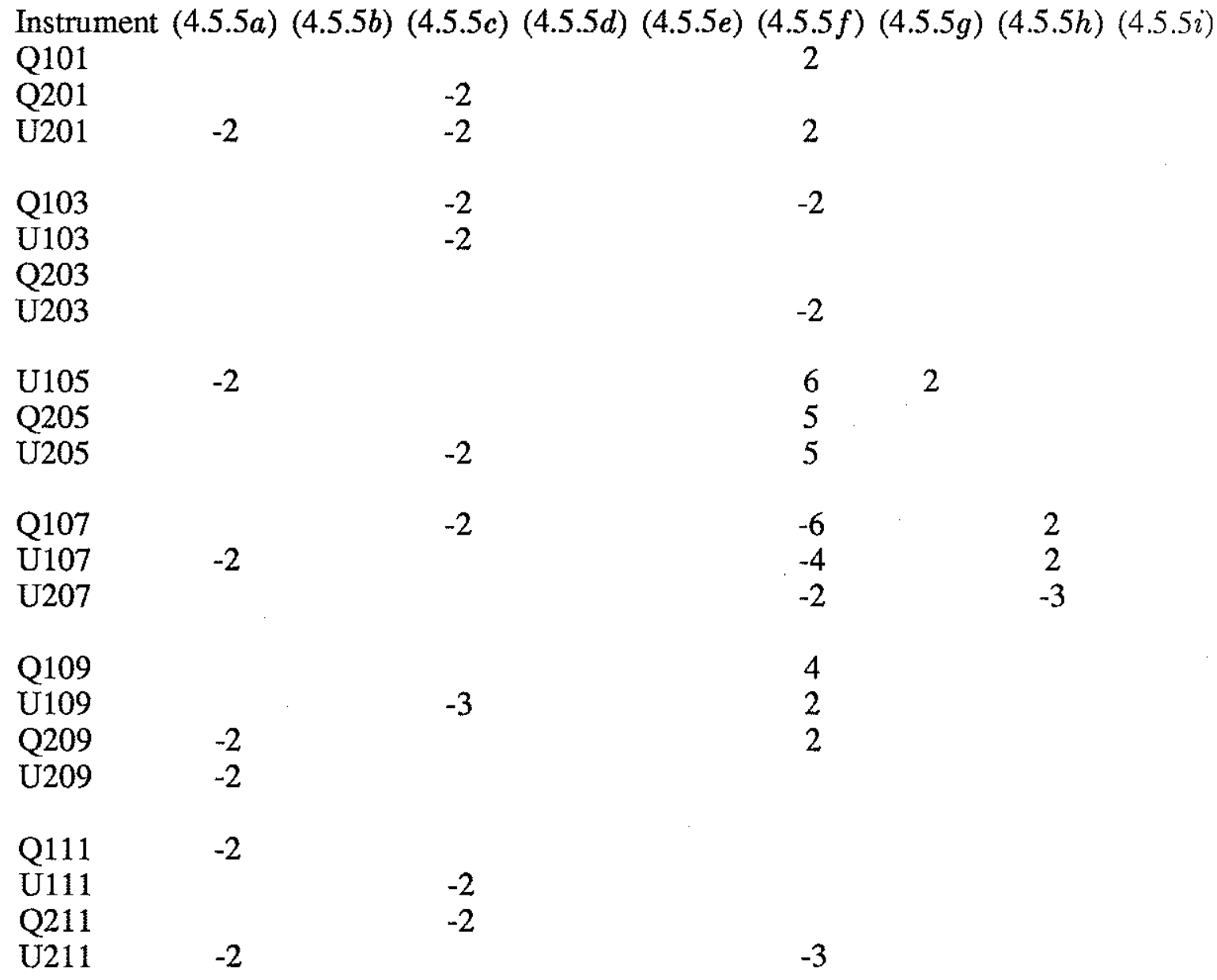

Figure 4.6.2 Table of averaged deviations

This table presents the deviations averaged over a frequency band that encompasses periods from 10 to 8 hours. The deviations are from the single current meter vs. GM79 plots. The numbers are standard deviations exceeded, that number being truncated to the nearest integer and numbers less than 2 are omitted. 

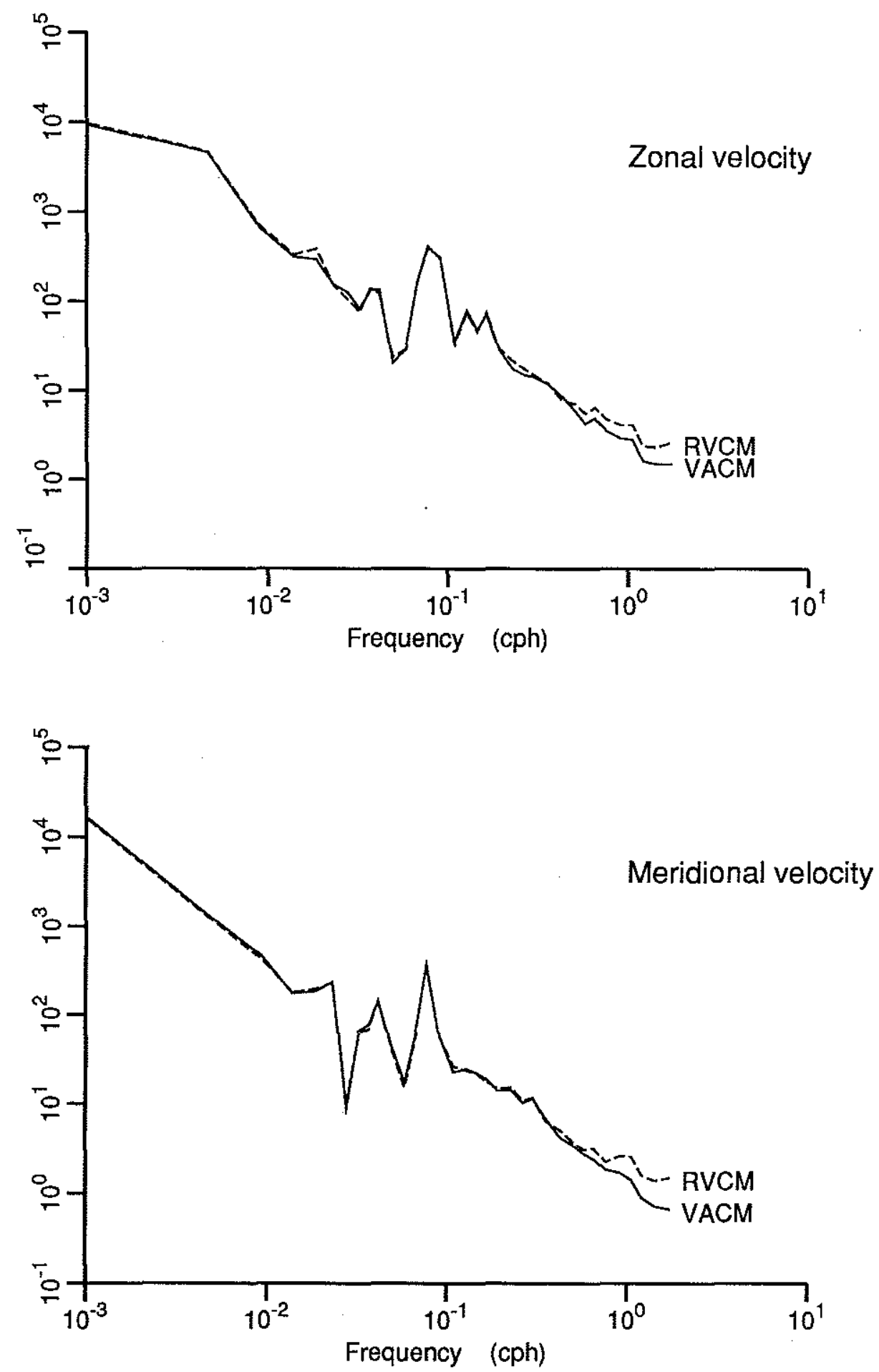

Figure 4.6.3 Current autospectra from VACM/RVCM

Autospectra of current comparing a vector averaged version of an INDEX79 record to a standard rotor-vane version of the same record. The top plot compares zonal kinetic energy calculated from vector-averaged data (VACM) to the rotor-vane version (RVCM). The rotor-vane version shows excess energy. The bottom plot compares meridional kinetic energy calculated from vector-averaged data (VACM) to the rotor-vane version (RVCM). Just as with the zonal kinetic energy case, the rotor-vane version shows excess energy. 
correlations should be evident if mooring motion is significant, but the temperaturepressure records do not show the simple phase lag that would be expected if the correlation were due to advection of the instrument. Furthermore, strong $\langle u T\rangle$ correlations are only found at the mid-depth instruments, but the mid-depth TP records are not particularly different from the other TP records.

Another sort of model modification that may help explain the model-data differences is to alter some of the symmetry assumptions of the isotropic internal wave models. The general internal wave models in chapter 5 define a four component generalized spectral level $(E, \Delta, P, Q)$ which allows a quantitative measure of the possible degrees of asymmetry in an internal wave model (see section 5.8). $E$ is the spectral level and is much the same as the $E$ in section 4.2. $\Delta$ is the difference between upward and downward propagating energy. $P$ and $Q$ are the two components of modal energy (the relative proportion of energy between $P$ and $Q$ gives the depths of the zero crossings). Thus the ratio of $\Delta$ to $E$ gives the percentage vertical asymmetry of the wavefield, while the ratio of $\sqrt{P^{2}+Q^{2}}$ to $E$ gives the percentage modal character of the wavefield. Thus the assumptions of vertical symmetry and WKBJ vertically propagating that were made in forming GM79 are equivalent to saying that three of the wave amplitude vector components $(\Delta, P, Q)$ are identically zero. By defining an equivalent vertical coordinate $\phi$

$$
\phi=2 \int_{z_{0}}^{z} m\left(z^{\prime}\right) d z^{\prime}
$$

and using the definition of $\lambda$ given by equation 4.2.3, a relatively general version of equation 4.2.2 that allows asymmetries can be written: equation 4.6.2.

It is clear from equation 4.6 .2 that this vertical asymmetry $(\Delta)$ affects only the imaginary parts of the current meter cross-spectra, thus it cannot account for either the discrepancies seen in the 4.5.5c estimator or the discrepancies seen in the 4.5.5 $f$ estimator. It could, however, account for the variations seen in $4.5 .5 \mathrm{~g}$ if they were sufficiently strong to consider modeling. Allowing non-zero $P$ and $Q$ would be equivalent to allowing modes in addition to the vertically propagating waves. Equation 5.9.6 suggests that a vertical array such as those discussed in chapters 5 and 6 would be much better at distinguishing between propagating waves and standing modes, since $E$ and $P$ and $Q$ are expressed differently in the measured cross-spectra that involve spatial separation. So most of the discussion will be delayed until those expanded data sets can be examined. But the 


$$
\begin{gathered}
{\left[\begin{array}{ccc}
\tilde{u}^{*} \tilde{u} & \tilde{u}^{*} \tilde{v} & \tilde{u}^{*} \tilde{T} \\
\tilde{v}^{*} \tilde{u} & \tilde{v}^{*} \tilde{v} & \tilde{v}^{*} \tilde{T} \\
\tilde{T}^{*} \tilde{u} & \tilde{T}^{*} \tilde{v} & \tilde{T}^{*} \tilde{T}
\end{array}\right]=\left[\begin{array}{ccc}
\frac{1}{2} \lambda N(1+\cos 2 \theta) & \frac{1}{2} \lambda N \sin 2 \theta & 0 \\
\frac{1}{2} \lambda N \sin 2 \theta & \frac{1}{2} \lambda N(1-\cos 2 \theta) & 0 \\
0 & 0 & \frac{1}{\lambda N}
\end{array}\right] \frac{\lambda_{0} E}{N_{0}}} \\
+\left[\begin{array}{ccc}
0 & 0 & \cos \theta \\
0 & 0 & \sin \theta \\
-\cos \theta & -\sin \theta & 0
\end{array}\right] i \frac{\lambda_{0} \Delta}{N_{0}} \\
+\left[\begin{array}{ccc}
-\frac{1}{2} \lambda N(1+\cos 2 \theta) \cos \phi & -\frac{1}{2} \lambda N \sin 2 \theta \cos \phi & -\cos \theta \sin \phi \\
-\frac{1}{2} \lambda N \sin 2 \theta \cos \phi & -\frac{1}{2} \lambda N(1-\cos 2 \theta) \cos \phi & -\sin \theta \sin \phi \\
-\cos \theta \sin \phi & -\sin \theta \sin \phi & \frac{1}{\lambda N} \cos \phi
\end{array}\right] \frac{\lambda_{0} P}{N_{0}} \\
+\left[\begin{array}{ccc}
-\frac{1}{2} \lambda N(1+\cos 2 \theta) \sin \phi & -\frac{1}{2} \lambda N \sin 2 \theta \sin \phi & \cos \theta \cos \phi \\
-\frac{1}{2} \lambda N \sin 2 \theta \sin \phi & -\frac{1}{2} \lambda N(1-\cos 2 \theta) \sin \phi & \sin \theta \cos \phi \\
\cos \theta \cos \phi & \sin \theta \cos \phi & \frac{1}{\lambda N} \sin \phi
\end{array}\right] \frac{\lambda_{0} Q}{N_{0}}
\end{gathered}
$$

possibility of using $P$ and $Q$ to explain the $u T$ in-phase correlations can be qualitatively explored with data limited to a single current meter. Equation 4.6 .2 gives the crossspectral matrix obtained from a single current meter in terms of the vector spectral level $(E, \Delta, P, Q)$. In particular, it gives the real part of the cross-power between $u$ and $T$ at zero separation as

$$
\operatorname{Re}\left\langle u^{*} T\right\rangle=\frac{\lambda_{0} T_{z}(z)}{N_{0}}[\langle\cos \theta \sin \phi P\rangle-\langle\cos \theta \cos \phi Q\rangle]
$$

For the particular case of understanding the results at the -05 and -07 instruments, choose a reference depth $z_{0}$ between them. Then $\phi$ will be positive at the depths of the -05 instruments, and negative at the depths of the -07 instruments. Thus the results that $u T$ in-phase correlations are positive at -05 instruments and negative at -07 instruments could be explained by $P$ modal energy that has a direction dependence $\cos \theta$. ( $P$ modal energy has an anti-node at the reference depth, which in this case would be positive.) If modal energy had simply been added to an isotropic wave field, then energy would be manifest both in $E$ and $P$ (see section 5.8). But a $\cos \theta$ dependence in $E$ does not affect the measured cross-spectra: equation 4.6.2 shows that only the isotropic and $\cos 2 \theta$ parts of $E$ get expressed in the single current meter cross-spectra. So a zonally oriented modal component to the wave field is consistent with the anomalies found. It remains to be seen in chapters 5 and 6 whether the model is still an adequate description when cross-spectra involving spatial separations are considered. 
Another type of possible model modification is to alter the physics behind the model. Small scale vertical temperature structure can be advected by internal waves, producing signals as measured by current meters that do not have the structure of simple internal waves. This temperature finestructure accounts for some types of differences between the GM79 model spectra and spectra from moored sensors (GM72) and is the generally accepted explanation for the phenomena characterized by Webster's rule (see section 1.2). Joyce and Desaubies (1977) conclude that finestructure contamination accounts for $10 \%$ of the auto-spectral level in the internal wave frequency band. This might help explain the excess temperature variance observed in the energy estimator plots of figure 4.5.2. But an enhanced auto-spectra cannot account for the large $u T$ coherence; it, in fact, would decrease that coherence.

A second possible modification to the physics would be to include the effects of shear on the internal wave field. The standard WKBJ theories of waves in a wind (Lighthill 1978) or shear flow (Olbers 1981) deal with the limit of wave packets that are small relative to the scale of the flow (for more references and discussion see section 5.1). Since the changes in the mean flow are gradual compared to the oscillations of the waves themselves, the dynamical balances which determine the interrelationships of $u$, $v$, and $T$ for the waves are unaffected by the shear: the mean shear only affects the amplitude as the waves travel from one part of the flow to another. The polarization relations are consequently unchanged, so the rejection of the isotropic no-mean-shear model also implies the rejection of the isotropic mean-shear model. Of course, the presence of mean shear implies the possibility of critical layers, in which case parts of the spectrum cannot propagate in certain regions, and the energy spectrum is no longer isotropic. But equation 4.6.2 clearly indicates that that would not lead to $u T$ correlations: non-zero $P$ or $Q$ is required. Furthermore, the shears at the fairly deep $(500 m+)$ PEQUOD instruments are not really sufficient for critical layers as far as the energetic (low wavenumber) waves are concerned (see section 5.6). 


\subsection{Shear-modified waves}

While the small wave-packet theory of the previous section does not alter the polarization relations and thus does not change the relation between the vector spectral level $(E, \Delta, P, Q)$ and the measured cross-spectra from a single current meter, sufficiently small scale shear modifications of internal waves can affect the polarization relations that we have been using in the model-data comparisons. The dynamics of internal waves in a mean shear flow are examined in section 5.5. The section discusses and justifies some scaling assumptions, and then calculates single wave solutions. Section 5.10 uses these single wave solutions along with a WKBJ solution to the vertical structure equation to outline how to expand the cross-spectral matrix in terms of the four-component spectral level $(E, \Delta, P, Q)$. Consider a propagating wave model, so that the modal components $P$ and $Q$ are assumed to be zero. Performing the wave expansion for the current meter cross-spectra then results in the following relations

$$
\begin{gathered}
u^{*} u+v^{*} v=\left[\frac{1}{2} K^{2} U_{z}^{2}+\left(N^{2}-\sigma^{2}\right) K^{2}+K \sigma U_{z z} \cos \theta-\frac{1}{2} K^{2} U_{z}^{2} \cos 2 \theta\right] E / m \sigma^{2} \\
u^{*} u-v^{*} v=\left[\frac{1}{4} K^{2} U_{z}^{2}+\frac{1}{2} K \sigma U_{z z} \cos \theta+\left(N^{2}-\sigma^{2}-\frac{1}{2} U_{z}^{2}\right) K^{2} \cos 2 \theta\right. \\
\left.+\frac{1}{2} K \sigma U_{z z} \cos 3 \theta+\frac{1}{4} K^{2} U_{z}^{2} \cos 4 \theta\right] E / m \sigma^{2} \\
u^{*} v=\left[\frac{1}{4} K \sigma U_{z z} \sin \theta+\frac{1}{2}\left(N^{2}-\sigma^{2}-\frac{1}{2} U_{z}^{2}\right) K^{2} \sin 2 \theta+\frac{1}{4} K \sigma U_{z z} \sin 3 \theta\right. \\
\left.+\frac{1}{8} K^{2} U_{z}^{2} \sin 4 \theta\right] E / m \sigma^{2}-i \Delta K \sin \theta U_{z} / \sigma \\
u^{*} \eta=\frac{1}{2}[-1+\cos 2 \theta] U_{z} K^{2} E / m \sigma^{2}-i \Delta K \cos \theta / \sigma \\
v^{*} \eta=\frac{1}{2} \sin 2 \theta U_{z} K^{2} E / m \sigma^{2}-i \Delta K \sin \theta / \sigma \\
\eta^{*} \eta=K^{2} E / m \sigma^{2}
\end{gathered}
$$

where the dispersion relation is

$$
m^{2}=\frac{N^{2}-\sigma^{2}}{\sigma^{2}} K^{2}+\frac{K \cos \theta}{\sigma} U_{z z}
$$

and $\eta$ can be related linearly to temperature fluctuations $T$. Equation 4.7.1d indicates that as long as the mean zonal velocity shear $U_{z}$ is non-zero an isotropic $E$ will result in a non-zero $u T$ correlation. So this is a possible mechanism for that part of the observations. Those correlations depend on the vertical gradient of mean velocity $U_{z}$. Without knowledge of what that gradient might be, there is no way to do the comparison 
as it was done for the stationary basic state case. It is possible, however, to estimate what sort of shear is necessary for the observed correlations, and compare those estimates with the direct measurements available, namely the White Horse velocity profiles.

\section{Estimating mean shear from high frequency internal waves}

The next step is to determine an estimator for the mean zonal velocity shear using the measured spectral quantities. Equation 4.7.1d gives the cross-spectrum of zonal velocity $u$ and displacement $\eta$. If one considers only the real part, the cross-spectra only depends on the $E$ component of the vector spectral level. One manipulation of the second moments in equation 4.7.1 that returns the mean velocity shear is then to take the ratio of the real part of the zonal velocity-vertical displacement cross-spectra and the displacement autospectra.

$$
\text { Real }\left[\frac{\left\langle u^{*} \eta\right\rangle}{\left\langle\eta^{*} \eta\right\rangle}\right]=-\frac{U_{z}}{2}+\frac{1}{2} \frac{\left\langle\cos 2 \theta U_{z} K^{2} E / m \sigma^{2}\right\rangle}{\left\langle K^{2} E / m \sigma^{2}\right\rangle}
$$

To the extent that the wave field does not have quadrapole symmetry horizontally (by being horizontally isotropic, for example), the second term is smaller than the first. Thus it is an approximate expression for the vertical gradient of mean velocity (as perceived by the internal wave field). The actual measurements are in terms of temperature, so the final expression is,

$$
U_{z}=2 T_{z} \operatorname{Real}\left(\frac{\left\langle u^{*} T\right\rangle}{\left\langle T^{*} T\right\rangle}\right)
$$

That ratio of cross spectra is the transfer (or admittance) function for velocity considered a function of temperature only, and selected plots from the PEQUOD data set are in figure 4.7.1. There are several things to be noted. At the depths where there are strong $\langle u T\rangle$ correlations, the strong correlations are present at both the moorings $(Q$ and $U)$. The ratio does not have much frequency dependence, which is what is expected from the theory. Finally, there is a striking sign reversal between the -05 and -07 instruments, instruments that are only separated by one hundred meters. 

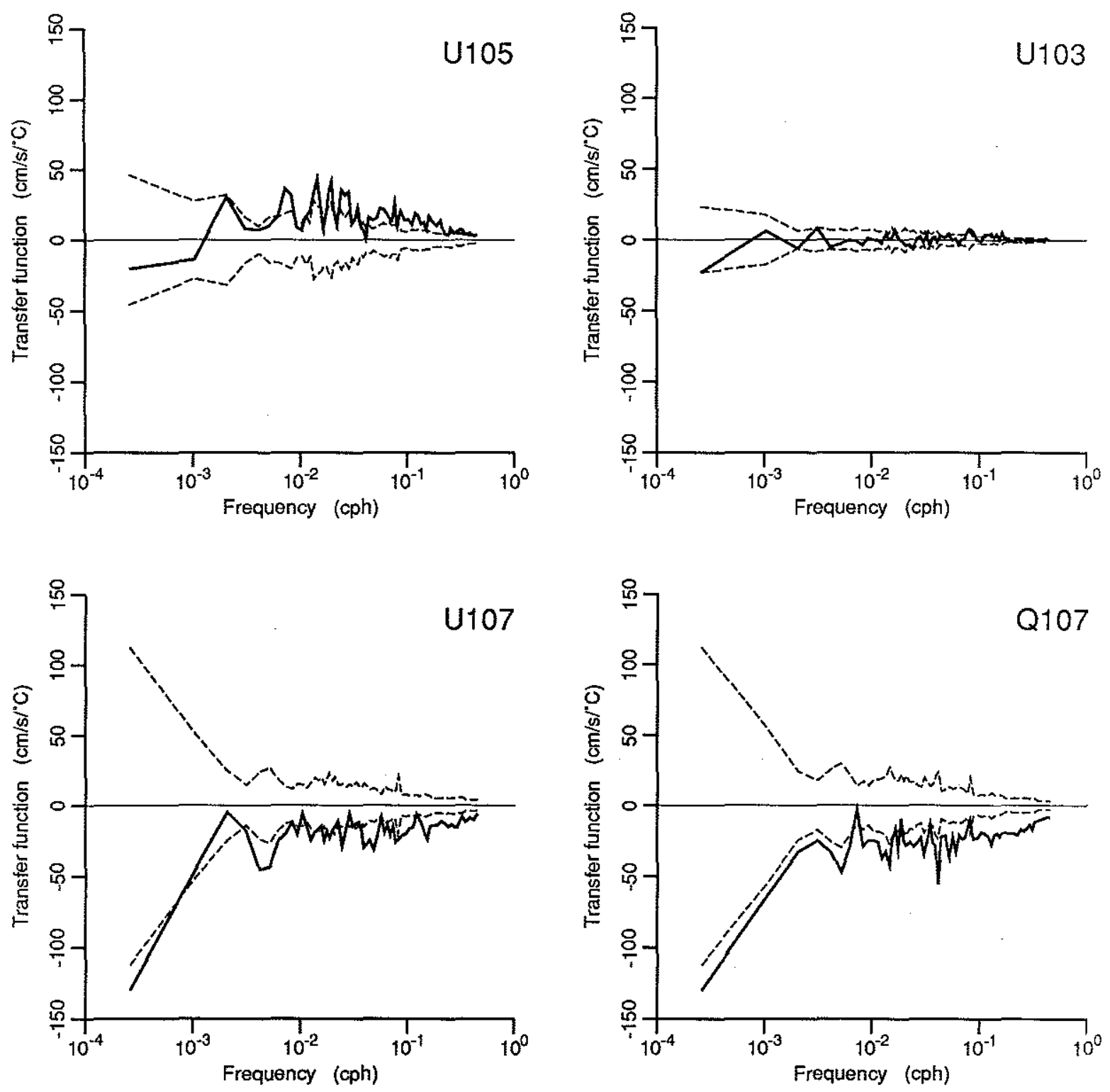

Figure 4.7.1 Transfer function of east velocity as a function of temperature

The solid curves give the real part of the transfer function of east velocity being considered a function of temperature. The dashed lines give the the zero significance level (doubled standard deviations). This noise level is taken from the covariance matrix of the cross-spectral estimators. It presumes a normal distribution, which is reasonable with high degrees of spectral averaging. Doubling one standard deviation gives the following expression for the smallest transfer function that can be distinguished from zero at $96 \%$ confidence:

$$
\sqrt{\left(\frac{2}{n_{r}}\right) \frac{\left\langle u^{*} u\right\rangle}{\left\langle T^{*} T\right\rangle}}
$$

This translates linearly to a smallest detectable mean shear. 


\section{Comparison with other mean shear estimates}

Finding independent measurements of these mean shears is rather difficult. The shears calculated from the internal wave spectra shears have been averaged for a year, and direct shear measurements were not made with the moored measurements. There are, however, instantaneous shear measurements made with the White Horse acoustic dropsonde. These are plotted in figure 2.1.5. The depths of the moored instruments are given in figure 2.1.3. Note that only the mid-depth instruments (depths between $1500-1600 \mathrm{~m}$, depths which correspond to instrument labels -05 and -07 ) are in a place where the shear could be constant from year to year: shear at other depths vary in sign as well as magnitude. This is consistent with the mean shear estimates calculated from the moored data, which have the strongest signals at mid-depths. The table of results (figure 4.7.2) confirms this result.

Several assumptions were made in deriving this result that merit further discussion. The assumption that there is less energy in the quadrapole symmetric part of $E$ than in the isotropic part of $E$ seems quite reasonable: were there any asymmetry in $E$ it seems more likely that it will be found oriented either along the zonal or the meridional direction, which would be dipole symmetry. The assumptions of zero $P$ and $Q$ are somewhat more arbitrary. As the discussion in section 4.6 points out, allowing a modal component (non-zero $P$ and $Q$ ) could account for the $u T$ correlation even without shear effects, so that allowing them to be non-zero means that both modes and mean shears could cause correlations. It remains to be seen whether the spatially separated cross-spectra used in chapters 5 and 6 can distinguish between the two possibilities. 
Figure 4.7.2 Table of Zonal Shear Estimates $U_{z}$ $\mathrm{cm} / \mathrm{s}$ per $100 \mathrm{~m}$

Whitehorse Velocity Profiles Total Jan $81 \quad$ Feb 82 Apr 82

Nets:

Depth

$\begin{array}{rrrrr}525 \mathrm{~m} & 1.50 & -9.30 & 3.60 & 20.00 \\ 600 \mathrm{~m} & -1.20 & 4.50 & -9.70 & 0.00 \\ 625 \mathrm{~m} & -4.80 & 9.60 & -22.00 & -8.40 \\ 1500 \mathrm{~m} & 1.60 & .80 & 11.00 & -11.00 \\ 1600 \mathrm{~m} & -10.00 & -12.00 & -9.50 & -7.20 \\ 1625 \mathrm{~m} & -13.00 & -12.00 & -9.70 & -21.00 \\ 3000 \mathrm{~m} & -0.84 & -2.90 & -6.70 & 12.00 \\ 3025 \mathrm{~m} & 3.80 & 5.50 & 2.00 & 3.00 \\ 3100 \mathrm{~m} & 0.09 & -2.40 & -.67 & 6.20 \\ 3125 \mathrm{~m} & -6.40 & -8.50 & -3.70 & -6.40\end{array}$

Internal wave estimates Jan 81 to Feb 82 estimate zero sig.

$\begin{array}{rr}20.00 & 10.00 \\ 5.50 & 4.80 \\ & \\ 6.10 & 3.00 \\ -6.00 & 2.90 \\ -8.20 & 2.80 \\ 2.10 & 1.40 \\ -1.80 & 1.70\end{array}$




\section{Chapter 5: Internal wave models}

\subsection{Introduction}

The Garrett and Munk models reviewed in chapter 1 interpret current meter data in terms of a horizontally isotropic, vertically symmetric, WKBJ vertically propagating internal wave field. But chapters 2 through 4 show that such a model is inadequate, and suggest four changes. Vertical asymmetry and horizontal anisotropy are suggested both by the theoretical considerations in chapter 2 and by the data fits of chapter 4 . A more careful determination of the vertical structure is suggested in chapter 2 on the grounds that the WKBJ approximation is inappropriate for determining the structure of low wavenumbers, a change that could be important since low wavenumbers contain most of the energy. Including both modes and propagating waves in the internal wave field could both explain the observations in chapter 3 and be consistent with the theoretical considerations which show that higher wavenumbers are scattered and thus lose the phase-locking characteristic of modes. Finally, mean shear effects on the internal wave field are both expected from a consideration of the relevant parameters (chapter 2) and offer a possible explanation for features in the single current meter cross-spectra (chapter 4). This chapter does the theory necessary to incorporate these generalizations of GM into the internal wave model.

Past work in internal waves has covered many parts of the problem, though the full set of changes suggested above have never been incorporated into a single model. Lighthill 1968 and Phillips 1980 both provide extensive theoretical discussions of the properties of internal waves in a motionless basic state. Müller et al. 1978 use a WKBJ version of such waves to analyze the IWEX data set. Their final fit to the data is strongly parameterized, but they do allow some vertical asymmetry and some horizontal anisotropy. While they do not attempt a model that includes both standing modes and propagating waves, they do try to see whether the IWEX data is better fit by a field of standing modes or a field of propagating waves, concluding that there was little evidence favoring modes apart from frequencies near the local buoyancy frequency. The model derived here will have the dual advantage that it will be much less parameterized and that it allows a mixture of modes and propagating waves.

Vertically standing modes frequently are computed without making the WKBJ approximation (see Phillips 1980, for example), but the equivalent calculation for vertically 
propagating waves is much less common. Philander 1978 and Gent and Luyten 1985 both calculate the vertical structure of non-WKBJ propagating waves in their respective attempts to determine how much energy propagates from the surface into the abyssal equatorial ocean. Despite using the same method, the two papers came to opposite conclusions. While the technique of determining the vertical structure used in this chapter is the same as these two earlier papers, the use of the vertical structure in modelling the data is quite different in that this chapter does not assume that all the energy is propagating downwards in the deep ocean, avoiding the problems that leave the two papers in total disagreement.

Work on internal waves in a parallel shear flow has generally been one of two sorts: either vertically propagating WKBJ waves in a slowly varying basic state or vertically standing modes in a basic state which is allowed to vary arbitrarily rapidly. (the one exception to this two part division is Olbers $1981 b$, a paper which uses a scattering approximation to find solutions in the limit that the variations in $U_{(z)}$ and $N(z)$ are much smaller than the scale of the internal waves (Born approximation)). WKBJ waves in a shear flow are examined in three papers by Francis Bretherton: Bretherton 1966, which is one of the earliest papers to deal with internal wave groups propagating in a shear flow, Bretherton and Garrett 1969, which considers general WKBJ waves in inhomogeneous basic states, and Booker and Bretherton 1966, which examines what happens to internal wave packets as they approach critical layers. Other papers that look at WKBJ wave packets include Olbers 1981a, which looks at internal wave packets in a geostrophic flow, and Jones 1968, which extends the results of Booker and Bretherton 1966 to include rotation.

Vertically standing modes in a mean shear flow are discussed by Phillips 1980: the eigenvalue problem that corresponds to shear modes is derived for a fairly general basic state. Peters 1983 uses shear modes to analyze upper ocean data from GATE (GARP Atlantic Tropical Experiment) (GARP is the Global Atmospheric Research Program). The work is similar to the Müller et al. 1978 interpretation of IWEX data in that the results are heavily parameterized, but the work is not a best fit of the data. Rather it is a qualitative comparison of the spectral features seen in the data and the features seen in the spectral models. Peters finds that while some spectral features are explained by kinematic shear effects, a horizontal anisotropy is required as well (since the analysis is in terms of modes there is no vertical anisotropy). 
While there are no papers that have considered both standing modes and propagating waves, the critical layer problems as they are examined by Booker and Bretherton 1966 and Jones 1968 are in some sense midway between the true WKBJ propagating solutions and vertically standing modes. The vertical structure equation that is solved (the TaylorGoldstein equation) is the same for both the critical layer problem and the shear mode problem, the primary difference being the choice of boundary conditions: the critical layer investigations match to a vertically propagating solution away from the critical layer while the shear mode calculations impose reflecting boundaries at the ocean top and bottom. The critical layer papers thus can be considered as bridging the gap between the papers that consider only vertically propagating waves and the papers that consider only vertically standing modes.

The analysis in this chapter is divided into three major parts. The first part determines the properties of the equatorial basic state and derives the equations of motion for small perturbations about that state. Section 5.2 examines the basic state itself: it is shown that it is possible to characterize the basic state by a mean zonal velocity $U(y, z)$ and a buoyancy frequency profile $N(y, z)$. Section 5.3 then derives the equations of motion for small perturbations to this basic state. But allowing the mean zonal velocity $U$ and the buoyancy frequency $N$ to depend on meridional position $y$ is more general than is necessary to interpret high-frequency current meter spectra. Scaling arguments in section 5.5 show that it is consistent to consider the basic state as being characterized by a mean zonal flow, $U_{(z)}$, and a buoyancy frequency profile $N(z)$ that are functions only of depth (i.e. the meridional variations of the two basic state functions $U(y, z)$ and $N(y, z)$ can be sufficiently small that they do not alter the first order dynamics of the wavelike perturbations).

The second part of the analysis focuses on the wave solutions that can exist in the equatorial basic state. Characterizing these wave solutions falls naturally into two parts: polarizations such as equation 1.3.4 which relate different dynamical variables $u, v, \eta, \ldots$ to the wave amplitude, and dispersion relations such as equation $1.3 .3 b$ which interrelate frequency and wavenumber. Because $U_{(z)}$ and $N(z)$ depend on the vertical coordinate $z$, the dispersion relation depends on $z$ as well. Consequently the dispersion relation is incorporated into the wave solutions in the process of finding solutions to the vertical structure equation (1.3.3a in section 1.3). Section 5.4 finds the polarization and dispersion relations for equatorial internal waves in a stationary basic state, while section 
5.5 finds the polarization and dispersion relations for the basic state where the mean zonal flow $U(z)$ is not necessarily zero. Section 5.6 then looks at the basic properties of the dispersion relation/vertical structure equation.

The third part of the analysis relates the wave solutions to the current meter crossspectra. Relating waves to cross-spectra involves a mixture of statistics and linear wave dynamics: sections 5.7 through 5.10 focus on wave properties, while the statistical aspects are dealt with in chapters 6 and 8. Sections 5.9 and 5.10 are essentially examples, showing what spectra look like using the internal waves found in section 5.4 and 5.5. Section 5.8 is more general: it develops the connection between the measured cross-spectra and the four component wave spectra $(E, \Delta, P, Q)$, the difference components of which give the relative strength of upward propagating waves, downward propagating waves, and standing modes. Section 5.8 not only shows that propagating waves and standing modes can co-exist, it shows how the measured cross-spectra can be used to distinguish between propagating waves and standing modes.

\subsection{Equatorial Basic State}

As an approximation to the Pacific background flow, consider a purely zonal flow $U(y, z)$ that is steady and zonally independent. Cane, 1980, discusses equatorial currents and their dynamics for more general forms of solution, but for the relevant case of steady zonally independent zonal flow the essential dynamics reduce to geostrophic balance of the zonal current and hydrostatic balance of vertical momentum. The corresponding equations are:

$$
\begin{gathered}
f U=-\frac{1}{\rho} P_{y} \\
-g \rho=P_{z} \\
P_{x}=0 \\
V=W=0
\end{gathered}
$$

where $(U, V, W)$ is the velocity vector, $\rho$ is the density, $P$ is the pressure, $(x, y, z)$ is the position with the $x$ direction being eastward, and subscripts denote differentiation. Combined, these equations give a thermal wind balance

$$
f \partial_{z}(\rho U)=g \rho_{y}
$$


As far as this chapter is concerned, this time independent flow is important only insofar as the internal waves are affected. To develop this connection, first consider a density field whose meridional dependence is only a small perturbation on a density field that has only vertical variation

$$
\rho(y, z)=\rho_{0}(z)+\rho^{\prime}(y, z) \quad \rho^{\prime} \ll \rho_{0}
$$

Further define a buoyancy frequency $N(y, z)$ such that

$$
N^{2}(y, z)=-\frac{g}{\rho} \partial_{z} \rho \approx-\frac{g}{\rho_{0}} \partial_{z} \rho
$$

As will become clear in section 5.3, as far as the internal waves are concerned the basic state can be characterized by two functions: the zonal velocity $U(y, z)$ and the buoyancy frequency $N(y, z)$. These functions are not independent, however. To see the connection, rewrite the thermal wind balance (equation 5.2.2 in terms of $N^{2}$.

$$
f \frac{1}{\rho_{0}} \partial_{z z}(\rho U)=-\partial_{y} N^{2}(y, z)
$$

The Boussinesq approximation leads to a further simplification

$$
f U_{z z}=-\partial_{y} N^{2}(y, z)
$$

Thus non-zero curvature in the zonal velocity requires meridional dependence in the buoyancy frequency. For example, consider a separable form for $U$ with a gaussian meridional dependence

$$
U(y, z)=U_{0}(z) e^{-\frac{1}{2}(y / L)^{2}}
$$

By making the equatorial $\beta$-plane approximation, $f=\beta y$, the equation 5.2 .6 becomes

$$
\beta y U_{z z}=-\partial_{y} N^{2}(y, z) .
$$

The chosen form for $U(y, z)$ has a first meridional derivative proportional to $y$ times itself, so equation 5.2.8 immediately suggests the solution

$$
N^{2}(y, z)=N_{0}^{2}(z)+\beta L^{2} U_{z z} .
$$

This makes quite explicit the connection between vertical curvature of velocity and meridional dependence of $N^{2}(y, z)$. However, in section 5.5 it is demonstrated that the effect on the waves of the meridional dependence of $N$ and $U$ are actually quite small. Thus meridional dependence of the basic state be ignored in determining the structure of the wavelike solutions. 


\subsection{Equatorial beta plane perturbation equations}

This section derives equations for perturbations around the basic state outlined in section 5.2. In particular, it scales the equations to show that, as far as the internal waves are concerned, the basic state is sufficiently characterized by the mean velocity $U(y, z)$ and the buoyancy frequency $N(y, z)$. The equations for perturbations around an equatorial mean zonal flow are given by Eriksen, 1985. The linear, inviscid, incompressible and Boussinesq equations are

$$
\begin{array}{r}
u_{t}+U u_{x}+w\left(U_{z}+f_{H}\right)-f v+P_{x}=0 \\
v_{t}+U v_{x}+f u+P_{y}=0 \\
w_{t}+U w_{x}-f_{H} u-b+P_{z}=0 \\
b_{t}+U b_{x}-v g \frac{\partial_{y} \bar{\rho}}{\rho_{0}}+w N^{2}=0 \\
u_{x}+v_{y}+w_{z}=0 \\
\rho(y, z)=\bar{\rho}(y, z)-\frac{\rho_{0}}{g} b \\
\bar{\rho}(y, z)=\rho_{0}(z)+\rho^{\prime}(y, z)
\end{array}
$$

where

$u, v, w \quad$ denote velocity perturbations,

$U(y, z) \quad$ denotes the basic state velocity,

$P \quad$ denotes reduced pressure,

$b$ denotes buoyancy fluctuations,

$f$ denotes the vertical component of the Coriolis vector $2 \Omega \sin \theta$,

$f_{H}$ denotes the horizontal component of the Coriolis vector $2 \Omega \cos \theta$, and

$\theta \quad$ is the latitude.

The buoyancy equation (5.3.1d) is the only equation where information about the mean density field other than that provided by the buoyancy frequency $N(y, z)$ is necessary. But a scaling argument shows that meridional advection of buoyancy can be neglected with respect to vertical advection. This can be seen by using the thermal wind relation to rewrite the ratio of the two terms

$$
\frac{v \frac{g}{\rho_{0}} \partial_{y} \bar{\rho}}{w N^{2}}=\frac{v}{w} \frac{\beta y U_{z}}{N^{2}}
$$


The equatorial $\beta$-plane approximation has been used to write $f=\beta y$. The ratio of the two terms being small is equivalent to a restriction on the ratio of meridional to vertical velocity,

$$
\left|\frac{v}{w}\right| \ll\left|\frac{N^{2}}{\beta y U_{z}}\right|
$$

As Eriksen, 1985, points out, for relevant values of the parameters $(y<40 \mathrm{~km}, N=$ $1 \mathrm{cph}$, and $U_{z}<10^{-3} \mathrm{~s}^{-1}$ ), the right hand side is 3000 or greater. Thus equation 5.3 .3 does not constrain $|v / w|$ very much. The revised version of the buoyancy equation is then

$$
b_{t}+U b_{x}+N^{2}(y, z) w=0 .
$$

Note that the set of equations 5.3.1 $a, b, c, d^{\prime}, e, f$ only depend on the basic state through the mean zonal velocity $U(y, z)$ and the buoyancy frequency $N(y, z)$.

\section{Boundary Conditions}

Boundary conditions have not yet been specified. If the solutions have horizontal scales much smaller than the basin, the ocean can be considered horizontally infinite. This assumption can be verified once the solutions have actually been found. The underlying argument behind this assumption is that, while in an inviscid, unforced, linear ocean moving the boundaries far away is not the same as removing them entirely, to the extent that the ocean is weakly viscous, forced, and non-linear, any phase-locking of waves (or other boundary effects) will be destroyed once far enough from the boundaries. It is in that sense, then, that the ocean can be considered infinite. Vertically the situation is similar. The bottom boundary layer and the top mixed layer are not properly described by the inviscid, adiabatic perturbation equations, and it is not clear what sort of apparent boundary conditions they might present to the interior flow. In previous work, investigators have used either radiation boundary conditions which lead to vertically propagating solutions, or they have used reflecting walls at the ocean top and bottom, which lead to modal solutions (see section 5.1). This chapter chooses to not impose top and bottom boundary conditions, allowing both the solutions to radiation boundary conditions (vertically propagating waves) and the solutions to perfectly reflecting top and bottom boundaries (modes). So the work that follows is new in the sense that the boundary conditions are not imposed: an inviscid, unforced, linear domain is chosen, and the boundary values remain unspecified. In order to have wave solutions that are separable 
in the vertical, however, the maximum depth of the region of interest must be constant. Since the region of interest need not intersect the bottom, this is not a strong constraint. Furthermore, assuming constant depth is appropriate in interpreting the PEQUOD data since the bottom is relatively flat in the area of the the current meter array (see chapter 2). So, while boundary values are arbitrary, they are specified at a single depth to allow vertical separation.

\subsection{Single wave solutions: motionless basic state}

Waves in a motionless basic state are presented here for two reasons. First of all, the derivation is more straightforward than the derivation for waves in the presence of a mean flow, so the problem is an important intermediate step to the more general problem. Secondly, in trying to understand the data it is important to show that a relatively simple model (i.e. waves in a motionless basic state) does not quite work before proceeding to the more complicated model (mean velocity shear modified waves). This section finds the wavelike perturbations when the mean velocity shear is identically zero.

In the case where there is no mean flow, equation 5.2.6 implies that the buoyancy frequency $N(y, z)$ is actually independent of meridional dependence $y$. That greatly simplifies finding wavelike solutions to the system of equations 5.3.1. The simplified equations are

$$
\begin{aligned}
u_{t}+f_{H} w-\beta y v & =-P_{x} \\
v_{t}+\beta y u & =-P_{y} \\
{\left[\partial_{t t}+N^{2}(z)\right] w-f_{H} u_{t} } & =-P_{z t} \\
u_{x}+v_{y}+w_{z} & =0
\end{aligned}
$$

At this point there are a number of ways to proceed (see Gill 1982, for an approach that differs from that used here, while Munk 1980 uses a scheme which is essentially the same). These equations are independent of $x$ and $t$, so zonal plane waves are possible solutions (see Lanczos 1961 for a very good presentation of how to deal with general linear systems). For a particular frequency $\sigma$, and if the terms involving the horizontal component of the Coriolis vector $f_{H}$ are neglected, the equations are separable in $\mathrm{z}$ (Nonzero $f_{H}$ will be dealt with in section 5.5). Choosing solutions of the form

$$
w=w^{*}(y) G(z) e^{i(k x-\sigma t)}
$$


give the following separations and equations

$$
\begin{aligned}
& u=u^{*}(y) G_{z}(z) e^{i(k x-\sigma t)} \\
& v=v^{*}(y) G_{z}(z) e^{i(k x-\sigma t)} \\
& P=P^{*}(y) G_{z}(z) e^{i(k x-\sigma t)} \\
&-i \sigma u^{*}-\beta y v^{*}+i k P^{*}=0 \\
&-i \sigma v^{*}+\beta y u^{*}+P_{y}^{*}=0 \\
& i k u^{*}+v_{y}^{*}+w^{*}=0 \\
&\left(N^{2}-\sigma^{2}\right) w^{*} G(z)-i \sigma P^{*} G_{z z}(z)=0
\end{aligned}
$$

Equation 5.4.4d is separable, which is easily seen when it is rewritten

$$
\frac{\left(N^{2}(z)-\sigma^{2}\right) G(z)}{G_{z z}(z)}=\frac{i \sigma P^{*}(y)}{w^{*}(y)}=-c^{2}
$$

The first expression is only a function of $z$, while the second is only a function of $y$; thus they are equal to a separation constant denoted $-c^{2}$. Consequently equation 5.4 .5 can be split into both an equation for the vertical structure and an equation for the horizontal structure. Two more equations are thus added to the list (equation 5.4.4),

$$
\begin{gathered}
w^{*}=-\frac{i \sigma}{c^{2}} P^{*} \\
G_{z z}(z)+\frac{\left(N^{2}-\sigma^{2}\right)}{c^{2}} G(z)=0
\end{gathered}
$$

and equation 5.4.4c can be rewritten

$$
i k u^{*}+v_{y}^{*}-\frac{i \sigma}{c^{2}} P^{*}=0
$$

The next step in finding wavelike solutions is to solve the three horizontal structure equations 5.4.4a,b, $c^{\prime}$. These equations can be combined into a single equation for $v$,

$$
\begin{gathered}
v_{y y}+l^{2}(y) v=0 \\
l^{2}(y)=\frac{\sigma^{2}-f^{2}}{c^{2}}-k^{2}-\beta k / \sigma
\end{gathered}
$$


Eriksen, $1980 b$, gives this equation along with a discussion of the equatorially confined solutions appropriate to a meridionally unbounded ( $\beta$-plane) ocean.

To explore the particular case of the high frequency solutions, rewrite equation 5.4.6b,

$$
\left((k+\beta / 2 \sigma)^{2}+l^{2}\right)=c^{-2}\left(\sigma^{2}\left(1+\left(\beta c / 2 \sigma^{2}\right)^{2}\right)-f^{2}\right)
$$

Writing the equation in this form makes it clear that, if $\sigma^{2} \gg \beta c / 2$ and $k \gg \beta / 2 \sigma$, the dispersion relation is the same as the one for $f$-plane internal waves (see Gill 1982).

$$
k^{2}+l^{2} \approx c^{-2}\left(\sigma^{2}-f^{2}\right)
$$

If in addition, $\sigma^{2} \gg f^{2}$, then $f$ can be dropped as well, and the irrotational dispersion relation is appropriate. Because of the equatorial $\beta$-plane approximation $f=\beta y$, this becomes a condition on $y$.

$$
\sigma \gg \beta y \text { implies } y \ll \sigma / \beta
$$

Since $\sigma^{2} \gg \beta c / 2$, at its most restrictive this condition says that $y \ll \sqrt{c / \beta}$, which is roughly $300 \mathrm{~km}$. If the frequencies of interest are higher, then this condition ceases to be a restriction because the limit approaches the size of the earth. For example, for frequencies $.1 \mathrm{cph}$ and higher, the constraint becomes $y \ll 7640 \mathrm{~km}$, which is threequarters the distance from pole to equator. So as long as $\sigma^{2} \gg \beta c / 2, k \gg \beta / 2 \sigma$, and the latitude of interest is sufficiently close to the equator (a constraint that disappears at high frequencies), the dispersion relation for internal waves in an ocean that is not rotating is an adequate approximation.

$$
\sigma^{2}=c^{2}\left(k^{2}+l^{2}\right)
$$

For the latitudes of the PEQUOD array, the conditions are easily met.

The polarization vector gives the amplitude for each of the dynamical variables $u, v, w, P$ in terms of a wave amplitude $A$. It can be found by using the simplified equations 5.4.4 with $\beta=0$ and meridional wavenumber, $l$, constant.

$$
\begin{aligned}
-i \sigma u^{*}+i k P^{*} & =0 \\
-i \sigma v^{*}+i l P^{*} & =0 \\
w^{*}+\frac{i \sigma}{c^{2}} P^{*} & =0
\end{aligned}
$$


Therefore

$$
\left[\begin{array}{c}
\tilde{u} \\
\tilde{v} \\
\tilde{\eta} \\
\tilde{w} \\
\tilde{P}
\end{array}\right]=\left[\begin{array}{c}
\cos \theta \\
\sin \theta \\
0 \\
0 \\
c
\end{array}\right] G_{z}^{i}(z) A^{i}+\left[\begin{array}{c}
0 \\
0 \\
1 / c \\
-i \sigma / c \\
0
\end{array}\right] G^{i}(z) A^{i}
$$

where

$$
\begin{aligned}
\alpha(x, y, t) & =\tilde{\alpha} e^{i(k x+l y-\sigma t)} \\
(k, l) & =K(\cos \theta, \sin \theta) \\
-i \sigma \eta & =w
\end{aligned}
$$

and $A^{i}$ is the amplitude corresponding to the $i^{t h}$ solution of the vertical structure equation. (Here $\alpha$ represents any of the dynamical variables $u, v, \eta, w$, or $P$ ). The vertical displacement $\eta$ has been introduced because it is roughly proportional to temperature. Since the system is linear, any quantity proportional to $A^{i}$ will function just as well as $A^{i}:$ it is an arbitrary choice to make it such that $P^{*}=c A$.

In future references, equation 5.4.12 will be written in more compact vector notation (using boldface small letters to denote vectors and boldface capital letters to denote matrices).

$$
\begin{gathered}
\tilde{\mathbf{u}}^{i}=\mathbf{s} G_{z}^{i}(z) A^{i}+\mathbf{r} G^{i}(z) A^{i} . \\
\mathbf{u}^{i}(x, y, z, t)=\tilde{\mathbf{u}}^{i}(z) e^{i(k x+l y-\sigma t)} .
\end{gathered}
$$




\subsection{Single wave solution: high frequency waves with mean flow}

Allowing a non-zero mean flow is one possible generalization of the problem solved in section 5.4. Not surprisingly, the equations of motion are somewhat less tractable when the non-zero mean flow is allowed. Fortunately, section 5.4 shows that the beta effect can be neglected as long as the frequency of interest is sufficiently high. By neglecting the beta effect from the beginning, the equations of motion 5.3.1 can be simplified while retaining the non-zero mean flow. (Note that Jones 1968 solves the equations while retaining both an $f$-plane rotation and a nonzero mean flow). Further simplification is desirable, however, since there is a meridional dependence in the equations from both the mean velocity $U(y, z)$ and the buoyancy frequency $N(y, z)$. This dependence eliminates the possibility of exact solutions that are wavelike in the meridional direction. But when the meridional scale of the basic state mean flow is much greater than the scale of the wave motion, the solutions are approximately wavelike. These are the sorts of solutions that are found in this section. The scaling for the most part is taken from Eriksen 1985.

Since we are interested in equatorial regions, neglecting beta effect terms means that the vertical component of the Coriolis vector is negligible. The equations of motion near the equator without the beta effect are, from equation 5.3.1,

$$
\begin{aligned}
\left(\partial_{t}+U \partial_{x}\right) u+w\left(U_{z}+f_{H}\right)+P_{x} & =0 \\
\left(\partial_{t}+U \partial_{x}\right) v+P_{y} & =0 \\
{\left[\left(\partial_{t}+U \partial_{x}\right)^{2}+N^{2}(y, z)\right] w-f_{H}\left(\partial_{t}+U \partial_{x}\right) u+\left(\partial_{t}+U \partial_{x}\right) P_{z} } & =0 \\
u_{x}+v_{y}+w_{z} & =0 \\
\left(\partial_{t}+U \partial_{x}\right) \eta & =w
\end{aligned}
$$

Combine 5.5.1a, 5.5.1b, and 5.5.1 $d$ to get

$$
v_{x} U_{y}+\left(\partial_{x x}+\partial_{y y}\right) P+w_{x}\left(U_{z}+f_{H}\right)-\left(\partial_{t}+U \partial_{x}\right)\left(w_{z}\right)=0
$$

In combining equation 5.5.2 with equation 5.5.1c, the meridional dependence of $U$ and $N^{2}$ create some additional terms (as compared to the case where $U$ and $N^{2}$ have no meridional dependence). These terms arise in the following context

$$
\begin{array}{r}
\left(\partial_{x x}+\partial_{y y}\right)\left[\left(\partial_{t}+U \partial_{x}\right)^{2}+N^{2}(y, z)\right] w=\left[\left(\partial_{t}+U \partial_{x}\right)^{2}+N^{2}(y, z)\right]\left(\partial_{x x}+\partial_{y y}\right) w \\
+\left\{\left[4 U_{y}\left(\partial_{t}+U \partial_{x}\right) \partial_{x y}+2 U_{y y}\left(\partial_{t}+U \partial_{x}\right) \partial_{x}+2\left(U_{y}\right)^{2} \partial_{x x}+\partial_{y y} N^{2}+2 \partial_{y}\left(N^{2}\right) \partial_{y}\right] w\right\}
\end{array}
$$


The terms in braces are the terms that arise because of the meridional dependence of the basic state. Similar terms arise in manipulating the the $\mathrm{P}$ term of equation 5.5.1c,

$$
\left(\partial_{x x}+\partial_{y y}\right)\left(\partial_{t}+U \partial_{x}\right) P=\left(\partial_{t}+U \partial_{x}\right)\left(\partial_{x x}+\partial_{y y}\right) P+\left\{\left[U_{y y} \partial_{x}+2 U_{y} \partial_{x}\right] P_{z}\right\}
$$

In addition, there is an extra term that arises when the total derivative $\left(\partial_{t}+U \partial_{x}\right)$ is applied to equation 5.5.2:

$$
\left(\partial_{t}+U \partial_{x}\right) v_{x} U_{y}=P_{y x} U_{y}
$$

a relation which follows from 5.5.1b. Given certain scaling assumptions, all these new terms are insignificant. The first term of the right hand side of equation 5.5.3 $a$ can be scaled as

$$
\left(N^{2}-\sigma^{2}\right) K^{2} w=\left(-K^{2} \sigma+U k \sigma K^{2}+N^{2} K^{2}\right) w
$$

where $K^{2}$ scales total horizontal wavenumber, and $\sigma$ scales intrinsic frequency $(\omega-$ $U(z) k)$. The terms in curly brackets can all be neglected with respect to terms in 5.5.4a as long as

$$
\begin{aligned}
& K \gg 1 / L_{U} \\
& K \gg 1 / L_{N^{2}}
\end{aligned}
$$

where

$$
\begin{gathered}
L_{U}=U / U_{y} \\
L_{N^{2}}=N^{2} / N_{y}^{2}
\end{gathered}
$$

Using the particular form of equation 5.2.7, these conditions can be rewritten

$$
\begin{aligned}
& K^{2} \gg 1 / L^{2} \\
& K^{2} \gg \beta U_{z z} / N^{2}
\end{aligned}
$$

Once these conditions are satisfied, the meridional dependence of $N^{2}$ and $U$ is unimportant relative to the other terms. Since the problem is now homogeneous in $x$ and $\mathrm{y}$, assume a wave-like solution: all variables have their $x, y, t$ dependence given by $e^{i(k x+l y-\omega t)}$ ( $\omega$ denotes the local frequency while $\sigma$ denotes the intrinsic frequency 
$\omega-k U)$. Then the equations 5.5 .1 can be rewritten

$$
\begin{aligned}
-i \sigma u+w\left(U_{z}+f_{H}\right)+i k P & =0 \\
-i \sigma v+i l P & =0 \\
\left(N^{2}-\sigma^{2}\right) w+i \sigma f_{H} u-i \sigma P_{z} & =0 \\
i k u+i l v+w_{z} & =0 \\
-i \sigma \eta & =w
\end{aligned}
$$

Combine 5.5.6a, 5.5.6b, and 5.5.6d to get

$$
\left(k^{2}+l^{2}\right) P-i k\left(U_{z}+f_{H}\right) w-i \sigma w_{z}=0
$$

Equation 5.5.7 can be substituted into 5.5.6a, $b$ to get polarization relations and into 5.5.6a, $c$ to get a vertical structure equation:

$$
\begin{aligned}
& u=-\frac{i l^{2}\left(U_{z}+f_{H}\right)}{K^{2} \sigma} w+\frac{i k}{K^{2}} w_{z} \\
& v= \frac{i k l\left(U_{z}+f_{H}\right)}{K^{2} \sigma} w+\frac{i k}{K^{2}} w_{z} \\
& \eta=(i / \sigma) w \\
& P= \frac{i k}{K^{2}}\left(U_{z}+f_{H}\right) w+\frac{i \sigma}{K^{2}} w_{z} \\
& w_{z z}+m^{2}(z) w=0
\end{aligned}
$$

where

$$
m^{2}(z)=\frac{N^{2}-\sigma^{2}}{\sigma^{2}} K^{2}+\frac{k}{\sigma} U_{z z}+\frac{l^{2}}{\sigma^{2}}\left(f_{H}+U_{z}\right) f_{H}
$$

The ratio of the term that is due to the horizontal Coriolis vector to the first term on the right-hand side is

$$
\frac{l^{2}}{K^{2}} \frac{f_{H}^{2}+f_{H} U_{z}}{N^{2}-\sigma^{2}} \approx \frac{f_{H}}{N} \mathrm{Ri}^{1 / 2}
$$

where $\mathrm{Ri}$ is the Richardson number (see appendix $D$ ). The approximation holds as long as. $N^{2}-\sigma^{2}$ is order $N^{2}$. Since $N$ is an order of magnitude greater than $f_{H}$, and the inverse Richardson number is less than four for stable flow, the term involving the horizontal Coriolis vector is negligible. Note that the ratio of the two remaining terms that contribute to $m^{2}$ is approximately

$$
\frac{\sigma^{2}}{N^{2} K^{2}} \frac{K}{\sigma} U_{z z}=\frac{\sigma}{N^{2} K} U_{z z}
$$


In order for this ratio to be order one and $5.5 .5 b^{\prime}$ to still be valid, $K^{2} \gg \beta K / \sigma$ or $\sigma^{2} \gg \beta \sigma / K(=\beta c)$, which is the same condition that allows use of the dispersion relation for a non-rotating ocean. (see the discussion leading up to equation 5.4.8). Thus it is reasonable to retain the curvature term. This is different from the WKBJ work such as Olbers $1981 a$ where the basic state is presumed to vary slowly, so slowly that its curvature is unimportant.

In the case where $m(z)$ varies slowly in the vertical $\left(m^{-2}(x) \partial_{z} m(z) \ll 1\right), m$ can be considered the local vertical wavenumber. In any case, equation $5.5 .8 e$ indicates that the solutions have a separable vertical structure. To bring out the parallels with the no mean current solutions (equations 5.4.12), equations 5.5.8 can be rewritten in vector form:

$$
\left[\begin{array}{c}
\tilde{u} \\
\tilde{v} \\
\tilde{\eta} \\
\tilde{w} \\
\tilde{P}
\end{array}\right]=\left[\begin{array}{c}
\cos \theta \\
\sin \theta \\
0 \\
0 \\
\sigma / K
\end{array}\right] G_{z}^{i} A^{i}+\left[\begin{array}{c}
-\sin ^{2} \theta K U_{z} / \sigma \\
\sin \theta \cos \theta K U_{z} / \sigma \\
K / \sigma \\
-i K \\
\cos \theta U_{z}
\end{array}\right] G^{i} A^{i}
$$

where

$$
\begin{gathered}
G_{z z}+m^{2}(z) G=0 \\
m^{2}(z)=\frac{N^{2}-\sigma^{2}}{\sigma^{2}} K^{2}+\frac{k}{\sigma} U_{z z}
\end{gathered}
$$

In compact vector notation this will be written as

$$
\begin{aligned}
& \tilde{\mathbf{u}}^{i}=\left(\mathbf{s} G_{z}^{i}+\mathbf{r} G^{i}\right) A^{i} \\
& \mathbf{u}^{i}=\tilde{\mathbf{u}}^{i} e^{i(k x+l y-\omega t)}
\end{aligned}
$$




\subsection{Vertical structure}

Both vertical structure equations $(5.4 .4 f, 5.5 .8 e)$ are of the form

$$
G_{z z}+m^{2}(z) G=0
$$

where $m$ is a specified real function of $\mathrm{z}$ (and frequency and wavenumber). Because this is a second order linear differential equation, it has two linearly independent solutions, the exact choice of which is somewhat arbitrary. In the case of depth-independent $m^{2}$, vertically propagating solutions take the form of complex exponentials $e^{ \pm i m z}$, while standing modes would be written as $\sin (m z)$ and $\cos (m z)$. The standing modes can thus be thought of as being a pair of vertically propagating solutions with a fixed phase relationship. The different solutions can be distinguished on the basis of boundary conditions. For example, in the $m^{2}$ constant case, the two independent solutions $\alpha(z)$ and $\gamma(z)$ satisfy:

$$
\begin{array}{cl}
\alpha=\alpha_{0} e^{-i m\left(z-z_{0}\right)} & \begin{cases}\alpha_{z}=-i m \alpha & \text { at } z=z_{0} \\
\alpha=\alpha_{0} & \text { at } z=z_{0}\end{cases} \\
\gamma=\gamma_{0} e^{i m\left(z-z_{0}\right)} & \begin{cases}\gamma_{z}=i m \gamma & \text { at } z=z_{0} \\
\gamma=\gamma_{0} & \text { at } z=z_{0}\end{cases}
\end{array}
$$

where $z_{0}$ could be the ocean surface or bottom or any other fixed reference point. In this thesis, the reference point will usually be taken to be near the ocean bottom.

In the more general cases of depth dependent $m(z)$, functions which satisfy the boundary conditions in equation 5.6 .2 can still be found.

$$
\begin{array}{ll}
\alpha_{z z}+m^{2}(z) \alpha=0 & \begin{cases}\alpha_{z}=-i m \alpha & \text { at } z=z_{0} \\
\alpha=\alpha_{0}(\sigma, \mathbf{k}) & \text { at } z=z_{0}\end{cases} \\
\gamma_{z z}+m^{2}(z) \gamma=0 & \begin{cases}\gamma_{z}=i m \gamma & \text { at } z=z_{0} \\
\gamma=\gamma_{0}(\sigma, \mathbf{k}) & \text { at } z=z_{0}\end{cases}
\end{array}
$$

where $\mathbf{k}$ denotes the horizontal wavenumber vector $(k, l)$, and the reference values $\alpha_{0}$ and $\gamma_{0}$ are real. Since the functions $\alpha$ and $\gamma$ are solutions to linear equations, they have arbitrary (complex) amplitude. So the choice of $\alpha_{0}$ and $\gamma_{0}$ real at $z=z_{0}$ is an arbitrary one. The choice of them as real, however, has the advantage that as long as $m(z)$ is real, the complex conjugate of $\alpha, \alpha^{*}$, satisfies the same equation and boundary conditions as $\gamma$. Thus an arbitrary solution can be expressed as $A \alpha(z)+B \alpha^{*}(z)$. The normalization $\alpha_{0}(\sigma, \mathbf{k})$ is chosen based on the desired units for $A$ and $B$ : In what follows it will usually 
be chosen so that $\alpha(z)$ is a mode with unit energy density. Note that unlike the constant $m$ case, the first boundary condition (linking $\alpha_{z}$ and $\alpha$ ) only holds at $z=z_{0}$. So while $\alpha$ has purely downward phase at $z=z_{0}$, it will be some mixture of upward and downward at another depth, a mixture that depends on $m(z)$. This idea is explored further in what follows.

To gain a better understanding of the function $\alpha(z)$, Gent and Luyten 1985 choose to decompose the vertical structure equation into two coupled first order linear differential equations. This decomposition is not unique, however the decomposition they have chosen is especially useful for buoyancy frequency profiles where $m(z)$ varies slowly in certain depth ranges. Suppose

$$
\begin{aligned}
\alpha(z) & =m^{-1 / 2}(z)[D(z)+U(z)] \\
\alpha_{z}(z) & =i m^{1 / 2}(z)[D(z)-U(z)]
\end{aligned}
$$

Then the following coupled first order linear differential equations are consistent with equation 5.6.3:

$$
\begin{aligned}
& \left(\partial_{z}-i m\right) D=U \frac{1}{2 m} \frac{d m}{d z} \\
& \left(\partial_{z}-i m\right) U=D \frac{1}{2 m} \frac{d m}{d z}
\end{aligned}
$$

So $D$ corresponds to upward phase (downward energy) while $U$ corresponds to downward phase (upward energy).

Equations 5.6.6 have an interesting physical interpretation. Within depth ranges where $m(z)$ is constant (or almost constant), the right-hand sides are essentially zero, and the two equations are decoupled. The solutions are then simple exponentials, $D=e^{i m z}$ and $U=e^{-i m z}$. For example, suppose that near a reference depth $U$ is non-zero, $D$ is zero, and $m(z)$ is constant. As equations 5.6.6 are integrated away from the reference point, $D \approx 0$ as long as $m$ varies negligibly. If $m$ has a sharp gradient, the right-hand sides of the equations grow large, and the variance in $U$ is transferred into $D$. At such a depth there is both downward and upward propagating energy. The problem is now to show that $\alpha(z)$ corresponds to this thought experiment. First rewrite $\alpha_{z z}$ to see that $\alpha$ 
does indeed satisfy equation 5.6.3.

$$
\begin{aligned}
\alpha_{z z} & =i m^{-1 / 2} \frac{1}{2} \frac{d m}{d z}(D-U)+i m^{1 / 2}\left(D_{z}-U_{z}\right) \\
& =i m^{-1 / 2} \frac{1}{2} \frac{d m}{d z}(D-U)+i m^{1 / 2}\left[i m(D+U)+(U-D) \frac{1}{2 m} \frac{d m}{d z}\right] \\
& =-m^{3 / 2}(D+U) \\
& =-m^{2} \alpha
\end{aligned}
$$

The first boundary condition of that equation rewritten in terms of $D$ and $U$ becomes

$$
i m^{1 / 2}(D-U)=-i m\left(m^{-1 / 2}(D+U)\right) \quad \text { at } \quad z=z_{0}
$$

therefore

$$
D\left(z_{0}\right)=0
$$

Thus the case of having the energy propagating exclusively upward at the reference depth $\left(D\left(z_{0}\right)=0, U\left(z_{0}\right) \neq 0\right)$ is equivalent to the solution designated $\alpha(z)$. Conversely, $\alpha^{*}(z)$ corresponds to the case of energy propagating exclusively downward at the reference depth $\left(D\left(z_{0}\right) \neq 0\right.$ and $\left.U\left(z_{0}\right)=0\right)$.

\subsection{Sum of single wave solutions}

The last three sections have looked at the properties of wavelike solutions to the equations of motion found in section 5.3. The form of these solutions was chosen to facilitate solving the equations, and it does not necessarily correspond to what might be found in the actual ocean. Because the equations are linear, these wavelike solutions can be linearly combined to find another solution. To analyze the measurements, one reverses the process: a given oceanic state is decomposed into a sum of wavelike solutions. Note that while it may be difficult to prove for a given linear system that this is always possible, in practice the proof is not necessary, since one can always check to see whether the sum of waves matches the measurements. Furthermore, to the extent that Fourier theory applies (Bracewell 1978), the proof can be given. In any case, this section examines properties of sums of wavelike solutions. Sections 5.4, 5.5, and 5.6 can be summarized mathematically as

$$
\begin{aligned}
\tilde{\mathbf{u}}=\left[\begin{array}{c}
\tilde{u} \\
\tilde{v} \\
\tilde{\eta}
\end{array}\right] & =\mathbf{r}\left(A \alpha(z)+B \alpha^{*}(z)\right)+\mathbf{s}\left(A \alpha_{z}(z)+B \alpha_{z}^{*}(z)\right) \\
& =\left(\mathbf{r} \alpha(z)+\mathbf{s} \alpha_{z}(z)\right) A+\left(\mathbf{r} \alpha^{*}(z)+\mathbf{s} \alpha_{z}^{*}(z)\right) B
\end{aligned}
$$


where

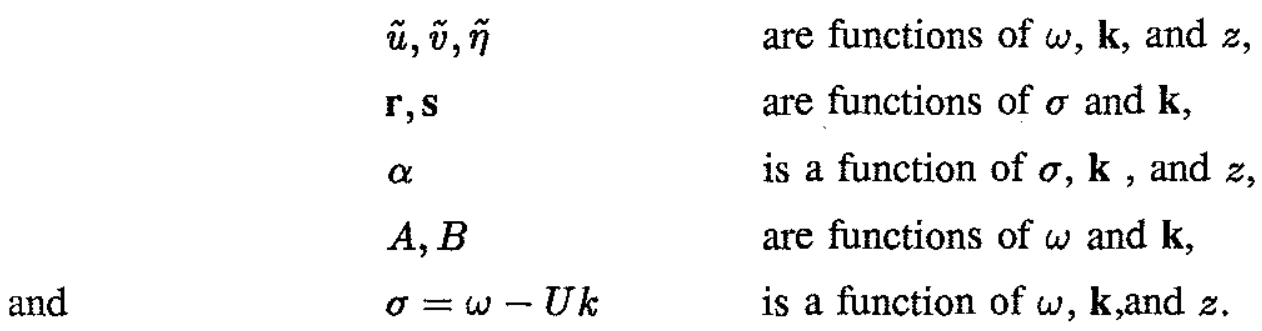

Equations 5.4.12 and 5.5.8 give explicit expressions for $\mathbf{r}$ and $\mathbf{s}$, while section 5.6 discusses the properties of $\alpha$. All three are functions determined by the equations of motion. $A$ is the upward propagating wave's amplitude, while $B$ is the amplitude of the downward propagating wave. So far, then, this a a sum of two waves. To express an arbitrary solution $\mathbf{u}(x, y, z, t)$ possibly a sum of many waves is needed. On an infinite domain the integral Fourier transform (Bracewell 1978) provides a mapping between frequency-wavenumber space and physical space.

$$
\begin{aligned}
& a(x, y, t)=\frac{1}{(2 \pi)^{3}} \iiint \tilde{a}(\omega, k, l) e^{i(k x+l y-\omega t)} d \omega d k d l \\
& \tilde{a}(\omega, k, l)=\iiint a(x, y, t) e^{-i(k x+l y-\omega t)} d x d y d t
\end{aligned}
$$

where $a$ represents any component of the dynamic vector $\mathbf{u}$, i.e. $u, v, \eta, w$, and $P$. Thus if we were considering a single component ( $u$, for example) at a single depth (so the the $z$ coordinate can be omitted), we could be certain that any solution $u(x, y, t)$ could be expressed as a sum of waves.

In practice, a measured function $a(x, y, t)$ is specified on a finite domain. With no loss of generality on that finite range, the function $a(x, y, t)$ can be represented as periodic outside the range of interest. That is, if the size of the area of interest is $L$ in $x$ and $y, T$ in time, the function is considered to repeat spatially with period $L$ and temporally with period $T$. This means the function can be represented as a sum of discrete Fourier components (see Koopmans 1974 p.22) (Koopmans extends the following results by showing that they are true for almost periodic functions as well.)

$$
a(x, y, t)=\sum_{i=-\infty}^{\infty} \sum_{j=-\infty}^{\infty} \sum_{n=-\infty}^{\infty} \tilde{a}_{i j n} e^{i\left(k_{i} x+l_{j} y-\omega_{n} t\right)}
$$

where $\left\{k_{i}\right\},\left\{l_{j}\right\}$, and $\left\{\omega_{n}\right\}$ are infinite sets of discrete wavenumbers and frequencies ordered so that their members monotonically increase with index $\left(k_{0}=l_{0}=\omega_{0}=0\right)$. 
The vector of dynamical variables can thus be represented as a sum of wave pairs ũ, each wave pair being determined by the pair of amplitudes $A_{i j n}, B_{i j n}$ and the deterministic wave functions $\mathbf{r}, \mathbf{s}$, and $\alpha$.

\section{Symmetry properties of $A$ and $B$}

The symmetry properties of A and B are determined by the symmetry properties of $\tilde{\mathbf{u}}$ and the coefficients in equation 5.7.1, namely $\mathbf{r}, \mathbf{s}$, and $\alpha$. Since $\tilde{\mathbf{u}}$ is the Fourier transform of a real vector, it has the property that

$$
\tilde{\mathbf{u}}(-\sigma,-\mathbf{k})=\tilde{\mathbf{u}}^{*}(\sigma, \mathbf{k})
$$

i.e., it is a vector of Hermitian functions. By inspecting equations 5.6.3, 5.5.9, and 5.4.12 it is clear that

$$
\begin{array}{r}
\mathbf{r}(-\sigma,-\mathbf{k})=\mathbf{r}^{*}(\sigma, \mathbf{k}) \\
\mathbf{s}(-\sigma,-\mathbf{k})=\mathbf{s}^{*}(\sigma, \mathbf{k}) \\
\alpha(-\sigma,-\mathbf{k})=\alpha^{*}(\sigma, \mathbf{k})
\end{array}
$$

Imposing 5.7.4 and 5.7.5 on 5.7.1 implies

$$
\begin{aligned}
& A(-\sigma,-\mathbf{k})=B^{*}(\sigma, \mathbf{k}) \\
& B(-\sigma,-\mathbf{k})=A^{*}(\sigma, \mathbf{k})
\end{aligned}
$$

which are the symmetry properties of $A$ and $B$. 


\subsection{Wave expansion of cross-spectra}

The eventual use of this wave expansion is to examine the ways in which the data and models constrain each other. The next step in that process is to express the cross-spectra in terms of the wave expansion of the last section. Define the inner product of two complex variables $a$ and $b$ as

$$
\langle a b\rangle=\lim _{\substack{T \rightarrow \infty \\ L \rightarrow \infty}} \frac{1}{L^{2} T} \int_{-T / 2}^{T / 2} d t \int_{-L / 2}^{L / 2} d x \int_{-L / 2}^{L / 2} d y \quad a^{*}(x, y, t) b(x, y, t)
$$

The covariance of $a$ and $b$ can then be expressed (see the discussion on autocovariance in Koopmans 1974)

$$
\operatorname{cov}(a, b)=\langle a(x, y, t) b(x+X, y+Y, t+\tau)\rangle
$$

Note that this is a purely deterministic definition of covariance: covariance is a triple integral over space and time normalized by the size of the area. In the actual comparison with data, statistical concepts make it possible to use ensemble averages to estimate these spatial (and temporal) integrals. But for the theoretical manipulations only the deterministic definition is necessary. If $a$ and $b$ are periodic the covariance can be expressed in terms of the Fourier coefficients $\tilde{a}_{i j n}, \tilde{b}_{i j n}$.

$$
\begin{aligned}
\langle a(x, y, t) b & (x+X, y+Y, t+\tau)\rangle \\
= & \sum_{i j n} \sum_{o p q} \tilde{a}_{i j n}^{*} \tilde{b}_{o p q} e^{i\left(k_{o} X+l_{p} Y-\omega_{q} \tau\right)}\left\langle e^{-i\left(k_{i} x+l_{j} y-\omega_{n} t\right)}, e^{i\left(k_{o} x+l_{p} y-\omega_{q} t\right)}\right\rangle \\
= & \sum_{i j n} \tilde{a}_{i j n}^{*} \tilde{b}_{i j n} e^{i\left(k_{i} X+l_{j} Y-\omega_{n} \tau\right)}
\end{aligned}
$$

This expression shows that the cross-spectrum between a pair of variables $a$ and $b$ is simply $\tilde{a}_{i j n}^{*} \tilde{b}_{i j n}$. (see Jenkins and Watts 1968 for a more statistical approach.) The important simplification in equation 5.8.3 is that the inner product of waves that have different frequencies or horizontal wavenumber vectors is zero, so that the six-fold sum reduces to a three-fold sum. In particular, cross-spectra due to solutions which correspond to each value of the frequency-wavenumber vector $(\omega, k, l)$ can be computed separately and the results combined to compute the total cross-spectra. The next part of this section applies this simplification to the wave expansion of section 5.7.

Because equation 5.8.3 applies equally to all the dynamic variables $\mathbf{u}$, the cross spectral matrix $\left\langle\tilde{\mathbf{u}}^{*} \tilde{\mathbf{u}}^{T}\right\rangle$ (using ${ }^{T}$ to denote vector transpose) can be expressed in terms 
of $A^{*} A, B^{*} B, A^{*} B$ through equation 5.7.1. Using $\left\langle\tilde{\mathbf{u}}_{1}^{*} \tilde{\mathbf{u}}_{2}^{T}\right\rangle$ to represent the cross-spectra between all the variables measured at location 1 and location 2 ,

$$
\begin{aligned}
& \left\langle\tilde{\mathbf{u}}_{1}^{*} \tilde{\mathbf{u}}_{2}^{T}\right\rangle \\
& \quad=\left(\mathbf{r}_{1}^{*} \alpha_{1}^{*}+\mathbf{s}_{1}^{*} \alpha_{1 z}^{*}\right)\left(\mathbf{r}_{2}^{T} \alpha_{2}+\mathbf{s}_{2}^{T} \alpha_{2 z}\right) A^{*} A+\left(\mathbf{r}_{1}^{*} \alpha_{1}+\mathbf{s}_{1}^{*} \alpha_{1 z}\right)\left(\mathbf{r}_{2}^{T} \alpha_{2}^{*}+\mathbf{s}_{2}^{T} \alpha_{2 z}^{*}\right) B^{*} B \\
& +\left(\mathbf{r}_{1}^{*} \alpha_{1}^{*}+\mathbf{s}_{1}^{*} \alpha_{1 z}^{*}\right)\left(\mathbf{r}_{2}^{T} \alpha_{2}^{*}+\mathbf{s}_{2}^{T} \alpha_{2 z}^{*}\right) A^{*} B+\left(\mathbf{r}_{1}^{*} \alpha_{1}+\mathbf{s}_{1}^{*} \alpha_{1 z}\right)\left(\mathbf{r}_{2}^{T} \alpha_{2}+\mathbf{s}_{2}^{T} \alpha_{2 z}\right) B^{*} A \\
& =\left[\begin{array}{lllll}
\mathbf{r}_{1}^{*} \mathbf{r}_{2}^{T} & \mathbf{r}_{1}^{*} \mathbf{s}_{2}^{T} & \mathbf{s}_{1}^{*} \mathbf{r}_{2}^{T} & \mathbf{s}_{1}^{*} \mathbf{s}_{2}^{T}
\end{array}\right]\left[\begin{array}{cccc}
\alpha_{1}^{*} \alpha_{2} & \alpha_{1} \alpha_{2}^{*} & \alpha_{1}^{*} \alpha_{2}^{*} & \alpha_{1} \alpha_{2} \\
\alpha_{1}^{*} \alpha_{2 z} & \alpha_{1} \alpha_{2 z}^{*} & \alpha_{1}^{*} \alpha_{2 z}^{*} & \alpha_{1} \alpha_{2 z} \\
\alpha_{1 z}^{*} \alpha_{2} & \alpha_{1 z} \alpha_{2}^{*} & \alpha_{1 z}^{*} \alpha_{2}^{*} & \alpha_{1 z} \alpha_{2} \\
\alpha_{1 z}^{*} \alpha_{2 z} & \alpha_{1 z} \alpha_{2 z}^{*} & \alpha_{1 z}^{*} \alpha_{2 z}^{*} & \alpha_{1 z} \alpha_{2 z}
\end{array}\right]\left[\begin{array}{c}
A^{*} A \\
B^{*} B \\
A^{*} B \\
B^{*} A
\end{array}\right]
\end{aligned}
$$

The notation of $5.8 .4 b$ requires some explanation. First and foremost, it is merely a rewriting of 5.8.4a, so in the event of any confusion consult 5.8.4a. The first term on the right hand side of $5.8 .4 b$ is a row vector which will be called $R S$. It is a vector of matrices, but each matrix is considered a single element as far as the matrix multiplication is concerned.

So far, this section has mapped from cross-spectra as a function of location to the cross-spectra of up and down wave amplitudes at a particular frequency and horizontal wavenumber vector. Of the four elements of the complex vector $\left(A^{*} A, B^{*} B, A^{*} B, B^{*} A\right)$, two are real, and the other two form a complex conjugate pair. That suggests the transformation

$$
\left[\begin{array}{c}
E \\
\Delta \\
P \\
Q
\end{array}\right]=\left[\begin{array}{cccc}
1 & 1 & 0 & 0 \\
1 & -1 & 0 & 0 \\
0 & 0 & 1 & 1 \\
0 & 0 & i & -i
\end{array}\right]\left[\begin{array}{c}
A^{*} A \\
B^{*} B \\
A^{*} B \\
B^{*} A
\end{array}\right]
$$

This transformation can be inverted as:

$$
\left[\begin{array}{c}
A^{*} A \\
B^{*} B \\
A^{*} B \\
B^{*} A
\end{array}\right]=\frac{1}{2}\left[\begin{array}{cccc}
1 & 1 & 0 & 0 \\
1 & -1 & 0 & 0 \\
0 & 0 & 1 & -i \\
0 & 0 & 1 & i
\end{array}\right]\left[\begin{array}{c}
E \\
\Delta \\
P \\
Q
\end{array}\right]
$$

$E, \Delta, P$, and $Q$ are all real variables. Their physical meanings will become more apparent in the next two sections, where this wave expansion of cross spectra is done for 
the particular physical models derived in sections 5.4 and 5.5. Some general statements, however, can be made. $E$ is, roughly speaking, the total (both up and down) wave action, while $\Delta$ is the difference (up minus down) between up and down wave action. $P$ is twice the cospectrum of up with down, and $Q$ is twice the quadrature spectrum of up with down. If $\Delta$ is zero, the wave field is vertically symmetric (there is as much upgoing energy as downgoing energy). Modes can be thought of as phase-locked vertically propagating waves (see section 5.3 ): the perfectly reflecting boundary condition requires phase matching at the boundary. Thus $P$ and $Q$ would be non-zero if modes were present. In fact the eigenvalues $\lambda_{i}$ of the wave amplitude covariance matrix

$$
\left[\begin{array}{cc}
A^{*} A & A^{*} B \\
B^{*} A & B^{*} B
\end{array}\right]
$$

are such that

$$
\begin{aligned}
\lambda_{1}+\lambda_{2} & =E \\
\left(\lambda_{1}-\lambda_{2}\right)^{2} & =\Delta^{2}+P^{2}+Q^{2}
\end{aligned}
$$

The polarization is a normalized difference in covariance eigenvalues. It can be written in terms of $E, \Delta, P, Q$ as well:

$$
\text { polarization }=\frac{\lambda_{1}-\lambda_{2}}{\lambda_{1}+\lambda_{2}}=\frac{\sqrt{\Delta^{2}+P^{2}+Q^{2}}}{E}
$$

So that the polarization being zero means that the two eigenvalues are identical (equal distribution of energy), and a polarization of one indicates one eigenvalue is zero (all the energy is in one mode). Note that there are different possibilities when the polarization is one. $E=\Delta$ implies all the action is propagating upwards, while $E^{2}=P^{2}+Q^{2}$ means there is a perfectly reflecting boundary, and a single modal solution contains all the energy. Section 8.3 does an explicit mapping between the four spectral parameters $E \triangle P Q$ and the energy of modes and propagating waves.

Rewriting equation $5.8 .4 b$ in terms of the real variables $E, \Delta, P, Q$ :

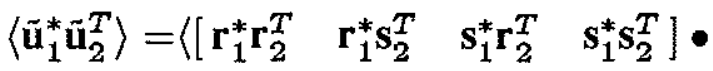

$$
\begin{aligned}
& \left.\left[\begin{array}{cccc}
\operatorname{Re}\left(\alpha_{1}^{*} \alpha_{2}\right) & i \operatorname{Im}\left(\alpha_{1}^{*} \alpha_{2}\right) & \operatorname{Re}\left(\alpha_{1} \alpha_{2}\right) & -\operatorname{Im}\left(\alpha_{1} \alpha_{2}\right) \\
\operatorname{Re}\left(\alpha_{1}^{*} \alpha_{2 z}\right) & i \operatorname{Im}\left(\alpha_{1}^{*} \alpha_{2 z}\right) & \operatorname{Re}\left(\alpha_{1} \alpha_{2 z}\right) & -\operatorname{Im}\left(\alpha_{1} \alpha_{2 z}\right) \\
\operatorname{Re}\left(\alpha_{1 z}^{*} \alpha_{2}\right) & i \operatorname{Im}\left(\alpha_{1 z}^{*} \alpha_{2}\right) & \operatorname{Re}\left(\alpha_{1 z} \alpha_{2}\right) & -\operatorname{Im}\left(\alpha_{1 z} \alpha_{2}\right) \\
\operatorname{Re}\left(\alpha_{1 z}^{*} \alpha_{2 z}\right) & i \operatorname{Im}\left(\alpha_{1 z}^{*} \alpha_{2 z}\right) & \operatorname{Re}\left(\alpha_{1 z} \alpha_{2 z}\right) & -\operatorname{Im}\left(\alpha_{1 z} \alpha_{2 z}\right)
\end{array}\right]\left[\begin{array}{c}
E \\
\Delta \\
P \\
Q
\end{array}\right]\right\rangle
\end{aligned}
$$


In both the single wave solutions considered (for internal waves that are in a basic state that is at rest, and for high frequency waves in a mean flow), $\mathbf{r}$ and $\mathbf{s}$ are both purely real when restricted to the measurable variables $u, v, \eta$ (see equations 5.4.10 and 5.5.3). Consequently, equation 5.8.6 implies that $\Delta(\omega, \mathbf{k})$ determines the imaginary part of the measured cross spectra, while $E, P, Q$ combine to determine the real part.

\section{Symmetry properties of $E, \Delta, P, Q$}

The symmetry properties of $A$ and $B$ (equation 5.7.6) quickly give the corresponding properties of $E, \Delta, P, Q$ :

$$
\begin{aligned}
E(-\sigma,-\mathbf{k}) & =E(\sigma, \mathbf{k}) \\
\Delta(-\sigma,-\mathbf{k}) & =-\Delta(\sigma, \mathbf{k}) \\
P(-\sigma,-\mathbf{k}) & =P(\sigma, \mathbf{k}) \\
Q(-\sigma,-\mathbf{k}) & =Q(\sigma, \mathbf{k})
\end{aligned}
$$

\subsection{Cross spectra for a basic state at rest}

This section derives the wave expansion of cross spectra for a particular choice of dynamics: high frequency internal waves in a resting, constant buoyancy frequency basic state. Then the variables $E, \Delta, P, Q$ defined in the last section can be related to the cross-spectra measurable from current meter data. The variables can also be related to energy densities and fluxes. Ultimately, then, the energy densities and fluxes can be related to the data, even though the densities and fluxes have not been directly measured.

The polarization vectors for high frequency internal waves are given in equation 5.4.12; the dispersion relation is given in equation 5.4.8, and the vertical structure equation is 5.4.10. The vertical structure function $\alpha$ is then:

$$
\begin{aligned}
\alpha & =\alpha_{0}(\sigma, \mathbf{k}) e^{-i m\left(z-z_{0}\right)} \\
\alpha_{z} & =-i m \alpha
\end{aligned}
$$

where

$$
\begin{gathered}
m=\frac{\sqrt{N^{2}-\sigma^{2}} K}{\sigma} \\
(k, l)=K(\cos \theta, \sin \theta)
\end{gathered}
$$


The normalization $\alpha_{0}(\omega, \mathbf{k})$ is chosen to be

$$
\alpha_{0}(\omega, \mathbf{k})=\frac{\sigma}{N K}
$$

This normalizes the waves to have unit energy density in a sense that will become explicit later. Define the following quantities to make the notation relatively compact

$$
\begin{gathered}
\lambda=\sqrt{1-\sigma^{2} / N^{2}} \\
\Delta z=z_{1}-z_{2} \\
\bar{z}=z_{1}+z_{2}-2 z_{0}
\end{gathered}
$$

where $z_{1}$ is depth of the first instrument, $z_{2}$ is depth of the second instrument, and $z_{0}$ is the reference depth. Note that if $\sigma \ll N, \lambda$ is fairly close to one. In terms of these variables the $\alpha$ matrix (see equation 5.8.9), is

$$
\left(\frac{\sigma}{N K}\right)^{2}\left[\begin{array}{cccc}
\cos m \Delta z & i \sin m \Delta z & \cos m \bar{z} & \sin m \bar{z} \\
m \sin m \Delta z & -i m \cos m \Delta z & -m \sin m \bar{z} & m \cos m \bar{z} \\
-m \sin m \Delta z & i m \cos m \Delta z & -m \sin m \bar{z} & m \cos m \bar{z} \\
m^{2} \cos m \Delta z & i m^{2} \sin m \Delta z & -m^{2} \cos m \bar{z} & -m^{2} \sin m \bar{z}
\end{array}\right]
$$

The row vector of matrices $R S$ (again see equation 5.8.9) is given in equation 5.9.5: the matrices are quite sparse. Then the matrix product suggested in equation 5.8 .9 is carried out, and the result is given in equation 5.9.6.

The $3 \times 3$ submatrix in the upper left corner corresponds to the quantities that can be measured with a current meter (if vertical displacement is calculated from temperature). The cross spectra calculated from a current meter array are functions of frequency and position: the expressions in equation 5.9.6 must be integrated over k. Short of doing that integration (and its inverse), some general comments can be made. First of all, the imaginary parts of the measured cross-spectra are dependent only on $\Delta$, and there is one linear combination of the imaginary parts that is constrained to be zero ( a consistency check)

$$
\operatorname{Im}\left(u_{1}^{*} u_{2}\right)+\operatorname{Im}\left(v_{1}^{*} v_{2}\right)-N^{2} \lambda^{2} \operatorname{Im}\left(\eta_{1}^{*} \eta_{2}\right)=0
$$

$E, P$ and $Q$ together determine the real part.

The energy equation for this system is

$$
\partial_{t}\left(\frac{1}{2}\left(u^{2}+v^{2}+w^{2}+N^{2} \eta^{2}\right)+\partial_{x}(u P)+\partial_{y}(v P)+\partial_{z}(w P)=0\right.
$$




\section{Equation 5.9.5}

$$
\begin{aligned}
\mathbf{r}_{1}^{*} \mathbf{r}_{2}^{T}= & {\left[\begin{array}{ccccc}
0 & 0 & 0 & 0 & 0 \\
0 & 0 & 0 & 0 & 0 \\
0 & 0 & \frac{1}{c^{2}} & -\frac{i \sigma}{c^{2}} & 0 \\
0 & 0 & \frac{i \sigma}{c^{2}} & \frac{\sigma^{2}}{c^{2}} & 0 \\
0 & 0 & 0 & 0 & 0
\end{array}\right] } \\
\mathbf{r}_{1}^{*} \mathbf{s}_{2}^{T}= & {\left[\begin{array}{ccccc}
0 & 0 & 0 & 0 & 0 \\
0 & 0 & 0 & 0 & 0 \\
\frac{1}{c} \cos \theta & \frac{1}{c} \sin \theta & 0 & 0 & 1 \\
\frac{i \sigma}{c} \cos \theta & \frac{i \sigma}{c} \sin \theta & 0 & 0 & i \sigma \\
0 & 0 & 0 & 0 & 0
\end{array}\right] } \\
\mathbf{s}_{1}^{*} \mathbf{r}_{2}^{T}= & {\left[\begin{array}{cccccc}
0 & 0 & \frac{1}{c} \cos \theta & \frac{-i \sigma}{c} \cos \theta & 0 \\
0 & 0 & \frac{1}{c} \sin \theta & \frac{-i \sigma}{c} \sin \theta & 0 \\
0 & 0 & 0 & 0 & 0 \\
0 & 0 & 0 & 0 & 0 \\
0 & 0 & 1 & -i \sigma & 0
\end{array}\right] } \\
\mathbf{s}_{1}^{*} \mathbf{s}_{2}^{T}= & {\left[\begin{array}{ccccc}
\cos { }^{2} \theta & \cos \theta \sin \theta & 0 & 0 & c \cos \theta \\
\cos \theta \sin \theta & \sin { }^{2} \theta & 0 & 0 & c \sin \theta \\
0 & 0 & 0 & 0 & 0 \\
0 & 0 & 0 & 0 & 0 \\
c \cos \theta & c \sin \theta & 0 & 0 & c^{2}
\end{array}\right] }
\end{aligned}
$$

The products of variables $u P, v P, \ldots$ can be thought of as covariances evaluated at zero separation. Consequently equation 5.8 .3 provides a way to write those products in terms of elements of the cross-spectral matrix,

$$
\langle u P\rangle=\int_{-\infty}^{\infty} \int_{-\infty}^{\infty} \int_{-\infty}^{\infty} \tilde{u}^{*} \widetilde{P} d \sigma d \mathbf{k}
$$

Cross-spectra are expanded in terms of $E, \Delta, P, Q$ in equation 5.9.6. This makes it possible to write the energy density and energy fluxes in terms of the spectral parameters $E, \Delta, P, Q$. Evaluated at a single depth, the cross spectral expansion gives 


\section{Equation 5.9.6}

$$
\begin{aligned}
& {\left[\begin{array}{lllll}
\tilde{u}_{1}^{*} \tilde{u}_{2} & \tilde{u}_{1}^{*} \tilde{v}_{2} & \tilde{u}_{1}^{*} \tilde{\eta}_{2} & \tilde{u}_{1}^{*} \tilde{w}_{2} & \tilde{u}_{1}^{*} \tilde{P}_{2} \\
\tilde{v}_{1}^{*} \tilde{u}_{2} & \tilde{v}_{1}^{*} \tilde{v}_{2} & \tilde{v}_{1}^{*} \tilde{\eta}_{2} & \tilde{v}_{1}^{*} \tilde{w}_{2} & \tilde{v}_{1}^{*} \tilde{P}_{2} \\
\tilde{\eta}_{1}^{*} \tilde{u}_{2} & \tilde{\eta}_{1}^{*} \tilde{v}_{2} & \tilde{\eta}_{1}^{*} \tilde{\eta}_{2} & \tilde{\eta}_{1}^{*} \tilde{w}_{2} & \tilde{\eta}_{1}^{*} \tilde{P}_{2} \\
\tilde{w}_{1}^{*} \tilde{u}_{2} & \tilde{w}_{1}^{*} \tilde{v}_{2} & \tilde{w}_{1}^{*} \tilde{\eta}_{2} & \tilde{w}_{1}^{*} \tilde{w}_{2} & \tilde{w}_{1}^{*} \tilde{P}_{2} \\
\tilde{P}_{1}^{*} \tilde{u}_{2} & \tilde{P}_{1}^{*} \tilde{v}_{2} & \tilde{P}_{1}^{*} \tilde{\eta}_{2} & \tilde{P}_{1}^{*} \tilde{w}_{2} & \tilde{P}_{1}^{*} \tilde{P}_{2}
\end{array}\right]=} \\
& {\left[\frac{1}{2} \lambda^{2}(1+\cos 2 \theta) \cos m \Delta z\right.} \\
& \frac{1}{2} \lambda^{2} \sin 2 \theta \cos m \Delta z \\
& \frac{1}{N} \lambda \cos \theta \sin m \Delta z \\
& \frac{i \sigma}{N} \lambda \cos \theta \sin m \Delta z \\
& \lambda^{2} \frac{\sigma}{K} \cos \theta \cos m \Delta z \\
& +\left[\begin{array}{c}
\frac{1}{2} \lambda^{2}(1+\cos 2 \theta) \sin m \Delta z \\
\frac{1}{2} \lambda^{2} \sin 2 \theta \sin m \Delta z \\
-\frac{1}{N} \lambda \cos \theta \cos m \Delta z \\
-\frac{i \sigma}{N} \lambda \cos \theta \cos m \Delta z \\
\lambda^{2} \frac{g}{K} \cos \theta \sin m \Delta z
\end{array}\right. \\
& \frac{1}{2} \lambda^{2} \sin 2 \theta \sin m \Delta z \\
& +\left[\begin{array}{c}
-\frac{1}{2} \lambda^{2}(1+\cos 2 \theta) \cos m \bar{z} \\
-\frac{1}{2} \lambda^{2} \sin 2 \theta \cos m \bar{z} \\
-\frac{1}{N} \lambda \cos \theta \sin m \bar{z} \\
-\frac{i \sigma}{N} \lambda \cos \theta \sin m \bar{z} \\
-\lambda^{2} \frac{\sigma}{K} \cos \theta \cos m \bar{z}
\end{array}\right.
\end{aligned}
$$




$$
\begin{aligned}
\frac{1}{2}\left(\tilde{u}^{*} \tilde{u}+\tilde{v}^{*} \tilde{v}+\tilde{w}^{*} \tilde{w}+\right. & \left.N^{2} \tilde{\eta}^{*} \tilde{\eta}\right)=\frac{1}{2}\left(\frac{1}{2} \lambda^{2}(1+\cos 2 \theta)+\frac{1}{2} \lambda^{2}(1-\cos 2 \theta)+\sigma^{2} / N^{2}+1\right) E \\
& +\frac{1}{2}\left(-\frac{1}{2} \lambda^{2}(1+\cos 2 \theta)-\frac{1}{2} \lambda^{2}(1-\cos 2 \theta)+\sigma^{2} / N^{2}+1\right) \cos m \bar{z} P \\
& +\frac{1}{2}\left(-\frac{1}{2} \lambda^{2}(1+\cos 2 \theta)-\frac{1}{2} \lambda^{2}(1-\cos 2 \theta)+\sigma^{2} / N^{2}+1\right) \sin m \bar{z} Q \\
& =E+\sigma^{2} / N^{2}(P \cos m \bar{z}+Q \sin m \bar{z}) \\
\tilde{u}^{*} \widetilde{P} & =\frac{N^{2}-\sigma^{2}}{N^{2}} \frac{\sigma}{K} \cos \theta(E-\cos m \bar{z} P-\sin m \bar{z} Q) \\
\tilde{v}^{*} \widetilde{P} & =\frac{N^{2}-\sigma^{2}}{N^{2}} \frac{\sigma}{K} \sin \theta(E-\cos m \bar{z} P-\sin m \bar{z} Q) \\
\tilde{w}^{*} \tilde{P} & =\frac{\sqrt{N^{2}-\sigma^{2}}}{N^{2}} \frac{\sigma^{2}}{K}(\Delta+i(\sin m \bar{z} P-\cos m \bar{z} Q))
\end{aligned}
$$

$P$ and $Q$ do not contribute to the average product $\langle w P\rangle$. This is because they do not contribute to the wavenumber-frequency integral of $5.9 .10 d$, since they are both even under frequency reffection (see equation 5.8.9), while their coefficients in 5.9.10d are odd under that reflection. The remaining terms in the expressions for the fluxes $(5.9 .10 b, c, d)$ have a simple interpretation as the products of group velocities and energy densities. By definition, the group velocity is the gradient of frequency in wavenumber space,

$$
c_{g}=\left(\frac{\partial \sigma}{\partial k}, \frac{\partial \sigma}{\partial l}, \frac{\partial \sigma}{\partial m}\right)
$$

This can be calculated explicitly by using the dispersion relation (equation 5.9.1c)

$$
c_{g}=\left(\frac{N^{2}-\sigma^{2}}{N^{2}} \frac{\sigma}{K} \cos \theta, \frac{N^{2}-\sigma^{2}}{N^{2}} \frac{\sigma}{K} \sin \theta, \frac{\sqrt{N^{2}-\sigma^{2}}}{N^{2}} \frac{\sigma^{2}}{K}\right)
$$

The expressions for the fluxes can then be rewritten

$$
\begin{aligned}
\tilde{u}^{*} \widetilde{P} & =\frac{\partial \sigma}{\partial k}(E-\cos m \bar{z} P-\sin m \bar{z} Q) \\
\tilde{v}^{*} \widetilde{P} & =\frac{\partial \sigma}{\partial l}(E-\cos m \bar{z} P-\sin m \bar{z} Q) \\
\tilde{w}^{*} \widetilde{P} & =\frac{\partial \sigma}{\partial m} \Delta
\end{aligned}
$$

Consider the case of vertically propagating wave $(P=Q=0)$. The two horizontal fluxes are simply the total energy density times the group velocity component in that direction. Note that positive $\Delta$ corresponds to net upward energy flux. 
Now consider the case of a perfectly reflecting boundary at $z=0$, i.e. $w=0$ at $z=0$. The polarization vectors (equation 5.4.12) translate that to the condition on wave amplitudes that $A=-B$. Thus the perfectly reflecting boundary at $z=0$ implies

$$
P=-E \text { and } \Delta=Q=0
$$

If the boundary were at some other depth other than zero, there would be a redistribution of energy between $P$ and $Q$. In either case the interpretation is again straightforward. The vertical flux is zero, as expected in the presence of a perfectly reflecting boundary. The zonal flux varies from 0 to $2 E$ depending on whether the mode has a node or a peak at that particular depth. The meridional flux behaves in the same way. Note that there is a small amount of additional energy $\left(\sigma^{2} / N^{2}\right.$ terms in equation 5.9.10a) due to the non-hydrostatic character of the dynamics. This energy is fixed in the sense that it is not involved in any of the fluxes.

In general solutions will have both modal and vertically propagating characteristics (partially reflecting boundaries). In that case a combination of the two flux interpretations is appropriate.

\subsection{Cross spectra for waves in a mean flow}

The variables $E, \Delta, P, Q$ have a slightly different physical interpretation in this case of high frequency waves in a mean shear flow. This section first outlines how those variables are related to measurable cross spectra, and then looks in detail at how they are related to energy density and fluxes.

The polarization relations, the dispersion relation, and the vertical structure equation for high frequency internal waves in a mean flow, are all given in equation 5.5.9. The vertical structure equation is presumed to have WKBJ solutions

$$
\alpha=\alpha_{0}(\sigma, \mathbf{k}) m^{-1 / 2}(z) e^{-i \int_{z_{0}}^{z} m(z) d z}
$$

From this an $\alpha$ matrix (see section 5.9) can be calculated. This matrix, combined with the $R S$ products (equation 5.10.4), and equation 5.8.6, gives the cross-spectra. Thus the measurable cross-spectra can be related to $E, \Delta, P$ and $Q$.

The energy equation for this system is

$$
\partial_{t} \hat{E}+\partial_{x}(U \hat{E}+u \hat{P})+\partial_{y}(v \hat{P})+\partial_{z}(w \hat{P})+U_{z} u w=0
$$




\section{Equation 5.10.4}

$$
\phi_{i} \equiv \frac{K U_{z i}}{\sigma_{i}}
$$

$$
\begin{aligned}
& \mathbf{r}_{1}^{*} \mathbf{r}_{2}^{T}= \\
& {\left[\begin{array}{ccccc}
\sin ^{4} \theta \phi_{1} \phi_{2} & -\sin ^{3} \theta \cos \theta \phi_{1} \phi_{2} & -\sin ^{2} \theta \frac{K}{\sigma_{2}} \phi_{1} & i \sin ^{2} \theta K \phi_{1} & -\sin ^{2} \theta \cos \theta \frac{\sigma_{3}}{K} \phi_{1} \phi_{2} \\
-\sin ^{3} \theta \cos \theta \phi_{1} \phi_{2} & \sin ^{2} \theta \cos ^{2} \theta \phi_{1} \phi_{2} & \sin \theta \cos \theta \frac{K}{\sigma_{2}} \phi_{1} & i \sin \theta \cos \theta K \phi_{1} & \sin \theta \cos ^{2} \theta \frac{\sigma_{2}}{K} \phi_{1} \phi_{2} \\
-\sin ^{2} \theta \frac{K}{\sigma_{1}} \phi_{2} & \sin \theta \cos \theta \frac{K}{\sigma_{1}} \phi_{2} & \frac{K_{1}}{\sigma_{1} \sigma_{2}} & -i \frac{K^{2}}{\sigma_{1}} & \cos \theta \frac{\sigma_{2}}{\sigma_{1}} \phi_{2} \\
-i \sin ^{2} \theta K \phi_{2} & i \sin \theta \cos \theta K \phi_{2} & i \frac{K^{2}}{\sigma_{2}} & K^{2} & i \cos \theta \sigma_{2} \phi_{2} \\
-\sin ^{2} \theta \cos \theta \frac{\sigma_{1}}{K} \phi_{1} \phi_{2} & \sin \theta \cos ^{2} \theta \frac{\sigma_{1}}{K} \phi_{1} \phi_{2} & \cos \theta \frac{\sigma_{1}}{\sigma_{2}} \phi_{1} & -i \cos \theta \sigma_{1} \phi_{1} & \cos ^{2} \theta \frac{\sigma_{1} \sigma_{2} \phi_{1} \phi_{2}}{K^{2}} \phi_{1}
\end{array}\right]} \\
& \mathbf{r}_{1}^{*} \mathbf{s}_{2}^{T}=\left[\begin{array}{ccccc}
-\sin ^{2} \theta \cos \theta \phi_{1} & -\sin ^{3} \theta \phi_{1} & 0 & 0 & -\sin ^{2} \theta \frac{\sigma_{2}}{K} \phi_{1} \\
\sin \theta \cos ^{2} \theta \phi_{1} & \sin ^{2} \theta \cos \theta \phi_{1} & 0 & 0 & \sin \theta \cos \theta \frac{\sigma_{2}}{K} \phi_{1} \\
\frac{K}{\sigma_{1}} \cos \theta & \frac{K}{\sigma} \sin \theta & 0 & 0 & \frac{\sigma_{2}}{\sigma_{1}} \\
i K \cos \theta & i K \sin \theta & 0 & 0 & i \sigma_{2} \\
\cos ^{2} \theta \frac{\sigma_{1}}{K} \phi_{1} & \sin \theta \cos \theta \frac{\sigma_{1}}{K} \phi_{1} & 0 & 0 & \cos \theta \frac{\sigma_{1} \sigma_{2} \phi_{1}}{K^{2}} \phi_{1}
\end{array}\right] \\
& \mathbf{s}_{1}^{*} \mathbf{r}_{2}^{T}=\left[\begin{array}{ccccc}
-\sin ^{2} \theta \cos \theta \phi_{2} & \sin \theta \cos ^{2} \theta \phi_{2} & \frac{K}{\sigma_{2}} \cos \theta & -i K \cos \theta & \cos ^{2} \theta \frac{\sigma_{2}}{K} \phi_{2} \\
-\sin ^{3} \theta \phi_{2} & \sin ^{2} \theta \cos \theta \phi_{2} & \frac{K}{\sigma_{2}} \sin \theta & -i K \sin \theta & \sin \theta \cos \theta \frac{\sigma_{2}}{K} \phi_{2} \\
0 & 0 & 0 & 0 & 0 \\
0 & 0 & 0 & 0 & 0 \\
-\sin ^{2} \theta \frac{\sigma_{1}}{K} \phi_{2} & \sin \theta \cos \theta \frac{\sigma_{1}}{K} \phi_{2} & \frac{\sigma_{1}}{\sigma_{2}} & -i \sigma_{1} & \cos \theta \frac{\sigma_{1} \sigma_{2}}{K^{2}} \phi_{2}
\end{array}\right] \\
& \mathbf{s}_{1}^{*} \mathbf{s}_{2}^{T}=\left[\begin{array}{ccccc}
\cos ^{2} \theta & \cos \theta \sin \theta & 0 & 0 & \frac{\sigma_{2}}{K} \cos \theta \\
\cos \theta \sin \theta & \sin ^{2} \theta & 0 & 0 & \frac{\sigma_{2}}{K} \sin \theta \\
0 & 0 & 0 & 0 & 0 \\
0 & 0 & 0 & 0 & 0 \\
\frac{\sigma_{1}}{K} \cos \theta & \frac{\sigma_{1}}{K} \sin \theta & 0 & 0 & \frac{\sigma_{1} \sigma_{2}}{K^{2}}
\end{array}\right]
\end{aligned}
$$


where

$$
\hat{E}=\frac{1}{2}\left(u^{2}+v^{2}+w^{2}+N^{2} \eta^{2}\right)
$$

and $\hat{P}$ is the pressure. Just as in section 5.9 , the products of variables in the energy equation can be thought of as covariances evaluated at zero separation. For example,

$$
\langle u \hat{P}\rangle=\int_{-\infty}^{\infty} \int_{-\infty}^{\infty} \int_{-\infty}^{\infty} \tilde{u}^{*} \tilde{\hat{P}} d \sigma d \mathbf{k}
$$

The next step is to write these cross-spectra using $E, \Delta, P$, and $Q$. The $\alpha$ matrix for zero separation is

$$
\left[\begin{array}{cccc}
m^{-1} & 0 & m^{-1} \cos \phi & m^{-1} \sin \phi \\
0 & -i & -\sin \phi & \cos \phi \\
0 & i & -\sin \phi & \cos \phi \\
m & 0 & -m \cos \phi & -m \sin \phi
\end{array}\right]
$$

where

$$
\phi=2 \int_{z_{0}}^{z} m(z) d z
$$

$\alpha_{0}$ has been chosen to be one.

The terms of the cross spectral matrix that are related to terms in the energy equation can now be expanded (omitting terms that are lost in the integration 5.9.9),

$$
\begin{aligned}
& \frac{1}{2}\left(\tilde{u}^{*} \tilde{u}+\tilde{v}^{*} \tilde{v}+\tilde{w}^{*} \tilde{w}+N^{2} \tilde{\eta}^{*} \tilde{\eta}\right)=\left(\frac{K^{2}}{\sigma^{2}}\left(N^{2}+U_{z}^{2} / 4\right)+\frac{K \cos \theta}{2 \sigma} U_{z z}-\frac{K^{2} U_{z}^{2}}{4 \sigma^{2}} \cos 2 \theta\right) \frac{E}{m} \\
& +\left(\frac{K^{2}}{\sigma^{2}}\left(\sigma^{2}+U_{z}^{2} / 4\right)-\frac{K \cos \theta}{2 \sigma} U_{z z}-\frac{K^{2}}{4 \sigma^{2}} U_{z}^{2} \cos 2 \theta\right) \frac{P \cos \phi+Q \sin \phi}{m} \\
& \tilde{u}^{*} \tilde{\hat{P}}=\left(\frac{1}{2} U_{z z}+\left[\left(N^{2}-\sigma^{2}\right) K / \sigma-\frac{K}{4 \sigma} U_{z}^{2}\right] \cos \theta+\frac{1}{2} U_{z z} \cos 2 \theta+\frac{K}{4 \sigma} U_{z}^{2} \cos 3 \theta\right) \frac{E}{m} \\
& -\left(\frac{U_{z z}}{2}+\left[\left(N^{2}-\sigma^{2}\right)+\frac{U_{z}^{2}}{4}\right] \frac{K}{\sigma} \cos \theta+\frac{U_{z z}}{2} \cos 2 \theta-\frac{K U_{z}^{2}}{4 \sigma} \cos 3 \theta\right) \frac{P \cos \phi+Q \sin \phi}{m} \\
& -\cos 2 \theta U_{z}(P \sin \phi-Q \cos \phi) \\
& \tilde{v}^{*} \tilde{\hat{P}}=\left(\left[\left(N^{2}-\sigma^{2}\right) K / \sigma-\frac{K}{4 \sigma} U_{z}^{2}\right] \sin \theta+\frac{1}{2} U_{z z} \sin 2 \theta+\frac{K}{4 \sigma} U_{z}^{2} \sin 3 \theta\right) \frac{E}{m} \\
& +\left(-\left[\left(N^{2}-\sigma^{2}\right) K / \sigma+\frac{K}{4 \sigma} U_{z}^{2}\right] \sin \theta-\frac{1}{2} U_{z z} \sin 2 \theta+\frac{K}{4 \sigma} U_{z}^{2} \sin 3 \theta\right) \frac{P \cos \phi+Q \sin \phi}{m} \\
& -\sin 2 \theta U_{z}(P \sin \phi-Q \cos \phi)
\end{aligned}
$$




$$
\begin{gathered}
\tilde{w}^{*} \tilde{\hat{P}}=\sigma \Delta \\
\tilde{u}^{*} \tilde{w}=K \cos \theta \Delta
\end{gathered}
$$

In this system of waves in a mean flow, energy is not conserved. This is manifest in equation 5.10 .2 by the interaction term $(u w) U_{z}$ which is the energy exchanged by the waves and the mean flow. There is, however, an action conservation equation for the system. Once the last two terms in equation $\mathbf{5 . 1 0 . 2}$ have been written in terms of $\Delta$, it becomes clear that they can be combined into the vertical derivative of a single quantity:

$$
\begin{aligned}
\partial_{z}(w \hat{P})+u w U_{z} & =\partial_{z}(\sigma \Delta)+K \cos \theta U_{z} \Delta \\
& =\partial_{z}(\sigma \Delta)-\sigma_{z} \Delta \\
& =\sigma \partial_{z} \Delta
\end{aligned}
$$

So the energy equation can be transformed into a conservation equation by dividing through by the intrinsic frequency $\sigma$,

$$
\partial_{t}(\hat{E} / \sigma)+\partial_{x}(U \hat{E} / \sigma+u \hat{P} / \sigma)+\partial_{y}(v \hat{P} / \sigma)+\partial_{z} \Delta=0
$$

This equation is the statement of wave action conservation: $\hat{E} / \sigma$ is the wave action, and $\Delta$ is the vertical flux of wave action.

\subsection{Summary}

This chapter examines properties of high frequency internal wave fluctuations in two possible oceanic basic states. In particular, single wave solutions are found for high frequencies in both a resting and zonally flowing mean state. General solutions are made up of sums of these single wave solutions. Statistical arguments show that the crossspectra of the general solutions are simply sums of the cross spectra of the single wave solutions, i.e. waves of differing frequency or horizontal wavenumber are orthogonal. Finally, properties of the single wave cross-spectra for the two basic states considered are demonstrated: how the wave parameters $E, \Delta, P, Q$ are related to the measurable cross spectra, and how these parameters are related to energy or action densities and fluxes. The next chapter examines how well different versions of these models fit the data. 


\section{Chapter 6: Consistency checks}

\subsection{Consistency checks}

Consistency checks are model-data comparisons that are independent of the values of the model parameters. They form necessary but insufficient conditions for models to be considered consistent with data: in order for the model and data to be considered consistent, the deviations of the consistency checks from the model predictions must be statistically insignificant. Without loss of generality, each consistency check defined here has been chosen to have a predicted value of zero. As an example, consider the incomplete set of consistency checks for the GM models that were discussed in section 1.5. Consistency checks that were discussed include Fofonoff's consistency check (which fixes the ratio of potential and kinetic energy), the velocity-displacement coherences (which GM predicts will be zero), and the zonal velocity-meridional velocity coherence (which is predicted to be a function of frequency and Coriolis parameter). Each of these predictions can be rewritten as a linear combination of cross-spectra, a combination whose expected value is zero. Furthermore, each of these predictions is independent of the spectral level $E(\omega)$, consequently each prediction is independent of the GM model parameters. Finally, while it is necessary that all of the consistency checks hold in order for the GM79 model to be considered consistent with a given data set, it is clearly not sufficient: among other things, the auto- and cross-spectral levels measured should agree with GM79 as well in order for the GM79 model to be considered correct.

For models with small numbers of parameters consistency checks are unnecessary, since any modeling of data can provide some measure of the model-data misfit. Consistency checks become useful when the number of consistency checks is (far) smaller than the number of model parameters (since the internal wave spectral level is a continuous function of wavenumber, the internal wave models have an infinite number of model parameters and this condition is satisfied). For complicated models consistency checks are useful in two closely related ways: model rejection and model reduction. In model rejection, if the consistency checks are violated the model can be rejected without going to the trouble of determining all the model parameters. Similarly, models can be reduced in size by arbitrarily eliminating some of the model parameters and using any consistency checks that result to see if the reduced model is possibly consistent with the data. These 
two uses differ more in degree than in kind: since most models can be considered to be special cases of some more general model, there is no essential difference between the two cases.

Müller and Siedler 1976 derive complete sets of consistency checks for a WKBJ vertically propagating internal wave field and for a field of standing modes. These results, in turn, are extended by Müller et al. 1978 in their systematic comparison of the IWEX data with a series of internal wave models. In both cases they write the consistency checks as linear combinations of the cross-spectra. The linearity allows the statistics of the consistency checks to be straightforwardly calculated from the statistics of the spectral estimators. This chapter adopts their techniques of considering and combining linear combinations of cross-spectra; in fact, many of the linear combinations are identical to those calculated in the IWEX analysis. But the analysis of these linear combinations done in this chapter is in terms of internal wave models that are more general than those used in the IWEX analysis. In particular, mixtures of vertically propagating and modal solutions are allowed, and the propagating solutions are not necessarily restricted to being WKBJ approximations. As it turns out, the PEQUOD data are inconsistent with a large and quite general class of internal wave models. This indicates that a more sophisticated model is required in order to explain these observations. Consistency checks constrain this new model only in that the new model cannot require the consistency checks that are violated. Quite naturally a wide class of models satisfy that condition. The class of models which fail is such that all the models consider the internal wave field to be in a resting basic state, i.e. there is no mean flow. One model which has no linear consistency checks is a general internal wave field in the presence of an unknown mean flow which has vertical shear. The violation of consistency checks for the no mean flow internal wave model and the absence of those checks in the model that includes a mean shear flow is taken as evidence that a model which includes the effects of a mean vertical shear flow could be adequate to model the PEQUOD data. This model is presented explicitly in chapter 8.

The remainder of this chapter looks in detail at the consistency check calculation. Consistency checks have three facets that need to be presented: their expression in terms of data, their expression in terms of the symmetries of the internal wave field, and the derivation that makes that connection between model and data possible. Section 6.2 presents consistency checks as they are calculated from data: it discusses the consistency 
check statistics and their evaluation using the framework used by Müller et al. 1978. Section 6.3 discusses consistency checks from a model perspective: it expresses the basic symmetries of an internal wavefield in terms of the generalized spectral level $(E, \Delta, P, Q)$ and shows by example how the symmetries can be related to consistency checks. Section 6.4 discusses the model simplification results from PEQUOD, showing how particular model parameters can be considered indistinguishable from zero. Section 6.5 examines the internal wave symmetries as measured using the PEQUOD results, essentially by combining many of the results of section 6.4. The examination concludes that the internal wave models without a mean shear flow are inadequate and thus an extended model is required. One possibly adequate model is an internal wave field that is subject to mean shear flow.

The sections following section 6.5 are appendical: they contain the derivations of consistency checks from various internal wave models. Section 6.6 derives the consistency checks for the general internal wave models presented in chapter 5. Section 6.7 considers the more restricted class of models that uses WKBJ approximations to solve the wave equation. Finally, section 6.8 demonstrates that the most general internal wave model that includes the effects of mean shear is not constrained by the consistency checks of the simpler resting basic state internal wave model, thus a field of internal waves modified by the presence of a mean shear flow it is a possible model for the internal wave field measured in PEQUOD. 


\subsection{Consistency check statistics and evaluation}

Following Müller et al. 1978, the consistency checks considered in this chapter are based on the linear combinations of the measured cross-spectra that the model predicts are zero. The internal wave model that is being evaluated for consistency with the data determines which linear combinations need be considered (sections 6.6, 6.7, and 6.8 give detailed derivations of the linear combinations appropriate for several internal wave models). Once the linear combinations with expected value zero have been determined, the statistics and evaluation of the consistency checks depend only on the statistics and values of the measured cross-spectra. This section is primarily concerned with the statistics and evaluation of the consistency checks, and for the most part will take the choice of linear combinations to be given by a general linear form.

Let the linear combinations that have expected value zero be written as a vector $\mathbf{e}$ given by a linear transformation of the measured cross-spectra $\mathbf{y}$ (the following discussion closely follows Müller et al. 1978). Writing the set of cross-spectral estimators as a vector $\mathbf{y}$ means that the linear transformation can be written as a matrix $\mathbf{L}$ so that

$$
\mathbf{e}=\mathbf{L y}
$$

Each element of e corresponds to a different linear consistency check. As an example, consider checking a horizontally isotropic resting basic state internal wave model against data from four current meters on the same mooring. Because the model wave field is horizontally isotropic, the model predicts the zonal spectra to be the same as the meridional spectra at any depth. This prediction is incorporated in the vector $\mathbf{e}$ by assigning to the first four elements of $\mathbf{e}$ the difference between the zonal velocity autospectra and the meridional velocity autospectra at each of the four depths. Furthermore, such a horizontally isotropic model predicts the cross-spectra between the zonal and meridional velocities to be zero as well. So all the cross-spectra between the zonal and meridional velocities at the same or different depths would be elements of $\mathbf{e}$ as well. And so on, until all the zero mean linear combinations of spectra have been included in the vector.

The next step is to relate the statistics of the linear combinations $\mathbf{e}$ to the statistics of the cross-spectral estimators $\mathbf{y}$. With no loss of generality, insist that the transformation matrix $\mathbf{L}$ be such that all the elements of e are real, and let $M_{\mathrm{cc}}$ be the number of elements of e. Then there are $M_{\mathrm{cc}}$ linear combinations and e consists of $M_{\mathrm{cc}}$ zero mean 
real random variables. The covariance matrix $\mathbf{T}$ of these random variables $\mathbf{e}$ is readily determined from the (true) covariance matrix $\mathbf{S}=\left\langle\mathbf{y}^{*} \mathbf{y}^{T}\right\rangle$ of the spectral estimators

$$
\mathbf{T}=\left\langle\mathbf{e e}^{T}\right\rangle=\mathbf{L}^{*} \mathbf{S} \mathbf{L}^{T}
$$

(The true covariance matrix of the spectral estimators is calculated following Jenkins and Watts 1968 , a calculation which is briefly summarized in appendix $C$ ). In order to efficiently combine the $M_{\mathrm{cc}}$ linear combinations into a single statistical quantity, Müller et al. calculate the statistic $\epsilon^{2}$ which is given by

$$
\epsilon^{2}=\frac{1}{M_{\mathrm{cc}}} \sum_{i=1}^{M_{\mathrm{cc}}} \frac{e_{i}^{2}}{T_{i i}}
$$

They argue that $\epsilon^{2}$ is distributed as a reduced $\chi^{2}$ variable (Bevington 1969) of $M_{\text {eff }}$ degrees of freedom, $\frac{1}{M_{\text {eff }}} \chi_{M_{\text {eff }}}^{2}$, where the effective number of degrees of freedom $M_{\text {eff }}$ is given by

$$
M_{\text {eff }}=M_{\mathrm{cc}}^{2}\left[\sum_{i i^{\prime}} \frac{T_{i i^{\prime}}^{2}}{T_{i i^{\prime}} T_{i^{\prime} i^{\prime}}}\right]^{-1}
$$

This means that $\epsilon^{2}$ has expected value unity and expected deviation from one that is determined from the properties of $\chi^{2}$. While considering the distribution of $\epsilon^{2}$ to be $\frac{1}{M_{\text {eff }}} \chi_{M_{\text {eff }}}^{2}$ is not rigorously justified for all possible covariance matrices $\mathbf{T}$, it is the rigorously correct distribution both in the limit of uncorrelated consistency checks (diagonal $\mathbf{T}$ ), and in the limit of all the linear combinations being included twice (i.e. the limit where all the linear combinations are perfectly correlated with exactly one other member of the set), making it at least a reasonable choice for the general case which presumably falls somewhere between those two extremes.

As an example of the statistic $\epsilon^{2}$, consider a vector of linear combinations e which has only two elements: the difference between zonal and meridional kinetic energy at depth 1 , and the difference between zonal and meridional kinetic energy at depth 2 .

$$
\begin{aligned}
& e_{1}=\left\langle u_{1}^{*} u_{1}\right\rangle-\left\langle v_{1}^{*} v_{1}\right\rangle \\
& e_{2}=\left\langle u_{2}^{*} u_{2}\right\rangle-\left\langle v_{2}^{*} v_{2}\right\rangle
\end{aligned}
$$

The true covariance of the cross-spectral estimators is a function of the true value of the cross-spectra and the degree of averaging $N_{r}$ (see appendix $C$ ). In this case suppose 
that the true values for both zonal and meridional velocity autospectra at both depths are all the same: denote that value by $\Phi_{0}$. Then the variance about the true autospectra for all of the autospectral estimators $\left\langle u_{i}^{*} u_{i}\right\rangle,\left\langle v_{i}^{*} v_{i}\right\rangle$ is given by $\frac{1}{N_{r}} \Phi_{0}^{2}$ and the covariance between any of the four spectral estimators is zero. This allows the covariance matrix $T_{i j}=\left\langle e_{i} e_{j}\right\rangle$ to be calculated,

$$
\mathbf{T}=\frac{1}{N_{\tau}}\left[\begin{array}{cc}
2 \Phi_{0}^{2} & 0 \\
0 & 2 \Phi_{0}^{2}
\end{array}\right]
$$

The two arises because the variance of the sum (or difference) of two independent random variables is simply the sum of the variances of each of the random variables. Now the statistic $\epsilon^{2}$ can be written explicitly as

$$
\epsilon^{2}=\frac{1}{2}\left[\frac{\left(\left\langle u_{1}^{*} u_{1}\right\rangle-\left\langle v_{1}^{*} v_{1}\right\rangle\right)^{2}}{\frac{2}{N_{r}} \Phi_{0}^{2}}+\frac{\left(\left\langle u_{2}^{*} u_{2}\right\rangle-\left\langle v_{2}^{*} v_{2}\right\rangle\right)^{2}}{\frac{2}{N_{r}} \Phi_{0}^{2}}\right]
$$

In this case, then, $\epsilon^{2}$ is simply the normalized average of the squares of the two differences. Thus it is entirely correct that $\epsilon^{2}$ is considered distributed as a reduced $\chi^{2}$ variable $\frac{1}{M_{\text {eff }}} \chi_{M_{\text {eff }}}^{2}$ with $M_{\text {eff }}=2$ effective degrees of freedom.

In practice, the true value $\Phi_{0}$ is not known, and an estimate must be used instead. If $N_{r}$ is large then the estimate is a good approximation to $\Phi_{0}$ and little error is introduced by the substitution. This means that the expression for $\epsilon^{2}$ changes from equation 6.2 .7 to

$$
\epsilon^{2}=\frac{1}{2}\left[\frac{\left(\left\langle u_{1}^{*} u_{1}\right\rangle-\left\langle v_{1}^{*} v_{1}\right\rangle\right)^{2}}{\frac{2}{N_{r}}\left(\frac{1}{2}\left[\left\langle u_{1}^{*} u_{1}\right\rangle+\left\langle v_{1}^{*} v_{1}\right\rangle\right]\right)^{2}}+\frac{\left(\left\langle u_{2}^{*} u_{2}\right\rangle-\left\langle v_{2}^{*} v_{2}\right\rangle\right)^{2}}{\frac{2}{N_{r}}\left(\frac{1}{2}\left[\left\langle u_{2}^{*} u_{2}\right\rangle+\left\langle v_{2}^{*} v_{2}\right\rangle\right]\right)^{2}}\right]
$$

Here the mean value at a particular depth is used instead of the true value.

It is important to note that, while perfect agreement between the model predictions and the data would mean that all the elements of $\mathbf{e}$ and thus $\epsilon^{2}$ would be identically zero, the expected value of $\epsilon^{2}$ is one, not zero. In fact, it is just as unlikely for $\epsilon^{2}$ to be very close to zero as it is to be very large. This is because it is expected that there be a certain amount of noise in the spectral estimators, thus it is expected that there be a certain amount of noise in the linear combinations used as consistency checks. It can be seen explicitly in equation 6.2 .7 , for example, that if the measured variance (numerators in the two fractions) matches the theoretical prediction for the variance in the spectral estimators (denominators in the two fractions) then the value of $\epsilon^{2}$ will be one. In effect, then comparing $\epsilon^{2}$ to its $95 \%$ confidence limits tests both the internal wave model and 
the statistical prediction of the size of the noise level. Only if both predictions are correct will $\epsilon^{2}$ be within its $95 \%$ confidence limits.

The consistency checks are presented by plotting the $\epsilon^{2}$ statistic for each model against its $95 \%$ confidence limits.

\subsection{Symmetries of the internal wave spectrum}

In order to systematically discuss the symmetries of the internal wave spectrum, this section relates some of the basic symmetries to the generalized spectral level $(E, \Delta, P, Q)$ defined in chapter 5. After listing the symmetries of interest, it is demonstrated that, by expanding each of the components $E, \Delta, P, Q$ in a Fourier series, each symmetry can be expressed as a constraint which forces a set of model parameters to zero. Since each model parameter can be expressed as a linear combination of the measured cross-spectra, this procedure gives a set of linear consistency checks of the form given in equation 6.2.1.

Even though the GM72 paper bases its discussion of the problem in terms of about a smeared set of modes, the GM internal wave spectral models essentially consider the internal wave field to consist of vertically propagating waves with as much energy propagating upwards as downwards. This allows the internal wave energy to be expressed as a single spectral level $E(\omega, K, \theta)$, a function only of frequency $\omega$, horizontal wavenumber amplitude $K$, and horizontal wavenumber direction $\theta$. In order to consider wavefields where there is more energy propagating in one vertical direction than another, chapter 5 introduced the spectral component $\Delta(\omega, K, \theta)$, which gives the difference in spectral level between waves propagating upwards and waves propagating downwards. $E$ remains the total upwards-plus-downwards spectral level. Generalizing still further in order to allow modes as well as vertically propagating waves, chapter 5 introduced the spectral components $P(\omega, K, \theta), Q(\omega, K, \theta)$ which give the level of the energy that has a modal character: $P$ gives the level of the energy that has a maximum at the reference level, which $Q$ gives the level of the energy that has a zero at the reference level (the reference level is some chosen depth, see section 5.6). $E$ is now the total upwards-plus-downwardplus-modal spectral level (For a concrete example, look at the end of section 5.9, which derives for a constant buoyancy frequency ocean expressions for the energy density and its fluxes in terms of $E, \Delta, P$, and $Q$ ).

We are now in a position to connect vertical symmetry properties of the wavefield to 
properties of the generalized spectral level $(E, \Delta, P, Q)$. When the wavefield has as much energy propagating upwards as it does downwards, the wavefield is said to be vertically symmetric. This is equivalent to $\Delta(\omega, K, \theta)$ being identically zero. If all the energy in a constant buoyancy frequency ocean is in vertically propagating waves, then there is no depth dependence of energy: the wavefield is then said to be vertically homogeneous. This is equivalent to $P$ and $Q$ being identically zero. In the more general case where the buoyancy frequency is not constant, to the extent that the WKBJ approximation provides adequate solutions to the vertical structure equation the measurements and vertical separations can be scaled to an equivalent constant buoyancy frequency ocean and the wavefield can be said to be (scaled) vertically homogeneous. Even in the more general case where the WKBJ approximation is inadequate, one can still talk about a purely propagating wavefield ( $P=Q=0)$ vs. a wavefield that is comprised of a mixture of vertically propagating waves and standing modes $(P, Q \neq 0)$.

The horizontal symmetry properties reduce to a set of zeroed model parameters in a similar way. If all four of the spectral level components are written as functions of frequency and horizontal wavenumber vector exclusively, we have implicitly assumed that there is no dependence of energy on absolute horizontal position: the wavefield is horizontally homogeneous. To the extent that the ocean is not horizontally homogeneous, this is a potential problem, but the scaling done in chapter $\mathbf{5}$ shows that as long as the frequencies of interest are above $1 \mathrm{cph}$ then horizontal homogeneity is appropriate. Furthermore, since the PEQUOD mooring measurements offer little horizontal resolution, horizontal inhomogeneities are effectively filtered out of the data and thus such inhomogeneities cannot be responsible for model-data misfits.

The GM models have the same amount of energy propagating in all directions, i.e. the wavefield is horizontally isotropic and the spectral level consequently shows no azimuthal dependence. Because the consistency checks are all in the form of linear combinations of cross-spectra that have expected value zero, it would be nice to express the existence of a symmetry as a set of linear combinations that have expected value zero. Horizontal isotropy can be expressed as a "zeroed set of parameters" if we consider each of the spectral level components to have a Fourier expansion in direction $\theta$

$$
X(\omega, K, \theta)=X_{0}(\omega, K)+2 \sum_{n=1}^{\infty}\left[X_{c n}(\omega, K) \cos n \theta+X_{s n}(\omega, K) \sin n \theta\right]
$$

where $X$ represents in turn $E, \Delta, P, Q$ and the normalizations have been chosen for later 
convenience. Given this expansion, there are rather simple expressions for the inverse transformation of calculating the coefficients $X_{c n}, X_{s n}, X_{0}$ from $X(\theta)$

$$
\begin{gathered}
X_{0}=\frac{1}{2 \pi} \int_{0}^{2 \pi} X(\theta) d \theta=\langle X\rangle \\
X_{o n}=\langle\cos n \theta X\rangle \\
X_{s n}=\langle\sin n \theta X\rangle
\end{gathered}
$$

Consequently the cosine and sine moments are directly equivalent to Fourier coefficients, and the notation $\langle\cos n \theta X\rangle$ (and $\langle\sin n \theta X\rangle$ ) will be used for the Fourier coefficients and for the cosine (and sine) moments, while $\langle X\rangle$ will be used for the zeroth Fourier coefficient which is also the average over all directions.

With this Fourier expansion horizontal, meridional, and zonal symmetries can be simply related to sets of zeroed Fourier coefficients. If the wavefield is zonally symmetric, then all of the cosine moments for the components of the generalized spectral level will be zero. If the wavefield is meridionally symmetric, then all of the sine moments of the components of the generalized spectral level will be zero. Finally, if the wavefield is horizontally isotropic, both the cosine and sine moments will be zero, leaving only the zeroth coefficient $\langle X\rangle$ non-zero for each of the spectral level components.

The results of this section are summarized in figure 6.3.1. 


\section{Wavefield symmetries in terms of generalized spectral level}

Wavefield property

Vertical symmetry

(Scaled) vertical homogeneity

Purely vertically propagating

Purely modal

Zonally symmetric

Meridionally symmetric

Horizontally isotropic
Expression in terms of $(E, \Delta, P, Q)$

$$
\begin{gathered}
\Delta=0 \\
P=Q=0 \\
P=Q=0 \\
\Delta=0 \\
\langle\cos n \theta X\rangle=0 \\
\langle\sin n \theta X\rangle=0 \\
\langle\cos n \theta X\rangle=\langle\sin n \theta X\rangle=0
\end{gathered}
$$

- $X$ represents any/all of the components $E, \Delta, P$, and $Q$.

- Horizontal homogeneity is presumed by all the models.

\section{Figure 6.3.1 Section 6.3 summary}

Summary of section 6.3 relating the basic symmetries of the field of internal waves to moments of the generalized spectral level. 


\subsection{Model reduction results from PEQUOD}

As will be derived in detail in the appendical half of this chapter (sections 6.6 and 6.7), some linear combinations of the spectra computed from current meters are directly related to the direction moments of the generalized spectral level. Zero values for these moments can be interpreted as statements on the symmetry properties of the internal wave field (see section 6.3). Using portions of the PEQUOD data set, this section checks whether some of the generalized spectral level moments of a no mean flow model for internal waves can be considered zero for the PEQUOD area. As it turns out, most of the moments are significantly non-zero. Furthermore, the violations in the PEQUOD data tend to be stronger along the zonal axis than along the meridional axis, an observation which is consistent with the effects being due to the presence of a mean zonal velocity shear.

The cross-spectra used in the comparison are taken from the first year current meter data from mooring $Q(\mathrm{Q} 1$ data) and the second year current meter data from mooring $U$ (U2 data). The two sets of year-long records were chosen because they have better data return and thus better depth coverage than the $Q 2$ and $U 1$ records. Only current meter data are used because the additional temperature measurements provided by the TP recorders do not provide additional consistency checks.

As demonstrated explicitly in section 6.6, the imaginary parts of the current meter cross-spectra are completely determined by the direction moments of $\Delta$. This is true both for internal wave models that include the effects of mean shear flow and for internal wave models that do not. The $\Delta$ direction moments for a no mean flow internal wave model calculated from the Q1 and U2 cross-spectra are presented in figures 6.4.1 and 6.4.2 respectively. Note that a series of very heavily averaged frequency bins are presented, and signals on the order of one percent can be distinguished from zero. Note that only the values for frequencies higher than $.1 \mathrm{cph}$ can be reasonably interpreted in terms of the high frequency internal wave models. This is because the no mean flow model used to connect the current meter spectra to the generalized spectral level makes assumptions valid only for these higher frequencies. Furthermore, the energy in the $.1 \mathrm{cph}$ bin is dominated by the tides, a forced motion not well modeled by the internal wave spectral model. The lower frequencies are included on the plots to help in the interpretation of the results for frequencies higher than $.1 \mathrm{cph}$ i.e. to clearly show the nature of the tidal peak and reveal whether the character of the energy at higher frequencies is of the same or very different nature than that tidal peak. 


\section{Q1 Moments}

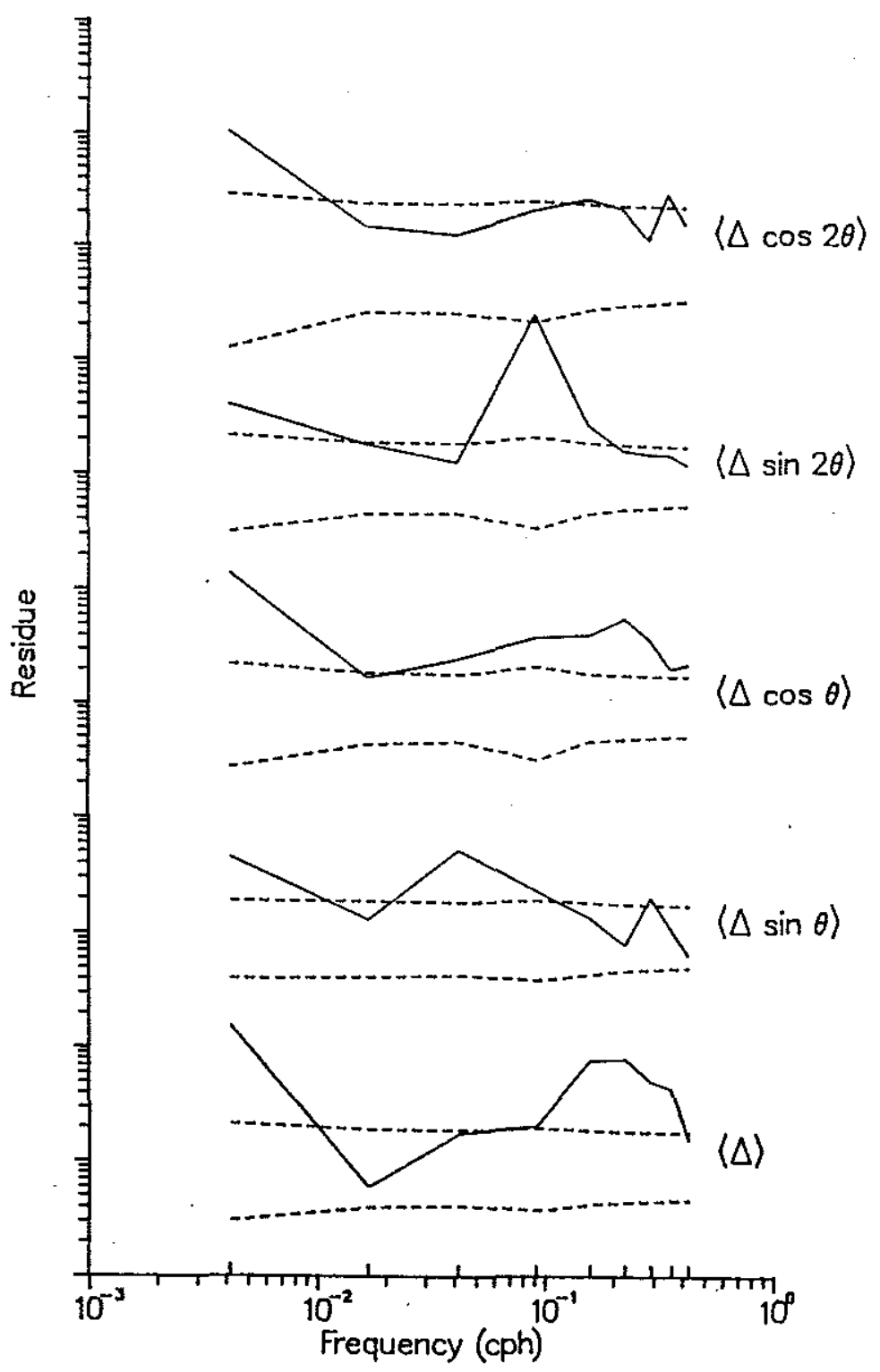

Figure 6.4.1 Zero $\Delta$ moment consistency checks using $Q 1$ data

The $\epsilon^{2}$ statistic is plotted for the five $\Delta$ moments using an internal wave model whose basic state has no mean flow. First year $Q$ mooring data are used to compute the cross-spectra. The pairs of dashed lines give the $95 \%$ confidence limits for each moment being zero, and the upward pointing tic marks on the frequency axis give the centers of the frequency bins.

Aside from the tidal energy included in the $.1 \mathrm{cph}$ frequency bin, which shows a strong violation in the meridional asymmetry $\langle\Delta \sin 2 \theta\rangle$, the violations in the supertidal frequencies are confined to the isotropic $\langle\Delta\rangle$ and first zonally asymmetric $\langle\Delta \cos \theta\rangle$ moments. The violations are strongly peaked above, not including, the tides. These results are consistent with the zonal mean flow being the causal agent. 


\section{U2 Moments}

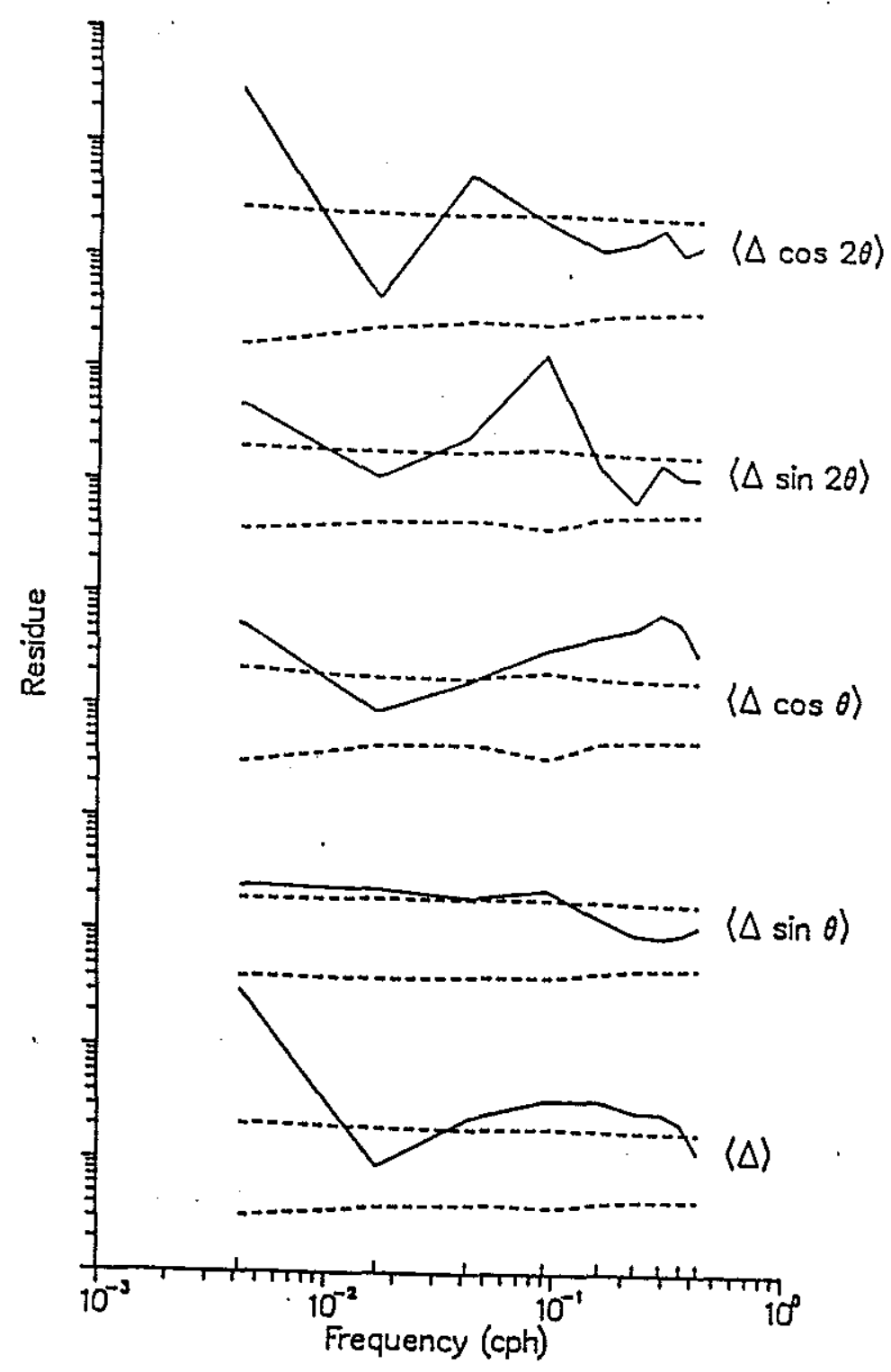

Figure 6.4.2 Zero $\Delta$ moment consistency checks using U2 data

The $\epsilon^{2}$ statistic is plotted for the five $\Delta$ moments using an internal wave model whose basic state has no mean flow. Second year $U$ mooring data are used to compute the cross-spectra. The pairs of dashed lines give the $95 \%$ confidence limits for each moment being zero, and the upward pointing tic marks on the frequency axis give the centers of the frequency bins.

Aside from the tidal energy included in the $.1 \mathrm{cph}$ frequency bin, which shows a strong violation in the meridional asymmetry $\langle\Delta \sin 2 \theta\rangle$, the violations in the supertidal frequencies are confined to the isotropic $\langle\Delta\rangle$ and first zonally asymmetric $\langle\Delta \cos \theta\rangle$ moments. The violations seem fairly uniform in strength at all frequencies above and including the tides. These results are consistent with the zonal mean flow being the causal agent. 
The bottom curve in figures 6.4.1 and 6.4.2 is the $\epsilon^{2}$ statistic for presuming that the isotropic moment for the upwards minus downwards spectral level difference $\langle\Delta\rangle$ is zero. Since the curve is outside the $95 \%$ confidence limits for frequencies above $.1 \mathrm{cph}$ in both figures, there is a significant difference between the horizontally isotropic portions of the upward and downward propagating energy at those frequencies.

The second curve from the bottom in each of the two figures 6.4.1 and 6.4.2 presents the results of presuming that the first sine moment of the up-down difference $\langle\Delta \sin \theta\rangle$ is zero. Since the curve is within the $95 \%$ confidence limits for the supertidal frequencies, the data is consistent with this moment being zero. To the extent that the only other sine moment of $\Delta$ resolved by the data $(\langle\Delta \sin 2 \theta\rangle)$ is zero as well, the up-down difference is meridionally symmetric: i.e. there is no difference between northward and southward vertically propagating energy.

The middle curve in each of the two figures presents the first cosine moment of the up-down difference $\langle\Delta \cos \theta\rangle$. In both plots the moment is significantly non-zero for the supertidal frequencies. This means that there is a significant difference between eastward and westward energy if the data are to be interpreted in terms of a no mean flow model.

The next curve in each of the two figures 6.4.1 and 6.4.2 shows the results for the second sine moment of the up-down difference $\langle\Delta \sin 2 \theta\rangle$. The moment is significantly non-zero for the tidally dominated $.1 \mathrm{cph}$ frequency bin, but drops rapidly and is consistent with zero for the supertidal frequencies. Since the first sine moment is consistent with zero as well, this shows that the up-down difference can be considered meridionally symmetric.

The top curve in each of the two figures 6.4.1 and 6.4.2 presents the second cosine moment of the up-down difference $\langle\Delta \cos 2 \theta\rangle$. In both cases it is consistent with zero. This means that the zonal asymmetry is sufficiently described by a $\cos \theta$ dependence on direction: the four-lobed character of the second cosine moment is not present in the data. Since chapter 5 showed that the higher direction moments $(n>2)$ are not related to the measured cross-spectra, the plots present a complete set of direction moments for the internal wave models that have a resting basic state.

In summary, then, there is a significant up-down difference in the PEQUOD data. Interpreted in terms of a no mean flow model, the isotropic and first cosine moments of the up-down difference are the only moments significantly different from zero for the supertidal frequencies. Even for an internal wave model that includes the effects of a 
mean shear flow, these results require a significant up-down difference (appendix $D$ ).

The remaining components of the generalized spectral level $(E, P, Q)$ are related to the real parts of the measured (current meter) cross-spectra. Again this is true for internal wave models with and without the effects of mean shear flow. While the current meter consistency checks do not distinguish between the different components $E, P, Q$, the checks do distinguish between the different direction moments of the grouped spectral level components. It is thus possible to test whether, for example, three of the first cosine moments $\langle E \cos \theta\rangle,\langle P \cos \theta\rangle$, and $\langle Q \cos \theta\rangle$ are all zero. As it turns out, all of the real part moments tested are significantly different from zero, with the zonal moments more strongly in violation of isotropy than the meridional moments. These results, like the up-down difference results, are possibly consistent with the effects of a mean zonal shear flow.

Because the consistency checks are unable to determine the relative contributions of $E, P, Q$, a new variable $R$ is used to represent the mixture (it can be thought of as representing the 'real part', since it is calculated from the real part of the current meter cross-spectra). Figures 6.4 .3 and 6.4.4 present the $R$ moments calculated from the $\mathrm{Q} 1$ and U2 data respectively. For the most part the results for the two data sets are the same, with zonal asymmetries (cosine moments) being distinctly stronger than meridional asymmetries (sine moments).

The top curve in figures 6.4 .3 and 6.4 .4 is the $\epsilon^{2}$ statistic for presuming that the isotropic $R$ moment is zero. Since the curve is far outside the $95 \%$ confidence limits for frequencies above $.1 \mathrm{cph}$ in both figures, there is significant isotropic energy of either propagating or modal character at these frequencies. That fact that the violation is so much stronger for the isotropic moments of $R$ than it is for the remaining moments of $R$ is consistent with the GM idea that the internal wave field is predominantly isotropic and vertically symmetric.

The bottom curve in each figure gives the $\epsilon^{2}$ statistic for the first sine moment $\langle R \sin \theta\rangle$, while the center curve gives the $\epsilon^{2}$ statistic for the second sine moment $\langle R \sin 2 \theta\rangle$. In both figures they are significantly non-zero for all frequencies above $.1 \mathrm{cph}$ : this means that the internal wave field is consistent with neither a meridionally symmetric wave field in a resting basic state nor a meridionally symmetric internal wave field that includes the effects of a zonal shear flow: some north-south differences in the generalized spectral level are required for consistency with the PEQUOD data. 


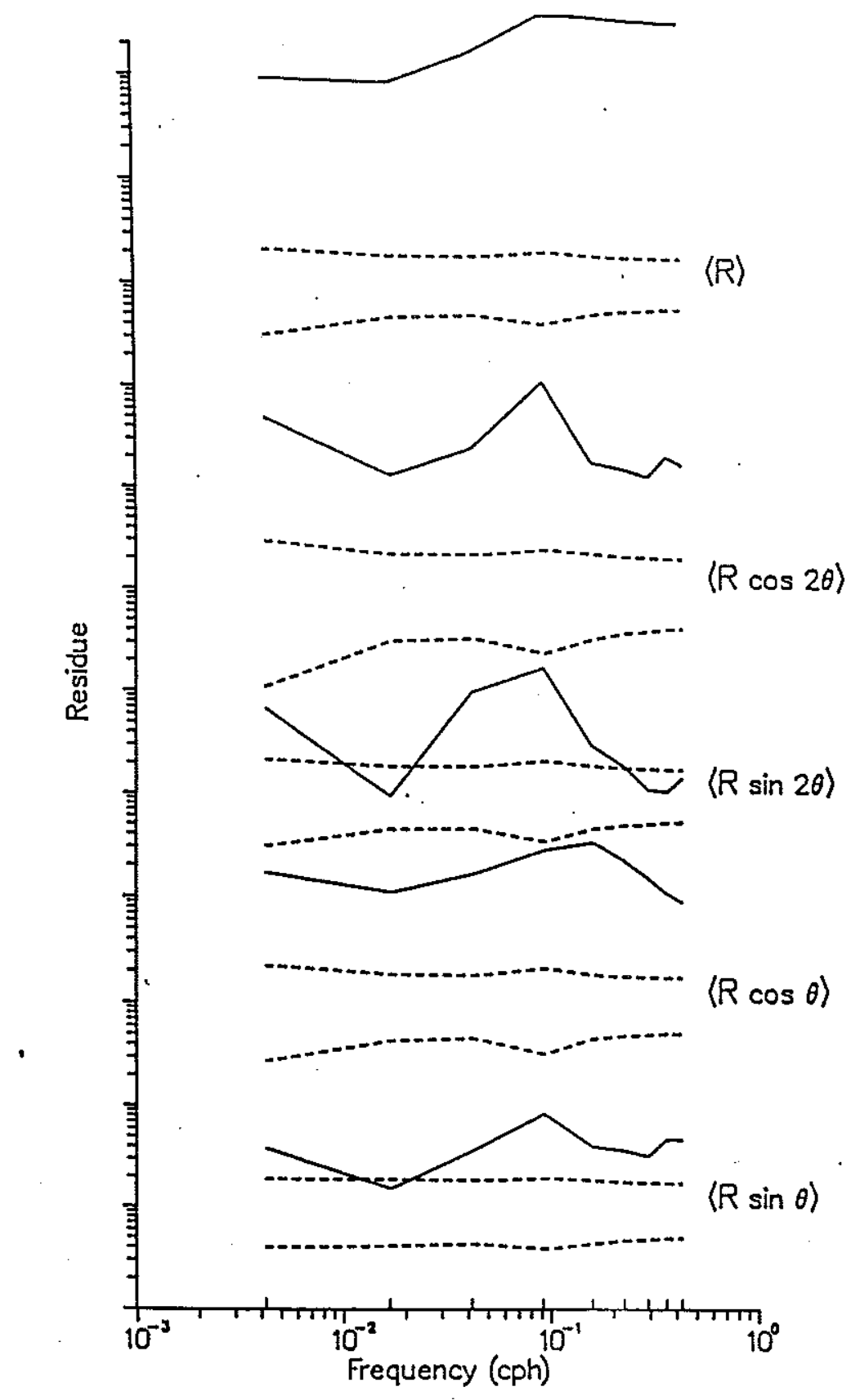

Figure 6.4.3 Zero $R$ moment consistency checks using Q1 data

The $\epsilon^{2}$ statistic is plotted for the five $R$ moments using an internal wave model whose basic state has no mean flow. First year $Q$ mooring data are used to compute the cross-spectra. The pairs of dashed lines give the $95 \%$ confidence limits for each moment being zero, and the upward pointing tic marks on the frequency axis give the centers of the frequency bins.

Comparison of the isotropic moment $\langle R\rangle$ with the other moments indicates that the wave field is predominantly isotropic, while the fact the the other moments are significantly non-zero indicates that an isotropic model is inadequate. 


\section{U2 Moments}

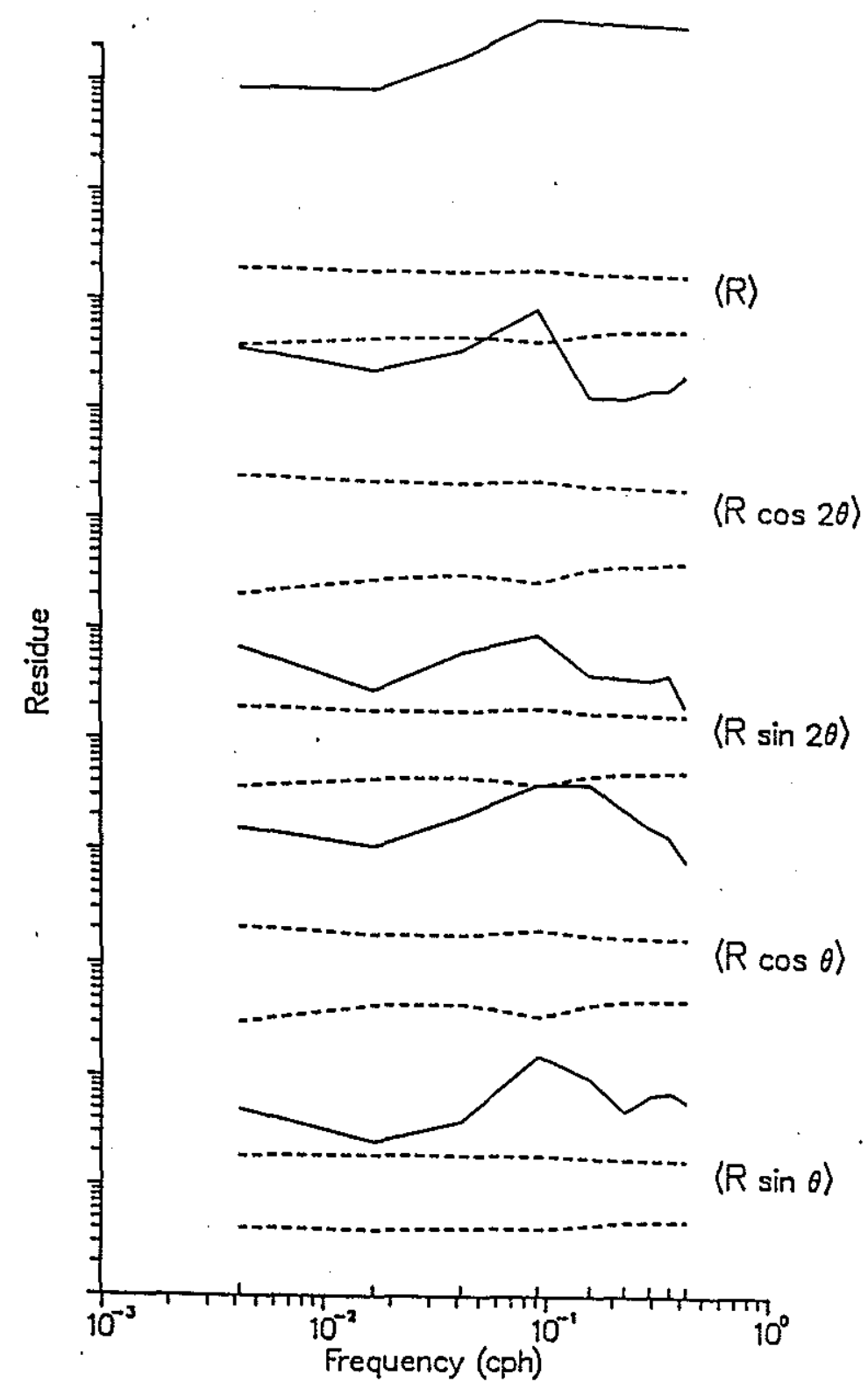

Figure 6.4.4 Zero $R$ moment consistency checks using $\mathrm{U} 2$ data

The $\epsilon^{2}$ statistic is plotted for the five $R$ moments using an internal wave model whose basic state has no mean flow. Second year $U$ mooring data are used to compute the cross-spectra. The pairs of dashed lines give the $95 \%$ confidence limits for each moment being zero, and the upward pointing tic marks on the frequency axis give the centers of the frequency bins.

Comparison of the isotropic moment $\langle R\rangle$ with the other moments indicates that the wave field is predominantly isotropic, while the fact the the other moments are significantly non-zero indicates that an isotropic model is inadequate. 
Counting from the top, the second and fourth curves give the $\epsilon^{2}$ statistic for the first cosine moment $\langle R \cos \theta\rangle$ and the second cosine moment $\langle R \cos 2 \theta\rangle$ respectively. They are significantly different from zero for all frequencies greater than $.1 \mathrm{cph}$, signifying that a strong zonal asymmetry in the generalized spectral level would be required for the no mean flow internal wave model to be consistent with the PEQUOD data, results that are also consistent with a internal wave model that includes the effects of a zonal shear flow. These cosine moments are distinctly larger than the sine moments, indicating that zonal asymmetries are much stronger than meridional asymmetries.

\subsection{Internal wave symmetry results from PEQUOD}

In addition to relating direction moments of the generalized spectral level to cross-spectra calculated from current meter data, the no mean flow internal wave models also predict that certain components of the current meter cross-spectra must be depth independent, and that certain components of the current meter cross spectra must be zero. After presenting the depth independence checks by themselves, this section goes on to combine linear consistency checks and look at whether certain simplified models are possibly consistent with the PEQUOD data. These simplified models include making all the symmetry assumptions of GM79, as well as various subsets thereof: thus all of the symmetries of section 6.3 are discussed. In light of the results of section 6.4 , it should not be surprising that many of these simplified models are not consistent with the data, and a more elaborate model, such as an asymmetric internal wave field that includes the effects of mean shear, is required.

Figure 6.5.1 checks the depth independence of $\left\langle u_{1}^{*} \eta_{1}\right\rangle$ (5 in table 3 of figure 6.6.3) and $\left\langle v_{1}^{*} \eta_{1}\right\rangle$ (7 in table 3 of figure 6.6.3) using both the $\mathrm{Q} 1$ and $\mathrm{U} 2$ datasets. In the no mean flow model depth independence of 5 and 7 are equivalent to depth independence of $\langle\Delta \cos \theta\rangle$ and $\langle\Delta \sin \theta\rangle$ (moments which are depth independent as a result of conservation of wave action); in the model of internal waves in a mean shear flow that equivalence no longer exists (so that while the moments $\langle\Delta \cos \theta\rangle$ and $\langle\Delta \sin \theta\rangle$ are still depth independent when the model includes the effects on mean shear, the quantities $\left\langle u_{1}^{*} \eta_{1}\right\rangle$ and $\left\langle v_{1}^{*} \eta_{1}\right\rangle$ are not predicted by the model to be depth independent). The plots show that in the meridional direction the cross-spectra are consistent with depth independence, while in the zonal direction the cross-spectra are not. It is important to note that figures 6.4 .1 and 6.4.2 show that $\Delta \sin \theta$ has a large peak at the tides but is indistinguishable from 
zero at supertidal frequencies: thus the new meridional information in figure 6.5.1 is that the tidal peak is in fact depth independent. The misfit in the zonal direction for higher frequencies is consistent with the idea that the effects are due to mean zonal currents.

Plotted in figures 6.5.2 and 6.5.3 are the $\epsilon^{2}$ statistics for several no mean flow internal wave models. The 'General no mean flow' checks include the linear combinations of the current meter cross-spectra for which all the internal wave models which have a resting basic state predict a value of zero. As will be shown in section 6.6 , these linear combinations have the form $\left\langle u_{1}^{*} v_{2}\right\rangle-\left\langle v_{1}^{*} u_{2}\right\rangle$, where the subscripts denote depth and all single depths and depth pairs are considered in turn. The depth independence checks looked at earlier in this section are included as well. The figures show that the consistency checks are violated for most of the supertidal frequencies, though the deviation is small compared to the less general models that follow. This inconsistency could be a consequence of the presence of a mean shear flow. First of all, the "all depth pair" check results from the isotropy of the basic state, thus it is violated when a mean shear flow is introduced. Likewise, the depth independence of 5 and 7 depends both on the isotropy of the basic state and on action flux conservation (action flux conservation is equivalent to $\Im\left(\alpha^{*} \alpha_{z}\right)$ being independent of depth). In the case of a mean shear flow, the isotropy is lost, which is sufficient to unconstrain $\mathbf{5}$ and $\mathbf{7}$. Note that is also possible to lose the constraint in the presence of forcing or dissipation. For example, were there a critical layer at some depth that acted as a sink of wave action, the action fluxes measured above and below the level would be different, and the consistency check that checks depth independence across the layer could become significantly nonzero.

The remaining consistency checks show that several of the assumptions used in simplifying the GM models are not valid for the PEQUOD data. 'Vertically symmetric' checks whether the up-down difference $\Delta$ can be considered zero. Plotted is the $\epsilon^{2}$ statistic that combines all the different $\Delta$ direction moment checks presented in section 6.4. Since the individual $\Delta$ moments were found to be significantly non-zero, it is not surprising that the $\Delta$ cannot be considered identically zero at almost all frequencies. Thus a vertically symmetric model cannot adequately explain the PEQUOD measurements.

'Horizontal isotropy' checks whether the generalized spectral level can be considered independent of direction. Plotted is the $\epsilon^{2}$ statistic that combines all of the $R$ and $\Delta$ cosine and sine moments that are presented in section 6.4 with the $u v$ check included in the generalized no mean flow check discussed above. As was noted in section 6.4, 


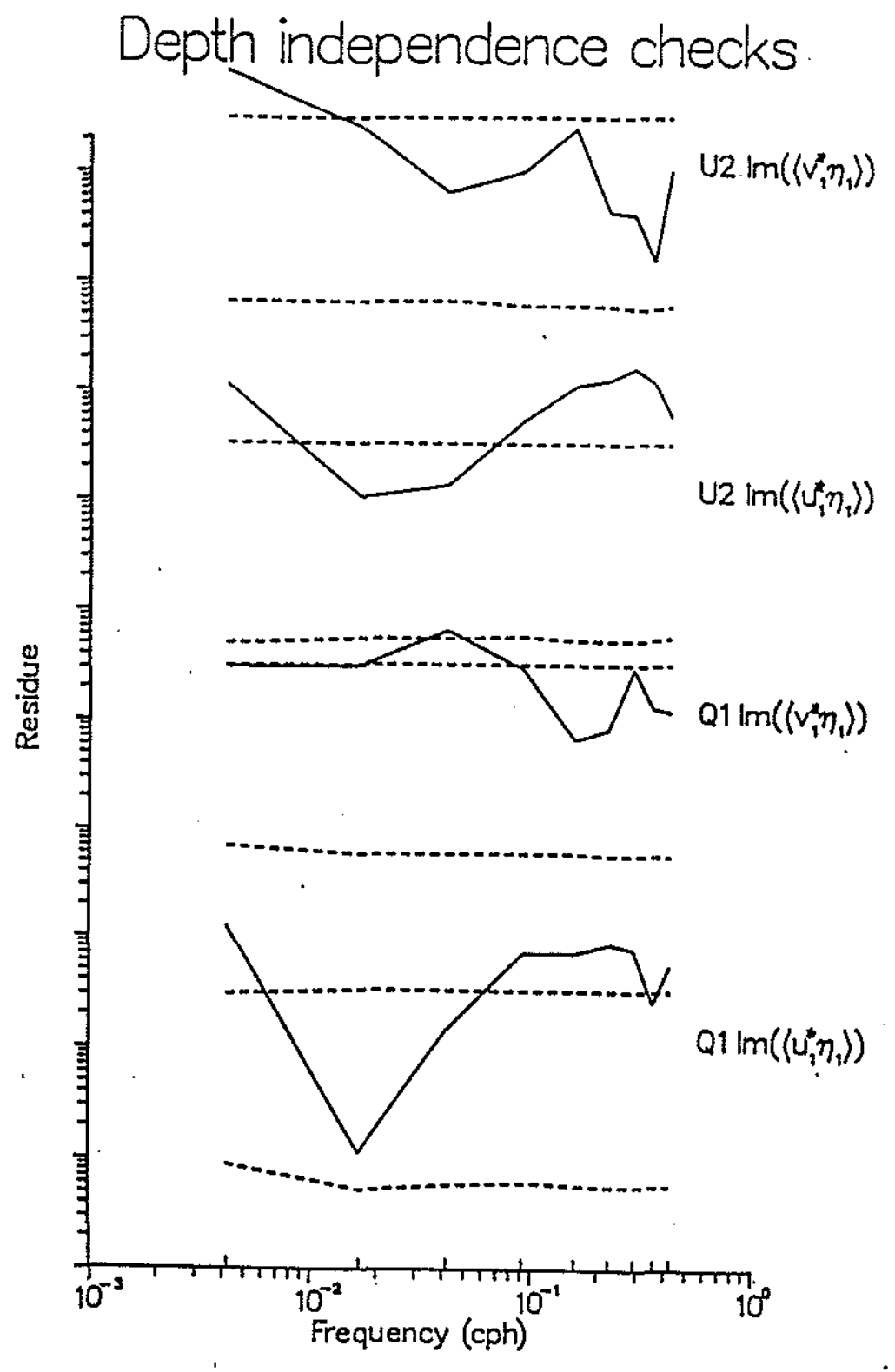

Figure 6.5.1 Depth independence consistency checks

The $\epsilon^{2}$ statistic is plotted for the two single current meter cross-spectra that should be depth independent in the general no mean flow model. First year $Q$ mooring data (Q1) and second year $U$ mooring data (U2) are used to compute the cross-spectra. The pairs of dashed lines give the $95 \%$ confidence limits for each model, and the upward pointing tic marks on the frequency axis give the centers of the frequency bins.

The plots show that the zonal component $\left\langle u_{1}^{*} \eta_{1}\right\rangle$ is not depth independent while the meridional component $\left\langle v_{1}^{*} \eta_{1}\right\rangle$ is depth independent. Since the calculations of section 6.4 show the measured meridional component to be indistinguishable from zero at all supertidal frequencies, the only new information about the meridional component is that the meridional component is depth independent at the tidal peak. The fact that the zonal component is not depth independent is consistent with mean zonal shear flow effects. 


\section{Q1 Consistency checks}

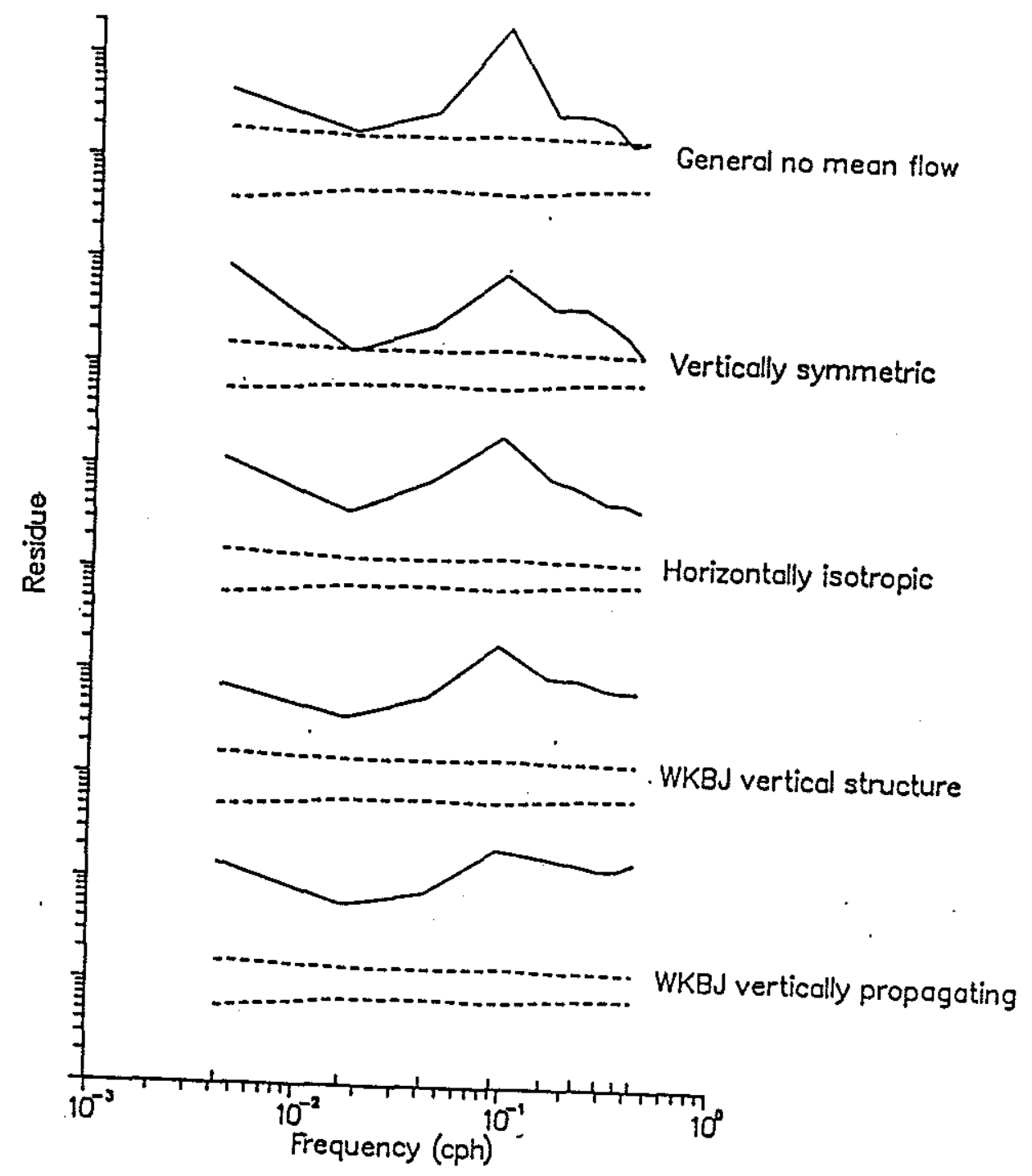

Figure 6.5.2 No mean flow model checks using Q1 data

The $\epsilon^{2}$ statistic is plotted for five internal wave models whose basic state has no mean flow. First year $Q$ mooring data (Q1) are used to compute the cross-spectra. The pairs of dashed lines give the $95 \%$ confidence limits for each model, and the upward pointing tic marks on the frequency axis give the centers of the frequency bins. The plots show that no mean flow models are inadequate to explain the PEQUOD data at supertidal frequencies. 


\section{U2 Consistency checks}

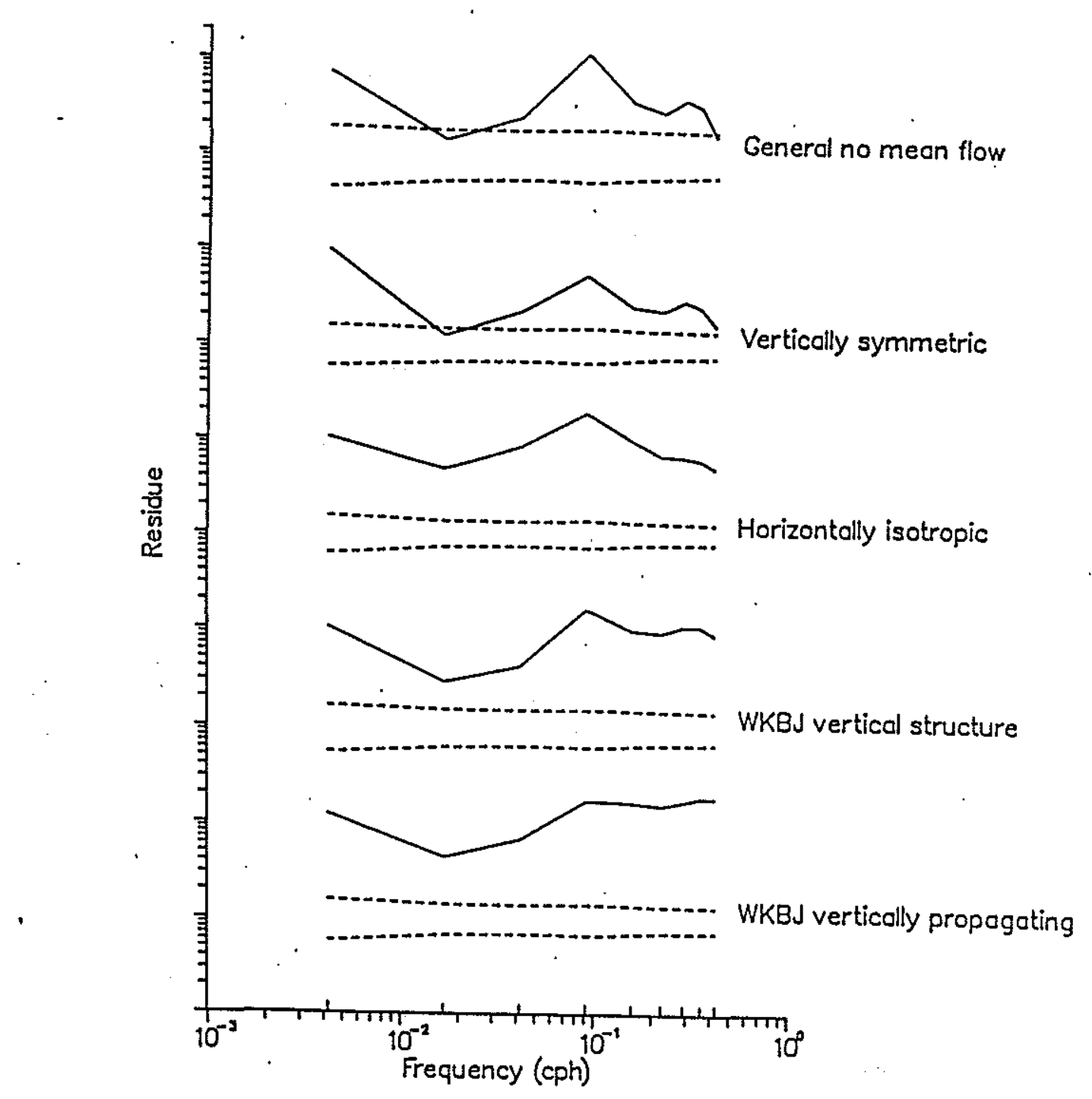

\section{Figure 6.5.3 No mean flow model checks using U2 data}

The $\epsilon^{2}$ statistic is plotted for five internal wave models whose basic state has no mean flow. Second year $U$ mooring data (U2) are used to compute the cross-spectra. The pairs of dashed lines give the $95 \%$ confidence limits for each model, and the upward pointing tic marks on the frequency axis give the centers of the frequency bins. The plots show that no mean flow models are inadequate to explain the PEQUOD data at supertidal frequencies. 
many of those moments are significantly non-zero, and thus the assumption of horizontal isotropy is strongly violated at all frequencies. Thus a horizontally isotropic model cannot be considered consistent with the PEQUOD data.

'WKBJ vertical structure' uses the consistency checks derived in section 6.7 to see whether WKBJ approximate solutions can be used to solve the vertical structure equation without destroying the ability of the model to fit the data. The strong violations seen at high frequencies show that such an approximation is not adequate. Finally, 'WKBJ vertically propagating' makes the further restriction to the WKBJ model that the solutions must be vertically propagating: the modal components of the generalized spectral level $(P, Q)$ are set to zero. Not surprisingly, the violation of this consistency check is even stronger than the violation of the 'WKBJ vertical structure' consistency check.

Combining these results shows that a internal wave model in a resting basic state is inconsistent with the PEQUOD data set, and many of the symmetry assumptions commonly made make the misfit very much worse. The fact that so many of the assumptions used in simplifying the GM model are not consistent with the PEQUOD data is only partially due to differences between the mid-latitude and equatorial environment. While the analysis presented in this chapter is quite close to the analysis done by Müller et al. 1978 on IWEX data, IWEX lasted for only forty days, while PEQUOD lasted for two years. It is possible that if these techniques of Muiller et al. 1978 were applied to a set of yearlong mid-latitude time series, violations of the basic symmetry assumptions might also be found. The fact, however, that the violations tend to be zonal in nature in PEQUOD suggests that at least some of the violations seen here are due to the equatorial environment. The next step is to see whether an internal wave model that includes the effects of a mean zonal shear flow is adequate to explain the spectral features seen in the PEQUOD data. 


\subsection{Consistency checks for no mean flow models with general vertical structure}

The remaining sections of this chapter are appendical. The consistency checks for the no mean flow basic state general solution $\alpha(z)$ to the vertical structure equation models are given in figure 6.6.1. As mentioned earlier, the consistency checks are derived by considering the expansion of the cross-spectra in terms of the generalized spectral level $(E, \Delta, P, Q)$ (see section 5.8). The first table in figure 6.6.1 gives the expansion of the cross-spectra between flow components vertically separated on the same mooring (e.g. two current meters that have a non-zero vertical separation), while the last table in figure 6.6.1 gives the expansion of cross-spectra between components at a single depth (e.g. sensors on a single current meter. The expansion for the general no mean flow model is derived using the equations of chapter 5: the polarization vectors given in equations 5.4.12 and 5.4.13 are used in the cross-spectral expansion given in equation 5.8.9. As an example of how the expansions are calculated and linear combinations chosen, compare the expansion for the cross-spectra between $u$ at depth $z_{1}$ and $v$ at depth $z_{2},\left\langle u_{1}^{*} v_{2}\right\rangle$, with the expansion for the cross-spectra between $v$ at depth $z_{1}$ and $u$ at depth $z_{2},\left\langle v_{1}^{*} u_{2}\right\rangle$.

$$
\begin{aligned}
\left\langle u_{1}^{*} v_{2}\right\rangle(\omega)=\frac{1}{2}\left\langle\operatorname { s i n } 2 \theta \left[\Re\left(\alpha_{1 z}^{*} \alpha_{2 z}\right) E+\Re\left(\alpha_{1 z} \alpha_{2 z}\right) P\right.\right. & \left.\left.-\Im\left(\alpha_{1 z} \alpha_{2_{z}}\right) Q\right]\right\rangle \\
& +\frac{1}{2} i\left\langle\Im\left(\alpha_{1 z}^{*} \alpha_{2 z}\right) \sin 2 \theta \Delta\right\rangle \\
\left\langle v_{1}^{*} u_{2}\right\rangle(\omega)=\frac{1}{2}\left\langle\operatorname { s i n } 2 \theta \left[\Re\left(\alpha_{1 z}^{*} \alpha_{2 z}\right) E+\Re\left(\alpha_{1 z} \alpha_{2 z}\right) P\right.\right. & \left.\left.-\Im\left(\alpha_{1 z} \alpha_{2_{z}}\right) Q\right]\right\rangle \\
& +\frac{1}{2} i\left\langle\Im\left(\alpha_{1 z}^{*} \alpha_{2 z}\right) \sin 2 \theta \Delta\right\rangle
\end{aligned}
$$

where $\Re(\ldots)$ denotes the real part and $\Im(.$.$) denotes the imaginary part. In this case, the$ expansions are identical, and the difference $\left\langle u_{1}^{*} v_{2}\right\rangle-\left\langle v_{1}^{*} u_{2}\right\rangle$ will be zero for all choices of generalized spectral level and vertical structure function. In the more common case where the expansions are not identical, the properties of the terms in the expansions need be considered. Though it is not written explicitly in figure 6.6.1, the generalized spectral level $(E, \Delta, P, Q)$ is a function of frequency $\omega$ and wavenumber $(k, l)=K(\cos \theta, \sin \theta)$, and the vertical structure function $\alpha_{1}$ is a function of frequency, horizontal wavenumber,and depth $\alpha\left(\omega, K, z_{1}\right)$, as is its vertical derivative $\alpha_{z}\left(\omega, K, z_{1}\right)$. Furthermore, the angle brackets $\langle\ldots\rangle$ represents an average over all waves (which can also be thought of as an integral over horizontal wavenumber and direction).

Since the generalized spectral levels are a priori unknown, the consistency checks have to be valid for all choices of functions $(E, \Delta, P, Q)$. An example which illustrates 
the use of these considerations is the kinetic energy difference. Consider the following expansions,

$$
\begin{aligned}
\left\langle u_{1}^{*} u_{2}\right\rangle=\frac{1}{2}\left\langle\operatorname { c o s } ^ { 2 } \theta \left[\Re\left(\alpha_{1 z}^{*} \alpha_{2 z}\right) E+\Re\left(\alpha_{1 z} \alpha_{2 z}\right) P-\right.\right. & \left.\left.\Im\left(\alpha_{1 z} \alpha_{2 z}\right) Q\right]\right\rangle \\
& +\frac{1}{2} i\left\langle\Im\left(\alpha_{1 z}^{*} \alpha_{2 z}\right) \cos ^{2} \theta \Delta\right\rangle \\
\left\langle v_{1}^{*} v_{2}\right\rangle=\frac{1}{2}\left\langle\operatorname { s i n } ^ { 2 } \theta \left[\Re\left(\alpha_{1 z}^{*} \alpha_{2 z}\right) E+\Re\left(\alpha_{1 z} \alpha_{2 z}\right) P-\right.\right. & \left.\left.\Im\left(\alpha_{1 z} \alpha_{2 z}\right) Q\right]\right\rangle \\
& +\frac{1}{2} i\left\langle\Im\left(\alpha_{1 z}^{*} \alpha_{2 z}\right) \sin ^{2} \theta \Delta\right\rangle
\end{aligned}
$$

Both expansions have identical vertical structure function dependence: they differ only in that the average over waves for the zonal velocity involves a $\cos ^{2} \theta$ weighing while the meridional velocity has $\sin ^{2} \theta$ weighing. By considering the Fourier properties of the generalized spectral level, this similarity of structure can be converted into a constraint on the possible symmetries of the generalized spectral level. Consider each of the spectral level components to have a Fourier expansion in direction $\theta$ as discussed in section 6.3. Then the sum and difference of the expressions in equations $6.6 .2 a, b$ are directly related to the Fourier coefficients of the generalized spectral levels. The first table in figure 6.6.1 presents expansions for a complete set of linear combinations of the cross-spectra between two vertically separated current meters. Those expansions are used to derive the second table in figure 6.6.1, a table which gives linear combinations that are zero for three of the models plotted in figures 6.5.1 and 6.5.2 (an entry of ' 0 ' means that the real part is zero for the model, while an entry of ' $i 0$ ' means that the imaginary part is zero). The 'General no mean flow' model includes only the consistency checks that hold for all the no mean flow models: in addition to the consistency check derived in the example (equation 6.6.1) there is the consistency check based on the conservation of vertical action flux (see section 6.5 ) that will be derived shortly. A relatively restrictive model 'Horizontal isotropy' assumes that there is no directional dependence in any of the generalized spectral levels. Another restrictive model 'vertical symmetry' is based on the assumption that the difference between upward and downward propagating spectral level $(\Delta)$ is zero: it results in zero imaginary part for all spectral components.

The consistency checks discussed so far are independent of the choice of the vertical structure function $\alpha(\omega, K, z)$ : none of the properties of the vertical structure equation have been used. As it turns out, there is one property of the general solution $\alpha$ to 
the vertical structure equation 5.6.1 that can be used to form consistency checks: the imaginary part $\Im\left(\alpha^{*}(z) \alpha_{z}(z)\right)$ is independent of depth. This is straightforwardly proven by taking the vertical derivative of the product

$$
\begin{aligned}
\partial_{z}\left(\alpha^{*} \alpha_{z}\right) & =\left|\alpha_{z}\right|^{2}+\alpha^{*} \alpha_{z z} \\
& =\left|\alpha_{z}\right|^{2}-m^{2}(z)|\alpha|^{2}
\end{aligned}
$$

The final expression is purely real, so that the imaginary part of the original expression must be zero. This relation holds as long as the equation is not singular; this means it holds even if $\mathrm{m}^{2}$ is negative (beyond a turning point), but it does not hold if $\mathrm{m}^{2}$ goes to infinity (a critical layer). In the no mean flow model, this independence of depth is equivalent to the vertical flux of energy being constant (see section 5.9). More generally, it is equivalent to the vertical action flux being constant (see section 5.10). In either case, the single current meter cross-spectra whose expansions are given in the last table in figure 6.6.1 can be used to take advantage of this property of $\alpha$. The table shows that there are two cross-spectra $(5,7)$ that are independent of depth as a consequence of equation 6.6 .5 . Therefore taking differences between the values of 5 and 7 at different depths results in additional consistency checks. While in theory one could take differences between all depths, because the same data would be used in many differences such consistency checks would be highly correlated. Thus in the figures depth independence is tested by checking whether nearest neighbor differences are statistically consistent with zero. 
Consistency checks for vertically separated instruments in a resting basic state General solutions $\alpha$ to the vertical structure equation

18 real moments per current meter pair

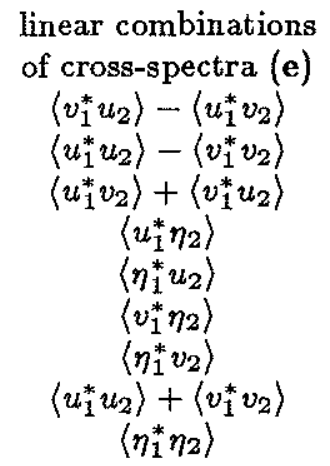

Spectral level expansion

$$
\begin{aligned}
& 0 \quad+\quad i 0 \\
& \left\langle\cos 2 \theta\left[\Re\left(\alpha_{1 z}^{*} \alpha_{2 z}\right) E+\Re\left(\alpha_{1 z} \alpha_{2 z}\right) P-\Im\left(\alpha_{1 z} \alpha_{2 z}\right) Q\right]\right\rangle+i\left\langle\Im\left(\alpha_{1 z}^{*} \alpha_{2 z}\right) \cos 2 \theta \Delta\right\rangle \\
& \left\langle\sin 2 \theta\left[\Re\left(\alpha_{1 z}^{*} \alpha_{2 z}\right) E+\Re\left(\alpha_{1 z} \alpha_{2 z}\right) P-\Im\left(\alpha_{1 z} \alpha_{2 z}\right) Q\right]\right\rangle+i\left\langle\Im\left(\alpha_{1 z}^{*} \alpha_{2 z}\right) \sin 2 \theta \Delta\right\rangle \\
& \left\langle\frac{1}{c} \cos \theta\left[\Re\left(\alpha_{1 z}^{*} \alpha_{2}\right) E+\Re\left(\alpha_{1 z} \alpha_{2}\right) P-\Im\left(\alpha_{1 z} \alpha_{2}\right) Q\right]\right\rangle+i\left\langle\Im\left(\alpha_{1 z}^{*} \alpha_{2}\right) \frac{1}{q} \cos \theta \Delta\right\rangle \\
& \left\langle\frac{1}{q} \cos \theta\left[\Re\left(\alpha_{1}^{*} \alpha_{2 z}\right) E+\Re\left(\alpha_{1} \alpha_{2 z}\right) P-\Im\left(\alpha_{1} \alpha_{2 z}\right) Q\right]\right\rangle+i\left(\Im\left(\alpha_{1}^{*} \alpha_{2 z}\right) \frac{\rho}{q} \cos \theta \Delta\right\rangle \\
& \left\langle\frac{1}{\sigma} \sin \theta\left[\Re\left(\alpha_{1 z}^{*} \alpha_{2}\right) E+\Re\left(\alpha_{1 z} \alpha_{2}\right) P-\Im\left(\alpha_{1 z} \alpha_{2}\right) Q\right]\right\rangle+i\left\langle\Im\left(\alpha_{1 z}^{*} \alpha_{2}\right) \frac{1}{q} \sin \theta \Delta\right\rangle \\
& \left\langle\frac{1}{\mathrm{c}} \sin \theta\left[\Re\left(\alpha_{1}^{*} \alpha_{2 z}\right) E+\Re\left(\alpha_{1} \alpha_{2 z}\right) P-\Im\left(\alpha_{1} \alpha_{2 z}\right) Q\right]\right\rangle+i\left\langle\Im\left(\alpha_{1}^{*} \alpha_{2 z}\right) \frac{1}{c} \sin \theta \Delta\right\rangle \\
& \left\langle\Re\left(\alpha_{1 z}^{*} \alpha_{2_{z}}\right) E+\Re\left(\alpha_{1 z} \alpha_{2 z}\right) P-\Im\left(\alpha_{1 z} \alpha_{2 z}\right) Q\right\rangle+i\left\langle\Im\left(\alpha_{1 z}^{*} \alpha_{2 z}\right) \Delta\right\rangle \\
& \left\langle\Re\left(\alpha_{1}^{*} \alpha_{2}\right) \frac{1}{c^{2}} E+\Re\left(\alpha_{1} \alpha_{2}\right) \frac{1}{c^{2}} P-\Im\left(\alpha_{1} \alpha_{2}\right) \frac{1}{c^{2}} Q\right\rangle+i\left\langle\Im\left(\alpha_{1}^{*} \alpha_{2}\right) \frac{1}{c^{2}} \Delta\right\rangle
\end{aligned}
$$

Consistency checks for single current meter measurements in a resting basic state General solutions $\alpha$ to the vertical structure equation

9 real moments per current meter

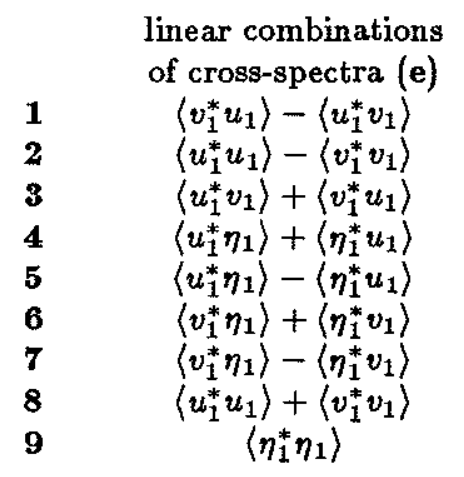

linear combinations

cross-spectra (e)

$\left\langle u_{1}^{*} u_{1}\right\rangle-\left\langle v_{1}^{*} v_{1}\right\rangle$

$\left\langle u_{1}^{*} v_{1}\right\rangle+\left\langle v_{1}^{*} u_{1}\right\rangle$

$\left\langle u_{1}^{*} \eta_{1}\right\rangle+\left\langle\eta_{1}^{*} u_{1}\right\rangle$

$\left\langle u_{1}^{*} \eta_{1}\right\rangle-\left\langle\eta_{1}^{*} u_{1}\right\rangle$

$\left\langle v_{1}^{*} \eta_{1}\right\rangle+\left\langle\eta_{1}^{*} v_{1}\right\rangle$

$\left\langle v_{1}^{*} \eta_{1}\right\rangle-\left\langle\eta_{1}^{*} v_{1}\right\rangle$

$\left\langle\eta_{1}^{*} \eta_{1}\right\rangle$
Vertical

symmetry

i0

$i 0$

$i 0$

$i 0$

io

i0

i0

20

20

\section{Depth independence checks}

$\Im\left(\alpha_{1 z}^{*} \alpha_{1}\right)$ is independent of depth, thus 5 and 7 are independent of depth. 'General no mean flow' checks the depth independence of 5 and 7 as well as the paired consistency check given in the second table of this figure.

Figure 6.6.1 General vertical structure consistency checks 


\section{Consistency checks for vertically separated instruments in a resting basic state}

General solutions $\alpha$ to the vertical structure equation

18 real moments per current meter pair

\section{Zero parameter checks}

Linear combinations

of the cross-spectra (e)

$$
\begin{gathered}
\left\langle v_{1}^{*} u_{2}\right\rangle-\left\langle u_{1}^{*} v_{2}\right\rangle \\
\left\langle u_{1}^{*} u_{2}\right\rangle-\left\langle v_{1}^{*} v_{2}\right\rangle \\
\left\langle u_{1}^{*} v_{2}\right\rangle+\left\langle v_{1}^{*} u_{2}\right\rangle \\
\left\langle u_{1}^{*} \eta_{2}\right\rangle \\
\left\langle\eta_{1}^{*} u_{2}\right\rangle \\
\left\langle v_{1}^{*} \eta_{2}\right\rangle \\
\left\langle\eta_{1}^{*} v_{2}\right\rangle \\
\left\langle u_{1}^{*} u_{2}\right\rangle+\left\langle v_{1}^{*} v_{2}\right\rangle \\
\left\langle\eta_{1}^{*} \eta_{2}\right\rangle
\end{gathered}
$$

linear combinations

of the cross-spectra (e)

$$
\begin{gathered}
\left\langle v_{1}^{*} u_{2}\right\rangle-\left\langle u_{1}^{*} v_{2}\right\rangle \\
\left\langle u_{1}^{*} u_{2}\right\rangle-\left\langle v_{1}^{*} v_{2}\right\rangle \\
\left\langle u_{1}^{*} v_{2}\right\rangle+\left\langle v_{1}^{*} u_{2}\right\rangle \\
\left\langle u_{1}^{*} \eta_{2}\right\rangle \\
\left\langle\eta_{1}^{*} u_{2}\right\rangle \\
\left\langle v_{1}^{*} \eta_{2}\right\rangle \\
\left\langle\eta_{1}^{*} v_{2}\right\rangle \\
\left\langle u_{1}^{*} u_{2}\right\rangle+\left\langle v_{1}^{*} v_{2}\right\rangle
\end{gathered}
$$$$
\left\langle\eta_{1}^{*} \eta_{2}\right\rangle
$$

$\langle R\rangle \quad\langle R \cos \theta\rangle \quad\langle R \sin \theta\rangle \quad\langle R \cos 2 \theta\rangle \quad\langle R \sin 2 \theta\rangle$

0

0
0

0

\section{0}

0

0

0
$\langle\Delta\rangle\langle\Delta \cos \theta\rangle \quad\langle\Delta \sin \theta\rangle \quad\langle\Delta \cos 2 \theta\rangle \quad\langle\Delta \sin 2 \theta\rangle$

$i 0$

$i 0$ $i 0$

$i 0$

i0

$i 0$

i0

Figure 6.6.2 Zero parameter checks for no mean flow models 


\subsection{Consistency checks for no mean flow models with WKBJ vertical structure}

If the vertical structure $\alpha$ is further restricted to WKBJ solutions (equation 5.10.1) are used, there are consistency checks in addition to those already found for general $\alpha$. The two linear combinations of second order $\alpha$ products that form the basis of the new consistency checks are,

$$
N_{2} \alpha_{1 z}^{*} \alpha_{2}=-N_{1} \alpha_{1}^{*} \alpha_{2 z}
$$

and

$$
\alpha_{1 z}^{*} \alpha_{2 z}=\frac{N_{1} N_{2}}{c^{2}} \alpha_{1}^{*} \alpha_{2}
$$

These relations are more conveniently incorporated into the consistency checks by defining a scaled vertical structure function

$$
\beta_{i}=\frac{\sqrt{N_{i}}}{c} \alpha_{i}
$$

By using these relations in the expansion of the cross-spectra, the set of WKBJ consistency checks are found. As expected, the set of WKBJ consistency checks is larger than the set of consistency checks for the general vertical structure case. The first table in figure 6.7.1 gives the cross-spectral expansion using WKBJ solutions, while the second table gives the resulting consistency checks for two WKBJ models: 'WKBJ vertical structure', which allows both modes and vertically propagating waves, and 'WKBJ vertically propagating', which allows only propagating waves $(P=Q=0)$. 


\section{The WKBJ single current meter consistency checks}

For the special case of relations between flow components at the same location (a 'single current meter'), the following are depth independent:

$$
\frac{\alpha_{1 z}^{*} \alpha_{1 z}}{N_{1}}=\frac{N_{1} \alpha_{1}^{*} \alpha_{1}}{c^{2}}=\frac{i}{c} \alpha_{1}^{*} \alpha_{1 z}=\beta_{1}^{*} \beta_{1}
$$

Consequently the WKBJ models predict that several of the single current meter moments can be scaled to be depth independent. 1 is zero even in the general vertical structure case (see section 6.6), and 5 and 7 are depth independent even in the general vertical structure case. 9 is depth independent for both WKBJ models. The remaining consistency checks all show differences between the model that allows modes and the model that is purely vertically propagating. 4 and 6 are zero only in the WKBJ vertically propagating case: they are the single current meter specialization of the WKBJ current meter pair relations 4 and 6. Likewise 8 is zero only for the vertically propagating model: it is the specialization of Fofonoff's consistency check (equation 1.5.3) to the equator. Finally, 2 and 3 are depth independent only for the WKBJ vertically propagating model.

Depth independence is tested by checking whether nearest neighbor differences are statistically consistent with zero. 
Consistency checks for vertically separated measurements in a resting basic state WKBJ solutions $\alpha$ to the vertical structure equation

18 real moments per current meter pair

$$
\begin{array}{ccc}
\begin{array}{c}
\text { linear combinations } \\
\text { of the cross-spectra }(\mathbf{e})
\end{array} & \text { Spectral level expansion } & + \\
\left\langle\left\langle v_{1}^{*} u_{2}\right\rangle-\left\langle u_{1}^{*} v_{2}\right\rangle\right. & 0 & i 0 \\
\frac{1}{\sqrt{N_{1} N_{2}}}\left[\left\langle u_{1}^{*} u_{2}\right\rangle-\left\langle v_{1}^{*} v_{2}\right\rangle\right] & \left\langle\cos 2 \theta\left[\Re\left(\beta_{1}^{*} \beta_{2}\right) E-\Re\left(\beta_{1} \beta_{2}\right) P+\Im\left(\beta_{1} \beta_{2}\right) Q\right]\right\rangle+i\left\langle\left(\beta_{1}^{*} \beta_{2}\right) \cos 2 \theta \Delta\right\rangle \\
\frac{1}{\sqrt{N_{1} N_{2}}}\left[\left\langle u_{1}^{*} v_{2}\right\rangle+\left\langle v_{1}^{*} u_{2}\right\rangle\right] & \left\langle\sin 2 \theta\left[\Re\left(\beta_{1}^{*} \beta_{2}\right) E-\Re\left(\beta_{1} \beta_{2}\right) P+\Im\left(\beta_{1} \beta_{2}\right) Q\right]\right\rangle+i\left\langle\Im\left(\beta_{1}^{*} \beta_{2}\right) \sin 2 \theta \Delta\right\rangle \\
\frac{1}{\sqrt{N_{1} N_{2}}}\left[N_{2}\left\langle u_{1}^{*} \eta_{2}\right\rangle+N_{1}\left\langle\eta_{1}^{*} u_{2}\right\rangle\right] & 2\left\langle\cos \theta\left[\Im\left(\beta_{1} \beta_{2}\right) P+\Re\left(\beta_{1} \beta_{2}\right) Q\right]\right\rangle & \\
\frac{1}{\sqrt{N_{1} N_{2}}}\left[N_{2}\left\langle u_{1}^{*} \eta_{2}\right\rangle-N_{1}\left\langle\eta_{1}^{*} u_{2}\right\rangle\right] & -2\left\langle\cos \theta \Im\left(\beta_{1}^{*} \beta_{2}\right) E\right\rangle & +2 i\left\langle\Re\left(\beta_{1}^{*} \beta_{2}\right) \cos \theta \Delta\right\rangle \\
\frac{1}{\sqrt{N_{1} N_{2}}}\left[N_{2}\left\langle v_{1}^{*} \eta_{2}\right\rangle+N_{1}\left\langle\eta_{1}^{*} v_{2}\right\rangle\right] & 2\left\langle\sin \theta\left[\Im\left(\beta_{1} \beta_{2}\right) P+\Re\left(\beta_{1} \beta_{2}\right) Q\right]\right\rangle & \\
\frac{1}{\sqrt{N_{1} N_{2}}}\left[N_{2}\left\langle v_{1}^{*} \eta_{2}\right\rangle-N_{1}\left\langle\eta_{1}^{*} v_{2}\right\rangle\right] & -2\left\langle\sin \theta \Im\left(\beta_{1}^{*} \beta_{2}\right) E\right\rangle & +2 i\left\langle\Re\left(\beta_{1}^{*} \beta_{2}\right) \sin \theta \Delta\right\rangle \\
\frac{1}{\sqrt{N_{1} N_{1}}}\left[\left\langle u_{1}^{*} u_{2}\right\rangle+\left\langle v_{1}^{*} v_{2}\right\rangle-N_{1} N_{2}\left\langle\eta_{1}^{*} \eta_{2}\right\rangle\right] & \left.2\left\langle-\Re\left(\beta_{1} \beta_{2}\right) P+\Im\left(\beta_{1} \beta_{2}\right) Q\right]\right\rangle & \\
\frac{1}{\sqrt{N_{1} N_{2}}}\left[\left\langle u_{1}^{*} u_{2}\right\rangle+\left\langle v_{1}^{*} v_{2}\right\rangle+N_{1} N_{2}\left\langle\eta_{1}^{*} \eta_{2}\right\rangle\right] & 2\left\langle\Re\left(\beta_{1}^{*} \beta_{2}\right) E\right\rangle & +2 i\left\langle\Im\left(\beta_{1}^{*} \beta_{2}\right) \Delta\right\rangle
\end{array}
$$

$\begin{array}{cccc} & \begin{array}{c}\text { linear combinations } \\ \text { of the cross-spectra (e) }\end{array} & \begin{array}{c}\text { WKBJ vertical } \\ \text { structure }\end{array} & \begin{array}{c}\text { WKBJ vertically } \\ \text { propagating }\end{array} \\ \mathbf{1} & \left\langle v_{1}^{*} u_{2}\right\rangle-\left\langle u_{1}^{*} v_{2}\right\rangle & 0+i 0 & 0+i 0 \\ \mathbf{2} & \left\langle u_{1}^{*} u_{2}\right\rangle-\left\langle v_{1}^{*} v_{2}\right\rangle & & \\ \mathbf{3} & \left\langle u_{1}^{*} v_{2}\right\rangle+\left\langle v_{1}^{*} u_{2}\right\rangle & i 0 & 0+i 0 \\ \mathbf{4} & N_{2}\left\langle u_{1}^{*} \eta_{2}\right\rangle+N_{1}\left\langle\eta_{1}^{*} u_{2}\right\rangle & & \\ \mathbf{5} & N_{2}\left\langle u_{1}^{*} \eta_{2}\right\rangle-N_{1}\left\langle\eta_{1}^{*} u_{2}\right\rangle & i 0 & 0+i 0 \\ \mathbf{6} & N_{2}\left\langle v_{1}^{*} \eta_{2}\right\rangle+N_{1}\left\langle\eta_{1}^{*} v_{2}\right\rangle & & \\ \mathbf{7} & N_{2}\left\langle v_{1}^{*} \eta_{2}\right\rangle-N_{1}\left\langle\eta_{1}^{*} v_{2}\right\rangle & i 0 & 0+i 0 \\ \mathbf{8} & \left\langle u_{1}^{*} u_{2}\right\rangle+\left\langle v_{1}^{*} v_{2}\right\rangle-N_{1} N_{2}\left\langle\eta_{1}^{*} \eta_{2}\right\rangle & & \\ \mathbf{9} & \left\langle u_{1}^{*} u_{2}\right\rangle+\left\langle v_{1}^{*} v_{2}\right\rangle+N_{1} N_{2}\left\langle\eta_{1}^{*} \eta_{2}\right\rangle & & \end{array}$

\section{Consistency checks for single current meters in a resting basic state} WKBJ solutions

9 real moments per current meter

$\begin{array}{cccc} & \text { linear combinations } & \text { WKBJ vertical } & \text { WKBJ vertically } \\ \text { of the cross-spectra (e) } & \text { structure } & \text { propagating } \\ \mathbf{1} & \left\langle v_{1}^{*} u_{1}\right\rangle-\left\langle u_{1}^{*} v_{1}\right\rangle & i 0 & i 0 \\ \mathbf{2} & {\left[\left\langle u_{1}^{*} u_{1}\right\rangle-\left\langle v_{1}^{*} v_{1}\right\rangle\right] / N_{1}} & & \text { depth independent } \\ \mathbf{3} & {\left[\left\langle u_{1}^{*} v_{1}\right\rangle+\left\langle v_{1}^{*} u_{1}\right\rangle\right] / N_{1}} & & \text { depth independent } \\ \mathbf{4} & \left\langle u_{1}^{*} \eta_{1}\right\rangle+\left\langle\eta_{1}^{*} u_{1}\right\rangle & & 0 \\ \mathbf{5} & \left\langle u_{1}^{*} \eta_{1}\right\rangle-\left\langle\eta_{1}^{*} u_{1}\right\rangle & \text { depth independent } & \text { depth independent } \\ \mathbf{6} & \left\langle v_{1}^{*} \eta_{1}\right\rangle+\left\langle\eta_{1}^{*} v_{1}\right\rangle & & 0 \\ \mathbf{7} & \left\langle v_{1}^{*} \eta_{1}\right\rangle-\left\langle\eta_{1}^{*} v_{1}\right\rangle & \text { depth independent } & \text { depth independent } \\ \mathbf{8} & {\left[\left\langle u_{1}^{*} u_{1}\right\rangle+\left\langle v_{1}^{*} v_{1}\right\rangle-N_{1}^{2}\left\langle\eta_{1}^{*} \eta_{1}\right\rangle\right] / N_{1}} & & 0 \\ \mathbf{9} & {\left[\left\langle u_{1}^{*} u_{1}\right\rangle+\left\langle v_{1}^{*} v_{1}\right\rangle+N_{1}^{2}\left\langle\eta_{1}^{*} \eta_{1}\right\rangle\right] / N_{1}} & \text { depth independent } & \text { depth independent }\end{array}$

Figure 6.7.1 WKBJ consistency checks for a no mean flow basic state 


\subsection{Consistency checks for internal waves in a mean shear flow}

The most general version of the model for internal waves in an unknown mean shear flow has no consistency checks. The search for such checks can be greatly shortened by noting that since the no mean flow models are special cases of the models that include a mean shear flow, any consistency checks for models that include mean velocity shear must be subsets of the consistency checks for the most general no mean flow model. This is because consistency checks are in some sense the complement of a model, thus the more general models have fewer consistency checks. The no mean flow model's consistency check has several components: one entry from the paired current meter consistency check table in figure 6.6.1 that holds for all separations, and two entries from the single current meter table in figure 6.6.1 that check the depth independence of linear combinations of the single current meter cross-spectra. As it turns out, none of these tests hold for the model that includes a mean shear flow. Consider first the imaginary parts of the crossspectra between velocity and displacement at a single point. The polarization vectors $\mathbf{r}$ and $\mathbf{s}$ for internal waves in a steady shear flow are given in equations 5.5.9 and 5.5.10. The cross-spectra of velocity and displacement can then be calculated by using $\mathbf{r}$ and $\mathbf{s}$ in equation 5.8.9. In particular, the imaginary parts are given by

$$
\begin{aligned}
& \Im\left(\left\langle u^{*} \eta\right\rangle(\omega, z)\right)=\left\langle\frac{1}{c} \cos \theta \Im\left(\alpha^{*}(\omega, \mathbf{k}, z) \alpha_{z}(\omega, \mathbf{k}, z)\right) \Delta(\omega, \mathbf{k})\right\rangle \\
& \Im\left(\left\langle v^{*} \eta\right\rangle(\omega, z)\right)=\left\langle\frac{1}{c} \sin \theta \Im\left(\alpha^{*}(\omega, \mathbf{k}, z) \alpha_{z}(\omega, \mathbf{k}, z)\right) \Delta(\omega, \mathbf{k})\right\rangle
\end{aligned}
$$

where $c=\omega / K-U(z) \cos \theta$ and the averaging process $\langle\ldots\rangle$ includes an integral over all wavenumber $\mathrm{k}$. If $c$ were depth-independent then the expressions 6.8 .1 and 6.8.2 would be independent of depth, and consistency checks could be formed by taking nearest neighbor differences. But because $c$ depends on depth in the model with a mean shear flow the two cross-spectra also depend on depth, and the consistency check is lost. The results do suggest, however, that if two depths have the same mean flow that the two cross-spectra ought to be the same. Similarly, when one compares the cross-spectra $\left\langle u_{1}^{*} v_{2}\right\rangle$ with the cross-spectra $\left\langle v_{1}^{*} u_{2}\right\rangle$ one also gets terms that depend on the mean flow,

$$
\begin{aligned}
\left\langle v_{1}^{*} u_{2}\right\rangle-\langle & \left.u_{1}^{*} v_{2}\right\rangle= \\
& \left.\left\langle\sin \theta \frac{U_{z}\left(z_{1}\right)}{c_{1}}\left[\Re\left(\alpha_{1}^{*} \alpha_{2 z}\right) E+\Re\left(\alpha_{1} \alpha_{2 z}\right) P-\Im\left(\alpha_{1} \alpha_{2 z}\right) Q\right]\right\rangle+i \Im\left(\alpha_{1}^{*} \alpha_{2 z}\right) \Delta\right\rangle \\
+ & \left.\left\langle\sin \theta \frac{U_{z}\left(z_{3}\right)}{c_{2}}\left[\Re\left(\alpha_{1 z}^{*} \alpha_{2}\right) E+\Re\left(\alpha_{1 z} \alpha_{2}\right) P-\Im\left(\alpha_{1 z} \alpha_{2}\right) Q\right]\right\rangle+i \Im\left(\alpha_{1 z}^{*} \alpha_{2}\right) \Delta\right\rangle
\end{aligned}
$$


Thus the possible presence of a mean shear flow means that none of the consistency checks need be satisfied. 


\section{Chapter 7: Single internal waves in a vertically varying basic state}

\subsection{Introduction}

Chapter 6 shows that the PEQUOD data set cannot be considered consistent with a field of internal waves in a resting basic state and thus a more sophisticated model, such as an internal wave field modified by the presence of a mean shear flow, is required. But chapter 6 merely says that a more sophisticated model could possibly fit the PEQUOD data, it does not calculate the internal wave spectrum that optimally fits. This chapter does the first step in calculating such a spectrum: it finds internal wave solutions both in a resting and in a shear-modified basic state. In considering one wave at a time, this chapter concentrates the basic physics of the internal wave solutions. This leaves the complexity of dealing with many waves together to chapter 8 , the chapter which fits sets of shear-modified waves to the mooring data.

Chapter 7 looks at a series of increasingly complicated problems culminating in the vertical structure of internal waves in a measured mean zonal shear flow. Each of the solutions to these problems are written in terms of the formalism developed in chapter 5. The formalism separates the solutions into several parts: the dispersion relation, the polarization vectors, the vertical structure function $G(\omega, \mathbf{k}, z)$ and its vertical derivative $G_{z}(\omega, \mathbf{k}, z)$. The dispersion relations and polarization vectors for internal waves with and without mean zonal shear flow have already been given in sections 5.4 and 5.5 , but $G$ and $G_{z}$ have not. The calculation of $G$ and $G_{z}$ starts in section 7.2, which introduces the vertical structure equation. The section primarily reviews section 5.6 , but it extends the earlier section's results by rewriting the equations in terms of amplitude and phase, a separation that helps clarify some of the later results of this chapter. Section 7.3 provides background by presenting the solution to a step profile in $m(z)$ : this solution is referred to in interpreting the more complicated numerical results. Section 7.4 considers internal waves in the measured resting basic state. It finds and compares numerically integrated and WKBJ approximate solutions. The WKBJ solutions are important not only because they are frequently used as approximate solutions in the literature, but also because by being simpler they clarify the physics of the more complicated numerical solutions. Furthermore, while the integrated solutions are more accurate for any given profile, some of the integrated solutions contain features which are sensitive to small changes in the 
buoyancy frequency profile. Given the presence of variability in the buoyancy frequency profile, it is not clear that these sensitive integrated solutions are better than the WKBJ solutions for modeling the spectra computed from a long time series.

Because of the sensitivity of certain solutions to small features in the buoyancy frequency profile, section 7.5 looks at the expected variability in the buoyancy frequency profile and its effect on the structure of the internal waves. While the PEQUOD CTD casts are inadequate to fully determine the vertical wavenumber structure of the buoyancy frequency fluctuations, some estimates can be made, and those results are extrapolated using the observations of Hayes and Powell 1980 and Toole and Hayes 1984: the density gradient variability at the PEQUOD site is consistent with what Hayes and Powell find and is an order of magnitude higher than what is expected at mid-latitudes (Desaubies and Smith 1982 present a buoyancy frequency variability calculation for mid-latitude regions). The enhancement of equatorial variability relative to mid-latitudes is probably due to low frequency waves trapped in the equatorial waveguide and thus the variability appears as frozen finestructure to the internal wave field. The effect of this enhanced variability on internal waves is computed both by using analytical techniques of WPRM (wave propagation in random media) theory and by using a straightforward numerical technique. It is found that waves which correspond to mode numbers greater than 15 are significantly scattered and thus will not display the phase-locking characteristic of modes.

Section 7.6 introduces the effects of mean flows by considering solutions to the equations that include a simplified mean zonal velocity. Section 7.7 continues by looking at waves in measured mean flows. It is found that the most important effect of the mean flows at the depths considered is the effects of mean shear $U_{z}$ through the polarization relations: doppler shifts $U k$ and the effects of mean velocity curvature $U_{z z}$ do not significantly affect the internal wave shapes for low wavenumbers, and effects of buoyancy frequency variability are much stronger than mean flow effects at higher wavenumbers.

The results of the chapter are summarized in section 7.8. Since scattering so strongly affects modes with high wavenumbers (the transition being at roughly mode 15 ), a precise determination of vertical structure is only relevant for the low modes. For these low modes the two effects of shear that change the dispersion relation are minimal: Doppler shifts are small and significant mean velocity curvature only occurs over small distances. Thus it is sufficient to use the dispersion relation for a resting basic state in determining the vertical structure functions $G(z)$ and $G_{z}(z)$. 


\subsection{The vertical structure equation revisited}

Both the internal waves in the no mean flow basic state and the internal waves in the basic state with a mean shear flow reduce to finding a solution $G$ to a vertical structure equation of the form

$$
G_{z z}+m^{2}(z) G=0
$$

where

$$
m^{2}(z)=\left\{\begin{array}{cl}
\frac{N^{2}(z)-\omega^{2}}{\omega^{2}} K^{2} & \text { no mean flow } \\
\frac{N^{2}(z)-\sigma^{2}}{\sigma^{2}} K^{2}+\frac{k U_{z z}}{\sigma} & \text { mean zonal flow } \\
\sigma=\omega-U k &
\end{array}\right.
$$

In the case of internal waves in a resting basic state, $G(z)$ is the vertical structure function for vertical displacement and vertical velocity, while $G_{z}(z)$ is the vertical structure function for horizontal velocities and reduced pressure (see equation 5.4.2). The situation where there is a mean zonal shear flow is more complicated (see equation 5.5.9): $G(z)$ is still the vertical structure function for vertical displacement and vertical velocity, but a linear combination of $G$ and $G_{z}$ is required to describe the the vertical structure of the horizontal velocities and reduced pressure. As section 5.6 discusses, since equation 7.2.1 is a second order linear differential equation, any solution $G(z)$ can be written as a linear combination of a pair of basis solutions, each of which satisfy the differential equation 7.2.1. There is some freedom in choosing these basis functions, and the choice made in section 5.6 is to use the solution that satisfies a pair of conditions at some reference depth $z_{0}$, as the first basis function, $\alpha(z)$, and its complex conjugate $\alpha^{*}(z)$ as the second. These conditions are

$$
\begin{gathered}
\alpha\left(z_{0}\right)=\alpha_{0}(\sigma, \mathbf{K}) \\
\alpha_{z}\left(z_{0}\right)=-i m(z) \alpha\left(z_{0}\right)
\end{gathered}
$$

$\alpha_{0}$ must be real in order for $\alpha^{*}(z)$ to be a complementary basis function for $\alpha$. In the case where $m(z)$ is a constant, $\alpha(z)$ is clearly the upward (energy) propagating wave (downward phase propagation), while its complex conjugate is the downward wave. In the general $m(z)$ case, the interpretation as upward and downward waves is more complicated, and some discussion loosely based on Gent and Luyten 1985 is in section 5.6. Essentially, while the wave $\alpha$ starts out at $z_{0}$ as a purely upward propagating wave, rapid variation in $m(z)$ causes reflections so that there is a mixture of both upward and downward energy. Its counterpart, $\alpha^{*}$, will have exactly the opposite mixture. 
Armed with that definition, it is straightforward to integrate equation 7.2.1 from initial conditions 7.2.3a,b for any given profile of $m(z)$ to find the solution $\alpha$ (a calculation that is done in Gent and Luyten 1985 and Philander 1978). While not necessary mathematically, it is instructive to rewrite the problem in terms of a polar decomposition for $\alpha(z)$,

$$
\alpha(z)=R(z) e^{i \theta(z)}
$$

This transformation is a variant of the Madelung transformation, which is suggested by Acton 1970 as appropriate for numerical integration of this equation. Its numerical properties aside, it transforms the complex differential equation 7.2.1 into a pair of real equations, one differential and one simple quadrature. The result is straightforwardly derived. By substituting 7.2.4 into 7.2.1, the following pair of equations result

$$
\begin{gathered}
R_{z z}-R \theta_{z}^{2}+m^{2}(z) R=0 \\
2 R_{z} \theta_{z}+R \theta_{z z}=0
\end{gathered}
$$

The second equation (7.2.5b) is separable and can be readily integrated. Using the initial conditions to determine the constant of integration gives the final results: a differential equation containing only the amplitude $R(z)$,

$$
R_{z z}+m^{2}(z) R-m_{0}^{2} \alpha_{0}^{4} R^{-3}=0
$$

with initial conditions

$$
\begin{aligned}
R\left(z_{0}\right) & =\alpha_{0} \\
R_{z}\left(z_{0}\right) & =0
\end{aligned}
$$

and a simple quadrature relation for phase $\theta(z)$,

$$
\theta(z)=-\int_{z_{0}}^{z} \frac{m_{0} \alpha_{0}^{2}}{R^{2}} d z
$$

The solution for constant $m^{2}(z) \quad\left(=m_{0}^{2}\right)$ can be found by inspection of 7.2.6: the amplitude $R(z)$ is equal to its initial value $\alpha_{0}$ throughout the water column, and the phase $\theta(z)$ is a linear function of depth. Several insights into the solutions, $R(z), \theta(z)$, for general $m^{2}(z)$ can be gained from the system of equations 7.2.6 as well. First of all, the phase $\theta(z)$ tends to be smoother than the amplitude, since the phase is an integral of a smooth function of the amplitude. This suggests that the phase results will be more 
robust than amplitude results in the presence of noise. At the very least, because the phase is an integral of a positive definite quantity, it is a single valued function of depth. Furthermore, equation 7.2.6a makes it clear that $R$ cannot smoothly go to zero. If forced towards zero, the amplitude $R$ responds by oscillating rapidly (an oscillation that would enhance mixing and dissipation effects were they included in the system).

In fact equation $7.2 .6 a$ is also the radial equation of motion for a mass moving in a plane while connected to a central pivot by a variable spring (see figure 7.2.1). While admittedly a somewhat contrived problem, it does provide an example where the physics is fairly clear.

$$
R_{t t}+K_{S}(t) R-A^{2} R^{-3}=0
$$

where $R(t)$ is the radius of the particle at time $t, K_{S}(t)$ is the spring constant normalized by the particle mass and is a given function of $t$, and $\mathrm{A}$ is the normalized angular momentum at the initial time. Since there are only radial forces, the angular momentum $A=R^{2}(t) \theta_{t}(t)$ is conserved. $R^{2}(z) \theta_{z}(z)$ is the analogous quantity for the oceanographic vertical structure problem, showing that as long as $m^{2}(z)$ is not infinite, neither the gradient of phase nor the amplitude can go to zero unless one of them is initially (and almost always) zero. Having both the amplitude and the vertical phase gradient nonzero is a very useful constraint, and it is important to realize that it is a result of the initial conditions 7.2.3b. There is no loss of generality, however, because arbitrary values of $G$ and $G_{z}$ at the reference depth $z_{0}$ can be matched by using linear combinations of $\alpha$ and $\alpha^{*}$.

It is also instructive to relate $\alpha_{z}(z)$ to $R(z)$ and $\theta(z)$. It is then possible to see that the phase behavior of $\alpha_{z}(z)$ is potentially different from the smooth, single-valued behavior of $\theta(z)$. Taking the vertical derivative of equation 7.2.4 gives

$$
\alpha_{z}(z)=i\left(\theta_{z}-i R_{z} / R\right) R e^{i \theta}
$$

It is then a straightforward matter to use the explicit solution for phase given in equation 7.2.6c to get an expression entirely in terms of $R(z)$ and $\alpha(z)$.

$$
\alpha_{z}(z)=-i \frac{m_{0} \alpha_{0}^{2}}{R^{2}(z)}\left(1+i \frac{R R_{z}}{m_{0} \alpha_{0}^{2}}\right) \alpha(z)
$$

As long as $m^{2}(z)$ does not go to infinity, $R(z)$ is positive definite, so the factoring done in going from equation 7.2.8a to equation 7.2.8b does not introduce any phase changes 


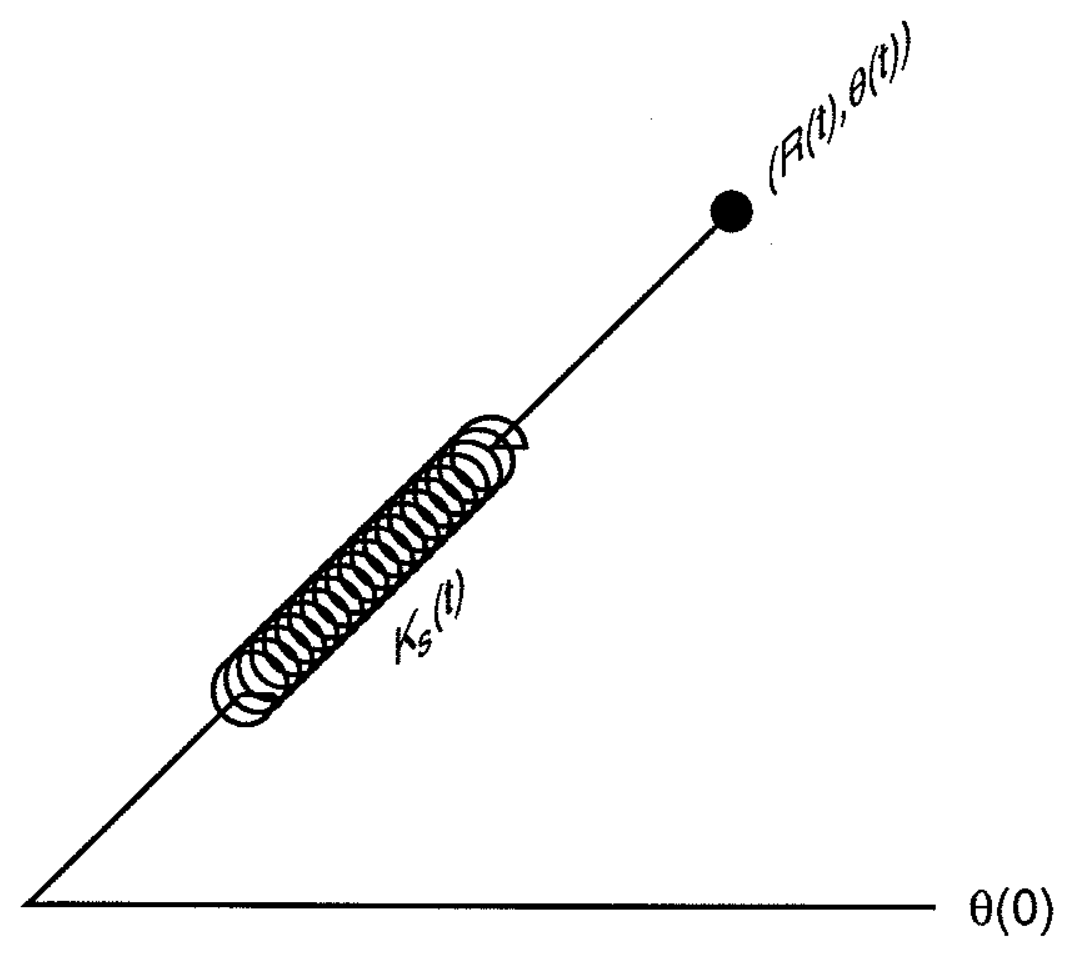

Figure 7.2.1 Mass moving in a plane

A problem analogous to the vertical structure equation with a non-constant $m^{2}(z)$ is the problem of a mass connected to a pivot by a variable spring. The normalized spring constant $K_{s}(t)$ corresponds to the variable local vertical scale $m^{2}(z)$. 
or singularities. Thus the phase difference between $\alpha(z)$ and $\alpha_{z}(z)$ is due entirely to the factor

$$
-i\left(1+i \frac{R R_{z}}{m_{0} \alpha_{0}^{2}}\right)
$$

This means that the phase difference will fluctuate around $-90^{\circ}$ in a manner determined by the rate of change of the amplitude $R$. In the case of constant $m^{2}(z)$, then, the phase difference will be a constant $-90^{\circ}$. In the more general case of a varying $m^{2}(z)$, the fluctuations will be non-zero. They will, however, be constrained to be less than $90^{\circ}$, since the phase of the factor in parentheses is within the interval $\left(-90^{\circ}, 90^{\circ}\right)$, the extreme values approached only as $R(z)$ rapidly goes to infinity.

\subsection{Step $m(z)$ profile}

To develop a basis for understanding the more complicated solutions to measured $m^{2}(z)$ profiles, consider the solutions to the vertical structure equations where the vertical dependence of $m(z)$ is a single step at some height of transition $z_{T}$. (Solutions to other theoretical profiles can be found in Gent and Luyten 1985 and Philander 1978). There are now two regions where $m(z)$ is constant: region 1 (above the transition height) which contains the reference height $z_{0}$, and region 2 (below the transition height). The solutions where $m(z)$ is constant are complex exponentials whose vertical scale is given by the local $m(z)$. Since region 1 contains the reference height, its solution is a purely upward propagating wave, while the solution in region 2 is going to be some mixture of the upward and downward propagating solutions.

$$
\alpha(z)= \begin{cases}e^{-i m_{1}\left(z-z_{0}\right)} & z>z_{T} \\ A e^{-i m_{2} z}+B e^{i m_{2} z} & z<z_{T}\end{cases}
$$

By matching $\alpha$ and $\alpha_{z}$ at the transition height, $A$ and $B$ are readily determined.

$$
\alpha(z)= \begin{cases}e^{-i m_{1}\left(z-z_{0}\right)} & z>z_{T} \\ e^{-i m_{1}\left(z_{T}-z_{0}\right)}\left[\cos m_{2}\left(z-z_{T}\right)-i\left(m_{1} / m_{2}\right) \sin m_{2}\left(z-z_{T}\right)\right] & z<z_{T}\end{cases}
$$

The amplitude of the solution in equation 7.3 .2 is

$$
|\alpha(z)|= \begin{cases}1 & z>z_{T} \\ \sqrt{\frac{1}{2}\left[1+\left(m_{1} / m_{2}\right)^{2}\right]+\frac{1}{2}\left[1-\left(m_{1} / m_{2}\right)^{2}\right] \cos 2 m_{2}\left(z-z_{T}\right)} & z<z_{T}\end{cases}
$$


The amplitude and phase of one such solution is plotted in figure 7.3.1. This solution suggests that rapid changes in $N(z)$ (resulting in rapid changes in $m(z)$ ) will result in solutions that have amplitudes that oscillate with twice the local vertical wavenumber, and the size of those oscillations will be proportional to the percentage change in $m(z)$. Phase, on the other hand, will be relatively smooth. Equation 7.2.6c shows that phase is an integral over amplitude. Furthermore, since the vertical scale of the variation in amplitude is half the vertical scale of the wave, the variations will tend to integrate out exactly over one wave period, resulting in a even greater smoothing effect than one would expect from integration through random noise.

\subsection{Integrated wave solutions in the absence of mean flow}

This section looks at the upward solutions $\alpha(z)$ to equation 7.2.1 with a dispersion relation appropriate for waves in a motionless basic state.

$$
m^{2}(z)=\frac{N^{2}(z)-\omega^{2}}{\omega^{2}} K^{2}
$$

Given the true buoyancy frequency profile $N_{(z)}$, it is straightforward to integrate from the boundary conditions (equation 7.2.3) given at the reference depth $z_{0}$ and calculate $\alpha$ for all $z$. There are two parameters in the dispersion relation and thus the results are functions of two parameters as well: frequency $\omega$ and horizontal wavenumber $K$. To the extent that the hydrostatic approximation is appropriate $\left(m^{2}(z)=N^{2}(z) K^{2} / \omega^{2}\right)$, these two parameters can be combined: only their ratio (the horizontal phase speed) affects the vertical structure equation. Since the solutions are in fact essentially hydrostatic (which will be demonstrated later in this section), all the solutions presented will arbitrarily be at the same frequency $(.2 \mathrm{cph})$ and different choices of horizontal wavenumber will be made to illustrate the possible behaviors of solutions.

Determining a 'true' buoyancy frequency profile is not particularly simple. In fact, it is not clear that such a concept has much validity: in the real ocean there are changes in the profile and those changes are reflected in the structure of the internal waves. For the integrations presented in this section, two profiles were calculated by averaging over the 28 PEQUOD CTD profiles listed in section 2.1: one profile is computed using a set of pressure bins spaced every $100 \mathrm{WKBJ}$ stretched meters (wsm), while the other profile is computed using a set of pressure bins that are spaced every $25 \mathrm{wsm}$. These WKBJ grids were chosen in order to be able to consider the variability in buoyancy frequency to 

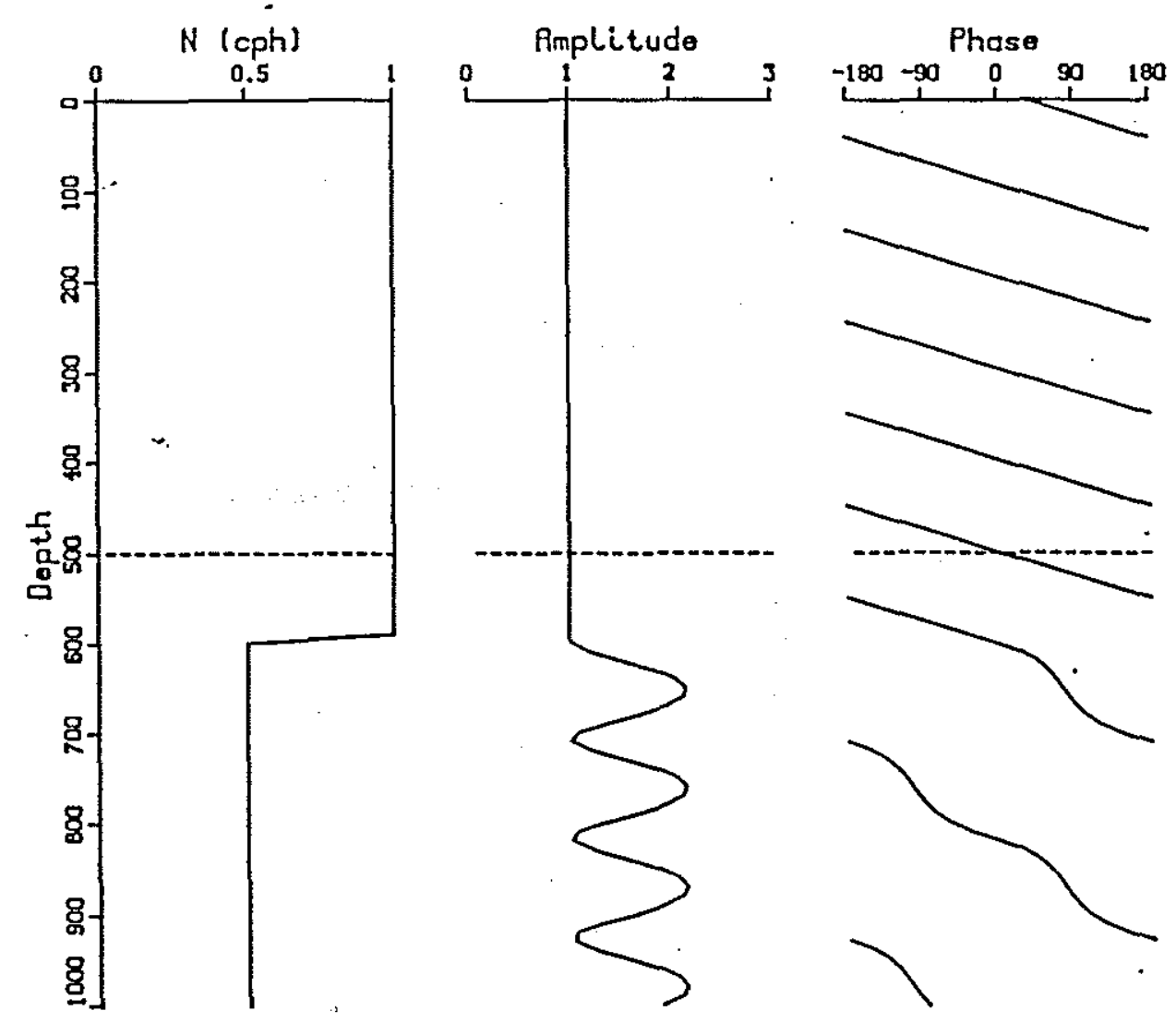

Figure 7.3.1 Solution to step change in $m(z)$

The plot gives the solution where the dispersion relation is

$$
m^{2}(z)=\frac{N^{2}(z)-\omega^{2}}{\omega^{2}} K^{2}
$$

and the reference height is chosen to be in region 1 (In this plot the frequency $\omega$ is .2 $\mathrm{cph}$ and the horizontal wavenumber $K$ is $2 \mathrm{cpkm}$ ). 
be stationary with depth, a point that will be greatly elaborated in section 7.5. As it turns out, the buoyancy frequency variability can quite strongly affect internal waves, so it is a factor that must be considered in order to correctly determine internal wave solutions.

Figure 7.4.1 shows an integration for a vertically propagating wave that corresponds to the first baroclinic mode (i.e. the first baroclinic mode is comprised of this upward propagating wave and its downward propagating counterpart at the same horizontal wavenumber). The left plot gives the buoyancy frequency profile computed on a 25 WKBJ stretched decibar grid: the mean profile is sharply peaked around $150 \mathrm{~m}$ and fairly smooth otherwise. The other two plots give amplitude $R(z)$ and phase $\theta(z)$. The phase decreases with height, as is appropriate for a wave with upward energy. The amplitude varies slowly as $N$ varies, though it is somewhat smoother: it never gets very far from the unit amplitude it has at $1000 \mathrm{~m}$ (the reference depth). The oscillations seen in the solution to a step profile found in section 5.3 are not readily apparent in figure 7.4 .1 because the water column does not extend sufficiently beyond the height of the thermocline. Furthermore, a single step profile is not a good approximation to the thermocline. An analytical solution much more analogous to the thermocline is done in Gent and Luyten 1985: there they consider a three layer stratified ocean.

Figure 7.4.2 shows an integration for a much higher horizontal wavenumber $K=.5$ : this corresponds to a mode number of 25 . The left-hand plot again gives the local vertical wavenumber $m(z)$ : The values are different (since the wave is much smaller) but the shape of the curve has not changed at all. The middle plot gives the amplitude: the curve has much more structure that the low wavenumber (first mode) solution's amplitude did. In light of the step profile solution of section 5.3 this solution makes a good deal of sense; the wiggles are caused by phase-locking between upward and downward propagating waves, their amplitude increasing as the value of the buoyancy frequency lessens. The right-hand plot gives the phase (the plot is such that the line is broken each time the phase goes from $-180^{\circ}$ to $180^{\circ}$ ). The phase plot shows much less structure than the amplitude plot: this is because phase is an integral over a function of amplitude, thus all the small scale fluctuations in amplitude are averaged out. As will be shown in section 7.5 , this large amount of structure in the amplitude is quite sensitive to changes in the buoyancy frequency profile. This is equivalent to saying that these waves are strongly scattered, which means these waves not only do not contribute to non-zero coherences over large separations, they are unable to form modes, making energy at these wavelengths 


\section{Upward propagating waves}

Run: P28W25

$$
\omega 0.2 \mathrm{cph}
$$

K $0.021 \mathrm{cpkm}$

$$
\begin{array}{ll}
\mathrm{N}\left(\mathrm{z}_{0}\right) & 1.3223 \mathrm{cph} \\
\mathrm{z}_{0} & 1000.0 \mathrm{~m}
\end{array}
$$
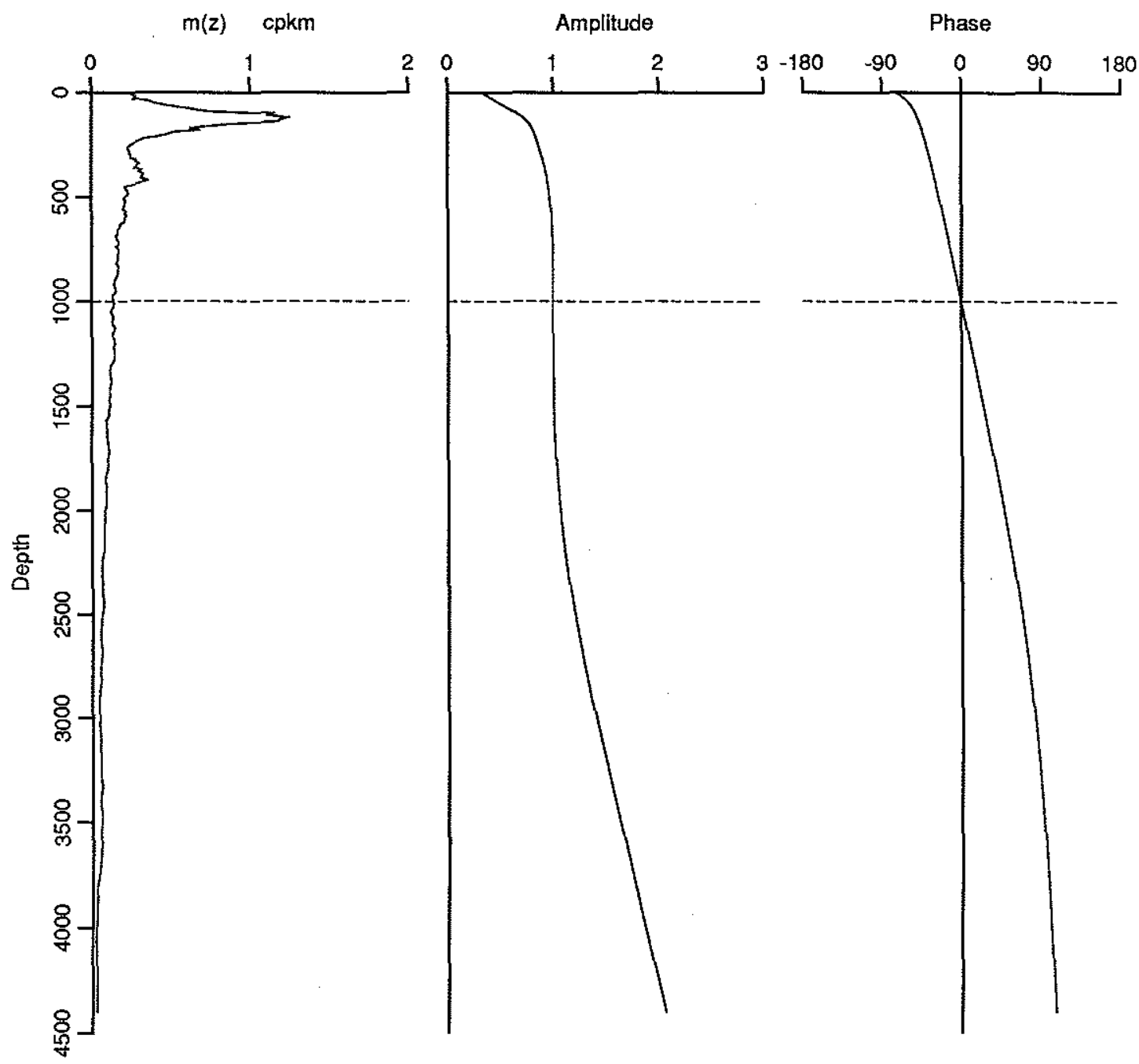

Figure 7.4.1 Low wavenumber wave integrated through a 25 wsm averaged $N$ profile

This plot gives the integrated solution for $\alpha(z)$ at a frequency $\omega$ of .2 $\mathrm{cph}$ and a horizontal wavenumber $K$ of $.021 \mathrm{cpkm}$. This wavenumber corresponds to the first baroclinic mode. The left plot gives the local vertical wavenumber profile (which is almost proportional to the buoyancy frequency profile): the buoyancy frequency profile is computed from PEQUOD data using bins every $25 \mathrm{wsm}$. The center plot gives the amplitude of $\alpha(z)$, and the right plot gives the phase of $\alpha(z)$. The solution is quite smooth and is basically not dependent on small fuctuations in the buoyancy frequency profiles. 


\section{Upward propagating waves}

Run: P28W25

$\omega 0.2 \mathrm{cph}$

$\mathrm{K} 0.5 \mathrm{cpkm}$
$\begin{array}{ll}\mathrm{N}\left(\mathrm{z}_{\mathrm{o}}\right) & 1.3223 \mathrm{cph} \\ \mathrm{z}_{0} & 1000.0 \mathrm{~m}\end{array}$

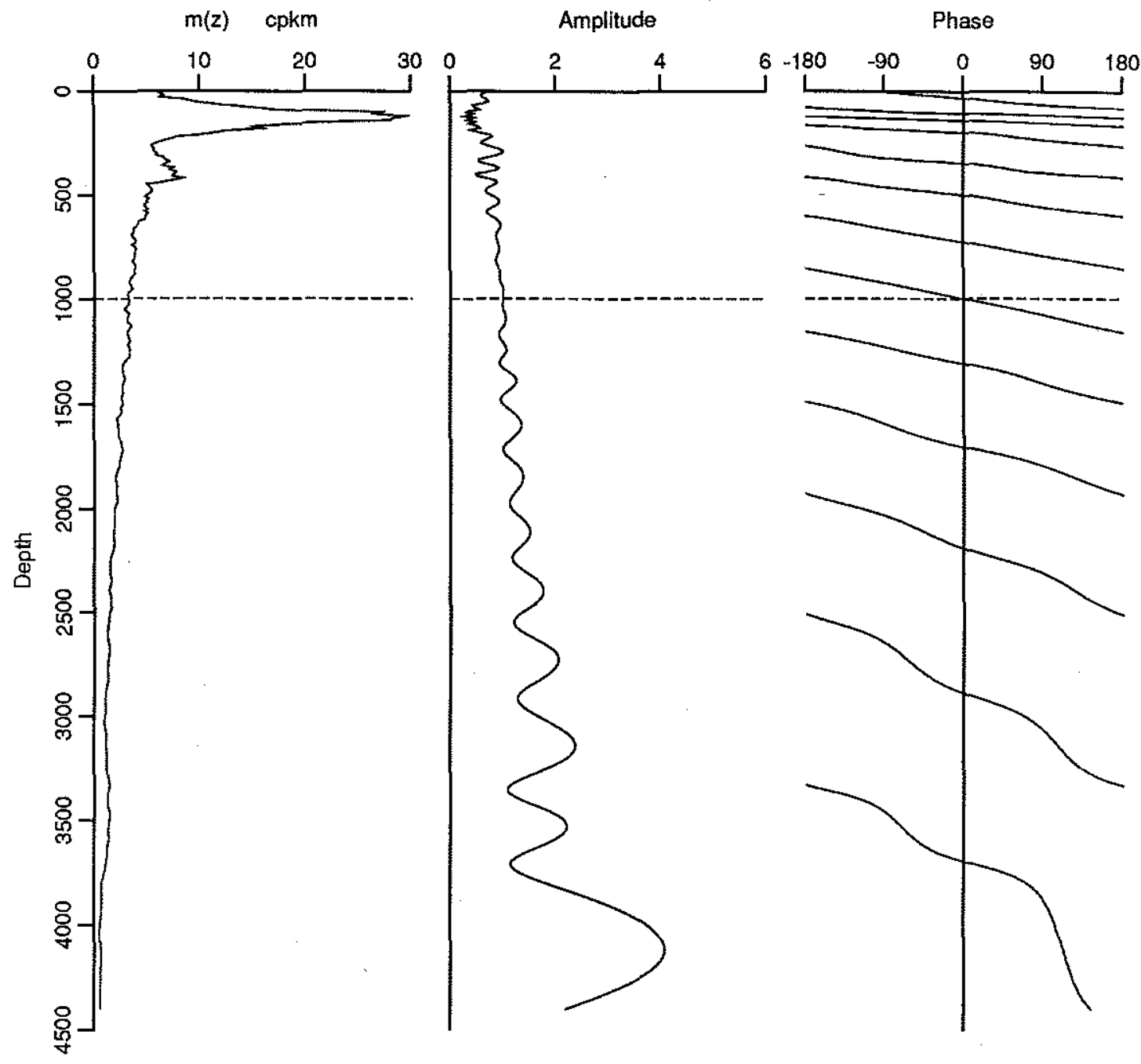

Figure 7.4.2 Medium wavenumber wave integrated through a 25 wsm averaged $N(z)$ profile

This plot gives the integrated solution for $\alpha(z)$ at a frequency $\omega$ of $.2 \mathrm{cph}$ and a horizontal wavenumber $K$ of $.5 \mathrm{cpkm}$. This wavenumber corresponds to mode 25 . The left plot gives the local vertical wavenumber profile (which is almost proportional to the buoyancy frequency profile): the buoyancy frequency profile is computed from PEQUOD data using bins every $25 \mathrm{wsm}$. The center plot gives the amplitude of $\alpha(z)$, and the right plot gives the phase of $\alpha(z)$. The solution show wiggles in amplitude that are quite similar to those seen in the step function solutions. Phase, on the other hand, is still quite smooth. 
essentially propagating in character.

While one could argue that mode 25 (which was discussed in the last paragraph) is as high as a mode as one would want to consider (a point of view which is given empirical and dynamical support in section 7.5), it is quite informative to consider higher modes as well. Figure 7.4 .3 presents the integration for the wave that corresponds to mode 100 . The buoyancy frequency profile used was computes with a grid of pressure bins that were spaced every $25 \mathrm{wsm}$. The wiggles in amplitude are even more pronounced than they were for the mode 25 wave. But now consider figure 7.4.4, which presents the same wave integrated through the buoyancy frequency profile that was computed using a 100 wsm grid. The wave in that figure is quite smooth: the amplitude varies slowly in inverse proportion to the variations in buoyancy frequency.

The smooth solution is in some sense the solution one might have expected: the wave scale is much smaller than that of the mean flow, and thus the wave amplitude varies in a WKBJ sense, inversely proportional to the square root of the local vertical wavenumber. $\dagger$ But the solution integrated through the $25 \mathrm{wsm}$ profile did not show the same character at all. The difference in the character of the high wavenumber solutions is due to the difference in high wavenumber content of the two buoyancy frequency profiles: the 100 wsm profile filters high wavenumber variability more thoroughly than the 25 wsm profile. For lower wavenumbers the high wavenumber content of the profile is less important: comparing figure 7.4.2 to figure 7.4.5 shows that solutions for medium wavenumbers do not depend on which profile is used. So the problem is accentuated for higher wavenumbers, and the difference between the two high wavenumber integrations points out the important of correctly determining the high wavenumber content of the $N^{2}(z)$ profile. That determination is in section 7.5 .

$\uparrow$ WKBJ solutions are discussed in more detail later in this section. 


\section{Upward propagating waves}

Run: P28W25

w $0.2 \mathrm{cph}$

$\mathrm{K} 2.0 \mathrm{cpkm}$

$\mathrm{N}\left(\mathrm{z}_{0}\right) 1.3223 \mathrm{cph}$

$z_{0} \quad 1000.0 \mathrm{~m}$
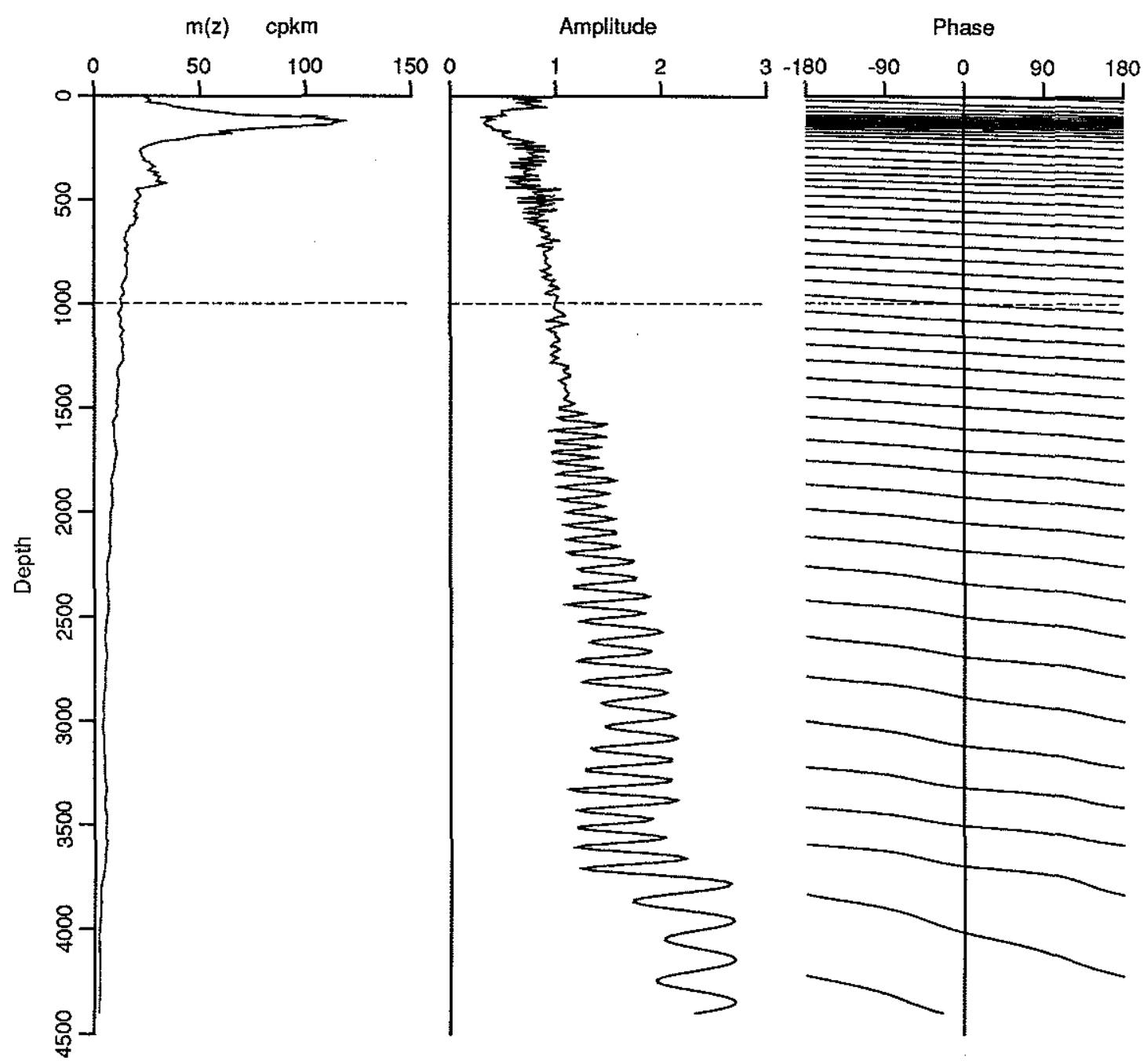

Figure 7.4.3 Very high wavenumber wave integrated through a 25 wsm averaged $N(z)$ profile

This plot gives the integrated solution for $\alpha(z)$ at a frequency $\omega$ of $.2 \mathrm{cph}$ and a horizontal wavenumber $K$ of $2 \mathrm{cpkm}$. This wavenumber corresponds to mode 100 . The left plot gives the local vertical wavenumber profile (which is almost proportional to the buoyancy frequency profile): the buoyancy frequency profile is computed from PEQUOD data using bins every $25 \mathrm{wsm}$. The center plot gives the amplitude of $\alpha(z)$, and the right plot gives the phase of $\alpha(z)$. The solution show wiggles in amplitude that are even bigger than those seen in the mode 25 solution. 


\section{Upward propagating waves}

Run: P28W100

$\omega 0.2 \mathrm{cph}$
K $2.0 \mathrm{cpkm}$

$\mathrm{N}\left(z_{0}\right) 1.3119 \mathrm{cph}$

$z_{0} \quad 1000.0 \mathrm{~m}$

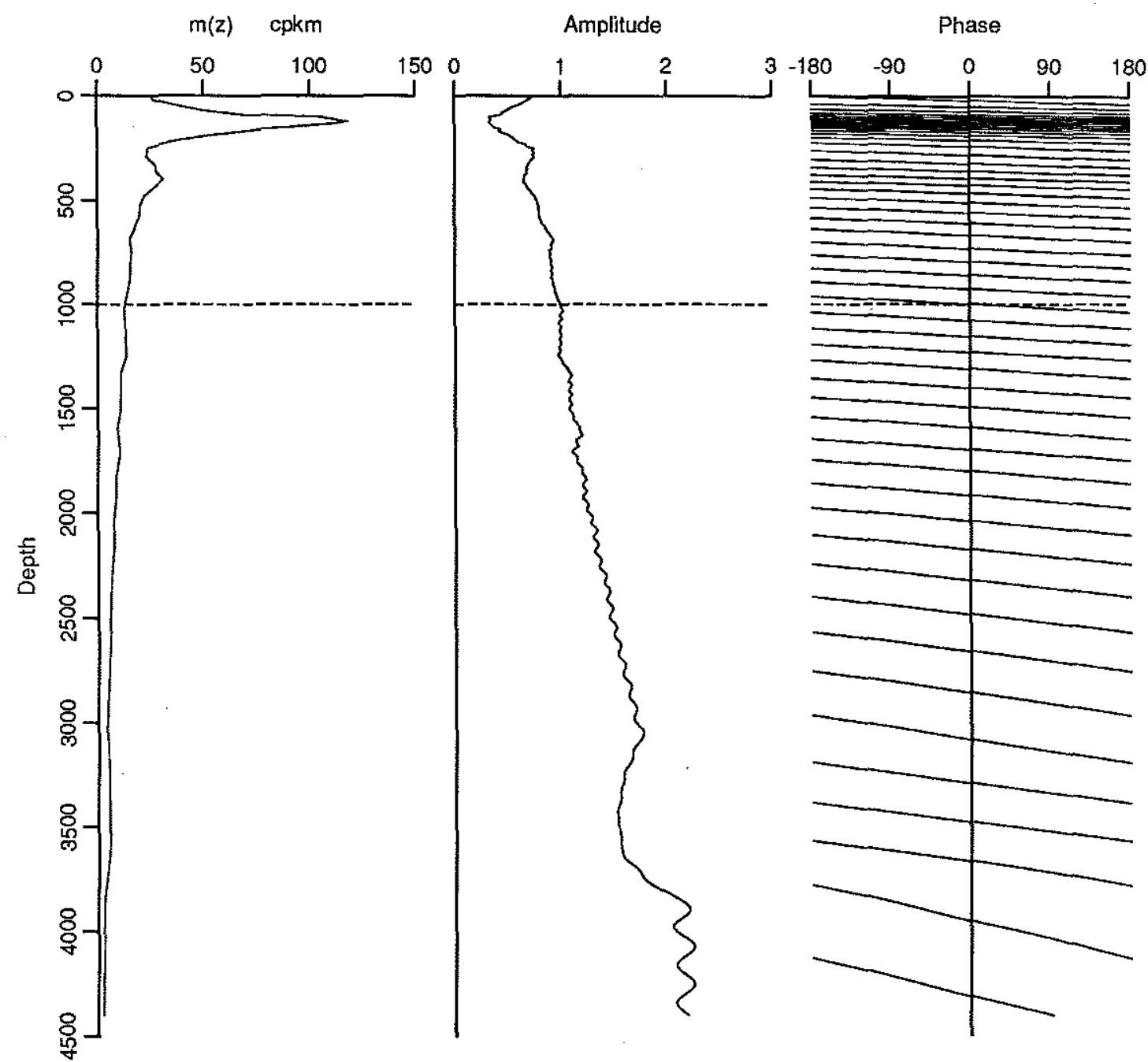

Figure 7.4.4 Very high wavenumber wave integrated through a 100 wsm averaged $N(z)$ profile

This plot gives the integrated solution for $\alpha(z)$ at a frequency $\omega$ of $.2 \mathrm{cph}$ and a horizontal wavenumber $K$ of $2 \mathrm{cpkm}$. This wavenumber corresponds to mode 100 . The left plot gives the local vertical wavenumber profile (which is almost proportional to the buoyancy frequency profile): the buoyancy frequency profile is computed from PEQUOD data using bins every $100 \mathrm{wsm}$. The center plot gives the amplitude of $\alpha(z)$, and the right plot gives the phase of $\alpha(z)$. The solution is quite smooth. The smoothness, however, is not necessarily a property of the ocean: the solution is smooth because the scale of the wave is smaller than the scale of the smoothing used to compute the buoyancy frequency profile. 


\section{Upward propagating waves}

Run: P28W100

$\omega 0.2 \mathrm{cph}$

$\mathrm{K} 0.5 \mathrm{cpkm}$
$\begin{array}{ll}N\left(z_{0}\right) & 1.3119 \mathrm{cph} \\ z_{0} & 1000.0 \mathrm{~m}\end{array}$

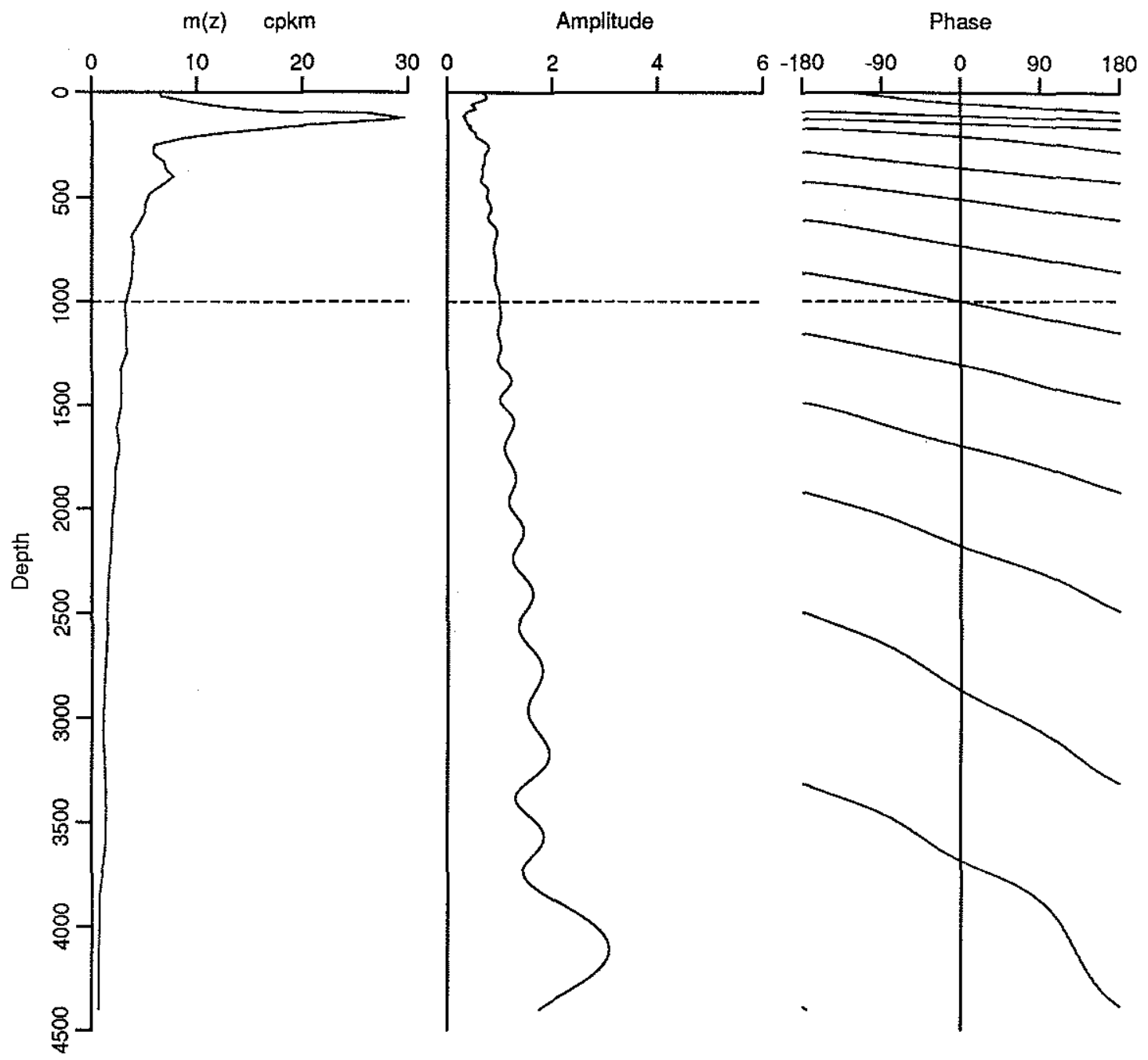

Figure 7.4.5 Medium wavenumber wave integrated through a $100 \mathrm{wsm} N(z)$ profile This plot gives the integrated solution for $\alpha(z)$ at a frequency $\omega$ of $.2 \mathrm{cph}$ and a horizontal wavenumber $K$ of $.5 \mathrm{cpkm}$. This wavenumber corresponds to mode 25 . The left plot gives the local vertical wavenumber profile (which is almost proportional to the buoyancy frequency profile): the buoyancy frequency profile is computed from PEQUOD data using bins every $100 \mathrm{wsm}$. The center plot gives the amplitude of $\alpha(z)$, and the right plot gives the phase of $\alpha(z)$. The solution show wiggles in amplitude that are quite similar to those seen in the integration through the $25 \mathrm{wsm}$ averaged buoyancy frequency profile plotted in figure 7.4.2. This is because, unlike the mode 100 case, the vertical wavelength of this wave is longer than the cutoff wavenumber of the filtered buoyancy frequency profile for both the $100 \mathrm{wsm}$ and the 25 wsm profiles. 


\section{Horizontal velocity structure function solutions}

The last part of section 7.2 points out that it is possible that the horizontal velocity structure function $\alpha_{z}(z)$ could have quite different phase behavior from the vertical velocity structure function $\alpha(z)$. But the computations with the measured profiles that are presented here indicate that the potential for peculiar phase behavior is not realized: the $\alpha_{z}$ plots are qualitatively the same as the $\alpha$ plots.

Figure 7.4.6 presents $\alpha_{z}$ for the first mode horizontal wavenumber (figure 7.4.1 presented $\alpha$ at the same wavenumber). The amplitude behavior of $\alpha_{z}$ is in some sense opposite to that of $\alpha$ : the amplitude of $\alpha_{z}$ decreases where the amplitude of $\alpha$ increases. The phase behavior is almost exact the same, except that there is an almost constant $90^{\circ}$ difference between the two. This is even more apparent in figure 7.4.9, where the left plot presents the deviations from the $90^{\circ}$ phase offset for this wavenumber.

Figure 7.4.7 presents a solution for medium wavenumbers. The wavenumber used is the same as for figure 7.4.2. The amplitude offers no surprises: the wiggles found in $\alpha$ are also in $\alpha_{z}$, the only difference being that wherever $\alpha$ increases, $\alpha_{z}$ decreases, and vice versa. The phase of $\alpha_{z}$, too, is qualitatively the same as the phase of $\alpha$ : it appears just as smooth, unlike the prediction of section 7.2. The resolution of this apparent conflict is in the center plot of figure 7.4.7. Here it is clear that the erratic phase behavior predicted in section 7.2 is going on, but because the phase is varying so rapidly, the random perturbations are so small as to be undetectable. In conclusion, then, the behavior of the horizontal velocity structure function $\alpha_{z}$ is qualitatively indistinguishable from the behavior of the vertical velocity structure function $\alpha$.

Figure 7.4.8 presents a solution in the very high wavenumber limit (roughly mode 100). The solution is computed using the profile that is averaged on a $25 \mathrm{wsm}$ grid, consequently it is not identical to the solution computed using the WKBJ approximation: the amplitude has wiggles that vary twice as fast as the wave itself. This solution is like the medium (mode 25) wavenumber solution in that the $\alpha_{z}(z)$ solution has the same character as the $\alpha(z)$ solution. It is interesting to note that, unlike the mode 25 solution, the phase difference between $\alpha(z)$ and $\alpha_{z}(z)$ does not continue increasing with distance from the reference depth $z_{0}$ : a maximum amplitude of $45^{\circ}$ is reached and more or less maintained. 


\section{Upward propagating waves}

Run: P28W25

w $0.2 \mathrm{cph}$
$\mathrm{K} 0.021 \mathrm{cpkm}$

$\begin{array}{ll}\mathrm{N}\left(\mathrm{z}_{0}\right) & 1.3223 \mathrm{cph} \\ \mathrm{z}_{0} & 1000.0 \mathrm{~m}\end{array}$
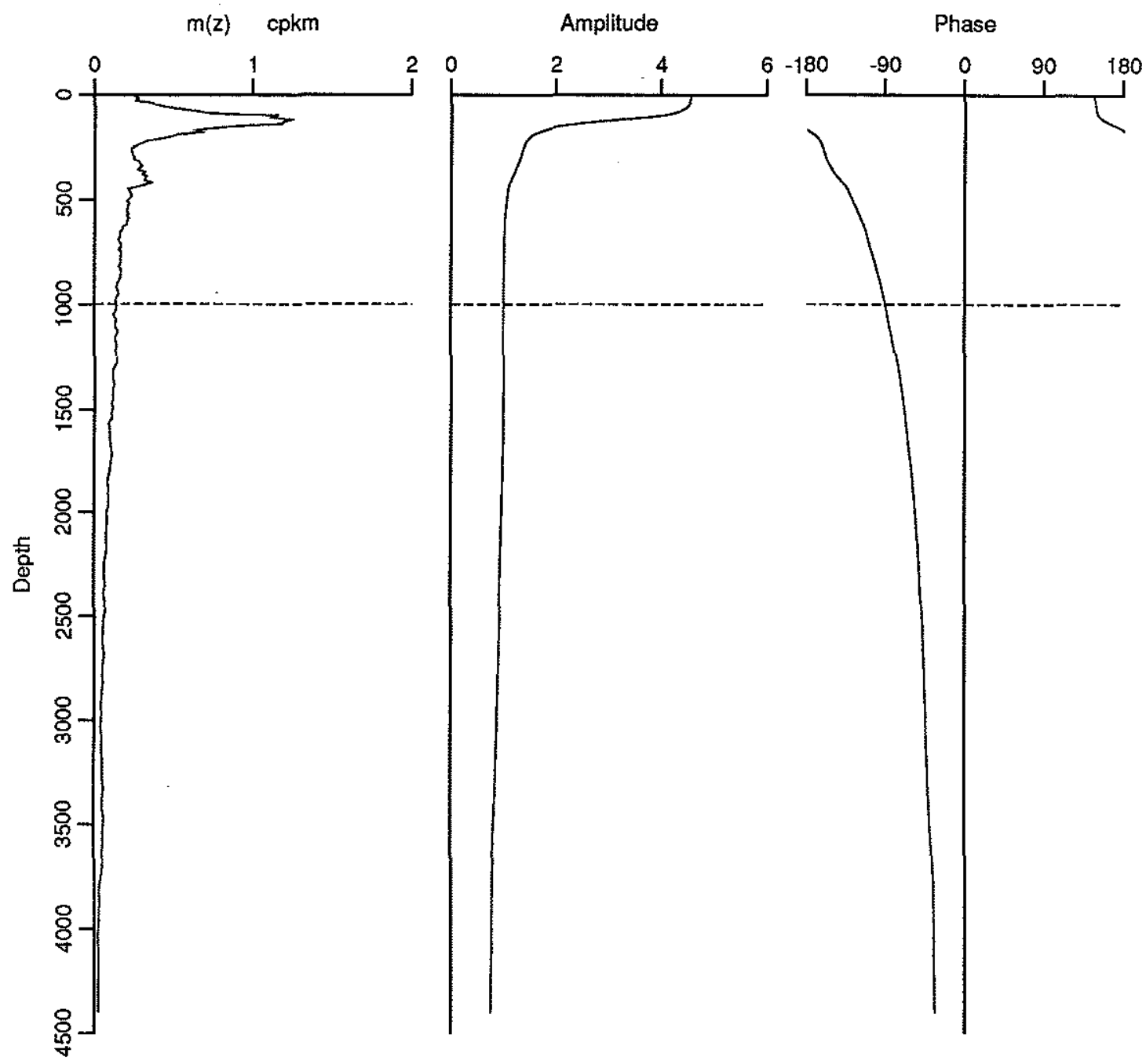

Figure 7.4.6 Low wavenumber wave integrated through an averaged $N$ profile

This plot gives the integrated solution for $\alpha_{z}(z)$ at a frequency $\omega$ of $.2 \mathrm{cph}$ and a horizontal wavenumber $K$ of $.021 \mathrm{cpkm}$. This wavenumber corresponds to the first baroclinic mode. The left plot gives the local vertical wavenumber profile (which is almost strictly proportional to the buoyancy frequency profile): the buoyancy frequency profile is computed from PEQUOD data using bins every $25 \mathrm{wsm}$. The center plot gives the amplitude of $\alpha_{z}(z)$, and the right plot gives the phase of $\alpha_{z}(z)$. The amplitude tends to vary with the local vertical wavenumber, and the structure of the phase is quite similar to the structure of the $\alpha(z)$ phase; there is an almost constant $90^{\circ}$ phase difference between the two. 


\section{Upward propagating waves}

Run: P28W25

$\omega 0.2 \mathrm{cph}$
$\mathrm{K} 0.5 \mathrm{cpkm}$

$\begin{array}{ll}N\left(z_{0}\right) & 1.3223 \mathrm{cph} \\ z_{0} & 1000.0 \mathrm{~m}\end{array}$
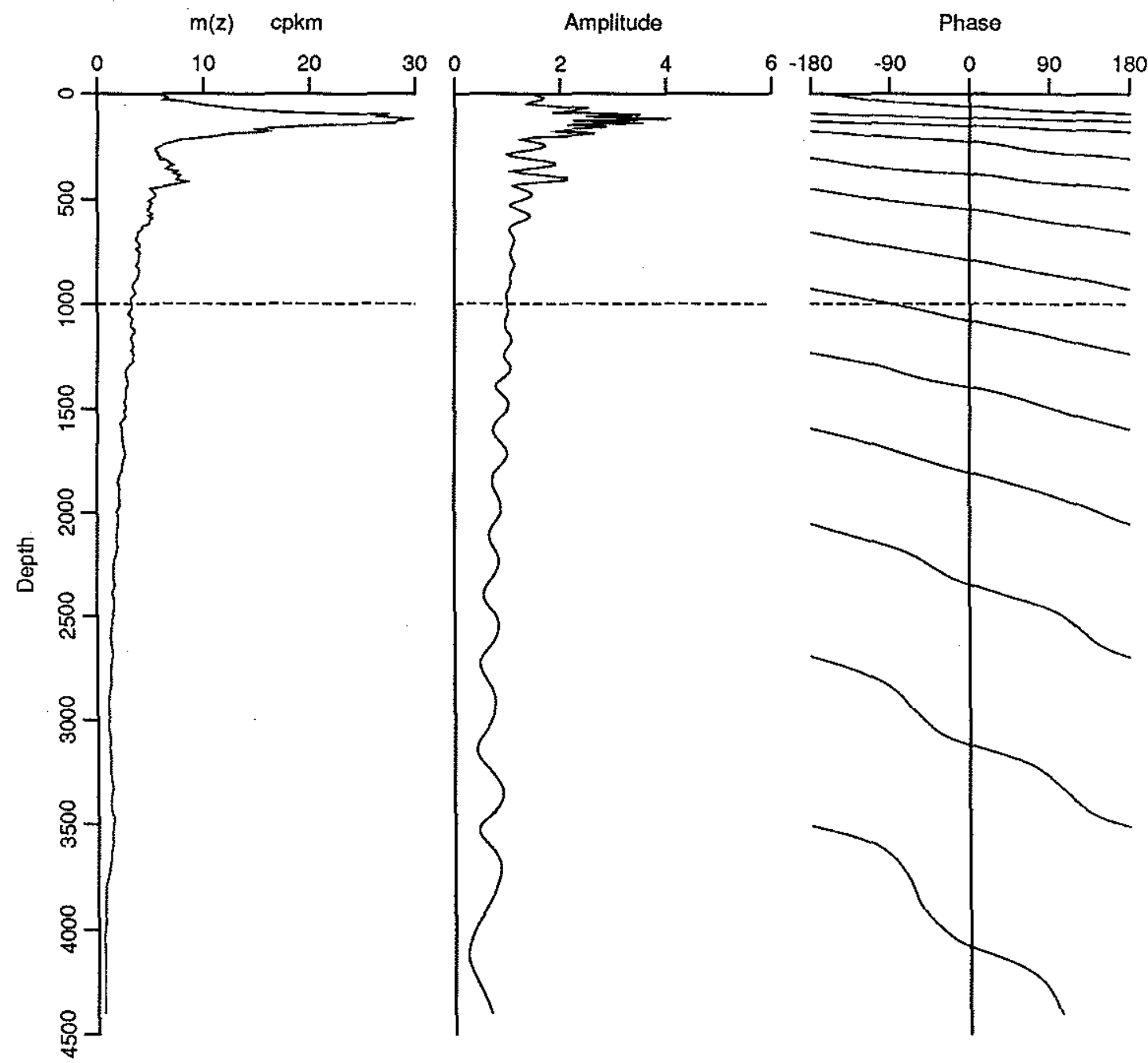

Figure 7.4.7 Medium wavenumber wave integrated through an averaged $N(z)$ profile

This plot gives the integrated solution for $\alpha_{z}(z)$ at a frequency $\omega$ of $.2 \mathrm{cph}$ and a horizontal wavenumber $K$ of $.5 \mathrm{cpkm}$. This wavenumber corresponds to mode 25 . The left plot gives the local vertical wavenumber profile (which is almost proportional to the buoyancy frequency profile): the buoyancy frequency profile is computed from PEQUOD data using bins every $25 \mathrm{wsm}$. The center plot gives the amplitude of $\alpha_{z}(z)$, and the right plot gives the phase of $\alpha_{z}(z)$. The solution show wiggles in amplitude that are quite similar to those seen in the solutions for $\alpha(z)$. Like the $\alpha(z)$ solutions, the phase is quite smooth. 
Upward propagating waves

Run: P28W25

$\omega 0.2 \mathrm{cph}$

$\mathrm{K} 2.0 \mathrm{cpkm}$

$\mathrm{N}\left(\mathrm{z}_{0}\right) 1.3223 \mathrm{cph}$

$\mathrm{z}_{\mathrm{O}} \quad 1000.0 \mathrm{~m}$

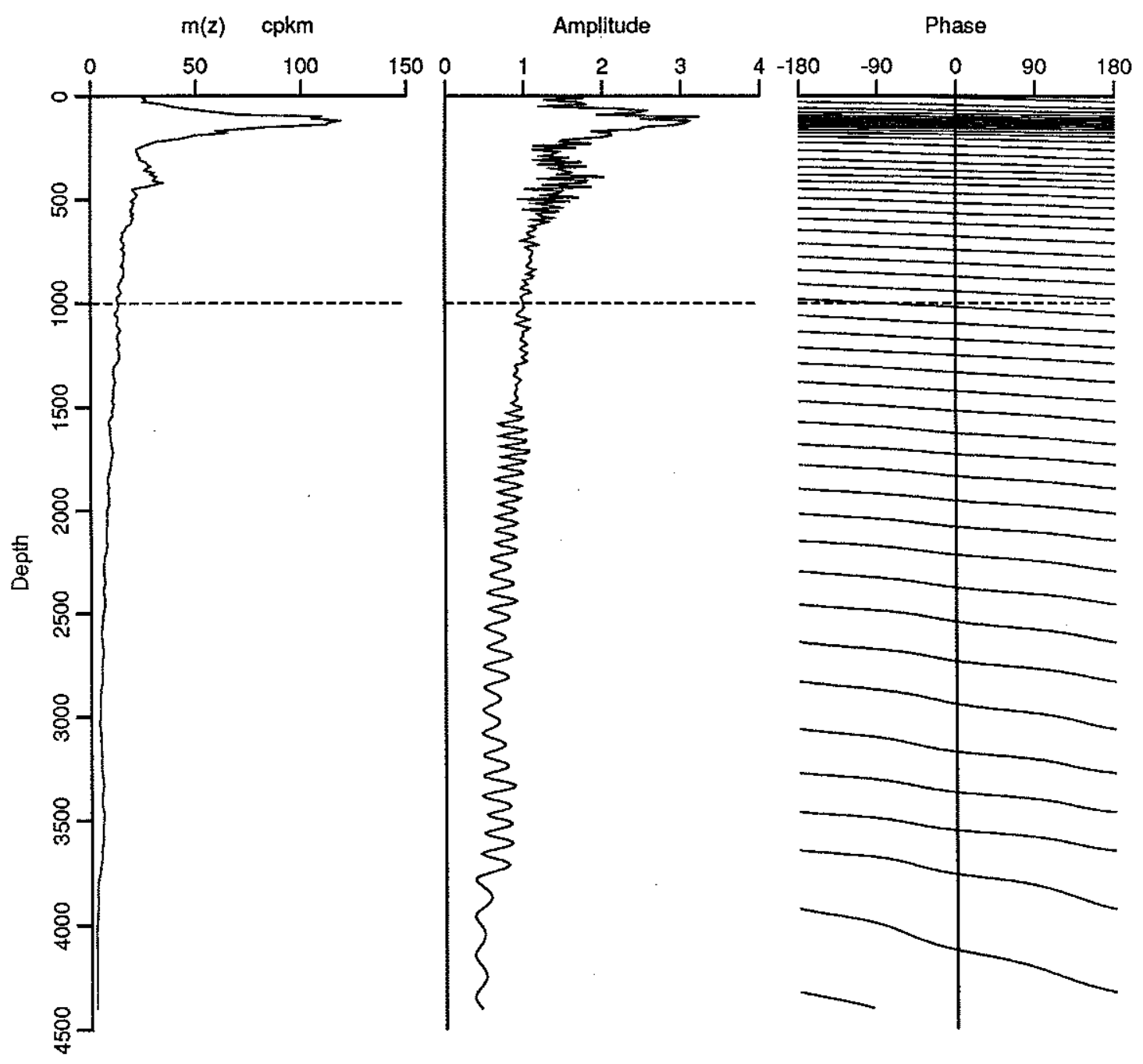

Figure 7.4.8 Very high wavenumber wave integrated through a variable $N(z)$ profile

This plot gives the integrated solution for $\alpha_{z}(z)$ at a frequency $\omega$ of $.2 \mathrm{cph}$ and a horizontal wavenumber $K$ of $2 \mathrm{cpkm}$. This wavenumber corresponds to mode 100 . The left plot gives the local vertical wavenumber profile (which is almost proportional to the buoyancy frequency profile): the buoyancy frequency profile is computed from PEQUOD data using bins every $25 \mathrm{wsm}$. The center plot gives the amplitude of $\alpha_{z}(z)$, and the right plot gives the phase of $\alpha_{z}(z)$. The solution show wiggles in amplitude that are quite similar to those seen in the solutions for $\alpha(z)$. The phase, however, is quite smooth. 


\section{Upward propagating waves}
$\mathrm{K} .021 \mathrm{cpkm}$
K $.500 \mathrm{cpkm}$
$\mathrm{K} 2.00 \mathrm{cpkm}$

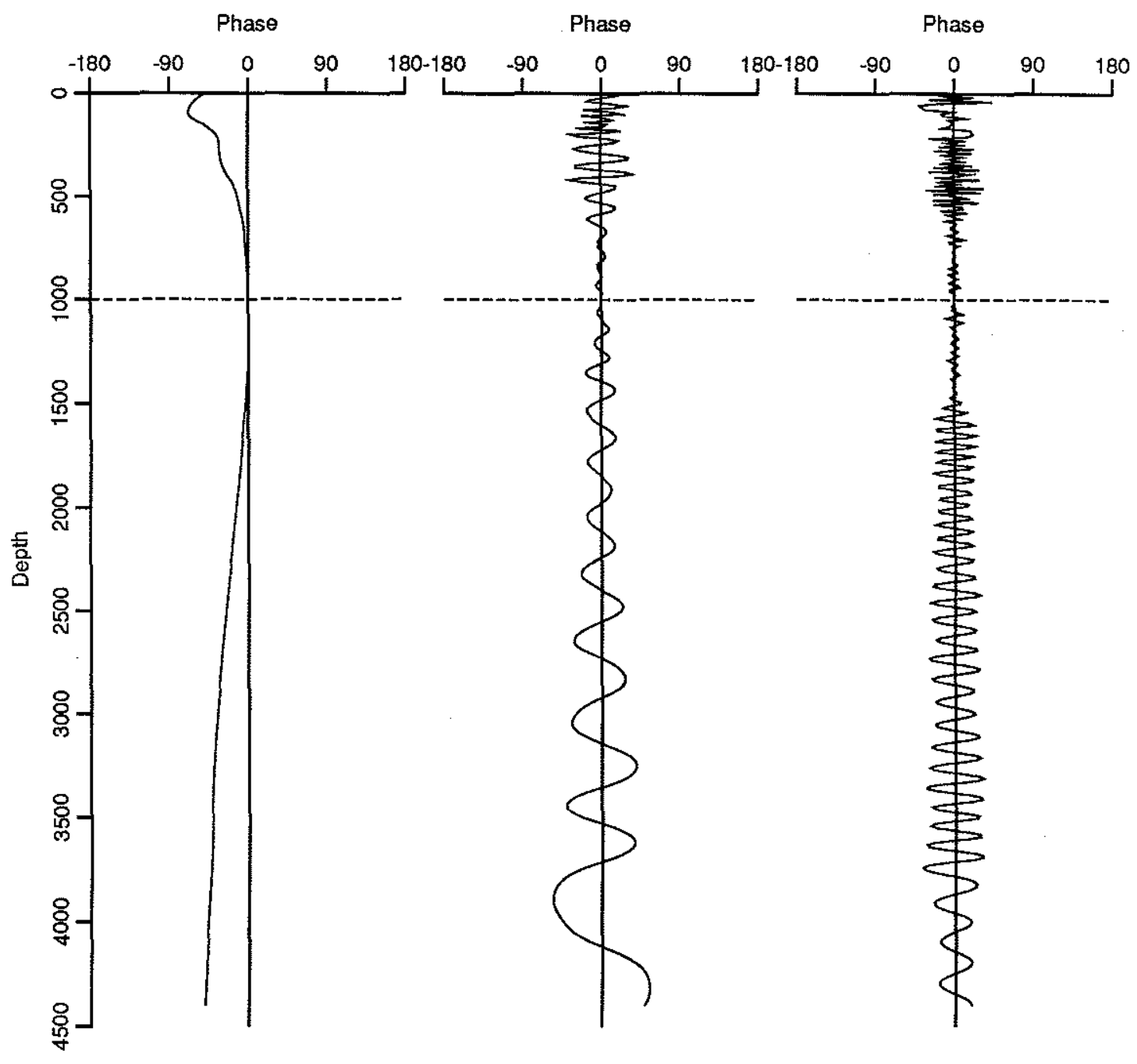

Figure 7.4.9 Variations in the phase difference between $\alpha$ and $\alpha$.

These three plots give the deviation from $-90^{\circ}$ of the phase difference between $\alpha$ and $\alpha_{z}$. The left plot gives this offset phase difference for a horizontal wavenumber $K$ of $.021 \mathrm{cpkm}$, the center plot gives the offset phase difference for a horizontal wavenumber $K$ of $.5 \mathrm{cpkm}$, and the right plot gives the offset phase for a horizontal wavenumber $K$ of 2 . cpkm. The plots show that there is some variability in the phase difference between $\alpha$ and $\alpha_{z}$ for the higher modes, even though the plots of the $\alpha_{z}$ and $\alpha$ show basically the same character. 


\section{Comparison with WKBJ solutions}

In many analyses of data, rather than integrating through the buoyancy frequency profile, as has been done so far, the analyses use the physical optics (WKBJ) approximation to solve the vertical structure equation. It is thus interesting to note the differences between these integrated solutions for $\alpha(z)$ and the WKBJ solutions that might otherwise be used. As it turns out, because of the presence of variability in the buoyancy frequency profile, for higher modes the WKBJ solution is in some ways better than the integrated solution, even though the integrated solution is more accurate for any given buoyancy frequency profile.

The WKBJ solution simply has the amplitude as inversely proportional to the square root of $m(z)$, while the phase is still given by equation 7.2.6c.

$$
R(z) \approx \sqrt{m\left(z_{0}\right) / m(z)} \alpha_{0}
$$

It is a good approximation as long as $m$ varies much more slowly than the scale of the wave, i.e.

$$
\frac{m_{z}}{m} \ll m
$$

This is clearly not the case in the low wavenumber limit, and figure 7.4 .10 compares the WKBJ solution to the integrated solution in that limit. The solid line is the integrated solution, the dashed line is the WKBJ solution, and the dotted line is an integrated solution using the hydrostatic dispersion relation

$$
m^{2}(z)=\frac{N^{2}(z)}{\omega^{2}} K^{2}
$$

The hydrostaticity is not an important issue for this frequency and wavenumber: there is no detectable difference between the integrated hydrostatic and non-hydrostatic solutions. The percentage differences between the WKBJ and integrated solutions appear to be large near the surface, and there is some phase difference between the solutions below the thermocline if the separation is sufficiently high.

In the very high wavenumber limit, if the $100 \mathrm{wsm}$ profile were used the integrated and WKBJ solutions would be indistinguishable. This is because the conditions for validity of the WKBJ approximation (equation 7.4.3) are satisfied. If, however, the 25 wsm profile is used, the solutions are quite different from one another, just as the difference medium wavenumber solutions differ from one another. Figure 7.4.11 gives 
Upward propagating waves

Run: P28W25

$$
\begin{aligned}
& \omega 0.2 \mathrm{cph} \\
& \text { K } 0.021 \mathrm{cpkm} \\
& \begin{array}{ll}
\mathrm{N}\left(\mathrm{z}_{\mathrm{o}}\right) & 1.3223 \mathrm{cph} \\
\mathrm{z}_{\mathrm{o}} & 1000.0 \mathrm{~m}
\end{array}
\end{aligned}
$$
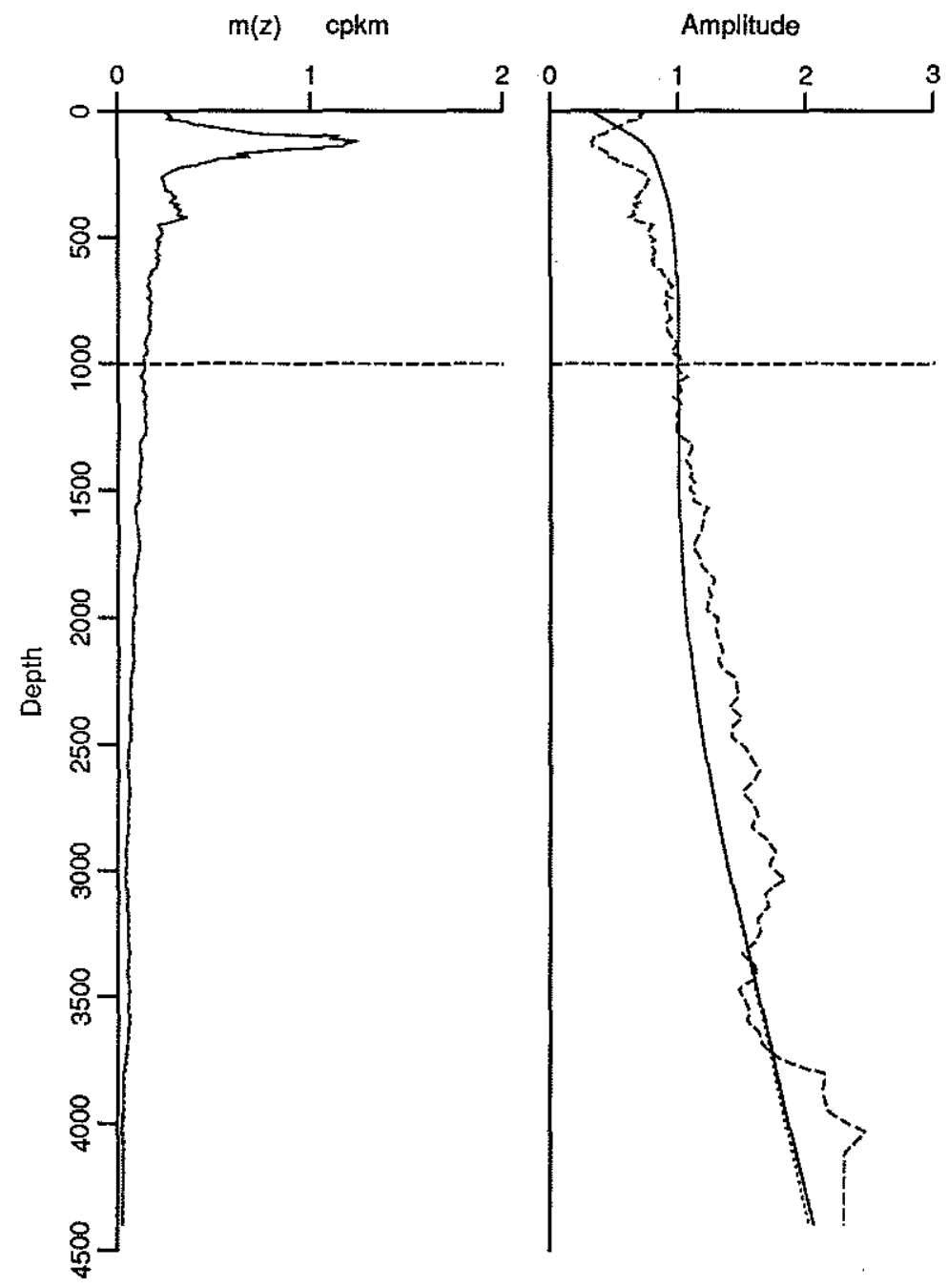

Phase
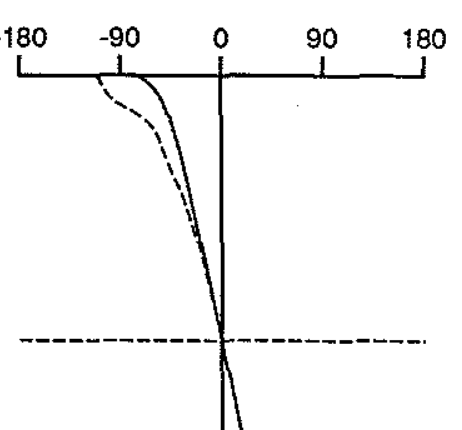

Figure 7.4.10 Comparison of WKBJ solutions with integrated solutions in the low wavenumber limit

The integrated solution for $\alpha(z)$ is given by the solid line, the WKBJ solution by the dashed line, and the integrated hydrostatic solution by the dotted line. In all three cases the frequency $\omega$ is $.2 \mathrm{cph}$ and the horizontal wavenumber $K$ is $.021 \mathrm{cpkm}$. The profile used is calculated on a 25 wsm grid. 
the comparison for the medium wavenumber (mode 25) wave. Again the WKBJ solution is given as the dashed line, the hydrostatic solution is given by the dotted line, and the integrated solution is given by the solid line. Note that in this case the hydrostatic solution is somewhat different that the non-hydrostatic solutions: in particular the phase is somewhat different when sufficiently far from the reference depth. On the other hand, the WKBJ and integrated solutions differ quite a bit in amplitude, but match quite well in phase. In fact, the amplitude of the WKBJ solution looks like a biased fit to the wiggles of the true solution. This presents an interesting question: it is precisely the wiggles of the true solution that are the most sensitive to changes in the $N$ profile, so that to the extent that there are small spatial and temporal variations in $N(z)$, the wiggles in the medium wavenumber waves are not well determined. Therefore in experimental situations, despite the fact that the WKBJ solution is not mathematically justified (i.e. it is not an accurate solution to the vertical structure equation), the WKBJ and the integrated solutions are equally (in)appropriate for medium to high wavenumbers. This becomes more evident in the next section, which looks at the effects of $N$ profile variations on the propagating waves.

\subsection{Effects of buoyancy frequency profile variability}

The local buoyancy frequency $N(z)$ is proportional to the local vertical density gradient. This section explores the effects of vertical density gradient variability on internal waves in a basic state with no mean shear flow. To some extent this problem has been considered by the previous section which argued that waves with vertical structure that is complicated is also highly dependent on the buoyancy frequency profile: to the extent that there is variability in that profile an averaged structure would be more appropriate than some very precise but also highly variable solution. Determining such an average structure is equivalent to determining the scattering of internal waves by buoyancy frequency fluctuations, a problem that is discussed in the vast literature on wave propagation in random media (WPRM). The WPRM literature deals both directly and indirectly with the problems of internal waves. After summarizing the buoyancy frequency variability by determining a vertical wavenumber spectrum for those fluctuations, this section reviews some of the relevant WPRM work and derives an analytic expression for the fluctuations effect on internal waves. This analytic analysis is somewhat limited because simplifying assumptions must be made in order to arrive at an analytic solution. Thus a numerical 


\section{Upward propagating waves}

Run: P28W25

w $0.2 \mathrm{cph}$

$\mathrm{K} 0.5 \mathrm{cpkm}$
$\begin{array}{ll}N\left(z_{0}\right) & 1.3223 \mathrm{cph} \\ z_{0} & 1000.0 \mathrm{~m}\end{array}$
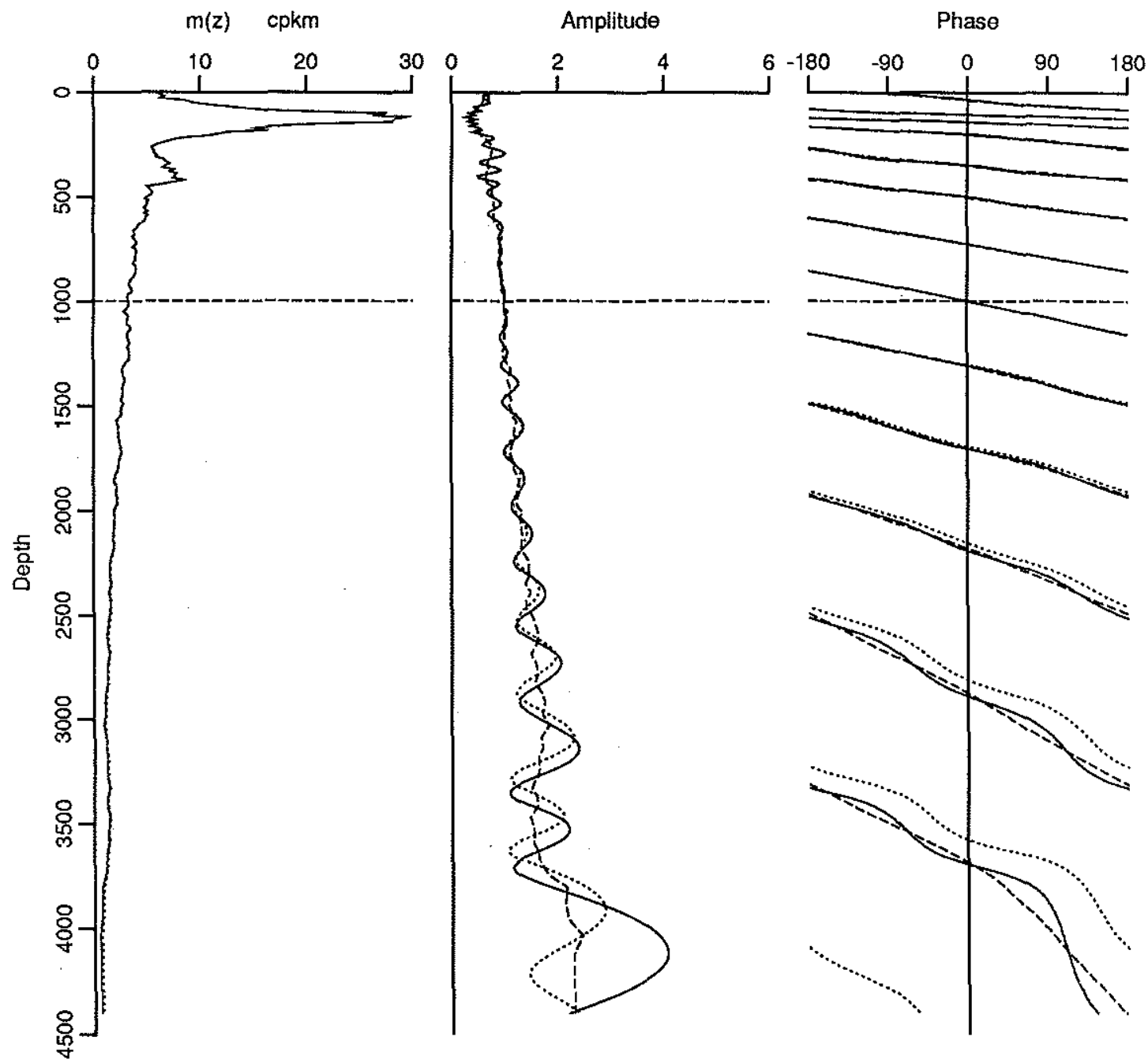

Figure 7.4.11 Comparison of WKBJ solutions with integrated solutions for medium wavenumber waves

The integrated solution for $\alpha(z)$ is given by the solid line, the WKBJ solution by the dashed line, and the integrated hydrostatic solution by the dotted line. In all three cases the frequency $\omega$ is $.2 \mathrm{cph}$ and the horizontal wavenumber $K$ is $.5 \mathrm{cpkm}$. The profile used is calculated on a 25 wsm grid. The plot shows the WKBJ solution to appear to be a best fit to the wiggles of the integrated solution: in a experimental situation, then, where the variability of the buoyancy frequency profile causes the wiggles to be poorly determined, the WKBJ solutions could very well be more appropriate then the relatively precise integrated solutions. 
analysis is made as well, verifying and extending the analytic results. It is found that low mode internal waves are not strongly affected by buoyancy frequency variability, while the higher modes are affected in two ways: the scattering decreases the measurable coherence due to the higher modes, and the scattering destroys the phase-locking of upward and downward waves characteristic of vertical modes, leaving the higher mode wavefield with a propagating rather than modal character.

As will be shown in the latter part of this section, the effect on internal waves of vertical density gradient variability is dependent on that variability's distribution as a function of vertical wavenumber. The variability of the vertical density gradient is here modeled as being due to advection of the mean density profile by oceanic motions. This model is discussed by Desaubies and Smith 1982 in their paper which models buoyancy frequency fluctuations as being due to a field of internal waves, a work which is reviewed in chapter 4 in its discussion of buoyancy frequency variability. Their results can be summarized by the following equation, an equation which relates the variance of the squared buoyancy frequency $N^{2}(z)$ to the variance of the vertical strain $\eta_{z}$,

$$
\frac{\left\langle\left(N^{2}(z)-N_{0}^{2}(z)\right)^{2}\right\rangle}{\left[N_{0}^{2}(z)\right]^{2}}=\left\langle\left[\eta_{z}(z)\right]^{2}\right\rangle
$$

where $N_{0}^{2}(z)$ is the true profile: $N_{0}^{2}(z)$ is best estimated by $\left\langle N^{2}(z)\right\rangle$. This expression also relates the vertical wavenumber spectrum of both the buoyancy frequency perturbations and the vertical strain. Furthermore, since vertical wavenumber spectrum of displacement $\left\langle\eta^{*} \eta\right\rangle(\beta)$ is easily related to the spectrum of vertical strain,

$$
\beta^{2}\left\langle\eta^{*} \eta\right\rangle(\beta)=\left\langle\eta_{z}^{*} \eta_{z}\right\rangle(\beta)
$$

buoyancy frequency variability can be related to displacement spectra as well.

Hayes and Powell 1980 characterize equatorial displacement spectra both on and off the equator in the eastern equatorial Pacific. They find that the displacement spectra is an order of magnitude higher directly on the equator as compared to five degrees north or south: this is shown in figure 7.5.1. The spectral level shown $\left(3 \times 10^{3} \mathrm{~m}^{3} / \mathrm{cyc}\right.$ at $.01 \mathrm{cpm}$ ) and the spectral slope of roughly -2 are equivalent to a strain spectrum that is white (spectral density independent of frequency) with a level of $11.8 \mathrm{cpm}^{-1}$. This matches calculations from the PEQUOD CTD data which show that the buoyancy frequency perturbation have a white spectrum with a level of $11 \mathrm{cpm}^{-1}$. 


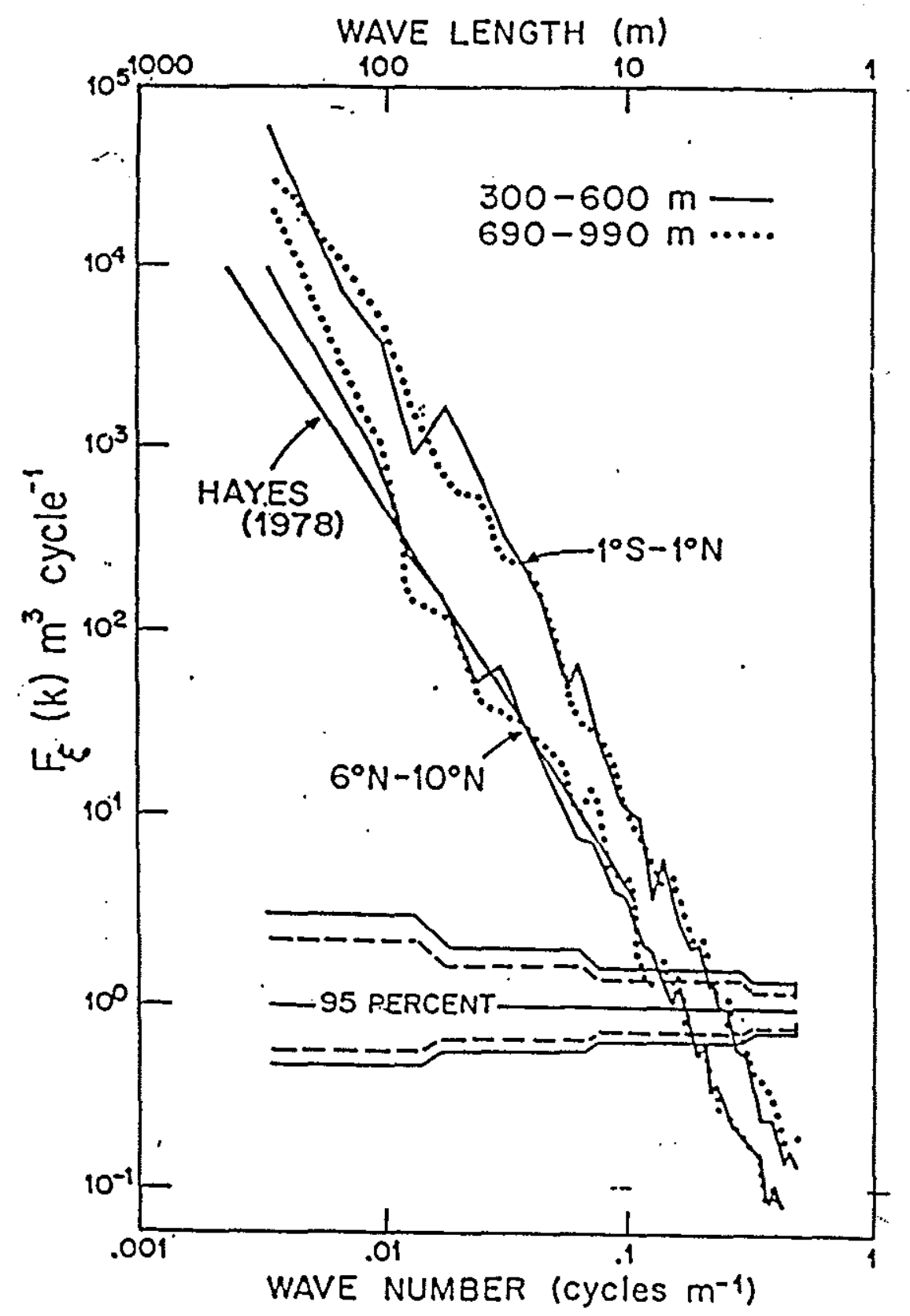

Figure 7.5.1 Equatorial Pacific displacement spectra

These curves compare vertical wavenumber spectra of displacement calculated from both equatorial $\left(1^{\circ} \mathrm{S}-1^{\circ} \mathrm{N}\right)$ and non-equatorial $\left(6^{\circ} \mathrm{N}-10^{\circ} \mathrm{N}\right)$ groups of stations: The straight line gives the GM75 model spectrum using parameters from Hayes 1978. The plots show that the equatorial displacement spectra is a factor of ten larger than the more mid-latitude displacement spectra. The equatorial spectra presented here are levels measured here are comparable in shape and level to calculations made from the PEQUOD data (this figure is figure 5 from Hayes and Powell 1980). 
Considering the strain spectrum to be white is neither a complete nor a sufficient characterization of the strain fluctuations as far as computing the effects on internal waves is concerned. Though it is not readily apparent from the Hayes and Powell figure (7.5.1), there is evidence that there is a $.1 \mathrm{cpm}$ vertical wavenumber cutoff of the displacement spectrum both on and off the equator. Toole and Hayes 1984 note that there is distinct evidence of a sharp drop in energy at $.1 \mathrm{cpm}$ in velocity shear spectra and less distinct evidence for the same cutoff in strain spectra: they further point out that aliasing effects could account for fact that the cutoff is less distinct in the strain spectra. The level they measure for strain spectra is also roughly comparable to the Hayes and Powell (and PEQUOD) levels. The measurements can thus be characterized as being constant up to $.1 \mathrm{cpm}$ and then dropping off quickly for higher wavenumbers. A simple model for the strain spectrum that is consistent with this characterization is

$$
\left\langle\eta_{z}^{*} \eta_{z}\right\rangle(\beta)=11 \mathrm{cpm}^{-1} \Pi\left(\frac{1}{2} \beta / \beta_{c}\right)
$$

where $\Pi(x)$ is the rectangle function $\dagger$ and the cutoff wavenumber $\beta_{c}$ is $.1 \mathrm{cpm}$.

$\dagger$ The rectangle function $\Pi(x)$ is defined to be

$$
\Pi(x)= \begin{cases}1 & |x|<\frac{1}{2} \\ \frac{1}{2} & |x|=\frac{1}{2} \\ 0 & |x|>\frac{1}{2}\end{cases}
$$

see Bracewell 1978. 
Analytic analysis of internal wave scattering due to buoyancy frequency variability The general problem of a wave propagating in a random medium (WPRM) has a vast literature with contributions from many of the sciences and the engineering fields. Mysak 1978 reviews some of the oceanic WPRM literature. It is a rather difficult presentation, mostly mathematics and statistics with little attention paid to dynamics, but it does survey many of the mathematical tools used in the subject. The book edited by Flatté (1979), on the other hand, is quite clear and spends quite a bit of time discussing physical properties: it unfortunately focuses exclusively on the problems posed by sound propagating through a fluctuating ocean. Not only is Flatté easier to comprehend, but it is more useful for our purposes because the methods presented by Flatte allow the calculation of the effect of scattering on two point statistics such as coherence and cross-spectra, while the methods reviewed by Mysak focus on one point averages such as expected wave amplitude or energy. Since the analysis will eventually be applied in interpreting measured crossspectra, the methods presented in Flatté 1979 are the ones utilized here.

Internal waves are solutions to the vertical structure equation 7.2.1. Random fluctuations in the buoyancy frequency profile lead to random fluctuations in the local vertical wavenumber $m(z)$. Rewriting equation 7.2.1 to explicitly include those fluctuations leads to the following equation:

$$
G_{z z}+m_{0}^{2}(z)[1+\mu(z)]^{2} G=0
$$

This is a fairly general equation, the only step that has been taken is to write the local vertical wavenumber as the sum of a deterministic part $m_{0}(z)$ and a normalized random part $\mu(z)$. For the particular case of internal waves in a resting basic state, and making the hydrostatic approximation, we can relate both the deterministic and random parts of the local vertical wavenumber to the mean and deviation from the mean buoyancy frequency,

$$
m_{0}^{2}(z)=\frac{N_{0}^{2}(z) K^{2}}{\omega^{2}}=\frac{\left\langle N^{2}(z)\right\rangle K^{2}}{\omega^{2}}
$$

and

$$
\mu=\frac{\left.\left[N(z)-N_{0}(z)\right\rangle\right]}{N_{0}(z)}
$$

Note that the 'true' buoyancy frequency $N_{0}(z)$ is estimated as the square root of the mean vertical density gradient instead of begin estimated as the mean buoyancy frequency. As demonstrated in the next section, the scattering of internal waves depends on the vertical 
wavenumber spectrum of the local vertical wavenumber fluctuations $\left\langle\mu^{*} \mu\right\rangle(\beta)$. Using equation 7.5.6, this spectrum can be related to the strain spectrum

$$
\left\langle\mu^{*} \mu\right\rangle_{(\beta)}=\frac{1}{4}\left\langle\eta_{z}^{*} \eta_{z}\right\rangle(\beta)
$$

where the fluctuations about the mean vertical density gradient have been presumed small so that a linearization of the square root could be made.

The next step is to solve the vertical structure equation 7.5.4 with non-zero random fluctuations $\mu$. The following discussion will demonstrate that

$$
G_{0}(z) e^{i \phi}
$$

is an adequate solution to the random differential equation 7.5.4, where $G_{0}(z)$ is the solution to the deterministic equation $(\mu \equiv 0)$ and $\phi$ is an integral over the random phase fluctuations

$$
\phi=\int_{z_{0}}^{z} m_{0}(z) \mu\left(z^{\prime}\right) d z^{\prime}
$$

This solution is in effect making the geometrical optics approximation for the part of the solution that is due to random fluctuations. As will be shown shortly, such a solution is certainly valid to the extent that the deterministic solution $G_{0}(z)$ is a valid geometrical optics solution; it is also valid in a statistical sense for a wider class of deterministic solutions.

In order to facilitate finding a solution $G(z)$ to the random differential equation 7.5.4, write $G(z)$ in the following form:

$$
G(z)=G_{0}(z) G_{1}(z)-\epsilon(z)
$$

where $G_{0}(z)$ is the solution to the deterministic problem, $G_{1}(z)$ is the part of the solution that we will eventually set equal to $e^{i \phi}$, and $\epsilon(z)$ is the part of the solution that is left over. After expanding out the curvature term and subtracting the deterministic equation for $G_{0}(z)$, equation 7.5.4 becomes

$$
2\left[G_{0 z} G_{1 z}+m_{0}^{2} \mu G_{0} G_{1}\right]+\left(G_{1 z z}+m_{0}^{2} \mu^{2} G_{1}\right) G_{0}=\left[\partial_{z z}+m_{0}^{2}\right] \epsilon
$$

Note the fluctuations $\mu$ are presumed to be small in order to make the operator operating on $\epsilon$ deterministic. Since we are only interested in the size of $\epsilon$ the accuracy of this approximation is not particularly important. 
By forcing $G_{1}(z)$ to be given by $e^{i \phi}$ and substituting into equation 7.5 .10 , the validity of the geometric optics solution 7.5.8 can be determined,

$$
\left[\partial_{z z}+m_{0}^{2}\right] \epsilon=2\left[i G_{0 z}+m_{0} G_{0}\right] m_{0} \mu e^{i \phi}
$$

This is a deterministic operator equation for $\epsilon(z)$ with random forcing modulated by the deterministic solution $G_{0}(z)$. The first thing to note about this equation is that when $G_{0}(z)$ is given by geometric optics and if $G_{0}$ has upward propagating phase (i.e. if $\left.G_{0 z}=i m_{0}(z) G_{0}\right)$, then $\epsilon$ is identically zero (the same could be said of a wave with downward propagating phase if $G_{1}$ were changed to $e^{-\phi}$ ). More generally, to the extent that geometric optics is appropriate for the deterministic solution $G_{0}$, equation 7.5.8 will provide an approximate solution to the random vertical structure equation. Furthermore, the equation for the statistical average of the error $\langle\epsilon\rangle$ indicates that in a statistical sense $\epsilon$ should stay small, i.e.

$$
\left[\partial_{z z}+m_{0}^{2}\right]\langle\epsilon\rangle=2\left[i G_{0 z}+m_{0} G_{0}\right] m_{0}\left\langle\mu e^{i \phi}\right\rangle
$$

and $\left\langle\mu e^{i \phi}\right\rangle$ should tend to remain small. Thus equation 7.5.8 is vindicated as being a reasonable solution.

Note that the weighing factor $m_{0}(z)$ in equation 7.5 .8 can be made implicit by defining a WKBJ stretched depth $\zeta$ as was done in chapter 3 ,

$$
\zeta\left(z_{1}, z_{2}\right)=\frac{1}{1 \mathrm{cph}} \int_{z_{1}}^{z_{2}} N\left(z^{\prime}\right) d z^{\prime}
$$

This means that equation $7.5 .8 b$ becomes

$$
\phi=q_{0} \int_{\varsigma_{1}}^{s_{2}} \mu\left(\zeta^{\prime}\right) d \zeta^{\prime}
$$

where $q_{0}$ is the wavenumber in a $1 \mathrm{cph}$ ocean, $q_{0} \equiv(1 \mathrm{cph}) \frac{K}{\sigma}$ (see chapter 3 ). Because the equations will then be simpler with no loss of generality, for the remainder of this section the depth will be taken to be WKBJ stretched, i.e. equation $7.5 .8 b^{\prime}$ will be used with $z$ written for $\zeta$.

Eventually these scattering-modified waves are used to compute spectra equivalent to the measured cross-spectra. So what is important is not so much how the individual waves are modified by scatter (equation 7.5.8), what is important is how the coherence 
structure that is the average over many realizations of such waves at many different frequencies and wavenumbers is modified by scatter. As a simplified problem, consider the scatter-induced modifications to the coherence structure of a single wave. In the absence of buoyancy frequency fluctuations, there is no scattering and if all motions at any given time are due to a single wave then all points are perfectly coherent with one another. In the presence of fluctuations, the vertical structure of the wave $G(z)$ can be written according to equation 7.5 .8 so that the cross power between two depths $z_{1}$ and $z_{2}$ is given by

$$
\left\langle G^{*}\left(z_{1}\right) G\left(z_{2}\right)\right\rangle=G_{0}^{*}\left(z_{1}\right) G_{0}\left(z_{2}\right)\left\langle e^{i \phi}\right\rangle
$$

where

$$
\phi=q_{0} \int_{z_{1}}^{z_{2}} \mu\left(z^{\prime}\right) d z^{\prime}
$$

Note that the integral no longer depends on the reference depth $z_{0}$ of the wave. This is a consequence of the geometric optics approximation in the solution for the random part of the solution, and would not hold true if either a more general method for solution had to be used, or if the problem ceased being one dimensional.

If we normalize the cross-power to get the coherence $\gamma\left(z_{1}, z_{2}\right)$ between the two depths, the dependence on the deterministic solution $G_{0}(z)$ drops out, leaving the simpler expression,

$$
\gamma\left(z_{1}, z_{2}\right)=\left|\frac{\left\langle G^{*}\left(z_{1}\right) G\left(z_{2}\right)\right\rangle}{\left|G\left(z_{1}\right)\right|\left|G\left(z_{2}\right)\right|}\right|=\left\langle e^{i \phi}\right\rangle
$$

Therefore, as long as we are primarily interested in calculating the scattering effects, the discussion can focus on the coherence $\gamma$ rather than the cross-power.

Equation 7.5.13a shows that the effect of scattering is to multiply the deterministic cross-spectral contribution by a factor that depends on the scattering: the ensemble average value $\left\langle e^{i \phi}\right\rangle$. Assume that the local vertical wavenumber fluctuations $\mu(z)$ are distributed as Gaussian random normal variables. It then follows that, because $\phi$ is simply a sum over the $\mu(z)$ at different depths, the random phase difference $\phi$ is also a Gaussian random norm variable. As it turns out, it is straightforward to show that as long as $\phi$ is 
Gaussian then $\left\langle e^{i \phi}\right\rangle$ is given by $e^{-\frac{1}{3} \sigma^{2}}$, where $\sigma^{2}$ is the variance of the phase $\phi$, namely

$$
\begin{aligned}
\left\langle e^{i \phi}\right\rangle & =\frac{1}{\sigma \sqrt{2 \pi}} \int_{-\infty}^{\infty} e^{i \phi} e^{-\phi^{2} / 2 \sigma^{2}} d \phi \\
& =\int_{-\infty}^{\infty} e^{\left.i 2 \pi \mid(2 \pi)^{-1 / 2} \sigma\right] k} e^{-\pi k^{2}} d k \\
& =e^{-\frac{1}{2} \sigma^{2}}
\end{aligned}
$$

So the problem has reduced to finding the variance $\sigma^{2}$ of the phase $\phi$ for a fixed range $\left[z_{1}, z_{2}\right]$.

In order to write the phase variance $\sigma^{2}$ in terms of the spectrum of local vertical wavenumber fluctuations $\left\langle\mu^{*} \mu\right\rangle(\beta)$, the double integral that is part of the definition of $\sigma^{2}$ must be expanded.

$$
\begin{aligned}
\sigma^{2} & =q_{0}^{2}\left\langle\int_{z_{1}}^{z_{2}} \mu(z) d z \int_{z_{1}}^{z_{2}} \mu\left(z^{\prime}\right) d z^{\prime}\right\rangle \\
& =q_{0}^{2}\left\langle\int_{z_{1}}^{z_{2}} \int_{z_{1}}^{z_{2}} \rho\left(\left|z-z^{\prime}\right|\right) d z d z^{\prime}\right\rangle
\end{aligned}
$$

Here $\rho$ represents the autocorrelation function for the local wavenumber variations (i.e. $\rho(z)$ is the Fourier transform of the spectrum). By changing the variables of integration from $z_{1}$ and $z_{2}$ to a suitably rescaled sum and difference of the two coordinates, the double integral can be reduced to the relatively compact form

$$
\begin{aligned}
\sigma^{2} & =q_{0}^{2} R^{2} \int_{-\infty}^{\infty} \bigwedge(\epsilon) \rho(R \epsilon) d(\epsilon) \\
& =q_{0}^{2} R \int_{-\infty}^{\infty} \operatorname{sinc}^{2}(\nu)\left\langle\mu^{*} \mu\right\rangle(\nu / R) d \nu
\end{aligned}
$$

where $R=z_{2}-z_{1}$ is the separation between the two points, $\nu$ is a dimensionless vertical wavenumber, $\bigwedge(z)$ is the triangle function $\dagger$, and $\operatorname{sinc}(x)$ is the sinc function. $\ddagger$ Some

$\dagger$ The triangle function is defined to be

$$
\bigwedge(x)= \begin{cases}1-|x| & |x|<1 \\ 0 & \text { otherwise }\end{cases}
$$

The triangle function's Fourier transform is the sinc function squared (Bracewell 1978).

$\ddagger$ The sinc function used here is defined for frequencies in cycles per unit, i.e.

$$
\operatorname{sinc}(x)=\frac{\sin \pi x}{\pi x}
$$


Fourier properties are used in going from equation 7.5.16a to equation 7.5.16b. The form of this final equation shows that the relation between the dominant scales of the fluctuations (information contained in $\left\langle\mu^{*} \mu\right\rangle(\beta)$ ) and the separation $R$ between the points of interest determine the strength of the correlation.

We are now in a position to calculate the effects of equatorial finestructure scattering on internal waves. By using the explicit form for the strain spectra given in equation 7.5.3, we find the following expression for the local vertical wavenumber fluctuations $\operatorname{spectra}\left\langle\mu^{*} \mu\right\rangle(\beta)$,

$$
\left\langle\mu^{*} \mu\right\rangle(\beta)=\rho_{0} \Pi\left(\frac{1}{2} \beta / \beta_{c}\right)
$$

where the amplitude $\rho_{0}$ is roughly $3 \mathrm{cpm}^{-1}$ and the cutoff wavenumber $\beta_{c}$ is $.1 \mathrm{cpm}$. This explicit function can then be used to calculate the phase variance $\sigma^{2}$ by using equation 7.5.16,

$$
\sigma^{2}=q_{0}^{2} R \int_{-\infty}^{\infty} \operatorname{sinc}(\nu) \rho_{0} \Pi\left(\frac{1}{2} \nu /\left[R \beta_{c}\right]\right)
$$

For realistic values of the parameters, this expression can be simplified considerably. As long as the separation being considered $R$ is much greater than the cutoff wavelength $\rho^{-1}$ (10m in this case), the rectangle function is unity over the range in $\nu$ that contributes significantly to the integral, making the integral in equation 7.5.17 essentially the infinite integral of $\operatorname{sinc}^{2}$. That integral turns out to be exactly one. $\nmid$ This means that for separations significantly greater than $10 \mathrm{~m}$, the phase variance is simply proportional to the (stretched) separation $R$, i.e. $\sigma^{2}=q_{0}^{2} \rho_{0} R$. This means that the coherence $\gamma\left(z_{1}, z_{2}\right)$ is a simple exponential

$$
\gamma\left(z_{1}, z_{2}\right)=e^{-\frac{1}{2} \sigma^{2}}=e^{-R / L_{E}}
$$

where the coherence decay scale $L_{E}$ is given by

$$
L_{E}=\frac{2}{\rho_{0} q_{0}^{2}}=.1 q_{0}^{-2} \mathrm{mcpm}^{2}
$$

The second expression uses the value of $3 \mathrm{cpm}^{-1}$ from equation 7.5 .17 for the strain spectral size $\rho_{0}$.

$\dagger$ The infinite integral is easily evaluated by recognizing that the integral is the fourier transform evaluated at zero. The fourier transform of $\operatorname{sinc}^{2}(k)$ is the triangle function $\bigwedge(x)$, thus the integral is $\Lambda(0)=1$. 
The results embodied in equation 7.5.20 suggest that long waves are unaffected by scatter, while shorter waves can be scattered quite strongly. The formula states that a wave with a vertical wavelength of $1 \mathrm{~km}$ will have a exponential decay scale of $100 \mathrm{~km}$, i.e. it will not decay at all. A $100 \mathrm{~m}$ wave, on the other hand, has an exponential decay of $1 \mathrm{~km}$, which means it would decay significantly in propagating between the top and bottom of the ocean. This suggests that while there may be a significant modal character to the $1 \mathrm{~km}$ waves (since they can feel the top and bottom), the $100 \mathrm{~m}$ waves will be strictly propagating, having lost all top and bottom phase information through scattering off finestructure. The transition wavelength is roughly $300 \mathrm{~m}$, which is the wave whose exponential decay is $10 \mathrm{~km}$ (roughly the distance from top to bottom and back again). This wave corresponds roughly to mode 30 , thus a model of the internal wave field that is primarily modal up to mode 30 , and primarily propagating for modes higher than 30 , is suggested. This is supported of the results of chapter 3, which showed that the long wavelength energy is modal in character. To the extent that the higher modes carry little energy, experimental verification of the propagating nature of the higher modes is difficult.

As a partial check on the assumptions made in deriving the analytic results for the scattering of internal waves due to equatorial finestructure, some numerical simulations were made using CTD data from the PEQUOD site. Because of the averaging necessary to compute buoyancy frequency from noisy data, the variability contained in the buoyancy frequency computed from the PEQUOD CTD profiles is reduced from the level that actually exists at the PEQUOD site. As discussed in section 7.4, the buoyancy frequency profiles were calculated on a pressure grid with bins such that there are estimates every 25 wsm (WKBJ stretched meters). Linear interpolation is then used in order to estimate the buoyancy frequency between grid points.

The process of spatial interpolation filters the spectrum of the buoyancy frequency variability, and the particular filter that corresponds to linear interpolation is readily computed using the Fourier properties of the relevant functions. Because linear interpolation is equivalent to convolution with a triangle function (see Bracewell 1978), the filtered spectrum is proportional to the Fourier transform of that triangle function squared. Since the Fourier transform of the triangle function is $\operatorname{sinc}^{2}$, the true spectrum has been multiplied by a function proportional to $\operatorname{sinc}^{4}$. Therefore, if the true strain spectrum were white, the filtered spectrum would be proportional to $\operatorname{sinc}^{4}$ (this is also true if the true 
spectral form is given by a 'top hat' shape such as equation 7.5.17 where the cutoff wavelength is smaller than $25 \mathrm{wsm}$ ),

$$
\left\langle\eta_{z}^{*} \eta_{z}\right\rangle(\beta)=\rho_{0} \operatorname{sinc}^{2}(\beta \delta z)
$$

where $\delta z=25$ wsm is the grid spacing of the interpolation scheme.

In order to get a analytical estimate for the effect of this filtered buoyancy frequency variability on internal waves, simply substitute this new form for the spectral shape in equation 7.5.16b and proceed as we did for the rectangle function spectral form. As it turns out, the manipulations are somewhat more tractable in the spatial rather than the Fourier domain, so we use equation 7.5.16 $a$ to get an expression for the phase variance,

$$
\sigma^{2}=\frac{q_{0}^{2} R^{2} \rho_{0}}{\delta z} \int_{-\infty}^{\infty} \Lambda(\epsilon) \Lambda * \bigwedge(\lambda \epsilon) d \epsilon
$$

where $\lambda=R / \delta z$ is the ratio of the separation to the grid spacing used in the linear interpolation, and $*$ denotes convolution. Nothing more than tedious algebra is required to evaluate this expression: the answer is

$$
\sigma^{2}=\rho_{0} q_{0}^{2} \delta z \begin{cases}\frac{2}{3} \lambda^{2}-\frac{1}{6} \lambda^{4}+\frac{1}{20} \lambda^{5} & \lambda<1 \\ -\frac{1}{10}+\frac{1}{3} \lambda+\frac{1}{3} \lambda^{2}-\frac{1}{60} \lambda^{5} & 1<\lambda<2 \\ \frac{1}{3} \lambda+\frac{7}{10} & 2<\lambda\end{cases}
$$

Note that for separations much large than the grid size, the leading behavior is an exponential decay $e^{-R / L_{\mathrm{s}}}$ where the decay scale is given by

$$
L_{E}=\frac{6}{q_{0}^{2} \rho_{0}}
$$

This decay scale is simply three times what was found for the 'top hat' spectral form (equation 7.5.17). A normalized version $\phi(\lambda)$ of the phase variance is plotted for small separation in figure 7.5.2.

$$
\phi(\lambda)=\sigma^{2} \frac{L_{E}}{2 \delta z}
$$

These analytic results now need to be compared to the numerical results. 


\section{Normalized phase variance}

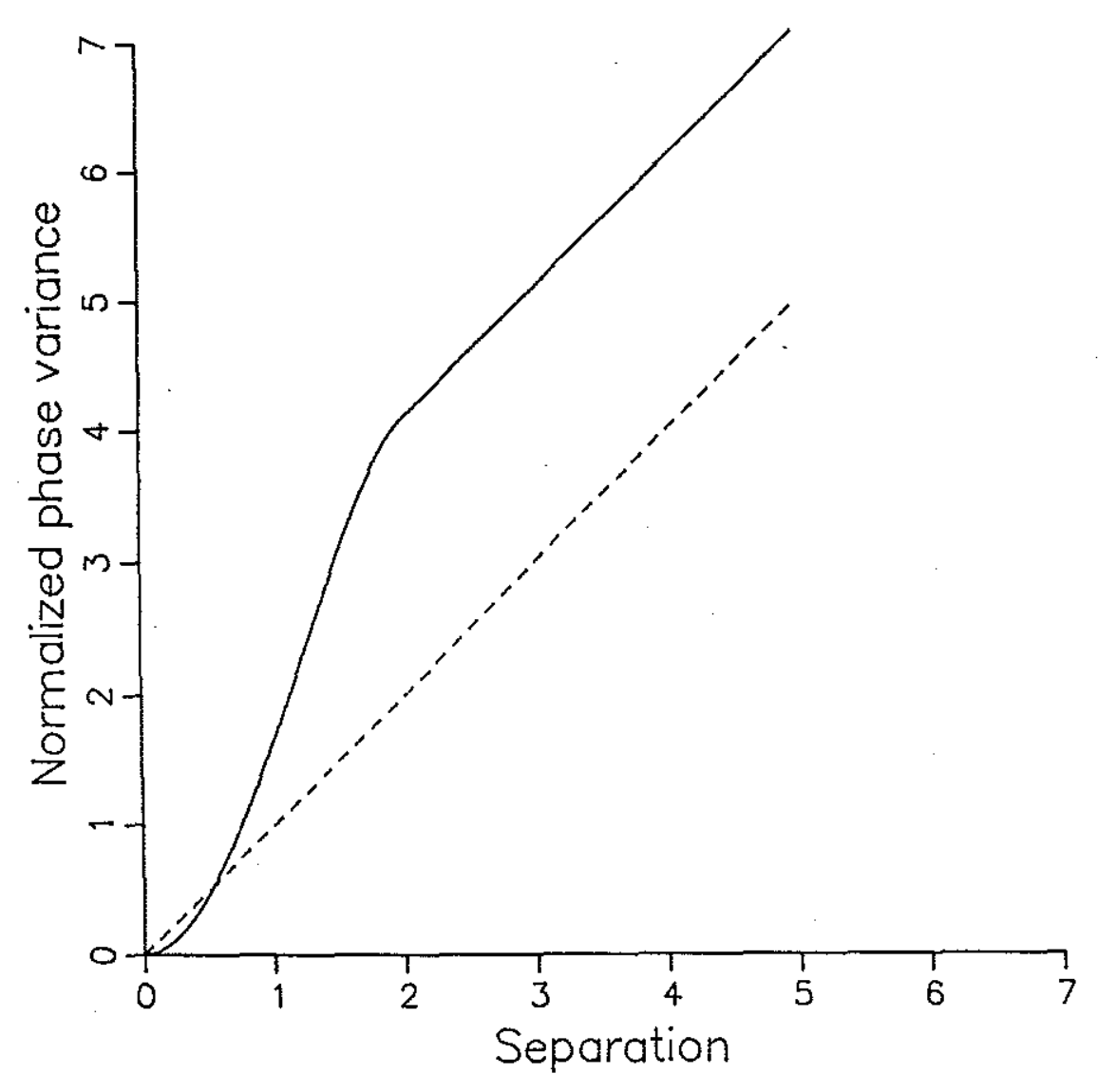

Figure 7.5.2 Phase variance for linearly interpolated profiles

Plotted is the normalized phase variance $\phi$ against the scaled separation $\lambda$ (the separation is scaled by the grid spacing of the interpolation). The curve is a line with slope one for large argument: that is the limit given in equation 7.5.24 which shows the phase variance is proportional to the separation $R$ with scale $L_{E}$. For smaller separations, there is some deviation from that simple relation. 
Numerical analysis of internal wave scattering off buoyancy frequency variability

The numerical calculations of buoyancy frequency variability are presented in three parts. First a rough estimate is made of the size of the effect on internal waves at various wavenumbers by overplotting integrations through different realizations of the buoyancy frequency profile. If the wave is not particularly dependent on which realization of the buoyancy frequency profile is used, then it can be safely concluded that the wave at that particular wavenumber is not strongly scattered. If, on the other hand, the wave varies considerably depending on which realization is used, then the wave is strongly scattered. The second part of the numerical calculation considers a more quantitative measure, the coherence between any two depths due to waves at a particular wavenumber. When considering motions at a single wavenumber, the coherence between any two points is computed by averaging over all realizations of the wave. If the wave shows little difference between realizations, then the coherence between all depths will be unity. If the wave varies wildly from realization to realization, then the average over all realizations of cross-power will be much smaller than the power itself, and the coherence will be small. One important result of these coherence calculations is that separation determines most of the loss of coherence: away from the thermocline average depth of the two points being considered in the coherence is not nearly as important as the difference in depth between the two points. This facilitates the third part of the numerical calculations, where it is assumed that the effects of scattering are primarily determined by separation so that numerical estimates of the $e$-folding length $L_{E}$ can be made. It turns out that the numerical estimates of the effects of scattering are from 4 to 10 times the estimates of the analytical calculation, though the form of the analytical result is not unreasonable. Thus a modified version of equation 7.5.20 is deemed adequate to summarize the effects of scattering.

As a rough measure of the effect of buoyancy frequency variability on internal waves, integrations through individual profiles were overplotted on the integrations through the 25 wsm mean profile that were discussed in section 7.4. The 28 profiles that go into the mean profile were each averaged on a pressure grid that corresponds to a grid equally spaced every $25 \mathrm{wsm}$ (WKBJ stretched meters). The profiles were then averaged together on pressure surfaces to get the mean profile. For figures 7.5.3 and 7.5.4, integrations through the individual profiles from the $K$ site (see chapter 2) were plotted on top of the integrations through the mean profile. Figure 7.5.3 shows the results for the first 
baroclinic mode $(K=.021 \mathrm{cpkm})$ : there is a factor of two variation in amplitude and almost no variation in phase. Despite the variation between realizations, each realization of the amplitude is quite smooth. But, as we shall see, the phase variations are much more important than amplitude variations, and variations such as these amplitude changes are not as important. The variations for the twenty-fifth mode $(K=.5 \mathrm{cpkm})$, on the other hand, are much larger than the mean. Figure 7.5.4 shows that not only are the amplitude variations very large, but the phase changes are significant as well. These wavenumbers are at opposite ends of a continuum of scattering effects: intermediate wavenumbers are affected by scattering to a degree somewhere between the two extremes.

In order to get a more quantitative measure of the effect of buoyancy frequency variability on internal waves, consider the coherence $\gamma\left(z_{1}, z_{2}\right)$ between two depths due to motion at a particular wavenumber.

$$
\gamma\left(z_{1}, z_{2}\right)=\left|\frac{\left\langle G^{*}\left(z_{1}\right) G\left(z_{2}\right)\right\rangle}{\left|G\left(z_{1}\right)\right|\left|G\left(z_{2}\right)\right|}\right|
$$

where the average $\langle\ldots\rangle$ is over all realizations, and $G$ is the wavefunction for the wavenumber of interest. If the wavefunction $G(z)$ shows little dependence on wavenumber, then the coherence will be one for all separations. This is illustrated in figure 7.5.5, which shows the coherence pattern due to a wave of horizontal wavenumber $K=.021$ cpkm (the first baroclinic mode). Plotted are contours of coherence with a contour interval of .1. The left and bottom axes given the WKBJ stretched depth, while the top axis gives the corresponding true depths. This wavenumber showed little dependence on realization in figure 7.5.3, so it is not surprising that the coherence is essentially one for all depth pairs. This is in stark contrast to the coherence pattern seen for horizontal wavenumber $K=.5 \mathrm{cpkm}$ (mode 25) given in figure 7.5.6. Here there is quite a lot of structure, but there are only a few features we need make note of for the remainder of this calculation. First of all, there is a sharp drop in coherence at the thermocline. The counterpoint to that is that away from the thermocline (below 500 true meters), the contours roughly parallel the 1 contour, which means that the coherence can be considered to be dependent primarily on separation in the deep water. Since all the PEQUOD instruments are below $500 \mathrm{~m}$, this result is sufficient to interpret the current meter spectra in chapter 8. Finally, while this plot is for the coherence calculated from horizontal velocity, the plot for displacement is essentially the same, and no distinction need be made between the two dynamic variables in computing scattering. This last point confirms the results of 


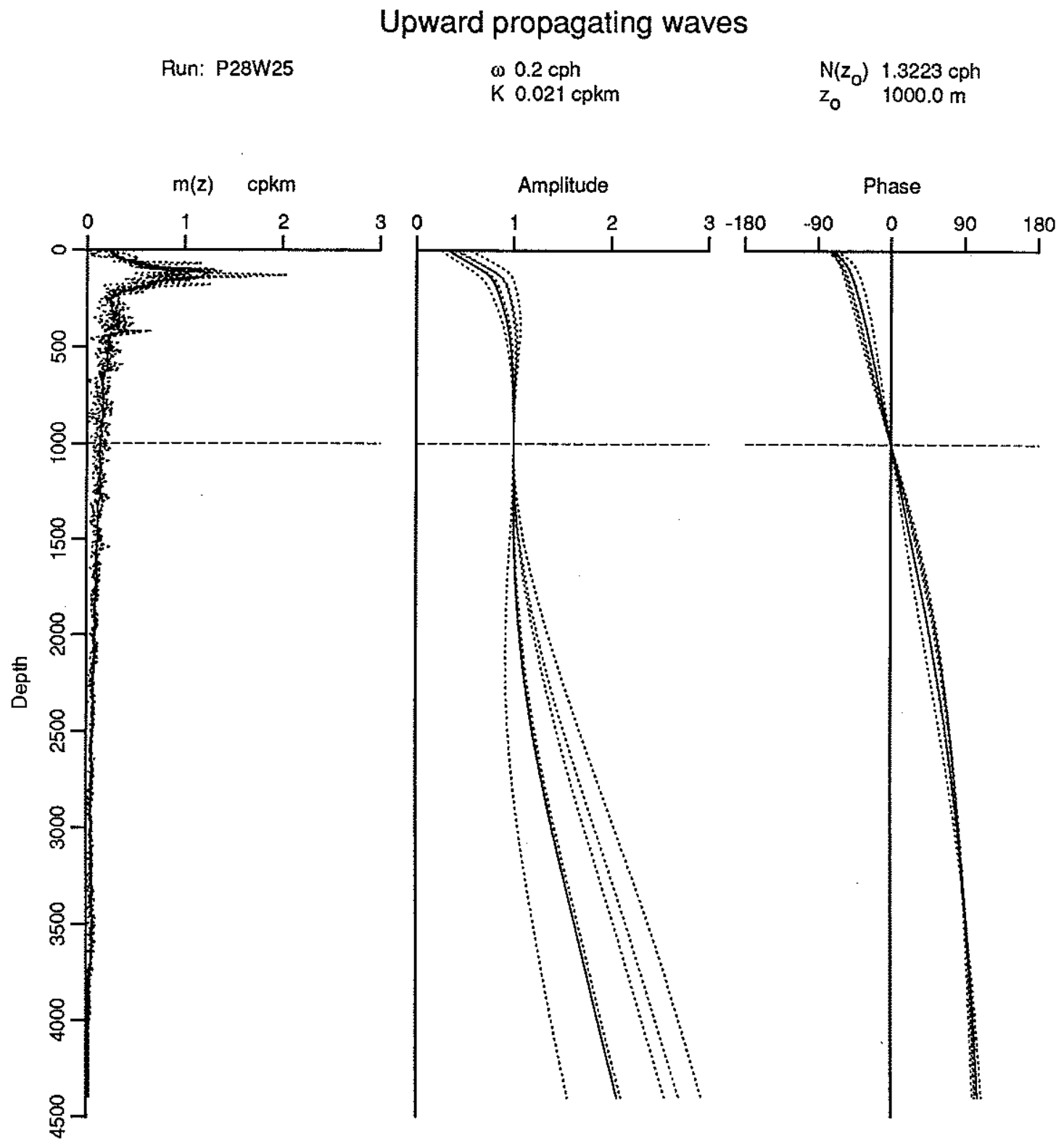

Figure 7.5.3 Effect of $N$ variability on low wavenumber waves

The solid line is the solution $\alpha(z)$ calculated for the averaged profile, while the dotted lines are integrations through individual profiles (see text for a more complete description). The frequency is $.2 \mathrm{cph}$ and the horizontal wavenumber is $.021 \mathrm{cpkm}$. 


\section{Upward propagating waves}

Run: P28W25

$$
\begin{aligned}
& \text { w } 0.2 \mathrm{cph} \\
& \begin{array}{ll}
N\left(z_{0}\right) & 1.3223 \mathrm{cph} \\
z_{0} & 1000.0 \mathrm{~m}
\end{array}
\end{aligned}
$$
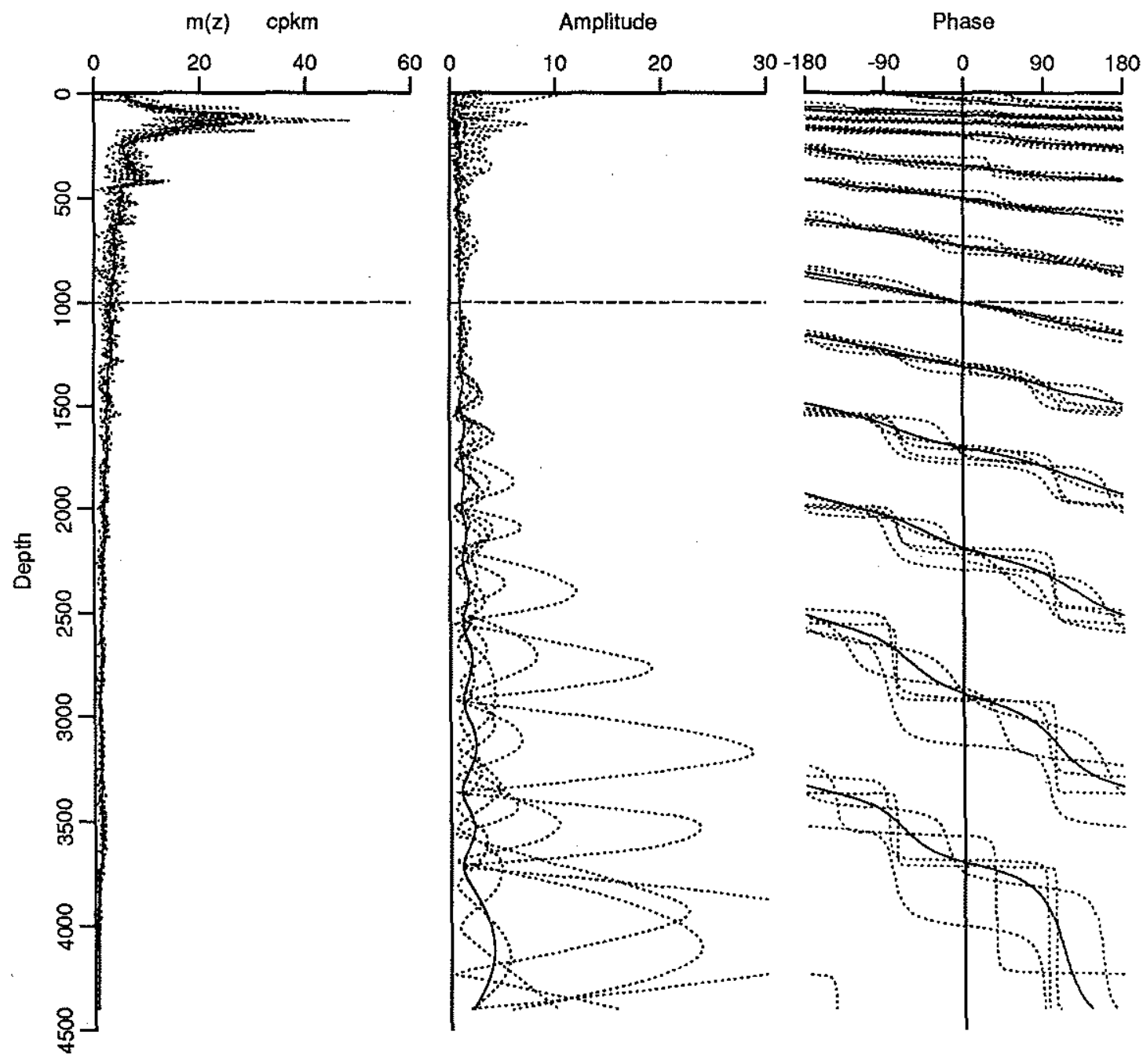

Figure 7.5.4 Effect of $N$ variability on medium wavenumber waves

The solid line is the solution $\alpha(z)$ calculated for the averaged profile, while the dotted lines are waves calculated for some individual profiles. All the solutions are calculated for a frequency $\omega$ of $.2 \mathrm{cph}$ and a horizontal wavelength $K$ of $.5 \mathrm{cpkm}$. (See text for a more complete description). 
section 7.4, which showed that the vertical structure function $\alpha$ and its vertical derivative $\alpha_{z}$ have roughly the same character.

The contour plot in figure 7.5.6 suggests that it is reasonable to consider coherence to be a function of separation for depths below the thermocline. Figure 7.5.7 shows a plot of coherence against separation for a horizontal wavenumber of $.3 \mathrm{cpkm}$ (roughly mode 15). The coherence shows regular oscillations about a fairly linear trend. The oscillations have a vertical wavenumber of $3 \mathrm{cpkm}$ which is exactly twice the wave's WKBJ stretched vertical wavenumber of $1.5 \mathrm{cpkm}$, the signature of reflection and subsequent interaction of upward and downward propagating energy (see section 7.3). At this horizontal wavenumber the coherence drops to .5 at a separation of roughly $3.2 \mathrm{~km}$, which means that the e-folding distance is roughly $6.3 \mathrm{~km}$.

Figure 7.5.8 shows coherence as a function of separation for a horizontal wavenumber of $.5 \mathrm{cpkm}$. The curve again shows half wavelength oscillations about a linearly decreasing mean, but the decease is distinctly more rapid in the $.5 \mathrm{cpkm}$ case than it is in the $.3 \mathrm{cpkm}$ case. The linear decrease on these two log-linear plots shows that the exponential form $e^{-R / L_{\varepsilon}}$ that the analytic analysis gives for the dependence of coherence on separation is quite reasonable the most serious omission being the half wavelength oscillations. Knowing that the form is reasonable, we can now compare numerical estimates of the $e$-folding length $L_{E}$ to the analytic calculations.

The table in figure 7.5.9 compares numerical estimates of the $e$-folding length $L_{E}$ with analytic estimates using geometric optics with two different choices of noise spectral form. The column marked 'Estimated $L_{E}$ ' is calculated from straight-line fits to curves such as 7.5.7 and 7.5.8 which consider coherence to be an exponential function of WKBJ stretched separation. The column marked 'Top hat $L_{E}$ ' is calculated using the spectral form given in equation 7.5.17, i.e. it is an analytic result assuming that the noise spectrum is white up to a cutoff of $.1 \mathrm{cpm}$. The column marked 'Linear $L_{E}$ ' is calculated using the noise spectrum after it has been filtered by the wavenumber equivalent of a $25 \mathrm{wsm}$ linear spatial interpolation. The resulting $L_{E}$ is simply three times the $L_{E}$ from the top hat spectrum. The final column gives the ratio between the top hat calculation and the estimated value of $L_{E}$ : it is more or less four, though there is quite a bit of scatter. It is not surprising that the numerical calculations show a more rapid decrease in coherence than the analytic calculations for any given wavenumber: the numerical calculations include more physics that the geometrical optics approximation allows the analytic solutions to 
H20WZ314

Coherence pattern due to scattering

$$
\begin{aligned}
& \omega \quad 0.200 \mathrm{cph} \theta \quad 0.000 \mathrm{cyc} \\
& \omega-\text { Uk } 0.200 \mathrm{cph} \quad \mathrm{z}_{0} \quad 1000.0 \mathrm{~m} \quad \mathrm{~N}\left(\mathrm{z}_{0}\right) \quad 1.322 \mathrm{cph}
\end{aligned}
$$

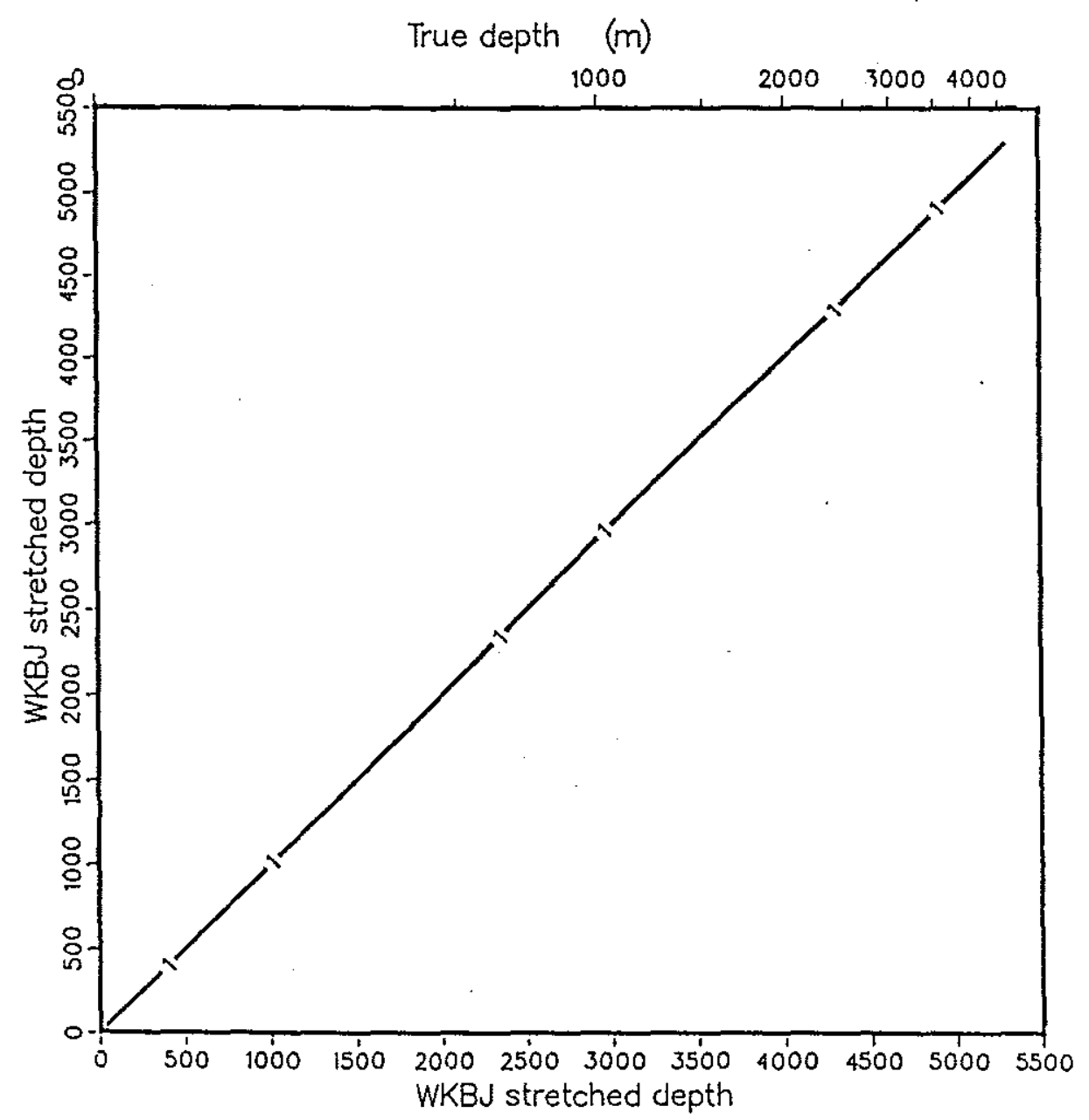

Figure 7.5.5 Coherence pattern due to scattering

This is the coherence pattern due to scattering for the first baroclinic mode $(K=.021$ $\mathrm{cpkm}$ ). The left and bottom axes give the WKBJ stretched depth; while the top axis gives the corresponding true depths. The plot shows that the first baroclinic mode is essentially not effected by scattering. 
P28W25H

Coherence pattern due to scattering

\author{
$\omega .=0.200 \mathrm{cph} \theta \quad 0.000 \mathrm{cyc} \quad \mathrm{K} \quad 0.500 \mathrm{cpkm}$ \\ $\omega-U k \quad 0.200 \mathrm{cph} \quad z_{0} 1000.0 \mathrm{~m} \quad \mathrm{~N}\left(\mathrm{z}_{0}\right) \quad 1.322 \mathrm{cph}$
}

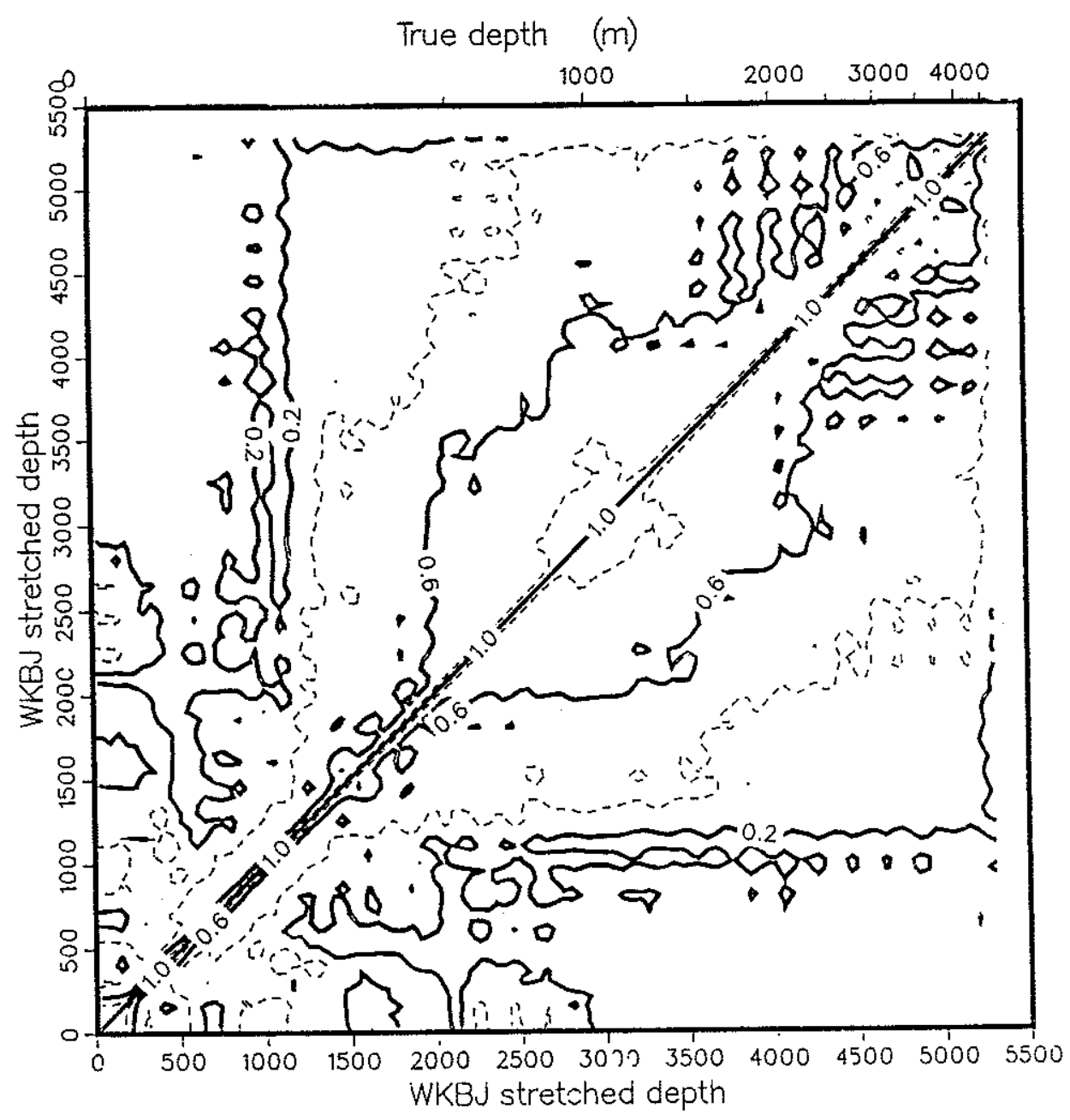

Figure 7.5.6 Coherence pattern due to scattering

This is the coherence pattern due to scattering for the twenty-fifth baroclinic mode $(K=.5 \mathrm{cpkm})$. The left and bottom axes give the WKBJ stretched depth, while the top axis gives the corresponding true depths. The plot is of the coherence calculated from horizontal velocity (the plot for coherence calculated from displacement is essentially the same). The plot shows that there is significant scattering at this vertical wavenumber, both at the thermocline and in the deep water (the deep water scattering seems to be primarily a function of separation). 
Coherence loss due to scattering

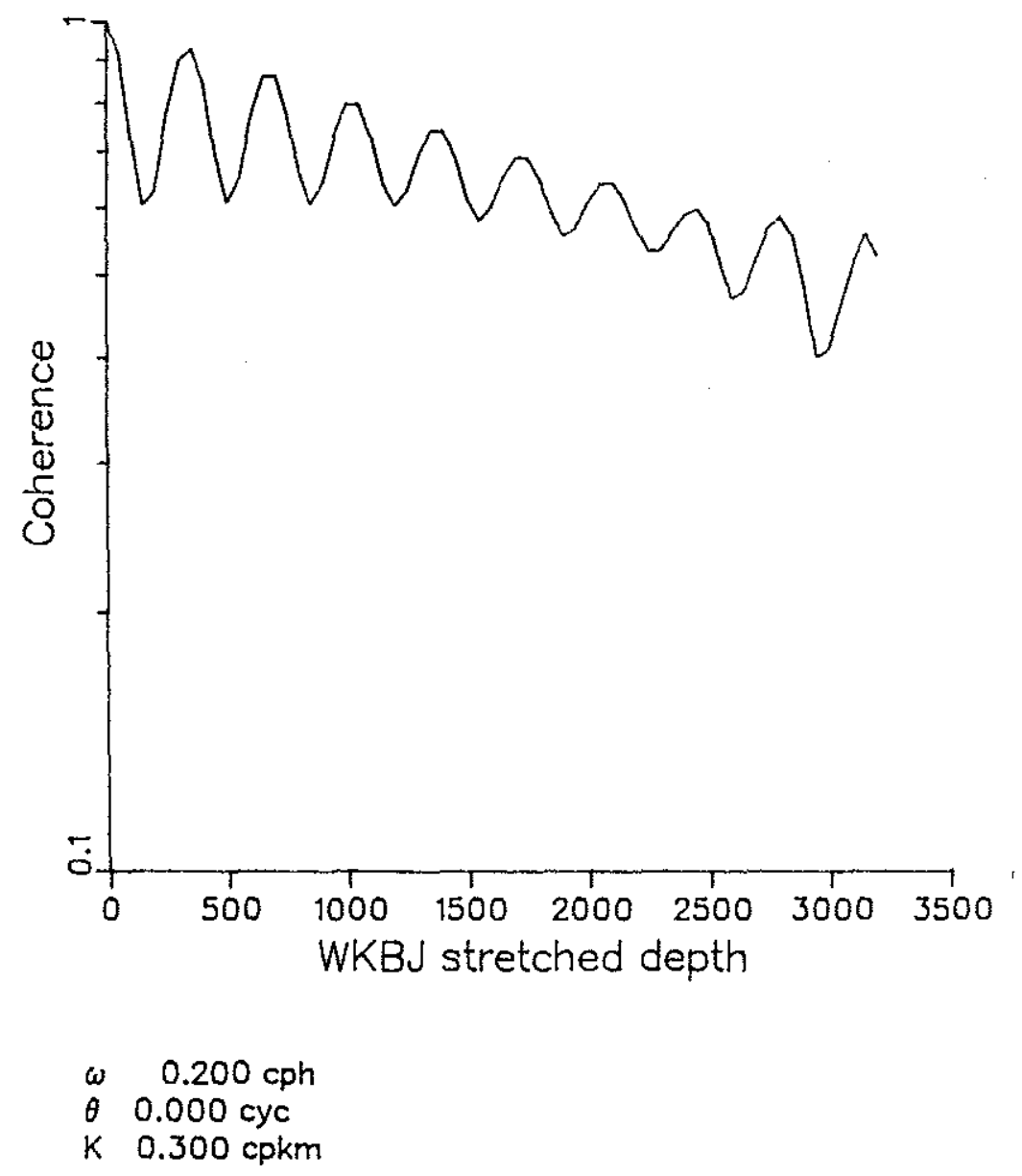

\section{Figure 7.5.7 Coherence as a function of separation}

This figure shows coherence as a function of WKBJ stretched vertical separation for a wave whose horizontal wavenumber is $.3 \mathrm{cpkm}$ (roughly mode 15 ). The buoyancy frequency for each profile was computed on a pressure grid which corresponded to an equally spaced grid of $25 \mathrm{wsm}$. Aside from the half wavelength oscillations, coherence is modeled well by a linear fit on this log-linear plot. This results in the same exponential dependence of coherence on separation that characterizes the analytic solutions. 
Coherence loss due to scattering

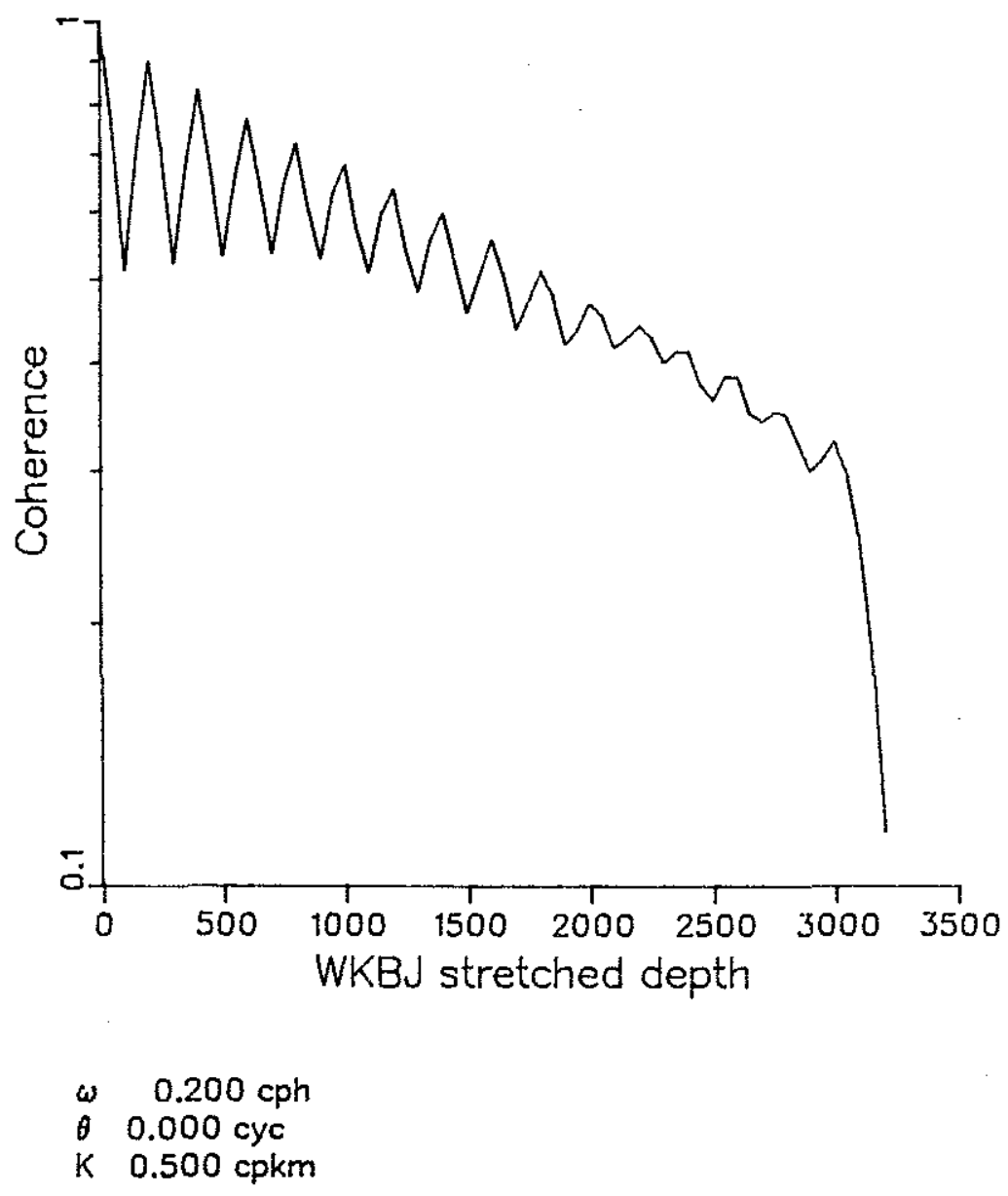

\section{Figure 7.5.8 Coherence as a function of separation}

This figure shows coherence as a function of WKBJ stretched vertical separation for a wave whose horizontal wavenumber is $.5 \mathrm{cpkm}$ (roughly mode 25 ). The buoyancy frequency for each profile was computed on a pressure grid which corresponded to an equally spaced grid of $25 \mathrm{wsm}$. Aside from the half wavelength oscillations, coherence is modeled well by a linear fit on this log-linear plot, a line which is distinctly steeper than the corresponding line on the $K=.3 \mathrm{cpkm}$ plot (figure 7.5.7). The linear fit results in the same exponential dependence of coherence on separation that characterizes the analytic solutions. 
include. But the fact that the ratio is roughly constant shows that the estimated $L_{E}$ is roughly proportional to the analytic calculations of $L_{E}$. Thus the analytic form can be used to extrapolate the numerical results to wavelengths and separations not directly measured.

While the last column of the table shows the ratio between the estimated values and the top hat estimates, another possible comparison is between the linear interpolation value of $L_{E}$ and the estimated value. Since the linear interpolation value is simply three times the top hat value, the linear interpolation value of $L_{E}$ is a factor of ten larger than the corresponding numerical value. At this point one has to make a choice. If one believes that the high wavenumber buoyancy frequency variability is due to high frequency motions, then the filtering done in the numerical simulations is appropriate and the ratio given in the table should be used to correct the analytic results. If, however, one believes that the high wavenumber variability is due to low frequency equatorial motions, then the filtering is inappropriate and some conversion of the numerical results is necessary. Since the analytic results for the 'top hat' and filtered 'top hat' spectra differ by a factor of three, it is reasonable to expect that the numerical results for the filtered and unfiltered cases would also differ by a factor of three. Thus the ratio should be considered closer to ten than to four. In either case, the calculations have shown that the $e$-folding length $L_{E}$ can be considered to have the wavenumber dependence predicted by the analytic calculation, but that the $e$-folding length $L_{E}$ has a factor of four to ten decrease in going from the analytic to the numeric results, a decrease that reduces coherence for any given separation. The important simplifying result is that the coherence can be considered to be given by the simple analytic form of the analytic theory $e^{-R / L_{E}}$ with a modified $e$-folding length $L_{E}$ that depends only on the inverse square vertical wavenumber $q_{0}^{-2}$. 


\section{Comparison of numerical and analytic results}

$\begin{array}{ccccccc}\begin{array}{c}\text { Vertical } \\ \text { Wavenumber } \\ (\mathrm{cpkm})\end{array} & \text { Mode } & \begin{array}{c}\text { Estimated } \\ \text { coherence } \\ \text { at 0 wsm }\end{array} & \begin{array}{c}\text { Estimated } \\ L_{E} \\ (\mathrm{~km})\end{array} & \begin{array}{c}\text { Top hat } \\ L_{E} \\ (\mathrm{~km})\end{array} & \begin{array}{c}\text { Linear } \\ L_{E} \\ (\mathrm{~km})\end{array} & \begin{array}{c}\text { Top hat } \\ \text { Estimate } \\ \text { ratio }\end{array} \\ .465 & 1 & 1 & & 9600 & 29000 & \\ .945 & 5 & 1 & & 490 & 1500 & \\ 1.5 & 10 & .91 & 30 & 119 & 360 & 4 \\ 2.5 & 15 & .83 & 6.3 & 47 & 140 & 7 \\ 3.0 & 25 & .80 & 3.5 & 17 & 51 & 5 \\ 3.5 & 30 & .71 & 3.9 & 12 & 35 & 3 \\ & 35 & .68 & 2.9 & 9 & 26 & 3\end{array}$

The vertical wavenumber $q_{0}$ is related to the horizontal wavenumber $K$ by $q_{0}=$ $1 \mathrm{cph} K / \omega$.

Figure 7.5.9 Numerical and analytic estimates of $L_{E}$

This table contains the numerical and two analytic estimates of the $e$-folding length $L_{E}$. The column marked 'Estimated $L_{E}$ ' is calculated from straight-line fits to curves such as 7.5.7 and 7.5.8 which consider coherence to be an exponential function of WKBJ stretched separation. The column marked 'Top hat $L_{E}$ ' is calculated using the analytic form given in equation 7.5.17, i.e. it is an analytic result assuming that the noise spectrum is white up to a cutoff of $.1 \mathrm{cpm}$. The column marked 'Linear $L_{E}$ ' is calculated using the noise spectrum after it has been filtered by a $25 \mathrm{wsm}$ linear interpolation. The resulting $L_{E}$ is simply three times the $L_{E}$ from the top hat spectrum. The final column gives the ratio between the top hat calculation and the estimated value of $L_{E}$ : it is more or less four, though there is quite a bit of scatter. 


\section{Summary of buoyancy frequency variability results}

The analysis of buoyancy frequency variability's effect on internal waves was split into three parts: characterizing the variability, an analytic calculation of the resulting scattering of internal waves, and a numerical calculation of the scattering. By using the works of Hayes and Powell 1980 and Toole and Hayes 1984 to extrapolate the PEQUOD CTD measurements, it was decided that the variability spectrum was adequately characterized by a rectangle function with amplitude of $3 \mathrm{cpm}^{-1}$ and cutoff wavenumber $\beta_{c}$ of $.1 \mathrm{cpm}$. The analytic calculation showed that the internal wave scattering resulted in a loss of coherence given by a exponential function of separation $R$, namely

$$
\gamma(R)=e^{-R / L_{E}}
$$

where the decay scale $L_{E}$ is proportional to the inverse square vertical wavenumber $q_{0}$,

$$
L_{E}=L_{0} q_{0}^{-2}
$$

The analytic calculation fixed the constant $L_{0}$ at $.1 \mathrm{~m} \mathrm{cpm}^{2}$ for the equatorial buoyancy frequency variability spectrum. The numerical calculation confirmed this functional form, though the constant $L_{0}$ is decreased by a factor between four and ten. Making the conservative choice $\left(L_{0}=.025 \mathrm{~m} \mathrm{cpm}^{2}\right)$ means that a vertical wavenumber of $1.5 \mathrm{cpkm}$ (corresponding to mode 15 ) has a decay scale of $6.3 \mathrm{~km}$, sufficient to prevent it (and all shorter waves) from showing much modal character. 


\subsection{Integrated wave solutions in an idealized mean flow}

This section and the next examine the upward solutions $\alpha(z)$ to the vertical structure equation (7.2.1) with a dispersion relation appropriate for waves in a basic state that has a mean zonal velocity shear. This section looks at solutions in a simplified mean velocity shear, while section 7.7 looks at solutions in a mean shear similar to that measured at the PEQUOD site.

The effects of a mean shear flow on internal wave can be expressed with a set of three non-dimensional parameters

$$
\begin{array}{ll}
d=U(z) K / \omega & \text { the Doppler shift, } \\
\lambda=U_{z}(z) / N(z) & \text { the inverse root Richardson number, and } \\
\mu_{z z}=\frac{\omega}{N^{2}(z)} \frac{U_{z z}(z)}{K} & \text { the scaled mean curvature. }
\end{array}
$$

In terms of the these parameters the dispersion relation $5.5 .8 f$ for a wave $(\omega, K, \theta)$ becomes

$$
m^{2}(z)=\frac{N^{2}(z) K^{2}}{\sigma^{2}}\left[1-\sigma^{2} / N^{2}+\mu_{z z}(\sigma / \omega)\right]
$$

where the intrinsic frequency $\sigma=\omega(1-d \cos \theta)$. Note that the dispersion relation 7.6.1 only depends on two of the mean velocity parameters: the Doppler shift $d(z)$ and the scaled curvature $\mu_{z z}(z)$. The inverse root Richardson number $\lambda(z)$ along with the Doppler shift determine the changes in the polarization relations (given originally in equation 5.5.9 and scaled in equation 8.2.8). So while $\lambda$ does not affect the dispersion relation, it does affect the prediction for measured (current-meter) cross-spectra.

The form of the vertical structure equation (7.2.1) is the same whether or not the basic state contains a mean shear flow, consequently it seems reasonable to expect the solutions in a mean flow to have much the same character as the solutions in a resting basic state as long as $m^{2}(z)$ has roughly the same character. But the presence of a mean velocity profile introduces some important dynamical changes. Because the intrinsic frequency $\sigma$ depends on vertical position through its dependence on the mean velocity, there is the possibility that, for sufficiently strong mean flows and/or slow waves, the intrinsic frequency may go to zero at certain depths. This causes the local vertical wavenumber to go to infinity, creating a singularity in the vertical structure equation. Such waves are called critical and the depth range near the depth where the intrinsic frequency approaches zero is called a critical layer.

In addition to critical layers, there now exists the possibility that $\left|\mu_{z z}\right|$ is larger than 
one, making the squared local vertical wavenumber negative for waves propagating in certain directions. This means that there will be parts of the water column where the solutions are exponentially growing/decaying (evanescent regions). While this was also a possibility in the no mean flow system (wherever the buoyancy frequency $N(z)$ became less than the frequency $\omega$ ), in the system with non-zero mean velocity curvature it can happen for all frequencies. This is because $\mu_{z z}$ is inversely proportional to the horizontal wavenumber $K$ so that, as the wavenumber $K$ goes to zero some angles of propagation have negative $m^{2}(z)$.

Consider waves in a basic state where the mean velocity is zero except for a small eastward jet at mid-depths as depicted in figure 7.6.1. This velocity jet was arbitrarily chosen to be gaussian, i.e. it is of the form

$$
U(z)=U_{m} e^{-\frac{1}{2}\left(\frac{z-z_{c}}{z_{w}}\right)^{2}}
$$

Here the maximum velocity $U_{m}$ is $10 \mathrm{~cm} / \mathrm{s}$, the width $z_{w}$ is $100 \mathrm{~m}$, and the center depth $z_{c}$ is $1000 \mathrm{~m}$. This parameters are chosen to give a mean profile that has properties of the same size as the measured profiles (which will be presented shortly). The buoyancy frequency $N$ is chosen to be a constant $1 \mathrm{cph}$, so that all the variations in the local vertical wavenumber $m(z)$ are due to the mean velocity profile.

As a rough measure of the effects of a mean shear flow on internal waves, the three non-dimensional shear parameters for the gaussian velocity profile are plotted in figure 7.6.2. The center plot presents the inverse root Richardson number $\lambda$. It shows a maximum of roughly $40 \%$, showing that the polarization relations will be significantly affected in the vicinity of the current jet. It is important to remember that changes in the polarization relations are purely local: in computing the coherence between an instrument in still water above the current jet with an instrument in still water below the jet, for example, the shear effects in the polarization relations would be zero since both instruments are in still water. This is in contradistinction to the dispersion relation changes, which can affect the coherence between the two instruments.

The right plot in figure 7.6.2 gives the scaled mean velocity curvature $\mu_{z z}$. The curvature is potentially important when $\mu_{z z}$ is greater than one so that $m^{2}(z)$ becomes negative for some choice of propagation direction. Plotted are $\mu_{z z}$ for wavenumbers than roughly correspond to modes 5,15 and 25 . The plot shows that for this simple profile mode 5 has a region of negative squared local vertical wavenumber $m^{2}(z)$ : a 


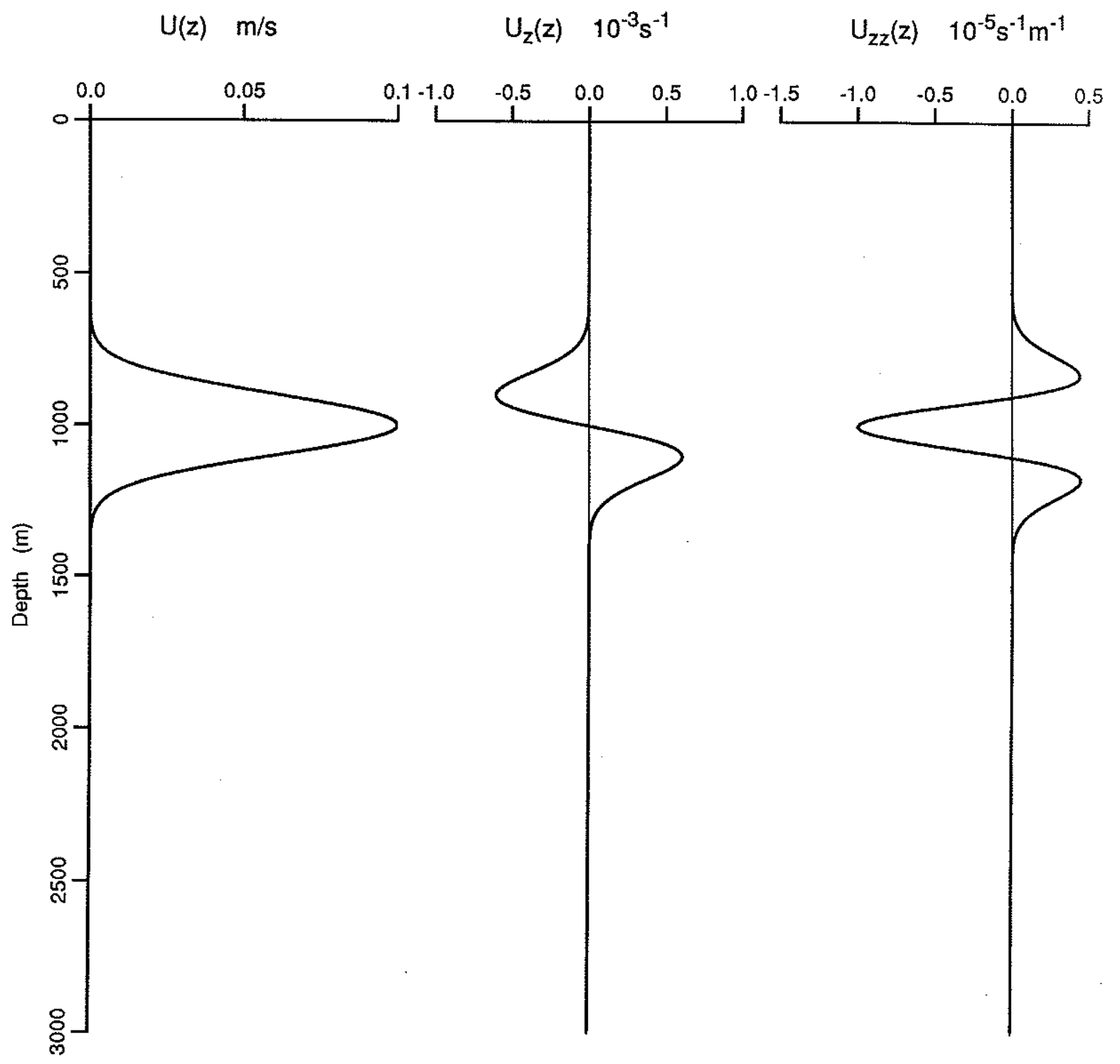

Figure 7.6.1 Gaussian velocity jet

These plots show the mean zonal velocity and its first and second vertical derivatives for the gaussian profile studied in this section. 


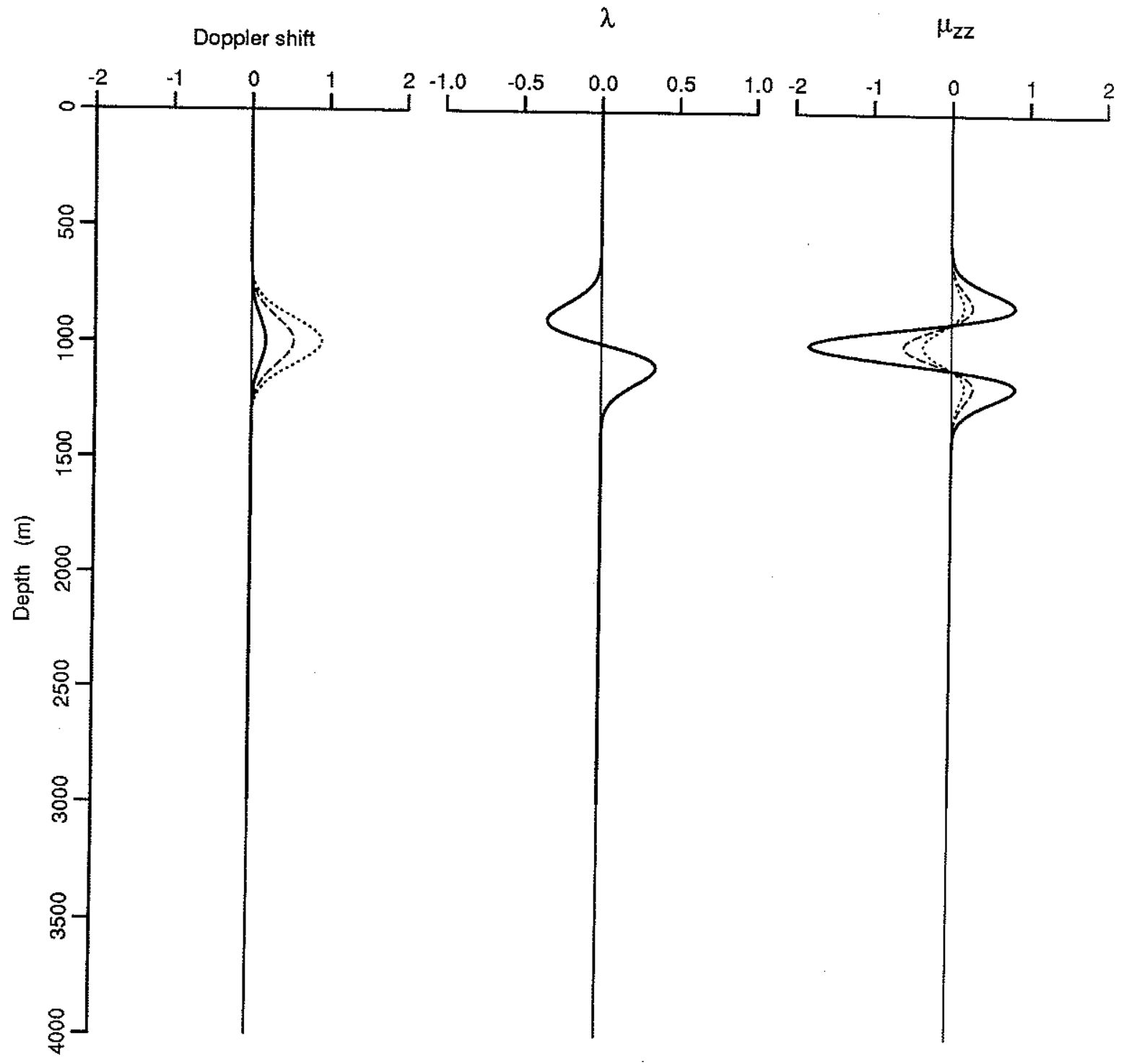

Figure 7.6.2 Normalized zonal velocity at $\omega=.2 \mathrm{cph}$.

These plots are normalized velocities in the sense that the profiles in figure 7.6.1 have been scaled to show their effect on internal waves. The left plot gives the Doppler shift for wavenumbers that correspond to modes 5 (solid line), 15 (dashed line), and 25 (dotted line). The right plot gives the normalized mean velocity curvature $\mu_{z z}$ for the same three wavenumbers. The center plot gives the inverse root Richardson number $\lambda$. 
more extensive study would show that modes with number 10 or less all have such a region.

The left-hand plot gives the Doppler shift for modes 5, 15 and 25. The plot shows that while mode 5 is not significantly affected by Doppler shift even at the peak of the jet, mode 25 has a Doppler shift close to 1 . This means that the intrinsic frequency is close to zero for waves propagating eastward, and a critical layer will exist for these waves at $1000 \mathrm{~m}$ depth. Essentially this critical layer represents a region where the linear inviscid statistically stationary dynamics of the model are inadequate. Several of the approximations made in deriving the equations (section 5.5) are invalid as well. Thus the critical layer is a region where the structure of the internal wave solutions no longer provides a reasonable framework for interpreting cross-spectra. As long as an instrument is not actually at a critical level, the details of what occurs inside the critical layer is unimportant for interpreting spectra. What is important is the structure of the wave on both sides of the critical layer, and whether or not motions on opposite sides of the layer are coherent. Booker and Bretherton 1967 find solutions to the problem of propagation through such a critical layer by using an initial value problem to avoid the singularity. They find solutions that exponentially decay within the critical layer, so that as long as the mean flow is stable (Richardson number much greater than .25), the wave cannot propagate past the depth at which the critical layer occurs - they are effectively absorbed. The initial value problem is mathematically equivalent to postulating a friction where the frictional force is proportional to the dynamical variable itself (Newtonian cooling for the all the dynamical variables). This friction leaves the dynamical equations (5.5.6) unchanged, except that the intrinsic frequency $\sigma$ now has a frictional component,

$$
\sigma=\omega-U(z) k-i r
$$

where $r$ is the coefficient of friction so that the frictional force in the $i^{t h}$ momentum (or buoyancy equation) is given by $r u_{i}(\mathbf{u}=(u, v, w, b))$. Because the presence of friction adds a purely imaginary component, the equations are no longer singular even when the real part of the intrinsic frequency vanishes, and one can actually envision propagating waves through such a region. But the friction acting on the rapidly varying solution quickly damps it out, and waves cannot propagate through a layer of finite thickness. Furthermore, in the presence of rotation (Jones 1968) or rotation with mean flow vorticity 
(Kunze 1985), the double singularity of equation 7.6.1 separates into two singularities,

$$
m^{2}(z)=\frac{N^{2}(z) K^{2}}{\omega^{2}-f_{\mathrm{eff}}^{2}}
$$

where $f_{\text {eff }}$ is given by Kunze as being the planetary $f$ plus a contribution from the mean flow. Unlike equation 7.6.1, where $m^{2}(z)$ is positive on both sides of the critical point (where $\sigma$ vanishes), here there are two separate critical points $\left(\sigma= \pm f_{\text {eff }}\right.$ ), and $m^{2}(z)$ is strongly negative between them. This would be a region of strong exponential growth/decay, suggesting that the solutions could be even more effectively stopped than the non-rotating case.

Away from the critical layers, the dynamics remain essentially frictionless to the extent the friction parameter makes an insignificant contribution to the intrinsic frequency equation 7.6.3.

Three solutions to the vertical structure equation for a fairly low wavenumber (mode 5) are presented in figure 7.6.3. The solid line shows the solution for a wave oriented perpendicular to the mean flow, while the dotted and dashed lines correspond to waves propagating along and against the mean flow. Note that the wave propagating perpendicular to the mean flow is completely unaffected by it: the solution is identical to that found in the no mean flow case. So comparing the solid line to the dotted and dashed gives some measure of how important the inclusion of the mean flow is to the dynamics.

The left-hand plot gives the local vertical wavenumber, i.e. the square root of the absolute value of equation 7.6.1 times the sign, so that negative $M=m(z)$ signifies a region of exponential growth/decay rather than a region where propagation takes place. The center and right-hand plots give the amplitude and phase of the upward propagating solutions (the reference depth is $3000 \mathrm{~m}$ ). The wave propagating along the mean flow (dotted line) has a roughly hundred meter region where $m(z)$ is negative, but the effect on the solution is fairly small, especially as far as phase in concerned. The wave propagating westward (dashed line) also nearly identical to the meridionally propagating waves. Note that $m(z)$ is not symmetrically altered for westward and eastward waves: this is due to Doppler shifting in the intrinsic frequency. Comparing all three curves suggests that the effects of curvature is not particularly important, since only waves with low wavenumber are affected and they are relatively immune to short variations in the vertical profile because of their long wavelength. 


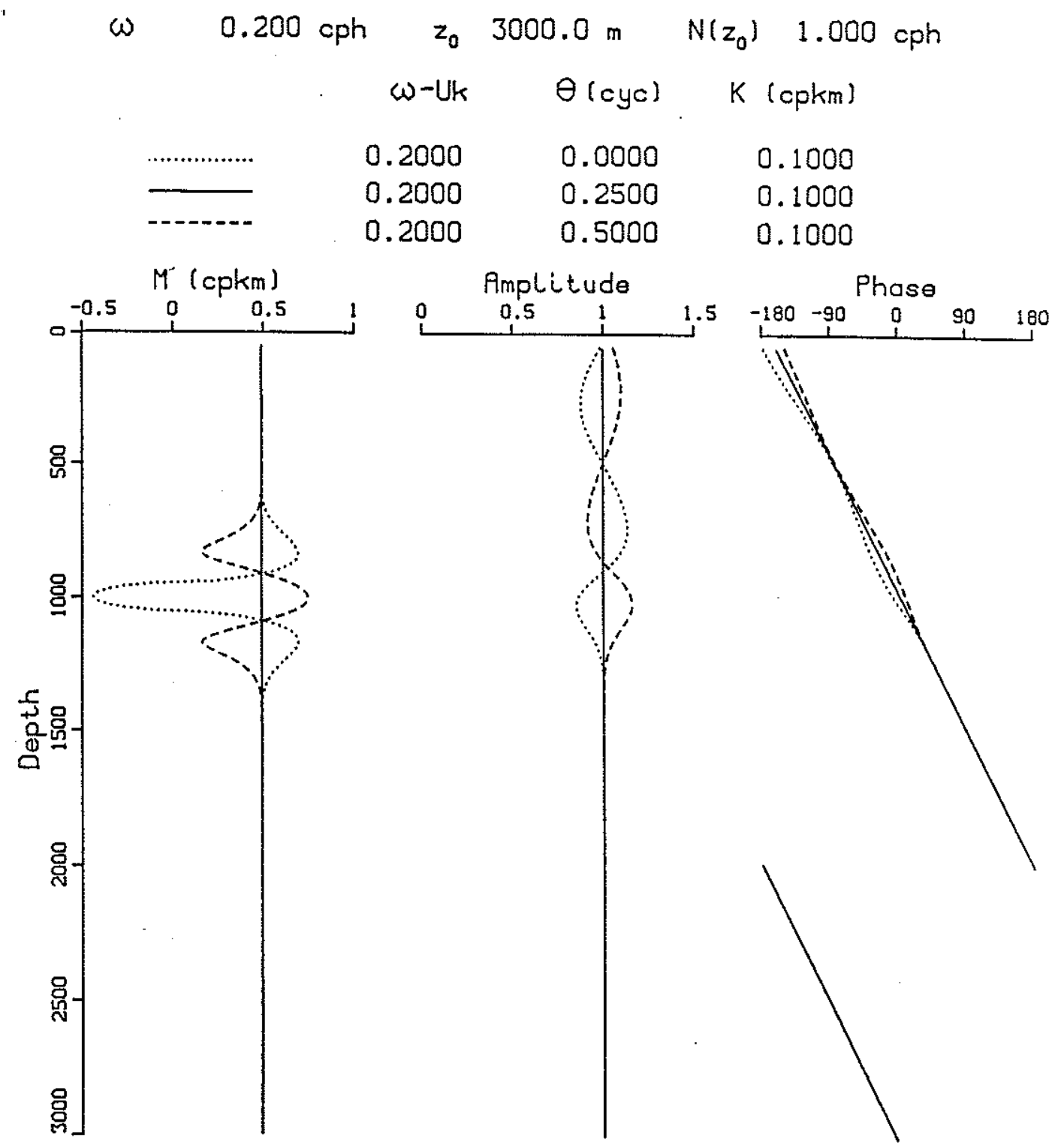

Figure 7.6.3 Low wavenumber solutions in an idealized mean flow.

The dotted lines are the solutions $\alpha$ for the wave propagating eastward (along the mean eastward flow), the solid lines are for a wave propagating meridionally, and the dashed lines are for a westward wave. 
Figure 7.6.4 shows wave solutions for waves with somewhat higher wavenumber. Here different directions show distinctly different behaviors. The meridional wave (solid line) is a simple complex exponential, the solution to a constant $N$ profile with no contribution from the mean flow. The westward wave (dashed line) shows the phase locking expected from a pair of bumps in $m(z)$ : the solution looks much like the step profile solution after encountering the first bump, then shifts so that the amplitude is centered on that of the meridional wave after encountering the second bump and the phase is shifted by roughly $30^{\circ}$. The eastward wave shows distinctly different behavior. The amplitude deviates from one only in the vicinity of the jet - the downward wave has not been excited. The phase shift is distinctly greater, being roughly $90^{\circ}$. The wavenumber is sufficiently high that the curvature effect for the eastward wave has been effectively eliminated: the double lumps that are in both the curvature profile (figure 7.6.1) and the $m(z)$ profile for the westward wave have disappeared.

The final figure of the section (figure 7.6.5) shows a critical layer start to form. The local vertical wavenumber for the eastward wave now gets quite large, and there are several complete cycles in the span of a few hundred meters. The amplitude for the eastward wave is essentially one once the velocity jet is passed, making the solution identical to that obtained from a WKBJ approximation. This is as expected, since the large local vertical wavenumber in the critical layer satisfies the validity requirements for WKBJ (equation 7.4.3).

In summary, there are two major changes to the local vertical wavenumber $m^{2}(z)$ due to the presence of mean flow. The existence of curvature in the local vertical wavenumber means that at all frequencies there will be horizontal wavevector choices which result in regions of negative $m^{2}(z)$. But the waves that are affected are all quite long, and the effect of a small region of negative $m^{2}(z)$ is unimportant. On the other hand, the presence of a mean flow introduces the possibility of critical layers, layers which radically alter the propagation and structure of high wavenumber waves. Whether these altered waves are important or not in terms of interpreting the data very much depends on the mean velocity profile (which will determine which waves are critical), and on the wavefield itself, which may or may not have significant energy at those high wavenumbers. 


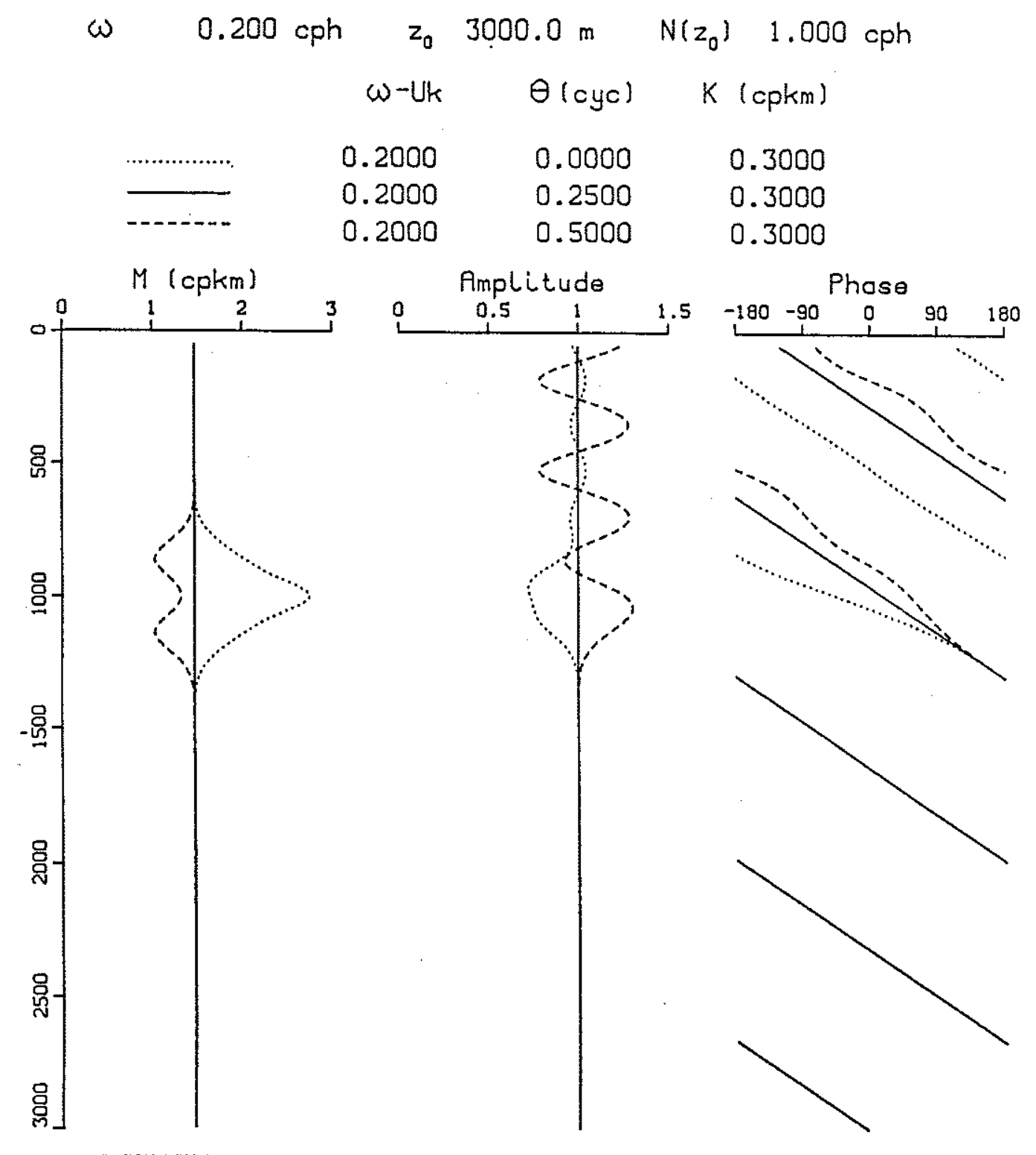

Figure 7.6.4 Mode 15 wavenumber solutions in an idealized mean flow.

The dotted lines are the solution $\alpha(z)$ for the wave propagating eastward (along the mean eastward flow), the solid lines are for a wave propagating meridionally, and the dashed lines are for a westward wave. 


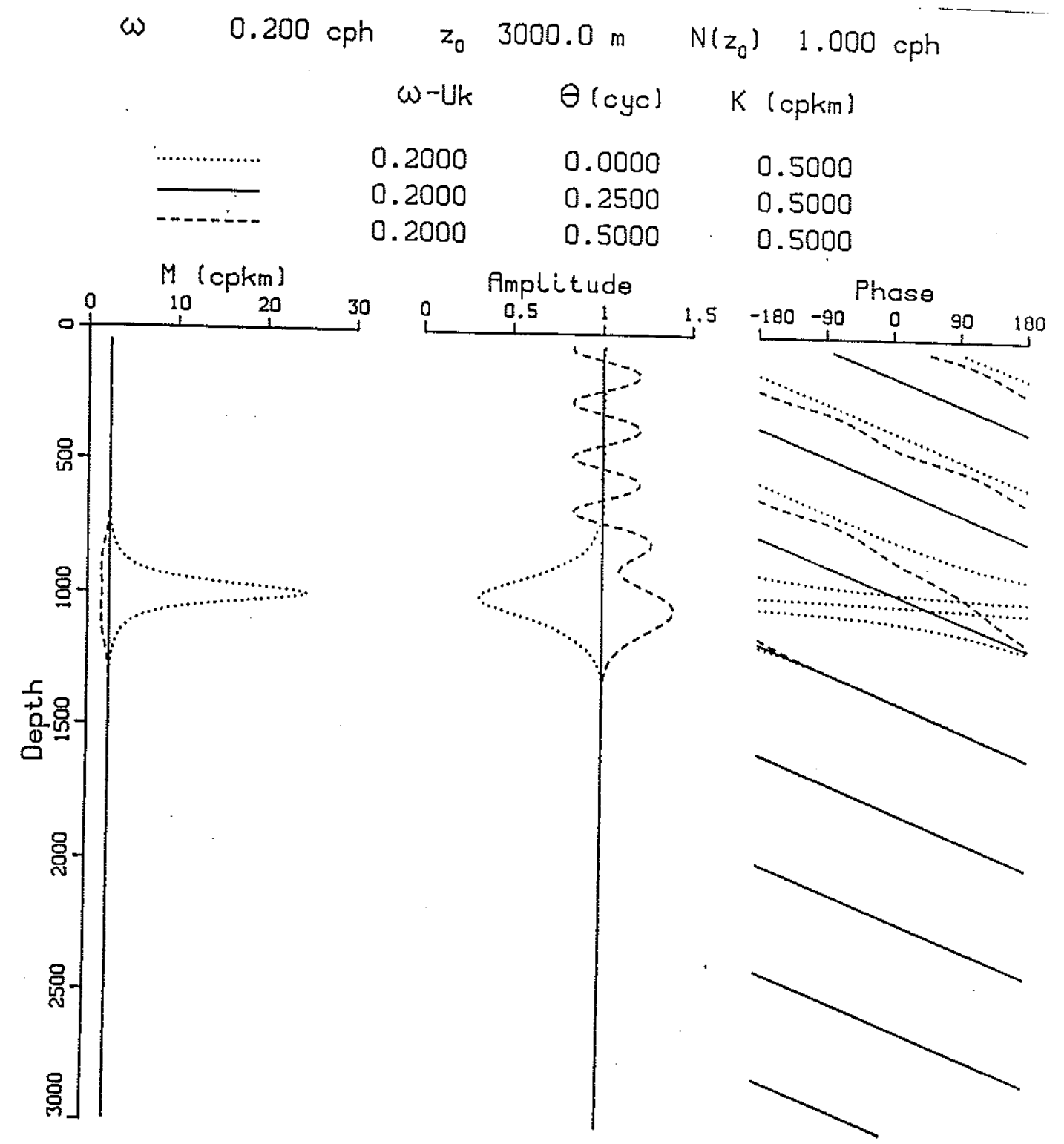

Figure 7.6.5 Nearly critical wavenumber solutions in an idealized mean flow.

The dotted lines are the solutions $\alpha$ for the wave propagating eastward (along the mean eastward flow), the solid lines are for a wave propagating meridionally, and the dashed lines are for a westward wave. 


\subsection{Integrated wave solutions in a measured mean flow}

Section 7.6 looks at the character of the effects of a mean shear flow on internal wave structures by considering waves in a single zonal velocity jet in a constant buoyancy frequency ocean. This section looks at the more complicated situation where measured velocity and buoyancy frequency profiles are used as the basic state.

The buoyancy frequency profiles used are identical to those used in the no mean flow analysis (section 7.4), and are discussed in section 2.1. The velocity profiles are taken from the White Horse casts as well. A glance at figure 2.1.5 shows the large variability in the velocity profiles, a variability which makes it quite difficult to determine mean profiles of velocity, velocity gradient, and velocity curvature. To some extent this variability is due to high frequency motions and thus truly is noise that the estimate of a mean velocity must filter out. Some of the variability, however, could be due to long-term time variability and spatial variations: that latter sort of variability, to the extent that the mean velocity significantly affects the waves, causes enhanced variability in the modes, amplifying the sort of noise seen in sections 7.4 and 7.5. Despite recognizing that this mechanism may be a source of misfit between measured cross-spectra and any fit to a set of internal waves, the mean profile still determines the basic internal wave field. A best fit spline with adjustable knots is used to get reasonable estimates of velocity, slope and curvature (see deBoor and Rice 1968). The knot spacing determines the amount of vertical smoothing that is done. Figure 7.7.1 compares two such spline fits with the data that is being fit. The dotted curve is the average profile computed from the Whitehorse drops. The solid curve is a spline fit that uses many (21) knots in an attempt to include all the structure in the profile. It is indistinguishable from the average profile everywhere except a small region near $1400 \mathrm{~m}$ depth. By choosing more knots (or different knot placements), even that discrepancy could have been eliminated. On the other hand, there is the dashed line, which shows a fit using many fewer knots. It misses most of the structure in the profile, responding to rapid variations with a gentle curve.

Choosing between such profiles requires knowing whether the variability is due to time dependent motions captured in the instantaneous profiles, or due to some significant long term or spatial variations. The rest of this section will focus on the solid curve which reproduces almost all of the structure of the averaged profile. This is because in some ways this is a worst case: if the mean flows are going to affect the internal wave field at all, they will do so when all the structure is included. This does not guarantee 


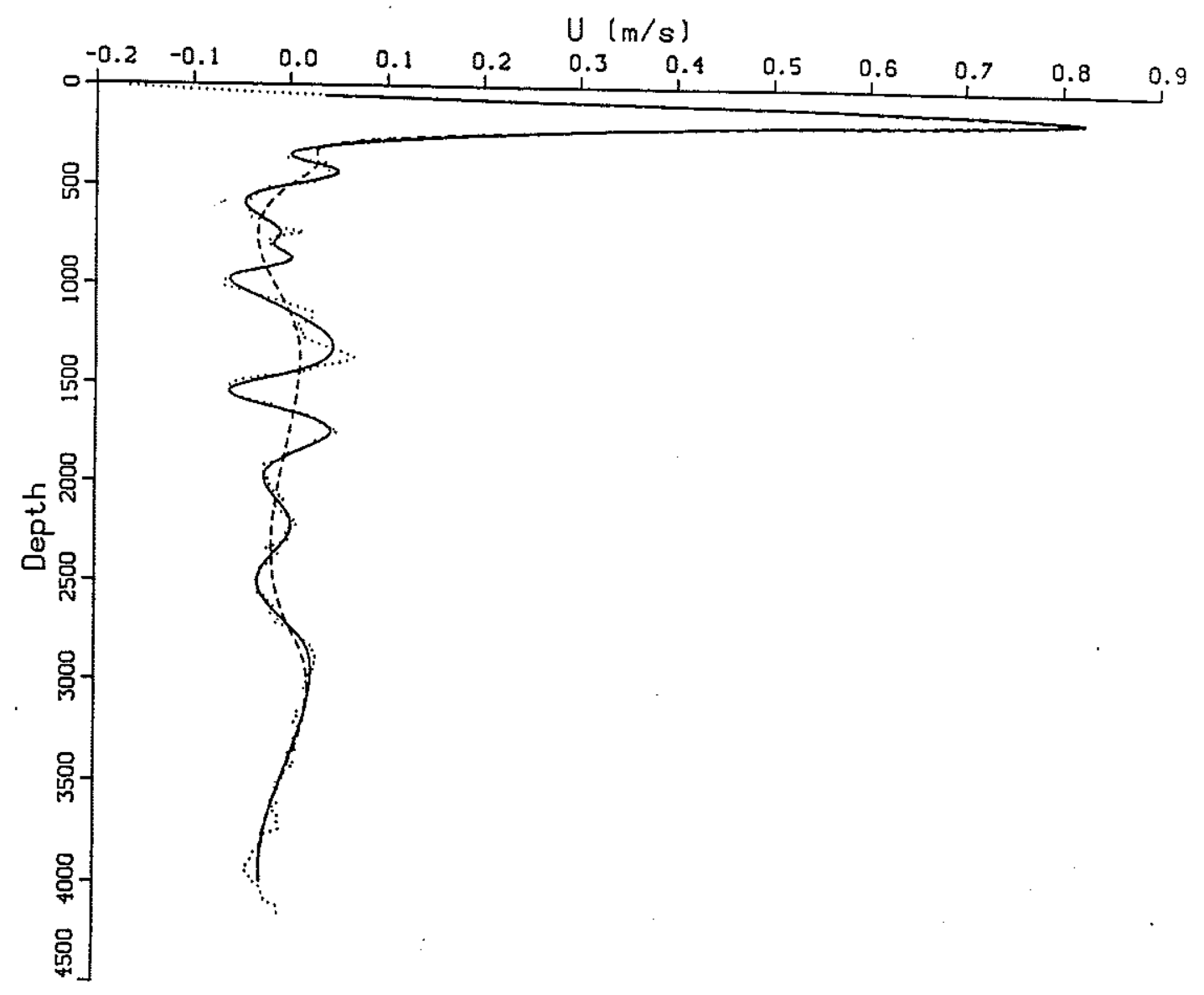

\section{Figure 7.7.1 Mean velocity spline fit comparison}

This is a plot of two spline fits and the averaged profile that they fit. The dotted curve is the averaged profile, the solid curve is a spline fit with relatively high vertical resolution, while the dashed curve is a spline fit with more vertical averaging. 
that the spectral fit that is done in chapter 8 will be improved by using waves with more structure. That result can only be determined by actually doing the fit, and if the fit is better, supports the choice of basic state.

Figure 7.7.2 shows the smoothed velocity profile corresponding to the solid curve of figure 7.7.1, along with its first and second derivatives. The depth range is confined to be between 500 and 3500 meters: this is the range that has instruments in the PEQUOD experiment, and confining the analysis to this range avoids the complications that occur in the strong current near the surface. The velocity magnitudes are fairly small, $5 \mathrm{~cm} / \mathrm{s}$ in either direction. The piecewise linear character of the curvature is an artifact: since the velocity is being fit with a piecewise cubic spline, its second derivative is being fit with a series of lines. But this is almost inevitable: the curvature is difficult to measure, and its calculated values will always reflect the vertical averaging required to get significant results.

Figure 7.7.3 shows the non-dimensional shear parameters calculated from the mean velocity and its derivatives for this measured profile, just as figure 7.6 .2 did for the idealized velocity profile of the last section. The inverse root Richardson number $\lambda$ is given in the center plot. While $\lambda$ is close to one above and below the Equatorial Undercurrent, below $500 \mathrm{~m} \lambda$ rarely gets as high as .5 . Since $\lambda$ only affects the polarization relations and is thus a local effect as far as interpreting cross-spectra is concerned, the values near the three groups of instruments $(600 \mathrm{~m}, 1600 \mathrm{~m}, 3100 \mathrm{~m}$ ) are the values of interest. The plot suggests that the instruments in the center group are the most strongly affected by Richardson number effects.

The scaled curvature $\mu_{z z}$ is given in the right-hand plot. The plot shows that curvature effects are fairly strong for mode 5 (solid curve) and mode 15 (dashed curve) has regions where $\mu$ is large though but less than one. Thus modes 10 and less certainly have regions of exponential growth/decay for certain wave propagation directions. But because these regions occur for only the longest (low mode) waves, small regions of exponential growth/decay could have little effect. Thus the actual solutions must be found before it can be determined that these regions actually have an important effect on the structure of the waves.

The left-hand plot in figure 7.7.3 gives the Doppler shift for modes 5, 15 and 25 . The plots show that even mode 5 is critical in the Undercurrent, but below $500 \mathrm{~m}$ none of the modes plotted are particularly effected. Away from the Undercurrent, then, criticality 


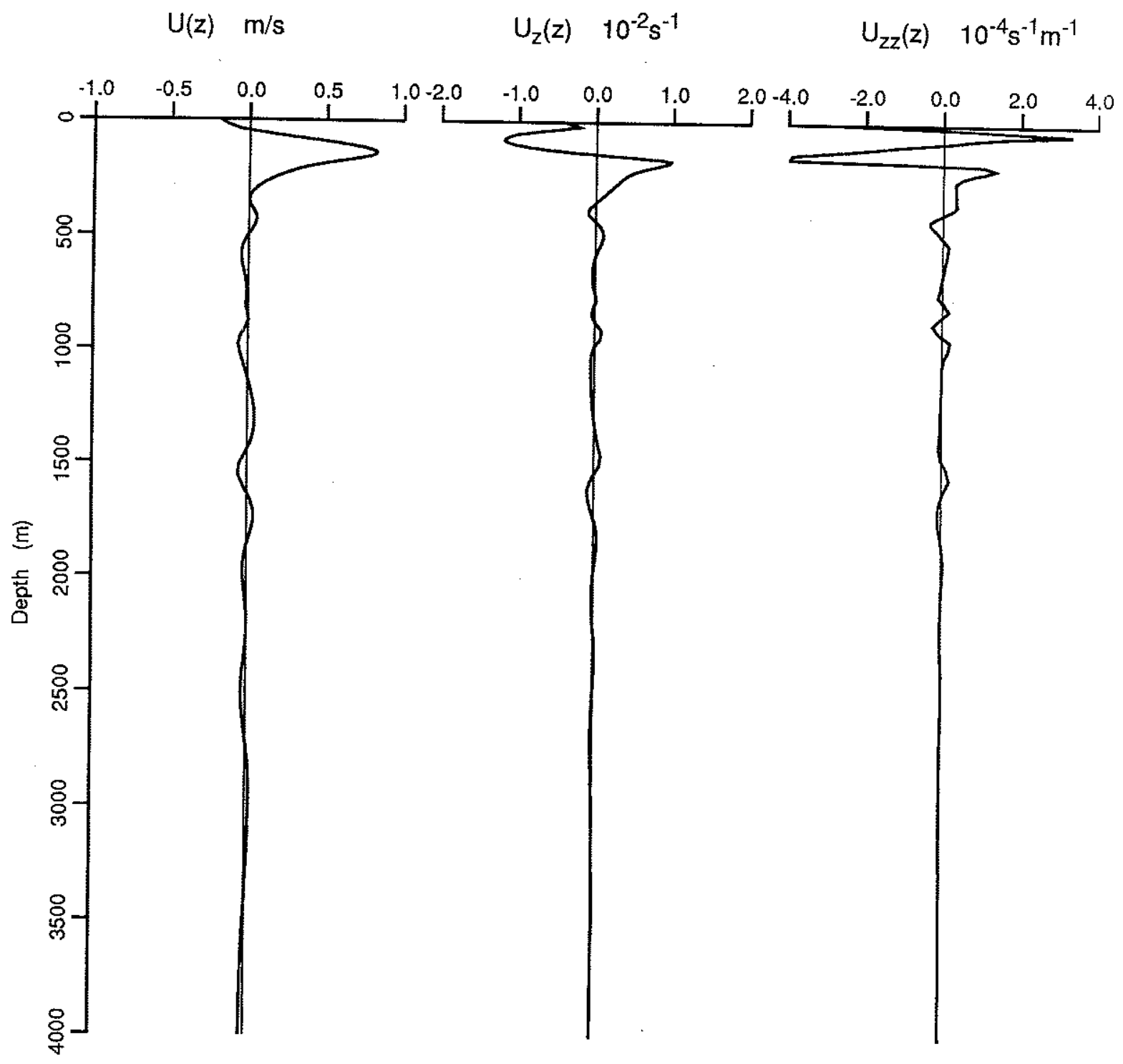

Figure 7.7.2 Mean velocity, slope and curvature from Whitehorse data

This are plots of the mean velocity and its first two vertical derivatives used in the wave calculations for the remainder of this section. The profile is a spline fit to an averaged White Horse velocity profile: it is the same as the solid curve in figure 7.7.1. 


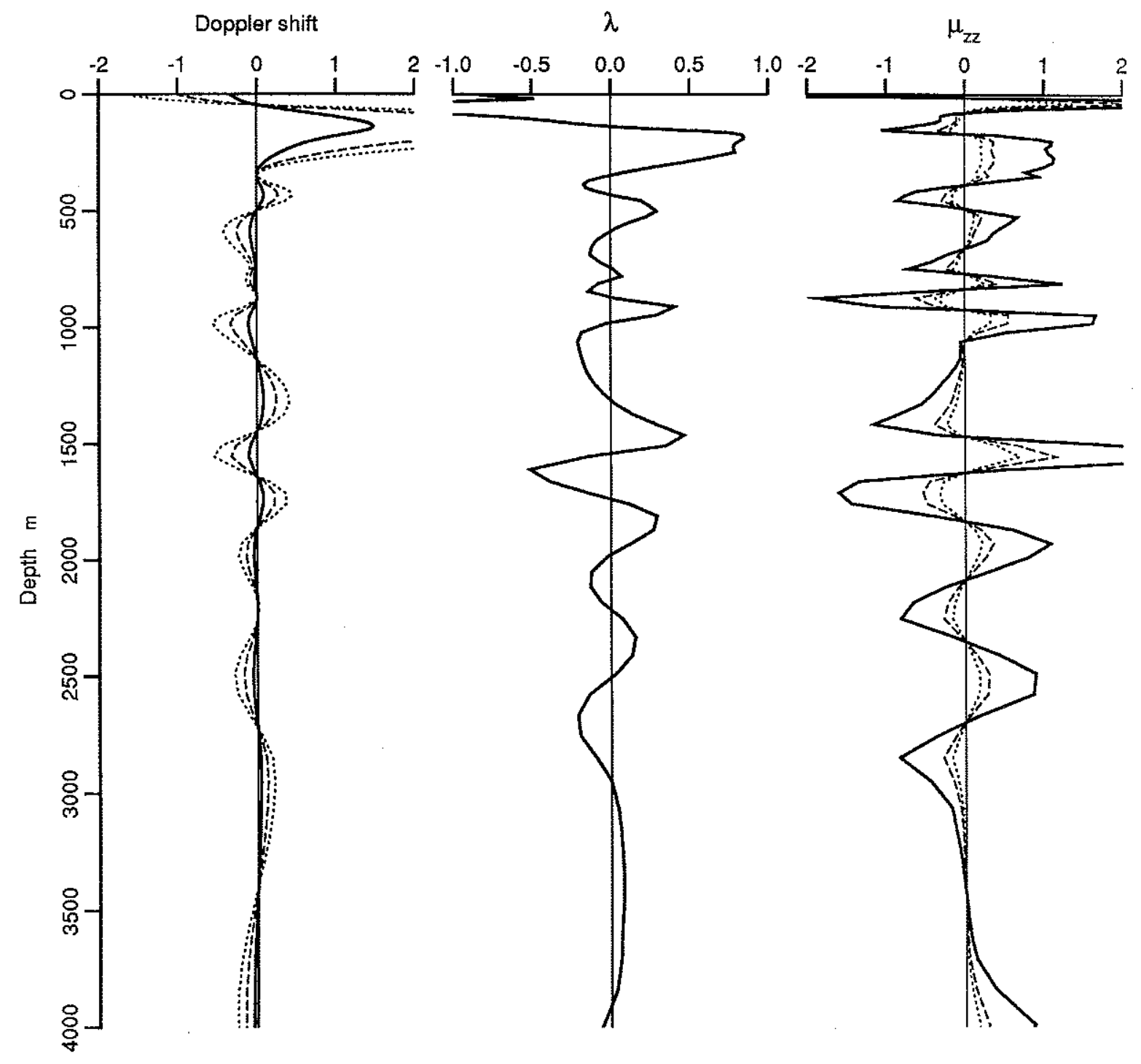

\section{Figure 7.7.3 Normalized measured zonal velocity}

These plots are normalized velocities in the sense that the profiles in figure 7.7.1 have been scaled to show their effect on internal waves. The left plot gives the Doppler shift for wavenumbers that correspond to modes 5 (solid line), 15 (dashed line), and 25 (dotted line). The right plot gives the normalized mean velocity curvature $\mu_{z z}$ for the same three wavenumbers. The center plot gives the inverse root Richardson number $\lambda$. To the extent that the low modes have most of the energy, the last two effect dominate measurable spectra. 
only involves quite high mode numbers. The fact that even mode 5 is critical in the Undercurrent suggests that only modes 1 through 3 can possibly show the phase-locking characteristic of modes: the strong velocity shear in the Undercurrent strongly affects higher modes.

Over the reduced depth range that spans the instruments $(500 \mathrm{~m}-3100 \mathrm{~m})$, vertical variations in the local vertical wavenumber profiles are primarily due to variations in the velocity profile. Figure 7.7.4 shows the local vertical wavenumber for a eastward wave whose wavelength is quite long (roughly mode 5). The buoyancy frequency is quite smooth, showing no major features and a gradual decrease from roughly $2 \mathrm{cph}$ at $500 \mathrm{~m}$ to $\frac{1}{2} \mathrm{cph}$ at $3000 \mathrm{~m}$. Some small scale variations in $N$ near the top appear in the local vertical wavenumber as well, but most of the small scale features of the local vertical wavenumber are due to the presence of a mean flow.

The wave solutions confirm some of the conclusions about the effects of Doppler shift and normalized velocity curvature $\mu$. Like the low wavenumber waves in the previous section, low wavenumber waves in these measured profiles show little dependence on the small scale variations in the basic state. Figure 7.7.5 shows eastward, northward, and westward waves with a horizontal wavelength of $10 \mathrm{~km}$ (roughly mode 5). The solid line represents the northward wave: for it there are no mean zonal velocity effects, so that the solid line can also be considered the reference point where the mean flow is eliminated entirely. The dotted line is the eastward propagating solution, while the dashed line is the westward propagating solution. In both cases, despite large changes in local vertical wavenumber the modes are quite smooth and similar to one another: the modes are quite independent of mean zonal shear effects as as far as the Doppler shift and the curvature are concerned. Thus the only effect of the shear flow on the low modes is though the polarization relations, and that is dominated by the local mean shear $(\lambda)$ effects. Since the low modes contain most of the energy, local mean shear effects will be most of the shear modifications in the internal wave structures.

Figure 7.7.6 shows solutions at horizontal wavenumber $K=.5 \mathrm{cpkm}$, and the character of the three waves is basically the same, even though the waves are by no means identical. There are in fact distinct phase differences, as well as quite a bit of amplitude wiggle. But this is the wavenumber range which section 5.4 shows to have large amplitude variations in the waves caused by variations in the local vertical wavenumber profile. The variations in amplitude and phase due to the presence of a mean zonal 

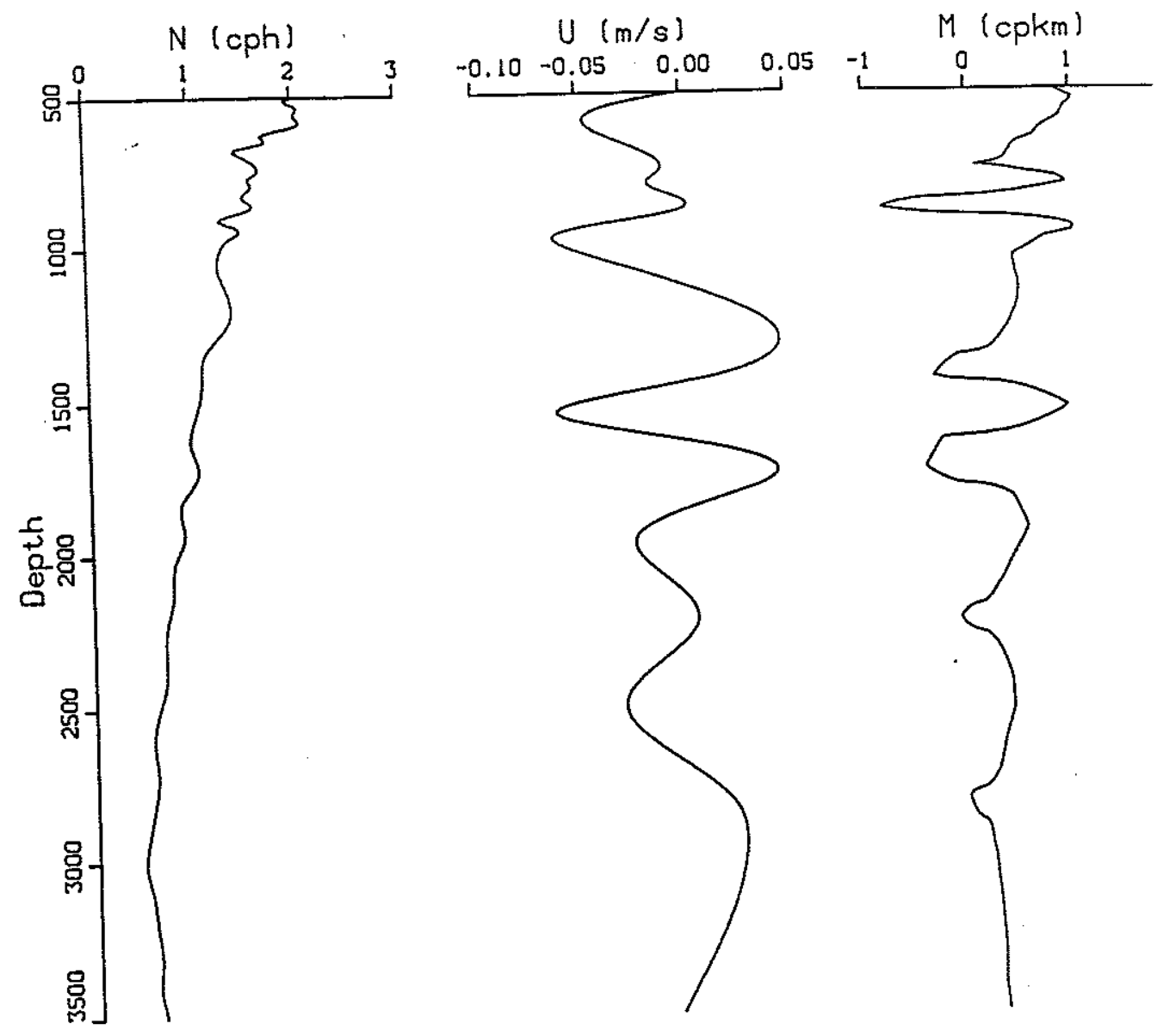

Figure 7.7.4 Local vertical wavenumber for mode 5

This plot provides an example of the local vertical wavenumber $m(z)$ can be affected by variations in the mean velocity profile. The wave is comparable to the eastward wave plotted in figure 7.7.5. 


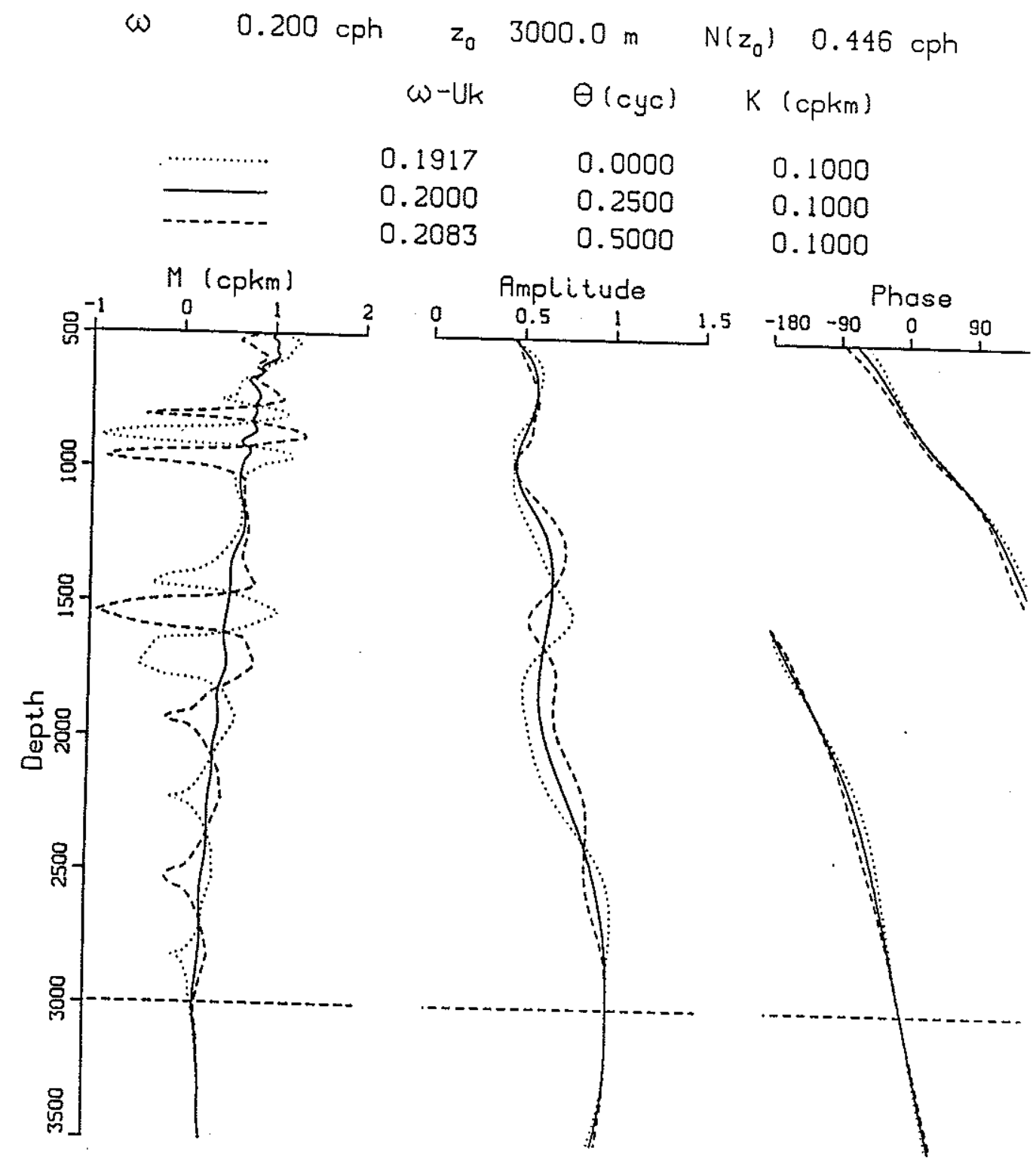

Figure 7.7.5 Low wavenumber solutions in a measured mean flow

The dotted lines correspond to the eastward wave, the dashed lines correspond to the westward wave, and the solid lines gives the solution for the northward wave (which is also the solution in the absence of zonal mean flow). The closeness of the three solutions suggests that local vertical wavenumber changes due to the presence of a mean flow are not very important for low modes. That means that only the local mean shear modifications of the polarization relations will be very important. 


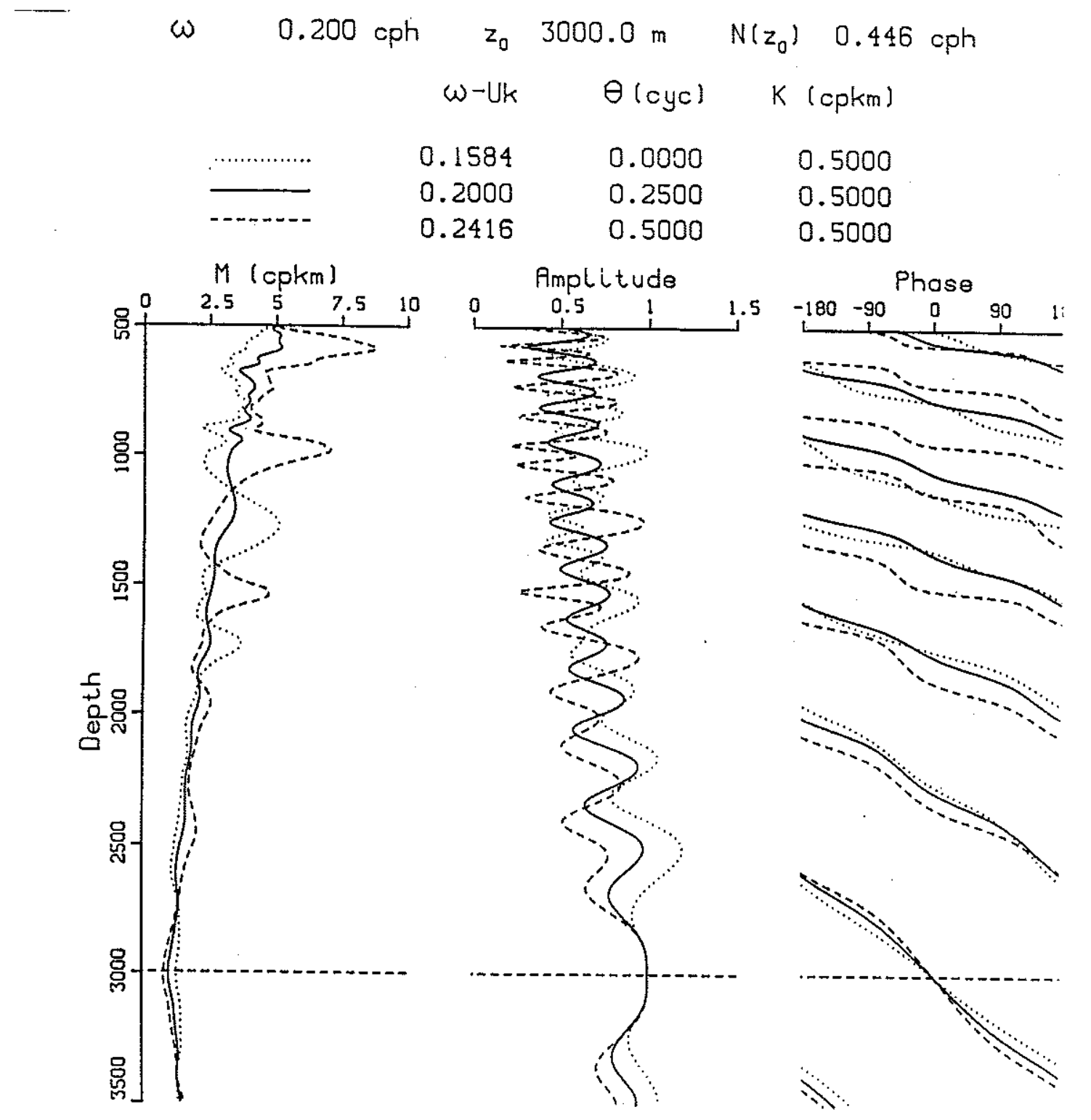

Figure 7.7.6 Mode 25 wavenumber solutions in a measured mean flow

The dotted lines correspond to the eastward wave, the dashed lines correspond to the westward wave, and the solid lines gives the solution for the northward wave (which is also the solution in the absence of zonal mean flow). The variations due to the presence of a mean shear flow seen in this plot are much smaller than the variations due to internal wave scattering seen in section 7.5 , thus the mean flow effects in the dispersion relation are not directly detectable in the current meter spectra. 
velocity shear flow are much small than the variations due to changes in the buoyancy frequency profile seen in section 7.5 , so that even if the changes due to the flow were present in the current meter spectra, they would be undetectable in the presence of the buoyancy frequency variability. Thus mean shear effects are not important for these higher modes as far as the measurements are concerned.

In conclusion, then, the low wavenumber waves are quite robust, showing little dependence on velocity or buoyancy frequency. The fact that mean curvature is such that the squared local vertical wavenumber is negative in many places for these waves turns out to not be particularly important, so that the only important effect of the mean shear flow is the local velocity shear in the polarization relations. Somewhat higher wavenumbers show more velocity profile effects, differentiating between waves propagating in different directions. Once fairly high wavenumbers are reached $(K \approx .5 \mathrm{cpkm})$, the waves are distinctly different, but variability in the buoyancy frequency profiles causes internal wave scattering that is much larger than the mean velocity shear effects. Thus as far as the fit of shear modes in chapter 8 is concerned, most of the effect of the presence of a zonal mean shear flow is through the polarization relations, effects that are fairly independent of mode number and thus affect even the energetic low modes.

\subsection{Summary}

This chapter determines the physical effects of the vertical structure equations that need to be included in the spectral inversion of chapter 8. By looking at solutions to the vertical structure equation in both a resting basic state and a basic state that includes a mean flow, the chapter is able to determine which features of the basic states significantly affect the internal wave field.

Section 7.4 looked at solutions in a resting basic state. It found that while low wavenumber solutions are quite robust, high wavenumber solutions are quite sensitive to the high wavenumber content of the buoyancy frequency profile. Because of this sensitivity, section 7.5 looked at the expected variability in the buoyancy frequency profile and its effect on the structure of the internal waves. The observations of Hayes and Powell 1980 and Toole 1984 show that the density gradient variability in the Equatorial Pacific is an order of magnitude higher than what is expected at mid-latitudes, results consistent with the measurements at the PEQUOD site. The enhancement of equatorial variability relative to mid-latitudes is probably due to low frequency waves trapped in the equatorial 
waveguide and thus appears as frozen finestructure to the internal wave field. The effect of this enhanced variability on internal waves is that waves which correspond to mode numbers greater than 15 are significantly scattered and thus will not display the phaselocking characteristic of modes.

Section 7.7 finds that the most important effects of the mean flows at depth act through the polarization relations: Doppler shifts $U k$ and the effects of mean velocity curvature $U_{z z}$ do not significantly affect the internal wave shapes for low wavenumbers, and effects of buoyancy frequency variability are much stronger than mean flow effects at higher wavenumbers. Furthermore, because of the strong shear effects in the Equatorial Undercurrent, only very low wavenumbers (modes 1 through 3 ) are likely to show the phase-locking expected of modes.

Since scattering so strongly affects modes with high wavenumbers, a precise determination of vertical structure is only relevant for the low modes. For these low modes the two effects of shear that change the dispersion relation are minimal: Doppler shifts are small and significant mean velocity curvature only occurs over small distances. Thus it is sufficient to use the dispersion relation for a resting basic state in determining the vertical structure functions $G_{(z)}$ and $G_{z}(z)$, though shear effects should be included in the polarization relations. To the extent that one is interested in higher wavenumbers, the WKBJ approximation should suffice, not because it is an accurate approximation but because the variability in the solution is rather high and the WKBJ solution is closer to the average than an integration through any particular realization. A careful study of high wavenumbers should include the enhanced variability as well the the WKBJ average structure. 


\section{Chapter 8: Spectral estimates}

\subsection{Introduction}

Chapter 1 presents the Garrett and Munk (GM) model as a universal description of the world's internal wave field. The calculations in chapters 3, 4, and 6 show that the GM model is an inadequate description of the equatorial internal wave field: a more sophisticated model is required to be consistent with the PEQUOD data. The changes in the model suggested by these chapters include considering standing modes in combination with propagating waves, including a mean shear flow in the basic state, and allowing vertical asymmetry and horizontal anisotropy in the wave field itself. This chapter uses a spectral inversion to optimally fit the PEQUOD central mooring data with an internal wave model that incorporates these changes.

Section 8.2 is a brief review of linear internal wave theory and scaling as it is needed for the spectral inversion. It uses the results of chapters 5 and 7 to construct the set of internal waves that are used to interpret the equatorial data.

Section 8.3 reviews the transition from a linear wave model to a spectral model derived in chapter 5 . The internal wave spectrum is written in terms of direction moments of the spectral parameters $E, \Delta, P, Q$, and an interpretation of $E, \Delta, P, Q$ in terms of propagating waves and modes is given to complement the earlier interpretation in terms of correlated propagating waves given in section 5.8 .

The next two sections consider the problems of spectral estimation and inversion. Section 8.4 is a review of wavenumber estimation and spectral estimator statistics. It considers some of the estimators reviewed by Bretherton and McWilliams 1980, though their rigorous formalism is not presented. The ideas used in formulating the beamforming and Capon estimators in particular provide the basis of the spectral inversion.

Section 8.5 presents the spectral inversion used to calculate the results of sections 8.6 and 8.7. This estimator improves upon the estimators reviewed in section 8.4 in that it copes with the problem of having many waves that cannot be fully resolved and manages to incorporate the spectral properties of the spectral parameters, namely that the energies of the waves represented by the parameters be positive definite. The inversion is a modification of the spectral inversion proposed by Bretherton and McWilliams 1980: it differs in that the model is less arbitrarily parameterized and the model-model covariance 
matrix is modified to include the spectral character of the estimates. The section finishes by deriving the particular model-model covariance structure necessary to calculate the direction moments of the $E, \Delta, P, Q$ parameters.

Section 8.6 looks at the resolving power of the PEQUOD current meter arrays, i.e. the array's ability to distinguish between internal waves that have different wavenumbers. It considers three measures of resolution: model rank, response to a smooth spectral model, and response to unit impulses at particular wavenumbers. An understanding of the resolution helps interpret the results of the final sections.

In order to understand which spectral features are necessary to interpret the PEQUOD data, section 8.7 applies a series of models to the data. It starts with the GM model of chapter 1: an internal wave model that is highly parameterized, horizontally isotropic, vertically symmetric, purely propagating and has a WKBJ approximate vertical structure. Section 8.7 then proceeds to relax each of these assumptions, noting the effects of each change on the fit to the PEQUOD data. The section concludes that the wavefield is both vertically asymmetric and horizontally anisotropic, with some evidence of both the kinematic effects of a mean shear flow and the phase locking that distinguishes between modes and propagating waves. The section also finds that some features of the internal wave field change in time, changes that are correlated with the phase of the 1982-1983 El Niño event. 


\subsection{Internal wave review and scaling}

This chapter interprets the current meter spectra as the linear superposition of internal waves. As far as the process of spectral estimation is concerned, the internal wave model is fully expressed by a linear relation

$$
d_{i}(\omega)=\sum_{\alpha} H_{i \alpha}(\omega) a_{\alpha}(\omega)
$$

i.e. at a particular frequency the Fourier transform (in time) of the $i^{\text {th }}$ time series $d_{i}$ can be expressed as a linear superposition of waves, the contribution of the $\alpha^{t h}$ wave being proportional to its amplitude $a_{\alpha}$. This section reviews the theory that goes into determining the wave structure $H_{i}$ for a wave, paving the way for the spectral (squared Fourier coefficient/squared wave amplitude) model considered in section 8.3.

Sections 5.4 and 5.5 show that the problem of determining wavelike solutions $A e^{i(k x+l y-\omega t)} G(z)$ to the equations for small perturbations to the equatorial basic state reduces to solving the vertical structure equation,

$$
G_{z z}(z)+m^{2}(z) G(z)=0
$$

where the squared local vertical wavenumber $m^{2}(z)$ for internal waves in a mean shear flow is given by

$$
m^{2}(z)=\frac{N^{2}(z) K^{2}}{\sigma_{I}^{2}}-K^{2}+\frac{K}{\sigma} U_{z z} \cos \theta+\frac{K^{2}}{\sigma^{2}}\left(f_{H}+U_{z}\right) f_{H} \sin ^{2} \theta
$$

$\sigma=\omega-K U \cos \theta$ is the intrinsic frequency, and the horizontal wavevector has been written in polar coordinates $(k, l)=K(\cos \theta, \sin \theta)$. As shown in chapter 7 , low wavenumber solutions to equation 8.2.2 are smooth and insensitive to the detailed structure of the local vertical wavenumber $m(z)$, while high wavenumber solutions can be quite intricate and that detailed structure is strongly dependent on small features in $m(z)$. Since the $m(z)$ profile is to some extent uncertain and is likely to vary if long periods of time are considered, the highly detailed structure of the high wavenumbers is both uncertain and not particularly useful for representing long-term spectral averages. For the purposes of spectral modeling, then, we are primarily interested in the structure of the low modes. Because of the focus on low modes, of the four terms on the right hand side of equation 
8.2.3, only the first is important in the analysis of equatorial internal waves. The fourth term includes the effects of the horizontal component of the Coriolis force - it can be neglected relative to the first term (see section 5.5). The third term contains the effects of curvature in the mean velocity profile. While the third term can make a significant contribution to the local vertical wavenumber if the wave is very long (small $K$ ), the calculations of chapter 7 show that very long waves are not affected much by short vertical scale perturbations in the mean velocity profile $U(z)$ or the buoyancy frequency profile $N(z)$. Thus the third term does not have a significant effect on the structure of solutions.

The second term contains the non-hydrostatic effects. The term is locally important where the intrinsic frequency $\sigma$ is a significant fraction of the local buoyancy frequency $N_{(z)}$. The term significantly affects the structure of solutions only if it is locally important over a vertical distance comparable to the vertical wavelength of the wave. The integrations of chapter 7 demonstrate that this term has little effect on the low baroclinic modes at the frequencies of interest $(\omega \approx .2 \mathrm{cph})$.

The upshot is that the vertical structure solutions necessary for low wavenumber analysis can be obtained by considering only the first term: i.e. solving the hydrostatic resting basic state vertical structure equation. Some solutions for upward propagating waves are plotted in chapter 7. Note, however, that neither a resting basic state nor the hydrostatic equations are used to simplify the polarization relations (which will be discussed later in this section): in the polarization relations the kinematic effects of mean shear are not smoothed out by the large vertical scales of the low modes. 


\section{Boundary conditions}

Section 5.6 discusses the expansion of the general solution of the vertical structure equation in terms of a pair of orthogonal solutions. What follows here is a brief review of that discussion.

Since the vertical structure equation 8.2.2 is a linear second order differential equation, there are two independent solutions which can be combined linearly to get the general solution. As was done in section 5.6, the choice made here is to use the generalized upward and downward propagating solutions as the pair of basis functions. So define the upward propagating solution $\alpha(z)$ such that it satisfies the following pair of conditions

$$
\begin{aligned}
\alpha_{z}\left(z_{0}\right) & =-i m\left(z_{0}\right) \alpha\left(z_{0}\right) \\
\alpha\left(z_{0}\right) & =1
\end{aligned}
$$

where the reference depth $z_{0}$ is here taken to be the bottom. Note that $\alpha(z)$ is the solution with upward energy - the phase propagates downwards. The solution with downward propagating energy is given by the complex conjugate of the upward propagating solution, namely $\alpha^{*}(z)$.

The discussion so far leaves untouched the question of appropriate top and bottom boundary conditions. If one believes that the ocean is essentially unbounded in the high frequency internal wave range (and there are a number of mechanisms that make this seem like a reasonable point of view, see chapter 5), then the two propagating solutions are the appropriate solutions and boundary conditions are unimportant. But the calculations in chapter 7 show that scattering in particular is not strong enough to affect low wavenumber energy, and the coherences in chapter 3 suggest that there are aspects of the current meter data that are better explained by a modal rather than a propagating model. Modes satisfy a no flow condition at the (flat) bottom boundary, namely

$$
G\left(z_{0}\right)=0
$$

which means that modes can be written as the difference of the two propagating solutions,

$$
G(z)=i\left(\alpha(z)-\alpha^{*}(z)\right)
$$

Modes also satisfy an upper boundary condition which has affects the solutions by only allowing discrete values of horizontal wavenumber $K$ for a given value of the frequency 
$\omega$. In the computations made in this chapter a free surface boundary condition is used at the surface, but the wavenumbers found differ little from the wavenumbers that would be found were the rigid lid approximation made, an approximation which gives the upper boundary condition

$$
G(0)=0
$$

In the model used to analyze the current meter data mixtures of propagating waves and modes are allowed, leaving the current meter data to determine whether the internal wave field is of a primarily modal or propagating character.

\section{Polarization relations}

Given an internal wave solution $a_{\alpha} e^{i(k x+l y-\omega t)} G^{\alpha}(z)$, the problem remains to write the Fourier transformed time series $u(\omega), v(\omega), \eta(\omega)$ in terms of the wave solution. This connection is made by using the polarization relations derived in section 5.5. For an array of sensors that includes only vertical separations, the Fourier coefficients $u, v, \eta$ at frequency $\omega$ and position $z_{i}$ are given by

$$
\left[\begin{array}{l}
u_{i} \\
v_{i} \\
d_{i}
\end{array}\right]=\left[\begin{array}{c}
\cos \theta \psi_{i}-\sin ^{2} \theta \lambda_{i} \phi_{i} \\
\sin \theta \psi_{i}+\sin \theta \cos \theta \lambda_{i} \phi_{i} \\
\phi_{i}
\end{array}\right] a_{\alpha}
$$

where

$$
\begin{aligned}
d_{i} & =N\left(z_{i}\right) \eta_{i} & & \text { normalized displacement } \\
\phi_{i} & =\left[K N\left(z_{i}\right) / \sigma_{i}\right] G\left(z_{i}\right) & & \text { dimensionless mode/wave shape } \\
\psi_{i} & =G_{z}\left(z_{i}\right) & & \text { vertical derivative of mode/wave shape } \\
\lambda_{i} & =\left(U_{z}\left(z_{i}\right)+f_{H}\right) / N\left(z_{i}\right) & & \text { inverse root Richardson number } \\
\sigma_{i} & =\omega-U\left(z_{i}\right) K \cos \theta & & \text { intrinsic frequency }
\end{aligned}
$$

Equation 8.2.8 gives the sought after linear relation between the wave amplitudes $a_{\alpha}$ and the Fourier coefficients $d_{i}$.

$$
d_{i}=H_{i \alpha} a_{\alpha}
$$

Note that here $d_{i}$ is used to represent any of the normalized current meter variables $u_{i}, v_{i}, d_{i} . H_{i \alpha}$ thus contains all of the internal wave physics expressed in the vertical structure equation 8.2.2 and the polarization relations 8.2.8. 


\subsection{Spectral model}

Section 8.2 uses the model matrix $H_{i \alpha}$ to relate the wave amplitudes $a_{\alpha}$ to the Fourier transform of the current meter data $d_{i}$. This section looks at how this relation translates to a relation between the current meter cross-spectral estimates $\left\langle d_{i}^{*} d_{j}\right\rangle$ and the energy in each internal wave $\left\langle a_{\alpha}^{*} a_{\alpha}\right\rangle$, a relation given earlier as equation 5.8.9. The derivation here differs from that given in section 5.8 in that it exactly matches the calculations performed on the data.

The essence of the spectral model is to average over many realizations of the internal wave field. For a particular realization $\gamma$, the current meter measurements $d_{i}^{(\gamma)}$ are related to the wave amplitudes $a_{\alpha}^{(\gamma)}$ by equation 8.2.9,

$$
d_{i}^{(\gamma)}=\sum_{\alpha} H_{i \alpha} a_{\alpha}^{(\gamma)}
$$

It is important to note that the model matrix $\mathbf{H}$ does not depend on realization, only the wave amplitudes and data change from one realization to another. By assuming ergodicity multiple realizations can be found by breaking a long time series into pieces and considering each piece as a separate realization. Thus for each piece equation 8.3.1 gives the relation between the Fourier transform of the current meter data and the wave amplitudes.

The current meter cross-spectral matrix is the set of products of the form $d_{i}^{*} d_{j}$ averaged over all realizations, namely $\left\langle d_{i}^{*} d_{j}\right\rangle$.

$$
\left\langle d_{i}^{*} d_{j}\right\rangle=\frac{1}{N_{r}} \sum_{\gamma=1}^{N_{r}} d_{i}^{(\gamma)^{*}} d_{j}^{(\gamma)}
$$

where $N_{r}$ is the number of realizations (pieces) averaged over. The same relation holds between the averaged squared wave amplitude $s_{\alpha}=\left\langle a_{\alpha}^{*} a_{\alpha}\right\rangle$ and the amplitudes for each realization $a^{(\gamma)}$,

$$
s_{\alpha}=\left\langle a_{\alpha}^{*} a_{\alpha}\right\rangle=\frac{1}{N_{r}} \sum_{\gamma=1}^{N_{r}} a_{\alpha}^{(\gamma)^{*}} a_{\alpha}^{(\gamma)}
$$

At this point it is a simple matter to combine equations 8.3.1-8.3.3 to relate the current meter cross-spectra to the averaged wave energy,

$$
\left\langle d_{i}^{*} d_{j}\right\rangle=\sum_{\alpha} H_{i \alpha}^{*} H_{j \alpha} s_{\alpha}
$$


This is the basic relation between wave energy and current meter cross-spectra, and the rest of this chapter is concerned with inverting this equation, i.e. determining as much as possible about the energy of each internal wave $s_{\alpha}$ given the cross-spectra computed from the current meter array.

There are several important features of equation 8.3.4. First of all, it has been assumed that different waves are uncorrelated, i.e. $\left\langle a_{\alpha}^{*} a_{\beta}\right\rangle=0$ whenever $\alpha \neq \beta$. This greatly simplifies the right-hand side of equation 8.3.4. This orthogonality comes not from any property of the linear system discussed in section 8.2 , rather it is due to the presumption that in a long term average the internal waves are forced randomly and without correlation. The presumption is based on the idea that, while a single forcing event creates a set of waves that are coherent with each other, when many unrelated forcing events are including by averaging over many realizations that coherence is lost. Furthermore, non-linear effects and dissapation will also destroy any coherence. If the forcing is deterministic (the tides, for example) then forced coherence is not lost by averaging, and the assumption of uncorrelated waves can break down.

The second important feature of equation 8.3.4 is that it is a linear relation between the current meter cross-spectra and the wave energy. Thus techniques of linear inversion can be used to calculate the wave energy $s_{\alpha}$ from the current meter cross-spectra. The spectral problem uses stationarity and egodicity to greatly condense the size of the modeling problem. The original linear problem (8.3.1) relates the $2 N_{r} N_{t g}$ real random variables that are the data to $2 N_{r} N_{M}$ wave amplitudes (again counting the real and imaginary parts separately). The spectral problem, on the other hand, relates $N_{t s}^{2}$ real random variables (the current meter cross-spectra) to $N_{M}$ real model parameters (the spectral energies $s_{\alpha}$ ). Since there are many more realizations $N_{r}$ than there are time series $N_{t s}$, the model is a quite concise description of the data set.

The internal waves propagating in a given direction $\theta$ consist of a mixture of upward propagating waves, downward propagating waves, and vertically standing modes. Thus the wave energy $s_{\alpha}$ is a function of several independent variables: frequency $\omega$ (which will be left implicit), wavenumber $K$, direction $\theta$, and a discrete parameter $g$ that covers the possibilities of being upward, downward, or modal. As will become quickly apparent if it is not obvious already, a current meter array cannot resolve energies corresponding to all values of these independent variables. In light of this lack of resolution it makes more sense to map the wave energy $s(K, \theta, g)$ to a different (and much smaller) set of 
parameters that are well measured by the array. This mapping will be done in two stages: first the mapping to the spectral parameters $E, \Delta, P, Q$ introduced in chapter 5 , then the mapping to the coefficients of a Fourier expansion in direction $\theta$, an expansion introduced in chapter 6 . The product of these two mappings results in the linear transformation $T_{\beta}(K, \theta, g)$ which gives the wave energies $s(K, \theta, g)$ in terms of the direction moments of the spectral parameters $e_{\beta}$, namely,

$$
s(K, \theta, g)=T_{\beta}(K, \theta, g) e_{\beta}
$$

When the continuous nature of direction $\theta$ is not important, this relation can also be written as

$$
s_{\alpha}=T_{\alpha \beta} e_{\beta}
$$

\section{$E, \Delta, P, Q$ parameterization}

At a particular frequency $\omega$ and vector horizontal wavenumber $K(\cos \theta, \sin \theta)$, the internal wave field can be considered to have three components,

$$
\begin{array}{ll}
a \alpha(z) & \text { upward propagating wave } \\
b \alpha^{*}(z) & \text { downward propagating wave } \\
c\left(i \alpha(z)-i \alpha^{*}(z)\right) & \text { mode }
\end{array}
$$

Note that the mode has a zero crossing at the bottom $z_{0}$ : more generally one could consider $i\left(e^{i \zeta} \alpha(z)-e^{-i \zeta} \alpha^{*}(z)\right)$ which has a zero crossing that depends on a phase $\zeta$. In what follows some use is made of a fourth wave called the "anti-mode" which is simply a mode whose zero crossing is given by $\zeta=\frac{\pi}{2}$. By assumption these three (or four) internal waves are statistically independent, so the current meter cross-spectra can be written (omitting the polarization vector factors for clarity),

$$
\begin{aligned}
\left\langle d_{i}^{*} d_{j}\right\rangle & =\alpha_{i}^{*} \alpha_{j}\left\langle a^{*} a+c^{*} c\right\rangle+\alpha_{i} \alpha_{j}^{*}\left\langle b^{*} b+c^{*} c\right\rangle-\left(\alpha_{i}^{*} \alpha_{j}^{*}+\alpha_{i} \alpha_{j}\right)\left\langle c^{*} c\right\rangle \\
& =\alpha_{i}^{*} \alpha_{j}\left\langle A^{*} A\right\rangle+\alpha_{i} \alpha_{j}^{*}\left\langle B^{*} B\right\rangle+\alpha_{i}^{*} \alpha_{j}^{*}\left\langle A^{*} B\right\rangle+\alpha_{i} \alpha_{j}\left\langle B^{*} A\right\rangle \\
& =\alpha_{i}^{*} \alpha_{j}(E+\Delta)+\alpha_{i} \alpha_{j}^{*}(E-\Delta)+\alpha_{i}^{*} \alpha_{j}^{*}(P-i Q)+\alpha_{i} \alpha_{j}(P+i Q)
\end{aligned}
$$

As an intermediate step, equation 8.3.6b shows the expansion in terms of the correlated up and down waves that were discussed in chapter 5. Equation 8.3.6c then uses that 
equation to write the expansion in terms of the spectral parameters $E, \Delta, P, Q$, spectral parameters that have the following meanings:

$E$ is the symmetric part of the spectrum,

$\Delta$ is the difference in energy between upward and downward propagating waves,

$P \quad$ is the modal component of the spectrum, and

$Q$ is the anti-modal component of the spectrum.

These labels follow from the mapping implied by the different parts of equation 8.3.6. Written in matrix form, that mapping is as follows,

$$
\left[\begin{array}{c}
\left\langle a^{*} a\right\rangle \\
\left\langle b^{*} b\right\rangle \\
\left\langle c^{*} c\right\rangle \\
\left\langle f^{*} f\right\rangle
\end{array}\right]=\frac{1}{2}\left[\begin{array}{cccc}
1 & 1 & 1 & 1 \\
1 & -1 & 1 & 1 \\
0 & 0 & -1 & 0 \\
0 & 0 & 0 & -1
\end{array}\right]\left[\begin{array}{c}
E \\
\Delta \\
P \\
Q
\end{array}\right]
$$

and

$$
\left[\begin{array}{c}
E \\
\Delta \\
P \\
Q
\end{array}\right]=\left[\begin{array}{c}
\left\langle a^{*} a\right\rangle+\left\langle b^{*} b\right\rangle+2\left\langle c^{*} c\right\rangle+2\left\langle f^{*} f\right\rangle \\
\left\langle a^{*} a\right\rangle-\left\langle b^{*} b\right\rangle \\
-2\left\langle c^{*} c\right\rangle \\
-2\left\langle f^{*} f\right\rangle
\end{array}\right]
$$

$\left\langle f^{*} f\right\rangle$ is the energy of the anti-mode. Equation 8.3.7b offers several insights into the spectral parameters $E, \Delta, P, Q$ in particular and the spectral problem in general. Clearly both $P$ and $Q$ are negative definite because the energy in each mode is positive: this is consistent with the correlation interpretation given in chapter 5 because $P$ and $Q$ are the correlation between up and down and negative correlation is required to produce cancellation at the bottom (reference depth $z_{0}$ ).

$E$ is the only parameter that receives contributions from all modes and waves. As a consequence if energy at any given wavenumber can be measured at all the $E$ component will be measurable. The ability to measure $\Delta, P, Q$ then determines whether we can distinguish between upward propagating, downward propagating, and modal solutions. The fact that the GM79 model spectrum is vertically symmetric and effectively propagating ( $\Delta=P=Q=0$ ) can thus be considered as more of a statement that $\Delta, P, Q$ are difficult to measure rather than a statement that the internal wave field is vertically symmetric and propagating. 


\section{Direction moments}

The wave energy $s_{\alpha}$ depends continuously on direction, which means that there are an infinite number of waves at a particular wavenumber $K$ and frequency $\omega$. Because the polarization relations (equation 8.2.6) depend to leading order only on low powers of $\cos \theta$ and $\sin \theta$, a Fourier series in direction $\theta$ provides an efficient parameterization of the wave energy's direction dependence. This expansion is the same as that done in section 6.3 ; the only difference being that the expansions in chapter 6 parameterize internal waves in a resting basic state while here the model includes some kinematic shear effects.

Let $X$ represent in turn $E, \Delta, P$, and $Q$. Then express $X(\theta)$ as a Fourier expansion in direction $\theta$,

$$
X(\omega, K, \theta)=X_{0}(\omega, K)+2 \sum_{n=1}^{\infty} X_{c n}(\omega, K) \cos n \theta+X_{s n}(\omega, K) \sin n \theta
$$

This transform is inverted by

$$
\begin{aligned}
X_{0} & =\langle X\rangle=\frac{1}{2 \pi} \int_{0}^{2 \pi} X(\theta) d \theta \\
X_{c n} & =\langle\cos n \theta X\rangle \\
X_{s n} & =\langle\sin n \theta X\rangle
\end{aligned}
$$

For a resting basic state (no mean shear) internal wave model, the expansions are given in chapter 6 . Those expansions show that

$\begin{array}{ccc}\left\langle u^{*} u\right\rangle+\left\langle v^{*} v\right\rangle & \text { depends on } & \langle X\rangle \\ \left\langle\eta^{*} \eta\right\rangle & \text { depends on } & \langle X\rangle \\ \left\langle v^{*} \eta\right\rangle & \text { depends on } & \langle X \sin \theta\rangle \\ \left\langle u^{*} \eta\right\rangle & \text { depends on } & \langle X \cos \theta\rangle \\ \left\langle u^{*} v\right\rangle & \text { depends on } & \langle X \sin 2 \theta\rangle \\ \left\langle u^{*} u\right\rangle-\left\langle v^{*} v\right\rangle & \text { depends on } & \langle X \cos 2 \theta\rangle\end{array}$

Here we see that the commonly computed "like" coherences (i.e. coherences of zonal velocity with zonal velocity, meridional velocity with meridional velocity, displacement with displacement) are measurements of the horizontally isotropic component of the spectra: it is the correlations between unlike variables that furnish direction information, primarily the correlations between velocity and displacement. Note that in this resting basic state model higher direction moments simply do not contribute to the current meter spectra. 
If we complicate the model by including the effects of a mean zonal flow, more direction moments contribute to the current meter spectra. If we include only the shear effects in the polarization relations and ignore Doppler shift effects (an approximation appropriate in the low mode limit), the first 9 direction moments (up to $\cos 4 \theta$ ) contribute, though the higher moments contributions are all proportional to the local inverse root Richardson number $\lambda_{i}$, so the contributions can be quite small. If one includes a linearized Doppler shift effect, the first 11 direction moments contribute, but again the higher moments make smaller and smaller contributions. Appendix $D$ gives the full expansions for the low mode shear effects and linearized Doppler shift models. But the most important moments are the first five given above.

\section{Summary of spectral model}

The main point of this section is expressed in equation 8.3.4. It relates the current meter cross-spectra to the energies $s_{\alpha}$ of a set of uncorrelated waves. The latter half of the section considers the resolution ability of a current meter array that has only vertical separations. These resolution considerations suggest changing the model parameters $s_{\alpha}$ to the spectral parameters $E, \Delta, P, Q$, each of these spectral parameters being expanded in a Fourier series in direction $\theta . E$ is the best resolved of the four spectral parameters since it measures the combined energy of propagating waves and standing modes. Essentially only the first five direction moments are resolved by the cross-spectra between the current meter variables $u, v$, and $\eta$, though the inclusion of mean shear effects means that there are other moments that contribute to the cross-spectra as well.

This leaves us the problem of inverting equation 8.3.4. In particular, we would like to take advantage of the spectral nature of the parameters $s_{\alpha}$ : since the $s_{\alpha}$ are energies they must be positive semi-definite, and by assumption the waves are uncorrelated. The problem becomes a little more subtle when 8.3.4 is rewritten in terms of the direction moments of $E, \Delta, P, Q$, since it is not immediately obvious how positive definiteness of the $s_{\alpha}$ and lack of wave-wave correlation constrain $E, \Delta, P, Q$ moments. This problem is solved in the next two sections. 


\subsection{Review of wavenumber estimation and spectral estimator statistics}

The problem of estimating the internal wave field from current meter data differs only slightly from the problem of spectral (wavenumber) estimation using an irregular array (the slight difference arises in using the polarization relations to combine the unlike measurements $u, v$, and $\eta$ in determining a single wave field). Wavenumber estimation is a ubiquitous problem in the geophysical sciences and there is a large literature on the subject. Bretherton and McWilliams 1980 systematically review the problem of wavenumber estimation, offering a statistical perspective that encompasses many of the estimators commonly used. That statistical perspective is used here as well, but the final choice of estimator differs from that of Bretherton and McWilliams. Rather than redevelop the rigorous formalism that is presented in that paper, I have chosen to present a series of increasingly sophisticated estimators, culminating in the estimator that is actually used in interpreting the PEQUOD data. Unlike earlier estimators, this estimator manages to both be optimal in a statistical sense and incorporate the positive energy and statistical independence of the underlying internal wave field.

The spectral estimators discussed here are all generalized least-squares (GaussMarkov) estimators. These estimators are all based on the idea that the optimal set of model parameters $a_{\alpha}$ is the set which minimizes a given quadratic norm. In particular, given the linear model

$$
\mathbf{d}^{(0)}=\mathbf{H a}^{(0)}
$$

which relates the true values of the vector of model parameters $\mathbf{a}^{(0)}$ to the true values of the measurements $\mathbf{d}^{(0)}$, we can define the vector of residuals $\epsilon_{j}$ by

$$
\epsilon=\mathbf{d}-\mathbf{H a}
$$

as the difference between the data and the prediction from the vector of model parameter estimates a. A general quadratic norm can be written as,

$$
\chi^{2}=\mathbf{a}^{\dagger} \mathbf{L a}+\epsilon^{\dagger} \mathbf{W} \epsilon
$$

where $\dagger$ denote the complex conjugate transpose and $\mathbf{L}$ and $\mathbf{W}$ are matrices whose eigenvalues are all positive definite. As will be shown shortly, in a statistical interpretation the matrices $\mathbf{L}$ and $\mathbf{W}$ are each the inverse of a covariance matrix. A simple least-squares proceedure (Menke 1984) shows that the model parameters a which minimize $\chi^{2}$ are

$$
\mathbf{a}=\left(\mathbf{L}+\mathbf{H}^{\dagger} \mathbf{W H}\right)^{-1} \mathbf{H}^{\dagger} \mathbf{W d}
$$


This is the Gauss-Markov inverse. Given this result, all that needs to be done in order to find an optimal estimator is to make an appropriate choice of inverse model covariance matrix $\mathbf{L}$ and inverse residual covariance matrix $\mathbf{W}$. The beamforming estimator, for example, solves for the amplitude of a single wave by inverting equation 8.3.1 with $\mathbf{L}$ identically zero and $\mathbf{W}$ the identity matrix (see appendix $G$ ). While this is a reasonable choice, by considering the statistical properties of the residuals $\epsilon_{i}$ one can choose the inverse covariance matrices such that the estimates a are optimal in a statistical sense.

\section{Fourier coefficient statistics}

Though we are eventually interested in the statistics of the spectral estimates $s_{\alpha}$, a first step is to understand the statistics of the Fourier transformed data $d_{i}(\omega)$. As discussed in Bretherton and McWilliams 1980 as well as any spectral analysis text (see Jenkins and Watts 1968), the Fourier coefficients have random components $\epsilon_{j}$ that can be considered to be joint normally distributed Gaussian random variables. This means that the probability of a given set of $\epsilon_{j}$ occurring is proportional to the exponential

$$
e^{-\frac{1}{2} \epsilon_{i}^{*} Q_{i j}^{*} \epsilon_{j}}
$$

where the matrix $Q_{i j}$ is the inverse of the true covariance matrix $f_{i j}^{(0)}$ that corresponds to the estimated covariance $\hat{f}_{i j}=\left\langle d_{i}^{*} d_{j}\right\rangle$. This means minimizing $\sum_{i j} \epsilon_{i}^{*} Q_{i j}^{*} \epsilon_{j}$ returns the most probable solution. Thus given a linear problem such as equation 8.3.1, finding the maximum likelihood solution for Fourier coefficients $\mathbf{a}$ is equivalent to finding the least squares solution using $\mathbf{Q}^{*}$ as the residual norm $\mathbf{W}$ in equation 8.4.4. The Capon estimator does exactly that in solving for the amplitude of a single wave by using $\mathbf{Q}^{*}$ as the residual norm $\mathbf{W}$ and setting the model space norm $\mathbf{L}$ to be zero (see appendix $G$ ).

Both the beamforming and Capon estimators estimate spectral energy by squaring and averaging least-square estimates of wave amplitudes (see appendix G). Thus both the beamforming and the Capon estimators insure that any energy estimate $s_{\alpha}$ is not negative. But while the Capon solution is optimal for the problem of fitting a single wave to data, it is not clearly the optimal estimator for fitting a set of waves. 


\section{Spectral estimator statistics}

An alternative to computing wave energy by squaring amplitude estimates is to use the Gauss-Markov inverse to invert a purely spectral problem such as the one given in equation 8.3.4.

$$
\left\langle d_{i}^{*} d_{j}\right\rangle=\sum_{\alpha} A_{i j \alpha} e_{\alpha}
$$

where in the case of equation 8.3.4 the model $A_{i j \alpha}$ is given by $H_{i \alpha}^{*} H_{j \alpha}$. Writing the model as $A_{i j \alpha}$ allows us to use spectral parameters $e_{\alpha}$ that cannot be written as a simple product. In either case, for a purely spectral problem the residual norm $\mathbf{W}$ is based on the statistical properties of the spectral estimator residuals rather than the Fourier coefficient residuals.

Define $\epsilon_{i j}$ to be the noise in the measured cross-spectral estimate $\left\langle d_{i}^{*} d_{j}\right\rangle$. Appendix $\mathrm{C}$ shows that the covariance between the spectral noise $\epsilon_{i j}$ and the noise $\epsilon_{k l}$ is given by

$$
C_{i j k l}=\operatorname{Cov}\left(\epsilon_{i j}, \epsilon_{k l}\right)=\frac{1}{N_{r}} f_{i l}^{(0)} f_{k j}^{(0)}
$$

where $f_{i j}^{(0)}$ is the true value of $\left\langle d_{i}^{*} d_{j}\right\rangle$. This is a consequence of spectral estimators being the averaged product of Fourier coefficients (see appendix $\mathrm{C}$ ). The norm $\mathbf{W}$ which corresponds to the inverse of $C_{i j k l}$ is (Bretherton and McWilliams 1980, Jenkins and Watts 1968),

$$
W_{i j k l}=N_{r} Q_{i k} Q_{l j}
$$

where $Q_{i j}$ is the inverse of $f_{i j}$. The generalization of the norm given in 8.4.3 to doublesubscripted data is

$$
\chi^{2}=e_{\alpha} M_{\alpha \beta} e_{\beta}+\epsilon_{i j}^{*} W_{i j k l} \epsilon_{k l}
$$

where all subscripts are summed over. The generalization of the Gauss-Markov solution given in 8.4.4 to double-subscripted data is

$$
e_{\alpha}=\left[M_{\beta \alpha}+F_{l i \beta} F_{i l \alpha}\right]^{-1} F_{l i \beta} Q_{i j}^{\prime}\left\langle d_{j}^{*} d_{l}\right\rangle
$$

where subscripts other than $\alpha$ are summed over, and the number of realizations has been absorbed in $Q_{i j}^{\prime}=\sqrt{N_{r}} Q_{i j}$. The normalized spectral model $F_{i l \alpha}$ is given by

$$
F_{i l \alpha}=Q_{i j}^{\prime} A_{j l \alpha}
$$

The only remaining undetermined quantity is the model space norm $M_{\alpha \beta}$. 
The reason that fitting many waves to the current meter spectra is more difficult than fitting single waves is that it is quite likely that there will be two or more more waves that to some extent indistinguishable. This introduces the problem of deciding how the energy should be distributed among the two partially indistinguishable waves. Bretherton and McWilliams 1980 solve the problem by choosing the model space norm to be diagonal, i.e. $M_{\alpha \beta}=\delta_{\alpha \beta}\left[s_{\alpha}^{(0)}\right]^{-2}$ and $s_{\alpha}^{(0)}$ is the expected size of the energy for the $\alpha^{t h}$ wave. $s_{\alpha}^{(0)}$ is the a priori spectrum. This choice of $M_{\alpha \beta}$ means that the matrix in square brackets in equation 8.4 .9 will be invertible and there will be a well-defined solution $e_{\alpha}$.

Note the second term in equation 8.4 .8 represents the norm that finds the most likely value of the residuals $\epsilon_{i j}$. The first term is minimum when all the wave energy estimates are zero. If the a priori sizes $\left(s_{\alpha}^{(0)}\right)$ tend towards infinity, then the contribution from the first term is small and the total residue $\chi^{2}$ is essentially due to the residuals $\epsilon_{i j}$. But if there is a wave $\alpha$ that is poorly resolved by the measurements, then the solution that minimizes 8.4.8 simply sets that wave amplitude to zero. In the more common case where two waves $\alpha$ and $\beta$ are indistinguishable, the solution that minimizes 8.4.8 allocates the energy according to the ratio between their a priori sizes $s_{\alpha}^{(0)}$ and $s_{\beta}^{(0)}$. Consequently the problem of computing the set of all $a_{\alpha}$ is now always well defined, though in interpreting the results we have to keep in mind that unresolved components are set according to the a priori notions embodied in $M_{\alpha \beta}$.

Note that in going from the Fourier coefficient representation of equation 8.3.1 to the spectral representation 8.4.6 the estimator can incorporate the constraint that correlations between waves are zero. This is an improvement over the beamforming and Capon estimators which, in the case where two waves are indistinguishable, will return a correlation of one between the two waves, i.e. the cross-power between the two waves will be the same as the power in each of them. The spectral estimator, on the other hand, can leave the different waves uncorrelated, uncorrelated being a more reasonable choice for the wave pair than complete correlation. The disadvantage of using the spectral representation 8.4.6 is that, unlike the beamforming and Capon estimators, the spectral estimates are not forced to be positive definite so that a lack of resolution may result in negative estimates of energy. Section 8.5 derives a spectral domain estimator that includes the positive semi-definiteness of the spectral estimates. 


\subsection{Spectral inversion}

This section derives the estimator used for fitting the PEQUOD data. It is nearly identical to the spectral estimator given at the end of section 8.4, the most important difference being that the model space norm $M_{\alpha \beta}$ is no longer arbitrarily chosen: it is a function of both the a priori spectrum $s_{\alpha}^{(0)}$ and the normalized model matrix $F_{i j \alpha}$. The model parameters are the $E, \Delta, P, Q$ direction moments discussed in section 8.3: combining equations 8.3.4 and 8.3.5 give the relation between the direction moments $e_{\beta}$ and the measured cross-spectra $\left\langle d_{i}^{*} d_{j}\right\rangle$,

$$
\left\langle d_{i}^{*} d_{j}\right\rangle=\sum_{\alpha \beta} H_{i \alpha}^{*} H_{j \beta} T_{\alpha \beta} e_{\beta}
$$

$H_{i \alpha}$ is the structure of the $\alpha^{t h}$ wave and $T_{\alpha \beta}$ is the matrix of tranformation from the direction moments $e_{\beta}$ to the wave energy $s_{\alpha}$. Therefore the normalized model matrix $F_{i l \beta}$ is given by

$$
F_{i l \beta}=\sum_{j \alpha} Q_{i j}^{\prime} H_{j \alpha}^{*} H_{l \alpha} T_{\alpha \beta}
$$

The model space metric $M_{\alpha \beta}$ is chosen to be

$$
M_{\gamma \delta}=\sum_{\alpha} T_{\alpha \gamma} T_{\alpha \delta}\left[\left(s_{\alpha}^{(0)}\right)^{-2}+2 F_{i l \beta} U_{\beta \alpha}\left(s_{\alpha}^{(0)}\right)^{-1}\right]
$$

where $U_{\beta \alpha}$ is the left inverse of $T_{\alpha \beta}$, i.e. $\sum_{\alpha} U_{\beta \alpha} T_{\alpha \gamma}=\delta_{\beta \gamma} . U_{\beta \alpha}$ will be discussed in more detail later in this section.

Plugging equations 8.5.2 and 8.5.3 into the Gauss-Markov inverse 8.4.9 gives estimates of the direction moments $e_{\beta}$ in terms of the measured cross-spectra $\left\langle d_{i}^{*} d_{j}\right\rangle$. Thus equations 8.4.9, 8.5.2, and 8.5.3 together form the basis of the solution used in interpreting the PEQUOD data. This estimator is similar to the spectral estimator advocated by Bretherton and McWilliams. It differs in three ways: here a zeroth order model is not subtracted from the data, the wavenumber parameterization is based on theoretical predictions rather than a parameterization chosen solely on the basis of resolution, and the model space norm (inverse model-model covariance) is not chosen to be diagonal. The remainder of this section is devoted to showing that the choice of model norm $M_{\alpha \beta}$ given in equation 8.5.3 is such that the estimator will tend to keep the energy estimates $s_{\alpha}$ positive semi-definite. 
The starting point for this derivation is equation 8.3.1 which relates the measured Fourier coefficients to the wave amplitudes for each realization,

$$
d_{i}^{(\gamma)}=H_{i \alpha} a_{\alpha}^{(\gamma)}
$$

If we use the Gauss-Markov inverse to find optimal sets of wave amplitudes $a_{\alpha}^{(\gamma)}$ for each realization, we get

$$
\left(L_{\alpha \beta}+H_{i \alpha}^{*} Q_{i j}^{*} H_{j \beta}\right) a_{\beta}^{(\gamma)}=H_{i \alpha}^{*} Q_{i j}^{*} d_{j}^{(\gamma)}
$$

where the term in parenthesis is the matrix that must be inverted in order to calculate $a_{\beta}^{(\gamma)}$, and there is an implicit summation over all the repeated indices $i, j, \alpha$. Rather than inverting first for the $a_{\alpha}^{(\gamma)}$ and then computing mean square amplitude as is done in the Capon estimator (see appendix $G$ ), consider reversing the procedure so that equation 8.5.4 is squared and averaged before inverting and solving for the wave spectra. It turns out that the reversed procedure suggests a way of dealing with the general parameterized spectral problem.

The spectral problem is to find the average value $\left\langle a_{\gamma}^{*} a_{\delta}\right\rangle$, i.e. the average over many realizations of the Fourier coefficient problem. As a first step towards finding a solution, square and average equation 8.5.4. Since the model stays the same over all the realizations, only the Fourier coefficients $a_{\gamma}^{*}$ and the data $d_{j}$ are affected by the averaging process and one is left with

$$
\begin{array}{r}
{\left[L_{\alpha \gamma}^{*} L_{\beta \delta}+H_{j \gamma}^{*} Q_{i j} H_{i \alpha} L_{\beta \delta}+L_{\alpha \gamma}^{*} H_{k \beta}^{*} Q_{l k} H_{l \delta}+H_{i \alpha} H_{k \beta}^{*} Q_{i j} Q_{l k} H_{l \delta} H_{j \gamma}^{*}\right]\left\langle a_{\gamma}^{*} a_{\delta}\right\rangle} \\
=H_{i \alpha} H_{k \beta}^{*} Q_{i j} Q_{l k}\left\langle d_{j}^{*} d_{l}\right\rangle
\end{array}
$$

If we now invert the matrix in square brackets we can solve for the wave amplitude cross-spectra $\left\langle a_{\gamma}^{*} a_{\delta}\right\rangle$. We would get exactly the same answer that one would get by inverting 8.5.4 and squaring the result. This is not a particularly useful procedure, since the spectral problem as written in equation 8.5.5 involves inverting a $N_{M}^{2}$ by $N_{M}^{2}$ matrix while the Fourier coefficient problem as given in equation 8.5.4 involves inverting a $N_{M}$ by $N_{M}$ matrix. But equation 8.5 .5 can be greatly simplified by including the fact that different waves are uncorrelated.

The constraint that all the waves are uncorrelated means the set of $N_{M}^{2}$ model parameters $\left\langle a_{\gamma}^{*} a_{\delta}\right\rangle$ is reduced to the set of $N_{M}$ parameters $\left\langle a_{\alpha}^{*} a_{\alpha}\right\rangle$. Now the spectral problem has the same number of parameters as the Fourier coefficient problem: to solve 
it a ( $N_{M}$ by $N_{M}$ ) matrix must be inverted. Furthermore, since the statistical independence of waves is part of the internal wave model, the spectral formulation of the problem has a distinct advantage over the beamforming and Capon estimators because the spectral formulation can use the statistical independence of waves to help resolve wavenumbers that are difficult to distinguish.

Equation 8.5.5 modified by the constraint of statistical independence of the waves becomes

$$
\begin{array}{r}
{\left[L_{\alpha \gamma}^{*} L_{\alpha \gamma}+H_{j \gamma}^{*} Q_{i j} H_{i \alpha} L_{\alpha \gamma}+L_{\alpha \gamma}^{*} H_{k \alpha}^{*} Q_{l k} H_{l \delta}+H_{i \alpha} H_{k \alpha}^{*} Q_{i j} Q_{l k} H_{l \delta} H_{j \gamma}^{*}\right]\left\langle a_{\gamma}^{*} a_{\gamma}\right\rangle} \\
=H_{i \alpha} H_{k \alpha}^{*} Q_{i j} Q_{l k}\left\langle d_{j}^{*} d_{l}\right\rangle
\end{array}
$$

(There is implicit summation over the data indices $i j k l$ and the model index $\gamma$ but not over the model index $\alpha$ ). As long as the assumption that the waves are uncorrelated is correct, the solution to this problem will correspond to the squared solution where all the energies are positive. One would thus expect that, while computationally the estimates of energy are possibly negative, there will not be any significantly negative estimates of energy. By comparing the solution in equation 8.5.6 with the Gauss-Markov solution (equation 8.4.10) to the problem given in equation 8.5.4, we see that solving the spectral estimator corresponds to solving the linear problem

$$
\sum_{\alpha} H_{i \alpha}^{*} H_{l \alpha}\left\langle a_{\alpha}^{*} a_{\alpha}\right\rangle=\left\langle d_{\jmath}^{*} d_{l}\right\rangle
$$

subject to the inverse covariance

$$
\begin{aligned}
C_{i j k l}^{-1} & =Q_{i j} Q_{l k} \\
Q_{i j} & =\left(f_{i j}^{*}\right)^{-1}
\end{aligned}
$$

for the residuals, and the inverse covariance

$$
M_{\beta \alpha}=\delta_{\beta \alpha}\left[\left(s_{\alpha}^{(0)}\right)^{-2}+2 F_{i i \alpha}\left(s_{\alpha}^{(0)}\right)^{-1}\right]
$$

for the model parameters ( $F_{i j \alpha}$ will be defined in a moment). The solution to this problem is

$$
s_{\alpha}=\left[M_{\beta \alpha}+F_{l i \beta} F_{i l \alpha}\right]^{-1} F_{l i \beta} Q_{i j}\left\langle d_{j}^{*} d_{l}\right\rangle
$$

where 


$$
\begin{array}{ll}
s_{\alpha}=\left\langle a_{\alpha}^{*} a_{\alpha}\right\rangle & \text { is the energy in the } \alpha^{t h} \text { wave, } \\
s_{\alpha}^{(0)} & \text { is the corresponding a priori value, and } \\
F_{i l \alpha}=Q_{i j} H_{j \alpha}^{*} H_{l \alpha} & \text { is the normalized spectral model. }
\end{array}
$$

Note that the first line of equation 8.5.4 gives the covariance of unaveraged spectral estimators $d_{j}^{*} d_{l}$ (see appendix $C$ or Jenkins and Watts 1968). The $\left\langle d_{j}^{*} d_{l}\right\rangle$ are the averaged spectral estimators: their inverse covariance is given in equation 8.4.8 and the elements are a factor of $N_{r}$ larger than the inverse covariance of unaveraged spectral estimators. That suggests replacing $Q_{i j}$ in equation 8.5.10 and in the definition of $F_{i j \alpha}$ with the normalized version $Q_{i j}^{\prime}$ defined in section 8.4. Now the estimator in equation 8.5.10 corresponds to the purely spectral Gauss-Markov estimator defined in equation 8.4.10. It differs in that the choice of model space norm $M_{\alpha \beta}$ is not purely arbitrary: the norm is dependent on both the a priori spectrum $s_{\alpha}^{(0)}$ and the normalized spectral model $F_{i j \alpha}$. Because this form of the model space covariance makes the purely spectral problem parallel to a squared coefficient problem, this form of the model space norm is a mechanism that returns positive energies whenever possible. 


\section{Model-Model covariance for $E, \triangle, P, Q$ direction moments}

The remaining step is to rewrite the spectra inversion in terms of the direction moments of $E, \Delta, P, Q$. While technically this is a necessary step, it provides no new understanding of the internal wave field or the estimation problem. The transformation of variables in the inversion follows directly from the linearity of the relation between the wave energies $s(\theta, g)$ and the spectral parameters $e_{\beta}$. Note that in transforming to direction moments of $E, \Delta, P, Q$ we are only concerned with the direction dependence $\theta$ and the model $/$ wave type index $g$. The other independent variables $(\omega, K)$ will thus be emitted for clarity.

As stated in equation 8.3.5, the transformation described in section 8.3 can be summarized by a linear transformation $T_{\beta}(\theta, g)$ (the transformation $\mathrm{T}$ can also be written as a matrix $T_{\alpha \beta}$ when the continuous nature of the direction $\theta$ is not important). The transformation $T_{\beta}(\theta, g)$ is not strictly invertible (because of the expansion of a finite series of direction moments to the continuous variable $\theta$ ), but some choice $\mathbf{U}$ can be made so that

$$
e_{\beta}=\sum_{g} \int_{0}^{2 \pi} U_{\beta}(\theta, g) s(\theta, g) d \theta
$$

and

$$
\sum_{g} \int_{0}^{2 \pi} U_{\beta}(\theta, g) T_{\alpha}(\theta, g) d \theta=\delta_{\beta \gamma}
$$

i.e. $\mathbf{U}$ is a left inverse of $\mathbf{T}$. With these two matrices of transition, the spectral inversion can be rewritten in terms of the direction moments. As noted in equation 8.5.1, the model equation 8.3 .4 becomes

$$
\begin{aligned}
\left\langle d_{j}^{*} d_{l}\right\rangle & =\sum_{\alpha} H_{i \alpha}^{*} H_{l \alpha} s_{\alpha} \\
& =\sum_{\alpha \beta} H_{i \alpha}^{*} H_{l \alpha} T_{\alpha \beta} e_{\beta}
\end{aligned}
$$

so that the measured cross-spectra $\left\langle d_{j}^{*} d_{l}\right\rangle$ are related to the direction moments $e_{\beta}$. The model space residue is rewritten in several steps. First the matrix of transition $T_{\alpha \beta}$ is used to change the model space residue from a function of wave energy $s_{\alpha}$ to a function of direction moments $e_{\beta}$,

$$
s_{\alpha} M_{\alpha \beta} s_{\beta}=e_{\gamma} T_{\alpha \gamma} M_{\alpha \beta} T_{\beta \delta} e_{\delta} \equiv e_{\gamma} N_{\gamma \delta} e_{\delta}
$$

The next step is to rewrite $M_{\alpha \beta}$ in terms of the true values of the spectral parameters $e_{\gamma}^{(0)}$ and the new normalized spectral model $R_{i l \beta}=F_{i l \alpha} T_{\alpha \beta}$. It is straightforward to 
incorporate the new form of the true values,

$$
s_{\alpha}^{(0)}=T_{\alpha \beta} e_{\beta}^{(0)}
$$

and it is readily verified that

$$
F_{i l \alpha}=R_{i l \beta} U_{\beta \alpha}
$$

Thus the inverse model-model covariance for the spectral parameters $e_{\beta}$ is

$$
N_{\gamma \delta}=T_{\alpha \gamma} T_{\alpha \delta}\left[\left(s_{\alpha}^{(0)}\right)^{-2}+2 R_{i l \beta} U_{\beta \alpha}\left(s_{\alpha}^{(0)}\right)^{-1}\right]
$$

which is what was given in equation 8.5.3. The explicit forms and derivations for the model-model covariance of the direction moments $\mathbf{e}$ are given in appendix $E$. By using $N_{\gamma \delta}$ as the model-model covariance, the direction moments $e_{\beta}$ represent a continuous field of uncorrelated internal waves.

\subsection{Spectral resolution}

This section is concerned with how well the inverse resolves the spectral parameters. Given any fit of an internal wave spectrum to a dataset, it is important to understand which parts of the solution are strongly determined and which parts of the solution are a result of no information. While a detailed understanding of the array resolution would be so tedious as to be useless, some quite useful insights can be gained through some simple analyses. The first analysis concentrates on simply determining the number of model parameters one expects to be able to resolve. The second examines the response of the inverse to a known smooth input spectrum (GM79). The third examines the response to impulse forcing. These three investigations of resolution provide a basis for interpreting the results of section 8.7 .

A simple characterization of resolution is the number of model parameters that can be resolved. In the literature of inverse methods this number is called the rank of the system.* Rough consideration of the array geometries for the $Q$ and $U$ moorings gives good predictions for the ranks of some simple internal wave models (the ranks are calculated using equation $F .3$ ).

* This is actually a slight generalization of the usual use of the word rank, see appendix $F$. 
The designs of the first year $Q$ mooring $(Q 1)$ and the second year $U$ mooring $(U 2)$ are identical, but instrument failures and timing problems made the usable portions of the arrays differ. The design was to have three groups of four instruments (see chapter 2): the top group starting at roughly 500 meters, the center group at 1500 meters, and the bottom group at $3000 \mathrm{~m}$. Each group consists of alternating temperature-pressure (TP) recorders with current meters, the spacings within the groups being order 50 meters. Both arrays end up with 20 usable time series out of a possible 24 . The $Q 1$ array is missing a current meter in the center group and a TP recorder in the bottom group, while the $U 2$ array is missing a TP recorder in the top group and a current meter in the bottom group.

Consider for a moment an isotropic vertically symmetric internal wave model. In this model, of the four spectral parameters $E, \Delta, P, Q$ only $E$ is non-zero, and of its five direction moments possibly resolved by the array, only its isotropic (zeroth) direction moment is non-zero. Both the displacement spectra (which is computed from temperature) and the kinetic energy spectrum furnish information about the isotropic moment of $E$. Furthermore, since displacement goes as the vertical structure function $G(z)$ while velocity goes as the vertical derivative $G_{z}(z)$, the velocity and displacement furnish independent pieces of information about the vertical structure of the motions. Since there are displacement measurements at 10 levels and velocity measurements at 5 , the largest number of modes one could expect to resolve is 15 .

In figure 8.6.1 is a table of ranks for a series of isotropic models applied to the $Q 1$ data (similar results are found for the $U 2$ array). The first row gives the results for three isotropic, vertically symmetric models. The models each have 16 parameters: the energy in each of the first fifteen baroclinic modes plus a spectral parameter that measures the level in modes 15 through 45 presuming a spectral slope of -2 (see figures 8.6.2-.4). All the models have an a priori spectrum $e^{(0)}(j)$ which is of the form

$$
e^{(0)}(j)=e_{*} \frac{2}{1+\left(j / j_{*}\right)^{-8}}
$$

where the roll-off mode number $j_{*}$ is always taken to be 3 . When the slope $s$ is taken to be -2 this is precisely the GM79 prediction discussed in chapter 1 . One would like the results to not depend on the choice of a priori spectrum, so much of this section is devoted to varying the a priori spectrum and characterizing the resulting changes.

The three models in figure 8.6.1 differ in the slope $s$ presumed for the a priori wavenumber spectrum. In order to investigate the properties of resolution without the 
complication of including the loss of resolution due to noise, an a priori spectrum that is a factor of ten higher than the true values is used. The first column in the table gives the results for a model where the a priori slope is zero. The slope of zero is equivalent to saying that wave energy is equally likely to be in any of the first fifteen modes. The rank of that system is 13.2 , which is somewhat short of predicted maximum value of 15 . Since the choice of mode spacing was done on theoretical grounds and had nothing to do with the shape of the array, it is not surprising that the the resolution does not reach the maximum. This is not a very realistic a priori spectrum, however. First of all, the a priori spectrum is much higher than that actually observed, thus the effects of noise are smaller than they would be for more realistic choices of spectrum. Increasing the effects of noise decreases the effective rank of the system. Secondly, to the extent that one believes that the GM79 spectrum essentially characterized the internal wave spectrum, there should be much less energy at higher wavenumbers than low wavenumbers (wavenumber slope of -2). Thus the less energetic high wavenumbers will be very hard to resolve, and the rank of the system will again decrease. The other two entries in the first row of the table demonstrate this effect. With an a priori wavenumber slope of -1 the rank of the system is reduced by 2.1 , and with an a priori slope of -2 the rank is reduced by 2.6 to arrive at a value of approximately 8 . Thus as far as measurements of a red wavenumber spectrum are concerned, the array is able to resolve half the number of model parameters given to it. Because noise effects have been minimized, lack of resolution is not so much an inability to measure energy at a particular wavenumber as it is an inability to distinguish between waves. This is the generalization of the well-known problem of aliasing that is found when doing Fourier analysis on periodic arrays. Since the array is far from periodic (and could never be, since the low modes cannot be characterized by a simple sinusoid), the confusion between waves is more complicated, but the principle remains the same. To the extent that the inverse cannot distinguish between waves, it uses the a priori spectrum to decide how distribute energy among them.

Since the model was presumed to be vertically symmetric, it only uses the real part of the data cross-spectra. If we complicate the model by allowing vertical asymmetry (non-zero $\Delta$ ), then it is possible to model the imaginary part of the array cross-spectra as well. The imaginary part, however, is much more strongly affected by noise than the real part. This can be understand by considering the coherence between two like (e.g. temperature with temperature) sensors. When the two sensors are very close together then 


\section{Estimates of rank for isotropic model fits}

$$
Q 1 \text { data }
$$

$\begin{array}{lcccc}\text { slope: } & 0 & -1 & -2 & \text { Number of parameters } \\ E & 13.2 & 11.1 & 8.4 & 16 \\ E, \Delta & 19.8 & 16.6 & 12.6 & 31 \\ E, \Delta, P & 22.2 & 18.5 & 14.2 & 46 \\ E, \Delta, P, Q & 23.3 & 18.9 & 14.3 & 61\end{array}$

\section{Estimates of rank for isotropic model fits} $U 2$ data

$\begin{array}{lcccc}\text { slope: } & 0 & -1 & -2 & \text { Number of parameters } \\ E & 13.2 & 11.2 & 8.5 & 16 \\ E, \Delta & 19.4 & 16.4 & 12.6 & 31 \\ E, \Delta, P & 21.7 & 18.3 & 14.3 & 46 \\ E, \Delta, P, Q & 23.0 & 18.9 & 14.5 & 61\end{array}$

\section{Figure 8.6.1 Estimates of rank}

The table gives estimates of rank for a series of isotropic internal wave models. All the models consisted of the first fifteen baroclinic modes plus a spectral parameter than allowed energy in modes 15 through 45 presuming a spectral slope of -2 (which matches GM79). The $Q 1$ and $U 2$ arrays are used for the calculation. Cross-spectra at $.158 \mathrm{cph}$ were used to calculate the noise structure, though spectra at other frequencies could have been used to obtain similar results. 
the imaginary part is very small (it must be zero for zero separation). When the sensors are far apart the imaginary part is also very small. There can be some intermediate maximum, but the tendency for the imaginary part to be small means that it is strongly affected by noise. The second row of the table in figure 8.6.1 gives the results for such a model. At a spectral slope of zero there are 6.6 model parameters added in going from a symmetric spectrum to a possibly asymmetric spectrum - in the more realistic case of slope -24.2 parameters are added. Thus the difference between up and down $(\Delta)$ is poorly resolved relative to the sum of up and down $(E)$.

Another component of the wave models (spectral parameter $P$ ) allows distinguishing between modes and propagating waves. Note that the energy of the mode is already included in the model: adding the parameter $P$ asks whether modes are distinguishable from propagating waves. The possible modal effects are best understood by considering a simplified example. In a uniform medium, when the solutions are complex exponentials, the purely propagating model predicts a cross-spectra that depends only on the separation between sensors. To the extent that the measurements depend on absolute as well as relative position, a modal component to the model would be required. In the more general case the mandate for modes is less clear, though in light of the simplified problem it is certainly reasonable that some features of the data require a modal character. The third and fourth rows of the table show the effects of adding the mode and anti-mode to the model. The anti-mode is so poorly resolved as to be useless in the slope -2 case: thus it will be dropped from consideration for the rest of the chapter. The mode adds two resolved parameters to the model for all three choices of slope. Though the modal resolution is not very good when one considers the entire wavenumber range, to the extent that the energy at one or two wavenumbers can be separated into modal and propagating components it is worthwhile to distinguish between the two. 


\section{Inversion results with a known input}

A second test of resolution is to look at the response of the estimator to a known input. A unit energy version of the GM79 spectrum discussed in chapter 1 was chosen as a test spectrum. The GM79 spectrum is isotropic and vertically symmetric, thus the only non-zero coherences in the current meter cross-spectra are between like variables. Furthermore, their coherence as a function of WKBJ scaled vertical separation is given by a simple exponential (see section 1.6). The GM79 spectrum is a fair representation of the actual spectrum, so the model's ability to reproduce the GM79 spectrum should be a pretty good measure of its ability to represent the PEQUOD data. The sensor arrangement of the $U 2$ array is used in the following calculations.

To verify that the spectral inverse will in fact return the correct level in the absence of noise and with a large a priori model variance, a one parameter fit was tried, that one parameter being the level of a GM79 shaped spectrum. Since the model spectral shape and the data spectral shape match perfectly, one would expect an accurate estimate of the spectral level, and that is in fact what happens: the spectral inverse returns the correct level. Note that if the a priori model variance were not set to be quite large, the inverse would reduce the estimate somewhat and allow some of the energy to be interpreted as noise. This is because the inverse has been told that there is some noise, and this test case actually has none, so some of the signal would be misinterpreted. If noise were added to the test data this problem would be avoided.

The GM79 spectrum was then used to test the somewhat more complicated model used in the calculations of rank. That model has 16 parameters: the energy in each of the first fifteen baroclinic modes plus a parameter that gives the energy in modes 15 through 45 presuming a wavenumber slope of -2 and a WKBJ vertical structure. There are two reasons why the 16 parameter model would not necessarily reproduce the test data even if it accurately represents the oceanic wave field. One source of discrepancy is that the test data presumes a WKBJ vertical dependence in determining the mode structure while the 16 parameter model uses integrated baroclinic modes and thus does not make the WKBJ assumption in determining the low mode baroclinic structure. The high mode spectral parameter, on the other hand, is based on a WKBJ approximation, and thus can be expected to match the test data more closely. A more serious discrepancy is the inclusion of zero wavenumber energy in the test data, energy that is not expected in the real ocean. The zeroth mode does not exist in the model, thus the energetic zeroth mode 
is aliased into the modes that are present.

In order to demonstrate that the inversion is not sensitive to the choice of a priori spectrum, four runs are made which start with the same test data but have different a priori spectra. A fifth run which uses WKBJ-approximate waves to fit a slightly different test spectrum is made as well. The first run is shown in figure 8.6.2. Here the a priori spectrum has been chosen so that the a priori wavenumber slope is the same as the true slope, but the level is chosen to be a factor of ten higher than the true values. The result is a relatively poor estimate. Estimates for modes 6 and 14 are much too low and the estimate for the parameterized part is slightly too high. Unlike the single parameter problem, which is overdetermined and thus where the a priori spectrum has little effect, the baroclinic modes are not well resolved and an appropriate a priori spectrum is necessary to get good results.

The second run is given in figure 8.6.3. Here the a priori spectrum is chosen to be closer (but still higher than) the expected values. The high mode parameterization is exactly correct, thus the dotted line that gives its extrapolation to mode 1 gives the true level as well. There is now distinctly more energy in modes 6 and 10, though there is still a dip in the spectrum at mode 6 . The estimates here are much better, and to the extent that there are real model differences, may be as close a match as is warranted. The next two runs, however, show that there are some resolution problems in the baroclinic modes. This was predicted in the first part of this section which showed that only 8 of the 16 model parameters were resolved when the a priori slope is -2 .

The third run is given in figure 8.6.4. It shows the results when the a priori spectrum is approximately correct at low wavenumbers, but rather than having a wavenumber slope of -2 it has a wavenumber slope of -1 so that the high wavenumber values are incorrect. The estimates are fairly close to the estimates which had a priori slope -2 , which is very good: the estimates are not very sensitive to the choice of a priori energy distribution among the low modes.

The fourth run is shown in figure 8.6.5. Here the a priori spectrum has a slope of zero, and the spectral estimates are somewhat difference: the mode 8 value is distinctly higher than in the a priori slope -2 run and the parameterized high mode level has increased slightly. But even though the one could get bad estimates by using this a priori slope, it is quite clear from the results that slope 0 is not appropriate for this test data. So while the estimates in this case are not optimal, the estimator clearly indicates that 


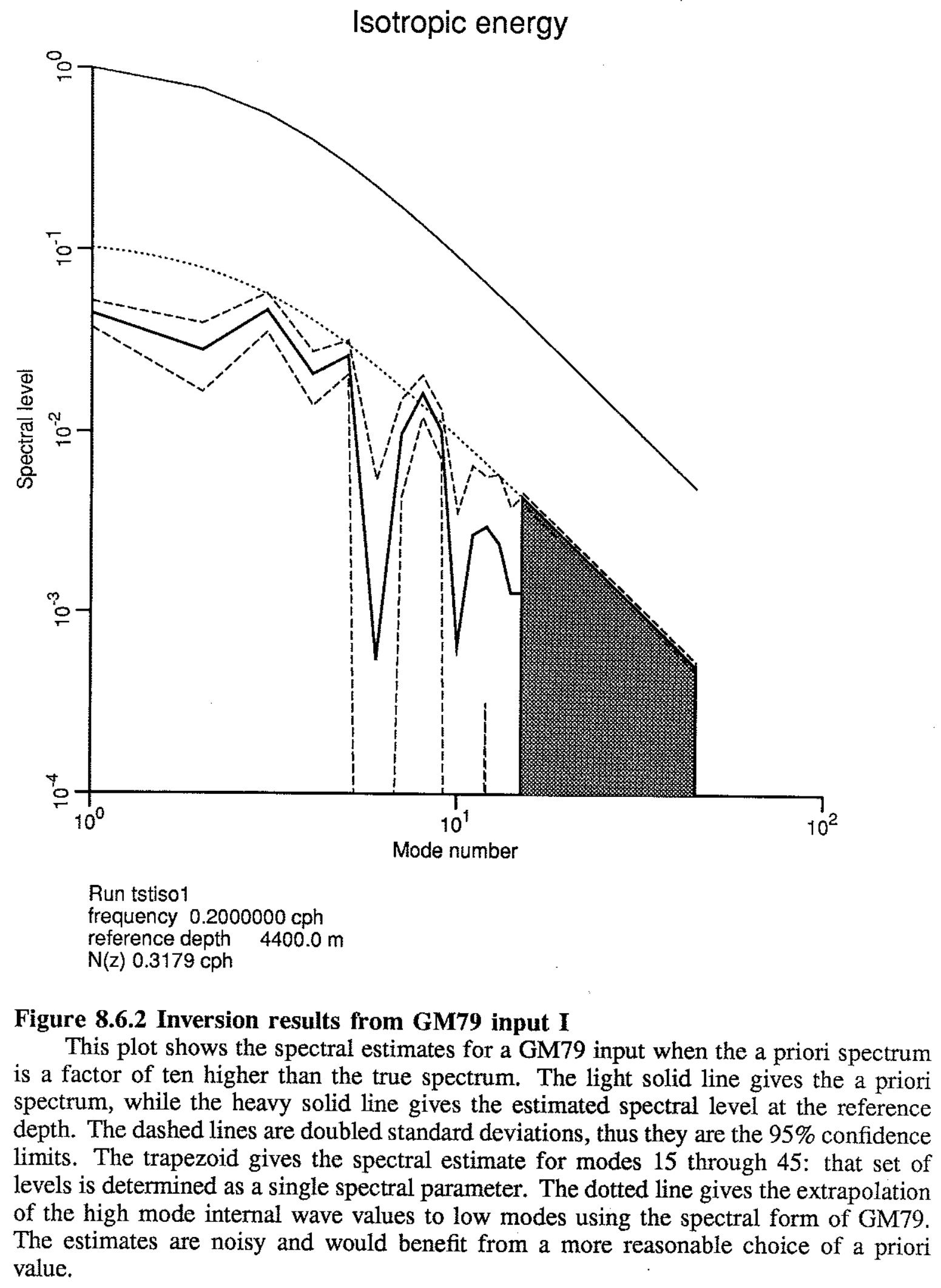




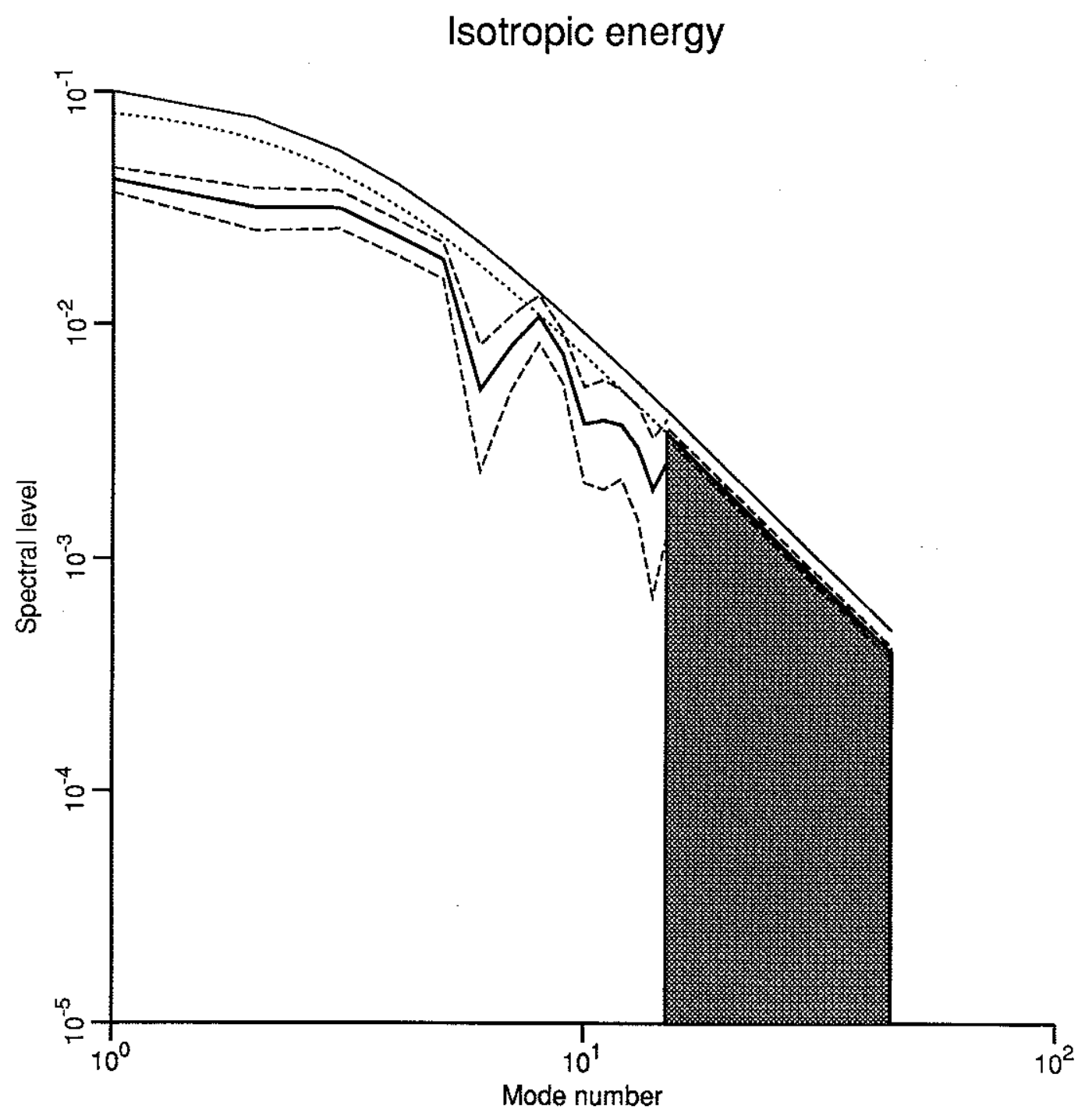

Run tstiso2

frequency $0.2000000 \mathrm{cph}$

reference depth $4400.0 \mathrm{~m}$

$\mathrm{N}(\mathrm{z}) 0.3179 \mathrm{cph}$

\section{Figure 8.6.3 Inversion results from GM79 input II}

This plot shows the spectral estimates for a GM79 input when the a priori spectrum is set slightly higher than the true spectrum. The light solid line gives the a priori spectrum, while the heavy solid line gives the estimated spectral level at the reference depth. The dashed lines are double standard deviations, thus they are the $95 \%$ confidence limits. The trapezoid gives the spectral estimate for modes 15 through 45: that set of levels is determined as a single spectral parameter. The dotted line gives the extrapolation of the high mode internal wave values to low modes. The estimates are similar to the estimates of figure 8.6.2, but the error bars are much smaller. 


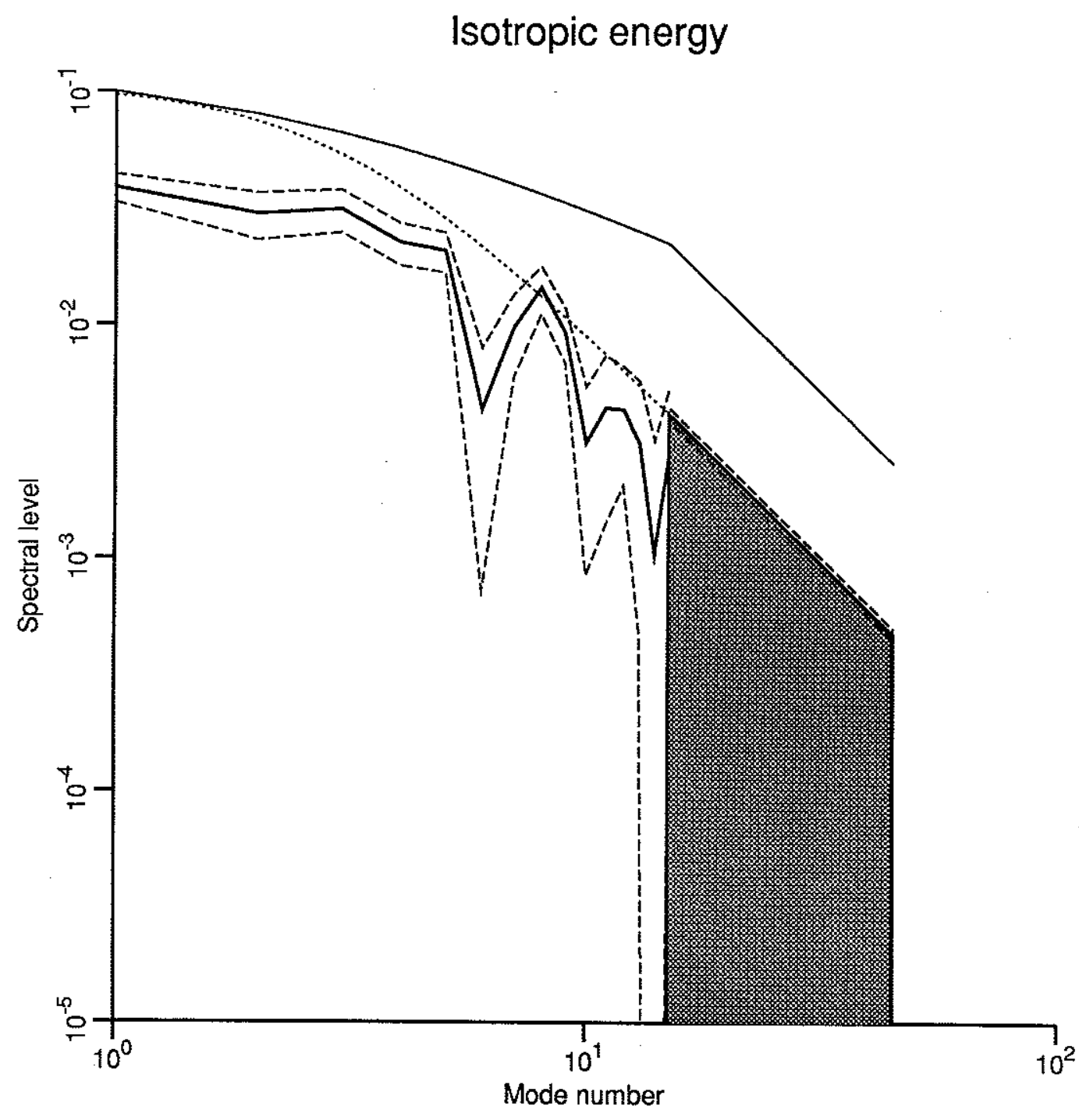

\section{Figure 8.6.4 Inversion results from GM79 input III}

This plot shows the spectral estimates for a GM79 input when the a priori slope for high wavenumbers is set to -1 rather than the true value of -2 . The light solid line gives the a priori spectrum, while the heavy solid line gives the estimated spectral level at the reference depth. The dashed lines are double standard deviations, thus they are the $95 \%$ confidence limits. The trapezoid gives the spectral estimate for modes 15 through 45 : that set of levels is determined as a single spectral parameter. The dotted line gives the extrapolation of the high mode internal wave values to low modes. The estimates differ primarily for modes 8 through 15: The low modes and the high mode parameterization are essentially unchanged. 
the choice of a priori spectrum is inconsistent with the true values.

By way of comparison, figure 8.6.6 show the fit of a 16 parameter WKBJ spectral model to a test spectrum that omits the zeroth mode. This is an important difference because the Desaubies 1976 form of the GM79 spectrum has most of the energy in the zeroth mode, a mode that Garrett and Munk explicitly exclude from their model. The zeroth mode is not in the 15 wave spectral model, thus any energy in that mode is misinterpreted as energy in other modes. The fit to the modified test spectrum is somewhat better and distinctly smoother despite the a priori level being much higher than the true value. This shows that most of the discrepancy seen in the other plots is due to side-lobes of the zeroth mode, a mode which should not be in the test spectrum. Thus one should be very careful in using the low wavenumber information in the Desaubies 1976 spectral form.

In conclusion, given test inputs the inverse returns exactly correct values when the model is identical to the test input, and roughly correct values when the model has a more approximate match. The model is not particularly sensitive to the choice of a priori level: it gives roughly the same results for a priori slopes of -2 and -1 and definitely indicates that an a priori slope of 0 is inappropriate for the test data. To the extent that the test data (GM79) is a good representation of the data, then, the inverse will return fairly accurate results for the isotropic vertically symmetric portion of the wavenumber spectrum. 


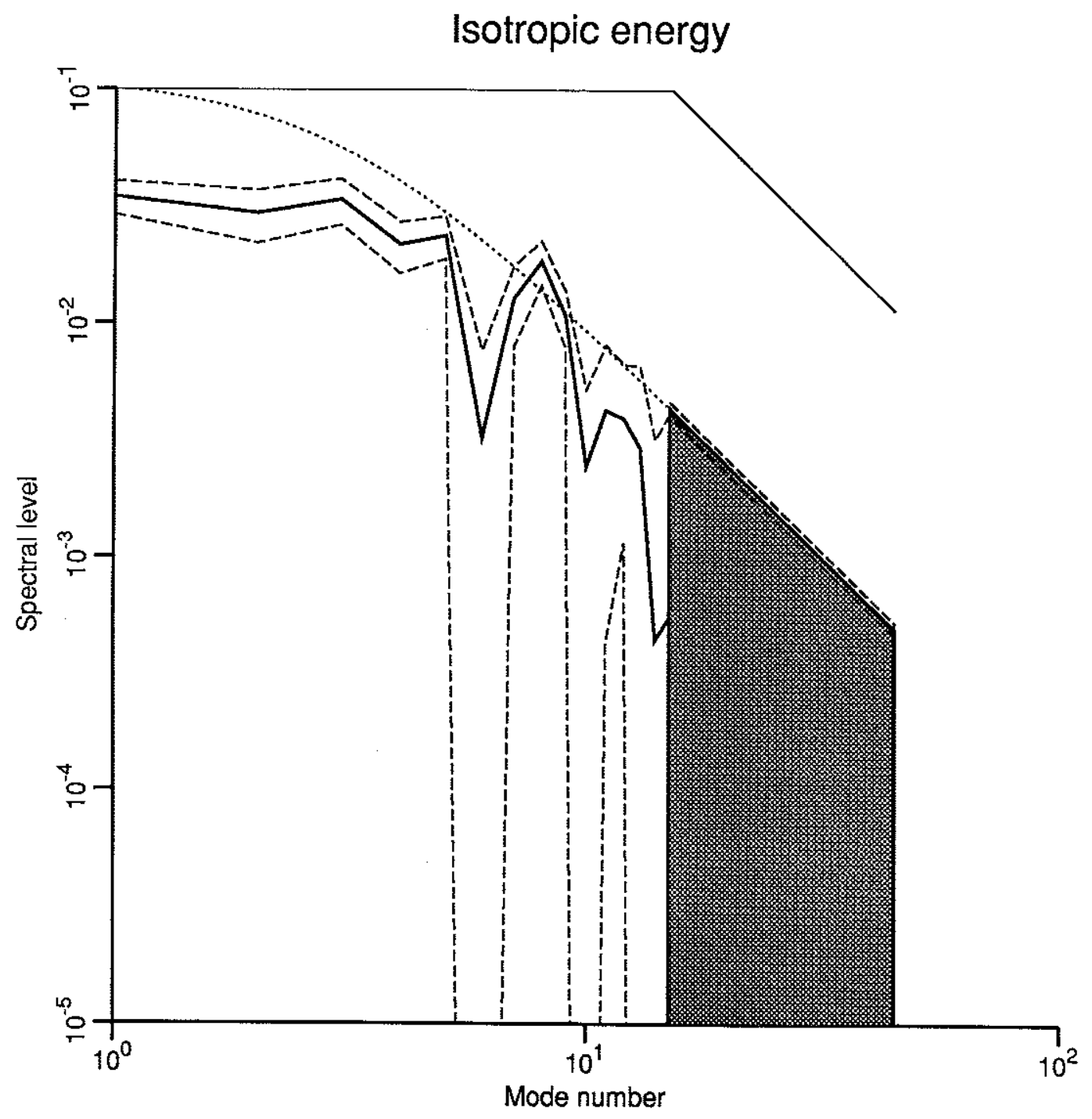

Run tstiso4

frequency $0.2000000 \mathrm{cph}$

reference depth $4400.0 \mathrm{~m}$

$\mathrm{N}(\mathrm{z}) 0.3179 \mathrm{cph}$

\section{Figure 8.6.5 Inversion results from GM79 input IV}

This plot shows the spectral estimates for a GM79 input when the a priori levels are such that energy is equally distributed among the first fifteen modes. The light solid line gives the a priori spectrum, while the heavy solid line gives the estimated spectral level at the reference depth. The dashed lines are double standard deviations, thus they are the $95 \%$ confidence limits. The trapezoid gives the spectral estimate for modes 15 through 45: that set of levels is determined as a single spectral parameter. The dotted line gives the extrapolation of the high mode internal wave values to low modes. The estimates show fairly clearly that a wavenumber slope of 0 is inappropriate for the test data. 


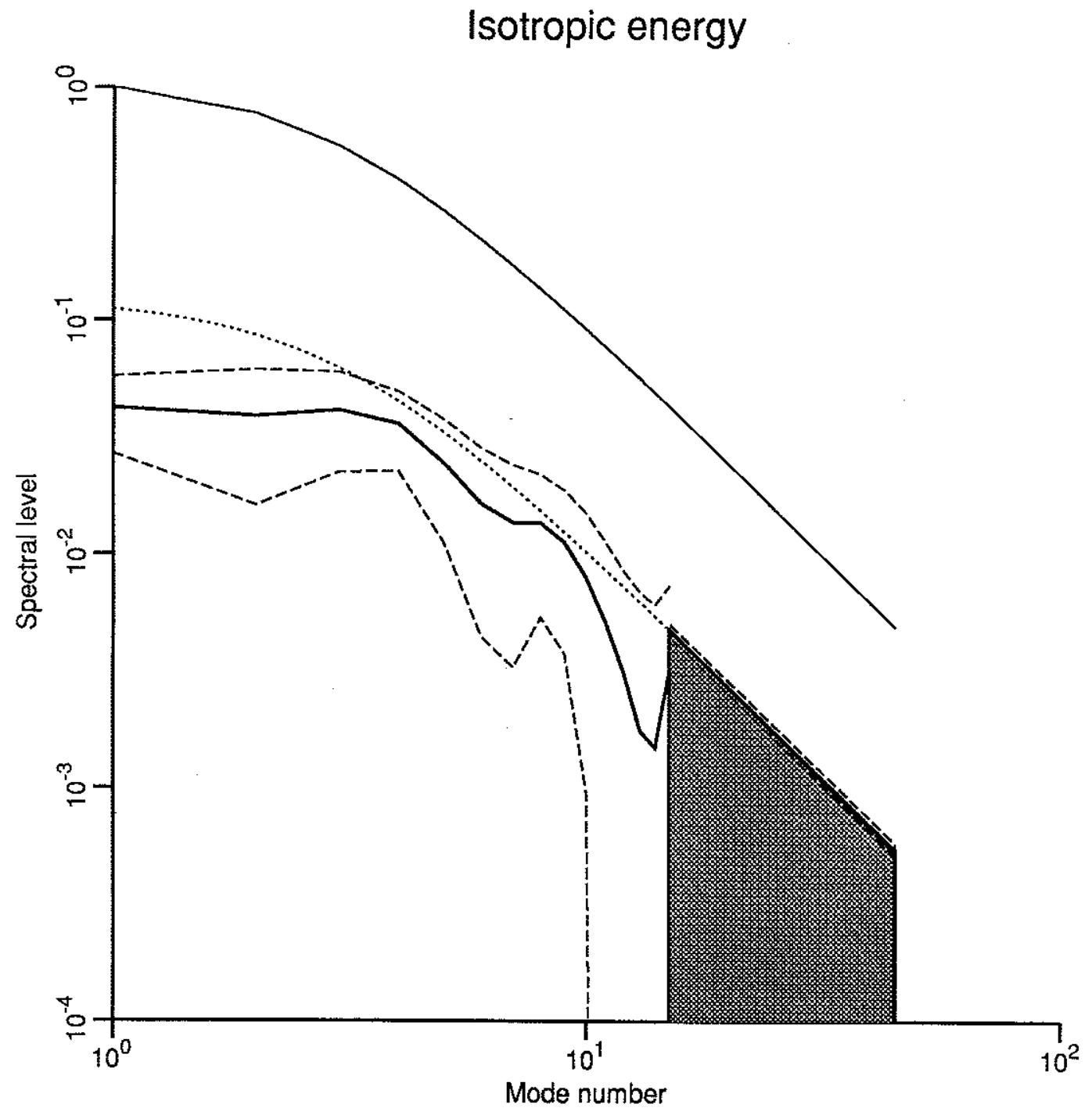

Run tstisoh1

frequency $0.2000000 \mathrm{cph}$

reference depth $4400.0 \mathrm{~m}$

$\mathrm{N}(\mathrm{z}) 0.3179 \mathrm{cph}$

Figure 8.6.6 Inversion results from modified GM79: WKBJ modes

This plot shows the spectral estimates for a GM79 input modified by removing the zeroth mode energy. The model has been modified in that it used a WKBJ approximation rather than a pure integration to determine the vertical structures that correspond to low wavenumbers. The light solid line gives the a priori spectrum, while the heavy solid line gives the estimated spectral level at the reference depth. The dashed lines are double standard deviations, thus they are the $95 \%$ confidence limits. The trapezoid gives the spectral estimate for modes 15 through 45: that set of levels is determined as a single spectral parameter. The dotted line gives the extrapolation of the high mode internal wave values to low modes. Despite the a priori spectrum being much higher than the estimates, the estimates are quite smooth, unlike figure 8.6.2. 


\section{Impulse response}

A third perspective on resolution is to consider the response to a unit impulse at a particular wavenumber. This is similar to calculating the "beam pattern" when using the beam forming estimator discussed in section 8.4. Since understanding all the impulse responses is sufficient to predict all the responses of the model, attaining such an understanding can be rather difficult. To simplify the problem somewhat, only the isotropic response will be considered. All the models discussed have the same 16 wavenumbers parameters as the model introduced in the discussion of model rank: wavenumbers that correspond to the first fifteen baroclinic modes plus a high mode spectral parameter which represents energy in modes 15 through 45.

In considering a series of impulses, it is most natural to consider a white spectrum, i.e. all the pulses are the same size. Note that this does not match our a priori notion that the wavenumber spectrum is red, but the calculation does provide useful information about the resolution of the array. Figure 8.6.7 shows the symmetric response $(E)$ to upward propagating impulses at each of fifteen mode numbers when the a priori spectrum is white and set very high. Note that the total response to each impulse is fairly close to one: this means that while there is some confusion between modes, none of the energy is interpreted as noise in this effectively noise free case. The response is concentrated along the diagonal as it should be, but the peak value along the diagonal is .8 , not the 1 of perfect resolution. There is also a low (.1) off diagonal peak that means some of the mode 9 through 11 energy is perceived as modes 1 through 3 . The fact that the peak value is .8 means that at best $20 \%$ of the energy is spread into other modes: for the modes that have peak values of .6 $40 \%$ the energy is lost. Note that much of the lost energy shows up in neighboring modes which mitigates the resolution problem as long as we find smooth solutions. The plot suggests that modes 4 though 14 are the best resolved of the array.

Figure 8.6.8 shows the $E$ response to upward impulses for an $E \Delta P$ model - i.e. the correlation between up and down that characterizes modes is allowed. Because the modal character is poorly resolved, the $E$ resolution is somewhat degraded. The total response for each upward impulse is not as close to one as it is in the $E \Delta$ (purely propagating) model. This is because two waves that are correlated generate more response than two independent waves, thus misinterpreting upward energy as modal energy results in a lower amplitude. The peak values of the symmetric response are now .6 rather than the 


\section{UZIAU2}

E response to up forcing

19.4 resolved model porometers out of 31 .

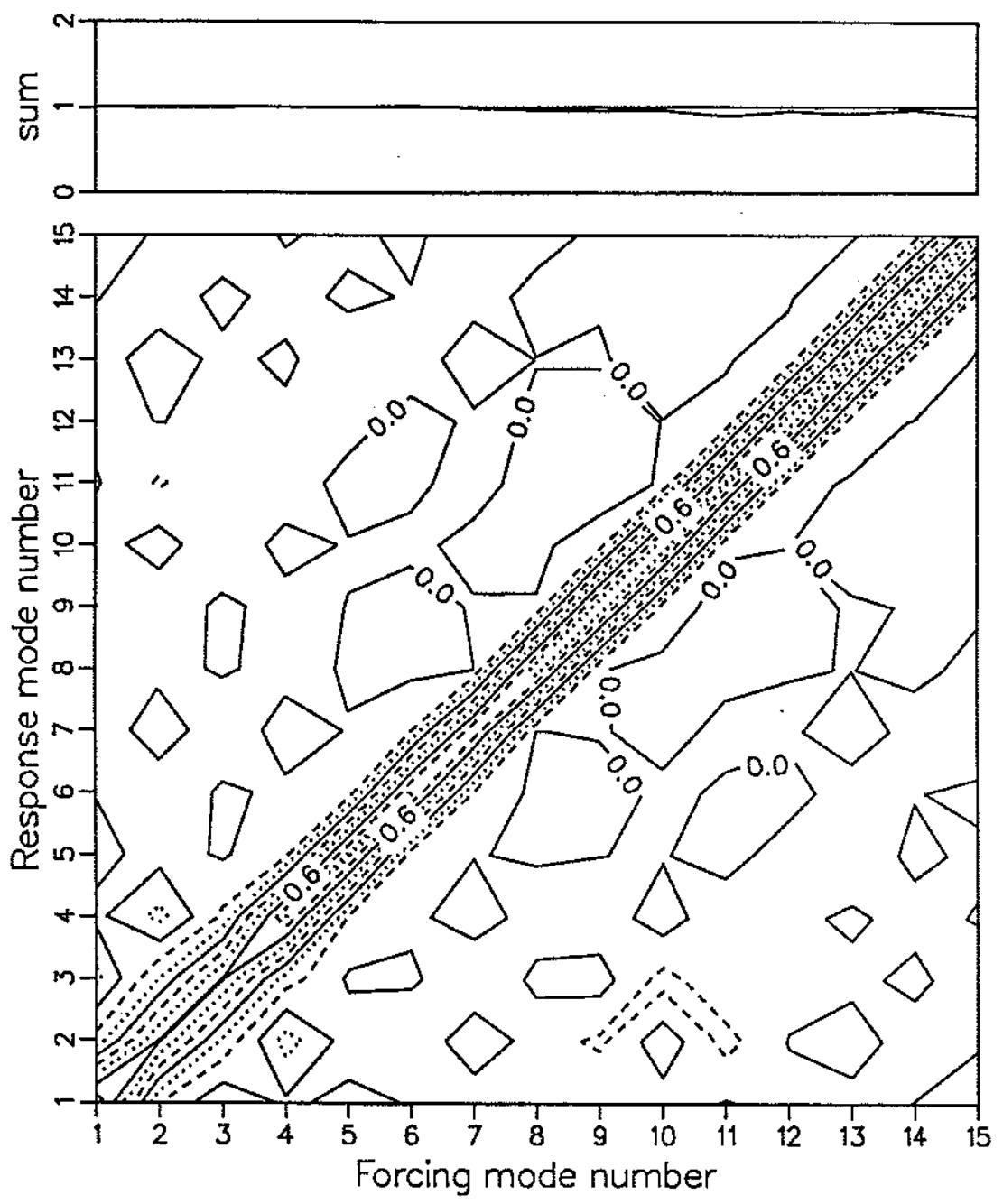

Figure 8.6.7 Isotropic $E$ response to unit impulses

This figure shows the $E$ response to unit energy upward propagating waves at wavenumbers that correspond to each of the first fifteen baroclinic modes. The upper plot gives the response summed over the first fifteen mode numbers while the bottom plot shows how that response is distributed with mode number. The total response is quite close to one, which means that little energy is lost to noise. The response is concentrated along the diagonal, which means that most of the energy in a particular mode is perceived as being in that mode, but the peak value of .8 indicates that at least $20 \%$ of the energy leaks into neighboring modes. 


\section{U2IAU3}

E response to up forcing

21.7 resolved model parameters out of 46 .

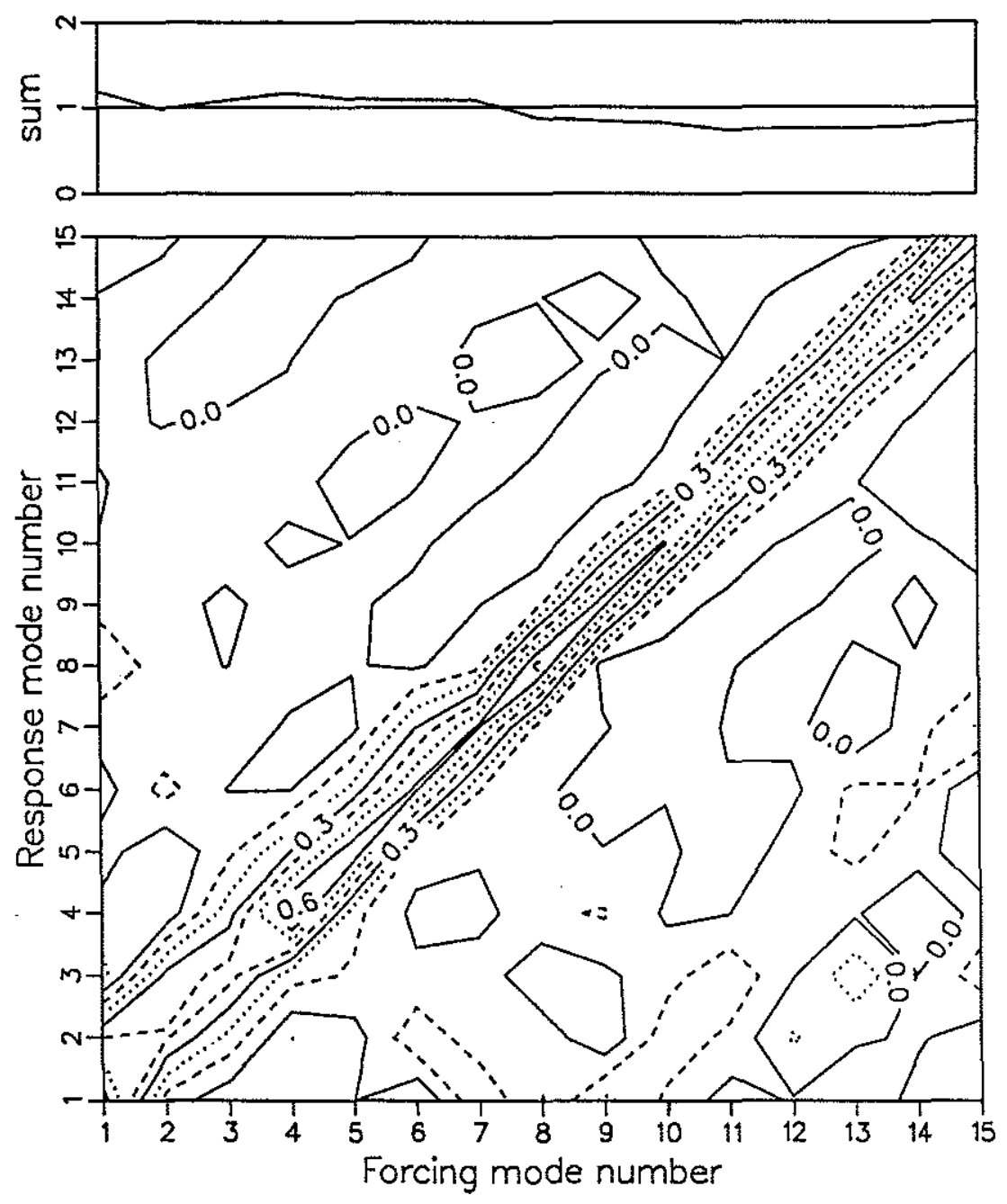

Figure 8.6.8 Isotropic $E$ response to unit impulses

This figure shows the $E$ response to unit energy upward propagating waves for an $E \Delta P$ model. Wavenumbers included correspond to each of the first fifteen baroclinic modes. The upper plot gives the response summed over the first fifteen mode numbers while the bottom plot shows how that response is distributed with mode number. The total response shows some variation from one, which means some energy is misinterpreted as being modal in character. The response is concentrated along the diagonal, which means that most of the energy in a particular mode is perceived as being in that mode, but the peak value of .6 indicates that at least $40 \%$ of the energy leaks into neighboring modes. 
.8 of the purely propagating model, which means that as least $40 \%$ of the energy can be misinterpreted as other waves or modes.

Figure 8.6.9 shows the $\Delta$ response to unit energy upward propagating waves. While this plot is calculated for a $E \Delta P$ model, the plot for a purely propagating $(E \Delta)$ model is nearly the same. This can be understood by recalling that $\Delta$ is determined by the imaginary part of the current meter cross spectra while $E, P, Q$ are determined by the real part. Thus there is no possibility of confusion between $P$ and $\Delta$, and the presence or absence of $P$ does not affect the resolution of $\Delta$.

Were the resolution of $\Delta$ perfect, the contours in figure 8.6 .9 would be concentrated near the diagonal and would have a peak value of one. As we know from the calculations of rank done earlier, the resolution of $\Delta$ is not perfect. This is seen in figure 8.6.9 as well. The total response to each impulse is distinctly less than one for all modes below mode 10, and, while the totals are better for modes 10 and higher, the resolution for higher modes is not particularly good. The peak values of the contours are somewhat less than .5 , which means that there is really not much ability to distinguish between neighboring wavenumbers. Note that there is very little aliasing per se: only wavenumbers 5 and 6 are partially perceived as negative (downward) mode 3 .

It is also possible to consider the resolution when the a priori spectrum is red rather than being white. This more closely corresponds with our a priori notions of what the wavenumber spectrum should be like. A red a priori spectrum means that the higher modes will be less well resolved because given a choice of putting energy into low wavenumbers or high wavenumbers the inverse will choose to put the energy into low wavenumbers. The resulting resolution patterns are much more complicated, Figure 8.6.10 shows the $E$ response to upward impulses for a $E \Delta$ model when the a priori spectrum has a slope of -2 . Note that the corresponding plot for a $E \Delta P$ model is pretty much the same though the peak values are slightly reduced: the slope of -2 has eliminated some of the ambiguity introduced by the modal parameter $P$. The best resolved modes are 1 and 2, 4 through 6, and much of the energy put in at high wavenumbers is interpreted as being in low wavenumbers. Figure 8.6.11 shows the $\Delta$ response for the same model. The integrated response to the three lowest mode numbers is close to one, somewhat closer than with the white a priori spectrum used to calculate figure 8.6.9. While the peak response for these wavenumbers is only .5, the contour plot indicates that most of the lost response is in neighboring modes, so the lack of resolution does not 


\section{U2IAU3}

$D$ response to up forcing

21.7 resolved model parameters out of 46 .
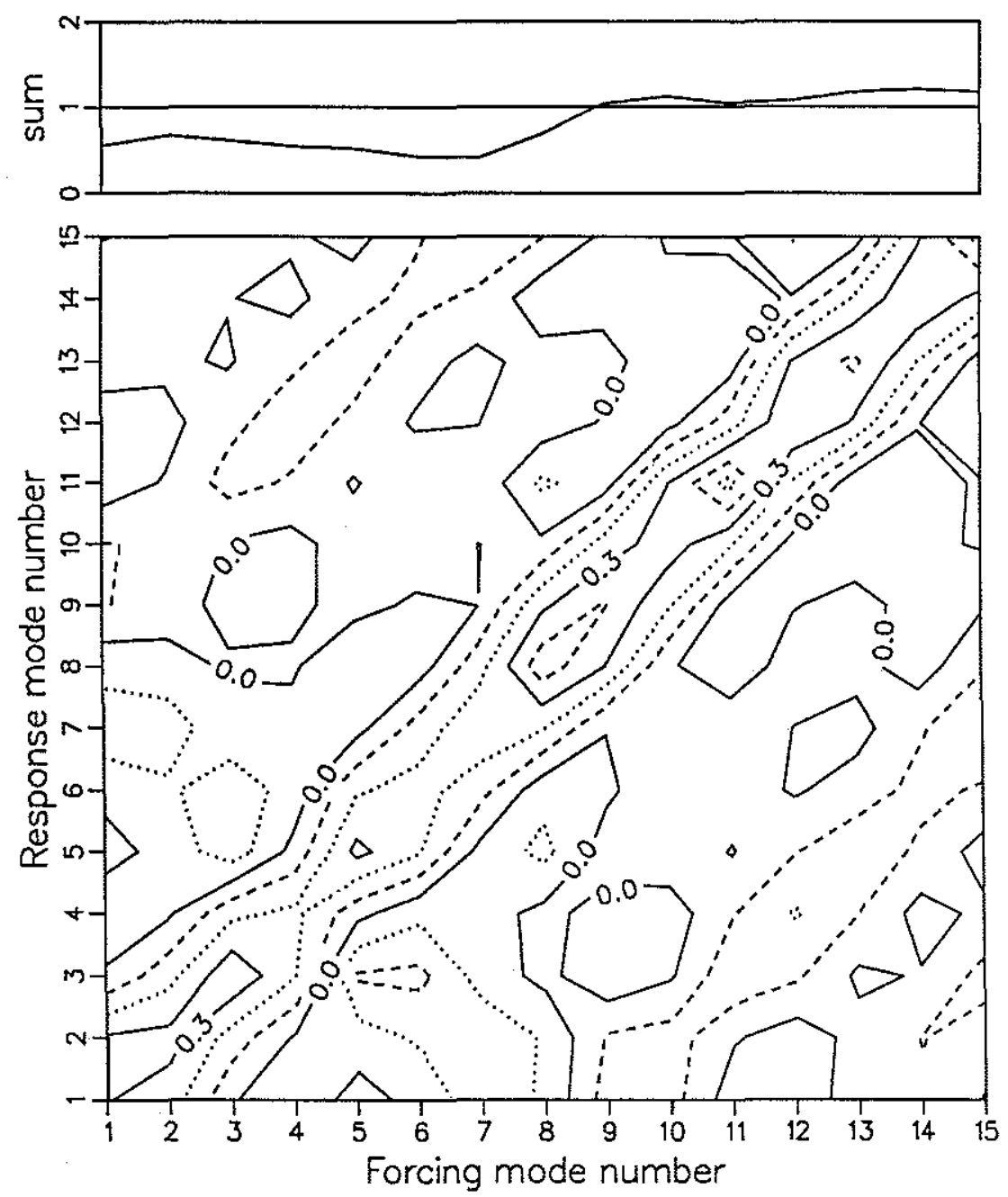

\section{Figure 8.6.9 Isotropic $\Delta$ response to unit impulses}

This figure shows the $\Delta$ response to unit energy upward propagating waves at wavenumbers that correspond to each of the first fifteen baroclinic modes. The upper plot gives the response summed over the first fifteen mode numbers while the bottom plot shows how that response is distributed with mode number. This plot confirms the calculations of rank which predicted that the resolution of $\Delta$ would not be particularly good. 


\section{U2IAS2}

E response to up forcing

12.6 resolved model parameters out of 31 .
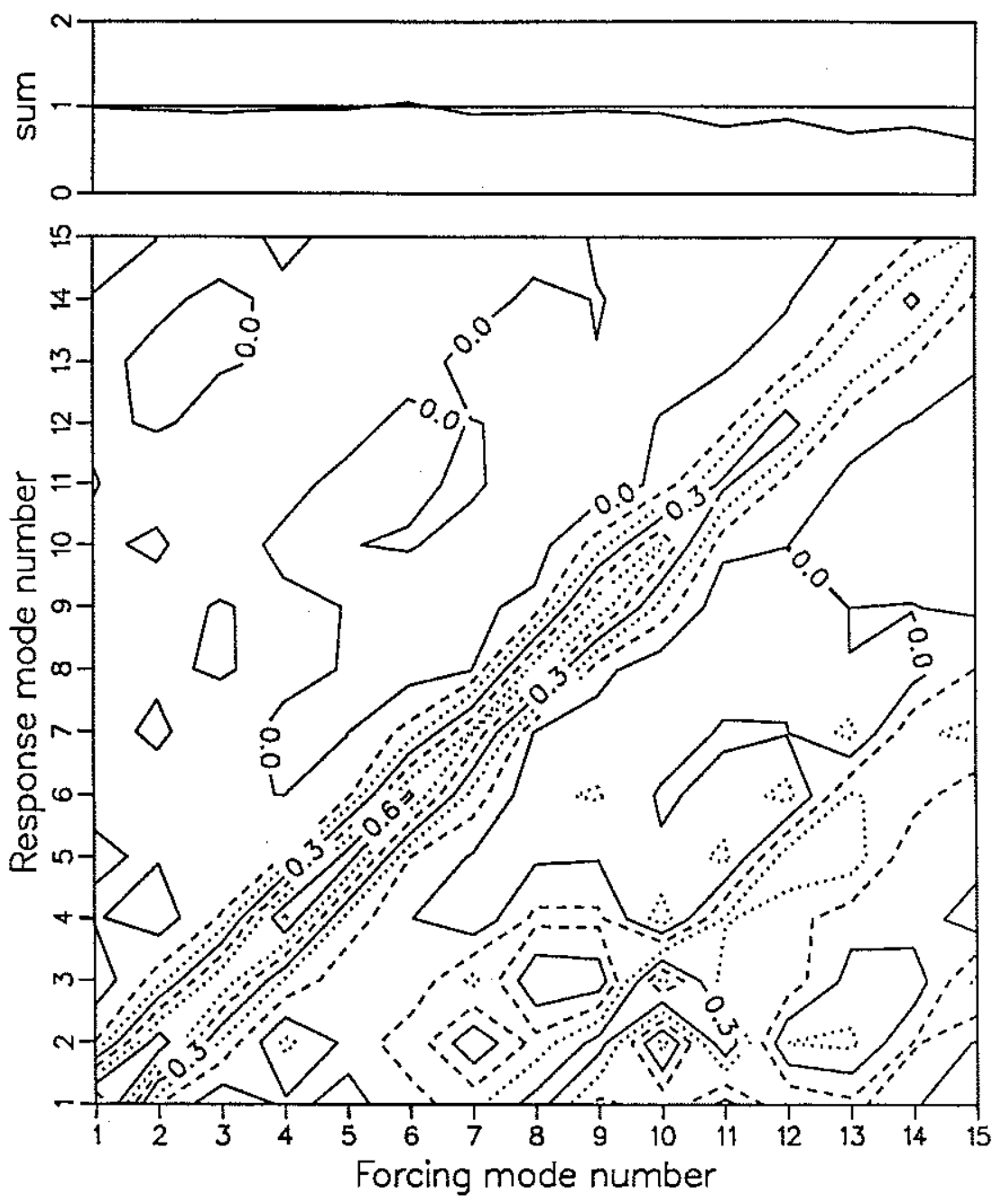

Figure 8.6.10 Isotropic $E$ response to unit impulses

This figure shows the $E$ response to unit energy upward propagating waves for a $E \Delta$ model which has a red a priori spectrum (slope -2). The upper plot gives the response summed over the first fifteen mode numbers while the bottom plot shows how that response is distributed with mode number. Modes 1,2 and 4 through 6 are best resolved, and most of the energy put in in the higher modes is interpreted as low mode energy. 
U2IAS2

$D$ response to up forcing

12.6 resolved model parameters out of 31 .
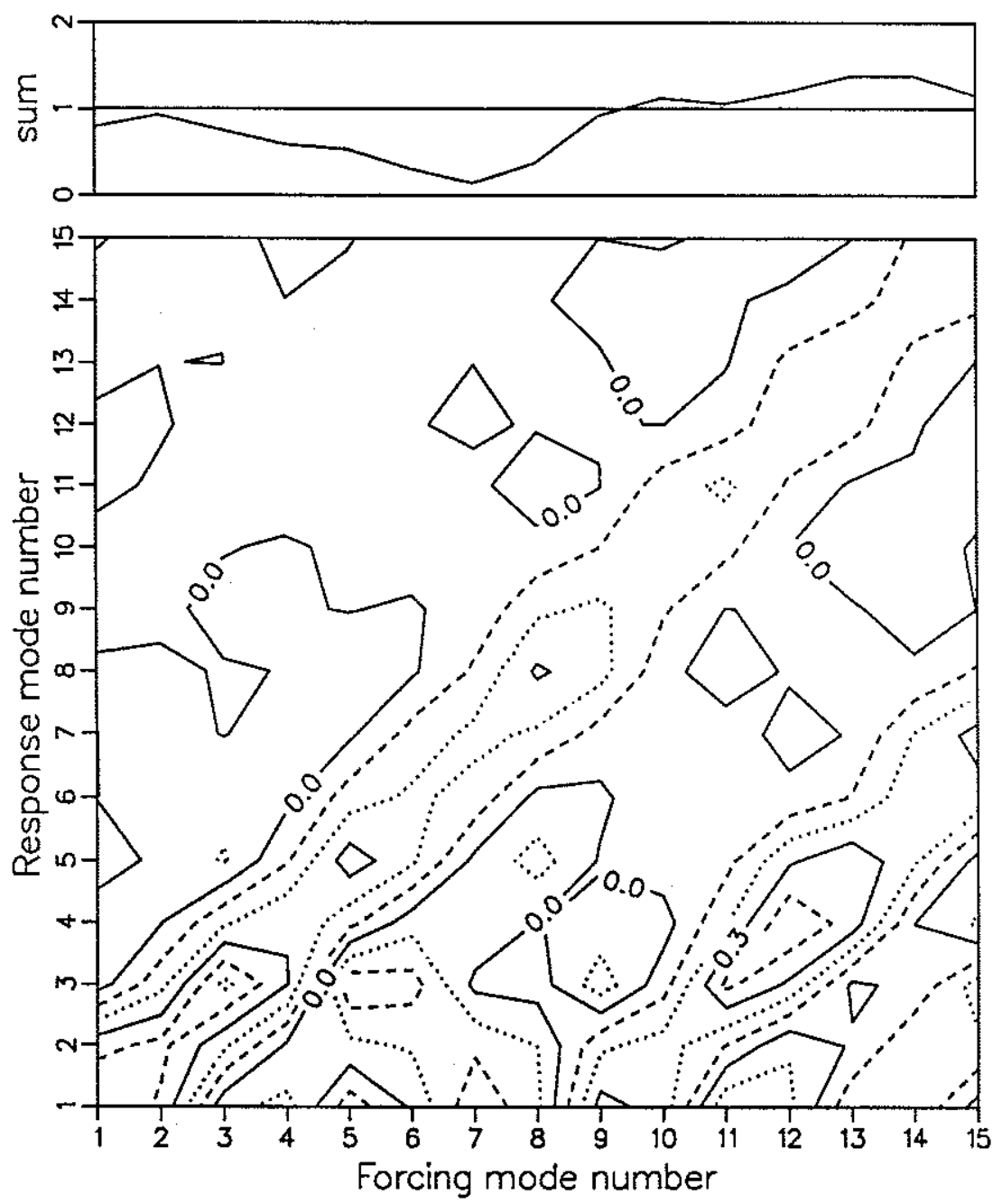

Figure 8.6.11 Isotropic $\Delta$ response to unit impulses

This figure shows the $\Delta$ response to unit energy upward propagating waves for a $E \Delta$ model which has a red a priori spectrum (slope -2). The upper plot gives the response summed over the first fifteen mode numbers while the bottom plot shows how that response is distributed with mode number. The plot shows that net vertical fluxes cannot be resolved at high wavenumbers. On the other hand, since most of the low wavenumber leakage is into neighboring wavenumbers, net vertical fluxes can be resolved at low wavenumbers. 
prevent measuring net vertical fluxes at low wavenumbers. High mode numbers, on the other hand, are strongly aliased into low mode numbers, thus net vertical fluxes at high wavenumbers are not measureable.

\subsection{Inversion results}

In chapter 1, the Garrett and Munk (GM) model is introduced as a universal description of the world's internal wave field. The calculations in the chapters that follow show that the GM model cannot fully characterize the internal wave field as measured by the PEQUOD mooring data. Different inadequacies are demonstrated in different chapters. In considering coherence as a function of vertical separation, chapter 3 finds that a WKBJ model with only propagating waves is inadequate and vertical standing modes must also be included. The single meter cross spectra of chapter 4 show that an isotropic internal wave field in a resting basic state cannot be considered consistent with the data: either the effects of horizontal anisotropy or the kinematic effects of a mean shear flow are necessary. The consistency checks of chapter 6 verify and expand upon the results of chapter 4, showing that not only are horizontal and vertical asymmetries required, some shear kinematics are required as well. This section constructs a series of models that progressively incorporate the spectral features that the earlier chapters suggest are required. The results of earlier chapters are reproduced in that the simpler models are shown to be inadequate, but this section goes further by actually constructing internal wave fields that have the additional structures necessary to improve the fit between the models and the measurements.

For the calculations in this section, the data has been broken down into eight threemonth pieces: four from the first year $Q$ mooring data, and four from the second year $U$ mooring data (see table 8.7.1). Considering model fits to subsets of the yearlong time series has two important advantages over considering only yearlong pieces. Since the usable portions of the $U$ and $Q$ moorings are slightly different, any differences between the internal wave fields measured by the two moorings could be due to differences in mooring arrangement or mooring placement as well as actual differences in the ocean state. However differences between three month pieces taken at the same array are due to differences in the ocean state. Secondly, the three month pieces offer time resolution of the internal wave field: long term changes in the mean ocean state (such as the onset of El Niño during the second half of the PEQUOD experiment) are potential modifiers of 


\section{Table 8.7.1 Times included in spectral pieces}

$\begin{array}{ccc}\text { Piece } & \text { Mooring } & \text { Time } \\ 1 & \mathrm{Q} & \text { 24 January 1981 through 24 April 1981 } \\ 2 & \mathrm{Q} & \text { 24 April 1981 through 23 July 1981 } \\ 3 & \mathrm{Q} & \text { 23 July 1981 through 21 October 1981 } \\ 4 & \mathrm{Q} & \text { 21 October 1981 through 19 January 1982 } \\ 5 & \mathrm{U} & \text { 18 February 1982 through 19 May 1982 } \\ 6 & \mathrm{U} & \text { 19 May 1982 through 17 August 1982 } \\ 7 & \mathrm{U} & \text { 17 August 1982 through 15 November 1982 } \\ 8 & \mathrm{U} & \text { 15 November 1982 through 13 February 1983 }\end{array}$

the internal wave field, and the time resolution offered by the three month pieces creates an opportunity to correlate changes in the wavefield with changes in the mean ocean state. In the PEQUOD data, some features observed in the internal wave field seem strongly tied to the phase of the El Niño cycle.

The residuals are the differences between a given model's prediction for the current meter cross spectra and the measured values. The usable portions of both the $Q$ and $U$ moorings both contain twenty time series, so the cross spectra to be modeled at each frequency (and thus the residuals) consist of 400 real numbers. In order to grossly characterize this large set of numbers, a single number, the residue, is defined to be,

$$
\chi^{2}=\sum_{i j k l}^{N_{t \beta}} \epsilon_{k i} Q_{i j} Q_{k l} \epsilon_{j l}
$$

where $\epsilon_{j l}$ is the residual that corresponds to the cross-spectral estimate $\left\langle d_{j}^{*} d_{l}\right\rangle$, and the inverse covariance $Q_{i j}$ is given in equation 5.4.8. There are several important points to be made about the residue. In order for a model fit to be considered successful, the residuals should correspond to noise. The noise in the cross spectral estimators can be considered to be approximately joint-normally distributed in the limit where the amount of averaging $N_{r}$ is high, where the covariance between the noise in any two spectral estimators is given by equation 5.4.8. Therefore, if the residuals are due purely to noise in the cross-spectral estimators, the residue $\chi^{2}$ is the properly weighed sum to evaluate the likelihood of those residuals occurring, i.e. the probability of that set of residuals occurring is proportional to $e^{-\frac{1}{2} x^{2}}$.

The maximum likelihood corresponds to the minimum value of the residue. The estimator used in this section and derived in section 8.5 minimizes the sum of the residue 
and a corresponding model space term, namely

$$
\chi^{2}+s_{\alpha} M_{\alpha \beta} s_{\beta}
$$

the second (model space) term being included to insure that the problem is well-posed (see sections 8.4 and 8.5). Thus as long as $M_{\alpha \beta}$ is chosen such that the second term does not dominate the first, the solution produced by the inversion will tend to minimize the residue $\chi^{2}$.

Figure 8.7.1 presents the residue as a function of frequency from fits of a series of models to the data of one three month period. Rather than plot $\chi^{2}$ directly, it turns out to be more convenient to plot a normalized version $\chi_{r}^{2}=\chi^{2} / N_{t s}^{2}$, i.e. the residue normalized by the number of real random variables involved. On the plot there are several lines that are included for reference. The line marked 'Theoretical signal' is the residue for a null model when the true covariances are known, i.e. the residuals are taken to be the true cross spectra. It can be computed explicitly, namely

$$
\begin{aligned}
\chi_{r}^{2} & =\sum_{i j k l}^{N_{t s}} f_{k i}^{(0)} Q_{i j} Q_{k l} f_{j l}^{(0)} / N_{t s}^{2} \\
& =N_{r} / N_{t s}
\end{aligned}
$$

Thus the theoretical signal turns out to be the number of spectral realizations divided by the number of time series one is attempting to resolve. The variation of the theoretical signal with frequency seen in the plot reflects the increased amount of spectral averaging used with higher frequencies.

The 'Null model' differs from the theoretical signal only in that it reflects our imperfect knowledge of the noise statistics of the cross-spectral operators. The residue as defined in equation 8.7.1 depends on knowing the inverse $Q_{i j}$ of the true covariance $f_{i j}^{(0)}$. In practice $Q_{i j}$ is not known and one has to make some choice. The choice made here was to estimate $Q_{i j}$ for the data from any particular three month period from the year long average which includes the period in question. In particular, $Q_{i j}$ is taken to be the inverse of

$$
\sqrt{N_{r}}\left(\left\langle d_{j}^{*} d_{l}\right\rangle+\lambda / \sqrt{N_{r}}\right)
$$

where the averaging is done over one year and $\lambda=\sum_{i}\left\langle d_{i}^{*} d_{i}\right\rangle / N_{t s}$ i.e. $\lambda$ is the mean energy. Note that the contribution of $\lambda$ is quite small once $N_{r}$ is large. The $\lambda$ term 


\section{Residues}

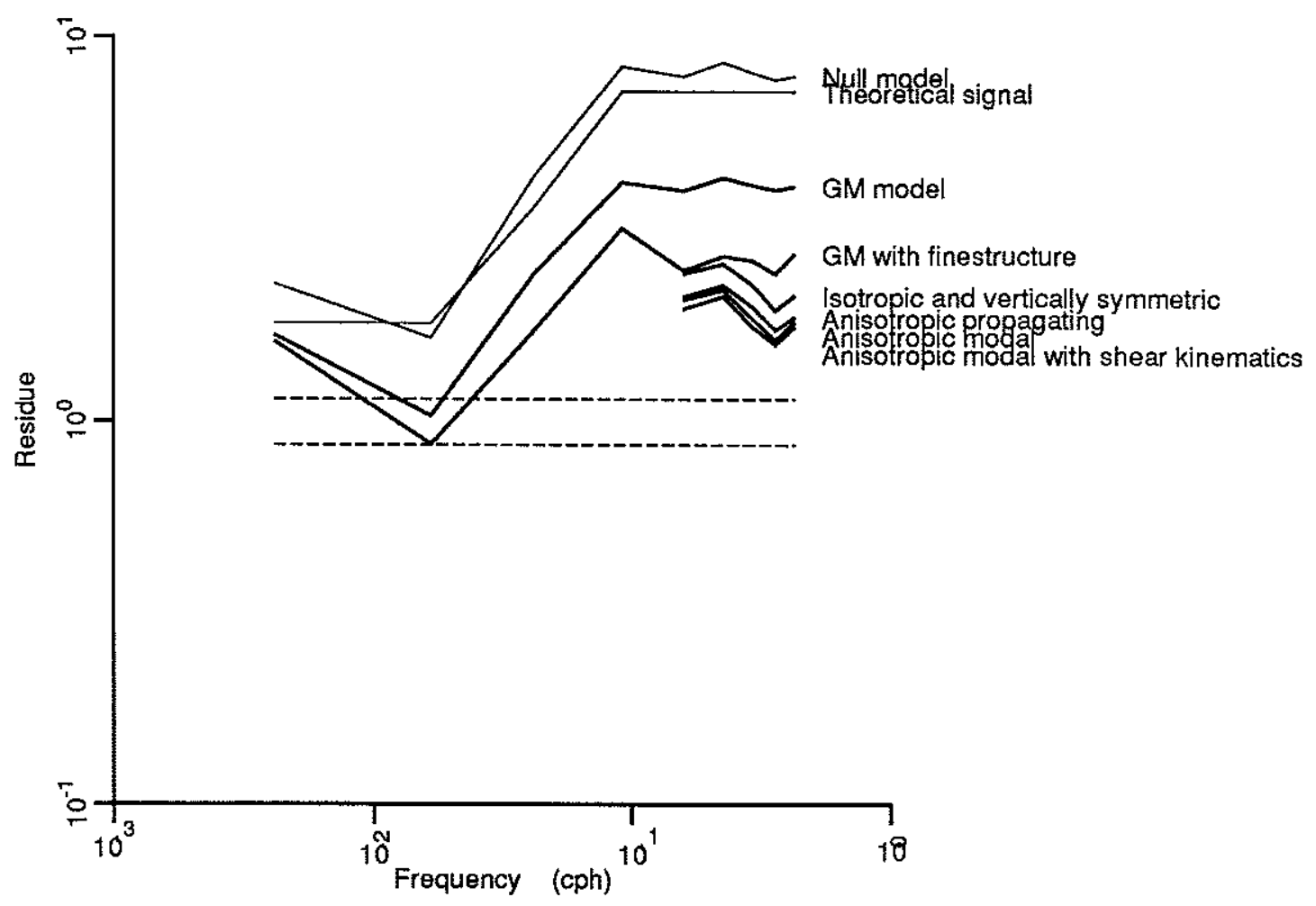

q1pin: 24 January 1981 through 24 April 1981 dashed lines are $95 \%$ confidence limits for noise

\section{Figure 8.7.1 Residues from piece 1}

The plot shows the residue from a series of model fits to the first spectral pieces, i.e. the $Q$ mooring from 24 January 1981 through 24 April 1981 . 
is included because it is the size of the uncertainty in the estimate of the cross spectra and thus represents the noise (and thus additional energy) introduced into the problem by using an estimate for $Q$ rather than the true value. The 'null model' line is thus the residue from the null model, i.e. the residuals are taken to be the actual data and the chosen form (8.7.5) is used for $Q$. For this piece the null model residue is quite close to the theoretical signal, suggesting that the choice of $Q_{i j}$ is appropriate.

The dashed lines give the theoretical value of the noise level: our imperfect knowledge of $Q$ means the calculation is not rigorous (the difference between 'Null model' and 'Theoretical signal' suggests the noise level should at least be doubled). The noise level is derived by noting that $\chi^{2}$ is the sum of $N_{t s}^{2}$ squared real Gaussian random variables, thus it is a random variable distributed according to a chi-squared distribution with $N_{t s}^{2}$ degrees of freedom. The normalized version of the residue $\chi_{r}^{2}$ is accordingly distributed as a reduced chi-squared random variable (see Bevington 1969). If the residuals are purely due to noise, the expected value of $\chi_{r}^{2}$ is one, while the $95 \%$ confidence limits about that value provide the basis of the dashed lines in the figure.

The remaining lines on the figure give the residue for various models of the equatorial internal wave field. The line marked 'GM model' gives the residue for a one parameter model that has the same coherence structure as the GM model (figures 1.6.1 and 1.6.2). The internal wave field is thus horizontally isotropic, vertically symmetric and purely propagating with a WKBJ approximate vertical structure. The one undetermined parameter is the GM level at the frequency of interest (GM level is defined to be the spectral level in a $1 \mathrm{cph}$ ocean, see section 4.3). Note that this computation of GM level differs from the computations done in chapter 4 . In chapter 4, the kinetic and potential energies are measured using a single current meter and combined to form an estimate of the GM level. Here all of the available cross spectra are considered and the GM level is chosen to minimize the total residue. Figure 8.7.1 shows that the GM model accounts for approximately half the null model residue. This result is typical of all eight pieces.

A plot of GM spectral level as a function of frequency and season is given in figure 8.7.2. GM79 predicts that the spectrum should have a slope of -2 with a level such that it is $6.4 \mathrm{~cm}^{2} / \mathrm{s}^{2} / \mathrm{cph}$ at a frequency of $.2 \mathrm{cph}$. Such a reference is given by the top line of each trapezoid. The heavy lines give the estimates of GM level: they are quite smooth with a frequency slope slightly less than -2 . This slope is similar to what was seen in the calculations of chapter 4 . The changes in level from one period to another are of 


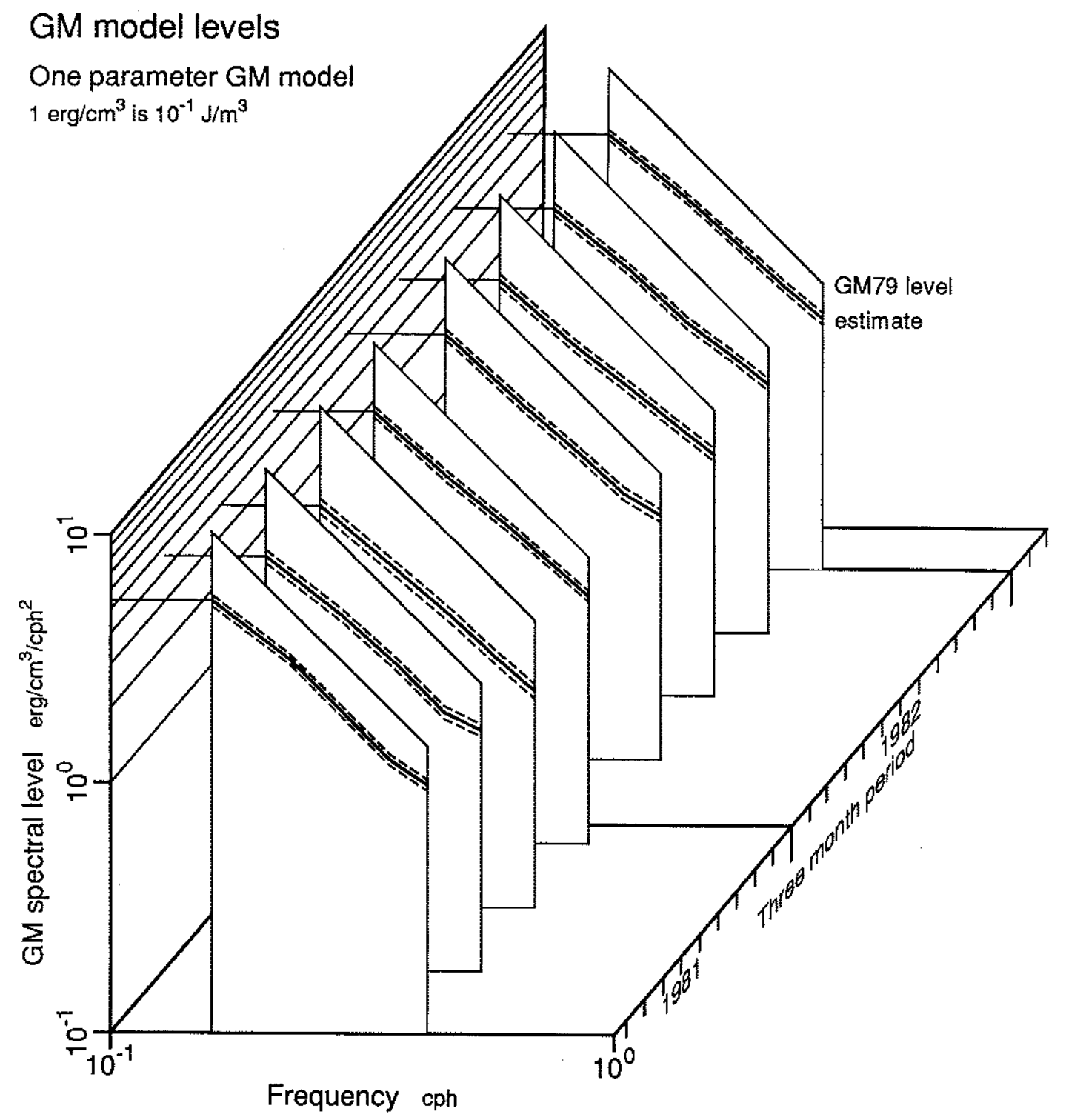

\section{Figure 8.7.2 GM spectral level}

GM spectral level $\left(\mathrm{erg} / \mathrm{cm}^{3} /(\mathrm{cph})^{-2}\right)$ is plotted as a function of frequency and period (each spectrum represents an average over three months). The solid lines give the estimates while the dashed lines give the $96 \%$ confidence limits). GM spectral level is the energy renormalized to an equivalent $1 \mathrm{cph}$ ocean, see chapter 1 . The top line of each trapezoid gives the GM79 prediction. The heavy lines are the estimates: they are quite smooth and have a frequency slope slightly less than -2 . The time dependence of the level at .158 cph shows a $20 \%$ variation with highs in the winter and lows in the summer. The frequency dependence has been computed using 5 equal width $(1 / 15 \mathrm{cph})$ frequency bins: the lowest bin is centered at $.158 \mathrm{cph}$ and the highest bin is centered at $.425 \mathrm{cph}$ (see chapter 3 for details). 
order $20 \%$, with higher values in the winter pieces and lower values in the summer. The estimated levels are half that of the GM prediction. This difference in level contrasts with the results of chapter 4 which found that the levels were approximately the same as the GM79 prediction. The differences occur because the GM model poorly reproduces the vertical coherence structure of the PEQUOD data. This can be most easily seen in the vertical coherence plots in chapter 3: for short separations, the PEQUOD coherences are smaller than the GM predictions, and for very long separations the PEQUOD coherences are higher than the GM predictions. The fit done here tried to match the entire coherence structure with a GM model and thus does not include as much of the energy as the chapter 4 calculations where the model only need match energy at a particular depth. To improve the fit between model and data both the large vertical scale and the short vertical scale behaviors of the model need to be changed.

In order to better model the short vertical scale behavior of the observations, 'GM with finestructure' adds a finestructure model to the GM model used above. This finestructure model assumes that the sharp drop in coherence at short separations is an artifact of the way the measurements were taken. Because the current meters are fixed in position with finite separations in the vertical between them, small scale vertical structure advected past the sensors is misinterpreted as high frequency motion. This problem is discussed in many papers, including the original Garrett and Munk paper GM72, and later papers devoted specifically to the problem such as Joyce and Desaubies 1977. In considering mid-latitude finestructure Joyce and Desaubies conclude that, at the frequencies considered here $(.1$ to $.5 \mathrm{cph}$ ), finestructure accounts for roughly $10 \%$ of the energy. As was pointed out in chapter 7 , the vertical wavenumber spectrum has a much higher level on the equator: this could extent to finestructure as well. So a priori we might expect a $25 \%$ effect. Here finestructure is modeled as isotropic energy with no vertical coherence, i.e. a white noise wavenumber spectrum. Thus fifteen finestructure parameters were calculated: a velocity finestructure level and a displacement finestructure level for each current meter, and a displacement finestructure level for each TP recorder. As can be seen in figure 8.7.1, the residue is greatly reduced. Figure 8.7.3 shows the changes in the estimates of GM level. Not surprisingly, the GM level estimates are somewhat reduced.

One possibility for improving the model fit is to change the wavenumber dependence of the spectral level from the rather smooth shape used by GM79 (section 1.6). The line marked 'Isotropic' gives the residue for a model internal wave field that substitutes the 


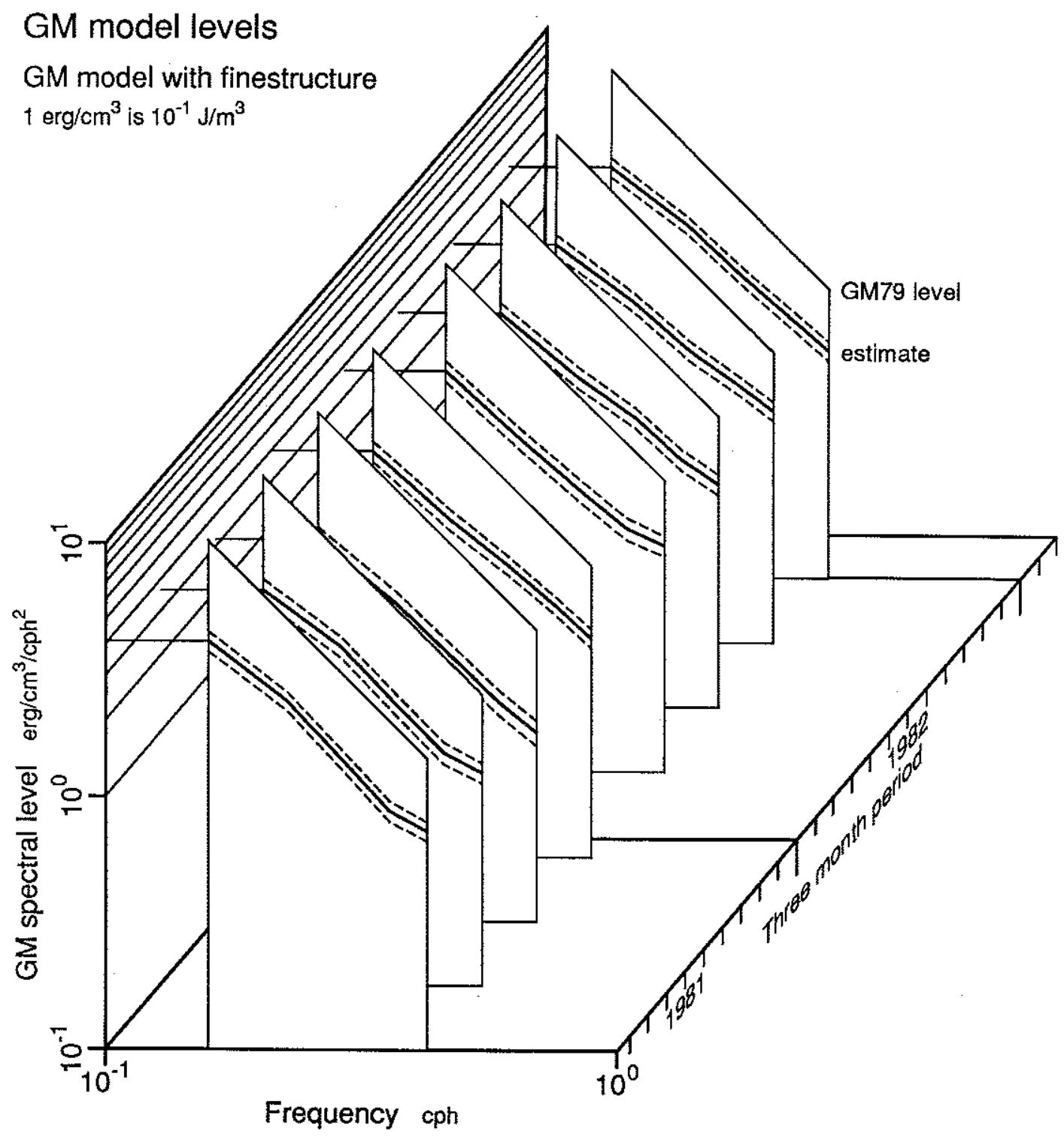

Figure 8.7.3 GM model with finestructure

GM spectral level $\left(\mathrm{erg} / \mathrm{cm}^{3} /(\mathrm{cph})^{-2}\right)$ is plotted as a function of frequency and (3 month) season for a model that includes both GM level and finestructure. The top line of each trapezoid gives the GM79 prediction. The heavy lines are the estimates: they are quite smooth and have a frequency slope slightly less than -2 . The time dependence of the level at .158 cph shows a $20 \%$ variation with highs in the winter and lows in the summer. The frequency dependence has been computed using 5 equal width $(1 / 15 \mathrm{cph})$ frequency bins: the lowest bin is centered at $.158 \mathrm{cph}$ and the highest bin is centered at $.425 \mathrm{cph}$. 
integrated wave shapes of chapter 7 for the first fifteen baroclinic modes, retaining the GM spectral form and WKBJ vertical structure for modes 16 and higher. Thus the energies at wavenumbers corresponding to each of the first fifteen baroclinic modes are determined as well as a high wavenumber spectral parameter that measures the energy in the higher modes. The discussion in section 8.6 shows that not all fifteen low wavenumbers are fully resolved: an a priori wavenumber slope of -2 is used by the inverse to allot energy between unresolved modes. Figure 8.7.1 shows that the residue for this model is not much smaller than the residue from the GM model with finestructure at a frequency of $.158 \mathrm{cph}$, but there is a distinct residue reduction for higher frequencies.

This isotropic model is similar to the GM model in that the wavefield is presumed to be horizontally isotropic, vertically symmetric and purely propagating (spectral parameters $\Delta, P$, and $Q$ are identically zero). Figure 8.7.4 presents the vertically symmetric spectral level estimate $(E)$ at a frequency of $.158 \mathrm{cph}$. The spectra has been normalized to correspond to energy at the ocean bottom: 4250 meters for the $Q$ mooring and 4400 meters for the $U$ mooring. For the GM model (and thus for the high wavenumber portion of the isotropic model) this means multiplying the GM level by the local buoyancy frequency. For both the $U$ and $Q$ moorings the buoyancy frequency at the bottom is $.32 \mathrm{cph}$. The thin line near the top given the a priori level. The level chosen is $10 \%$ smaller than the GM prediction plotted in figures 8.7.2 and 8.7.3. The heavy line gives the spectral level estimates while the dashed lines give the doubled standard deviations (roughly $95 \%$ confidence). The shaded portion of each trapezoid gives the high wavenumber portion of the wavenumber spectrum that has been parameterized by a GM model (i.e. the mode number slope is fixed at -2). The remaining points are the energies in the wavenumbers than correspond to the low baroclinic modes. Comparing the estimates to the a priori line shows that the estimates are slightly larger than half the GM prediction, which is the same size as the prediction of the GM model that includes finestructure.

Because the residue was not significantly reduced at $.158 \mathrm{cph}$ by allowing the modes to separately determine their individual energies, one suspects that the solution should be very much like the GM spectral form. The dotted line is the extrapolation of the high wavenumber energy parameterization to low wavenumbers using the GM spectral form. Except for the wavenumber that corresponds to the first baroclinic mode, the low wavenumber energies tend to be statistically indistinguishable from the dotted line. Thus to the extent that the PEQUOD arrays can resolve the lower modes, the GM spectral 


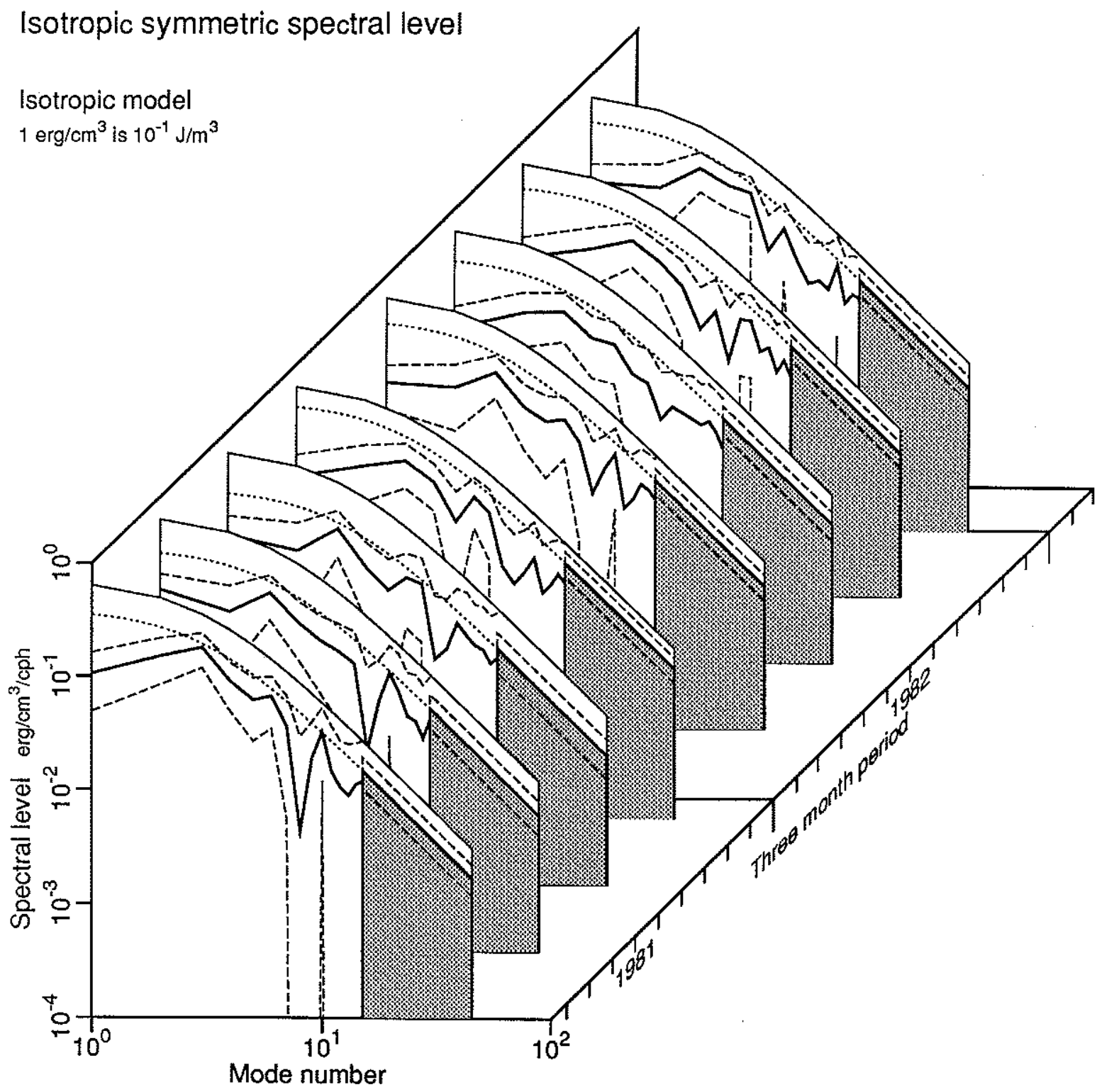

Figure 8.7.4 Symmetric energy from an isotropic model

The symmetric spectral level $E$ at a frequency of $.158 \mathrm{cph}$ is plotted as a function of wavenumber and season (each spectrum represents an average over 3 months). The light line (top of each cutout) is the a priori level, and the shaded trapezoid gives the wavenumber range that has been parameterized with a GM spectral form. The heavy line gives the estimates themselves while the dashed lines are the doubled standard deviations. The dotted line gives the extrapolation of the high wavenumber GM level to lower wavenumbers. For the most part the low wavenumber estimates do not deviate significantly from the dotted line, showing the GM spectral form is a good representation of the distribution of isotropic and symmetric internal wave energy. 
form is a fairly good representation of the isotropic energy. While the energy of first baroclinic mode is consistently lower than the GM extrapolation, the difference is not particularly great and is possibly due to array resolution: the array resolves the second and third modes somewhat better than the first.

Both the single current meter calculations of chapter 4 and the consistency checks of chapter 6 show show strong evidence for horizontal anisotropy in the internal wave field. The line marked 'anisotropic propagating' modifies the isotropic model by allowing some horizontal anisotropy and vertical asymmetry in the first fifteen baroclinic modes. This anisotropy is introduced by allowing the first five direction moments of $E$ and $\Delta$ (moments through $\sin 2 \theta$ ) to be non-zero (see section 8.3 for a discussion of direction moments). The residue for the anisotropic model is distinctly smaller than the residue for the isotropic model at all frequencies.

The estimates of isotropic symmetric energy $E$ for this model are essentially the same as the estimates for the isotropic model. This is not surprising since the isotropic moments of the internal wave spectral level are given by the real parts of the 'like' current meter spectra (zonal velocity with zonal velocity, displacement with displacement, etc.) while the anisotropy is given by the 'unlike' components and the vertical asymmetry is given by the imaginary part of the 'like' spectra (this is discussed further in section 8.3). Thus adding anisotropy to the model enables it to model a new portion of the current meter cross spectral matrix, and does not change the modeling of the portion of the cross spectral matrix that was modeled by the isotropic model. But the presence of anisotropy does change other aspects of the internal wave spectral level.

In a vertically symmetric wavefield there is no net vertical energy flux because the amount of energy propagating upwards is the same as the amount propagating downwards. Plotted in figure 8.7.5 is the net vertical energy flux for this asymmetric purely propagating model. The flux has been integrated over mode number so that it is only a function of frequency and time. The vertical flux is strongly dependent on time, being significantly different from zero only during the first year (1981). Since the two years are measured with different moorings one cannot be certain that the difference signifies a change in the oceanic state: it is possible that the results are due to mooring differences.' This difference between 1981 and 1982 vertical flux can also be seen in the vertical coherence calculations of chapter 3: phase differences in first year large separation coherences computed from velocity are roughly $-135^{\circ}$ while the second year large separation velocity coherences 


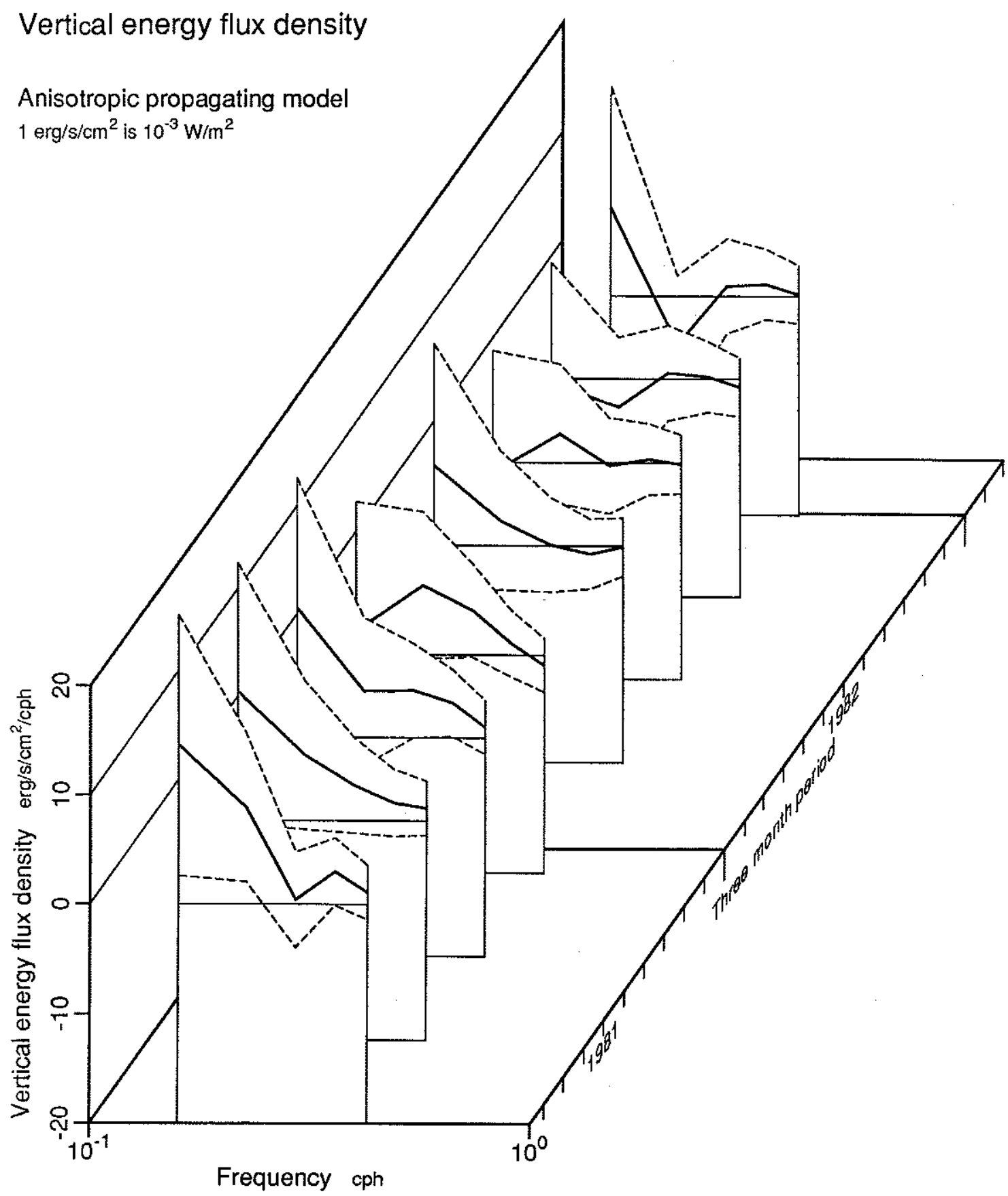

Figure 8.7.5 Vertical flux from a propagating model

The vertical energy flux density is plotted against frequency and season (each spectrum represents a 3 month average). The solid lines give the estimate while the dashed lines are doubled standard deviations. The zonal flux has some dependence on time, being significantly different from zero only during the first year. This could be due to mooring differences or 1982-83 El Niño effects. 
are $-180^{\circ}$.

In an isotropic wavefield there are no net horizontal energy fluxes because the same amount of energy is propagating in all directions. Allowing the wave field to be anisotropic introduces the possibility of a net horizontal energy flux. Plotted in figure 8.7.6 is the net vertically averaged zonal energy flux for this model. The flux has been integrated over mode number so that it is a function of frequency and time. The zonal flux shows a strong dependence on both time and frequency. For the periods where the flux is significant the lower frequencies contribute most of the energy with a frequency slope somewhat steeper than the -2 of the isotropic energy, suggesting that lower frequencies carry proportionally more of the zonal flux of energy. The time dependence shows a dramatic difference between pieces from the first year and pieces from the second year. While pieces 1 and 5 both show eastward fluxes that are slightly larger than one standard deviation, the remaining pieces from the second year show successively stronger zonal fluxes as the year progresses while the first year pieces show no significant flux at all. The increase in zonal flux as the second year progresses correlates well with the onset of the 1982-83 El Niño event, a point that will be explored further once it has been demonstrated that including kinematic shear effects does not significantly alter the flux estimates.

With the anisotropic model, it is also possible to get a net meridional flux. But the calculations reveal that the meridional flux is not significant, and rather than show two sets of null results, discussion will be postponed to a later model.

The coherence calculations of chapter 3 showed that modal effects must be included in the GM model in order to explain the large vertical separation coherences. 'Anisotropic modal' modifies the propagating model by allowing modal effects (spectral parameter $P$ is possibly non-zero). Figure 8.7.1 shows that the change in residue resulting from that modification is not particularly large, showing that the model modification is not statistically important as far as fitting the entire data set is concerned. But the estimates of modal component are significantly non-zero. Figure 8.7 .7 gives the percentage of the isotropic energy the inversion has characterized as modal for period 1 (24 January 1981 through 24 April 1981). The estimates tend to be close to 50\%, with a slightly higher value of $65 \%$ at mode 1 . Far more important that the estimates are the $95 \%$ confidence lines, lines which show that only the very lowest estimates are distinguishable from zero at $95 \%$ confidence. The high wavenumber estimates are at $50 \%$ simply because the a priori 


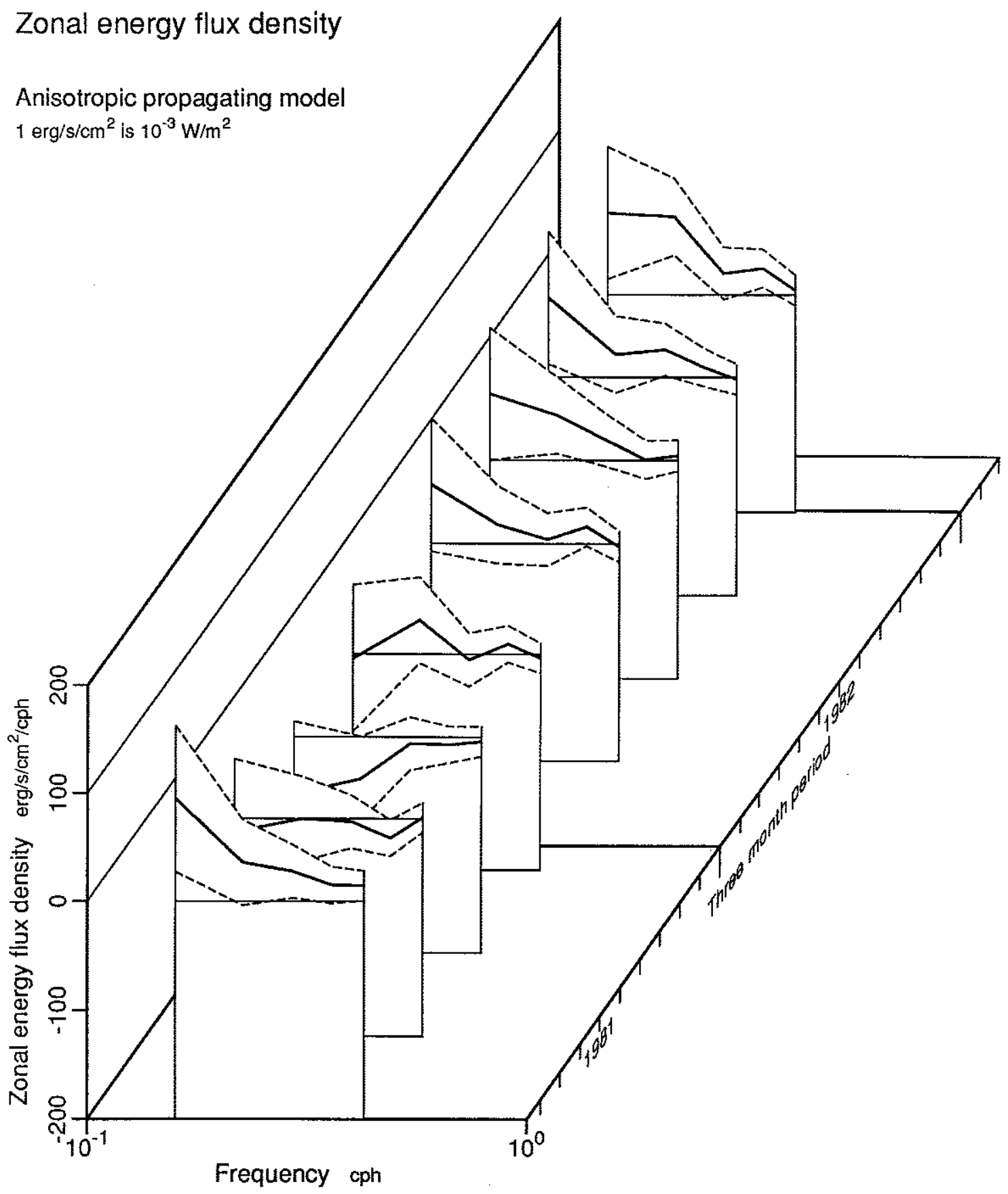

Figure 8.7.6 Zonal flux from a propagating model

The zonal energy flux density is plotted against frequency and season (each spectrum represents a 3 month average). The solid lines give the estimate while the dashed lines are doubled standard deviations. The zonal flux has a strong dependence on time, being much greater as the 1982-83 E1 Niño event progresses. This flux is confined to the lower frequencies, the higher frequencies showing little change between the two years. 
Percentage modal

Run q1p1sf 1981 through 24 April 1981

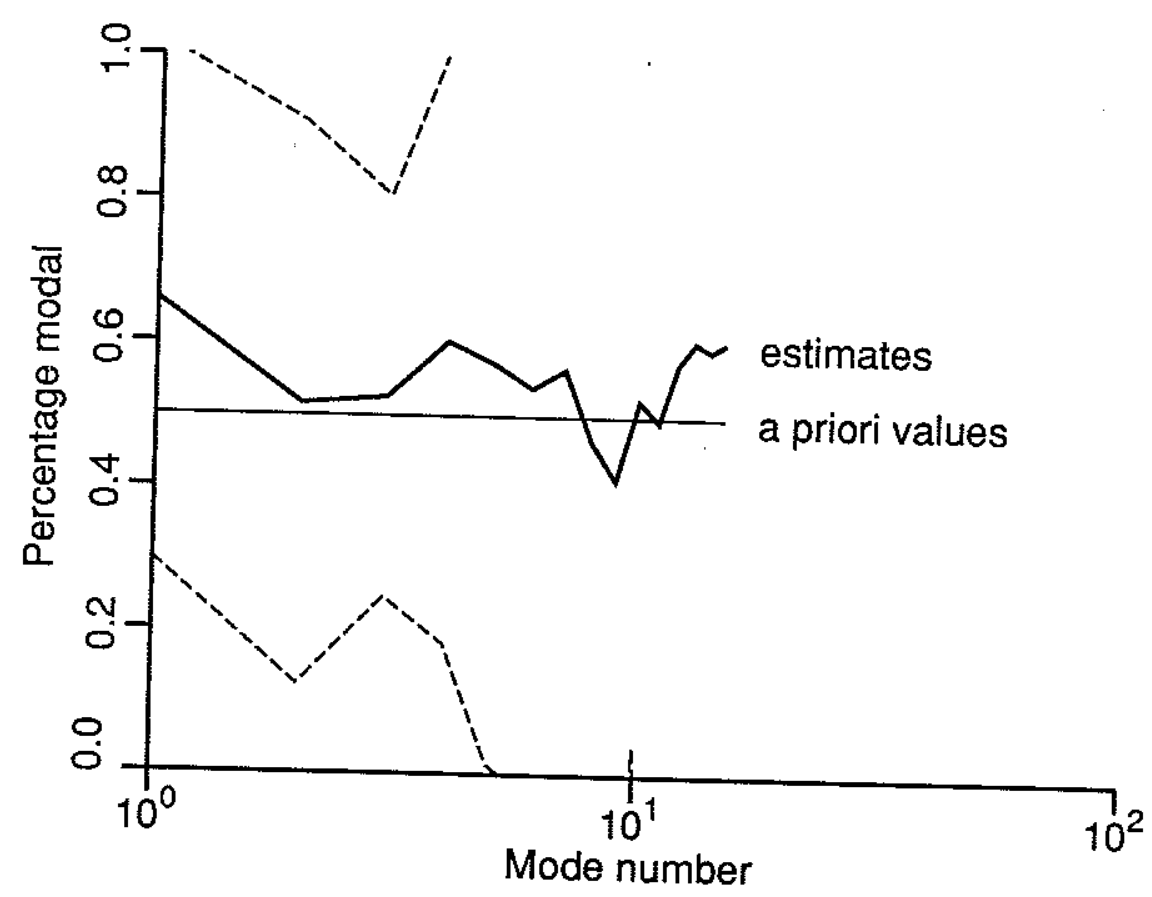

\section{Figure 8.7.7 Percentage modal from the shear kinematic model}

This figure shows the percentage of the isotropic energy that the shear kinematic model characterizes as modal. The heavy solid line gives the estimate of this percentage as a function of mode number; the dashed lines give the doubled standard deviations ( $96 \%$ confidence) about the estimates. The plot indicates that the modal component is resolved only for the very lowest wavenumbers. 
value of the percentage is $50 \%$ (energy is equally likely to be modal or propagating). Two conclusions can be drawn: the modal character is marginally resolved by this array, and the low wavenumbers have a significant modal component.

In chapter 4 it is shown that the observed strong zonal velocity-temperature correlations could be due to either a strong zonal asymmetry or the kinematic effects of a mean shear flow. In chapter 6 it is shown that in fact some shear effects are necessary. The line 'Anisotropic modal with shear kinematics' gives the residue for a model that modifies the anisotropic modal model by including kinematic shear effects in the structure of the low wavenumber waves. While the change in residue is very small, it does show that the model with shear kinematics does better than the model without shear kinematics. Note that the two models have exactly the same number of model parameters to be determined by the inversion: only the structure of the lower modes has been changed.

The residues in figure 8.7.1 are from model fits to the first period of data (24 January 1981 through 24 April 1981). For comparison figure 8.7.8 gives the residues as a function of time for both the shear kinematic model and the null model. While there is some time dependence in both the null model residue and the shear model residue, the residues in the frontmost piece of each plot do not differ in structure from the residues in the later pieces. Thus the residues in figure 8.7.1 are typical of the residues from all eight pieces.

Throughout this series of models, only the physics of the fifteen low modes has been changed: the high mode model is identical to the GM model that started the sequence. The fact that the shear effects are more strongly expressed at higher wavenumbers (the Doppler shift in particular) means that shear effects should be included for high wavenumbers as well. This was not done on the PEQUOD data set because the instrument spacing is such that the array resolves both low and very high modes but there is a lack of resolution at intermediate scales. While it might be possible to deal with the problems of resolution when there is an intermediate scale gap, it seems more prudent to concentrate on low wavenumbers and roughly parameterize intermediate and high wavenumbers. This is especially reasonable to the extent that the wavenumber spectrum is red, a prediction that the GM79 model makes and thus is consistent with a large number of measurements.

Figure 8.7.9 gives the isotropic and symmetric spectral level $(E)$ at a frequency of $.158 \mathrm{cph}$ for the model that includes shear kinematics. The level is the energy at the reference depth, thus it is equivalent to figure 8.7 .4 which presents the results of the isotropic model. The difference between the two figures is primarily in the first 


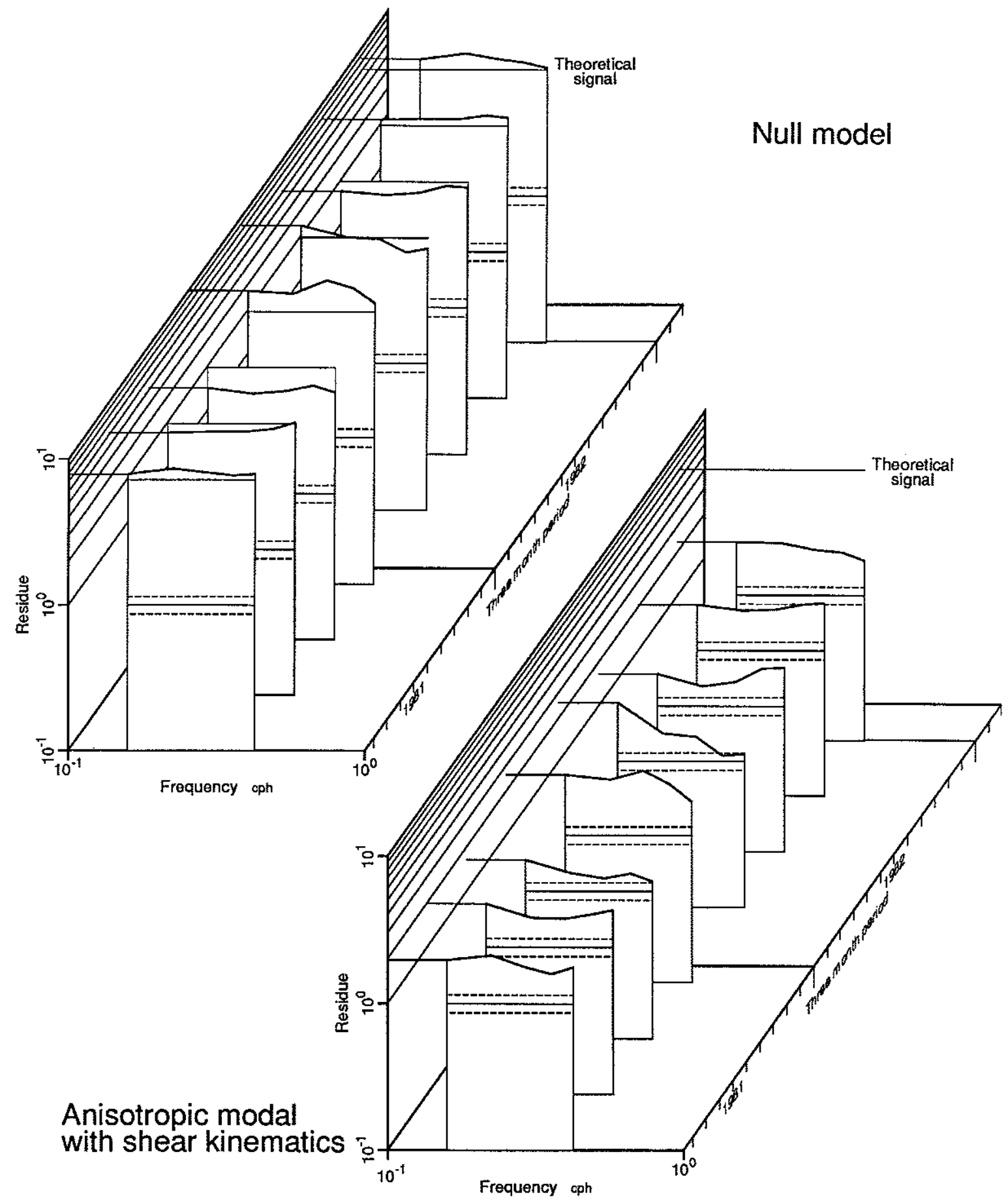

Figure 8.7.8 Residue for the shear kinematic model

This figure presents the residue for both the null model and the shear kinematic model as a function of time (the frontmost curves are a subset of the residues presented in figure 8.7.1). The heavy solid line gives the residue for each model, while the dashed lines give the theoretical $95 \%$ confidence limits for noise (see the discussion of figure 8.7.1 for more details). The plots show that figure 8.7.1 is indeed typical of the ensemble of eight pieces. 


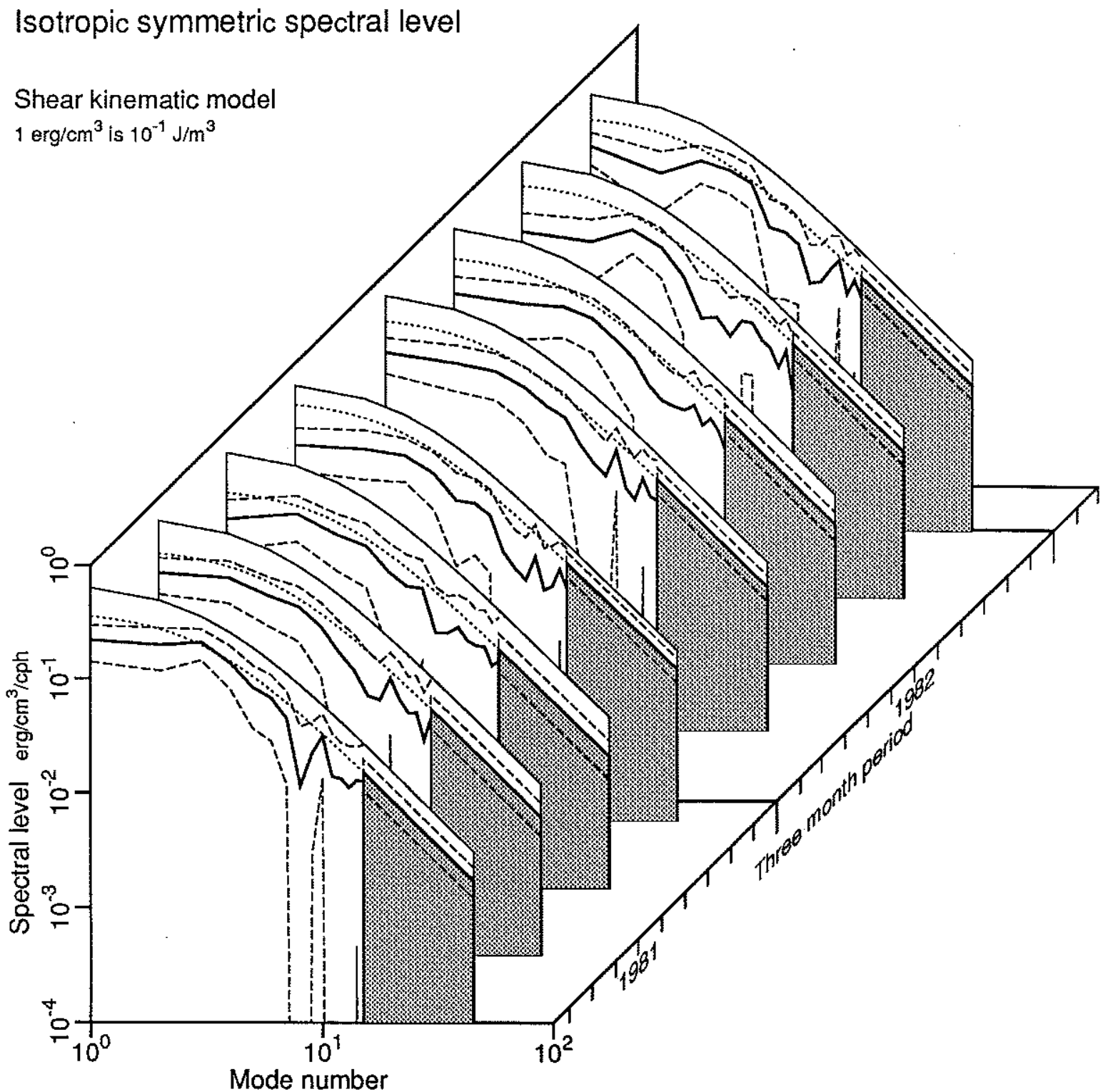

Figure 8.7.9 Isotropic symmetric spectral level from shear kinematic model

The isotropic moment of the symmetric spectral level $E$ at a frequency of .158 $\mathrm{cph}$ is plotted as a function of wavenumber and season (the spectra are averaged over 3 month pieces, see text). The light line (top of each cutout) is the a priori level; the shaded trapezoid gives the wavenumber range that has been parameterized with a GM spectral form. The heavy line gives the estimates themselves while the dashed lines are the doubled standard deviations. The dotted line gives the extrapolation of the high wavenumber GM level to lower wavenumbers. For the most part the low wavenumber estimates do not deviate significantly from the dotted line, showing the GM spectral form is a good representation of the distribution of isotropic and symmetric internal wave energy when the integrated wave structures are used. 
baroclinic mode, which has higher energies in the shear kinematic modal model. This suggests that the first baroclinic mode shows significant modal character, the fit being distinctly improved by the addition of phase-locking $P$. Note that the error bars for the modes 10 through 15 are a much larger proportion of the estimate than the error bars for modes 1 through 5: this is due in part to a lack of resolution which has been resolved by the a priori spectrum being red. Thus the low group of modes is favored over the high group.

Figure 8.7.10 presents the high wavenumber GM spectral level as a function of period and frequency. While the quantity plotted is the same as in figure 8.7.3, the calculation is quite different: in figure 8.7.3 the GM model is used to fit the entire data set, while in figure 8.7.10 the model is used only to fit the energy that corresponds to modes numbered higher than 15 . The estimates are much closer to the GM prediction, suggesting that using GM for high wavenumbers is more appropriate than using GM to model the entire wavenumber spectrum. The error bars are much larger as well: this is due to difficulty in resolving high wavenumber energy in the presence of the energetic low wavenumber waves. The time dependence of the estimates, however is very much the same as in figure 8.7.3: the time dependence at $.158 \mathrm{cph}$ shows a $20 \%$ variation with highs in the winter and lows in the summer.

Figure 8.7.9 gives the spectral level at the reference depth for the frequency bin centered at $.158 \mathrm{cph}$. It is possible to use the vertical structure of each wavenumber to translate those values to be the spectral level at any depth. Figure 8.7.11 gives the spectral level averaged over the entire depth and integrated over all wavenumber. Only the energy in the finestructure estimates and in the residuals is excluded. This can be compared to the GM spectral level estimates by multiplying the GM spectral level by the vertically averaged buoyancy frequency $(\approx 1 \mathrm{cph})$. The dependence on time is roughly the same as the dependence shown by the GM level in figure 8.7.10, which means that the entire wavenumber spectrum shows a $20 \%$ variation with lows in the summer and highs in the winter.

Plotted in figure 8.7.12 is the net vertical energy flux for the shear kinematic model. The figure is comparable to figure 8.7.5 which gives the vertical flux for the anisotropic purely propagating model. The results are fairly close, demonstrating that the additional degrees of freedom in the shear kinematic model introduced by allowing some modal character did not affect the vertically asymmetry. This is not surprising, since modes 


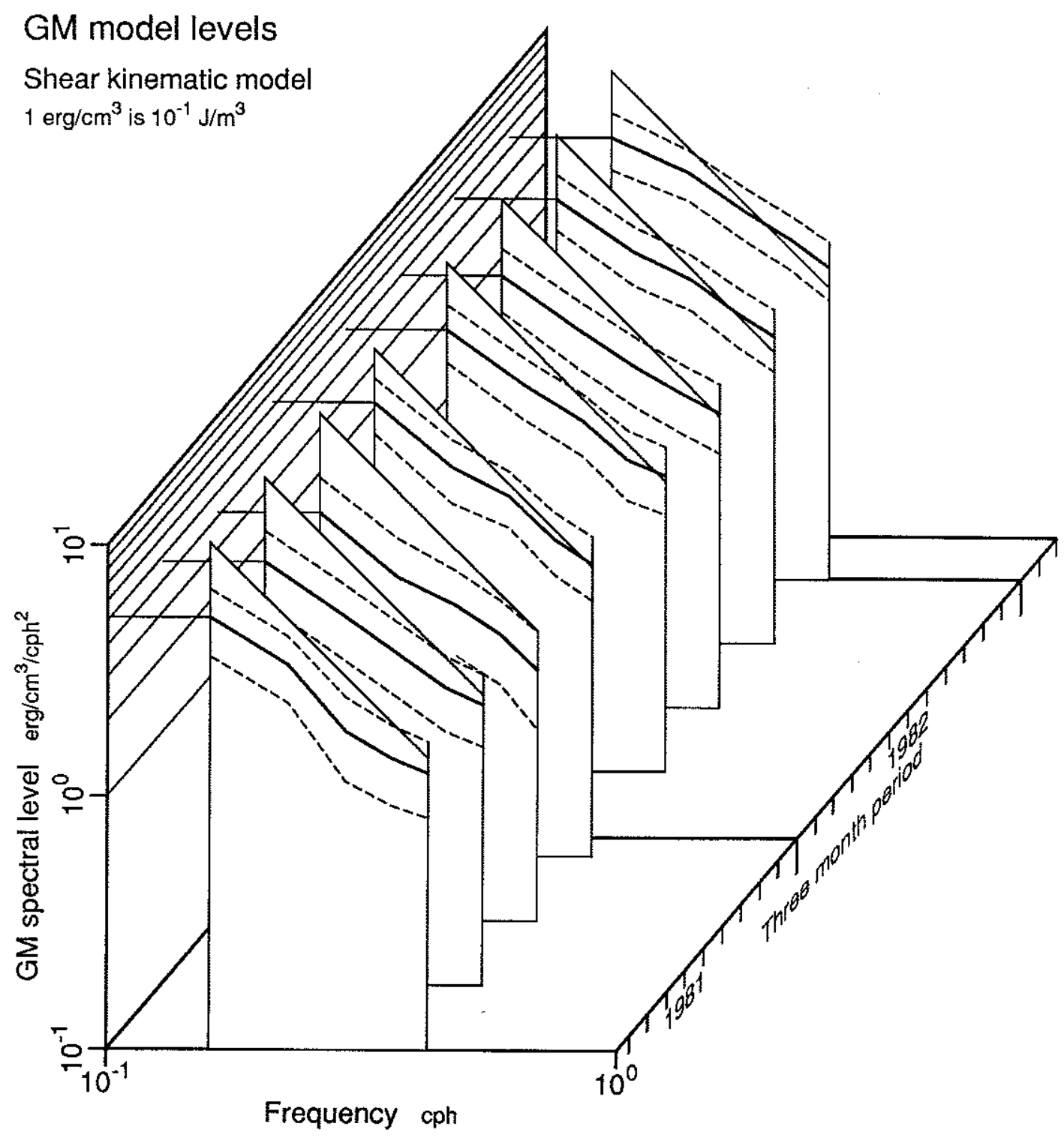

Figure 8.7.10 GM spectral level from shear kinematic model

GM spectral level is plotted as a function of frequency and season (each spectrum represents an average over three months). The top line of each trapezoid gives the GM79 prediction. The heavy lines are the estimates: they are quite smooth and have a frequency slope slightly less than -2 . The time dependence of the level at $.158 \mathrm{cph}$ is projected on the $y z$ plane. It shows a $20 \%$ variation with highs in the winter and lows in the summer. This figure corresponds to figure 8.7.3, the difference being that here the GM level is the high wavenumber component of shear kinematic model while in figure 8.7.3 the GM level is used to represent the entire internal wave spectrum. 


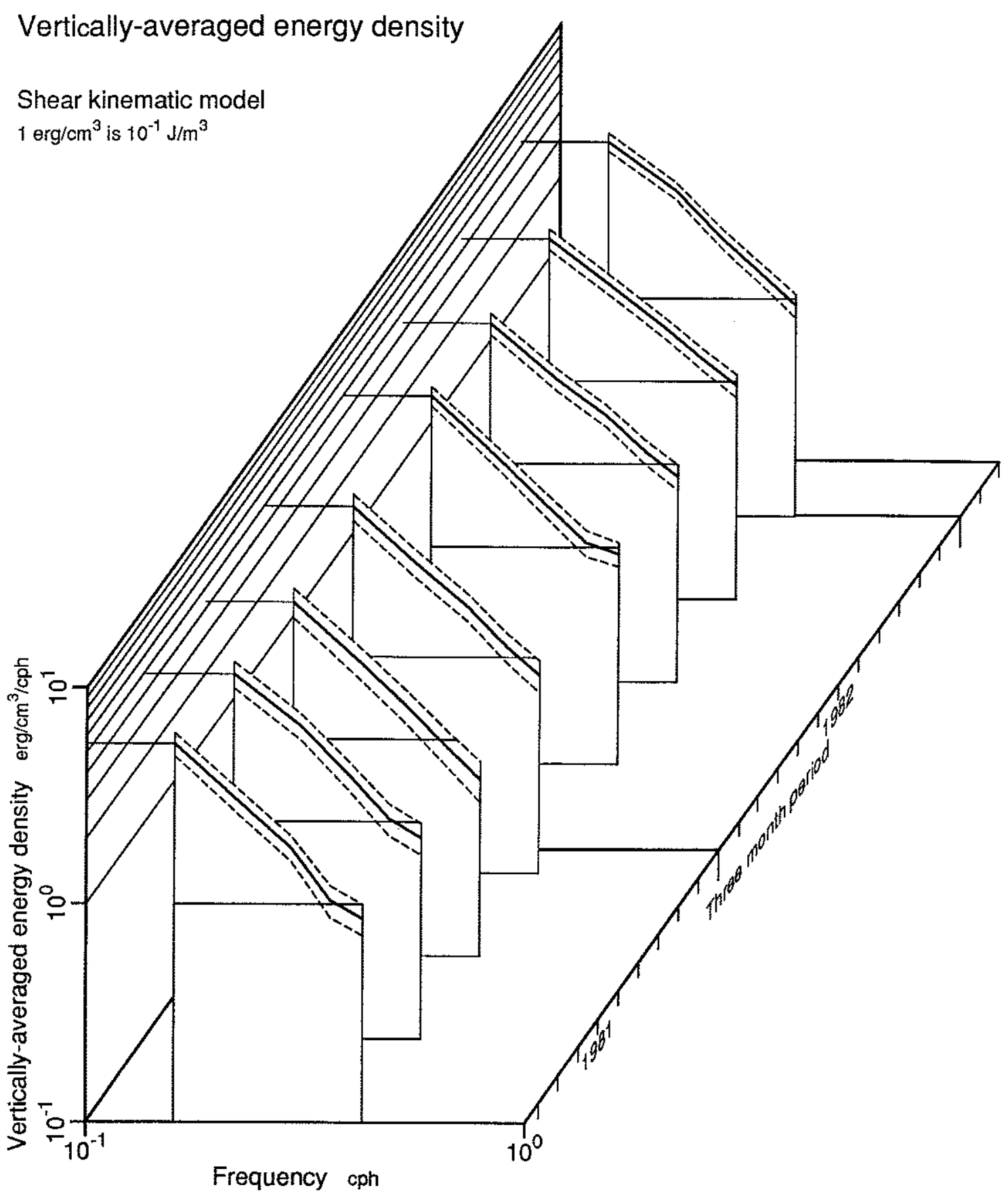

Figure 8.7.11 Vertically averaged energy from shear kinematic model

Vertically averaged energy density is plotted as a function of frequency and season (each spectrum represents an average over three months). The solid lines give the estimate while the dashed lines are doubled standard deviations. The vertically averaged energy shows time dependence with a variation of order $20 \%$. 


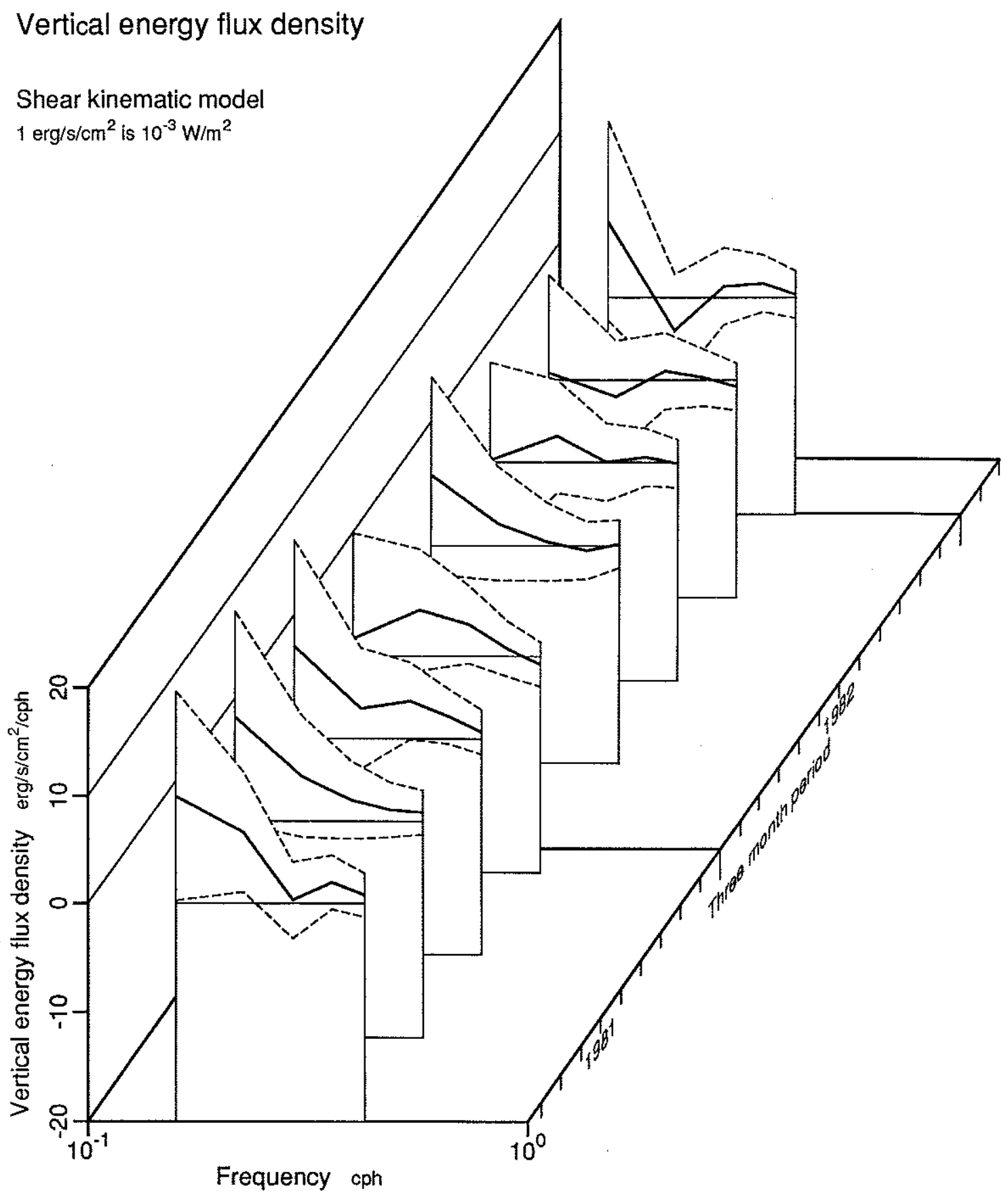

Figure 8.7.12 Vertical energy flux from shear kinematic model

Vertical energy flux density is plotted as a function of frequency and season (each spectrum represents an average over three months). The solid lines give the estimate while the dashed lines are doubled standard deviations. The size of the fluxes during the first year are consistent with the generation of internal waves by tidal currents flowing over a rough bottom. The disappearance during the middle of the second year suggests a counterbalancing flux. 
by their very nature have no net vertical flux. At the beginning of the first year the vertical energy flux is significantly upwards at $96 \%$ confidence (error bars are doubled standard deviations) the vertical energy flux is also upwards at the beginning of the second year, though the flux then is not significantly different from zero. Pieces 6 and 7, which correspond to the periods where the 1982-83 El Niño is setting up, show zero to downward fluxes, though the sizes are not significantly different from zero.

The vertical energy flux in each of the first three pieces integrated over the five frequencies (the total bandwidth considered is $1 / 3 \mathrm{cph}$ ) is roughly $1 \mathrm{erg} / \mathrm{s} / \mathrm{cm}^{2}$, the same size as many of the possible sources of energy of the internal wave field (Olbers 1983, Thorpe 1975). The fact that the flux is upwards suggests that the source should either be at the bottom or that there is a sink near the top. One bottom source of roughly $1 \mathrm{erg} / \mathrm{s} / \mathrm{cm}^{2}$ is the generation of internal waves by tidal currents going over bottom irregularities (Bell 1975, Olbers 1983). This mechanism generates internal waves at the tidal frequency, but non-linear effects could transfer the energy to neighboring frequencies. This is consistent with the fact that figure 8.7 .12 shows that the vertical flux is strongest at $.158 \mathrm{cph}$, the bin closest to the tidal frequency. The disappearance of this flux during the onset of El Niño suggests that a counterbalancing downward flux has been introduced during the transition from the non-El Niño oceanic state to the El Niño oceanic state.

While the measured flux of $1 \mathrm{erg} / \mathrm{s} / \mathrm{cm}^{2}$ is the same size as many of the possible sources of internal wave energy, it is much smaller than the vertical flux would be if the entire internal wave field were propagating in the same direction. This vertical energy flux can be computed by integrating the product of energy density and vertical group velocity over frequency and wavenumber: such a computation using the GM79 spectral form gives

$$
\begin{aligned}
\int_{.125 \mathrm{cph}}^{.458 \mathrm{cph}} d \omega \sum_{j=1}^{\infty}\left(\mathbf{c}_{g} \cdot \hat{\mathbf{z}}\right) E(\omega, j) & =\int_{.125 \mathrm{cph}}^{.458 \mathrm{cph}} d \omega \sum_{j=1}^{\infty}(2.16 \mathrm{~m} / \mathrm{s}) / j \omega B(\omega) H(j) \\
& =30 \mathrm{erg} / \mathrm{s} / \mathrm{cm}^{2}
\end{aligned}
$$

Thus the measured vertical flux is roughly $4 \%$ of the maximum vertical flux the GM79 internal wave field can carry. The fact that such a small percentage of the internal wave field can carry a dynamically significant energy flux underscores the difficultly of measuring dynamically significant energy fluxes.

Figure 8.7.13 gives the meridional energy flux as a function of frequency and time. It shows that the net meridional flux is not significantly different from zero at $96 \%$ 


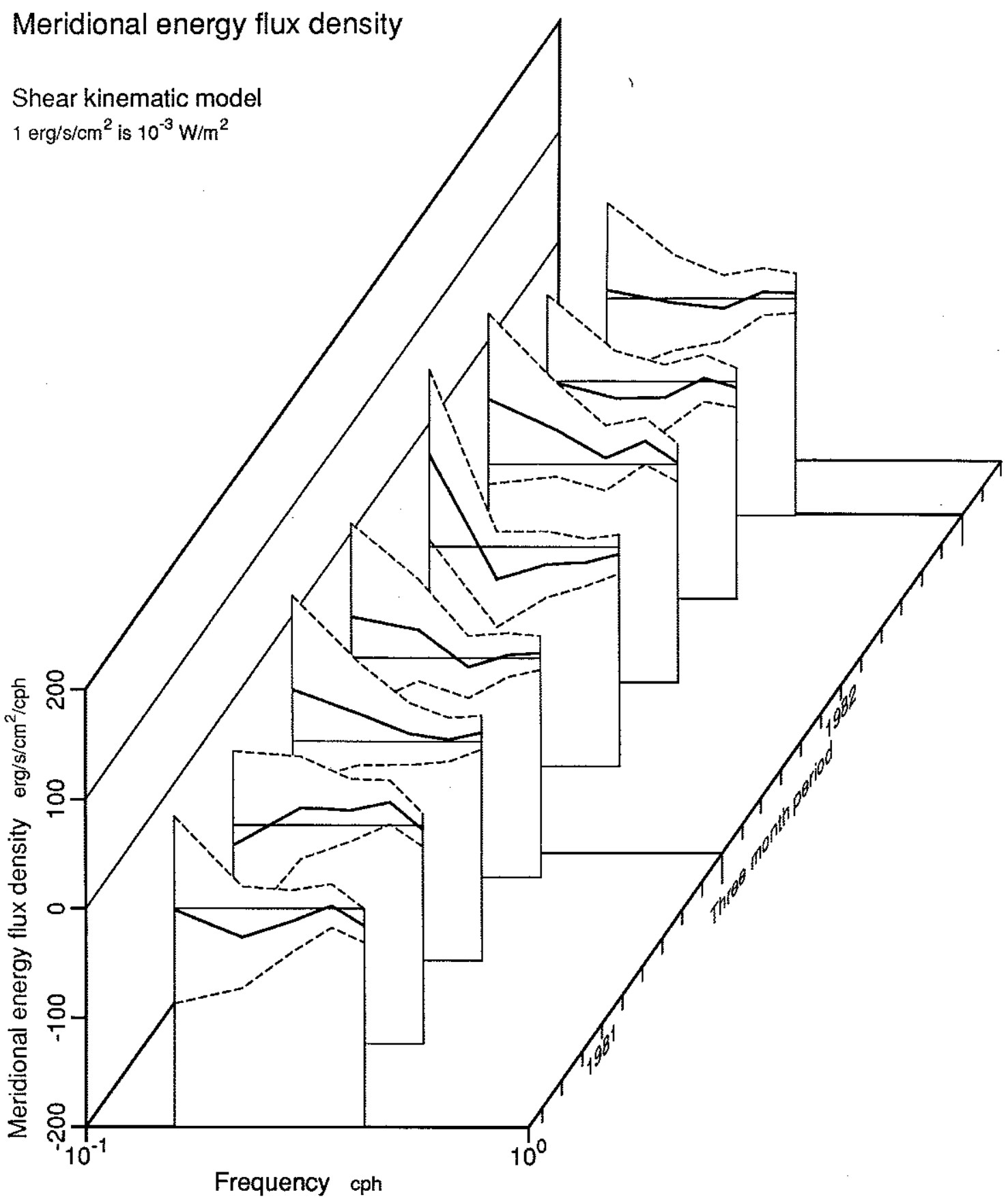

Figure 8.7.13 Meridional energy flux from shear kinematic model

Meridional energy flux density is plotted as a function of frequency and season (each spectrum represents an average over three months). The solid lines give the estimate while the dashed lines are doubled standard deviations. The estimates are not significantly different from zero, so that the assumption of symmetry about the equator seems fairly reasonable. 
confidence. This is not particularly surprising, since a priori we expect the wave field to be symmetric with respect to the equator, and thus expect zero net meridional energy flux in the internal wave field.

Plotted in figure 8.7.14 is the net zonal energy flux for the shear kinematic model. This is comparable to figure 8.7.6, which gives the zonal energy flux for the purely propagating anisotropic model. The figure shows that the inclusion of shear kinematics has not reduced the zonal flux estimates. Therefore, while both the shear kinematic effects and the zonal asymmetry lead to the correlations between zonal velocity and displacement observed in chapter 4 , the shear kinematic effects are not sufficient and some zonal energy flux is required to match the data.

In the earlier discussion of zonal flux, it was hinted that the flux may be due to the onset of the 1982-1983 El Niño event. While any statement made here about forcing is pure speculation, the wind events that herald the start of El Niño in the western Pacific seem to be a likely forcing mechanism. It has been shown that such wind events generate eastward propagating Kelvin waves, and indeed such Kelvin waves have been followed across the Pacific (Lukas et al. 1984, Eriksen et al. 1983). It would not be particularly surprising if a corresponding energy transport could be seen at higher frequencies as well.

As in figure 8.7.6, the zonal flux in figure 8.7.14 shows a strong dependence on both time and frequency. For the periods where the flux is significant, the lower frequencies contribute most of the energy with a frequency dependence somewhat redder than the -2 of the isotropic energy. This suggests that lower frequencies carry proportionally more of the zonal flux of energy. The zonal flux shows is significantly eastward during the second year, but it also shows a significant eastward flux at the beginning of the first year. This correlates well with the Western Pacific zonal wind index presented by Lukas $e$ t al. 1984, which shows eastward anomalies in February March of 1981 as well as strong eastward winds from June until December of 1982.

The estimates of energy level given in figures 8.7.11 and 8.7.15 show little dependence on time, a result that at first seems to contradict the zonal flux results that show a strong dependence on the phase of the El Niño cycle. The resolution of this apparent conflict is that the zonal flux observed is a small fraction of what the zonal flux would be if the entire internal wavefield were propagating in the same direction. The latter flux is computed by integrating the depth-averaged product of energy density and zonal group velocity over frequency and wavenumber. Using the GM79 spectral form for energy 


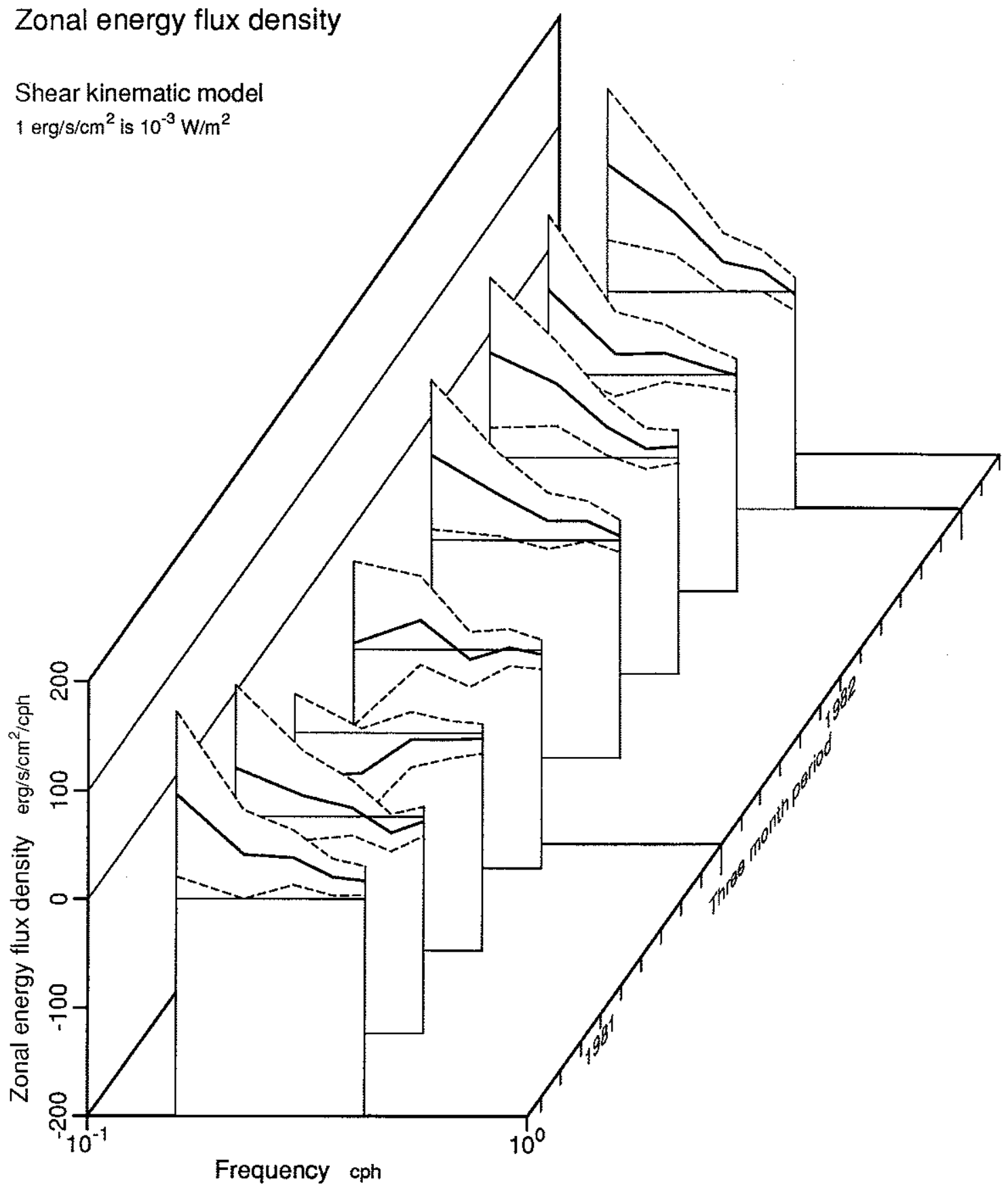

Figure 8.7.14 Zonal energy flux from shear kinematic model

Zonal energy flux density is plotted as a function of frequency and season (each spectrum represents an average over three months). The solid lines give the estimate while the dashed lines are doubled standard deviations. The flux shows a strong time and frequency dependence, properties that are consistent with remote forcing by Western Pacific wind anomalies. 
density gives the following estimate of zonal fiux,

$$
\begin{aligned}
\int_{.125 \mathrm{cph}}^{.458 \mathrm{cph}} d \omega \sum_{j=1}^{\infty}\left(\mathbf{c}_{g} \cdot \hat{\mathbf{x}}\right) \bar{E}(\omega, j) & =\int_{.125 \mathrm{cph}}^{.458 \mathrm{cph}} d \omega \sum_{j=1}^{\infty}(2.16 \mathrm{~m} / \mathrm{s}) / j \bar{N} B(\omega) H(j) \\
& =130 \mathrm{erg} / \mathrm{s} / \mathrm{cm}^{2}
\end{aligned}
$$

where $\bar{N}$ is the buoyancy frequency averaged over depth (approximately $1 \mathrm{cph}$ ). Thus the measured zonal flux is roughly $10 \%$ of the maximum zonal flux the internal wave field can carry, which is consistent with the $10 \%$ variability in energy level shown in figure 8.7.15.

As a summary of the energy and flux results, figure 8.7 .15 presents the energy and fluxes integrated over the five frequency bins. The energy plot shows a minimum in the 1981 summer but not during the 1982 summer. If the minimum in the 1981 summer is part of a regular seasonal cycle, then the 1982-1983 El Niño results in an excess of internal wave energy for that part of its cycle. The vertical energy flux is significantly positive through much of 1981, but is indistinguishable from zero in 1982 . A simple model suggests that the upward fluxes in 1981 are due to non-linear spectral transfer from the current-topography interaction given in Bell 1985, and El Niño forces a counterbalancing downward flux for 1982. The vertical fluxes are, of course, consistent with other sources as well. The meridional energy flux tends to be indistinguishable from zero as noted earlier in figure 8.7.13. The zonal flux appears to have a simple seasonal cycle in 1981, but is consistently eastward throughout 1982. This time dependence is similar to the time dependence of the Western Pacific zonal wind index presented by Lukas et al. 1984, but the index more closely resembles the behavior of the first mode zonal flux density shown in figure 8.7.14 than the behavior of the integrated zonal flux in that the winds increase towards the end of 1982 while the integrated flux tends to be relatively constant throughout 1982 . The presence of the 1982-83 El Niño seems to have significantly altered the zonal flux of energy carried by the internal wave field. 

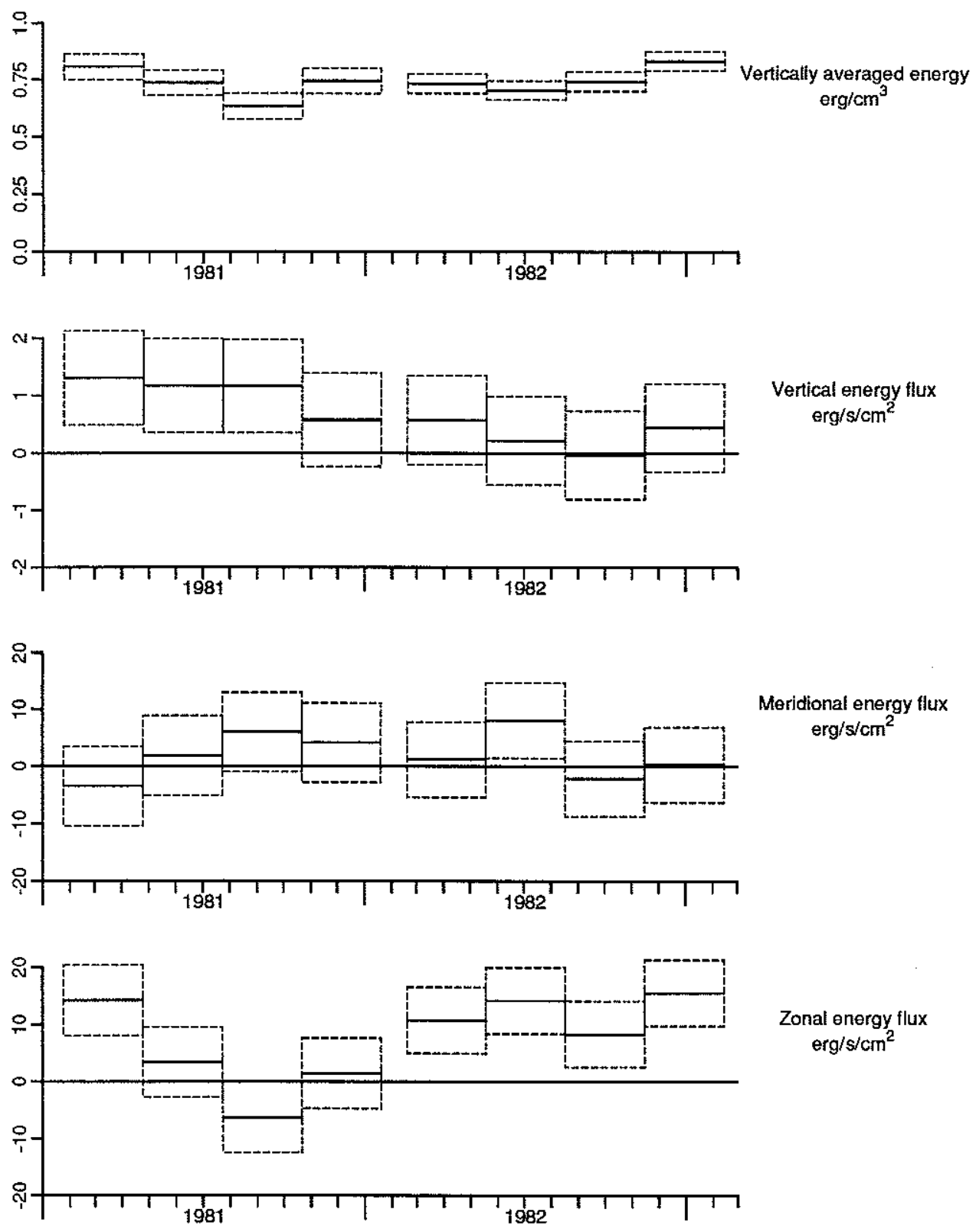

Figure 8.7.15 Total energy and fluxes from shear kinematic model

These four plots present the energy and net fluxes integrated over the frequency band from .125 to $.458 \mathrm{cph}$. They are thus integrations over frequency of the densities presented in figures 8.7.11 through 8.7.14, and show basically the same results. 


\subsection{Summary}

This chapter presents results from the spectral inversions applied to the PEQUOD current meter data, in the process reviewing many of the results of earlier chapters. The results reveal several previously unmeasured properties of the internal wave field. The first point is that with the year long time series available now (and not available during the formulation of the GM models), an isotropic and vertically symmetric WKBJ propagating model is not adequate to model the data. Nor should it be on theoretical grounds: many of the assumptions were made simply because lesser amounts of data were unable to show the contrary. In particular, the modal character of low wavenumber waves can be seen even at the relatively high frequencies considered here. The modal character is clearly evident in the chapter 3 comparisons of velocity and temperature coherences. The chapter 3 results are supported in section 8.7 which shows that the differences in the coherence patterns can be attributed to the difference between purely propagating and partially modal models even when the models include fairly general vertical structures. This result should be fairly universal, though the deeper thermocline in the Atlantic ocean means that coherences over long separations with smoothly varying buoyancy frequency profiles cannot be calculated there.

The results also show that there are significant vertical and horizontal asymmetries. The vertical energy flux is upwards during 1981 of a size that is consistent with the flux expected from the interaction of tidal currents with a spectrum of bottom topography. This flux disappears in the middle of the second year, suggesting that there may be a counterbalancing downward flux brought on by the onset of the 1982-83 El Niño event. The zonal flux is significantly eastward during the first three months of 1981 and all of 1982, with the flux increasing from the beginning of 1982 to the end. This behavior is consistent with remote forcing by the Western Pacific winds in the sense that anomalously eastward winds are observed at the times necessary to cause the enhanced zonal fluxes.

What is not observed in the data are large changes in the isotropic and vertically symmetric portion of the internal wave field $\langle E\rangle$. For that aspect of the spectrum, then, the GM models models provide an accurate summary of the internal wave field when combined with integrated (non-WKBJ) modes. 


\section{Chapter 9: Summary}

This thesis uses data from the two central moorings of the PEQUOD current meter array to examine the equatorial Pacific internal wave field. The analysis reveals several previously unmeasured properties of the internal wave field, properties that are both difficult to measure and important for understanding the dynamics of the internal wave field.

The details of the PEQUOD current meter array are given in chapter 2. The thesis analyzes the data from moorings $\mathrm{Q}$ and $\mathrm{U}$, both being instrumented with six current meters and six temperature-pressure (TP) recorders organized into three widely-spaced groups of four closely-spaced instruments (instrument failures reduced the arrays to five current meters and five TP recorders). Because the groups are widely spaced and are located outside of the dynamically complicated upper ocean, the array is well suited for looking at low mode internal waves. As pointed out in section 8.6, because temperature and velocity measurements contain complementary information, such an array can resolve up to fifteen modes: ten modes because temperature is measured at ten depths, and five more modes because velocity is measured at five depths. This gain in modal resolution is one of several advantages accrued by considering velocity and temperature spectra together rather than the more common practice of considering temperature and velocity spectra separately.

The internal wave spectra produced by Garrett and Munk provide the modeling starting point for the thesis; the latest version (GM79) is compared to the physically more sophisticated models in analyzing the PEQUOD data. The GM79 model is horizontally isotropic, vertically symmetric, purely propagating, and universal in both time and space. It is also highly parameterized, predicting a smooth dependence of internal wave energy on both frequency and wavenumber. As Wunsch 1975 points out, this set of properties effectively eliminates all the interesting physics, since such a model cannot have forcing that varies from place to place or time to time. Thus an important step in understanding the dynamics of the internal wave field is to make measurements of deviations from the simple GM79 spectrum.

Chapters 3 and 4 use subsets of the current meter cross-spectra to show that GM79 is not sufficient to model the PEQUOD data. Chapter 3 examines the coherence as a function of vertical separation. Calculations using PEQUOD data show that for large 
separations coherence computed from velocity is distinctly different from the coherence computed from temperature. This difference is inconsistent with the purely propagating nature of the GM79 model, and can be explained by the inclusion of standing modes in the internal wave model. Section 3.3 shows similar differences between velocity and temperature coherence can be seen in mid-latitude (LOTUS) data as well, showing that the partially modal character of the internal wave field is not limited to the equator. Though the modal character has not been observed prior to this thesis, the result is expected on theoretical grounds since there are no strong dynamical reasons why the very lowest modes should be purely propagating.

Chapter 4 shows that single current meter cross-spectra from PEQUOD are also inconsistent with GM79 internal wave model. The strongest deviations observed are the strongly non-zero $u T$ coherence for the middle instrument cluster. As shown in later chapters, particularly chapter 6, such spectral features can be modeled by both including the kinematic effects of a mean shear flow and allowing horizontal and vertical asymmetries.

The demonstrated inadequacy of GM79 prompts chapter 5, which derives the tools necessary to construct more general internal wave models and understand how the new model features are expressed in current meter cross-spectra. As shown in chapter 3, the difference between WKBJ standing modes and WKBJ propagating waves is expressed in current meter spectra as a difference in the coherence computed from velocity and the coherence computed from temperature. As shown in chapter 6, horizontal and vertical asymmetries are expressed in that 'like' coherences (velocity with velocity, temperature with temperature, etc.) depend on the isotropic component of the internal wave spectrum, 'unlike' coherences (zonal velocity with temperature, etc.) only depend on the horizontally anisotropic component of the internal wave spectrum, and the imaginary parts of the cross-spectra only depend on the difference between upward propagating energy and downward propagating energy.

The more general internal wave models fit the PEQUOD data better than the simple GM79 model. This is first shown in chapter 6 , which uses the consistency check techniques of Müller et al. 1978 to check models against the full set of cross-spectra from each mooring. The time series in PEQUOD are much longer than those Müller et al. 1978 had in IWEX, thus it is not particularly surprising that in PEQUOD the deviations from the simple symmetries of GM79 are more significant than deviations in IWEX. 
Chapter 8 also shows that the more general models do better than GM79 in modeling the PEQUOD data. The chapter considers a series of models, starting with a model almost as simple as the GM79 model and culminating in a low wavenumber model that includes horizontal and vertical asymmetries, a mixture of modes and propagating waves, and some shear kinematic effects. The more complicated models have smaller residues than the simple models, suggesting that the additional physics contained in the more general models is appropriate.

In chapter 8 , the more general models concentrate on low vertical wavenumbers. This is for several reasons. First of all, the arrangement of instruments into three groups of four means that the array can resolve low wavenumbers $(.1 \mathrm{cpkm})$ and high wavenumbers (10 cpkm) but not medium wavenumbers. This spectral gap makes resolving both low and high wavenumbers rather complicated. Secondly, earlier measurements (such as those incorporated in GM79) show that wavenumber spectra are red: low wavenumbers contain more energy than high wavenumbers. Thus to the extent that the analysis must arbitrarily decide (because of a lack of resolution) that some energy is either low wavenumber energy or high wavenumber energy, the energy probably belongs to low wavenumbers. Finally, while the effects of scattering on low wavenumbers are weak, scattering becomes quite important for high wavenumbers (see section 2.2 and section 7.5). High scattering means that it is not entirely appropriate to model high wavenumber energy with a simple linear model, thus it is not possible to learn much about high wavenumber physics by considering the linear wave structure.

The models of chapter 8 reveal several important features of the internal wave field. The energy content and fluxes of the internal wave field averaged over the frequency band from $.125 \mathrm{cph}$ to $.458 \mathrm{cph}$ are shown in figure 8.7 . In 1981, the vertically averaged energy shows a seasonal cycle with a $10 \%$ amplitude and minimum in the summer. This is consistent with other observations of a seasonal cycle in the internal wave field (Briscoe 1984). The seasonal cycle is not apparent in the 1982 data, but 1982 is the beginning of the 1982-1983 El Niño event and thus is quite likely to be anomalous.

The net vertical energy flux also shows differences between the two years. The first nine months of 1981 all show a net upward flux of $1.2( \pm .4) \times 10^{-3} \mathrm{~W} / \mathrm{m}^{2}$, while the following year and a quarter do not show a significant net vertical energy flux. This is a small fraction of the $30 \times 10^{-3} \mathrm{~W} / \mathrm{m}^{2}$ that the GM79 spectrum would carry were all the internal wave energy propagating in the same direction. On the other hand, it is 
the same size as many of the postulated sources of energy for the internal wave field. Olbers 1983 reviews many of those sources of energy, most of which are approximately $1 \times 10^{-3} \mathrm{~W} / \mathrm{m}^{2}$. The measured vertical flux is the same size as some of the other energy fluxes expected in the ocean: Fofonoff 1981 estimates that the energy flux from the gyre scale winds to the geostrophic flow is roughly $2 \times 10^{-3} \mathrm{~W} / \mathrm{m}^{2}$. Therefore, despite being a small fraction of the maximum flux the internal wave field can carry, the measured vertical fluxes are large enough to be dynamically important.

The net zonal energy flux is also different in the two years. 1981 shows what appears to be a simple seasonal cycle with a significant eastward flux only in the first three months. On the other hand, there is a significantly eastward flux throughout 1982 of roughly $12( \pm 3) \times 10^{-3} \mathrm{~W} / \mathrm{m}^{2}$. While this is a small fraction of the $130 \times 10^{-3} \mathrm{~W} / \mathrm{m}^{2}$ that the GM79 spectrum would carry were all the internal wave energy propagating in the same horizontal direction, it is much larger than the internal wave energy sources reviewed by Olbers 1983. The size of the energy flux into the internal wave field from the wind during parts of JASIN is roughly $4 \times 10^{-3} \mathrm{~W} / \mathrm{m}^{2}$ for a wind of $8 \mathrm{~m} / \mathrm{s}$ (Briscoe 1983). It is conceivable that the large net zonal fluxes are due to the anomalous winds observed in the Western Pacific during early 1981 and most of 1982 (Lukas et al. 1984) since there is a geometrical concentration effect that could make the zonal fluxes greater than the vertical fluxes. If the horizontal extent of wind anomalies is much greater than the depth of the ocean, a vertical flux applied to the ocean surface over a zonal extent of $1000 \mathrm{~km}$ could result in a flux out of the sides of a depth averaged box such that there is a factor of 40 increase between the flux through the top and the flux out the east and west ends of the box. The meridional flux is not enhanced by the same mechanism both because the meridional extent is not as great as the zonal extent and because the wind anomalies are fairly symmetric with respect to the equator.

All of the deviations from GM79-the modal character, the zonal and horizontal asymmetries, the shear kinematic effects-are a fairly small percentage of the internal wave spectrum. Aside from the shear kinematic effects, which are small by assumption because only low wavenumbers were allowed to deviate from GM79, and the modal character, which is small because of a lack of resolution, the smallness is a property of the internal wave field. But the small deviations from GM79 are important in that they are related to the sources and sinks of energy in the internal wave field. The situation is much like that of geostrophy and quasi-geostrophic motions: while geostrophy is a 
very good first order balance and it is quite difficult to measure large-scale deviations from it, it is the small quasi-geostropic deviations that give us information about how the geostrophic flows evolve. In the internal wave case, the small asymmetries and time variability of the internal wave field give us the information about how the internal wave field evolves and interacts. It is only through further analyses of the sort performed in this thesis that the flow of energy in the ocean will ever be adequately measured and understood. 


\section{Appendix A: RVCM measurements of equatorial data}

The PEQUOD moorings were instrumented with temperature-pressure (TP) recorders and Aanderaa current meters, the latter measuring speed, direction, and temperature. The Aanderaa is a standard rotor-vane (RV) current meter: it measures velocity by periodically storing direction and rotor count. By differencing rotor counts, an averaged speed is calculated, this speed can then be combined with the instantaneous direction to calculate velocity components. Because the direction measurement is unaveraged, it is subject to aliasing error, and because the direction information is used in a non-linear way to calculate velocities, it is not immediately obvious how that aliasing affects the two components of velocity. This appendix calculates the errors in velocity components induced by aliasing of the direction measurement. Because of the non-linear nature of the problem, the error is dependent on the true mean as well as the variance of the measurements. By sorting through the possible error sources, and running simulations on data sets similar to PEQUOD, these errors can be estimated. This discussion will first look at the relevant characteristics of Aanderaa current meters, then examine some possible sources of error other than the direction aliasing problem. Finally, the aliasing problem is examined in detail, both theoretically and with simulations that use records similar to those in the PEQUOD data set. 


\section{RVCM description}

The properties of the Aanderaa current meter that are considered here are properties of an idealized RVCM current meter. (This is not an important assumption, since other errors are considered as well, and the relative error sizes compared.) Rather than give a complete description of the instrument (a description which can be found in NOIC 1974), I will concentrate on the features that are considered in the error analysis.

The RVCM measures current speed (water displacement, actually) and direction. Displacement is measured by counting rotor revolutions. Direction is measured in a two-step process: A vane orients the instrument into the current, and the direction of the instrument is measured by a magnetic compass. Temperature, rotor count, and direction are recorded once a sampling interval. Since speed is calculated by differencing rotor counts, it is effectively averaged over the sampling interval, unlike temperature and direction. Consequently the speed is not as sensitive to aliasing. Likewise, the thermistor has a finite mass, which tends to average the thermal measurement. In addition to aliasing, there is another possible problem in that speed lags direction by half a sampling interval. This phase lag can be eliminated by differencing every other rotor count, which unfortunately decreases time resolution. This is only necessary when the frequencies of interest approach the sampling frequency, and there are enough other problems with the high-frequency measurements that this lag is not a real issue.

\section{Competing effects}

The literature on systematic errors in RVCM's has focused on pumping-up of nearsurface instruments by wave action. The pumping-up occurs because the rotor rectifies high frequency variability and distributes that energy over the spectrum. The general consensus is that such pumping-up is minimal for subsurface moorings. There also is evidence of the opposite problem: mean flows altering high frequency flows. In analyzing RVCM data at high frequencies where there was a strong mean, (the same situation as the PEQUOD data set), Barry Ruddick 1977 found a completely artificial enhancement of high-frequency cross-current spectral energy. To understand how this enhancement comes about, define the following variables:

$$
\begin{array}{ll}
u & \text { along-(mean) current } \\
v & \text { cross-(mean) current } \\
S & \text { speed }
\end{array}
$$


$\theta \quad$ direction

Ruddick shows that the errors in direction and speed result in errors in $u$ and $v$ of the form

$$
\begin{aligned}
& d u=d S+\text { h.o.t. } \\
& d v=u d \theta+\text { h.o.t. }
\end{aligned}
$$

where

$$
\begin{aligned}
& u=S \cos \theta \\
& v=S \sin \theta
\end{aligned}
$$

so that if there are significant directional errors one would expect more error variance in the cross-current component. He further points out a possible source of such directional error is a high-frequency (.1 Hertz) torsional oscillation found on some moorings. These errors, however, are clearly white-noise in nature (see figure A.1), and thus the mechanism cannot account for anisotropy seen at lower frequencies (where the spectra are clearly red in nature).

\section{Theoretical error estimate}

A rigorous error calculation quickly becomes ridiculously complicated; an approximate calculation is much more useful. Such an exercise has two benefits: it gives an approximate error estimate, and it provides a framework for understanding the numerical simulation. Since a large fraction of the calculation's utility comes from the light it sheds on the numerical calculation, the conventions used in the theoretical calculation are close analogs of the numerical conventions, and the early discussion will focus on choosing these conventions. The discussion then looks at the approximations necessary to make the problem analytically tractable. Finally, the distribution of error with frequency is calculated, both theoretically and using measurements. 


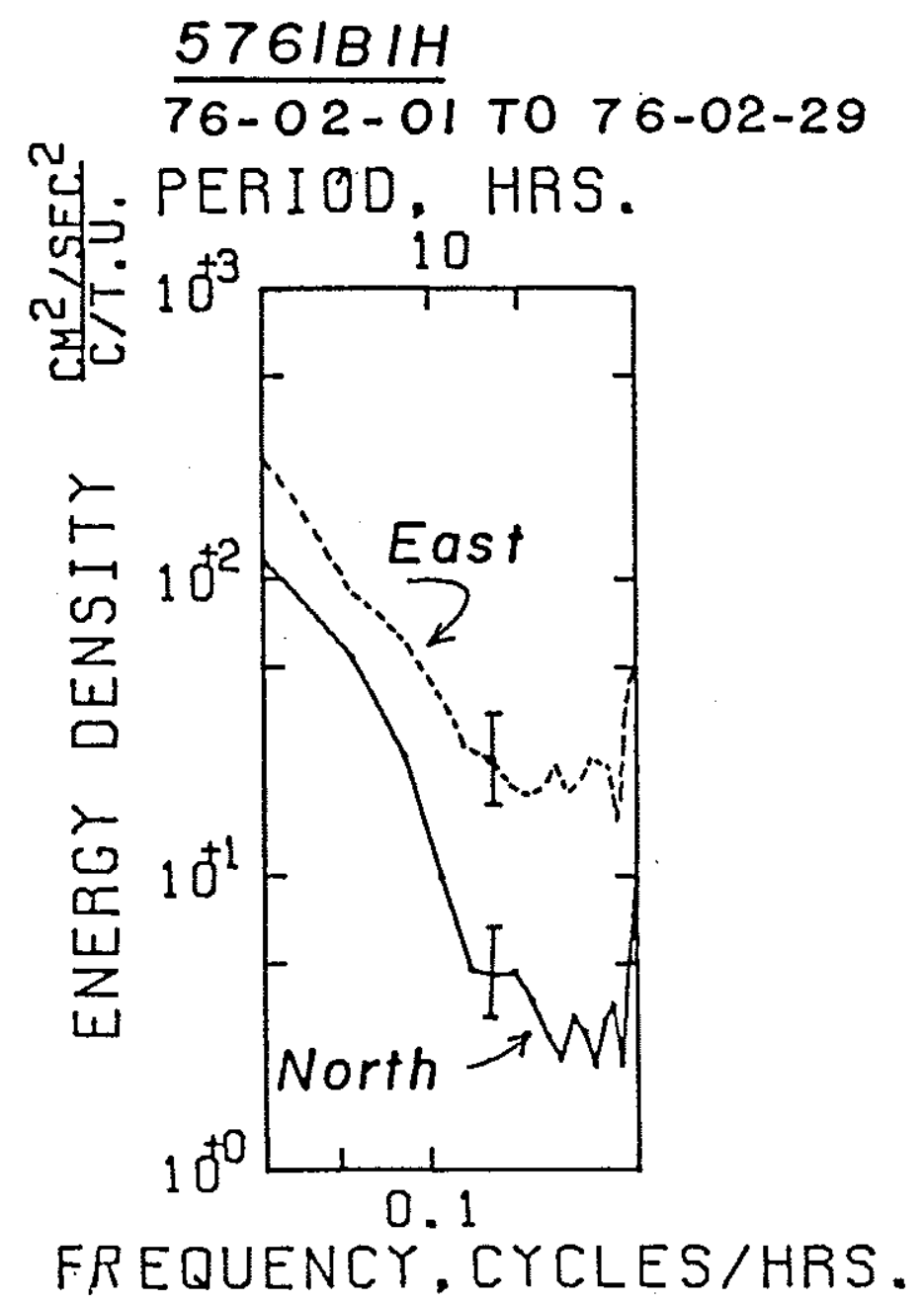

Figure A.1 Power spectra with enhanced cross-current component

The spectra shows an artificial enhancement of cross-current spectra which can be explained by instrumental error. From Ruddick (1977). 


\section{Variables}

The variables used in the discussion are:

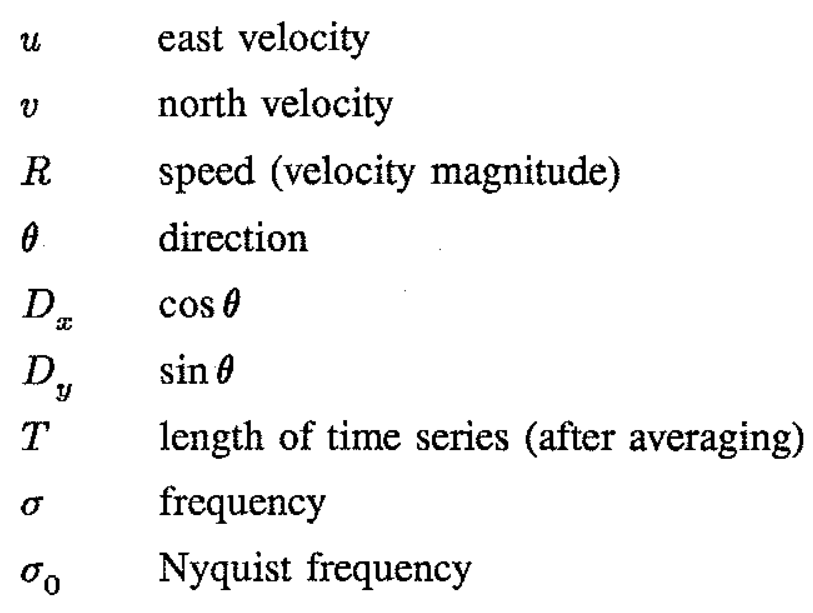

where

$$
\begin{aligned}
& u(t)=R(t) D_{x}(t) \\
& v(t)=R(t) D_{y}(t)
\end{aligned}
$$

The choice of $D_{x}$ and $D y$ rather than the direction $\theta$ is simply to make the calculation more tractable. It also makes the calculations for $u$ and $v$ entirely parallel; so that the calculations need be done explicitly only for $u$. The first six variables are separated into a mean plus deviation: $x^{\prime}$ denotes the deviation, while $\langle x\rangle$ denotes the mean. The spectral convention is such that the one-sided $(0$ to $\infty)$ integral over frequency contains all the energy. It follows that the spectral density can be considered twice the Fourier transform of the auto-correlation integral, making it well defined for both positive and negative frequency. 


\section{Removing means}

Spectra and their corresponding correlation integrals are usually calculated on demeaned data. So equation $A .3 a$ needs to be translated to relations between the spectra $\left(\left\langle u^{*} u\right\rangle(\sigma)\right.$, $\left\langle R^{*} R\right\rangle(\sigma)$, and $\left.\left\langle D_{x}^{*} D_{x}\right\rangle(\sigma)\right)$ and the means $\left(\langle u\rangle,\langle R\rangle,\left\langle D_{x}\right\rangle\right)$. To make things as simple as possible, all the manipulations will be made first in the time domain, then the final relation will be transformed into the spectral domain.

The auto-correlation function is

$$
C(\tau)=\frac{1}{T} \int_{-T / 2}^{T / 2}(u(t)-\langle u\rangle)(u(t+\tau)-\langle u\rangle) d t
$$

which is more concisely written

$$
C(\tau)=\langle(u(t)-\langle u\rangle)(u(t+\tau)-\langle u\rangle)\rangle
$$

The mean velocity is given by the average of equation $A .3 a$,

$$
\langle u\rangle=\langle R\rangle\left\langle D_{x}\right\rangle+\left\langle R^{\prime} D_{x}^{\prime}\right\rangle
$$

Rewriting the correlation function is somewhat more complicated. First define the following shorthand

$$
\begin{aligned}
\lambda(t) & =R^{\prime}(t) D_{x}^{\prime}(t)-\left\langle R^{\prime} D_{x}^{\prime}\right\rangle \\
\langle a b\rangle(\tau) & =\left\langle a^{\prime}(t) b^{\prime}(t+\tau)\right\rangle
\end{aligned}
$$

Then the auto-correlation function can be written as

$$
\begin{aligned}
C(\tau)= & \left\langle\left[\lambda(t)+\langle R\rangle D_{x}(t)+\left\langle D_{x}\right\rangle R(t)\right]\left[\lambda(t+\tau)+\langle R\rangle D_{x}(t+\tau)+\left\langle D_{x}\right\rangle R(t+\tau)\right]\right\rangle \\
= & \langle\lambda \lambda\rangle(\tau)+\langle R\rangle^{2}\left\langle D_{x} D_{x}\right\rangle(\tau)+\left\langle D_{x}\right\rangle^{2}\langle R R\rangle(\tau)+\langle R\rangle\left[\left\langle\lambda D_{x}\right\rangle(\tau)+\left\langle D_{x} \lambda\right\rangle(\tau)\right] \\
& +\left\langle D_{x}\right\rangle[\langle\lambda R\rangle(\tau)+\langle R \lambda\rangle(\tau)]+\langle R\rangle\left\langle D_{x}\right\rangle\left[\left\langle R D_{x}\right\rangle(\tau)+\left\langle D_{x} R\right\rangle(\tau)\right]
\end{aligned}
$$

This is the correlation integral version of squaring a trinomial.

Given some assumptions about the distribution of $R$ and $D_{x}$, the higher moments $\langle\lambda \lambda\rangle(\tau),\left\langle\lambda D_{x}\right\rangle(\tau)$, and $\langle\lambda R\rangle(\tau)$ - can be calculated. Assuming that $(R(t), R(t+\tau)$, 
$D_{x}(t)$, and $\left.D_{x}(t+\tau)\right)$ are joint-normally distributed means the fourth order moments can be rewritten in terms of the second order moments (see appendix B).

$$
\begin{aligned}
\langle\lambda(t) \lambda(t+\tau)\rangle & =\left\langle R^{\prime}(t) D_{x}^{\prime}(t) R^{\prime}(t+\tau) D_{x}^{\prime}(t+\tau)\right\rangle-\left\langle R^{\prime} D_{x}^{\prime}\right\rangle^{2} \\
& =\left\langle R^{\prime}(t) R^{\prime}(t+\tau)\right\rangle\left\langle D_{x}^{\prime}(t) D_{x}^{\prime}(t+\tau)\right\rangle+\left\langle R^{\prime}(t) D_{x}^{\prime}(t+\tau)\right\rangle\left\langle D_{x}^{\prime}(t) R^{\prime}(t+\tau)\right\rangle \\
& =\langle R R\rangle(\tau)\left\langle D_{x} D_{x}\right\rangle(\tau)+\left\langle R D_{x}\right\rangle(\tau)\left\langle D_{x} R\right\rangle(\tau)
\end{aligned}
$$

Joint-Normality means the third order moments are zero.

$$
\langle\lambda R\rangle(\tau)=\left\langle\lambda D_{x}\right\rangle(\tau)=0
$$

While there are cases where the assumption of joint-normality is clearly false (the speed spectra of two zero mean independent random velocities, for example), in the case of strong mean flow the assumption seems reasonable.

In terms of the shorthand defined in equation $A .7 b$ Fourier transforms are particularly simple; the transformation to the spectral domain becomes the following transcription:

$$
\operatorname{FT}(\langle a b\rangle(\tau))=\frac{1}{2}\left\langle a^{*} b\right\rangle(\sigma)
$$

By using the equality that relates the Fourier transform of a product to the convolution of Fourier transforms (Bracewell 1978)

$$
\mathrm{FT}(a(t) b(t))=a(\sigma) * b(\sigma)
$$

(where * denotes convolution), the velocity spectra can be expanded in terms of the measured quantities.

$$
\begin{aligned}
\left\langle u^{*} u\right\rangle(\sigma)= & \frac{1}{2}\left\langle R^{*} R\right\rangle(\sigma) *\left\langle D_{x}^{*} D_{x}\right\rangle(\sigma)+\langle R\rangle^{2}\left\langle D_{x}^{*} D_{x}\right\rangle(\sigma)+\left\langle D_{x}\right\rangle^{2}\left\langle R^{*} R\right\rangle(\sigma) \\
& +\langle R\rangle\left\langle D_{x}\right\rangle\left(\left\langle D_{x}^{*} R\right\rangle(\sigma)+\left\langle R^{*} D_{x}\right\rangle(\sigma)\right)+\frac{1}{2}\left\langle R^{*} D_{x}\right\rangle(\sigma) *\left\langle D_{x}^{*} R\right\rangle(\sigma)
\end{aligned}
$$




\section{Aliasing estimate}

To estimate the effect of direction aliasing on velocity spectra, separate the direction spectrum into a true spectrum plus an error.

$$
<D_{x}^{*} D_{x}>(\sigma)=<D_{x}^{*} D_{x}>(\sigma)_{\text {true }}+\Delta<D_{x}^{*} D_{x}>(\sigma)
$$

The resulting error in the velocity spectrum is found by plugging equation $A .14$ into equation $A .13$.

$$
\begin{aligned}
\Delta<u^{*} u>(\sigma)=\frac{1}{2}<R^{*} R>(\sigma) * \Delta & <D_{x}^{*} D_{x}>(\sigma) \\
& +<R>^{2} \Delta<D_{x}^{*} D_{x}>(\sigma)+\text { h.o.t }
\end{aligned}
$$

The cross-power terms have been dropped since the aliased energy is not expected to be coherent with the unaliased speed measurements. Thus the velocity spectrum error has two parts, a lump at the Nyquist frequency scaled by the mean speed, and a part which is spread out over the spectrum by a convolution with the spectrum of speed.

\section{Aliasing error form}

To write out the velocity error explicitly, the form for the aliased spectrum and the speed spectrum must be known. Rather than choose an arbitrary form for aliased energy, it is better to choose a spectral form for which the aliased energy can be calculated. Aliasing occurs as a consequence of finite sampling of a continuous time series, $f(t)$. This process of finite sampling can be represented by

$$
\operatorname{III}(x)=\sum_{n} \delta(x-n) \quad n \in[-\infty, \infty]
$$

in that

$$
\mathrm{III}(t / \Delta t) f(t)=\sum f(n \Delta t) \delta(t / \Delta t-n)
$$

In the Fourier domain this becomes

$$
\Delta t \operatorname{III}(\sigma \Delta t) * f(\sigma)=\sum_{n} f(t-n \Delta t)
$$

However this is not a good representation of the original function $f$,. since it has the function vanishing between sampling points. A representation that connects sampling 
points smoothly seems more appropriate. An interpolation such that no energy is added to the spectrum beyond what is measured (i.e. there is no energy beyond the Nyquist frequency), is one such choice. In the Fourier domain this is written as

$$
\Pi(\sigma \Delta t) \Delta t \Pi \Pi(\sigma \Delta t) * f(\sigma)
$$

where $\Pi$ is a rectangle function (equal to unity on the interval $\left[-\frac{1}{2}, \frac{1}{2}\right]$ and zero elsewhere). In the time domain the function becomes

$$
\frac{1}{\Delta t} \operatorname{sinc}(t \Delta t) * \operatorname{III}(t / \Delta t) f(t)
$$

which can be rewritten

$$
\sum_{n} f(n \Delta t) \operatorname{sinc}(t / \Delta t-n)
$$

A distinctly better interpolation than equation $A .18$. The sampled Fourier transform is then

$$
\Pi(\sigma \Delta t) \sum_{n} f(\sigma-n / \Delta t)
$$

Koopmans 1974 shows this is also true for the power spectra.

$$
\left\langle f^{*} f\right\rangle(\sigma)=\Pi(\sigma \Delta t) \sum_{n}\left\langle f^{*} f\right\rangle_{\mathrm{true}}(\sigma-n / \Delta t)
$$

For red spectra, the terms in the sum become rapidly less important. The first three terms suffice for most purposes $(n=-1,0,1)$. Solving for the difference from the true spectra then gives

$$
\Delta\left\langle f^{*} f\right\rangle(\sigma)=2 \Pi(2 \Delta t) \Delta t \Pi(2 \Delta t \sigma) \star\left\langle f^{*} f\right\rangle_{\text {true }}(\sigma)
$$

where

$$
\Pi(x)=\frac{1}{2}\left(\delta\left(x-\frac{1}{2}\right)+\delta\left(x+\frac{1}{2}\right)\right)
$$

Equation $A .24$ and equation $A .25$ together can be used to calculated the aliased fraction of the direction spectrum. 


\section{Model Spectrum}

Since the PEQUOD spectra have a slope of roughly -2 at high frequencies, choose model spectra of the form

$$
f(\sigma)=\frac{1}{\gamma^{2}+\left(\sigma / \sigma_{0}\right)^{2}}
$$

where $\gamma$ is a small constant. In fact, if the spectra is sufficiently red so that a small inverse spectral range, $\epsilon$, is expected, where

$$
\epsilon=f\left(\sigma_{0}\right) / f(0)
$$

then the normalized roll-off frequency $\gamma$ is such that

$$
\gamma^{2}=\epsilon /(1-\epsilon) \approx \epsilon
$$

an expression for $\gamma$ in terms of the expected spectral range in amplitude. Realistic values of $\epsilon$ are $10^{-3}$ to $10^{-4}$, so realistic $\gamma$ are indeed small. A spectrum of this form corresponds to the following auto-correlation function:

$$
\frac{\pi \sigma_{0}}{\gamma} e^{-\left|2 \pi \gamma \sigma_{0} t\right|}
$$

A few basic theorems for Fourier transforms (Bracewell 1978) give the convolution of equation $A .26$ with itself:

$$
f(\sigma) \star f(\sigma)=\frac{2 \pi \sigma_{0}}{\gamma\left(4 \gamma^{2}+\left(\sigma / \sigma_{0}\right)^{2}\right)}
$$

Choose model spectra

$$
\begin{aligned}
\left\langle R^{*} R\right\rangle(\sigma) & =\frac{R}{\gamma^{2}+\left(\sigma / \sigma_{0}\right)^{2}} \\
\left\langle D_{x}^{*} D_{x}\right\rangle(\sigma) & =\frac{D_{x}}{\gamma^{2}+\left(\sigma / \sigma_{0}\right)^{2}}
\end{aligned}
$$

Equation $A .30$ combined with equation $A .24$ gives the error $\Delta\left\langle D_{x}^{*} D_{x}\right\rangle(\sigma)$. The expression for velocity error can then be completed to give

$$
\Delta\left\langle u^{*} u\right\rangle(\sigma)=G\left(\sigma+2 \sigma_{0}\right)+G\left(\sigma-2 \sigma_{0}\right)
$$

where

$$
G(\sigma)=\frac{\pi R D_{x} \sigma_{0}}{\gamma\left(4 \gamma^{2}+\left(\sigma / \sigma_{0}\right)^{2}\right)}+\frac{\langle R\rangle^{2} D_{x}}{\gamma^{2}+\left(\sigma / \sigma_{0}\right)^{2}}
$$


Clearly this is well approximated by

$$
G(\sigma)=G\left(\sigma_{0}\right)\left(\sigma / \sigma_{0}\right)^{-2} \quad \sigma / \sigma_{0} \gg \gamma
$$

where

$$
G\left(\sigma_{0}\right) \approx\left[\left(\pi \sigma_{0} / \gamma\right)\left\langle R^{*} R\right\rangle\left(\sigma_{0}\right)+\langle R\rangle^{2}\right]\left\langle D_{x}^{*} D_{x}\right\rangle\left(\sigma_{0}\right)
$$

An approximate expression for velocity spectra then follows

$$
\left\langle u^{*} u\right\rangle(\sigma)=\frac{1}{2}\left\langle R^{*} R\right\rangle(\sigma) \star\left\langle D_{x}^{*} D_{x}\right\rangle(\sigma)+\langle R\rangle^{2}\left\langle D_{x}^{*} D_{x}\right\rangle(\sigma)
$$

an expression which ignores contributions from several terms. A percentage error can also be calculated

$$
\frac{\Delta\left\langle u^{*} u\right\rangle(\sigma)}{\left\langle u^{*} u\right\rangle(\sigma)}=\frac{4 \gamma^{2}+\left(\sigma / \sigma_{0}\right)^{2}}{\left(2-\sigma / \sigma_{0}\right)^{2}} \times 100 \%
$$

This goes from $100 \%$ at $\sigma_{0}$ to $10 \%$ at $\sigma_{0} / 2$ and $2 \%$ at $\sigma_{0} / 4$. As $\sigma_{0}$ approaches zero the percentage error goes to $\gamma^{2} \times 100 \%$. This would indicate the effects of aliasing could be ignored two octaves below the Nyquist frequency and lower.

\section{Simulation using VACM's}

VACM's, while using the same physical apparatus for measuring currents as standard RVCM's, use a quite different sampling scheme. They average east and north velocity components separately, usually every $1 / 8$ rotor count, which eliminates some of the problems found with standard RVCM's. They are interesting in light of interpreting RVCM's because they also record the same information as a standard RVCM (rotor count and direction). This allows a direct comparison of the two averaging schemes, and taking the vector averaged version as 'truth', gives a handle on the errors induced by the RVCM's sampling scheme. Such an intercomparison has already been done for instruments modified to measure wind (see Weller et al. 1983). Their results show that the spectra calculated using the two different methods but with the same data are significantly different in the top several octaves (see figure A.2). So one might expect problems in interpreting data within two octaves of the Nyquist frequency. Since the problem is so non-linear, however, to estimate errors in particular records it is prudent to consider records which have similar spectra. The relevant features for the PEQUOD dataset are: 


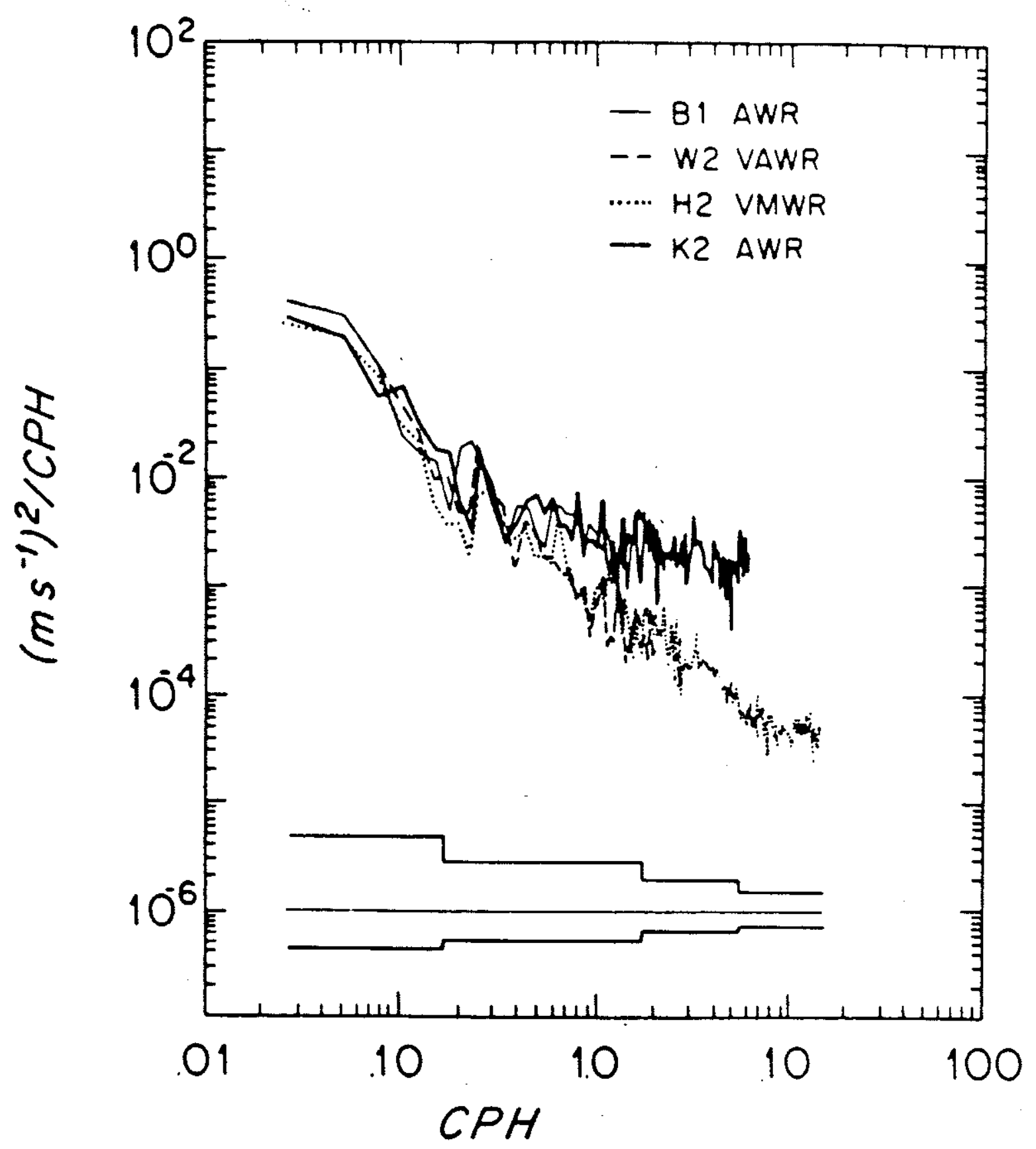

Figure A.2 Wind velocity autospectra from JASIN

Autospectra of wind velocity records from 27 August to 2 September 1978. The coverage of the high frequencies differs because of the different sampling rates of the instruments; all autospectra have been calculated from the original time series and band averaged in the same way. Instrument B1 has a 3.5 minute sampling period, W2 and H2 have 2 minute sampling periods, and $\mathrm{K} 2$ has a 10 minute sampling period. 95 percent confidence limits are shown at the bottom. (From Weller et al. 1983). 
- they are on subsurface moorings

- there is a fairly constant mean current

- there is enhanced spectral energy in the cross current component

Though the PEQUOD moorings are equatorial, there is no strong dynamical effect at sufficiently short scales and sufficiently high frequencies. So the above criteria should be sufficient to insure a useful comparison.

\section{Results}

Figure $A .3$ shows an intercomparison made using a $200 \mathrm{~m}$ record from INDEX79, an equatorial experiment in the Indian ocean. The spectra are enhanced in both components when averaged as a RVCM, which is as expected in the presence of aliasing. At $\sigma_{0}$ the enhancement is $100 \%$; at $\sigma_{0} / 3$ the enhancement is $30 \%$. Note that the error is systematic: the RVCM spectra are always greater than VACM spectra. Furthermore, the enhancement is not the same for meridional and zonal velocities: At $\sigma_{0}$ RVCM zonal velocity is $1.1(\mathrm{~cm} / \mathrm{s})^{2} / \mathrm{cph}$ larger than VACM zonal velocity, while RVCM meridional velocity is $.8(\mathrm{~cm} / \mathrm{s})^{2} / \mathrm{cph}$ larger than VACM meridional velocity. This results in a $40 \%$ increase in the difference between the spectra. That is expected with aliased energy, since larger spectra should increase more.

\section{Conclusions}

In sampling oceanic currents dominated by mean flows (such as are found at the equator), the standard RVCM seriously alters the data. Theoretical estimates using a simplified spectrum indicate that the error generated by the RVCM sampling scheme is spread throughout the spectrum at a fairly constant level. Because of the red nature of the spectra, however, such a noise level becomes inconsequential at frequencies below roughly $\sigma_{0} / 4$. The simulations run on VACM data bear this tendency out: they also indicate that the error is much reduced at two octaves below the Nyquist frequency, (though the actual numbers are only in rough agreement with the theoretical simulation). An important point is that the error is such that it enhances differences between zonal and meridional spectra that may already exist, possibly making a non-significant difference appear significant. In light of this, it is seems wise to avoid depending on autospectra at frequencies higher than $\sigma_{0} / 4$. 

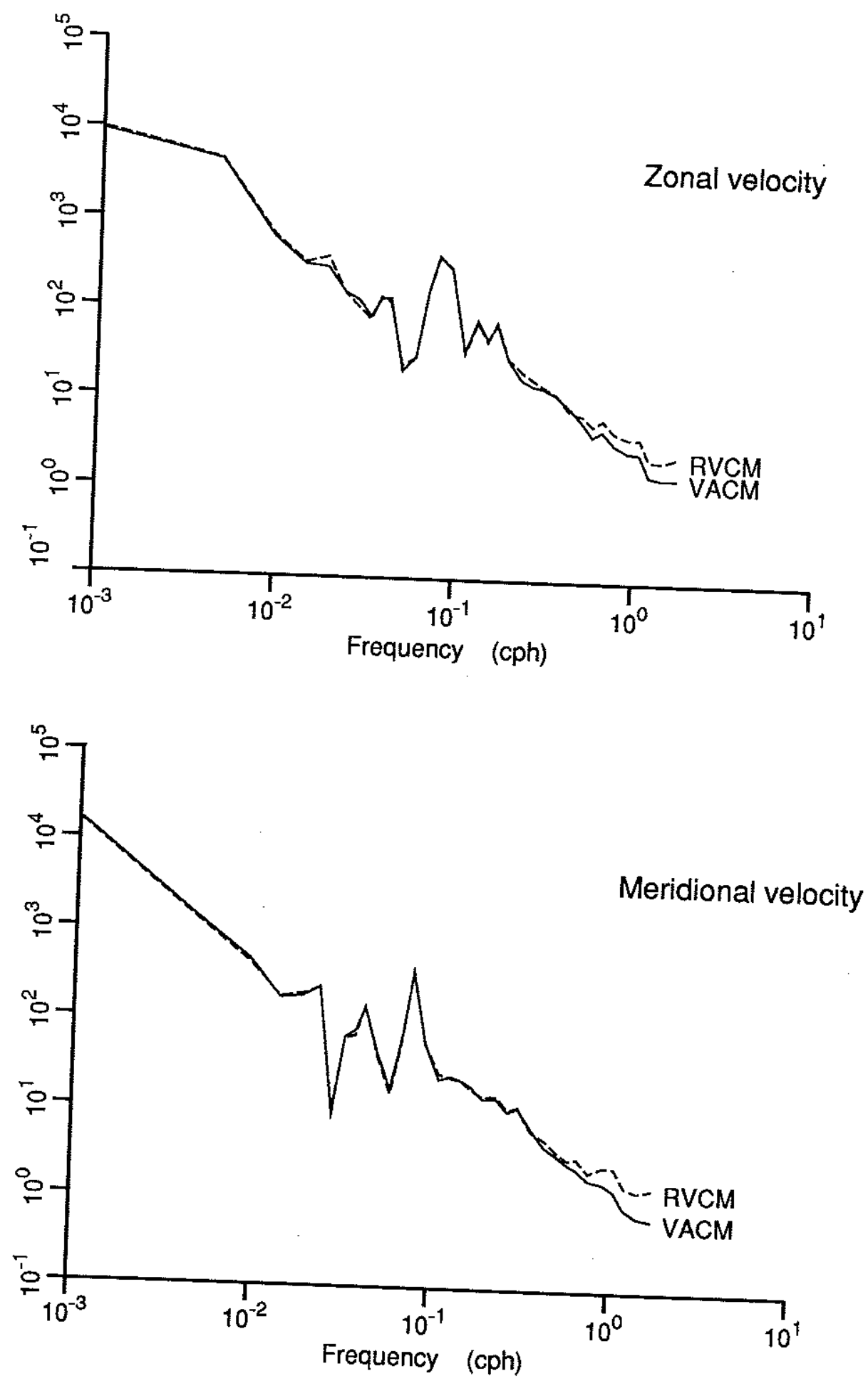

\section{Figure A.3 Current autospectra from VACM/RVCM}

Autospectra of current comparing a vector averaged version of an INDEX79 record to a standard rotor-vane version of the same record. The top plot compares zonal kinetic The rotor-vane version shows excessed data (VACM) to the rotor-vane version (RVCM). energy calculated from vector-averaged energy. The bottom plot compares meridional kinetic Just as with the zonal kinetic energyed data (VACM) to the rotor-vane version (RVCM). 


\section{Appendix B: Fourth order moments for joint-normal variables}

Calculating the fourth order moments of real joint-normal variables is fairly straightforward, it only requires some simple matrix manipulations. The moment is needed both in appendix $A$ (which deals with RVCM current meter aliasing), and appendix $C$ (which calculates the covariance of cross- spectral estimators). Define the following variables:

$\mathbf{z}$ a vector of joint-normal, zero mean random variables

$d \mathbf{z} \quad d z_{1} d z_{2} \ldots d z_{n}$

C covariance matrix $\left\langle z_{i} z_{j}\right\rangle$

$\mathrm{C}^{-1} \quad$ inverse of the covariance matrix

The probability distribution for joint-normal random variables $z_{i}$ is

$$
P(\mathbf{z})=\frac{1}{\sqrt{(2 \pi)^{n}(\operatorname{det} \mathbf{C})}} e^{\frac{1}{2} \mathbf{z}^{x} \mathbf{C}^{-1} \mathbf{z}}
$$

The expected value for the fourth moment is then

$$
\left\langle z_{i} z_{j} z_{k} z_{l}\right\rangle=\frac{1}{\sqrt{(2 \pi)^{n}(\operatorname{det} C)}} \int_{-\infty}^{\infty} \ldots \int_{-\infty}^{\infty} z_{i} z_{j} z_{k} z_{l} e^{\frac{1}{2} \mathbf{z}^{T} \mathbf{C}^{-1} \mathbf{z}} d \mathbf{z}
$$

where repeated indices denote summation. To evaluate this integral, perform a change of basis from $\mathbf{z}$ to the normalized eigenvector basis $\mathbf{y}$ (i.e. the $y_{i}$ are all independent with unit variance). Therefore

$$
\begin{aligned}
\mathbf{y} & ={ }^{-1} \mathbf{U}^{T} \mathbf{z} \\
d \mathbf{y} & =(\operatorname{det} \mathbf{C})^{-1 / 2} d \mathbf{z}
\end{aligned}
$$

where ${ }^{2}$ is the diagonal matrix of eigenvalues $\sigma_{i}^{2}$, and $\mathbf{U}$ is the matrix of eigenvectors. Thus equation $B .2$ becomes

$$
\begin{aligned}
& \left\langle z_{i} z_{j} z_{k} z_{l}\right\rangle= \\
& \quad \frac{1}{\sqrt{(2 \pi)^{n}}} U_{i m} \sigma_{m m} U_{j n} \sigma_{n n} U_{k o} \sigma_{o o} U_{l p} \sigma_{p p} \int_{-\infty}^{\infty} \ldots \int_{-\infty}^{\infty} y_{m} y_{n} y_{o} y_{p} e^{\frac{1}{2} y^{T} \mathbf{y}} d \mathbf{y}
\end{aligned}
$$

The $y_{i}$ are all independent, so the integral is non-zero only if the $y_{i}$ are pairwise identical, or if all four indices are the same $(m=n=o=p)$. The univariant moments for zero mean, unit variance random variables are

$$
\langle y y\rangle=1 \quad \text { and } \quad\left\langle y^{4}\right\rangle=3
$$


so that the fourth moment for joint-normal (Gaussian) independent variables is

$$
\left\langle y_{m} y_{n} y_{o} y_{p}\right\rangle=\delta_{m n} \delta_{o p}+\delta_{m o} \delta_{n p}+\delta_{m p} \delta_{n o}
$$

Plugging this into equation $B .4$ and performing the change of basis gives the desired result,

$$
\left\langle z_{i} z_{j} z_{k} z_{l}\right\rangle=\left\langle z_{i} z_{j}\right\rangle\left\langle z_{k} z_{l}\right\rangle+\left\langle z_{i} z_{k}\right\rangle\left\langle z_{j} z_{l}\right\rangle+\left\langle z_{i} z_{l}\right\rangle\left\langle z_{j} z_{k}\right\rangle
$$




\section{Appendix C: Covariance of cross-spectral estimators}

The covariance of the cross-spectral estimators is a straight-forward result of their being the covariance of complex joint-normal random variables. To see this, start with a vector of complex joint-normal random variables $m_{i}$. They are zero mean variables, so that $\left\langle m_{i}\right\rangle=0$ for all $i$. Thus their covariance is given simply by $\left\langle m_{i}^{*} m_{j}\right\rangle$. Consider for a moment the fourth moment of these complex quantities, $\left\langle m_{i}^{*} m_{j} m_{k}^{*} m_{l}\right\rangle$. Since the $m_{i}$ are joint-normal (Gaussian) quantities, this fourth moment can be rewritten in terms of second moments,

$$
\left\langle m_{i}^{*} m_{j} m_{k}^{*} m_{l}\right\rangle=\left\langle m_{i}^{*} m_{j}\right\rangle\left\langle m_{k}^{*} m_{l}\right\rangle+\left\langle m_{i}^{*} m_{l}\right\rangle\left\langle m_{k}^{*} m_{j}\right\rangle+\left\langle m_{i}^{*} m_{k}^{*}\right\rangle\left\langle m_{j} m_{l}\right\rangle
$$

This is derived by first writing the complex variables in terms of their real and imaginary parts, then using the results for real variables (appendix $B$ ). The covariance of the unaveraged cross-spectral estimators can be written in terms of this fourth moment,

$$
\begin{aligned}
\left\langle\left(m_{i}^{*} m_{j}-\left\langle m_{i}^{*} m_{j}\right\rangle\right)^{*}\left(m_{k}^{*} m_{l}-\left\langle m_{k}^{*} m_{l}\right\rangle\right)\right\rangle & =\left\langle m_{j}^{*} m_{i} m_{k}^{*} m_{l}\right\rangle-\left\langle m_{i}^{*} m_{j}\right\rangle^{*}\left\langle m_{k}^{*} m_{l}\right\rangle \\
& =\left\langle m_{k}^{*} m_{i}\right\rangle\left\langle m_{j}^{*} m_{l}\right\rangle+\left\langle m_{i} m_{l}\right\rangle\left\langle m_{j}^{*}, m_{k}^{*}\right\rangle
\end{aligned}
$$

The second term in equation $C .2 b$ is identical to the first term if the $m_{i}$ are real. If the $m_{i}$ are complex, however, the term is zero. The latter follows from the assumption that the $m_{i}$ can be represented as a linear transformation of an underlying set of independent complex random variables $y_{i}$. Complex random variables are simply a special case of linear combinations of real random variables (real and imaginary parts have equal variance and are uncorrelated). Thus if we write

$$
y_{i}=R_{i}+i I_{i} \quad\left\langle R_{i} R_{j}\right\rangle=\left\langle I_{i} I_{j}\right\rangle=\frac{1}{2} \lambda_{i} \delta_{i j} \quad\left\langle R_{i} I_{j}\right\rangle=0
$$

we find that the second moments of $y_{i}$ are simply

$$
\left\langle y_{i}^{*} y_{j}\right\rangle=\lambda_{i} \delta_{i j} \quad\left\langle y_{i} y_{j}\right\rangle=\left\langle R_{i} R_{j}\right\rangle-\left\langle I_{i} I_{j}\right\rangle=0
$$

Therefore, if the linear relation between $m_{i}$ and $y_{i}$ is $m_{i}=u_{i j}^{*} y_{j}$, the second moments of $m_{j}$ are

$$
\left\langle m_{i}^{*} m_{j}\right\rangle=u_{i k} u_{j k}^{*} \lambda_{k}
$$


and

$$
\left\langle m_{i} m_{j}\right\rangle=u_{i k}^{*} u_{j l}^{*}\left\langle y_{k} y_{l}\right\rangle=0
$$

Thus as long as the $m_{i}$ are complex numbers,

$$
\begin{aligned}
&\left\langle\left(m_{i}^{*} m_{j}-\left\langle m_{i}^{*} m_{j}\right\rangle\right)^{*}\left(m_{k}^{*} m_{l}-\left\langle m_{k}^{*} m_{l}\right\rangle\right)\right\rangle=\left\langle m_{k}^{*} m_{i}\right\rangle\left\langle m_{j}^{*} m_{l}\right\rangle \\
&\left\langle\left(m_{i}^{*} m_{j}-\left\langle m_{i}^{*} m_{j}\right\rangle\right)\left(m_{k}^{*} m_{l}-\left\langle m_{k}^{*} m_{l}\right\rangle\right)\right\rangle=\left\langle m_{i}^{*} m_{l}\right\rangle\left\langle m_{k}^{*} m_{j}\right\rangle
\end{aligned}
$$

There is only a small difference between equations $C .6 a$ and $C .6 b$. In the first case, the conjugate of the $m_{i} m_{j}$ differences is averaged, while in the second case the unaltered differences are averaged. Both are acceptable definitions for covariance, but it is somewhat less confusing if the complex conjugate is put in explicitly, i.e.

$$
\operatorname{Cov}\left(\left(m_{i} m_{j}\right)^{*},\left(m_{k} m_{l}\right)\right)=\left\langle\left(m_{i}^{*} m_{j}-\left\langle m_{i}^{*} m_{j}\right\rangle\right)^{*}\left(m_{k}^{*} m_{l}-\left\langle m_{k}^{*} m_{l}\right\rangle\right)\right\rangle
$$

and

$$
\operatorname{Cov}\left(\left(m_{i} m_{j}\right),\left(m_{k} m_{l}\right)\right)=\left\langle\left(m_{i}^{*} m_{j}-\left\langle m_{i}^{*} m_{j}\right\rangle\right)\left(m_{i}^{*} m_{j}-\left\langle m_{k}^{*} m_{l}\right\rangle\right)\right\rangle
$$

In the case of averaged estimates of the cross-spectral matrix, the variance is reduced by the amount of independent realizations averaged together, $N_{r}$. Denoting the averaged estimate by $\hat{f}_{i j}$, and the true value by $f_{i j}^{\text {true }}=\left\langle m_{i}^{*} m_{j}\right\rangle$, this results in an amended version of equation C.6,

$$
\operatorname{Cov}\left(\hat{f}_{i j}, \hat{f}_{k l}\right)=\frac{1}{N_{r}} f_{i l}^{\text {true }} f_{k j}^{\text {true }}
$$




\section{Appendix D: Current meter spectra in terms of $E \triangle P Q$ direction moments}

The starting equation is the polarization relations for an internal wave $A e^{i(k x+l y-\omega t)} G(z)$ in a mean zonal shear flow,

$$
\left[\begin{array}{c}
u \\
v \\
\eta \\
p^{\prime}
\end{array}\right]=\left[\begin{array}{c}
\cos \theta \\
\sin \theta \\
0 \\
\frac{\sigma}{K}
\end{array}\right] G_{z}^{i}(z) A+\left[\begin{array}{c}
-\sin ^{2} \theta U_{z} / N_{0} \\
\sin \theta \cos \theta U_{z} / N_{0} \\
N_{0}^{-1} \\
\frac{\sigma U_{z}}{K N_{0}} \cos \theta
\end{array}\right] \frac{K N_{0}}{\sigma} G^{i}(z) A
$$

where $\sigma=\omega-U K \cos \theta$ is the intrinsic frequency. This is rewritten in terms of normalized variables as

$$
\left[\begin{array}{l}
u \\
v \\
d \\
p
\end{array}\right]=\left[\begin{array}{c}
\cos \theta \\
\sin \theta \\
0 \\
1
\end{array}\right] \psi_{i} A+\left[\begin{array}{c}
-\sin ^{2} \theta \lambda_{i} \\
\sin \theta \cos \theta \lambda_{i} \\
1 \\
\cos \theta \lambda_{i}
\end{array}\right] \phi_{i} A
$$

where

$$
\begin{array}{ll}
d=N \eta & \text { normalized displacement } \\
p=p^{\prime} K / \sigma & \text { normalized pressure } \\
\phi_{i}=\frac{K N\left(z_{i}\right)}{\sigma_{i}} \alpha\left(z_{i}\right) & \text { normalized vertical structure } \\
\psi_{i}=\alpha_{z}\left(z_{i}\right) & \text { vertical derivative of } \alpha \\
\lambda_{i}=\frac{U_{z}\left(z_{i}\right)}{N\left(z_{i}\right)} & \text { inverse root Richardson number }
\end{array}
$$

$p^{\prime}$ is the reduced pressure; $p$ is the reduced pressure normalized by the phase speed. Low modes (which are the most energetic) are such that their $\alpha_{z}$ and $\phi$ only weakly depend on direction $\theta(\sigma \gg U k)$. Thus we proceed to expand in terms of the direction moments of $E \Delta P Q$, ignoring the effects of direction on $\psi$ and $\phi$.

The first step in computing spectra for these waves is to rewrite equation $D .2$ in vector notation

$$
\mathbf{u}=\mathbf{s} \psi+\mathbf{r} \phi
$$

This equation can be squared to get the cross spectra

$$
\left\langle\mathbf{u}_{1}^{*} \mathbf{u}_{2}\right\rangle^{T}=\left(\mathbf{s}_{1}^{*} \mathbf{s}_{2}^{T} \psi_{1}^{*} \psi_{2}+\mathbf{r}_{1}^{*} \mathbf{s}_{2}^{T} \phi_{1}^{*} \psi_{2}+\mathbf{s}_{1}^{*} \mathbf{r}_{2}^{T} \psi_{1}^{*} \phi_{2}+\mathbf{r}_{1}^{*} \mathbf{r}_{2}^{T} \phi_{1}^{*} \phi_{2}\right) A^{*} A
$$

which is the same expansion found in chapter 5 . What follows is merely the explicit 
algebra implied by this matrix equation. Note that

$$
\left\langle\mathbf{u}_{1}^{*} \mathbf{u}_{2}\right\rangle=\left[\begin{array}{cccc}
u^{*} u & u^{*} v & u^{*} d & u^{*} p \\
v^{*} u & v^{*} v & v^{*} d & v^{*} p \\
d^{*} u & d^{*} v & d^{*} d & d^{*} p \\
p^{*} u & p^{*} v & p^{*} d & p^{*} p
\end{array}\right]
$$

The matrices in figure $D .1$ are subsets of what was given in chapter 5 ( $w$ has been omitted since it is proportional to the normalized displacement $d$ ). Plugging $D .7$ into $D .5$ and using the direction moment expansion given in equation 8.3.7 gives the expansions in figure D.2. Note that since products involving reduced pressure $p$ are only needed to compute energies (see sections 5.9 and 5.10), the forms given for those products do not include shear effects.

\section{Linearized Doppler shift}

In assuming that $\phi_{i}$ does not depend on direction $\theta$, the derivation so far assumes that the Doppler shifts that differentiate the intrinsic frequency $\sigma$ from the true frequency $\omega$ are essentially zero. By doing the following linearization the first order effects of Doppler shift can be included in the above expansions.

$$
\begin{aligned}
\phi_{i}^{\prime} & =\frac{K N\left(z_{i}\right)}{\sigma_{i}} \alpha\left(z_{i}\right) \\
& \approx \frac{K N\left(z_{i}\right)}{\omega} \alpha\left(z_{i}\right)\left[1+U\left(z_{i}\right) /(\omega / K) \cos \theta\right] \\
& =\phi_{i}\left[1+\tau_{i} \cos \theta\right]
\end{aligned}
$$

where $\tau_{i}$ is the Doppler shift $U_{\left(z_{i}\right) K / \omega, \phi^{\prime}}$ is the vertical structure function that includes the effects of Doppler shift, and $\phi$ is the vertical structure function that does not include those effects. Going from $\phi^{\prime}$ to $\phi$ modifies two of the three types of vertical structure function products

$$
\begin{aligned}
& \psi_{1} \phi_{2}^{\prime}=\psi_{1} \phi_{2}+\psi_{1} \phi_{2} \tau_{2} \cos \theta \\
& \phi_{1}^{\prime} \phi_{2}^{\prime} \approx \phi_{1} \phi_{2}+\phi_{1} \phi_{2}\left(\tau_{1}+\tau_{2}\right) \cos \theta
\end{aligned}
$$

where terms in squared Doppler shift have been dropped. The expressions in equation $D .9$ can be substituted into equation $D .5$ to get expansions that include the effects of small Doppler shifts on the low modes. 
Figure D.1 Coefficient matrices

$$
\begin{aligned}
& \mathbf{s}_{1}^{*} \mathbf{s}_{2}^{T}=\left[\begin{array}{cccc}
\cos ^{2} \theta & \cos \theta \sin \theta & 0 & \cos \theta \\
\cos \theta \sin \theta & \sin ^{2} \theta & 0 & \sin \theta \\
0 & 0 & 0 & 0 \\
\cos \theta & \sin \theta & 0 & 1
\end{array}\right] \\
& =\left[\begin{array}{cccc}
\frac{1}{2}+\frac{1}{2} \cos 2 \theta & \frac{1}{2} \sin 2 \theta & 0 & \cos \theta \\
\frac{1}{2} \sin 2 \theta & \frac{1}{2}-\frac{1}{2} \cos 2 \theta & 0 & \sin \theta \\
0 & 0 & 0 & 0 \\
\cos \theta & \sin \theta & 0 & 1
\end{array}\right] \\
& \mathbf{r}_{1}^{*} \mathbf{s}_{2}^{T}=\left[\begin{array}{cccc}
-\cos \theta \sin ^{2} \theta \lambda_{1} & -\sin ^{3} \theta \lambda_{1} & 0 & -\sin ^{2} \theta \lambda_{1} \\
\sin \theta \cos ^{2} \theta \lambda_{1} & \sin ^{2} \theta \cos \theta \lambda_{1} & 0 & \sin \theta \cos \theta \lambda_{1} \\
\cos \theta & \sin \theta & 0 & 1 \\
\cos ^{2} \theta \lambda_{1} & \sin \theta \cos \theta \lambda_{1} & 0 & \cos \theta \lambda_{1}
\end{array}\right] \\
& =\left[\begin{array}{cccc}
\frac{1}{4}(-\cos \theta+\cos 3 \theta) \lambda_{1} & \frac{1}{4}(-3 \sin \theta+\sin 3 \theta) \lambda_{1} & 0 & \frac{1}{2}(-1+\cos 2 \theta) \lambda_{1} \\
\frac{1}{4}(\sin \theta+\sin 3 \theta) \lambda_{1} & \frac{1}{4}(\cos \theta-\cos 3 \theta) \lambda_{1} & 0 & \frac{1}{2}(\sin 2 \theta) \lambda_{1} \\
\cos \theta & \sin \theta & 0 & 1 \\
\frac{1}{2}(1+\cos 2 \theta) \lambda_{1} & \frac{1}{2}(\sin 2 \theta) \lambda_{1} & 0 & \cos \theta \lambda_{1}
\end{array}\right] \\
& \mathbf{s}_{1}^{*} \mathbf{r}_{2}^{T}=\left[\begin{array}{cccc}
-\cos \theta \sin ^{2} \theta \lambda_{2} & \sin \theta \cos ^{2} \theta \lambda_{2} & \cos \theta & \cos ^{2} \theta \lambda_{2} \\
-\sin ^{3} \theta \lambda_{2} & \cos \theta \sin ^{2} \theta \lambda_{2} & \sin \theta & \sin \theta \cos \theta \lambda_{2} \\
0 & 0 & 0 & 0 \\
-\sin ^{2} \theta \lambda_{2} & \sin \theta \cos \theta \lambda_{2} & 1 & \cos \theta \lambda_{2}
\end{array}\right] \\
& =\left[\begin{array}{cccc}
\frac{1}{4}(-\cos \theta+\cos 3 \theta) \lambda_{2} & \frac{1}{4}(\sin \theta+\sin 3 \theta) \lambda_{2} & \cos \theta & \frac{1}{2}(1+\cos 2 \theta) \lambda_{2} \\
\frac{1}{4}(-3 \sin \theta+\sin 3 \theta) \lambda_{2} & \frac{1}{4}(\cos \theta-\cos 3 \theta) \lambda_{2} & \sin \theta & \frac{1}{2}(\sin 2 \theta) \lambda_{2} \\
0 & 0 & 0 & 0 \\
\frac{1}{2}(-1+\cos 2 \theta) \lambda_{2} & \frac{1}{2}(\sin 2 \theta) \lambda_{2} & 1 & \cos \theta \lambda_{2}
\end{array}\right] \\
& \mathbf{r}_{1}^{*} \mathbf{r}_{2}^{T}=\left[\begin{array}{cccc}
\sin ^{4} \theta \lambda_{1} \lambda_{2} & -\sin ^{3} \theta \cos \theta \lambda_{1} \lambda_{2} & -\sin ^{2} \theta \lambda_{1} & -\sin ^{2} \theta \cos \theta \lambda_{1} \lambda_{2} \\
-\sin ^{3} \theta \cos \theta \lambda_{1} \lambda_{2} & \sin ^{2} \theta \cos ^{2} \theta \lambda_{1} \lambda_{2} & \sin \theta \cos \theta \lambda_{1} & \sin \theta \cos ^{2} \theta \lambda_{1} \lambda_{2} \\
-\sin ^{2} \theta \lambda_{2} & \sin \theta \cos \theta \lambda_{2} & 1 & \cos \theta \lambda_{2} \\
-\sin ^{2} \theta \cos \theta \lambda_{1} \lambda_{2} & \sin \theta \cos ^{2} \theta \lambda_{1} \lambda_{2} & \cos \theta \lambda_{1} & \cos ^{2} \theta \lambda_{1} \lambda_{2}
\end{array}\right]= \\
& {\left[\begin{array}{cccc}
\left(\frac{3}{8}-\frac{1}{2} \cos 2 \theta+\frac{1}{8} \cos 4 \theta\right) \lambda_{1} \lambda_{2} & \left(-\frac{1}{4} \sin 2 \theta+\frac{1}{8} \sin 4 \theta\right) \lambda_{1} \lambda_{2} & \left(-\frac{1}{2}+\frac{1}{2} \cos 2 \theta\right) \lambda_{1} & \frac{1}{4}(-\cos \theta+\cos 3 \theta) \lambda_{1} \lambda_{2} \\
\left(-\frac{1}{4} \sin 2 \theta+\frac{1}{8} \sin 4 \theta\right) \lambda_{1} \lambda_{2} & \left(\frac{1}{8}-\frac{1}{8} \cos 4 \theta\right) \lambda_{1} \lambda_{2} & \frac{1}{2} \sin 2 \theta \lambda_{1} & \frac{1}{4}(\sin \theta+\sin 3 \theta) \lambda_{1} \lambda_{2} \\
\left(\frac{1}{2}+\frac{1}{2} \cos 2 \theta\right) \lambda_{2} & \frac{1}{2} \sin 2 \theta \lambda_{2} & 1 & \cos \theta \lambda_{2} \\
\frac{1}{4}(-\cos \theta+\cos 3 \theta) \lambda_{1} \lambda_{2} & \frac{1}{4}(\sin \theta+\sin 3 \theta) \lambda_{1} \lambda_{2} & \cos \theta \lambda_{1} & \frac{1}{2}(1+\cos 2 \theta) \lambda_{1} \lambda_{2}
\end{array}\right]}
\end{aligned}
$$


$\left\langle u_{1}^{*} u_{2}\right\rangle=\left(\frac{1}{2} \Re\left(\psi_{1}^{*} \psi_{2}\right)+\frac{3}{8} \lambda_{1} \lambda_{2} \Re\left(\phi_{1}^{*} \phi_{2}\right)\right) E_{0}-\left(\frac{1}{4} \lambda_{1} \Re\left(\phi_{1}^{*} \psi_{2}\right)+\frac{1}{4} \lambda_{2} \Re\left(\psi_{1}^{*} \phi_{2}\right)\right) E_{1 c}+\left(\frac{1}{2} \Re\left(\psi_{1}^{*} \psi_{2}\right)-\frac{1}{2} \lambda_{1} \lambda_{2} \Re\left(\phi_{1}^{*} \phi_{2}\right)\right) E_{2 c}+\left(\frac{1}{4} \lambda_{1} \Re\left(\phi_{1}^{*} \psi_{2}\right)+\frac{1}{4} \lambda_{2} \Re\left(\psi_{1}^{*} \phi_{2}\right)\right) E_{3 c}+\frac{1}{8} \lambda_{1} \lambda_{2} \Re\left(\phi_{1}^{*} \phi_{2}\right) E_{4 c}$ $+\left(\frac{1}{2} \Im\left(\psi_{1}^{*} \psi_{2}\right)+\frac{3}{8} \lambda_{1} \lambda_{2} \Im\left(\phi_{1}^{*} \phi_{2}\right)\right) i \Delta_{0}-\left(\frac{1}{4} \lambda_{1} \Im\left(\phi_{1}^{*} \psi_{2}\right)+\frac{1}{4} \lambda_{2} \Im\left(\psi_{1}^{*} \phi_{2}\right)\right) i \Delta_{1 c}+\left(\frac{1}{2} \Im\left(\psi_{1}^{*} \psi_{2}\right)-\frac{1}{2} \lambda_{1} \lambda_{2} \Im\left(\phi_{1}^{*} \phi_{2}\right)\right) i \Delta_{2 c}+\left(\frac{1}{4} \lambda_{1} \Im\left(\phi_{1}^{*} \psi_{2}\right)+\frac{1}{4} \lambda_{2} \Im\left(\psi_{1}^{*} \phi_{2}\right)\right) i \Delta_{3 c}+\frac{1}{8} \lambda_{1} \lambda_{2} \Im\left(\phi_{1}^{*} \phi_{2}\right) i \Delta_{4} i$ $+\left(\frac{1}{2} \Re\left(\psi_{1} \psi_{2}\right)+\frac{3}{8} \lambda_{1} \lambda_{2} \Re\left(\phi_{1} \phi_{2}\right)\right) P_{0}-\left(\frac{1}{4} \lambda_{1} \Re\left(\phi_{1} \psi_{2}\right)+\frac{1}{4} \lambda_{2} \Re\left(\psi_{1} \phi_{2}\right)\right) P_{1 c}+\left(\frac{1}{2} \Re\left(\psi_{1} \psi_{2}\right)-\frac{1}{2} \lambda_{1} \lambda_{2} \Re\left(\phi_{1} \phi_{2}\right)\right) P_{2 u}+\left(\frac{1}{4} \lambda_{1} \Re\left(\phi_{1} \psi_{2}\right)+\frac{1}{4} \lambda_{2} \Re\left(\psi_{1} \phi_{2}\right)\right) P_{3 c}+\frac{1}{8} \lambda_{1} \lambda_{2} \Re\left(\phi_{1} \phi_{2}\right) P_{4 c}$ $-\left(\frac{1}{2} \Im\left(\psi_{1} \psi_{2}\right)+\frac{8}{8} \lambda_{1} \lambda_{2} \Im\left(\phi_{1} \phi_{2}\right)\right) Q_{0}+\left(\frac{1}{4} \lambda_{1} \Im\left(\phi_{1} \psi_{2}\right)+\frac{1}{4} \lambda_{2} \Im\left(\psi_{1} \phi_{2}\right)\right) Q_{1 c}-\left(\frac{1}{2} \Im\left(\psi_{1} \psi_{2}\right)-\frac{1}{2} \lambda_{1} \lambda_{2} \Im\left(\phi_{1} \phi_{2}\right)\right) Q_{2 v}-\left(\frac{1}{4} \lambda_{1} \Im\left(\phi_{1} \psi_{2}\right)+\frac{1}{4} \lambda_{2} \Im\left(\psi_{1} \phi_{2}\right)\right) Q_{3 c}-\frac{1}{8} \lambda_{1} \lambda_{2} \Im\left(\phi_{1} \phi_{2}\right) Q_{4 c}$

$\left\langle u_{1}^{*} v_{2}\right\rangle=\left(-\frac{3}{4} \lambda_{1} \Re\left(\phi_{1}^{*} \psi_{2}\right)+\frac{1}{4} \lambda_{2} \Re\left(\psi_{1}^{*} \phi_{2}\right)\right) E_{1 s}+\left(\frac{1}{2} \Re\left(\psi_{1}^{*} \psi_{2}\right)-\frac{1}{4} \lambda_{1} \lambda_{2} \Re\left(\phi_{1}^{*} \phi_{2}\right)\right) E_{2 s}+\left(\frac{1}{4} \lambda_{1} \Re\left(\phi_{1}^{*} \psi_{2}\right)+\frac{1}{4} \lambda_{2} \Re\left(\psi_{1}^{*} \phi_{2}\right)\right) E_{9 s}+\frac{1}{8} \lambda_{1} \lambda_{2} \Re\left(\phi_{1}^{*} \phi_{2}\right) E_{4 s}$ $+\left(-\frac{3}{4} \lambda_{1} \Im\left(\phi_{1}^{*} \psi_{2}\right)+\frac{1}{4} \lambda_{2} \Im\left(\psi_{1}^{*} \phi_{2}\right)\right) i \Delta_{1 s}+\left(\frac{1}{2} \Im\left(\psi_{1}^{*} \psi_{2}\right)-\frac{1}{4} \lambda_{1} \lambda_{2} \Im\left(\phi_{1}^{*} \phi_{2}\right)\right) i \Delta_{2 s}+\left(\frac{1}{4} \lambda_{1} \Im\left(\phi_{1}^{*} \psi_{2}\right)+\frac{1}{4} \lambda_{2} \Im\left(\psi_{1}^{*} \phi_{2}\right)\right) i \Delta_{9 s}+\frac{1}{8} \lambda_{1} \lambda_{2} \Im\left(\phi_{1}^{*} \phi_{2}\right) i \Delta_{4 s}$ $+\left(-\frac{3}{4} \lambda_{1} \Re\left(\phi_{1} \psi_{2}\right)+\frac{1}{4} \lambda_{2} \Re\left(\psi_{1} \phi_{2}\right)\right) P_{1 s}+\left(\frac{1}{2} \Re\left(\psi_{1} \psi_{2}\right)-\frac{1}{4} \lambda_{1} \lambda_{2} \Re\left(\phi_{1} \phi_{2}\right)\right) P_{2 s}+\left(\frac{1}{4} \lambda_{1} \Re\left(\phi_{1} \psi_{2}\right)+\frac{1}{4} \lambda_{2} \Re\left(\psi_{1} \phi_{2}\right)\right) P_{3 s}+\frac{1}{8} \lambda_{1} \lambda_{2} \Re\left(\phi_{1} \phi_{2}\right) P_{4 s}$ $-\left(-\frac{9}{4} \lambda_{1} \Im\left(\phi_{1} \psi_{2}\right)+\frac{1}{4} \lambda_{2} \Im\left(\psi_{1} \phi_{2}\right)\right) Q_{1 s}-\left(\frac{1}{2} \Im\left(\psi_{1} \psi_{2}\right)-\frac{1}{4} \lambda_{1} \lambda_{2} \Im\left(\phi_{1} \phi_{2}\right)\right) Q_{2 s}-\left(\frac{1}{4} \lambda_{1} \Im\left(\phi_{1} \psi_{2}\right)+\frac{1}{4} \lambda_{2} \Im\left(\psi_{1} \phi_{2}\right)\right) Q_{s s}-\frac{1}{8} \lambda_{1} \lambda_{2} \Im\left(\phi_{1} \phi_{2}\right) Q_{4 s}$

$\left\langle u_{1}^{*} d_{2}\right\rangle=-\frac{1}{2} \lambda_{1} \Re\left(\phi_{1}^{*} \phi_{2}\right) E_{0}+\Re\left(\psi_{1}^{*} \phi_{2}\right) E_{1 c}+\frac{1}{2} \lambda_{1} \Re\left(\phi_{1}^{*} \phi_{2}\right) E_{2 c}$

$=-\frac{1}{2} \lambda_{1} \Im\left(\phi_{1}^{*} \phi_{2}\right) i \Delta_{0}+\Im\left(\psi_{1}^{*} \phi_{2}\right) i \Delta_{10}+\frac{1}{2} \lambda_{1} \Im\left(\phi_{1}^{*} \phi_{2}\right) i \Delta_{2 c}$

$=-\frac{1}{2} \lambda_{1} \Re\left(\phi_{1} \phi_{2}\right) P_{0}+\Re\left(\psi_{1} \phi_{2}\right) P_{1}+\frac{1}{2} \lambda_{1} \Re\left(\phi_{1} \phi_{2}\right) P_{2 c}$

$=\frac{1}{2} \lambda_{1} \Im\left(\phi_{1} \phi_{2}\right) Q_{0}-\Im\left(\psi_{1} \phi_{2}\right) Q_{1 c}-\frac{1}{2} \lambda_{1} \Im\left(\phi_{1} \phi_{2}\right) Q_{2 c}$

$\left\langle v_{1}^{*} u_{2}\right\rangle=\left(\frac{1}{4} \lambda_{1} \Re\left(\phi_{1}^{*} \psi_{2}\right)-\frac{3}{4} \lambda_{2} \Re\left(\psi_{1}^{*} \phi_{2}\right)\right) E_{1 s}+\left(\frac{1}{2} \Re\left(\psi_{1}^{*} \psi_{2}\right)-\frac{1}{4} \lambda_{1} \lambda_{2} \Re\left(\phi_{1}^{*} \phi_{2}\right)\right) E_{2 s}+\left(\frac{1}{4} \lambda_{1} \Re\left(\phi_{1}^{*} \psi_{2}\right)+\frac{1}{4} \lambda_{2} \Re\left(\psi_{1}^{*} \phi_{2}\right)\right) E_{9 s}+\frac{1}{8} \lambda_{1} \lambda_{2} \Re\left(\phi_{1}^{*} \phi_{2}\right) E_{4 s}$ $+\left(\frac{1}{4} \lambda_{1} \Im\left(\phi_{1}^{*} \psi_{2}\right)-\frac{3}{4} \lambda_{2} \Im\left(\psi_{1}^{*} \phi_{2}\right)\right) i \Delta_{1 v}+\left(\frac{1}{2} \Im\left(\psi_{1}^{*} \psi_{2}\right)-\frac{1}{4} \lambda_{1} \lambda_{2} \Im\left(\phi_{1}^{*} \phi_{2}\right)\right) i \Delta_{2 s}+\left(\frac{1}{4} \lambda_{1} \Im\left(\phi_{1}^{*} \psi_{2}\right)+\frac{1}{4} \lambda_{2} \Im\left(\psi_{1}^{*} \phi_{2}\right)\right) i \Delta_{9 s}+\frac{1}{8} \lambda_{1} \lambda_{2} \Im\left(\phi_{1}^{*} \phi_{2}\right) i \Delta_{4}$ $+\left(\frac{1}{4} \lambda_{1} \Re\left(\phi_{1} \psi_{2}\right)-\frac{3}{4} \lambda_{2} \Re\left(\psi_{1} \phi_{2}\right)\right) P_{1 s}+\left(\frac{1}{2} \Re\left(\psi_{1} \psi_{2}\right)-\frac{1}{4} \lambda_{1} \lambda_{2} \Re\left(\phi_{1} \phi_{2}\right)\right) P_{2 s}+\left(\frac{1}{4} \lambda_{1} \Re\left(\phi_{1} \psi_{2}\right)+\frac{1}{4} \lambda_{2} \Re\left(\psi_{1} \phi_{2}\right)\right) P_{3 s}+\frac{1}{8} \lambda_{1} \lambda_{2} \Re\left(\phi_{1} \phi_{2}\right) P_{4 s}$

$-\left(\frac{1}{4} \lambda_{1} \Im\left(\phi_{1} \psi_{2}\right)-\frac{3}{4} \lambda_{2} \Im\left(\psi_{1} \phi_{2}\right)\right) Q_{1 s}-\left(\frac{1}{2} \Im\left(\psi_{1} \psi_{2}\right)-\frac{1}{4} \lambda_{1} \lambda_{2} \Im\left(\phi_{1} \phi_{2}\right)\right) Q_{2 s}-\left(\frac{1}{4} \lambda_{1} \Im\left(\phi_{1} \psi_{2}\right)+\frac{1}{4} \lambda_{2} \Im\left(\psi_{1} \phi_{2}\right)\right) Q_{3 s}-\frac{1}{8} \lambda_{1} \lambda_{2} \Im\left(\phi_{1} \phi_{2}\right) Q_{4}$

$\left\langle v_{1}^{*} v_{2}\right\rangle=\left(\frac{1}{2} \Re\left(\psi_{1}^{*} \psi_{2}\right)+\frac{1}{8} \lambda_{1} \lambda_{2} \Re\left(\phi_{1}^{*} \phi_{2}\right)\right) E_{0}+\left(\frac{1}{4} \lambda_{1} \Re\left(\phi_{1}^{*} \psi_{2}\right)+\frac{1}{4} \lambda_{2} \Re\left(\psi_{1}^{*} \phi_{2}\right)\right) E_{1 c}-\frac{1}{2} \Re\left(\psi_{1}^{*} \psi_{2}\right) E_{2 c}-\left(\frac{1}{4} \lambda_{1} \Re\left(\phi_{1}^{*} \psi_{2}\right)+\frac{1}{4} \lambda_{2} \Re\left(\psi_{1}^{*} \phi_{2}\right)\right) E_{9 c}-\frac{1}{8} \lambda_{1} \lambda_{2} \Re\left(\phi_{1}^{*} \phi_{2}\right) E_{4 c}$ $+\left(\frac{1}{2} \Im\left(\psi_{1}^{*} \psi_{2}\right)+\frac{1}{8} \lambda_{1} \lambda_{2} \Im\left(\phi_{1}^{*} \phi_{2}\right)\right) i \Delta_{0}+\left(\frac{1}{4} \lambda_{1} \Im\left(\phi_{1}^{*} \psi_{2}\right)+\frac{1}{4} \lambda_{2} \Im\left(\psi_{1}^{*} \phi_{2}\right)\right) i \Delta_{1 c}-\frac{1}{2} \Im\left(\psi_{1}^{*} \psi_{2}\right) i \Delta_{2 c}-\left(\frac{1}{4} \lambda_{1} \Im\left(\phi_{1}^{*} \psi_{2}\right)+\frac{1}{4} \lambda_{2} \Im\left(\psi_{1}^{*} \phi_{2}\right)\right) i \Delta_{3 c}-\frac{1}{8} \lambda_{1} \lambda_{2} \Im\left(\phi_{1}^{*} \phi_{2}\right) i \Delta_{4 c}$ $+\left(\frac{1}{2} \Re\left(\psi_{1} \psi_{2}\right)+\frac{1}{8} \lambda_{1} \lambda_{2} \Re\left(\phi_{1} \phi_{2}\right)\right) P_{0}+\left(\frac{1}{4} \lambda_{1} \Re\left(\phi_{1} \psi_{2}\right)+\frac{1}{4} \lambda_{2} \Re\left(\psi_{1} \phi_{2}\right)\right) P_{1 c}-\frac{1}{2} \Re\left(\psi_{1} \psi_{2}\right) P_{2 c}-\left(\frac{1}{4} \lambda_{1} \Re\left(\phi_{1} \psi_{2}\right)+\frac{1}{4} \lambda_{2} \Re\left(\psi_{1} \phi_{2}\right)\right) P_{3 c}-\frac{1}{8} \lambda_{1} \lambda_{2} \Re\left(\phi_{1} \phi_{2}\right) P_{4 c}$ $-\left(\frac{1}{2} \Im\left(\psi_{1} \psi_{2}\right)+\frac{1}{8} \lambda_{1} \lambda_{2} \Im\left(\phi_{1} \phi_{2}\right)\right) Q_{0}-\left(\frac{1}{4} \lambda_{1} \Im\left(\phi_{1} \psi_{2}\right)+\frac{1}{4} \lambda_{2} \Im\left(\psi_{1} \phi_{2}\right)\right) Q_{1 c}+\frac{1}{2} \Im\left(\psi_{1} \psi_{2}\right) Q_{2 c}+\left(\frac{1}{4} \lambda_{1} \Im\left(\phi_{1} \psi_{2}\right)+\frac{1}{4} \lambda_{2} \Im\left(\psi_{1} \phi_{2}\right)\right) Q_{3 c}+\frac{1}{8} \lambda_{1} \lambda_{2} \Im\left(\phi_{1} \phi_{2}\right) Q_{4 c}$ 
Figure D.2 Expansions in terms of $E \Delta P Q$ direction moments (cont)

$$
\begin{aligned}
\left\langle v_{1}^{*} d_{2}\right\rangle & =\Re\left(\psi_{1}^{*} \phi_{2}\right) E_{1 s}+\frac{1}{2} \lambda_{1} \Re\left(\phi_{1}^{*} \phi_{2}\right) E_{2 s} \\
& +\Im\left(\psi_{1}^{*} \phi_{2}\right) i \Delta_{1 s}+\frac{1}{2} \lambda_{1} \Im\left(\phi_{1}^{*} \phi_{2}\right) i \Delta_{2 s} \\
& +\Re\left(\psi_{1} \phi_{2}\right) P_{1 s}+\frac{1}{2} \lambda_{1} \Re\left(\phi_{1} \phi_{2}\right) P_{2 s} \\
& -\Im\left(\psi_{1} \phi_{2}\right) Q_{1 s}-\frac{1}{2} \lambda_{1} \Im\left(\phi_{1} \phi_{2}\right) Q_{2 s} \\
\left\langle d_{1}^{*} u_{2}\right\rangle & =-\frac{1}{2} \lambda_{2} \Re\left(\phi_{1}^{*} \phi_{2}\right) E_{0}+\Re\left(\phi_{1}^{*} \psi_{2}\right) E_{1 c}+\frac{1}{2} \lambda_{2} \Re\left(\phi_{1}^{*} \phi_{2}\right) E_{2 c} \\
& -\frac{1}{2} \lambda_{2} \Im\left(\phi_{1}^{*} \phi_{2}\right) i \Delta_{0}+\Im\left(\phi_{1}^{*} \psi_{2}\right) i \Delta_{1 c}+\frac{1}{2} \lambda_{2} \Im\left(\phi_{1}^{*} \phi_{2}\right) i \Delta_{2 c} \\
& -\frac{1}{2} \lambda_{2} \Re\left(\phi_{1} \phi_{2}\right) P_{0}+\Re\left(\phi_{1} \psi_{2}\right) P_{1 c}+\frac{1}{2} \lambda_{2} \Re\left(\phi_{1} \phi_{2}\right) P_{2 c} \\
& +\frac{1}{2} \lambda_{2} \Im\left(\phi_{1} \phi_{2}\right) Q_{0}-\Im\left(\phi_{1} \psi_{2}\right) Q_{1 c}-\frac{1}{2} \lambda_{2} \Im\left(\phi_{1} \phi_{2}\right) Q_{2 c} \\
\left\langle d_{1}^{*} v_{2}\right\rangle & =\Re\left(\phi_{1}^{*} \psi_{2}\right) E_{1 s}+\frac{1}{2} \lambda_{2} \Re\left(\phi_{1}^{*} \phi_{2}\right) E_{2 s} \\
& +\Im\left(\phi_{1}^{*} \psi_{2}\right) i \Delta_{1 s}+\frac{1}{2} \lambda_{2} \Im\left(\phi_{1}^{*} \phi_{2}\right) i \Delta_{2 s} \\
& +\Re\left(\phi_{1} \psi_{2}\right) P_{1 s}+\frac{1}{2} \lambda_{2} \Re\left(\phi_{1} \phi_{2}\right) P_{2 s} \\
& -\Im\left(\phi_{1} \psi_{2}\right) Q_{1 s}-\frac{1}{2} \lambda_{2} \Im\left(\phi_{1} \phi_{2}\right) Q_{2 s} \\
\left\langle d_{1}^{*} d_{2}\right\rangle & =\Re\left(\phi_{1}^{*} \phi_{2}\right) E_{0}+\Im\left(\phi_{1}^{*} \phi_{2}\right) i \Delta_{0}+\Re\left(\phi_{1} \phi_{2}\right) P_{0}-\Im\left(\phi_{1} \phi_{2}\right) Q_{0} \\
\left\langle u_{1}^{*} p_{1}\right\rangle & =\Re\left(\psi_{1}^{*} \psi_{1}\right) E_{1 c}+\Re\left(\psi_{1} \psi_{1}\right) P_{1 c}-\Im\left(\psi_{1} \psi_{1}\right) Q_{1 c} \\
\left\langle v_{1}^{*} p_{1}\right\rangle & =\Re\left(\psi_{1}^{*} \psi_{1}\right) E_{1 s}+\Re\left(\psi_{1} \psi_{1}\right) P_{1 s}-\Im\left(\psi_{1} \psi_{1}\right) Q_{1 s} \\
\left\langle w_{1}^{*} p_{1}\right\rangle & =\Im\left(\phi_{1}^{*} \psi_{1}\right) \Delta_{0} \sigma / N_{1} \\
w &
\end{aligned}
$$

where the vertical velocity $w$ is given by

$$
w_{j}=-i \sigma_{j} d_{j} / N_{j}
$$




\section{Appendix E: Model-Model covariance for the $E \triangle P Q$ direction moments}

The theory needed to compute the model-model covariance of the direction moments so that they properly represent a field of statistically independent waves is given in section 8.5. What follows here is an explicit derivation of the model-model covariance needed to calculate the results of chapter 8 .

Because the linear relation $T_{\alpha}(\theta, g)$ between the direction moments $e_{\beta}$ and the wave energy $s(\theta, g)$ factors into separate functions of direction $\theta$ and wave/mode index $g$,

$$
T_{\alpha}(\theta, g)=T_{\alpha}(\theta) T_{\alpha g}
$$

it suffices to consider the $E \triangle P Q$ transformation $T_{\alpha g}$ and direction moment transformation $T_{\alpha}(\theta)$ separately.

First consider the $E \triangle P Q$ transformation. The transformation matrix $T_{\alpha g}$ is given by equation 8.3.7 $a$ and the inverse transformation $U_{\alpha g}$ is given by equation 8.3.7b. Plugging these two matrices into the expression for model space norm 8.5.16 gives the model space norm for the direction moments $N_{\alpha \beta}$ in terms of the model space norm for the wave energies $M_{\alpha \beta}$,

$$
\frac{1}{4}\left[\begin{array}{cccc}
M_{11}+M_{22} & M_{11}-M_{22} & \left(M_{11}+M_{22}\right) & \left(M_{11}+M_{22}\right) \\
M_{11}-M_{22} & M_{11}+M_{22} & \left(M_{11}-M_{22}\right) & \left(M_{11}-M_{22}\right) \\
\left(M_{11}+M_{22}\right) & \left(M_{11}-M_{22}\right) & \left(M_{11}+M_{22}+M_{33}\right) & \left(M_{11}+M_{22}\right) \\
\left(M_{11}+M_{22}\right) & \left(M_{11}-M_{22}\right) & \left(M_{11}+M_{22}\right) & \left(M_{11}+M_{22}+M_{44}\right)
\end{array}\right]
$$

The diagonal (and only non-zero) elements of $M_{\psi \phi}$ are

$$
\begin{aligned}
& M_{11}=4\left[E^{(0)}+\Delta^{(0)}+P^{(0)}+Q^{(0)}\right]^{-2}+2\left[R_{i i E}+R_{i i \Delta}\right]\left[E^{(0)}+\Delta^{(0)}+P^{(0)}+Q^{(0)}\right]^{-1} \\
& M_{22}=4\left[E^{(0)}-\Delta^{(0)}+P^{(0)}+Q^{(0)}\right]^{-2}+2\left[R_{i i E}-R_{i i \Delta}\right]\left[E^{(0)}-\Delta^{(0)}+P^{(0)}+Q^{(0)}\right]^{-1} \\
& M_{33}=4\left[P^{(0)}\right]^{-2}-2\left[2 R_{i i E}-2 R_{i i P}\right]\left[P^{(0)}\right]^{-1} \\
& M_{44}=4\left[Q^{(0)}\right]^{-2}-2\left[2 R_{i i E}-2 R_{i i Q}\right]\left[Q^{(0)}\right]^{-1}
\end{aligned}
$$




\section{Derivation of model model covariance matrix for direction}

The derivation of the model-model covariance for direction moments is somewhat complicated by the continuous nature of the direction $\theta$. But by using continuous analogs of the equations in section 8.5 it is possible to treat the discrete set of direction moments so that they represent a continuous field of statistically independent waves.

To simplify the problem somewhat, I am going to presume that I have already transformed into the energy space of some vertical structure, and now all $\mathrm{I}$ have to do is go from a set of moments $\left\{a_{0}, a_{1 s}, a_{1 c}, \ldots\right\}$ to energy as a function of direction $a(\theta)$. Consider the Fourier transform relations,

$$
\begin{aligned}
a_{n} & =\frac{1}{2 \pi} \int_{0}^{2 \pi} a(\theta) e^{-i n \theta} d \theta \\
a(\theta) & =\sum_{n=-\infty}^{\infty} a_{n} e^{i n \theta}
\end{aligned}
$$

These Fourier transform relations are written in terms of complex Fourier coefficients $a_{n}$. These can be translated to coefficients of sines and cosines by

$$
\begin{aligned}
a_{n} & =a_{n c}-i a_{n s} \\
a_{-n} & =a_{n c}+i a_{n s} \\
a_{n c} & =\left(a_{n}+a_{-n}\right) / 2 \\
a_{n s} & =i\left(a_{n}-a_{-n}\right) / 2
\end{aligned}
$$

Also very important is the finite delta function relation

$$
\frac{1}{2 \pi} \int_{0}^{2 \pi} e^{i(m-n) \theta} d \theta=\delta_{n m}
$$

The continuous version of the model model covariance product given in equation 8.5.13 is

$$
\epsilon^{2}=\frac{1}{2 \pi} \int_{0}^{2 \pi} a(\theta) M(\theta) a(\theta) d \theta
$$

There are two important features of this expression. First of all, unlike the covariance product for the Fourier coefficients there is no complex conjugate on $a(\theta)$. This is because $a(\theta)$ is real (energy). The single integral over direction is a result of presuming that difference directions are uncorrelated.

Substituting the Fourier expansion of $a(\theta)$ into equation $E .7$, we get

$$
\epsilon^{2}=\frac{1}{2 \pi} \int_{0}^{2 \pi} \sum_{n=-\infty}^{\infty} \sum_{m=-\infty}^{\infty} a_{n} a_{m} M(\theta) e^{i(n+m) \theta} d \theta
$$


$M(\theta)$ is the model model covariance for an independent mode/wave, i.e. it has the form

$$
M(\theta)=\frac{1}{\hat{a}^{2}}+\frac{2 F_{i i}(\theta)}{\hat{a}}
$$

where $\hat{a}$ is the a priori value for the energy $a(\theta)$, and $F_{i i}(\theta)$ is the sum over the diagonal elements of the model prediction for the wave at $\theta$. If the wavefield is presumed to be isotropic, $\hat{a}$ is to be independent of direction. Our understanding of $F_{i j}(\theta)$ comes from the linear expression of the problem, namely

$$
\left\langle d_{i}^{*} d_{j}\right\rangle=\frac{1}{2 \pi} \int_{0}^{2 \pi} F_{i j}(\theta) a(\theta) d \theta=\sum_{n=-\infty}^{\infty} F_{-n} a_{n}
$$

where we have written $\left\{F_{n}\right\}$ as the Fourier transform coefficients of $F_{i i}(\theta)$. This means we can write the Fourier transform of $M(\theta)$ as

$$
M_{n}=\frac{1}{\hat{a}^{2}} \delta_{n 0}+\frac{2 F_{n}}{\hat{a}}
$$

This allows us to rewrite the residue $\epsilon^{2}$ in terms of Fourier coefficients

$$
\epsilon^{2}=\sum_{n=-\infty}^{\infty} \frac{a_{n} a_{-n}}{\hat{a}^{2}}+\sum_{n=-\infty}^{\infty} \sum_{m=-\infty}^{\infty} a_{n} a_{m} \frac{2 F_{-(n+m)}}{\hat{a}}
$$

The problem remains to translate this result so that it is in terms of the cosine and sine moments rather than the complex Fourier coefficients.

The translation is

$$
\begin{aligned}
a_{n} & =a_{n c}-i a_{n s} \\
F_{-n} & =F_{n c}+i F_{n s}
\end{aligned}
$$

Do the first term first, since it is simpler. The answer becomes

$$
\begin{aligned}
\sum_{n=-\infty}^{\infty} \frac{a_{n} a_{-n}}{\hat{a}^{2}} & =\sum_{n=-\infty}^{\infty} \frac{a_{n c}^{2}+a_{n s}^{2}}{\hat{a}^{2}} \\
& =\sum_{n=0}^{\infty} \frac{a_{n c}^{2}+a_{n s}^{2}}{\hat{a}^{2}} \begin{cases}1 & n=0 \\
2 & \text { otherwise }\end{cases}
\end{aligned}
$$

The second term is more complicated. First expand the object of the double summation,

$$
\begin{aligned}
a_{n} a_{m} F_{-(n+m)}= & \left(a_{n c}-i a_{n s}\right)\left(a_{m c}-i a_{m s}\right)\left(F_{(n+m) c}+i F_{(n+m) s}\right) \\
= & \left(a_{n c} a_{m c}-a_{n s} a_{m s}-i\left[a_{n c} a_{m s}+a_{n s} a_{m c}\right]\right) F_{(n+m) c} \\
& +\left(a_{n c} a_{m s}+a_{n s} a_{m e}+i\left[a_{n c} a_{m c}-a_{n s} a_{m s}\right]\right) F_{(n+m) s}
\end{aligned}
$$


Next consider applying the double summation. Since the sine coefficients are odd under index reflection while the cosine coefficients are even under the same transformation, any term that has an odd number of sine coefficients will sum to zero. This eliminates both imaginary parts. This leaves us with

$$
\begin{array}{r}
\sum_{n=-\infty}^{\infty} \sum_{m=-\infty}^{\infty} a_{n} a_{m} F_{-(n+m)}=\sum_{n=-\infty}^{\infty} \sum_{m=-\infty}^{\infty}\left(a_{n c} a_{m c}-a_{n s} a_{m s}\right) F_{(n+m) c} \\
+\left(a_{n c} a_{m s}+a_{n s} a_{m c}\right) F_{(n+m) s}
\end{array}
$$

The next simplification involves changing the sums to go from 0 rather than $-\infty$. As is usual with sines and cosines, $n=0$ is a special case.

$$
\begin{aligned}
& \sum_{n=-\infty}^{\infty} \sum_{m=-\infty}^{\infty} a_{n} a_{m} F_{-(n+m)}= \\
& \sum_{n=0}^{\infty} \sum_{m=0}^{\infty}\left(a_{n c} a_{m c}-a_{n s} a_{m s}\right) F_{(n+m) c}+\left(a_{n c} a_{m s}+a_{n s} a_{m c}\right) F_{(n+m) s} \\
& +\left(a_{n c} a_{m c}+a_{n s} a_{m s}\right) F_{(n-m) c}+\left(-a_{n c} a_{m s}+a_{n s} a_{m c}\right) F_{(n-m) s} \begin{cases}\frac{1}{2} & n=m=0 \\
1 & n=0 \text { or } m=0 \\
2 & n \neq 0 \text { and } m \neq 0\end{cases}
\end{aligned}
$$

Gathering terms so that the terms correspond to the desired matrix product

$$
\begin{aligned}
& \sum_{n=-\infty}^{\infty} \sum_{m=-\infty}^{\infty} a_{n} a_{m} F_{-(n+m)}= \\
& \sum_{n=0}^{\infty} \sum_{m=0}^{\infty}\left(F_{(n-m) c}+F_{(n+m) c}\right) a_{n c} a_{m c}+\left(F_{(n-m) c}-F_{(n+m) c}\right) a_{n s} a_{m s} \\
& +\left(F_{(n+m) s}-F_{(n-m) s}\right) a_{n c} a_{m s}+\left(F_{(n+m) s}+F_{(n-m) s}\right) a_{n s} a_{m c} \begin{cases}\frac{1}{2} & n=m=0 \\
1 & n=0 \text { or } m=0 \\
2 & n \neq 0 \text { and } m \neq 0\end{cases}
\end{aligned}
$$

The final result is then

$$
\epsilon^{2}=\sum_{n=0}^{\infty} \frac{a_{n c}^{2}+a_{n s}^{2}}{\hat{a}^{2}} \begin{cases}1 & n=0 \\ 2 & \text { otherwise }\end{cases}
$$




$$
\begin{gathered}
+\frac{2}{\hat{a}} \sum_{n=0}^{\infty} \sum_{m=0}^{\infty}\left(F_{(n-m) c}+F_{(n+m) c}\right) a_{n c} a_{m c}+\left(F_{(n-m) c}-F_{(n+m) c}\right) a_{n s} a_{m s} \\
+\left(F_{(n+m) s}-F_{(n-m) s}\right) a_{n c} a_{m s}+\left(F_{(n+m) s}+F_{(n-m) s}\right) a_{n s} a_{m c} \begin{cases}\frac{1}{2} & n=m=0 \\
1 & n=0 \text { or } m=0 \\
2 & n \neq 0 \text { and } m \neq 0\end{cases}
\end{gathered}
$$




\section{Appendix F: Generalized rank}

In chapter 8 rank is defined to be the number of model parameters that can be resolved. In the literature rank is used to characterized unbiased estimators and thus is always an integer: the rank is the number of non-redundant equations or the number of model parameters, whichever is smaller. A somewhat better definition that encompasses both possibilities is that rank is the number of non-zero eigenvalues (or singular values) of the model matrix A. The definition used here is that the rank is the trace of the model space resolution matrix: given a linear forward problem that gives data $\mathbf{d}$ as a function of model parameters $\mathbf{m}$,

$$
\mathbf{d}=\mathbf{A} \mathbf{m},
$$

and a linear inverse to that problem,

$$
\widehat{\mathbf{m}}=\mathbf{B d},
$$

then the model space resolution matrix is the matrix product $\mathbf{B A}$, and the rank $r$ is given by

$$
r=\sum_{i j} B_{j i} A_{i j}
$$

If an unbiased estimator such as the Singular Value Decomposition (Lanczos 1963) is used to find the generalized inverse $\mathbf{B}$, then equation $F .3$ returns the number of non-zero eigenvalues. If a biased estimator such as Gauss-Markov (Menke 1984) is used to find $\mathbf{B}$, then $F .3$ returns the number of model parameters that are significantly greater than noise: i.e. eigenvalues much greater than the noise count as one and eigenvalues much less than the noise count as zero. Thus $r$ is again roughly the number of parameters that can be resolved, though with the biased estimator the effects of noise have been included. Since equation $F .3$ coincides with the standard use of the word rank in all situations where the standard use is applicable, and measures a similar property in the biased case where the standard usage is not directly applicable, equation $F .3$ is taken to define rank. 


\section{Appendix $G$ Beamforming and Capon estimators}

There are two commonly used wavenumber estimation schemes that can be classified as single wave methods: beamforming and Capon (maximum likelihood) (Capon 1969, Bretherton and McWilliams 1980). These are single wave methods in that they fit one wave at a time to the data, repeating the process with many wavenumbers to arrive at a wavenumber spectrum. Equation 8.3.1 thus reduces to the vector relation which relates the measurements to single wave amplitude

$$
d_{i}^{(\gamma)}=H_{i} a^{(\gamma)}
$$

Note that in wavenumber estimation the effects of non-trivial polarization relations are absent and the vertical structure equation has propagating solutions that are simple exponentials. Consequently the model vector $H_{j}$ is given by a simple exponential $e^{i m z_{j}}$, where $m$ is the wavenumber that corresponds to the wave $\alpha$ being considered.

Equation 8.3.3 shows that the spectral energy $s_{\alpha}$ can be considered to be the mean square value of the wave amplitude $a_{\alpha}^{(\gamma)}$, that average being computed over all realizations. The beamforming estimation scheme effectively estimates a wave amplitude for each realization by a simple least squares inversion and then calculates the mean square wave amplitude,

$$
\begin{aligned}
a^{(\gamma)} & =\left(\mathbf{H}^{\dagger} \mathbf{H}\right)^{-1} \mathbf{H}^{\dagger} d^{(\gamma)} \\
& =N_{t s}^{-1} \sum_{j} e^{-i m z_{j}} d_{j}^{(\gamma)}
\end{aligned}
$$

(Note that $\mathbf{H}$ is actually a vector and not a matrix). This estimator is the same as the Gauss-Markov estimator (equation 8.4.4) with model space norm $\mathbf{L}$ identically zero and residual norm $\mathbf{W}$ being the identity matrix. When this relation is squared and averaged a more familiar form for the beamforming estimator is recovered,

$$
\left\langle a^{*} a\right\rangle=N_{t g}^{-2} \sum_{j=1}^{N_{t g}} \sum_{l=1}^{N_{t g}} e^{i m\left(z_{j}-z_{l}\right)}\left\langle d_{j}^{*} d_{l}\right\rangle
$$

While the beamforming estimate is essentially a best fit of the wave amplitude to the data for each realization, no attempt has been made to consider the covariance structure of the data. As it turns out, the beamforming estimate treats correctly the data noise covariance if the noise in each Fourier coefficient is uncorrelated. To the extent that the data is well 
modeled by a small set of waves, the data noise covariance will be more structured, a structure that should be considered in forming an optimal estimator.

The Capon wavenumber estimator (Capon 1969) differs from the beamforming estimator only in that a different residual covariance matrix $\mathbf{W}$ is used to estimate $a^{(\gamma)}$ from the data in each realization: equation G.2 is replaced by

$$
\boldsymbol{a}^{(\gamma)}=\left(\mathbf{H}^{\dagger} \mathbf{Q}^{*} \mathbf{H}\right)^{-1} \mathbf{H}^{\dagger} \mathbf{Q}^{*} \mathbf{d}
$$

so that the complex conjugate inverse $Q_{i j}^{*}$ of the true cross-spectral matrix $f_{i j}^{(0)}$ is used for the residual norm $W_{i j}$.

$$
\begin{aligned}
Q_{i j} & =f_{i j}^{(0)}-1 \\
\hat{f}_{i j} & =\left\langle d_{i}^{*} d_{j}\right\rangle
\end{aligned}
$$

$\hat{f}_{i j}$ is the estimate of the true value $f_{i j}^{(0)}$. A more familiar form of the Capon estimator is found by calculating the mean square amplitude as was done for the beamforming estimator. In addition, rather than using the inverse $Q_{i j}$ of the true covariance, the inverse $\widehat{Q}_{i j}$ of the estimated covariance is used. This simplifies the algebraic form of the estimator greatly,

$$
\begin{aligned}
s_{\alpha}=\left\langle a^{*} a\right\rangle & =\mathbf{H}^{\dagger} \widehat{\mathbf{Q}}^{*} \mathbf{H}^{-1} \\
& =\left(\sum_{j l} e^{i\left(z_{j}-z_{l}\right) m} \widehat{Q}_{j l}^{*}\right)^{-1}
\end{aligned}
$$

As show in section 8.4 , choosing the residual norm $W_{i j}$ to be the complex conjugate inverse covariance $Q_{i j}^{*}$ means that the Capon estimator is optimal in a statistical sense. But while the Capon estimator is optimal for the problem of fitting a single wave to data, it is not necessarily the optimal estimator for fitting a set of waves to data. 


\section{References}

Abramowitz, M, and I Stegun, 1972 Handbook of mathematical functions Dover, New York, NY. $\langle 1.6\rangle$

Acton, FS, 1970 Numerical methods that work. Harper and Row, New York, NY. $\langle 7.2\rangle$

Bateman, H, 1954 Table of integral transforms. McGraw-Hill, New York, NY. $\langle 1.6\rangle$

Bell, TH, 1975 Topographically generated internal waves in the open ocean Journal of Geophysical Research 80:320-327. $\langle 8.7\rangle$

Bevington, PR, 1969 Data reduction and error analysis for the physical sciences. McGraw-Hill, New York, NY. $\langle 6.2,6.5,8.7\rangle$

Booker, JR, and FP Bretherton, 1967 The critical layer for internal gravity waves in a shear flow. Journal of Fluid Mechanics 27:513-554. $\langle 5.1,7.6\rangle$

Bracewell, R, 1978 The Fourier transform and its applications, 2nd ed. McGraw-Hill, New York, NY. $\langle 5.7,7.5\rangle$

Bretherton, FP, 1966 The propagation of groups of internal gravity waves in a shear flow. Quarterly Journal of the Royal Meteorlogical Society 92:466-480. $\langle 5.1\rangle$

Bretherton, FP, and C Garrett, 1969 Wavetrains in inhomogeneous moving media. Proceedings of the Royal Society A 302:466-480. $\langle 5.1\rangle$

Bretherton, FP, and JC McWilliams, 1980 Estimation from irregular arrays. Reviews of Geophysics and Space Physics 18:789-812. 〈8.1, 8.4, 8.5〉

Briscoe, M, 1977 Gaussianity of Internal Waves. Journal of Geophysical Research $82: 2117-2126 .\langle 1.3\rangle$

Capon, J, 1969 High resolution frequency-wavenumber spectrum analysis. Proceedings of the IEEE 57:1408-1418. $\langle 8.4, \mathrm{G}\rangle$

Cane, MA, 1980 On the dynamics of equatorial currents, with applications to the Indian Ocean. Deep-Sea Research 27A:525-544. $\langle 5.2\rangle$

Cairns, JL, 1975 Internal wave observations from a midwater float. Journal of Geophysical Research 80:299-305. 〈1.2〉

Cairns, JL, and GO Williams, 1976 Internal wave observations from a midwater float,2. Journal of Geophysical Research 81:1943-1950. 〈1.0, 2.2, 3.1, 3.2〉

Charnock, H, 1965 A preliminary study of the directional spectrum of short period internal waves. Proceedings of the second US Navy Symposium on Military Oceanography 175-178. $\langle 1.2\rangle$

Davis, R, and L Regier, 1977 Methods for estimating directional wave spectra from multi-element arrays. JMR 35:453-477. $\langle 6.1\rangle$ 
deBoor, C, and JR Rice, 1968 Cubic spline approximation II - variable knots. Computer sciences department TR21, Purdue University. $\langle 7.6\rangle$

Desaubies, YJF, 1975 A linear theory of internal wave spectra and coherences near the Väisälä frequency. Journal of Geophysical Research 80:895-899. 〈2,2 3.4〉

Desaubies, YJF, 1976 Analytical representation of internal wave spectra. Journal of Physical Oceanography 6:976-981. $\langle 1.0,1.6\rangle$

Desaubies, YJF, and MG Gregg, 1981 Reversible and irreversible finestructure. Journal of Physical Oceanography 11:541-566. $\langle 1.1\rangle$

Desaubies, YJF, and W Smith, 1982 Statistics of Richardson number and instability in oceanic internal waves. Journal of Physical Oceanography 12:1245-1259. $\langle 2.2,4.4,7.1,7.5\rangle$

Eriksen, CC, 1980 $a$ Deep currents and their interpretation as equatorial waves in the Western Pacific Ocean. Journal of Physical Oceanography 11:48-70. $\langle 2.1\rangle$

Eriksen, CC, $1980 b$ Evidence for a continous spectrum of equatorial waves in the Indian Ocean. Journal of Geophysical Research 85:3285-3303. $\langle 4.3\rangle$

Eriksen, CC, 1982 Observations of internal wave reflections off sloping bottoms. Journal of Geophysical Research 87:525-538. $\langle 1.2\rangle$

Eriksen, CC, MB Blumenthal, SP Hayes, and P Ripa, 1983 Wind-Generated equatorial Kelvin waves observed across the Pacific Ocean. Journal of Physical Oceanography 13:1622-1640. $\langle 8.7\rangle$

Eriksen, CC, 1985 Some characteristics of internal gravity waves in the equatorial Pacific. Journal of Geophysical Research 90:7243-7255. 〈5.3〉

Flatté, SM, R Dashen, WH Munk, KM Watson, and F Zachariasen, 1979 Sound transmission through a fluctuating ocean. Cambridge University Press, Cambridge England. $\langle 7.5\rangle$

Fofonoff, NP, 1966 Oscillation modes of a deep-sea mooring. Geo-Marine Technology 2:13-17. $\langle 1.2\rangle$

Fofonoff, NP, 1969 Spectral characteristics of internal waves in the ocean. Deep-Sea Research 16 suppl. 59-72. $\langle 1.2,1.5,4.5\rangle$

Fofonoff, NP, 1969 Role of the NDBS in future natural variability studies of the North Atlantic. First Science Advisory Meeting, National Data Buoy Development Project, US Coast Guard. $\langle 1.2,1.5\rangle$

Fu, LL, 1980 Observations and models of inertial waves in the deep ocean. $\mathrm{PhD}$ Thesis, MIT-WHOI. $\langle 1.1,1.6\rangle$

Garrett, C, and W Munk, 1972 Space-time scales of internal waves. Geophysical Fluid Dynamics 2:225-264. $\langle 1.0\rangle$ 
Garrett, C, and W Munk, 1975 Space-time scales of internal waves: A progress report. Journal of Geophysical Research 80:291-297. $\langle 1.0\rangle$

Garrett, C, and W Munk, 1979 Internal waves in the ocean. Annual Review of Fluid Mechanics 11:339-369. $\langle 1.0\rangle$

Gent, P, and J Luyten, 1985 How much energy propagates vertically in the equatorial oceans? Journal of Physical Oceanography 15:997-1007. $\langle 5.1,5.6,7.2,7.3,7.4\rangle$

Gill, AE, 1982 Atmosphere-Ocean Dynamics. Academic Press, New York, NY. $\langle 1.3,5.4\rangle$

Goodman, NR, 1957 On the joint estimation of the spectra, cospectrum, and quadrature spectrum of a two-dimensional stationary gaussian process. Science Paper No. 10, Engineering Statistics Laboratory, New York University, New York, NY. $\langle 2.2\rangle$

Gould, WJ, 1971 Spectral characteristics of some current records from the eastern North Atlantic . Philosophical Transactions of the Royal Society . $\langle 1.2\rangle$

Howard, LN, 1961 Note on a paper of John W. Miles. Journal of Fluid Mechanics 10:509-512. $\langle 7.6, \mathrm{D}\rangle$

Hayes, SP, 1978 Temperature finestructure observations in the tropical North Pacific Ocean. Journal of Geophysical Research 83:5099-5104. $\langle 7.5\rangle$

Hayes, SP, and CJ Powell, 1980 Vertical wavenumber spectra of temperature finestructure in the Equatorial Pacific. Journal of Geophysical Research 85:4029-4035. $\langle 1.0,7.5\rangle$

Jenkins, GM, and DG Watts, 1968 Spectral Analysis and its Applications. Holden Day, San Francisco, CA. $\langle 1.2,4.4,6.2,8.4\rangle$

Johnson, RA, and DW Wichern, 1982 Multivariate Statistical Analysis. Prentice-Hall, Inc., Englewood Cliffs, NJ. $\langle 2.2\rangle$

Jones, WL, 1968 Reflexion and stability of waves in stably stratified fluids with shear flow: a numerical study. Journal of Fluid Mechanics 34:609-624. $\langle 5.1,7.6\rangle$

Joyce, TM, and YJF Desaubies, 1977 Discrimination between internal waves and temperature finestructure. Journal of Physical Oceanography 7:22-32. $\langle 7.5\rangle$

Katz, E, 1975 Tow spectra from MODE. Journal of Geophysical Research 80:1163-1167. $\langle 1.2\rangle$

Koopmans, LH, 1974 The spectral analysis of time series. Academic Press, New York, NY. $\langle 5.7,5.8\rangle$

Kunze, E, 1985 Near-Inertial wave propagation in geostrophic shear. Journal of Physical Oceanography 15:544-565. $\langle 7.6\rangle$

LaFond, EC, and KG LaFond, 1971 Thermal structure through the California front. Report N.U.C. TP 244,133.〈1.2

Lanczos, C, 1961 Linear Differential Operators. D. Van Nostrand Company, Princeton NJ. $\langle 5.4,8.4, \mathrm{~F}\rangle$ 
Levine, M, 1985 Internal wave climatology: an update. Internal gravity waves and small-scale turbulence, Proceedings of the 'Aha Huliko' a Hawaiian Winter Workshop University of Hawaii at Manoa, January 17-20 1984. eds. Peter Müller and Rita Pujalet Hawaii Institute of Geophysics Special Publication. $\langle 3.4\rangle$

Lighthill, MJ, 1968 Waves in Fluids. Cambridge Press. $\langle 4.6,5.1\rangle$

Lukas, R, SP Hayes, and $\mathbf{K}$ Wyrtki, 1984 Equatorial sea level response during the 1982-1983 E1 Niño Journal of Geophysical Research 89:10425-10430. 〈8.7〉

Luyten, JR, and JC Swallow, 1976 Equatorial undercurrents. Deep-Sea Research 23:999-1001. $\langle 2.1\rangle$

McComas, CH, and FP Bretherton, 1977 Resonant interaction of oceanic internal waves. Journal of Geophysical Research 82:1397-1412. $\langle 2.2\rangle$

Martin, S, WF Simmons, and CI Wunsch, 1969 Resonant internal wave interactions. Nature 224:1014-1016. $\langle 1.3\rangle$

Menke, W, 1984 Geophysical Data Analysis: Discrete Inverse Theory. Academic Press. $\langle 6.4,8.4\rangle$

Miles, JW, 1967 On the stability of heterogeneous shear flows. Journal of Fluid Mechanics 10:496-508. $\langle 7.5, \mathrm{D}\rangle$

Mueller, P, 1976 On the diffusion of momentum and mass by internal gravity waves. Journal of Fluid Mechanics 77:789-823. 〈1.2〉

Müller, P, and DJ Olbers, 1975 On the dynamics of internal waves in the deep ocean. Journal of Geophysical Research 80:3848-3860. $\langle 2.2\rangle$

Müller, P, DJ Olbers, and J Willebrand, 1978 The IWEX spectrum. Journal of Geophysical Research 83:479-500. $\langle 1.2,5.1,6.1,6.2\rangle$

Müller, P, and G Siedler, 1976 Consistency relations for internal waves. Deep-Sea Research 23:613-628. $\langle 6.1\rangle$

Munk, WH, 1980 Internal wave spectra at the buoyancy and inertial frequencies. Journal of Physical Oceanography 10:1718-1728. $\langle 1.0,1.1,5.4\rangle$

Munk, W, 1981 "Internal waves and Small Scale Processes" Evolution of Physical Oceanography, eds. Warren and Wunsch MIT Press, Cambridge MA. $\langle 1.0,1.6,4.3\rangle$

Munk, WH, and N Phillips, 1968 Coherence and band-structure of inertial motion in the sea. Rev. Geophys. 6:447-472. $\langle 1.4\rangle$

National Oceanographic Instrumentation Center, 1974 Instrument Fact Sheet: Models RCM-4 and RCM-5 Aanderaa Recording Current Meters. NOAA July, 1974. 〈A〉

Olbers, DJ, 1981 The propagation of Internal Waves in a Geostropic Current. Journal of Physical Oceanography 11:1224-1099. $\langle 4.6,5.1\rangle$ 
Olbers, DJ, 1981 A formal theory of internal wave scattering with applications to ocean fronts. Journal of Physical Oceanography 11:1078-1099. 〈5.1〉

Olbers, DJ, 1983 Models of the Oceanic Internal Wave Field. Reviews of Geophysics and Space Physics 21.7:1567-1606. $\langle 3.4,8.7\rangle$

PEQUOD , 1980 PEQUOD cover document, OMNET Boston MA. $\langle 2.1\rangle$

Perkins, HT, 1970 Inertial oscillations in the Mediterranean. MIT-WHOI thesis. $\langle 1.2\rangle$

Peters, H, 1980 Dispersion curves and modes of high frequency internal waves during JASIN '78. Jasin News 18:1-3. 〈3.4〉

Peters, H, 1983 The kinematics of a stochastic field of internal waves modified by a mean shear current. Deep-Sea Research 30.2A:119-148. $\langle 3.4,5.1\rangle$

Philander, G, 1978 Forced oceanic motions. Reviews of Geophysics and Space Physics 16:15-46. $\langle 5.1,7.3\rangle$

Phillips, OM, 1980 The dynamics of the upper ocean, 2ed. Cambridge University Press, Cambridge England. $\langle 1.3,1.4,5.1\rangle$

Pinkel, R, 1975 Upper ocean internal wave observations from FLIP. Journal of Geophysical Research 80:3892-3910. $\langle 3.4\rangle$

Ruddick, BR, 1977 Observations of interaction between the internal wavefield and lowfrequency flows in the North Atlantic. PhD thesis MIT-WHOI Joint Program in Oceanography and Ocean Engineering. $\langle 1.2\rangle$

Ruddick, BR, and TM Joyce, 1979 Observations of interaction between the internal wavefield and low-frequency flows in the North Atlantic. Journal of Physical Oceanography 9:500-517. $\langle 2.1\rangle$

Tarbell, SA, ET Montgomery, and MG Briscoe, 1985 A compilation of moored current meter and wind recorder data volume XXXVIII Long-Term Upper Ocean Study (LOTUS) (Moorings 787788789790 792) April 1983-May 1984. WHOI technical report WHOI-85-39. $\langle 3.3\rangle$

Thorpe, SA, 1975 The excitation, dissipation, and interaction of internal waves in the deep ocean. Journal of Geophysical Research 80:328-338. 〈8.7〉

Toole, JM, and SP Hayes, 1984 Finescale velocity-density characteristics and Richardson number statistics of the Eastern Equatorial Pacific Journal of Physical Oceanography 14:712-726. $\langle 7.1\rangle$

Voorhis, A, 1968 Measurements of vertical motion and the partition of energy in the New England slope water. Deep-Sea Research 15:599-608. $\langle 1.2\rangle$

Webster, F, 1968 Observations of inertial period motions in the deep sea. Reviews of Geophysics 6:473-490. $\langle 1.2\rangle$ 
Webster, F, 1968 Vertical profiles of horizontal ocean currents. Deep-Sea Research $16: 85-98 .\langle 1.2\rangle$

Webster, F, 1970 Lectures. Liege University Second Colloq. on the Hydrodynamics of the Ocean 20-53. $\langle 1.2\rangle$

Webster, F, 1971 Estimates of the coherences of ocean currents over vertical distances. Deep-Sea Research 19:35-44. $\langle 1.2\rangle$

Weller, RA, RE Payne, WG Large, and W Zenk, 1983 Wind measurements from an array of oceanographic moorings and from F.S. METEOR during JASIN 1978. Journal of Geophysical Research 88:9689-9705. 〈4.6〉

Wunsch, C, 1975 Deep ocean internal waves: What do we really know? Journal of Geophysical Research 80:339-343. $\langle 1.2\rangle$

Wunsch, C, 1976 Geographic variability of the internal wave field: A search for sources and sinks. Journal of Physical Oceanography 6:471-485. $\langle 1.0,4.4\rangle$

Wunsch, C, and J Dahlen, 1974 A moored temperature and pressure recorder. Deep-Sea Research 21:145-154. $\langle 2.1\rangle$

Wunsch, C, and S Webb, 1979 The climatology of deep ocean internal waves. Journal of Physical Oceanography 9:235-243. $\langle 1.2,3.1,4.3\rangle$ 
